\title{
Energy Availabilities for State and Local Development: 1973 Data Volume
}

$$
\begin{aligned}
& \text { D. P. Vogt } \\
& \text { P. L. Rice } \\
& \text { V. P. Pai }
\end{aligned}
$$

\section{OAK RIDGE NATIONAL LABORATORY}




\section{DISCLAIMER}

This report was prepared as an account of work sponsored by an agency of the United States Government. Neither the United States Government nor any agency Thereof, nor any of their employees, makes any warranty, express or implied, or assumes any legal liability or responsibility for the accuracy, completeness, or usefulness of any information, apparatus, product, or process disclosed, or represents that its use would not infringe privately owned rights. Reference herein to any specific commercial product, process, or service by trade name, trademark, manufacturer, or otherwise does not necessarily constitute or imply its endorsement, recommendation, or favoring by the United States Government or any agency thereof. The views and opinions of authors expressed herein do not necessarily state or reflect those of the United States Government or any agency thereof. 


\section{DISCLAIMER}

Portions of this document may be illegible in electronic image products. Images are produced from the best available original document. 


\section{Printed in the United States of America. Available from National Technical Information Service \\ U.S. Department of Commerce 5285 Port Royal Road, Springfield, Virginia 22161}

Price: Printed Copy $\$ 10.75$; Mlcrulicle $\$ 3.00$

This report was prepared as an account of work sponsored by the United States Government. Neither the United States nor the Energy Research and Development Administration/United States Nuclear Regulatory Commission, nor any of their employees, nor any of their contractors, subcontractors, or their employees, makes any warranty, express or implied, or assumes any legal liability or responsibility for the accuracy, completeness or usefulness of any information, apparatus, product or process disclosed, or represents that its use would not infringe privately owned rights. 
ORNL/TM-5890/S 2

Contract No. W-7405-eng-26

ENERGY AVAILABILITIES FOR STATE AND LOCAL DEVELOPMENT: 1973 DATA VOLUME

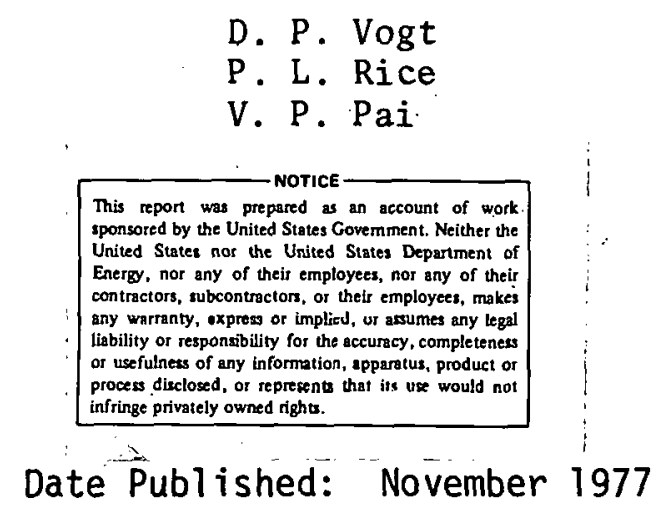
Regional and Urban Studies Section
Energy Division

Research sponsored by the Economic Development. Administration of the

U.S. Department of Commerce

\author{
OAK RIDGE NATIONAL LABORATORY \\ OAK RIDGE, TENNESSEE 37830 \\ operated by \\ UNION CARBIDE CORPORATION \\ for the \\ DEPARTMENT OF ENERGY
}




\section{THIS PAGE}

WAS INTENTIONALLY

LEFT BLANK 
CONTENTS

$\underline{\text { Page }}$

ABSTRACT .......................... . $v$

I. INTRODUCTION . . . . . . . . . . . . . . . . . . 1

I I. THE STRUCTURE OF THE REgIONAL ENERGY BALANCE STATEMENTS . . . 4

Regional Consumption . . . . . . . . . . . . . 4

Regional Supply... . . . . . . . . . . . . 8

Regional Imports . . . . . . . . . . . . . . 10

III. SPECIAL CHARACTERISTICS OF THE 1973 DATA TABLES . . . . . . . 12

The National Data Table . . . . . . . . . . . . 12

Regional Consumption of Coal . . . . . . . . . . . . 14

Summary . . . . . . . . . . . . . . . . . . . 14

IV. DATA SOURCES . . . . . . . . . . . . . . . . . 15

Demand . . . . . . . . . . . . . . . . . 15

Supply . . . . . . . . . . . . . . . . . 17

V. CONVERSION FACTORS . . . . . . . . . . . . . . . 20

VI. REGIONAL POPULATION . . . . . . . . . . . . . . . . 22

FOOTNOTES . . . . . . . . . . . . . . . . . . 26

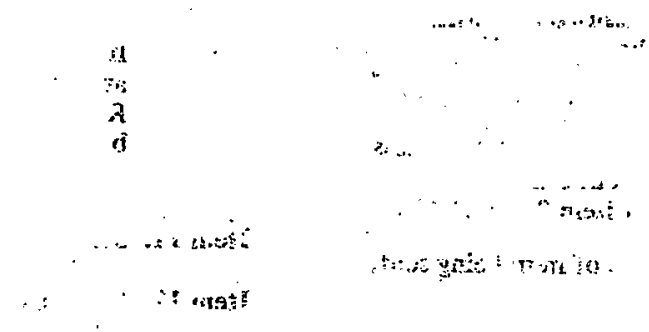




\section{THIS PAGE}

\section{WAS INTENTIONALLY \\ LEFT BLANK}




\begin{abstract}
This report is one of a continuing series developed by ORNL with financial support from the Economic Development Administration to present the supply, demand and net imports of seven fuel types for four final consuming sectors of BEAs, states, census regions, and the nation in 1973 . The data are formatted to present regional energy availability from primary extraction as well as from regional transformation processes. As constructed, the tables depict energy balances between availability and use for each of the specific fuels. The long-term objective of the program is to pinpoint those regions where economic development potentials will most likely be affected by the availability of energy. This information coupled with specific knowledge of projected economic growth and employment distribution patterns can assist EDA in developing its grantin-áid investment strategy.
\end{abstract}




\section{INTRODUCTION}

This volume supplements Energy Availabilities for State and Local Development: A Methodological and Data Overview (ORNL/TM-5890) published by Oak Ridge National Laboratory in June 1977 and Supplement I published in August 1977. The 1973 volume contains energy supply and demand information for the U.S., 9 census regions, 50 states, and 173 BEAs. Energy demand in 1973 for four final consuming sectors as well as donestic supply and net imports of seven fuel types are presented in the form of energy balance statements for each of these geographic regions in this volume.

The regional energy statements developed present a consistent format for monitoring and reporting changes in historic and projected energy availability and consumption patterns. Marginal changes in energy availability have generally been measured at the national level. However, at the regional level the unequal distribution and impact of such changes have been more difficult to measure. The identification of areas with significant differences in energy supply and use patterns could pinpoint potential shortages of energy and provide the Economic Development Administration and other users with information to plan and implement economic development programs at the national and regional level.

ORNL has developed a computerized regional energy analysis system. In designing this system we have focused primarily on the relationship belween economic activity and energy usage. The selection of an appropriate regional grid was, therefore, based on a system of functional economic regions for which reliable economic data were available. BEA regions by design represent labor market boundaries and are approximate energy load centers, a concept commonly used in electricity consumption 
analysis (Fig. 1). Moreover, OBERS employment-population projections are reported at the BEA spatial grid. BEAs can be disaggregated by county groups and these BEA portions then reaggregated consistent with state boundaries to facilitate comparisons by state energy offices. By using the $173 \mathrm{BEA}$ regions as a basic unit of analysis we have retained a sufficient level of detail to conduct meaningful regional economic analysis at the substate, state, and multistate level.

A brief description of the regional energy statements included in this report precedes the presentation of the data. A blbllographic li3t of the major data sources and an appendix showing the relevant Btu conversion factors are also included.

One caveat is in urder. The numbers reported herein should be interpreted as our best estimates of regional energy consumption and availability given available published sources and the adequacy of our procedure. The data do not represent an aggregation of exact information in a11 cases. 
ORNL-DWG 76-2734

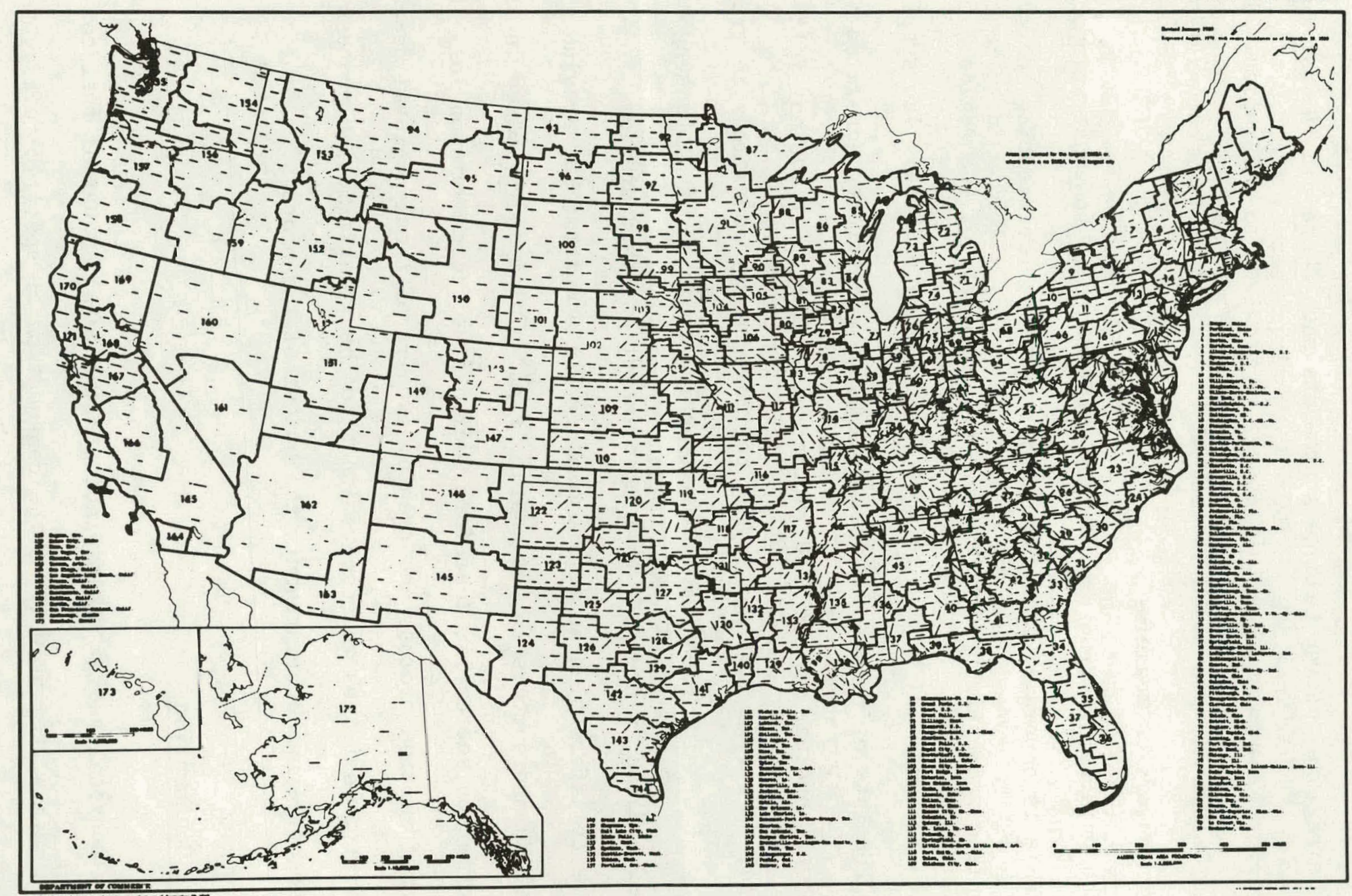

Fig. 1. BEA areas in the United States. 
II. THE STRUCIURE OF THE REGIONAL ENERGY BALANCE STATEMENTS

ihe regional energy balance statements show where and in what fue 1 form energy is used and how this energy is provided to the region. The data is internally processed in thermal equivalent units incorporating average conversion measures.

The underlying structure of these statements is somewhat unique. Each table is separated into three major sections representing cnergy consumption, domestic production and energy imports of the region. Consumption in the final demand sectors is for final end use. The supply sector consists of fossil fuel production and electricity gencrated by hydropower, nuclear, solar, and geothermal sources. It was not felt essential to our goal of portraying regional energy conditions to trace the flow of uranium from point of extraction to point of processing to point of end use, sinec most BEAs must import enriched uranium.

The transformation sectors use energy in one form, but provide it to final demand sectors in yet another form. Total energy usage in the transfurmation soctor is, therefore, defined as the conversion loss in the transformation process and treated as part of final regional demand. A positive number thus represents input to the transformation process and a negative number represents the output of the process.

\section{Regional Consumption}

The regional energy tables indicate the total amount of fuel consumed in each sector as well as the consumption of specific fuels. Table I illustrates 1973 energy production and usage in the United States. 
REGIONAL ENERGY BALANCE STATEGEHT

BY SECTOR AHD FOEL TYPE

\begin{tabular}{|c|c|c|c|c|c|c|c|c|c|c|}
\hline SECTOR & $\begin{array}{l}\text { DISTILLATE } \\
\text { OIL }\end{array}$ & $\begin{array}{l}\text { RESI DOAL } \\
\text { OIL }\end{array}$ & $\begin{array}{l}\text { GASOLI AE } \\
\text { (ALL O }\end{array}$ & $\begin{array}{c}\text { HYDRO- } \\
\text { CARBONS } \\
\text { WITS IN 10* } * 9\end{array}$ & $\begin{array}{c}\text { CRODE } \\
\text { OIL } \\
\text { BTO'S) }\end{array}$ & $\begin{array}{l}\text { NAT URAL } \\
\text { GAS }\end{array}$ & $\operatorname{COAL}$ & BLECT RICITY & $\begin{array}{l}\text { SECTOR } \\
\text { TOTAL }\end{array}$ & \\
\hline $\begin{array}{l}\text { PIHAL DEHAHD SECTORS } \\
\text { RESIDENTIAL, COUH. } \\
\text { INDOSTRIAL } \\
\text { TRANSPORTATION } \\
\text { HISCELLANEOOS OSES }\end{array}$ & $\begin{array}{r}3.127 .001 \\
802.455 \\
2.159 .099 \\
69.305\end{array}$ & $\begin{array}{r}1.020 .388 \\
1.275 .558 \\
732.492 \\
245.118\end{array}$ & $13,255,897$ & $\begin{array}{r}2.404 .000 \\
3.997 .743 \\
2.044 .400 \\
105.302\end{array}$ & $\begin{array}{l}\overline{-} \\
\overline{-}\end{array}$ & $\begin{array}{r}7.189 .959 \\
10.654 .299 \\
743.604 \\
443.958\end{array}$ & $\begin{array}{r}296.515 \\
4.376 .593 \\
2.991 \\
-\end{array}$ & $\begin{array}{r}3.301,138 \\
2,391,149 \\
17.015 \\
84,395\end{array}$ & $\begin{array}{r}17,339,001 \\
23,497,797 \\
18,955,498 \\
948,078\end{array}$ & $\begin{array}{r}248 \\
328 \\
26 \% \\
1 \%\end{array}$ \\
\hline $\begin{array}{l}\text { TOTAL P IHAL } \\
\text { DEAAND SECTORS }\end{array}$ & 6.157 .860 & $3.273,556$ & $13,255,897$ & $8,551,445$ & $\overline{-}$ & $19,031,820$ & $4,676,099$ & $5,793,697$ & $60,740,374$ & $84 \%$ \\
\hline $\begin{array}{l}\text { TRANSPOREATIOH } \\
\text { ELECTRICITY GER. } \\
\text { PETROLEUM PRODOCTS } \\
\text { HATORAL GAS } \\
\text { SYN-GAS }\end{array}$ & $\begin{array}{r}263,019 \\
-5,995,925 \\
-\end{array}$ & $\begin{array}{r}3,219,627 \\
-2,229.350 \\
- \\
-\end{array}$ & $\begin{array}{r}-12,589,07 \% \\
-\end{array}$ & $\begin{array}{r}78,774 \\
-6,531,169 \\
-1,339,919 \\
-\end{array}$ & $26,316,07 \overline{-}$ & $\begin{array}{r}3.706 .394 \\
1.199 .634 \\
1.539 .992 \\
-\end{array}$ & $\begin{array}{r}8,638,960 \\
= \\
=\end{array}$ & $\begin{array}{r}-4,994.555 \\
- \\
-\end{array}$ & $\begin{array}{r}10,912,219 \\
170,194 \\
200.073 \\
-\end{array}$ & $\begin{array}{r}15 \% \\
0 \% \\
0 \%\end{array}$ \\
\hline $\begin{array}{l}\text { NET POEL USED } \\
\text { IN TRABSFORGATION }\end{array}$ & -5.732 .906 & 990.277 & $-12,589,071$ & $-7.792,314$ & $26,316,075$ & 6.446 .020 & $8,638,960$ & $-4,994,555$ & $11,282,486$ & $16 \%$ \\
\hline $\begin{array}{l}\text { TOTAL GROSS PLONS } \\
\text { LOSSES } \& \text { OHISSIONS }\end{array}$ & $\begin{array}{r}6.420,879 \\
390,068\end{array}$ & $\begin{array}{r}6,493,183 \\
-65,890\end{array}$ & $\begin{array}{r}13.255,897 \\
-419,670\end{array}$ & $\begin{array}{r}8.630 .219 \\
-407,764\end{array}$ & $\begin{array}{r}26.316 .075 \\
39,898\end{array}$ & $\begin{array}{r}25.477 .840 \\
96.475\end{array}$ & $\begin{array}{r}13,315,059 \\
221,390\end{array}$ & $\begin{array}{r}5.793 .697 \\
463,354\end{array}$ & $\begin{array}{r}72.022 .860 \\
317.862\end{array}$ & $\begin{array}{r}100 \% \\
0 \%\end{array}$ \\
\hline TOTAL NET & 815.022 & $4.197,942$ & 247.155 & 351,366 & $26,355,973$ & $25,574,315$ & 13.536 .449 & 1.262 .496 & 72.340 .722 & \\
\hline
\end{tabular}

SUP ELY OF ENERGY

POSSIL POEL

HYDROEL.ECTRIC

NOCLEAE:

GEO.E SOLAR

-
-
-

$\begin{array}{ll}- & - \\ - & -\end{array}$

-

$-$

$-19,492,839 \quad 24,481,289 \quad 14,821,811 \quad 1,213,769 \quad 60,009,708$

$\quad-19,492,83924,481,289 \quad 14,821,811 \quad 1,213,769 \quad 60,009,708$

$927,948 \quad 58,795,939 \quad 98 \%$

$\begin{array}{lll}927.948 & 927,948 & 2 \% \\ 278,350 & 278,350 & 0 \%\end{array}$

$\begin{array}{rrr}78.350 & 278,350 & 08 \\ 7.471 & 7.471 & 08\end{array}$

-

\section{NET IMPOETS}

OF REGION

$815,022 \quad 4,197,942$

247,155

351,366

$6,863,134$

$1,093,026 \quad-1,285,361$

$48,727 \quad 12,331,014$

NOTES:

1 IRANS FORHATIOY LOSS POR IRANSPORAATION LOSS POR TRANS PORAATION LOSS POR TRANSPORAATION LOSS POR

ELECTRICITY GEH. $\quad=68.60 \pi$

NATORAL GAS

SYN-G AS
$=68.60 \%$

$=12.99 \%$

$=0.0 \%$ 
The 1972 table is illustrated in Table II to facilitate a comparison. ' In both years the residential sector consumed 17 quadrillion Btus (quads) of energy of which 7.2 was natural gas. The total energy consumption of all sectors $(60.7)$, presented in the row labeled "Total Final Demand," increased $3.8 \%$. Natural gas consumption (19.0 quads) accounted for the greatest single share of total energy demand in 1973. Our analysis assumes that none of the final demand sectors consume unprocessed crude oil.

A portion of electricity generation is included as à eransformation sector in which the output is total generated electricity by fossil fuel plants (in Btus) less the Btu content of the fuels consumed, where the conversion rate for electrical output is that of end use rather than fossil fuel equivalents. The output (indicated as negative demand) of electricity by fossil fuel plants in 1973 was 5.0 quads requiring total fuel consumption of 3.7 quads of natural gas, 8.6 quads of coal, and 3.6 quads of petroleum products. The fuel luss ill lie conversion process, treated as consumption of this sector, was 10.9 quads, Implynng ani efficiency in transformation of fossil fuels of approximately $31 \%$.

The net consumption of fuel used in all transformation sectors is presented in the row labeled "Net Fuel Used in Transformation." In 1973 the transformation sectors converted 26.3 quads of oil, 6.4 quads of gas, and 8.6 quads of coal into 25.1 quads of refined petroleum products and 5.0 quads of electricity. The transformation process required the consumption of 11,3 quads of energy.

Two additional summary rows are presented in the consumption portion of the tables. The row labeled "Total Gross Flows," is the column sum 
REGIONAL ENERGY BALANCE STATEKENT

BY SECTOR AND FOEL TYEF

\begin{tabular}{|c|c|c|c|c|c|c|c|c|c|c|}
\hline SECTOB & $\begin{array}{c}\text { DISTIILATE } \\
\text { OIL }\end{array}$ & $\begin{array}{l}\text { EES EDOAL } \\
\text { OIL }\end{array}$ & $\begin{array}{l}\text { GASOLIHE } \\
\text { (ALL OI }\end{array}$ & $\begin{array}{r}\text { OTBE } \\
\text { HYDBO- } \\
\text { CARBONS } \\
\text { NTS IN 10* }\end{array}$ & $\begin{array}{c}\text { CRODE } \\
\text { OIL } \\
9 \text { BTO०SI }\end{array}$ & $\begin{array}{l}\text { NAT ORAL } \\
\text { GAS }\end{array}$ & $\operatorname{COAL}$ & ELECT RI CITY & $\begin{array}{l}\text { SECTOR } \\
\text { TOTAL }\end{array}$ & \\
\hline $\begin{array}{l}\text { FIRAL DEBANC SECTORS } \\
\text { RESIDENTIAL, COUH. } \\
\text { IRCOSTEIAL } \\
\text { TRAHSPORTATIOR } \\
\text { GISCELIANECOS OSES }\end{array}$ & $\begin{array}{r}3.190 .998 \\
722.063 \\
1.886 .576 \\
63.603\end{array}$ & $\begin{array}{r}994.665 \\
1.198,249 \\
651,499 \\
237.415\end{array}$ & $12,821,097$ & $\begin{array}{r}2,349,297 \\
3,774,231 \\
1,902,911 \\
30,306\end{array}$ & $\bar{z}$ & $\begin{array}{r}7.175,481 \\
10,549,493 \\
787.003 \\
439.083\end{array}$ & $\begin{array}{r}233,844 \\
3,989,680 \\
-\end{array}$ & $\begin{array}{r}3,094.003 \\
2,270,661 \\
15,3 \varepsilon 3 \\
77,7 \leq 7\end{array}$ & $\begin{array}{r}17,038,288 \\
22,504,377 \\
18,064, .469 \\
848,204\end{array}$ & $\begin{array}{r}249 \\
328 \\
268 \\
19\end{array}$ \\
\hline $\begin{array}{l}\text { TOTAL PINAL } \\
\text { DERAND SECTOES }\end{array}$ & $5,8 \in 3,240$ & $3,081.828$ & $12,821,097$ & 8.056 .745 & - & 18.951 .060 & $4,223,524$ & $5.457,844$ & $58,455,338$ & E4 \\
\hline $\begin{array}{l}\text { TEARSPOEHATIOH } \\
\text { ELECT BICITY GEN. } \\
\text { PETROIEOH FROCOCTS } \\
\text { MATUEAL GAS } \\
\text { SYH-GAS }\end{array}$ & $\begin{array}{r}291.059 \\
-5.606 .005 \\
-\end{array}$ & $\begin{array}{r}2.762 .604 \\
-1.839 .069 \\
- \\
-\end{array}$ & $-12,153,152$ & $\begin{array}{r}146.987 \\
-6,245.761 \\
-1,517,690\end{array}$ & 24.829 .008 & $\begin{array}{r}3,990,025 \\
1.219,647 \\
1,674,427\end{array}$ & $\begin{array}{r}7,771,795 \\
- \\
-\end{array}$ & $\begin{array}{r}-4.801 .902 \\
=\end{array}$ & $\begin{array}{r}10.160 .608 \\
204.668 \\
156.737 \\
-\end{array}$ & $\begin{array}{r}15 \% \\
08 \\
6\end{array}$ \\
\hline $\begin{array}{l}\text { MET FOEL OSED } \\
\text { IA TRANSPORHLTION }\end{array}$ & $-5,314,946$ & $92 \lesssim .575$ & $-12,153,152$ & $-7,616,464$ & $24.829, c c 8$ & 6.884 .099 & $7,771.795$ & $-4,801,902$ & 10.522 .013 & $15 *$ \\
\hline $\begin{array}{l}\text { TCTAL GROSS PLCIS } \\
\text { LCSSES } 8 \text { OUISSIOUS }\end{array}$ & $\begin{array}{r}6,154,299 \\
-168.293\end{array}$ & $\begin{array}{r}5.846 .472 \\
-7 \equiv .403\end{array}$ & $\begin{array}{r}12.821,097 \\
-541,944\end{array}$ & $\begin{array}{r}8.203 .732 \\
44.719\end{array}$ & $\begin{array}{r}24,829,008 \\
-147,3 \leq 1\end{array}$ & $\begin{array}{r}25.835,159 \\
-588,880\end{array}$ & $\begin{array}{r}11.995 .319 \\
1,638.826\end{array}$ & $\begin{array}{r}5.457 .844 \\
492.8 \leq 8\end{array}$ & $\begin{array}{r}68.577 .351 \\
656,528\end{array}$ & $\begin{array}{r}999 \\
19\end{array}$ \\
\hline TCTAL NET OSAGE & 380.000 & $2.931,999$ & 126,000 & 485,000 & 24.681 .656 & $25,246,278$ & $13,634,145$ & $1,148, E C 0$ & $69,633,879$ & \\
\hline $\begin{array}{l}\text { SOFELY CF ERERGY } \\
\text { POSSI I POEL } \\
\text { BYDROEL ECTEIC } \\
\text { HOCLE AR } \\
\text { GEO.E SOLAB }\end{array}$ & - & $\bar{z}$ & $\overline{-}$ & 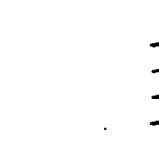 & $\begin{array}{r}19,976,656 \\
= \\
=\end{array}$ & $\begin{array}{r}24,224,278 \\
= \\
=\end{array}$ & $\begin{array}{r}14,998,624 \\
- \\
-\end{array}$ & $\begin{array}{r}936,953 \\
184,636 \\
7 \in 3\end{array}$ & $\begin{array}{r}59.199,558 \\
936.953 \\
184.636 \\
783\end{array}$ & $\begin{array}{r}989 \\
29 \\
09 \\
09\end{array}$ \\
\hline TCTAL SOPPLY & - & - & - & - & $19,976,656$ & $24.224,278$ & $14,998,624$ & $1,122,372$ & $60,321,930$ & \\
\hline
\end{tabular}

NET IHPCRTS
OP REGION

NOTES:

TBAHS FORHAT ICH LOSS FOR TRA HSFOBAATICB LOSS POB TRAAS POBAAT ION LOSS POR TRA HSPOREATICB LOSS POB 
of the positive elements in both the final demand and transformation sectors. This value represents the gross consumption of each fuel (neglecting the regional production of transformation energy products). This row is used primarily to allocate losses and omissions. A positive error value indicates an under-assignment of fuels to specific categories, inventory accumulations, or distribution loss. A negative error value indicates an over-assignment of consumption to a particular fuel or scctor, an understatement of production, or a depletion of stocks. The overall error, indicated in the "Sector Total" column, was $0 \%$ of gross flows in 1973, due to cancellation of positive and negative errors associated with specific products.

The total regional demand for fuel less the supply of energy by the transformation sector, presented in the row labeled "l'otal Net Usage," increased $3.9 \%$ between 1972 and 1973 to 72.3 quads. ${ }^{2}$ This energy demand is met by primary regional production and regional 1mports.

\section{Regional Supply}

The total supply of all forms of primary ellergy is given in the row labeled "Total Supply." Distillate oil, residual oil, gasoline, and other hydrocarbons have zero values, indicating that these products are not regarded as primary energy sources. The domestic production of crude oil, natural gas, and coal declined by . 7\% between 1972 and 1973 . The other primary fuels considered in the analysis include electricity generated by hydroelectric power, nuclear, geothermal, and solar sources. ${ }^{3}$ 
Primary electricity generation from hydro and nuclear sources was converted to Btus by the end use rate of 3412.8 Btus per kilowatt hour, rather than fossil fuel equivalents in order to maintain overall balance in the tables. The fossil fuel equivalent conversion rates are often used in supply-oriented analysis to address the potential effects of fuel switching in electrical generation. This approach would artificially inflate the total Btus produced in a region, however, and might lead to a different impression of the importance of each fuel and its contribution to end use electrical consumption.

To investigate the implications of fuel switching in electrical generation, the increase in generation by hydro and nuclear power and the reduction in demand for fossil fuels can be derived by using the appropriate transformation loss for the region. No effort was made in this study to provide a direct comparison of the fossil fuel savings attained from substituting geothermal, solar, or hydroelectric inputs, though this can be done easily.

For example, the average transformation loss for fossil fuel generation of electricity is approximately $68 \%$. For every kilowatt hour generated by hydro or nuclear sources, there is a net saving in the system of energy of 7252 BTUs. To get the same end use availability of electricity, 7252 BTUs of energy do not have to be produced. Since the uranium refinement process itself requires electricity, the net saving in nuclear generation would be somewhat less. 


\section{$\underline{\text { Regional Imports }}$}

The data in the tables represent the spatial patterns of consumption and production, but do not characterize the interrelationships of the flows. The concept of regional imports considered is a simple accounting statement of the difference between how much is used in a region and how much is produced.

The tables were constructed to balance such that the sum of row totals equals the sume of column totals. The last row thus represcnts the net regional imports by fuel (a negativi number indicating exporta). The number in the last row and column provides a measure of the net energy imports of the region, characterizing it as to its external reliance upon energy supplies.

At the national level, the tables report estimated net imports equal to the actual data (12.3 quads in 1973), a $14.2 \%$ increase over 1972. Any initial differences between estimated and measured net imports at the national level are then allocated to regions based on each region's share of total gross usage of epach fuel. These differences are reported in the row labeled "Loss, Errors, and Omissions."

Net regional imports by fuel type are computed as total net usage less regional supply. The regional imports, unlike the national imports, are nnt. a direct measure of the actual import/export flows of energy, but are derived from the consumption and production data.

The interpretation of these regional imports must be qualified. A region may show up as a net importer of energy, though these imports may be supplied under long-term contractual agreement with producers in other 
regions. Consequently, even though a region may appear vulnerable to changes in the energy distribution system, the region could in actuality have a fairly stable energy position. In a number of cases there are substantial counterflows of energy, wherein a region may export as well as import a given fuel. Certainly not all of the regionally produced energy is used to satisfy local demand, nor should that be inferred from the tables.

Though a region might not have its own electrical utility, it would still have access to electricity by importing it from adjacent regions. The tables indicate the amount of electricity imported, but do not indicate the source. To reiterate, there is no attempt made in the regional energy balance tables to depict energy flows among BEAs or states. We do hope to address the issue of energy flows in subsequent analysis for the Department of Commerce. 


\section{SPECIAL CHARACTERISTICS OF THE 1973 DATA TABLES}

The presentation format of the data and the methodology used in constructing the Regional Energy Balance Statements provide a consistent framework for examining the variety of interactions involved in energy production and consumption. There are, however, several conceptual differences in our approach to measuring energy input which result in slightly different estimates of aggregate energy consumption than those often reported. An interpretation of these differences and the1r effect upon regional energy patterns is provided to facilitate comparisons with our tables.

\section{The National Data Table}

The Bureau of Mines reports 61.3 quads of "net energy inputs" which may be roughly compared to our estimate of 60.7 quads of total energy consumption. The primary difference between the two values stems from our treatment of "Lusses and Onission3." Tho Bureau of Mines inriudes energy consumption which camiot be speclfieally alloeated to individizl states and the distribution loss of electricity in its éstimares of national energy consumption. In our tables both of these components appear in the "Losses and Omissions" row. By adjusting our tables in a similar manner, we estimate 61.6 quads of total energy consumptiun, a difference of less than .5\%.

The difference between our estimate of 72.3 quads of total net energy consumption and the 74.8 quads of "gross energy inputs" of the Bureau of Mines results from alternative conversion factors for electricity generated from nuclear or hydro sources. The Bureau of Mines uses a fossil fuel equivalents approach (the Btu value of fossil fuels needed 
to generate this electricity by conventional fossil fuel plants), whereas we convert electricity at the end use rate of 3412.8 Btus per kilowatt hour. By using the fossil fuel equivalents approach, we would obtain an additional 2.7 quads of energy input such that our estimate of the total energy consumption in the U.S. in 1973 would be 75.0 quads (a difference of $0.3 \%$.

One further caution in comparing our estimates with others is in order. The regional consumption data is estimated by an allocation prom cedure, while the energy supply and transformation data is generally obtained by accumulating region-specific information. The structure for generating the balance statements uses the information contained in the more reliable energy supply and import data to provide a measure of the error in the consumption estinates. Once energy supply and transformation data are accumulated and net imports determined, the amount of energy available for consumption is known. The difference between availability and consumption is estimated as "Losses and Omissions." Since these consumption estimates are developed by a non-statistical process, a traditional measure of error for each component is not available. An average medsure of the uncertainty associated with the data can be constructed, however, by applying the ratio of "Losses and Omissions" to total consumption to individual sector consumption estimates.

A large number of significant digits were retained in generating the tables to ensure a summation of BEAs which would be consistent with the national totals presented in the U.S. table. The individual consumption figures should be viewed as "best" point estimates, but not interpreted as exact figures. 
Regional Consumption of Coal

In computing the 1972 data table we observed a relatively large error for "Losses and Omissions" of $13.7 \%$ of gross flows of coal. Most of this error was accounted for by unreported shipments and changes in inventories as explained in Supplement I. In the 1973 table we computed a relatively small error (1.7\% of gross flows) due to offsetting factors.

A portion of the difference between the production and consumption data for coal is attributable to unreported shipments of coal (provided to mine-mouth operations or delivered from captive mines). It appears that an annual average of about $5.7 \%$ of gross flows should be accounted for by this coal consumption of industrial users .

Secondly, changes in inventories are reported in the "Losses and Omissions" row since this coal is reported as shipped, but not consumed. Historical aggregate time series indicate an error of $-4 \%$ of gruss flows should be accounted fur by the dccrcase in storks wh1ch uccurred in 1973. Following an inventory depletiun in 1971, the utilities increased their coal stocks in 1972 . The $19 \overline{7} \overline{3}$ data indıcate a subsequent depletion of these accumulated stocks. The negative error due to stock depletion offset.s the average error of $5.7 \%$ due to unreported shipments yielding a net error of $1.7^{\circ}$ of gross flows.

\section{Summary}

The Regional Energy Balance Statements wero developod to provide an overall view of the relationship of a region's net energy consumption and production patterns. To interpret the complete energy picture for a 
single region, one may wish to bring additional data to bear such as information as to actual flows and prior commitments.

The tables are not intended to suggest historical or potential limits on specific regions, but to provide a consistent base of energy information. Such a framework should aid regional policy makers in their consideration of the regional growth implications of the regional energy system. The general framework must be supplemented by region-specific information which only the local policy analyst can bring to bear in his assessment of the energy conditions which characterize each region.

\section{DATA SOURCES}

Demand

The input data required to perform the regional demand disaggregation is extensive and difficult to document in detail. To perform the regional disaggregation of the basic Bureau of Mines state level data series, 236 level variables ${ }^{4}$ and 12,036 state relative variables ${ }^{5}$ were estimated. The published primary data sources do not provide a consistent series for all seclurs and fuels considered, such that many approximations and independent judgments were necessary. Consequently, the computer allocation procedure was in some cases overridden to provide an appropriate estimate.

For purposes of this report only the major primary sources are listed below. One of the outputs of the computer program is a complete list of the specific sources used to determine the values for the relative and level variables. Since this is a voluminous listing, it is not included in this document, but is available at Oak Ridge National Laboratory as supporting documentation. 
- State Energy Consumption Data:

Fuel and Energy Data: United States by States and Census Divisions, 1973, Information Circular 8722, U.S. Department of the Interior, Bureau of Mines, Government Printing Office, Washington, D.C., 1976.

- Regional Activity Data:

County Business Patterns: 1971, 1972, U.S. Department of Commerce, Government Printing Office, Washington, D.C., 1972 and 1973.

Annual Survey of Manufactures: 1970-1971, U.S. Department of Commerce, Bureau of the Census, Government Printing Office, Washington, D.C., 1973.

- Regional Population Data:

Statistical Abstract of the United States: 1971, 1972, 1973, U.S. Department of Commerce, Government Printing Office, Washington, D.C., $1972,1973,1974$.

Federal-State Cooperative: 1973, Program for Population Estimates.

- Regional Energy Consumption Data:

Edison Electric Institute, Statistical Yearbook of the Electric Utility Industry, Edison Electric Institute, New York, New York, 1973.

Federal Power Commission, form 4 Data Tape, Cupuivity, Generation, Fuel Consumption by Month: 1972-1975, Washington, D.C.

Marquis R. Seidel, State Projections of Industrial Fuel Needs, Federal Power Commission, Office of Energy Systems, Washington, D.C., August $19 \% 6$.

Federal Power Commission, Steam-Electric Plant, Air, and Water Qualiity Control Data: 1971, Washington, D.C., June 1974.

American Gas Association, 1972 Gas Facts, Arlington, Virginia, 1973.

Mineral Industry Surveys: Sales of Fuel Oil and Kerosene in 1972, U.S. Department of the Interior, Bureau of Mines, Government Printing Office, Washington, D.C., 1973 .

Mineral Industry Surveys: Sales of Liquefied Petroleun Gases and Ethane in 1972, U.S. Department of the Interior, Bureau of Mines, Government Printing Office, Washington, D.C., 1973.

Highaway Statistics: 1972, U.S. Department of Transportation, Government Printing Office, Washington, D.C., 1973. 
Minerals Yearbook, Volume I, Metals, Minerals, and Fuels: 1971, 1972, U.S. Department of the Interior, Bureau of Mines, Government Printing Office, Washington, D.C., 1973, 1974.

Fuels and Electric Energy Consumed, Special Report Series MC72(SR-6), 1972 Census of Manufactures, U.S. Department of Commerce, Bureau of the Census, Government Printing Office, Washington, D.C., 1973.

National Energy Accounts: Energy Flows in the United States 19471972, Jack Faucett Associates, Inc., Chevy Chase, Maryland, November 1975. This document was prepared for the Federal Energy Administration.

Supply

As was the case with the demand data, the documentation of each supply variable is difficult given the variety of sources utilized and different allocation techniques employed. The primary distinguishing feature between the supply and demand data, however, is that the majority of the supply data is available at the county level, such that the allocation schemes play a minor role in determining BEA energy production. The numbers generally represent actual rather than estimated production. The data sources employed and a short description of the methodology are indicated below for coal, oil, natural gas, and associated petroleum and natural gas products.

- Coal Production

U.S. Bureau of Mines, Minerals Yearbook: Area Reports - Domestic, 1973 edition, Government Printing Office, Washington, D.C., 1975.

- Crude Uil and Natural Gas

Al1 oil and gas producing states were surveyed to obtain county (or field) estimates of their 1973 production of oil and natural gas. New York was the only state which did not respond to the survey. The oil and gas production in that state was allocated to BEA 9 (Cattaraugus and Allegany counties) based on the location of oil and gas fields in the state. West Virginia reported that they do not maintain county or fieldlevel information for o11 and gas production. 0il and gas production was 
allocated based on 1972 levels, which were in turn determined from information in the Minerals Yearbook. These two states accounted for less than $0.1 \%$ of the total oil production and aboul $0.9^{\circ}$ of the total gas production in 1973 .

The oil and gas production estimates by county for Louisiana, Texas, and Mississippi were used to allocate the reported state totals of the Bureau of Mines to eliminate definitional inconsistencies between the state agencies and the Bureau of Mines as to condensate, casinghead gas, gas from wet zones, etc.

The estimates of all other state agencies were assumed to supersede those of the Bureau of Mines when differences occurred.

For natural gas estimates, it was necessary to allocate the Bureau of Mines state totals for Kentucky, Illinois, Indiana, North Dakota, Pcnnsylvania, and Maryland, since these states reported crude oil production by county, but no information was provided on natud'd gas. Togcther these states accounted for only $0.8 \%$ of the total natural gas production. The 1972 estimates upon which the allocations were based were determined from a regression analysis utilizing information on drilling activity such as footage, success ratios, the number of proven gas and oil wells, the percentage of gas from associated wells, etc. Even with these allocations, 99.3\% of the total natural gas produced in 1973 was distributed among counties exactly according to state agency reports.

These state agencies are as follows:

Alabama State 0il and Gas Board

Alaska Department of Natural Resources

Arizona $\mathrm{Oil}$ and Gas Conservation Commisston

Arkansas $0 i 1$ and Gas Commission

California Resources Agency, Division of $\mathrm{Oil}$ and Gas

Colorado Oil and Gas Conservation Commission

Florida Department of Natural Resources

Illinois State Geological Survey

Indiana Department of Natural Resources

Kansas Geological Survey

Kentucky Department of Mines and Minerals

Louisiana Department of Conservation

Michigan Department of Natural Resources

Mississippi State Oil and Gas Board

Missouri State Oi1 and Gas Counc11

Montana $\mathrm{Oil}$ and Gas Conservation Division

Nebraska Oil and Gas Conservation Commission

Nevada Department of Conservation and Natural Resources

New Mexico Oil Conservation Commission

North Dakota Geological Survey

Ohio Department of Natural Resources

Oklahoma Corporation Commission - Oil and Gas Conservation Division

Pennsylvania Department of Environmental Resources 
South Dakota Geological Survey

Tennessee Department of Conservation - Division of Geology

Texas Railroad Commissiü1

Utah Division of Oil and Gas Conservation

West Virginia Geological and Economic Survey

Wyoming $\mathrm{Oil}$ and Gas Conservation Division

29 of the 30 oil producing states accounted for $99.9 \%$ of oil production.

- Petroleum Refining

The output of jet fuel, kerosene, distillates, residual oil, asphalt, and other hydrocarbons is reported by refining district (11) rather than by state in the Minerals Yearbook. Gasoline production is reported by state.6 County production of each output, excluding asphalt, was determined by using refining capacity (available by plant) and crude runs to stills (available by state) to characterize each county. Asphalt production by county was determined based upon a plant's specific capacity for asphalt production. The gasoline production numbers, then are controlled at the state level, and the other hydrocarbons controlled at the district level. The demand for natural gas of refiners was allocated based upon the refiner's demand for crude oil and overall refining capacity.

- Natural Gas Processing

Annual reports containing information as to the number of plants, their capacity, throughput, and production are submitted to the oil and Gas Joumal, Petroleum Publishing Company, Tulsa, Oklahoma, when capacity is in excess of 75 millions of cubic feet per day. The journal thus served as the primary data source for determining processing activity and supplied specific information in 1973 on $76 \%$ of the total gas throughput. A complete survey was conducted in 1974 and published in the 1975 International Petroleum Encyclopedia, Petroleun Publishing Company, Tulsa, Oklahoma. The data are given alphabetically by plant and were aggregated to counties to serve as the basis for allocating the capacity of the smaller plants (less than 75 mmcf daily).

Since a complete survey was not available for 1973, some simplifying assumptions were made to determine county-level throughput and production. If the number of operating plants was unchanged between 1973 and 1974 , the assumption was made that these were the same plants. It was then assulled that the county had the same share of the state's capacity in 1973 as in 1974. This share was then applied to the unallocated portion of the 1973 capacity fur the state. 'lhe output of the processing plants is sent to refineries for use as feedstock and to ultimate consumers. The estimated share of capacity was used for the allocation of the output of the smaller plants, which was then adjusted by the proportion of output going directly to consumers. Throughput, defined to include extraction loss, gas used at the plants, returned to formations, or vented and flared, was similarly adjusted by the proportion of output going directly to consumers and allocated to smaller plants based on their capacity. 
When the number of plants in operation in 1974 in a given state or county wa greater than in 1973, these plants were simply subtracted from the 1974 total before the weights were constructed. Generdlly, liuwever, the number of plants in 1974 was less than the number in 1973, and when the throughput of the plant was less than 75 mmcf daily, there was no way to determine with certainty which plant had ceased operation during the period. Since the number of plants and total capacity represented in these cases was relatively small, it was felt that the potential error generated was minimal.

\section{CONVERSION FACTORS}

Crude Oil

Natural Gas (consumption)

Natural Gas (production)

Electricity

Distillate Oil

Residual 0il

Gasoline

Asplialt

Kerosene and Jet Fue1

Natural Gas Liquids

Coal (production)
Alabama
Alaska
Arizona
Arkansas
Colorado
I11.inois
Indiana
Iowa

Kansas

kentucky

Maryland

Missouri

Montana

New Mexico

North Dakota

Ohio

Oklahoma

Pennsylvania.

Tennessee

Texas

Utah

Virginia

Washington

West Virginia

Wyoming
$5,800,000$ Btu per barre 1

1,021,000 Btu per thousand cubic feet

$1,093,000$ Btu per thousand cubic feet

3,412,000 Btu per thousand kilowatt hours

$5,825,000$ Btu per barrel.

$6,287,000$ Btu per barrel

$5,248,000$ Btu per barrel

$36,500,000$ Btu per ton

$5,670,000$ Btu per barrel

$4,032,629$ Btu per barrel

$25,740,000$ Btu per short ton

$22,860,000$ Btu per short ton

$21,840,000$ Btu per short ton

$28,760,000$ Bt.u per short ton

$22,330,000$ Btu per short ton

$22,780,000$ Btu per short ton

$23,200,000$ BTu per short ton

$19,420,000 \mathrm{Btu}$ per short ton

$24,340,000$ Btu per short ton

$24,812,000$ Btu per short ton

$25,440,000$ Btu per short ton

$21,800,000$ Bt.u per short ton

$21,336,000$ Btu per short ton

$23,080,000 \mathrm{Btu}$ per short ton

$14,160,000$ Btu per short ton

$24,600,000 \mathrm{Btu}$ per short ton

$25,240,000$ Btu per short ton

$26,720,000 \mathrm{Btu}$ per short ton

$26,200,000$ Btu per short ton

$13,200,000$ Btu per short ton

$25,440,000$ Btu per short ton

$26,360,000$ Btu per short ton

$19,340,000$ Btu per short ton

$26,800,000 \mathrm{Btu}$ per short ton

$20,620,000 \mathrm{Btu}$ per short ton 
The conversion factors for coal consumption are the same with the following exceptions: Massachusetts, Maine, New Hampshire, Rhode Island, Vermont, Delaware, District of Columbia and Maryland - 26.8; Kentucky, South Carolina, Tennessee, Florida, and Georgia - 25.46; Idaho, Montana, and Minnesota - 20.58; Iowa, Missouri, and Wisconsin - 22.78; New Jersey and New York - 26.72; Oregon and Washington - 20.58; California - 25.4; Connecticut - 26.12; Michigan - 24.6; North Carolina - 24.34 million Btus per ton.

To convert each of these factors to metric units, the appropriate rate of conversion is $1 \mathrm{Btu}=1054.8$ joules. 
VI. REGIONAL POPULATION

PEA REgION POPULATIOM IV 1973

BE ANO

BEA

PERsons

UNITED STATES TOTAL

EANGTR MAINE

PORTL AND-SOUTH PORTLAND MAINE

BURLINGTON VERMONT

BOSTON MASSACHUSETT

HARTFDRD CGNNECTICUT

AL BANY-SCHENECT AOY-TROY NEW YORK

SYRACUSE NFW YORK

FOCHESTER NEW YORK

BUFFALD NFW YRRK

ERIE PE NNSYYLVANIA

WILLI AMSPORT PE NNSYLVANT A

GINGHAMTON NEW YRRK-PFNNSYL VANTA

WTLKES-EARRE-HAZLET ON PENNSYLVANTA

NEW YORK NEW YORK

PHILADELPHIA PENNSYLVANIA-VEW JERSEY

HARRTSRURG PENNSYLVANTA

BALTIMORE MARYLAND

WASHING TON-DC MARYLAND-VIRGINIA

STAUNTON VIRGINIA

ROANOKE VIRGINIA

RICHMOND VIRGINIA

NORFOLK-PORTSMOUTH VIRGINIA

RALFIGH NORTH CAROLINA

WILMING TON NORTH CAROLINA

GRFENSB ORO-WINSTON SALEM-MIGH POIVT N.C.

CHARLOT TE NORTH CAROLINA

ASHEVILLE NORTH CAPBL INA

GREENVILLE SOUTH CAROLINA

COLUMBI A SOUTH CAROLINA

FLORENCE SOUTH CAROL INA

CHARLESTON SOUTH CAROLINA

AUGUSTA GEORGI A

SAVANNAH GE ORGI A

JACKS ONV ILLE FLORIDA

ORLANDO FLORIDA

MI AMI FLORDI A

TAMPA-SAINT PETERSBURG FLOR IDA

TALLAHASSEE FLORIDA

PENSÁCOLA FLORTDA

MONTGOMERY ALARAMA

ALSANY GEURGIA

MACON GEORGIA

COLUMBUS GEORGI A-ALABAMA

ATLANTA GEORGIA

GIRMI NGHAM AL AEAMA

MEMPHIS TENNESSEE-ARK ANSAS

HUNTSVTLLE ALABAMA

CMATTANOOGA TENNESSEE-GEORGIA

NASHVILLE TENNESSEE

KNOXVILLE TENNESSEE

BRISTOL VIRGINI A-TENNESSEE

MUNTI NG TON-ASHLAND WEST $\forall I R G I N I A-K E N T U C K Y-O M I O$

LEXINGT ON KENTUCKY

209821900

334500

771200

524200

6520600

3034500

1365400

1468800

1020000

1804600

475700

431900

774500

707700

10277100

7409900

1722300

2744300

3224900

412800

056100

1033800

1249100

$167750^{\circ}$

499500

1188100

1 SEst 00

495400

670100

643000

- 1.8200

45260

454400

41 res

11340

100600

$272 \pi 00$

21 1000

37200

$+1200$

70070

- aboe

soreos

471000

2467500

$177=300$

1787700

693800

roweo

1512700

004200

798509

1382206

53

797000 


\begin{tabular}{|c|c|}
\hline $\begin{array}{l}54 \\
55 \\
55 \\
57 \\
58 \\
59 \\
60 \\
61 \\
62 \\
63 \\
64 \\
65 \\
65 \\
67 \\
58 \\
69 \\
70 \\
71 \\
72 \\
73 \\
74 \\
75 \\
76 \\
77 \\
78 \\
79 \\
80 \\
81 \\
82 \\
83 \\
84 \\
85 \\
86 \\
87 \\
39 \\
89 \\
90 \\
91 \\
92 \\
93 \\
94 \\
95 \\
96 \\
97 \\
98 \\
99 \\
100 \\
101 \\
102 \\
103 \\
104 \\
105 \\
106 \\
107\end{array}$ & 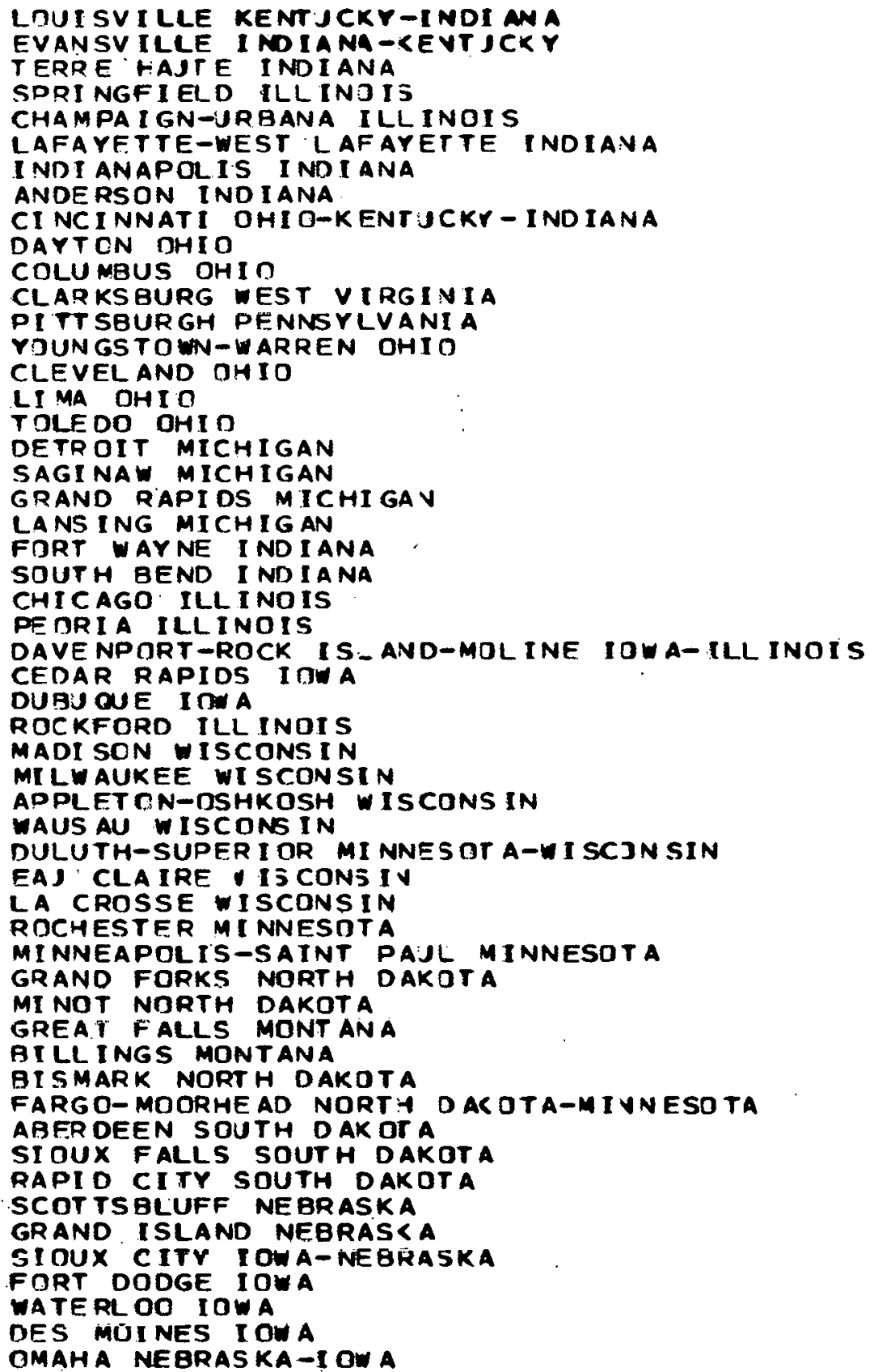 \\
\hline
\end{tabular}




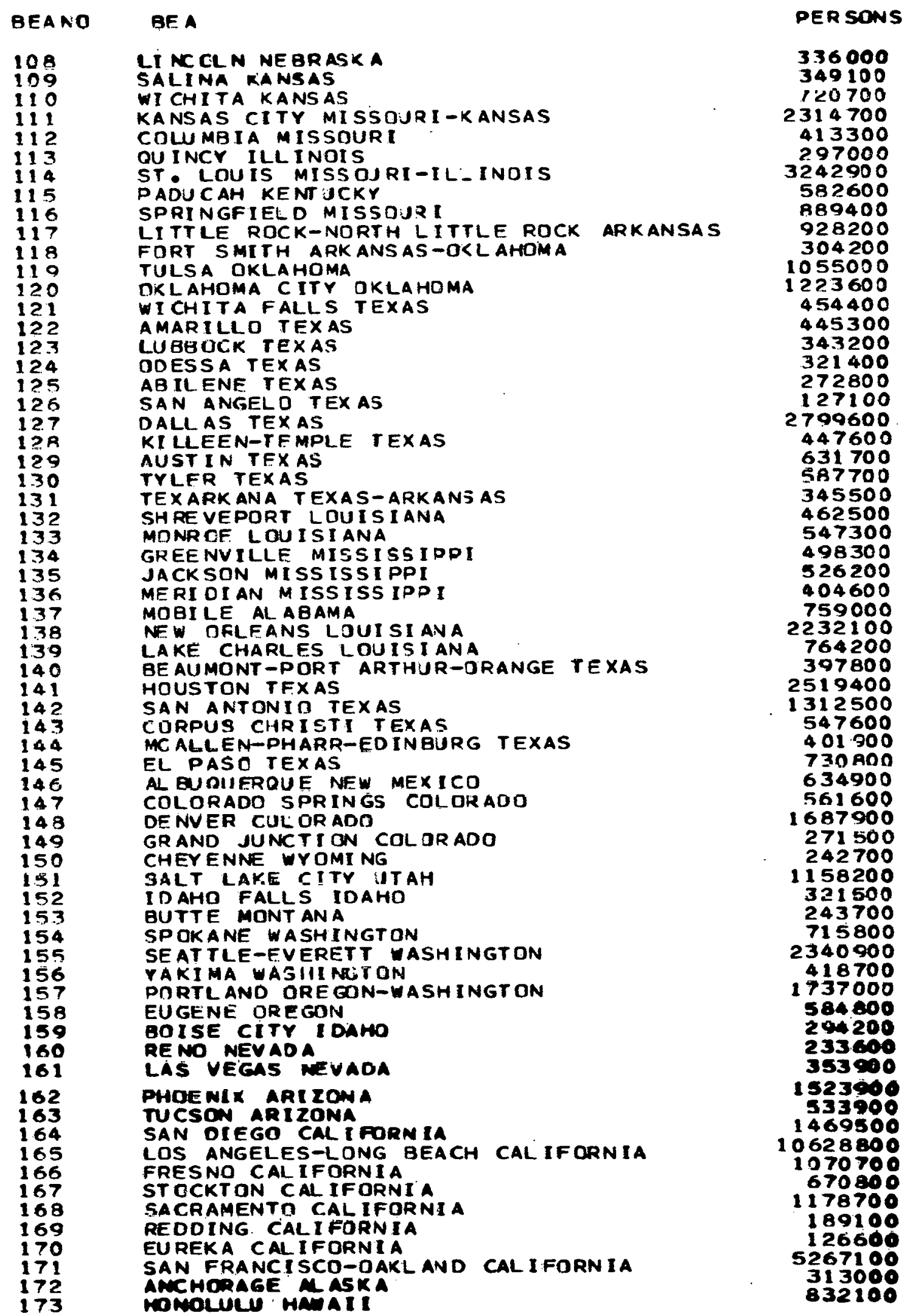


STATE POPULATION IN 1973

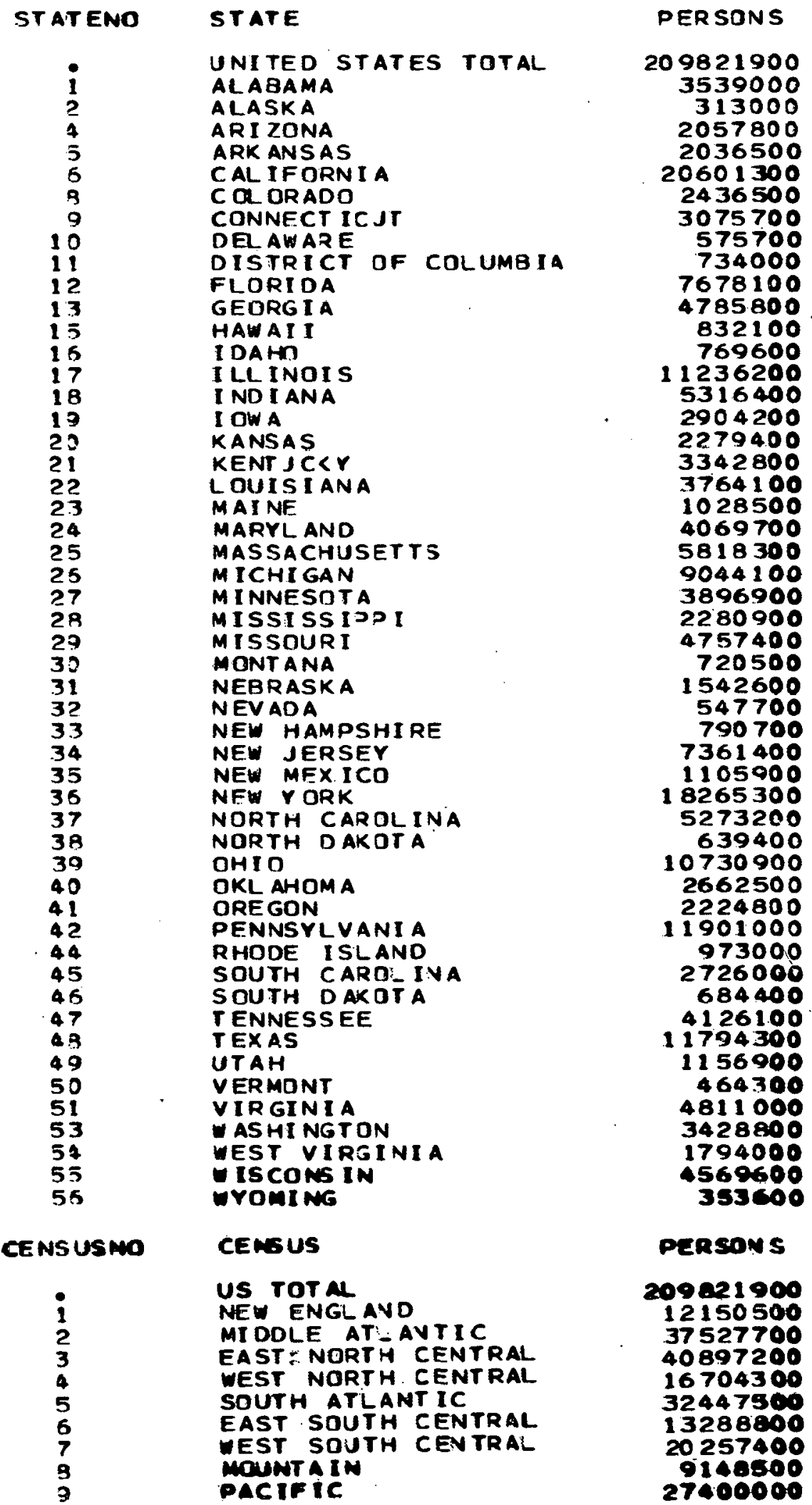




\section{FOOTNOTES}

1. The 1972 national table published herein is a revised version of that found in Vol. I.

2. The difference between our estimated value of 72.3 quads and the Bureau of Mines' estimated value of 74.8 quads is discussed in Section III.

3. Electricity generated from waste heat, refuse, and wood, has been included with geothermal and solar. The sharp increase of geothermal and solar generated electricity from 783 billion Btus to a 1973 value of 7.471 billion Btus was due primarily to an improvement in our siting of existing plants. In particular, the "Geysers" geothermal plant located in BEA 171 in California which gencrated 6,708 billion Btus in 1973 was excluded in the 1972 data volume, but has been included in the 1973 data.

4. The level variables measure the average per worker (per capita) use of a fuel in each consuming sector.

5. The relative variables measure the energy use per unit of activity for a state relative to the national average. The way in which the level and relative variables are used in the demand estimates is explained in Volume $I$ of this series.

6. Some states are lumped together in the reporting of both gasoline production and crude runs to stills (demand for crude). The disaggregation to states was then based upon reported plant refining capacity. 
REGIONAL ENERGY BALANCE STATEMENTS 
REGTONAL ENERGY BALAHCZ STATEGENT

BY SECTOR AND POBL TYPE

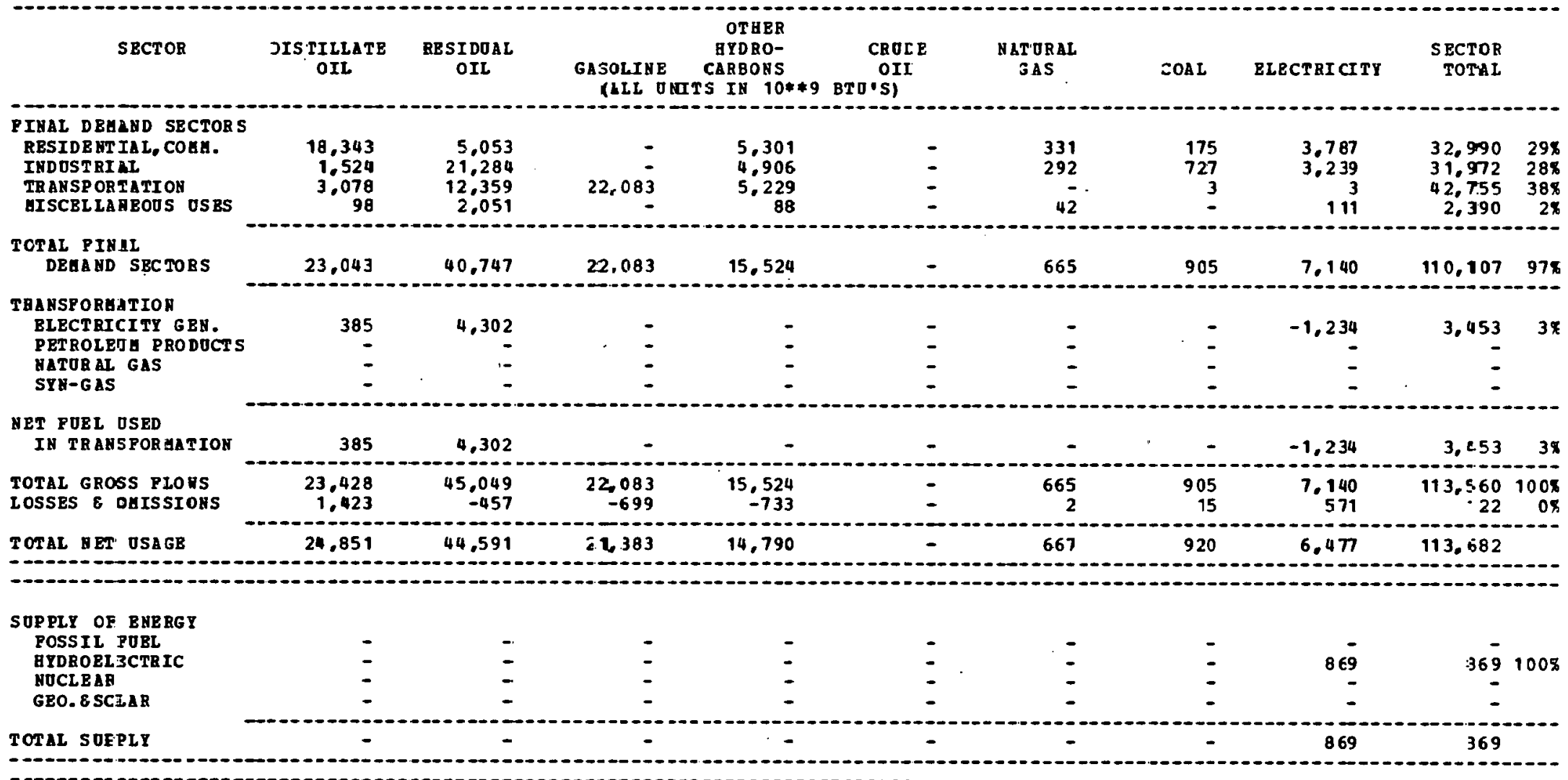

\section{MET IAPOETS}

24,851

44.591

$2 i, 383$

14.790

667

920

5.608

112,313

NOTBS:
1 TRAMS PORGATIOA LOSS POR TRA ASPORAATYY LOSS FOR TRA USPORAATIOA LOSS POR
RLBCTRICJTY GBN.
$=73.678$
$=0.08$
TBASPORGATIOH LOSS FOR
MATUR AL GAS
$=0.0 \mathrm{x}$
$S$ WN-G AS 
RBGIONAL EHEBGI BALANCE STATEEEHT

BY SBCTOB AND POEL TYPE

\begin{tabular}{|c|c|c|c|c|c|c|c|c|c|c|}
\hline SBCTOR & $\begin{array}{l}\text { DISTILLATB } \\
\text { OIL }\end{array}$ & $\begin{array}{l}\text { BBSIDOAL } \\
\text { OIL }\end{array}$ & $\begin{array}{l}\text { GASOLIAE } \\
\text { (ALI O }\end{array}$ & $\begin{array}{c}\text { OTHER } \\
\text { BYDRO- } \\
\text { CABBOHS } \\
\text { TS IH 10*\%9 }\end{array}$ & $\begin{array}{c}\text { CRODE } \\
\text { OIL } \\
\text { BTO'SI }\end{array}$ & $\begin{array}{l}\text { HAT ORAL } \\
\text { SAS }\end{array}$ & COAL & ELECTBICITY & $\begin{array}{r}\text { SECTOR } \\
\text { TOTAL }\end{array}$ & \\
\hline 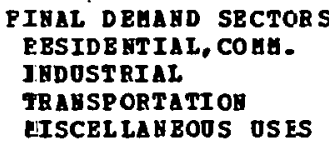 & $\begin{array}{r}42.285 \\
2.279 \\
6,387 \\
212\end{array}$ & $\begin{array}{r}14,540 \\
26,869 \\
9,845 \\
3,579\end{array}$ & $50,75 \overline{7}$ & $\begin{array}{r}12,083 \\
11.807 \\
1,300 \\
222\end{array}$ & $\begin{array}{l}- \\
\\
-\end{array}$ & $\begin{array}{r}1.338 \\
473 \\
107\end{array}$ & $\begin{array}{r}424 \\
2.631 \\
8 \\
-\end{array}$ & $\begin{array}{r}9,537 \\
5,045 \\
7 \\
185\end{array}$ & $\begin{array}{l}80,207 \\
49,104 \\
68,304 \\
4,305\end{array}$ & $\begin{array}{r}368 \\
228 \\
318 \\
28\end{array}$ \\
\hline $\begin{array}{l}\text { TCTAL PIBAL } \\
\text { DEAA SBCTORS }\end{array}$ & 51,163 & 54,833 & 50.757 & 25,412 & - & 1,918 & 3,063 & 14.774 & 201.920 & 918 \\
\hline $\begin{array}{l}\text { TRABSPORAATION } \\
\text { ELECTRICITY GEA. } \\
\text { PETROLEOE PRODOCTS } \\
\text { MATORAL GAS } \\
\text { SYH-GAS }\end{array}$ & $\begin{array}{r}432 \\
- \\
-\end{array}$ & $\begin{array}{r}26.342 \\
= \\
=\end{array}$ & $=$ & $\begin{array}{l}- \\
-\end{array}$ & $\begin{array}{l}- \\
\\
-\end{array}$ & - & I & $\begin{array}{r}-7.802 \\
- \\
- \\
-\end{array}$ & $\begin{array}{r}18,972 \\
= \\
=\end{array}$ & $9 x$ \\
\hline $\begin{array}{l}\text { NET POEL OSBD } \\
\text { IA TRABSPORAATIOY }\end{array}$ & 432 & 26.342 & - & - & - & - & - & $-7,802$ & 18,972 & 98 \\
\hline $\begin{array}{l}\text { TOTAL GROSS PLOHS } \\
\text { LOSSES } 8 \text { OLISSIOHS }\end{array}$ & $\begin{array}{r}51.595 \\
3.134\end{array}$ & $\begin{array}{r}81.175 \\
-823\end{array}$ & $\begin{array}{l}50,757 \\
-1,606\end{array}$ & $\begin{array}{l}25,412 \\
-1,200\end{array}$ & - & $\begin{array}{r}1,918 \\
7\end{array}$ & $\begin{array}{r}3.063 \\
50\end{array}$ & $\begin{array}{r}14.774 \\
1.181\end{array}$ & $\begin{array}{r}220,892 \\
742\end{array}$ & $\begin{array}{r}1008 \\
08\end{array}$ \\
\hline TOTAL NET OSA & 54.729 & 80,351 & 49,150 & 24.211 & - & 1.925 & 3,113 & 8,153 & 221.634 & \\
\hline
\end{tabular}

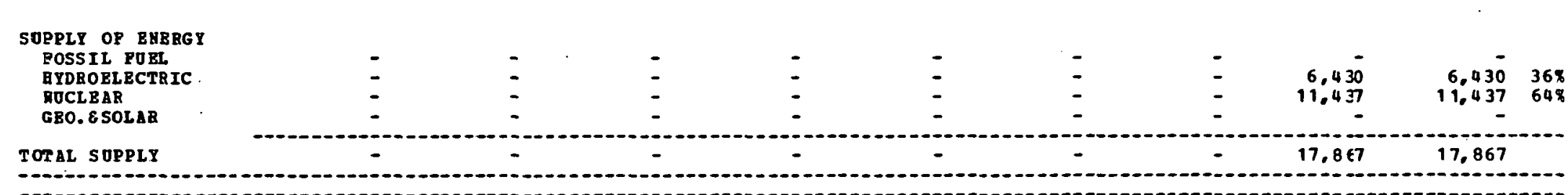

\section{BBT IMPORTS}

OP BRGIO

$54.729 \quad 80.351$

49,150

24.211

1,925

3,113

$-9.713$

203.767

-

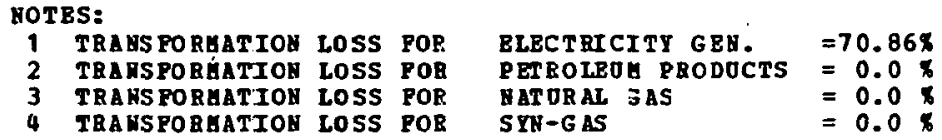

3 TRAMSPORHAT'TO LOSS POR

4 TRANSPORGATION LOSS POB

ATORAL SAS $=0.0$ 
REGIONAL ENERGY BALANCE STATELENT

BY SECTOR AND POEL TYPE

\begin{tabular}{|c|c|c|c|c|c|c|c|c|c|c|}
\hline SECTOR & $\begin{array}{c}\text { DISTILLATE } \\
\text { OIX }\end{array}$ & $\begin{array}{l}\text { RESIDOILL } \\
\text { OIL }\end{array}$ & $\begin{array}{l}\text { GASOLINE } \\
\text { (ALL OHIT }\end{array}$ & $\begin{array}{l}\text { HTDRO- } \\
\text { OABBONS } \\
\text { S IN 1C**9 }\end{array}$ & $\begin{array}{c}\text { CRODE } \\
\text { OIL } \\
\text { BTO'S) }\end{array}$ & $\begin{array}{l}\text { NATORAL } \\
\text { GAS }\end{array}$ & COAL & ELECTRICITY & $\begin{array}{l}\text { SECTOR } \\
\text { TOTAL }\end{array}$ & \\
\hline $\begin{array}{l}\text { INAL DEIAND SECTORS } \\
\text { RESIDENTIAL, COE!. } \\
\text { INDUSTRIAL. } \\
\text { TRANSPORTATION } \\
\text { GISCELLAKEOS USES }\end{array}$ & $\begin{array}{r}32.047 \\
1.021 \\
2.355 \\
.275\end{array}$ & $\begin{array}{r}3,737 \\
4,899 \\
12 \\
630\end{array}$ & $\$ 4.483$ & $\begin{array}{l}6.136 \\
6.997 \\
1.176 \\
168\end{array}$ & $\overline{-}$ & $\begin{array}{r}2,253 \\
1.655 \\
10 \overline{1}\end{array}$ & $\begin{array}{r}307 \\
1,833 \\
5 \\
-\end{array}$ & $\begin{array}{r}7,797 \\
4.419 \\
\quad 6 \\
166\end{array}$ & $\begin{array}{r}52,277 \\
20,744 \\
38,537 \\
1,310\end{array}$ & $\begin{array}{l}456 \\
185 \\
337 \\
156\end{array}$ \\
\hline $\begin{array}{l}\text { TOTAL PINAL } \\
\text { DEHA KD SECTOBS }\end{array}$ & 36,198 & 9.248 & 34,483 & $14,3 \cdot 37$ & - & 4.009 & 2,145 & 12,388 & 112,868 & $97 \%$ \\
\hline $\begin{array}{l}\text { TRANSPOAHATION } \\
\text { ELECTEICITY GEN. } \\
\text { PETROLEOL PRODOCTS } \\
\text { NATORAL GAS } \\
\text { STN-GAS }\end{array}$ & $\begin{array}{r}1,421 \\
- \\
-\end{array}$ & $\begin{array}{r}26 \\
- \\
-\end{array}$ & $\overline{-}$ & $\begin{array}{l}- \\
-\end{array}$ & $\begin{array}{l}- \\
-\end{array}$ & $\begin{array}{r}618 \\
- \\
-\end{array}$ & $\begin{array}{r}867 \\
- \\
-\end{array}$ & $\begin{array}{r}-597 \\
- \\
-\end{array}$ & $\begin{array}{r}2,335 \\
- \\
-\end{array}$ & كه \\
\hline $\begin{array}{l}\text { NET POEL OSED } \\
\text { IN TRAKSFORBAEION }\end{array}$ & 1.421 & 26 & - & $\therefore$ & - & 618 & 867 & -597 & 2,335 & 28 \\
\hline $\begin{array}{l}\text { TOTAL GROSS PLOTS } \\
\text { LOSSES E OHISSIJNS }\end{array}$ & $\begin{array}{r}37,619 \\
2,285\end{array}$ & $\begin{array}{r}9.274 \\
-94\end{array}$ & $\begin{array}{l}.34,483 \\
-1,091\end{array}$ & $\begin{array}{r}1+., \equiv 97 \\
-680\end{array}$ & - & $\begin{array}{r}4.627 \\
17\end{array}$ & $\begin{array}{r}3,012 \\
50\end{array}$ & $\begin{array}{r}12.388 \\
990\end{array}$ & $\begin{array}{r}115,203 \\
1,477\end{array}$ & $\begin{array}{r}978 \\
1 \%\end{array}$ \\
\hline
\end{tabular}

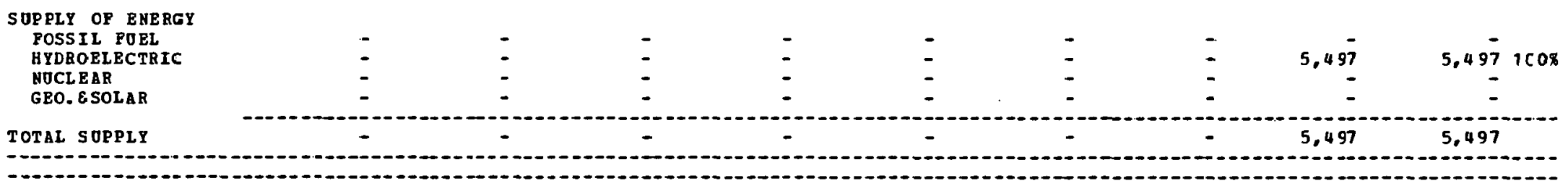

\section{NST IHPORTS}

3. ., 9014

9,179

33,391

13.716

4,644

3,062

7.284

111,183

\begin{tabular}{|c|c|c|c|c|c|}
\hline \\
\hline 2 & $\begin{array}{l}\text { TRANS FJRAAT IOH } \\
\text { TRANSP JRHAT IOH }\end{array}$ & $\begin{array}{l}\text { Loss } \\
\text { Los: }\end{array}$ & $\begin{array}{l}\text { POR } \\
\text { POR }\end{array}$ & $\begin{array}{l}\text { ELECT RICITY GEN. } \\
\text { PETROLEO PROLUCTS }\end{array}$ & $\begin{array}{l}=79.648 . \\
=0.08\end{array}$ \\
\hline 3 & TRA AS TO RAAT IOH & LoS: & POR & NAT JRAL GAS & $=0.0 \%$ \\
\hline 4 & TRANS POR HATION & Los & POR & $\subseteq Y N-G$ AS & $=0.0 \mathrm{6}$ \\
\hline
\end{tabular}




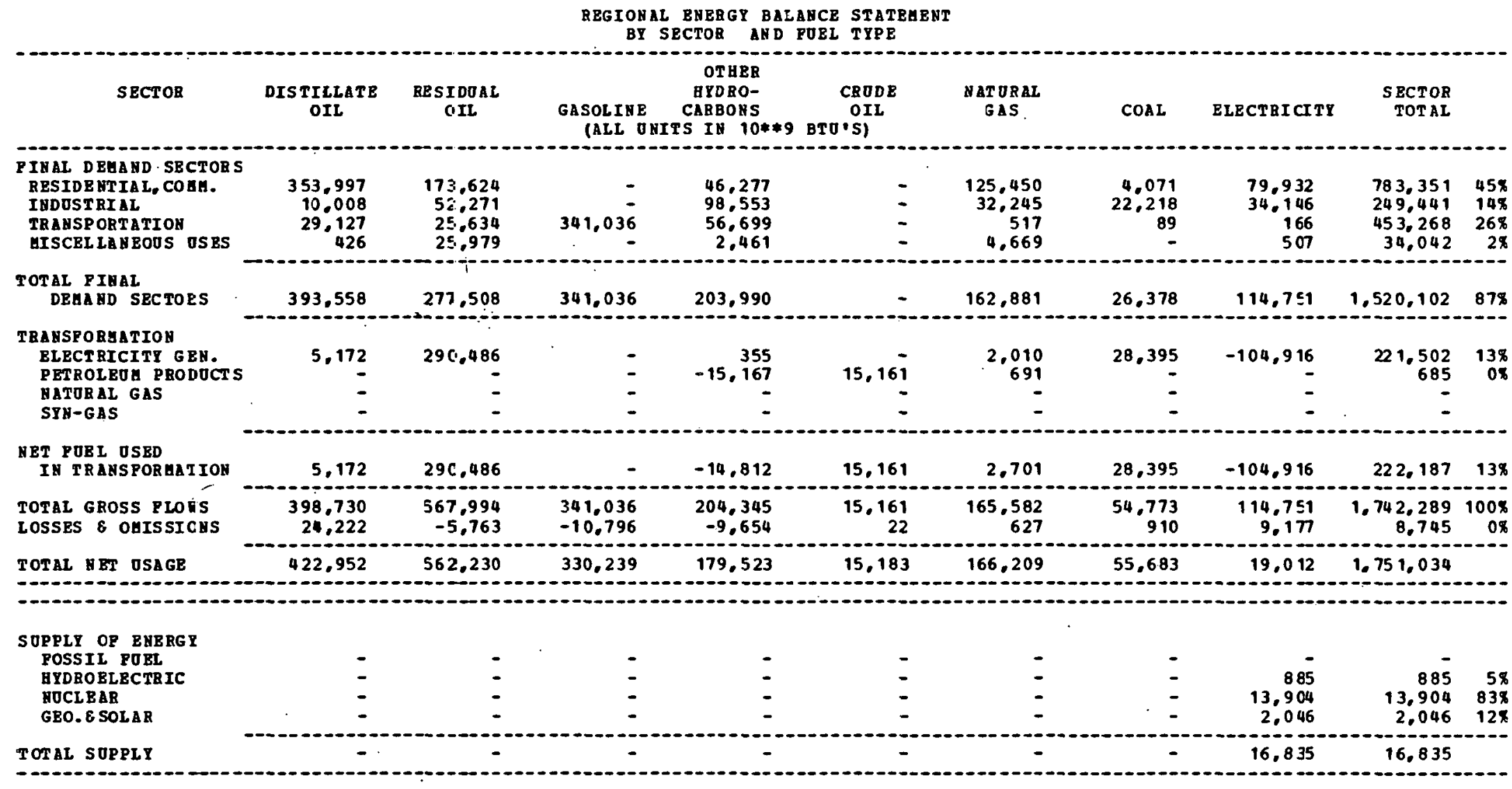

\begin{tabular}{|c|c|c|c|c|c|c|}
\hline \multicolumn{2}{|c|}{$\begin{array}{l}\text { BET IAPORTS } \\
\text { OP REGION }\end{array}$} & \multicolumn{2}{|c|}{422,952} & 562,230 & \multicolumn{2}{|c|}{330,239} \\
\hline & BS: & & & & & \\
\hline $\begin{array}{l}1 \\
2 \\
3 \\
4\end{array}$ & 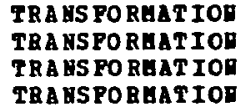 & $\begin{array}{l}\text { LOSS } \\
\text { LoSS } \\
\text { LoSS } \\
\text { LoSS }\end{array}$ & $\begin{array}{l}\text { POR } \\
\text { POR } \\
\text { POR } \\
\text { POR }\end{array}$ & $\begin{array}{l}\text { BLECTRICITY } \\
\text { PETROLEOA PI } \\
\text { GATORAL GAS } \\
\text { SYU-GAS }\end{array}$ & $\begin{array}{l}\text { GEN. } \\
\text { RODOCTS }\end{array}$ & $\begin{array}{l}=67.868 \\
=4.328 \\
=0.0 \times \\
=0.0 \times\end{array}$ \\
\hline
\end{tabular}




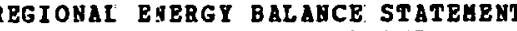

BY SECTOR AND POEL TYPE

\begin{tabular}{|c|c|c|c|c|c|c|c|c|c|}
\hline SECTOR & $\begin{array}{c}\text { DISTILLATE } \\
\text { OIL }\end{array}$ & $\begin{array}{c}\text { EBSIDOAL } \\
\text { OIL }\end{array}$ & $\begin{array}{l}\text { GASOLINE } \\
\text { (ALI OUIS }\end{array}$ & $\begin{array}{l}\text { AYDBO- } \\
\text { CABBOHS } \\
\text { IS IN } 10 * * 9\end{array}$ & $\begin{array}{c}\text { CRODE } \\
\text { OIL } \\
\text { BTO'SI }\end{array}$ & $\begin{array}{l}\text { MATORAL } \\
\text { GLS }\end{array}$ & COAL & BLECTRICITY & $\begin{array}{l}\text { SECTOR } \\
\text { TOTAL }\end{array}$ \\
\hline $\begin{array}{l}\text { PIHAL DEHAYD SECTORS } \\
\text { RBSIDE HTIAL, COBA. } \\
\text { IEDOSTRIAL } \\
\text { TRANSPORTRTION } \\
\text { GISCELLABOOS OSBS }\end{array}$ & $\begin{array}{r}1.6 .925 \\
-0.135 \\
14.511 \\
287\end{array}$ & $\begin{array}{r}39.874 \\
46,194 \\
4.067 \\
6.797\end{array}$ & 165.554 & $\begin{array}{r}17.570 \\
58.016 \\
15.052 \\
1.249\end{array}$ & : & $\begin{array}{r}47.331 \\
13.209 \\
1,68 \\
1.781\end{array}$ & $\begin{array}{r}: .672 \\
14.290 \\
35 \\
-\end{array}$ & $\begin{array}{r}42,365 \\
20,463 \\
34 \\
59\end{array}$ & $\begin{array}{r}275,737 \\
167,287 \\
199,321 \\
10,173\end{array}$ \\
\hline $\begin{array}{l}\text { TOTAL PIRAE } \\
\text { DEHABD SBCTORS }\end{array}$ & 151,858 & 96.932 & 165,554 & 91,887 & - & 67,389 & 15,997 & 62,901 & 652,518 \\
\hline $\begin{array}{l}\text { TRANSFORGATION } \\
\text { ELECTRICI TY GBH. } \\
\text { PETROLBOI PRODOCTS } \\
\text { NATORAL GAS } \\
\text { SYH-GAS }\end{array}$ & $\begin{array}{r}3,281 \\
=\end{array}$ & $\begin{array}{r}139,769 \\
=\end{array}$ & : & $\begin{array}{r}527 \\
-\end{array}$ & $\begin{array}{l}- \\
-\end{array}$ & $\begin{array}{r}3.631 \\
-\end{array}$ & $\begin{array}{r}999 \\
-\end{array}$ & $\begin{array}{r}-39,650 \\
= \\
=\end{array}$ & $\begin{array}{r}108,557 \\
= \\
-\end{array}$ \\
\hline $\begin{array}{l}\text { MET PUBL OSED } \\
\text { IH TRAHS PORGAT ION }\end{array}$ & 3.281 & 139,769 & - & 527 & - & 3.631 & 999 & $-39,650$ & 108,557 \\
\hline $\begin{array}{l}\text { TOTAL GROSS PLORS } \\
\text { LOSSES \& OAISSTONS }\end{array}$ & $\begin{array}{r}155.139 \\
9.424\end{array}$ & $\begin{array}{r}236,701 \\
-2,401\end{array}$ & $\begin{array}{r}165.554 \\
-5,241\end{array}$ & $\begin{array}{l}92,414 \\
-4,366\end{array}$ & - & $\begin{array}{r}71.020 \\
268\end{array}$ & $\begin{array}{r}15.996 \\
282\end{array}$ & $\begin{array}{r}62,901 \\
5,030\end{array}$ & $\begin{array}{r}761,075 \\
2,997\end{array}$ \\
\hline
\end{tabular}

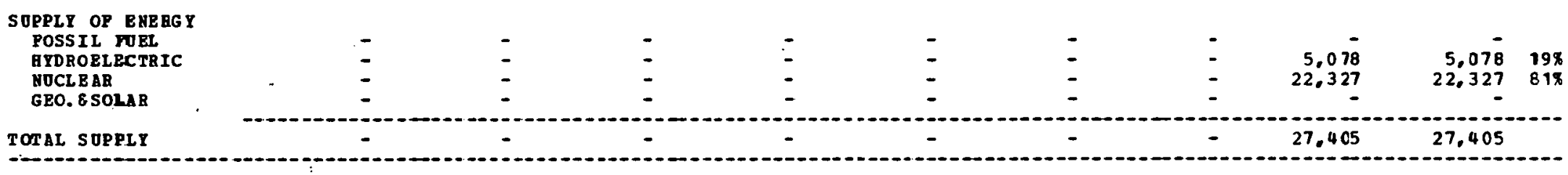

\section{BET IAPORIS}

$164,563 \quad 234,299 \quad 160,312$

88,047

11.288

17,278

876

736,667

NOT ES:

\begin{tabular}{|c|c|c|c|c|}
\hline 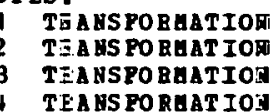 & $\begin{array}{l}\text { L Lass } \\
\text { Lass } \\
\text { Lass } \\
\text { Lass }\end{array}$ & $\begin{array}{l}\text { POR } \\
\text { POQ } \\
\text { POR } \\
\text { POE }\end{array}$ & 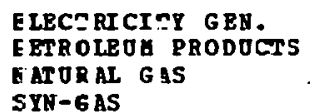 & $\begin{array}{l}=73.25 x \\
=0.08 \\
=0.08 \\
=0.08\end{array}$ \\
\hline
\end{tabular}


REGIONAL ENERGY BALANCE STATEAENT

BI SBCTOR AND FOBE TRPE

\begin{tabular}{|c|c|c|c|c|c|c|c|c|c|}
\hline SECTOR & $\begin{array}{l}\text { DISTILLATE } \\
\text { OIL }\end{array}$ & $\begin{array}{l}\text { RESIDOAL } \\
\text { OIL }\end{array}$ & $\begin{array}{l}\text { GASOLI I) } \\
\text { (ALL }\end{array}$ & $\begin{array}{c}\text { OTAER } \\
\text { GYDRO- } \\
\text { CARBOES } \\
\text { ITS IN 10**9 }\end{array}$ & $\begin{array}{c}\text { CRODE } \\
\text { OII } \\
\text { BTO'SI }\end{array}$ & $\begin{array}{l}\text { MATURAL } \\
\text { GAS }\end{array}$ & COAL & ELECTRI CITY & $\begin{array}{r}\text { SECTOR } \\
\text { TOTAL }\end{array}$ \\
\hline $\begin{array}{l}\text { PIHAL DEHAHD SECTORS } \\
\text { RESIDE NTIAL, COBA. } \\
\text { IHDDSTRIAL } \\
\text { TRANSPORTATI ON } \\
\text { GISCEL LAHEOUS OSES }\end{array}$ & $\begin{array}{r}46.240 \\
2.565 \\
3.193 \\
239\end{array}$ & $\begin{array}{r}19,629 \\
8,689 \\
1,799 \\
5,411\end{array}$ & 61.543 & $\begin{array}{r}7.658 \\
22.766 \\
3.091 \\
432\end{array}$ & . & $\begin{array}{r}32,906 \\
10,072 \\
220 \\
1.616\end{array}$ & $\begin{array}{r}848 \\
21,371 \\
13 \\
-\end{array}$ & $\begin{array}{r}14,403 \\
8,983 \\
142 \\
916\end{array}$ & $\begin{array}{r}121,684 \\
74,446 \\
70,001 \\
8,614\end{array}$ \\
\hline $\begin{array}{l}\text { TOTAL PIHAL } \\
\text { DEGA SD SBCTOBS }\end{array}$ & 52,237 & 35.528 & 61,543 & 33.947 & 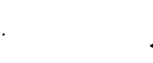 & 44,814 & 22,232 & 24.444 & 274.745 \\
\hline $\begin{array}{l}\text { TRANSPORHATIOH } \\
\text { ELECT RICITY GEH. } \\
\text { PBTROLEOE PRODOCTS } \\
\text { NATORAL GAS } \\
\text { SYN-GAS }\end{array}$ & $\begin{array}{r}3.007 \\
- \\
-\end{array}$ & $\begin{array}{r}26,133 \\
= \\
=\end{array}$ & $\begin{array}{l}- \\
-\end{array}$ & - & . & $\begin{array}{r}365 \\
- \\
-\end{array}$ & $\begin{array}{l}\overline{-} \\
\overline{-}\end{array}$ & $\begin{array}{r}-9.654 \\
- \\
-\end{array}$ & $\begin{array}{r}19,851 \\
:-\end{array}$ \\
\hline $\begin{array}{l}\text { MBT POEL OSED } \\
\text { IN TRABSPORGATIOH }\end{array}$ & 3.007 & 26.133 & - & - & 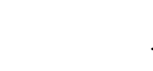 & 365 & - & $-9,654$ & 19,851 \\
\hline $\begin{array}{l}\text { TOTAL GROSS PLORS } \\
\text { LOSSES } 8 \text { OUISSI OAS }\end{array}$ & $\begin{array}{r}55,244 \\
3,356\end{array}$ & $\begin{array}{r}61.661 \\
-625\end{array}$ & $\begin{array}{r}61,543 \\
-1,948\end{array}$ & $\begin{array}{r}33,947 \\
-1,603\end{array}$ & tha & $\begin{array}{r}45.179 \\
171\end{array}$ & $\begin{array}{r}22,232 \\
369\end{array}$ & $\begin{array}{r}24.444 \\
1.954\end{array}$ & $\begin{array}{r}294.596 \\
1.673\end{array}$ \\
\hline TOTAL NET OSAGB & 58.600 & 61.035 & 59.594 & 32.343 & & 45,350 & 22,601 & 16.744 & 296.269 \\
\hline
\end{tabular}

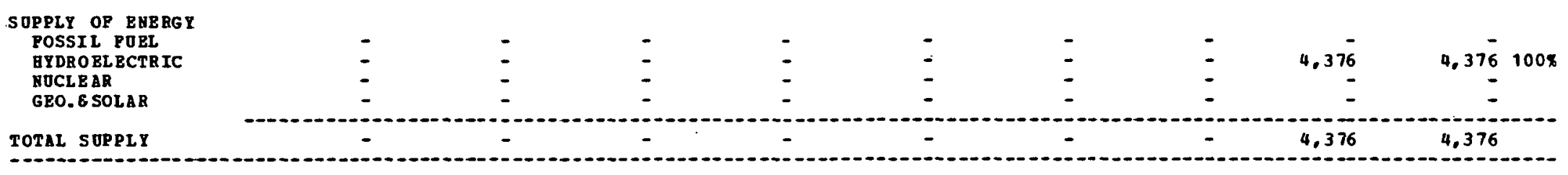

HET IHPORTS

58.600

61,035

59.594

32.343

45,350

22,601

12,368

291,893

-

NOTBS:

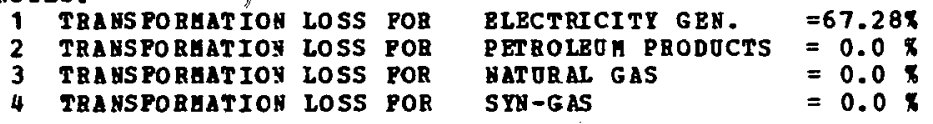




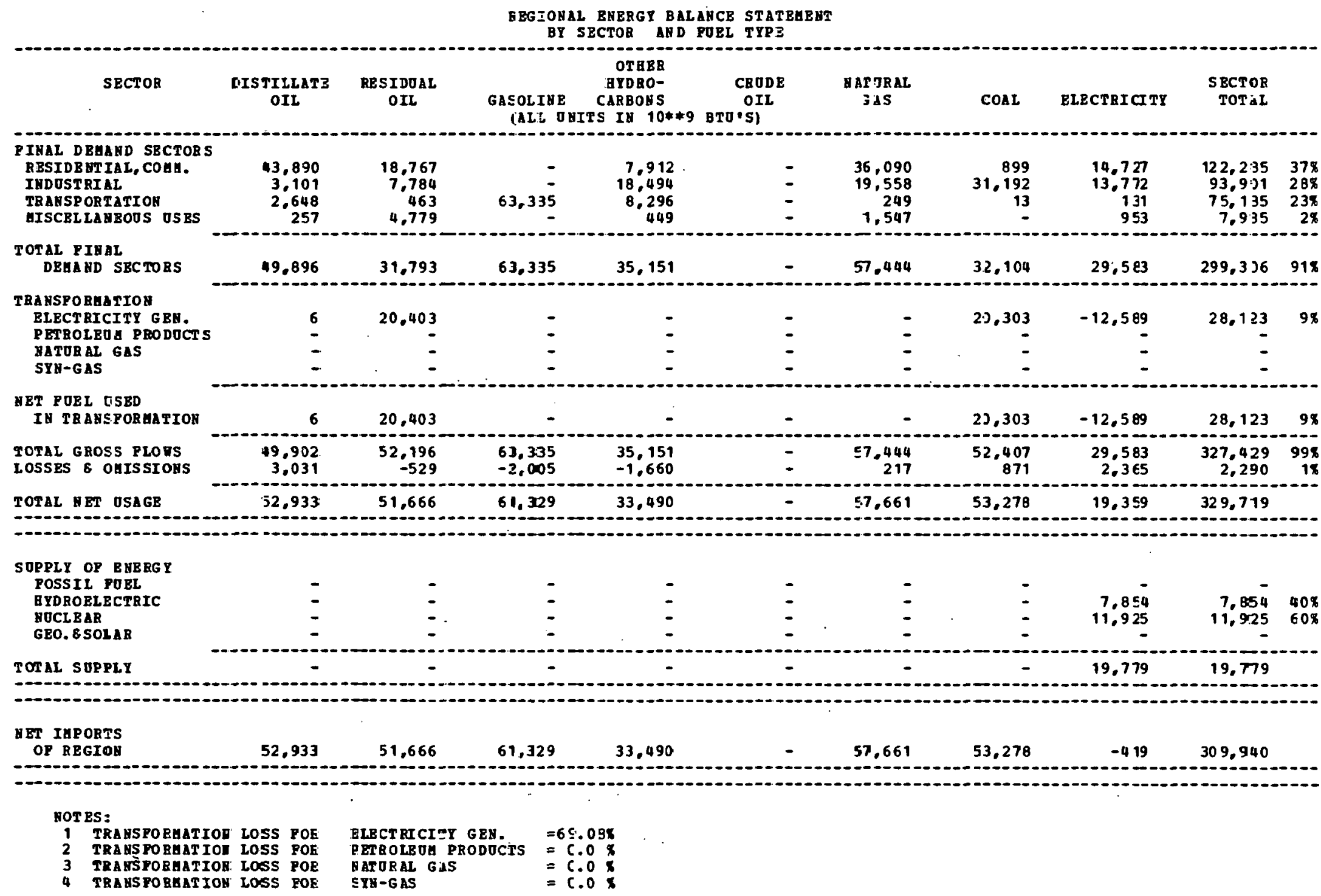

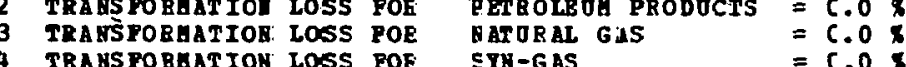


REGIOHAL BNEBGY BALANCE STATEHENT

BY SECTOR ABD FOBL TYPE

\begin{tabular}{|c|c|c|c|c|c|c|c|c|c|c|}
\hline SECTOR & $\begin{array}{l}\text { DISTILLATE } \\
\text { OIL }\end{array}$ & $\begin{array}{l}\text { RES I DO AL } \\
\text { OIL }\end{array}$ & $\begin{array}{r}\text { GASOLINE } \\
\text { (ALL O }\end{array}$ & $\begin{array}{l}\text { HYDRO- } \\
\text { CARBOHS } \\
\text { TS IH 10**9 }\end{array}$ & $\begin{array}{c}\text { CRODE } \\
\text { OIL } \\
\text { BTO'S) }\end{array}$ & $\begin{array}{l}\text { NATURAL } \\
\text { GAS }\end{array}$ & COAI & ELECTRI CITY & $\begin{array}{l}\text { SECTOR } \\
\text { TOTAL }\end{array}$ & \\
\hline $\begin{array}{l}\text { TOTAL PIMAL } \\
\text { DEHA SD SECTORS }\end{array}$ & 35.253 & 22,079 & 43,982 & 23,951 & - & 39,161 & 28,404 & 18,839 & 211,669 & $90 \%$ \\
\hline $\begin{array}{l}\text { TRANSPORAATIOA } \\
\text { ELBCT RICITY GEM. } \\
\text { RETROLEOA PRODOCTS } \\
\text { BATORAL GAS } \\
\text { STH-GAS. }\end{array}$ & $\begin{array}{r}69 \\
- \\
-\end{array}$ & $\begin{array}{r}2.065 \\
-\end{array}$ & $=$ & - & $\begin{array}{l}- \\
\overline{-} \\
\overline{-}\end{array}$ & $\begin{array}{r}80 \\
- \\
-\end{array}$ & $\begin{array}{r}26,796 \\
- \\
-\end{array}$ & $\begin{array}{r}-8,345 \\
- \\
-\end{array}$ & $\begin{array}{r}21,265 \\
= \\
=\end{array}$ & $9 \%$ \\
\hline $\begin{array}{l}\text { YET FOEL OSBD } \\
\text { IE TBAHSPORGA TIOH }\end{array}$ & 69 & 2,665 & $\overline{-}-$ & - & $\overline{-}$ & 80 & 26,796 & $-8,345$ & 21,265 & $9 \%$ \\
\hline $\begin{array}{l}\text { OOTAL GROSS FLORS } \\
\text { LOSSES } 6 \text { OUISSTOES }\end{array}$ & $\begin{array}{r}35,322 \\
2,145\end{array}$ & $\begin{array}{r}24.744 \\
-251\end{array}$ & $\begin{array}{l}43,982 \\
-1,392\end{array}$ & $\begin{array}{l}23,951 \\
-1,131\end{array}$ & - & $\begin{array}{r}39.241 \\
148\end{array}$ & $\begin{array}{r}55,200 \\
917\end{array}$ & $\begin{array}{r}18.839 \\
1.506\end{array}$ & $\begin{array}{r}232,934 \\
1.943\end{array}$ & $\begin{array}{r}99 \% \\
1 \%\end{array}$ \\
\hline
\end{tabular}

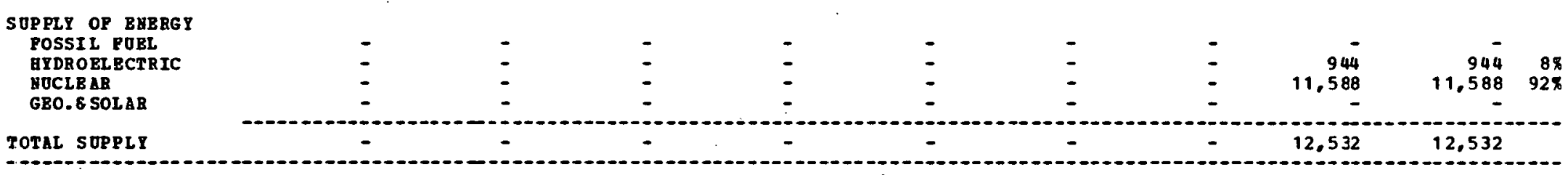

\section{NET IHPOBTS}

OP RBG1

$37,467 \quad 24,492 \quad 42,589$

22.819

39,389

56,117

$-531$

222.345

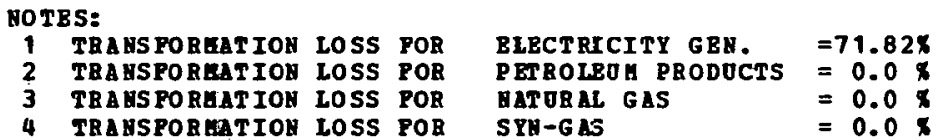

T TRA BSPORAAT IOH LOSS POR

MATORAL

$=0.0 \times$ 
REGIOHAL BHERGI BALABCE STATEGENT

BY SECTOR AND POEL TYPS

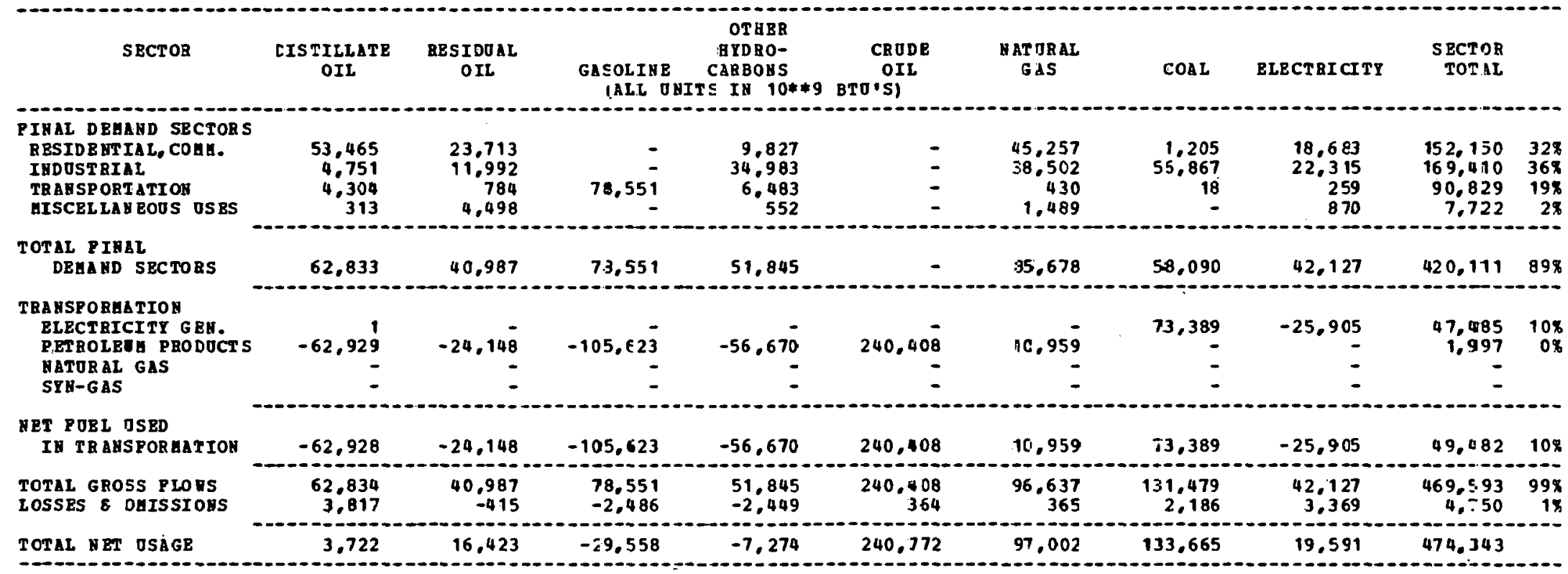

-

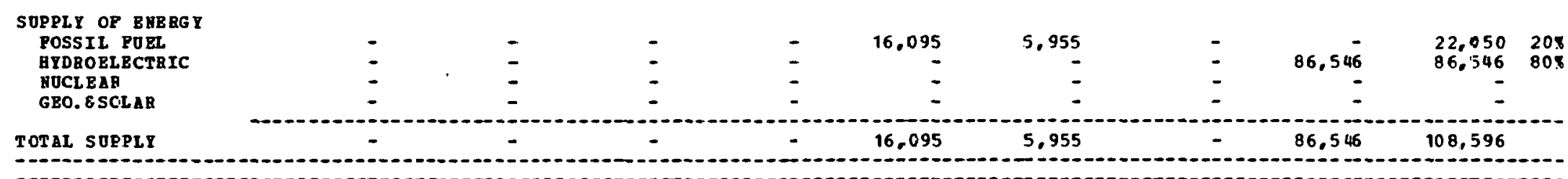

\begin{tabular}{|c|c|c|c|c|c|c|}
\hline \multicolumn{2}{|c|}{$\begin{array}{l}\text { NET IAPORTS } \\
\text { OP REGION }\end{array}$} & \multicolumn{2}{|c|}{3,722} & $16,42 \vdots$ & \multicolumn{2}{|c|}{-29.558} \\
\hline NOT & ES: & & & & & \\
\hline $\begin{array}{l}1 \\
2 \\
3 \\
4\end{array}$ & 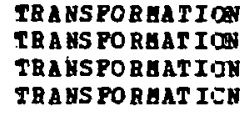 & $\begin{array}{l}\text { LOSS } \\
\text { LOSS } \\
\text { LOSS } \\
\text { LOSS }\end{array}$ & $\begin{array}{l}\text { POR } \\
\text { POR } \\
\text { POR } \\
\text { POR }\end{array}$ & $\begin{array}{l}\text { ELECT RICETY } \\
\text { PETBOLEOA P } \\
\text { BRTORAL GAS } \\
\text { SYH-GAS }\end{array}$ & $\begin{array}{l}\text { GEN. } \\
\text { RODOCTS }\end{array}$ & $\begin{array}{l}=69.708 \\
=0.798 \\
=0.08 \\
=0.08\end{array}$ \\
\hline
\end{tabular}


REgIONAL ENGRGY BALANCE STATEHENT

BY SECTOR AND FOEL TYPE

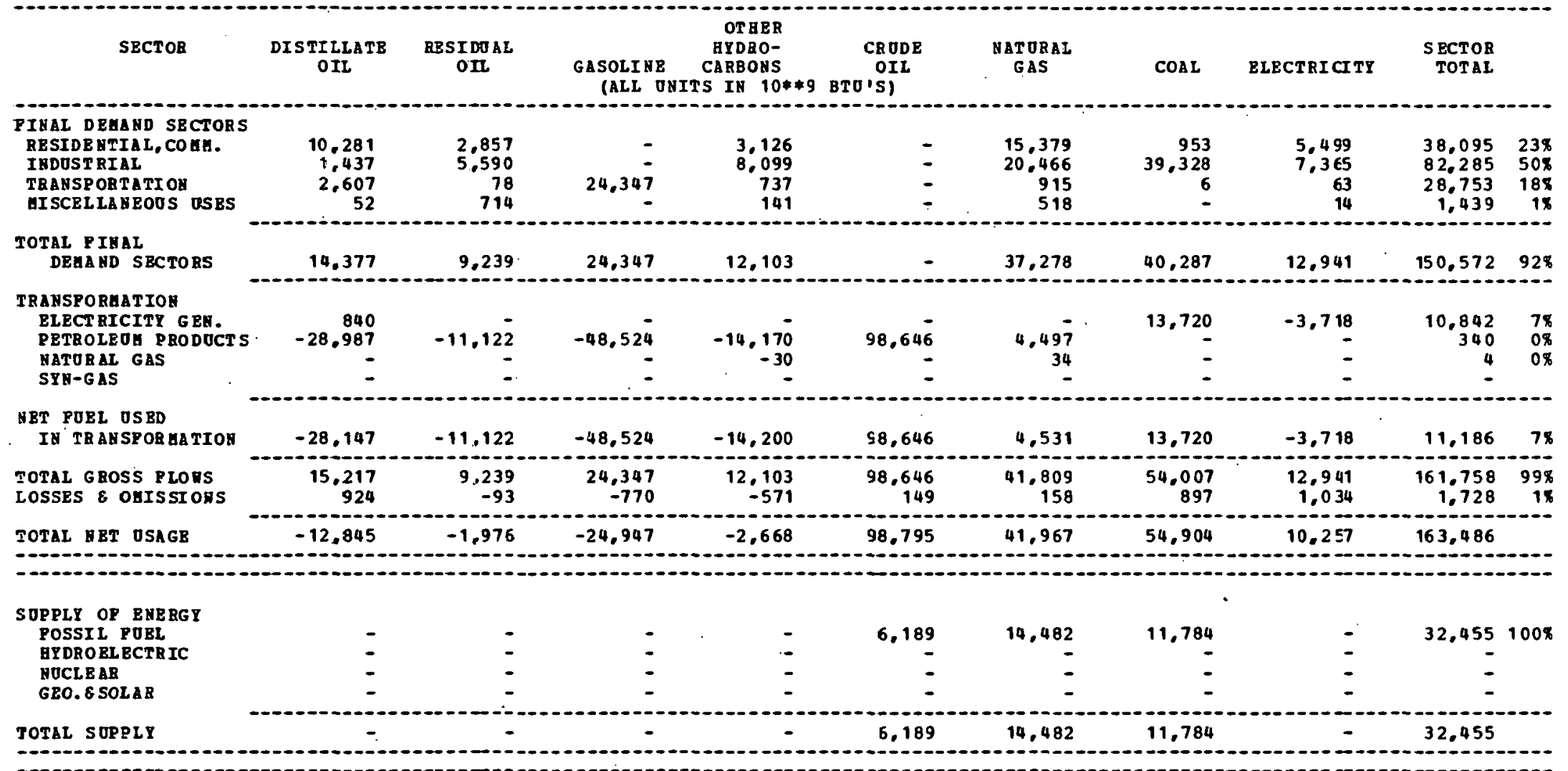

MET IUPORTS
OF BEGIOA


REGI JNAL BLERGY BALABCE STATEBEHT
BY SBCTOB ALD POBL TYPE

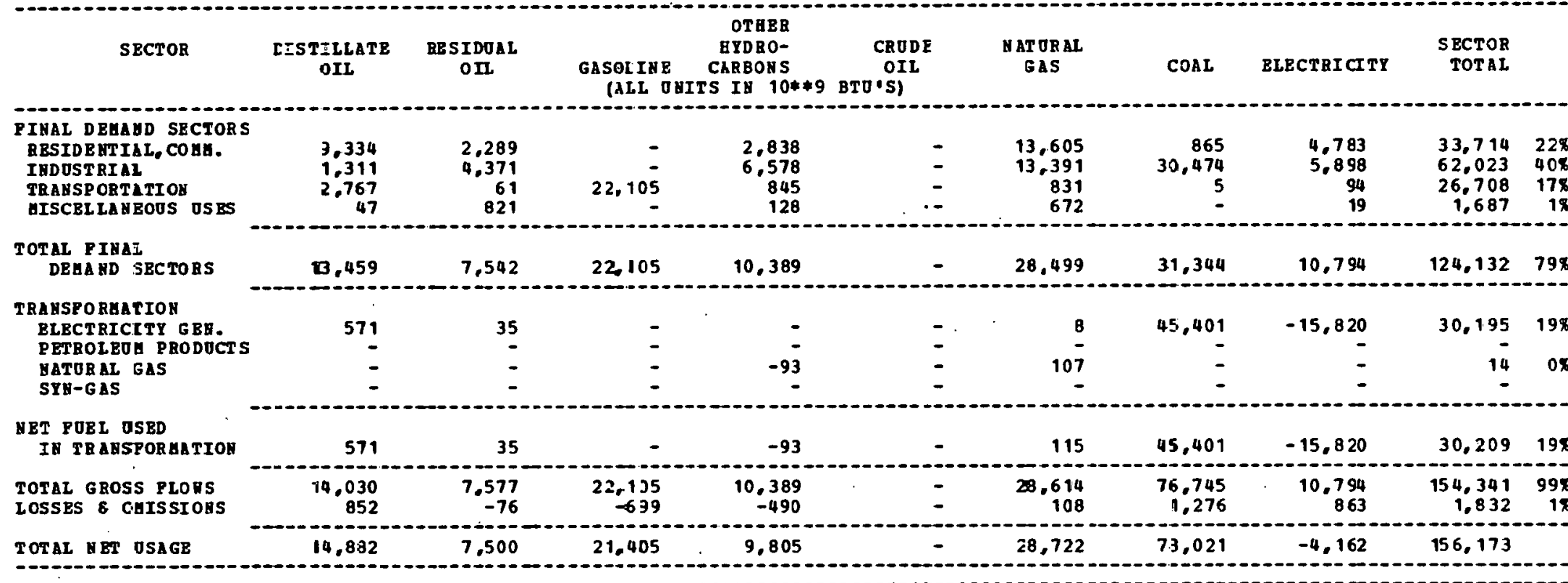

\begin{tabular}{|c|c|c|c|c|c|c|c|c|c|}
\hline $\begin{array}{l}\text { SOPPLY OP ESBRG } \\
\text { POSSIL POBL } \\
\text { QYDROELECTRIC } \\
\text { HOCLEAR } \\
\text { GEO.ESOLAR }\end{array}$ & $\bar{E}$ & : & $\bar{z}$ & $=$ & $\begin{array}{r}116 \\
- \\
-\end{array}$ & $\begin{array}{r}29.181 \\
- \\
-\end{array}$ & $\begin{array}{r}273,800 \\
= \\
=\end{array}$ & $\begin{array}{l}- \\
-\end{array}$ & $\begin{array}{r}303,097 \\
= \\
=\end{array}$ \\
\hline
\end{tabular}

$\begin{array}{ll}-10 & \end{array}$

BET IMPORTS

14,882

7.500

21,405

9,805

$-116$

$-45 \varepsilon \quad-195.778$

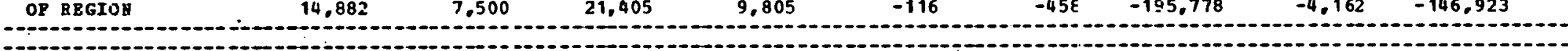

NOT BS:

\begin{tabular}{|c|c|c|c|}
\hline 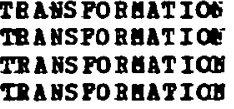 & $\begin{array}{l}\text { LC SS } \\
\text { LC SS } \\
\text { LC.SS } \\
\text { LCSS }\end{array}$ & $\begin{array}{l}\text { POZ } \\
\text { POa } \\
\text { PO3 } \\
\text { POB }\end{array}$ & $\begin{array}{l}\text { EIECTRICITY GEH. } \\
\text { PETROLEOR: PRODOCTS } \\
\text { IATORAL GAS } \\
\text { S TH-GAS }\end{array}$ \\
\hline
\end{tabular}


REgIONAL EXERG BALANCE STATEGENT

BY SECTOB AND POEL TYPE

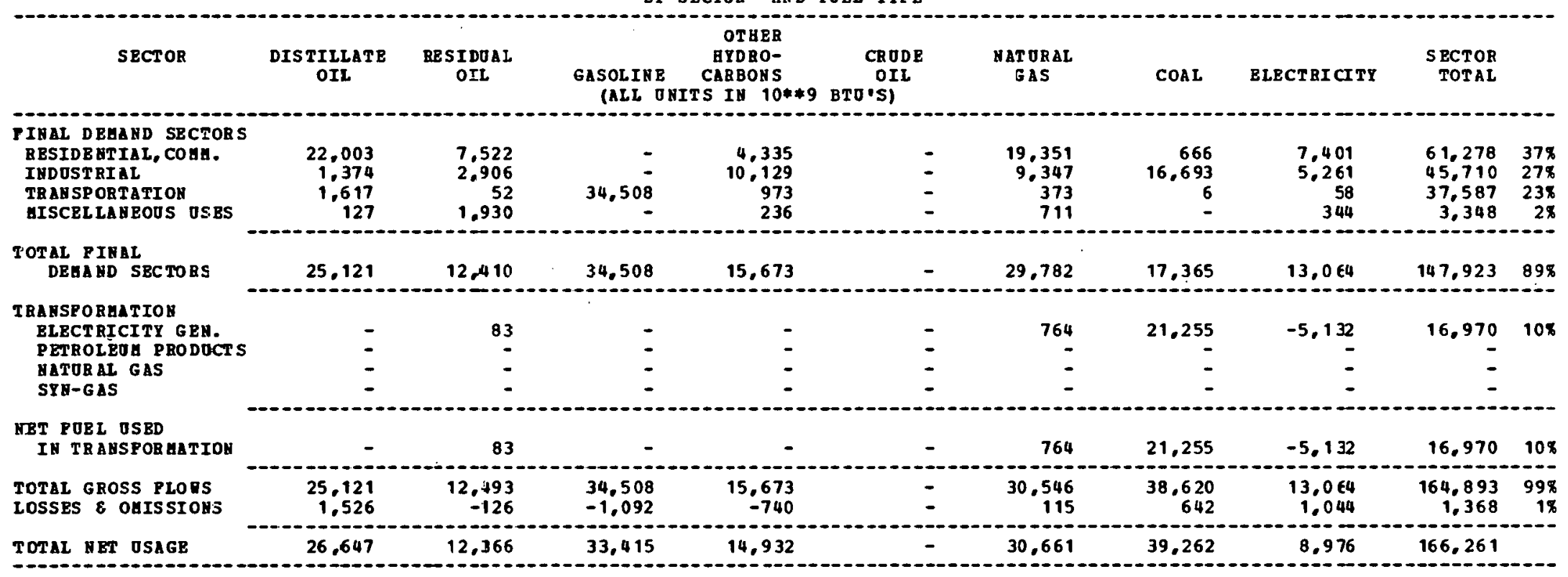

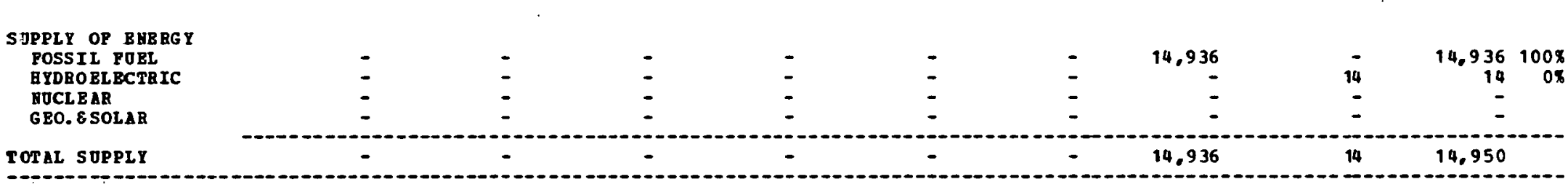

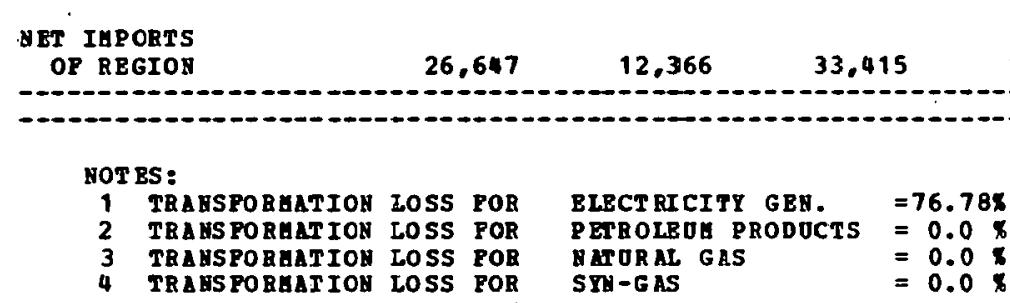


REgIONAL ENETGY BALANCE STATEAENT

BT SECTOS AND FOEL TYPE

\begin{tabular}{|c|c|c|c|c|c|c|c|c|c|c|}
\hline SBCTOR & $\begin{array}{l}\text { DISTIILATE } \\
\text { OIL }\end{array}$ & $\begin{array}{l}\text { BESI DOAL } \\
\text { OII }\end{array}$ & $\begin{array}{l}\text { GASOLIME } \\
\text { (ALL OBI }\end{array}$ & $\begin{array}{c}\text { OPGBQ } \\
\text { BYORO- } \\
\text { CARBONS } \\
\text { TS IB 10**9 }\end{array}$ & $\begin{array}{c}\text { CRIDE } \\
\text { OIL } \\
\text { BTO'S) }\end{array}$ & $\begin{array}{l}\text { MATURAL } \\
\text { GAS }\end{array}$ & COLL & ELECTRICITY & $\begin{array}{r}\text { SECTOR } \\
\text { TOTAL }\end{array}$ & \\
\hline $\begin{array}{l}\text { PIBAL DBEAHD SECTORS } \\
\text { RESIDB HTIAL, COBH. } \\
\text { INDOST RIAI } \\
\text { TRAHSP ORTATIOA } \\
\text { GISCEL LAHEOS OSBS }\end{array}$ & $\begin{array}{r}15.295 \\
1.774 \\
4.821 \\
77\end{array}$ & $\begin{array}{l}4.299 \\
4.932 \\
111 \\
1.047\end{array}$ & $36.22 \overline{-}$ & $\begin{array}{r}4.650 \\
10.708 \\
1.460 \\
209\end{array}$ & $\begin{array}{l}- \\
-\end{array}$ & $\begin{array}{r}22.934 \\
13.420 \\
1.362 \\
753\end{array}$ & $\begin{array}{r}1.418 \\
36.853 \\
8 \\
-\end{array}$ & $\begin{array}{r}8.229 \\
7.505 \\
150 \\
21\end{array}$ & $\begin{array}{r}56,825 \\
75,192 \\
44,132 \\
2,107\end{array}$ & $\begin{array}{r}31 \% \\
40 \% \\
24 \% \\
1 \%\end{array}$ \\
\hline $\begin{array}{l}\text { TOTAL FIRAL } \\
\text { DEHA AD SECTORS }\end{array}$ & 21,967 & 10,389 & 36.220 & 17.027 & - & 38,459 & 38,279 & 15.905 & 178,256 & $96 \%$ \\
\hline $\begin{array}{l}\text { TRARSPORBATIOB } \\
\text { BLECT RICITI GRN. } \\
\text { PETROLBOA PRODOCTS } \\
\text { MATORAL GAS } \\
\text { SYH-GAS }\end{array}$ & $\begin{array}{r}467 \\
- \\
-\end{array}$ & $\begin{array}{l}- \\
-\end{array}$ & $\begin{array}{l}- \\
-\end{array}$ & $\begin{array}{l}- \\
-\end{array}$ & $\begin{array}{l}- \\
-\end{array}$ & $\begin{array}{l}- \\
-\end{array}$ & $\begin{array}{r}6,351 \\
- \\
-\end{array}$ & $\begin{array}{r}-1,252 \\
- \\
-\end{array}$ & $\begin{array}{r}6,066 \\
- \\
-\end{array}$ & $3 x$ \\
\hline $\begin{array}{l}\text { ABT FOBL OSED } \\
\text { IH TRAHSFCIB GA TIOA }\end{array}$ & 467 & - & - & - & - & - & 6,851 & $-1,252$ & 6.066 & $3 x$ \\
\hline $\begin{array}{l}\text { TOTAL GROSS PLODS } \\
\text { LOSSES } 8 \text { OUISSIORS }\end{array}$ & $\begin{array}{r}22.434 \\
1.362\end{array}$ & $\begin{array}{r}10,389 \\
-105\end{array}$ & $\begin{array}{l}36,220 \\
-1,146\end{array}$ & $\begin{array}{r}17.027 \\
-804\end{array}$ & - & $\begin{array}{r}38.469 \\
145\end{array}$ & $\begin{array}{r}45,130 \\
750\end{array}$ & $\begin{array}{r}15,905 \\
1.272\end{array}$ & $\begin{array}{r}184,322 \\
1,474\end{array}$ & $\begin{array}{r}99 \% \\
18\end{array}$ \\
\hline $\begin{array}{l}\text { TOTAL DET OSAGE } \\
\end{array}$ & 23.796 & 10.283 & 35.073 & 16,222 & - & 38,614 & 45,880 & 15,925 & 185,796 & \\
\hline $\begin{array}{l}\text { SOPPLI OF BHERGI } \\
\text { POSSIL FOEL } \\
\text { HYDROELECIRIC } \\
\text { HOCLB AR } \\
\text { GEO.8 SOLAB }\end{array}$ & $\begin{array}{l}\overline{-} \\
\overline{-}\end{array}$ & $\begin{array}{l}- \\
- \\
-\end{array}$ & $\dot{-}$ & $\begin{array}{l}- \\
-\end{array}$ & $\overline{-}$ & $=$ & $\begin{array}{r}59,479 \\
= \\
=\end{array}$ & 34 & $\begin{array}{r}59.47 \mathrm{C} \\
340 \\
= \\
-\end{array}$ & $\begin{array}{r}998 \\
18\end{array}$ \\
\hline TOTAL SUPPLT & - & - & - & - & - & - & 59.479 & 344 & $59,82 \equiv$ & \\
\hline
\end{tabular}

NET IEPORTS

OF REGIOH

23,796

10,283

35,073

16,222

38.614

$-13,598$

$15,581 \quad 125,973$

HOTES:

1 TRAFS PORHATION LOSS POR

TRAAS POBAATION LOSS

TRAISS PORAATION COSS $P O B$

TRAISPOREATION LOSS POR

BLECTRICITY GEH.

PEROLEOL PRODOCTS

SYU-GAS

$=82.89 x$
$=0.0$
$=0.0 \%$
$=0.0 x$ 
BEgIONAL BNERG BALAHCE STATBHBTT

BY SBCTOQ AHD FOEL TYPE

\begin{tabular}{|c|c|c|c|c|c|c|c|c|c|c|}
\hline SECTOR & $\begin{array}{l}\text { DIS TILLATB } \\
\text { OIL }\end{array}$ & $\begin{array}{l}\text { RESIDOAL } \\
\text { OIL }\end{array}$ & $\begin{array}{l}\text { GASOL INE } \\
\text { (ALL O }\end{array}$ & $\begin{array}{c}\text { BYDRO- } \\
\text { CARBONS } \\
\text { IS IB 10*9 }\end{array}$ & $\begin{array}{c}\text { CRODE } \\
\text { OIL } \\
\text { BTO'S) }\end{array}$ & $\begin{array}{l}\text { MATORAL } \\
\text { GAS }\end{array}$ & $\operatorname{COAL}$ & ELECTRICITY & $\begin{array}{r}\text { SECTOR } \\
\text { TOTAL }\end{array}$ & \\
\hline 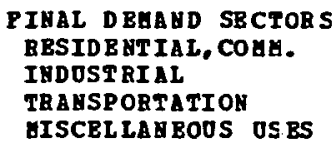 & $\begin{array}{r}610.945 \\
33.063 \\
101.911 \\
3.796\end{array}$ & $\begin{array}{r}316.984 \\
117.957 \\
167.861 \\
34.275\end{array}$ & $862,96 \overline{-}$ & $\begin{array}{r}115,104 \\
250,159 \\
259,124 \\
5,524\end{array}$ & $\overline{-}$ & $\begin{array}{r}484.559 \\
135,419 \\
2.574 \\
13.766\end{array}$ & $\begin{array}{r}11.080 \\
142,332 \\
354 \\
-\end{array}$ & $\begin{array}{r}235,744 \\
106,175 \\
8,431 \\
5,018\end{array}$ & $\begin{array}{r}1,774.416 \\
785,105 \\
1.403 .215 \\
62.379\end{array}$ & $\begin{array}{r}38 \% \\
17 \% \\
308 \\
18\end{array}$ \\
\hline $\begin{array}{l}\text { TOTAL FINAL } \\
\text { DEAA SD SBCTORS }\end{array}$ & 749.715 & 637.077 & 862,960 & 629,911 & - & 636,318 & 153,766 & 355,368 & 4.025 .115 & $86 \%$ \\
\hline $\begin{array}{l}\text { TRANSPORHATION } \\
\text { ELECTRICITY GRH. } \\
\text { PETROLED PRODUCTS } \\
\text { HATORAL GAS } \\
\text { SYH-GAS }\end{array}$ & $\begin{array}{r}92,863 \\
-222,078 \\
-\end{array}$ & $\begin{array}{r}711,971 \\
-85,210 \\
- \\
-\end{array}$ & $\begin{array}{r}-367,450 \\
-\end{array}$ & $\begin{array}{r}413 \\
-229.167 \\
- \\
-\end{array}$ & $\begin{array}{r}869.015 \\
-\end{array}$ & $\begin{array}{r}87,847 \\
39,615 \\
-\end{array}$ & $\begin{array}{r}26,034 \\
- \\
-\end{array}$ & $\begin{array}{r}-265,766 \\
- \\
-\end{array}$ & $\begin{array}{r}653.262 \\
4.725 \\
-\end{array}$ & $\begin{array}{r}14 \% \\
0 \%\end{array}$ \\
\hline $\begin{array}{l}\text { HET POBL OSED } \\
\text { IH TRABSFORAATION }\end{array}$ & -129.215 & 626.661 & $-367,450$ & $-228,754$ & 869.015 & 127.462 & 26.034 & $-265,766$ & 657.987 & 148 \\
\hline $\begin{array}{l}\text { TOTAL GROSS FLOUS } \\
\text { LOSSES } \varepsilon \text { OAISSIOH }\end{array}$ & $\begin{array}{r}842,578 \\
51.186\end{array}$ & $\begin{array}{r}1,348,948 \\
-13,688\end{array}$ & $\begin{array}{r}862,960 \\
-27,320\end{array}$ & $\begin{array}{l}630,324 \\
-29,781\end{array}$ & $\begin{array}{r}869,015 \\
1,317\end{array}$ & $\begin{array}{r}763,780 \\
2,892\end{array}$ & $\begin{array}{r}179,800 \\
2,989\end{array}$ & $\begin{array}{r}355,368 \\
28.420\end{array}$ & $\begin{array}{r}4,683,102 \\
16,015\end{array}$ & $\begin{array}{r}1008 \\
0 \%\end{array}$ \\
\hline
\end{tabular}

(1)

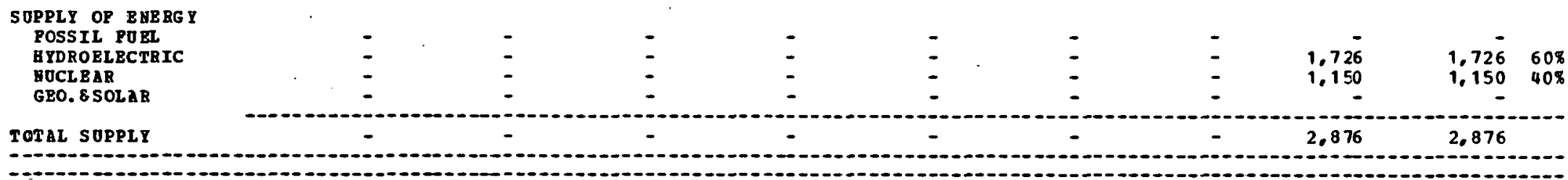

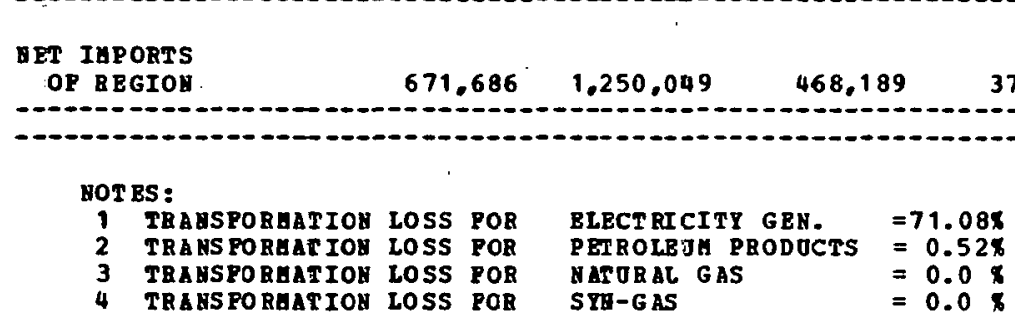


RBGICHAL BN $3 R G$ BALAICE STE TBEENT

EY. SECTOR AND FOBL TYPE

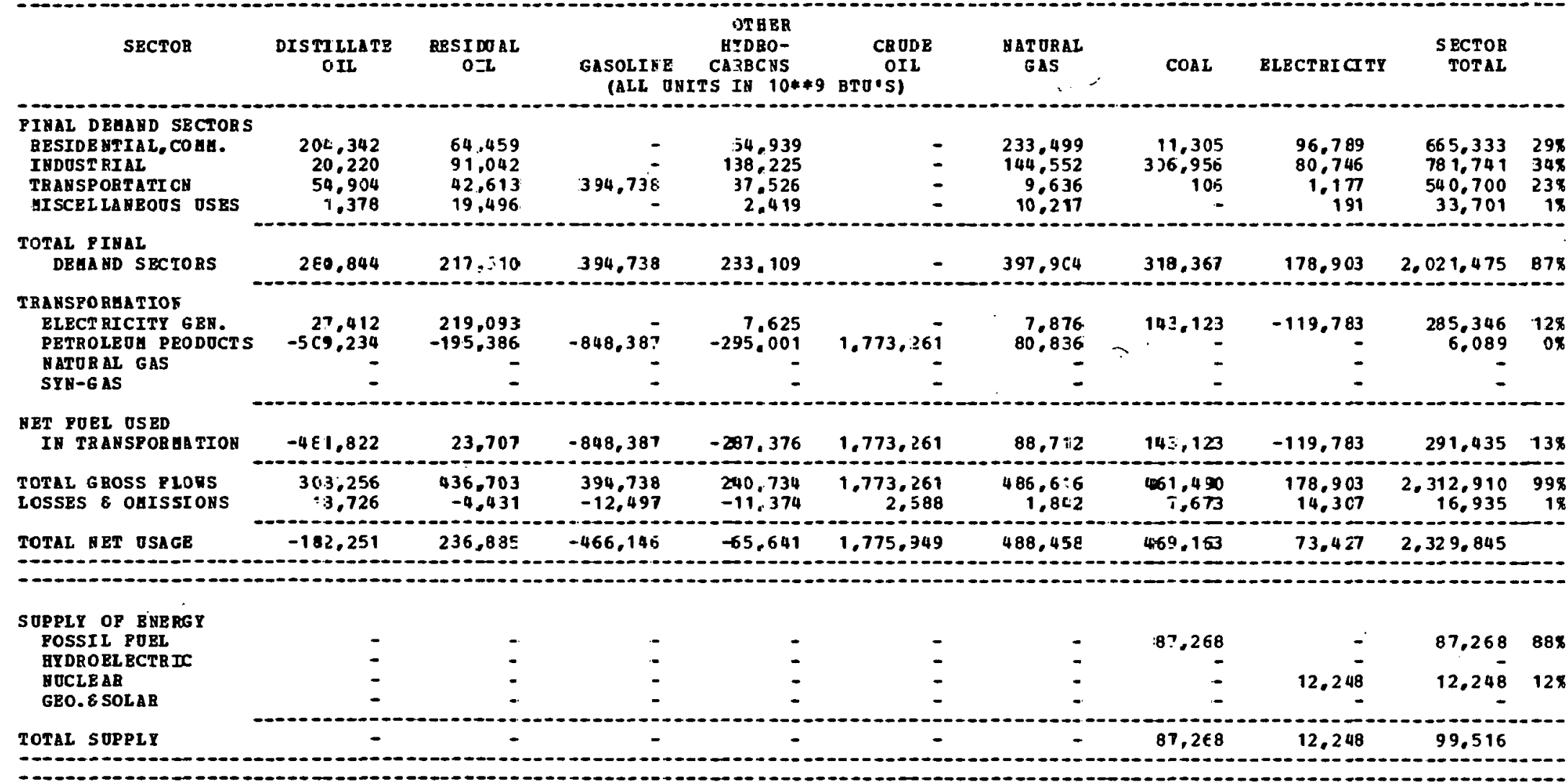

VET IEPORTS

OP REGIO

$-182.251$

236.885

$-466,146$

$-65,641 \quad 1,775,949$

488.458

$\Xi 81,8 \subseteq 5$

$61,179 \quad 2,230,329$

NOTES:

1 TRABS FOREATION LOSS FOB TRA RS PORAATION LOSS POR
TRA ESORATION LOSS POB TRA AS FORAATION LOSS POR
TRA NSFORATION LOSS POR
ELRCTRIC $-T Y$ G 3 .

. $=0.435$

STOF AL 6 AS

$\begin{array}{ll}S Y H-G A S & =0.09 \\ & =0.0\end{array}$ 
REGIONAL ENERGT BAIAHCE STATEGEHT

BY SECTOR AHD POEL TYPE

\begin{tabular}{|c|c|c|c|c|c|c|c|c|c|c|}
\hline SECTOR & $\begin{array}{l}\text { DIS TILLATE } \\
\text { OIL }\end{array}$ & $\begin{array}{l}\text { RESIDOAL } \\
\text { OIL }\end{array}$ & $\begin{array}{l}\text { GASOLINE } \\
\text { (ALL ONI }\end{array}$ & $\begin{array}{l}\text { AYDRO- } \\
\text { CABBONS } \\
\text { TS IN 10**9 }\end{array}$ & $\begin{array}{c}\text { CBODE } \\
\text { OIL } \\
\text { BTO'S) }\end{array}$ & $\begin{array}{l}\text { MAT ORAL } \\
\text { GAS }\end{array}$ & COAL & RLECTRICITY & $\begin{array}{r}\text { SECTOR } \\
\text { TOTAL }\end{array}$ & \\
\hline 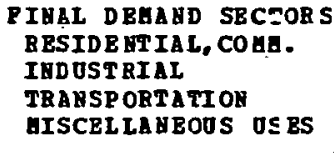 & $\begin{array}{r}38,518 \\
4,982 \\
16,646 \\
195\end{array}$ & $\begin{array}{r}12,186 \\
15,924 \\
326 \\
3,312\end{array}$ & 91,219 & $\begin{array}{r}11.712 \\
30,255 \\
5.249 \\
527\end{array}$ & $\overline{-}$ & $\begin{array}{r}59.349 \\
44.077 \\
3.430 \\
2.685\end{array}$ & $\begin{array}{r}3,571 \\
107,296 \\
25 \\
-\end{array}$ & $\begin{array}{r}21.690 \\
21.486 \\
366 \\
76\end{array}$ & $\begin{array}{r}147.026 \\
224.020 \\
117.261 \\
6.795\end{array}$ & $\begin{array}{r}228 \\
348 \\
18 \% \\
1 \%\end{array}$ \\
\hline $\begin{array}{l}\text { TOTAL PINAL } \\
\text { DEEA AD SECTORS }\end{array}$ & 60.341 & 31.748 & 91,219 & 47,743 & - & 109.541 & 110,892 & 43,618 & 495,102 & 758 \\
\hline $\begin{array}{l}\text { TRANSPOREATIOY } \\
\text { ELECTRICITY GEH. } \\
\text { PETROLEOA PRODOZTS } \\
\text { HATORAL GAS } \\
\text { SYB-GAS }\end{array}$ & $\begin{array}{r}3.574 \\
= \\
-\end{array}$ & $\begin{array}{r}3,184 \\
= \\
=\end{array}$ & : & - & E & $\begin{array}{r}404 \\
- \\
-\end{array}$ & $\begin{array}{r}214,513 \\
- \\
-\end{array}$ & $\begin{array}{r}-66.791 \\
- \\
-\end{array}$ & $\begin{array}{r}154,884 \\
= \\
=\end{array}$ & $24 \%$ \\
\hline $\begin{array}{l}\text { NET POEL OSED } \\
\text { IH TRANSPOREATIOH }\end{array}$ & 3.574 & 3,184 & - & - & - & 404 & 214,513 & -66.791 & 154,884 & $24 x$ \\
\hline $\begin{array}{l}\text { TOTAL GROSS PLORS } \\
\text { LOSSES } 8 \text { OHISSIOHS }\end{array}$ & $\begin{array}{r}63,915 \\
3,882\end{array}$ & $\begin{array}{r}34.932 \\
-354\end{array}$ & $\begin{array}{r}91.219 \\
-2.887\end{array}$ & $\begin{array}{l}47,743 \\
-2.255\end{array}$ & - & $\begin{array}{r}109,945 \\
416\end{array}$ & $\begin{array}{r}325,405 \\
5,410\end{array}$ & $\begin{array}{r}43.618 \\
3.488\end{array}$ & $\begin{array}{r}649,986 \\
7,699\end{array}$ & $\begin{array}{r}998 \\
18\end{array}$ \\
\hline TOTAL VET USAGB & 67.797 & 34.577 & 88,331 & 45,487 & - & 110,361 & 330,815 & $-19,684$ & 657,685 & \\
\hline $\begin{array}{l}\text { SUPPLY OF BHERGY } \\
\text { FOSSII FOBI. } \\
\text { BYDROELBCTEIC } \\
\text { NOCLEAR } \\
\text { GEO.ESOLAR }\end{array}$ & : & : & :- & $=$ & - & $\begin{array}{l}4 \\
-\end{array}$ & $\begin{array}{r}36,072 \\
- \\
-\end{array}$ & $\begin{array}{r}6.878 \\
611 \\
-\end{array}$ & $\begin{array}{r}36.076 \\
6.878 \\
611 \\
-\end{array}$ & $\begin{array}{r}838 \\
168 \\
17\end{array}$ \\
\hline TOTAL SUPPLI & $\begin{array}{c}- \\
-\end{array}$ & - & - & - & - & 4 & 36,072 & 7.489 & 43,565 & \\
\hline
\end{tabular}

\section{NET IH PORT}

67.797

34.577

88,331

45,487

110,357

294.743

$-27,173$

614,120

NOT BS:

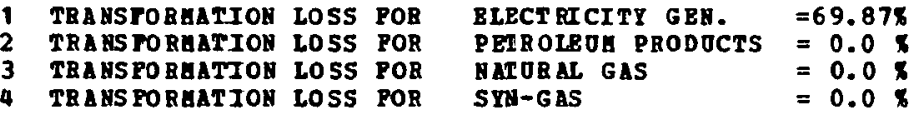


REGIOYAL EHERGI BALANCE STATELE NT

BI SECTC AND POEL TYPE

\begin{tabular}{|c|c|c|c|c|c|c|c|c|c|c|}
\hline SEC:OR & $\underset{0=L}{D I S T I L L A T E}$ & $\begin{array}{l}\text { RESI DOAL } \\
\text { OIL }\end{array}$ & $\begin{array}{l}\text { GASOLIHE } \\
\text { (ALL ONI? }\end{array}$ & $\begin{array}{c}\text { OTBER } \\
\text { ASDBO- } \\
\text { =APBONS } \\
\text { TS IN } 10 * * 9\end{array}$ & $\begin{array}{c}\text { CRODE } \\
\text { OII } \\
\text { BTO'SI }\end{array}$ & $\begin{array}{l}\text { NATURAL } \\
\text { GAS }\end{array}$ & $\cos L$ & ELECTRICITY & $\begin{array}{r}\text { SECTOR } \\
\text { TOTAL }\end{array}$ & \\
\hline 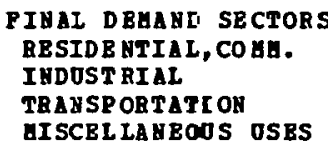 & $\begin{array}{r}51,495 \\
14.828 \\
26.009 \\
977\end{array}$ & $\begin{array}{r}18,133 \\
39,875 \\
24,583 \\
7,600\end{array}$ & 158.740 & $\begin{array}{r}26.316 \\
37.264 \\
21.274 \\
1.216\end{array}$ & $\begin{array}{l}\overline{-} \\
\overline{-}\end{array}$ & $\begin{array}{r}80.701 \\
57.449 \\
1.544 \\
4.434\end{array}$ & $\begin{array}{r}2,577 \\
140,360 \\
58 \\
-\end{array}$ & $\begin{array}{r}44.749 \\
27.037 \\
588 \\
134\end{array}$ & $\begin{array}{r}223,971 \\
316,813 \\
232,796 \\
14,361\end{array}$ & $\begin{array}{r}25 \% \\
35 \% \\
26 \% \\
2 \%\end{array}$ \\
\hline $\begin{array}{l}\text { TOTAL EIVAL } \\
\text { DEHA BD SECTORS }\end{array}$ & 93,309 & 90,991 & 158,740 & $E 6.070$ & - & 144,128 & 142,995 & 72,508 & 787.941 & $86 \%$ \\
\hline $\begin{array}{l}\text { TRANSPORHATHOA } \\
\text { ELECT RICITY GEH. } \\
\text { PETROLBOH PRODOCTS } \\
\text { MATORAL GAS } \\
\text { SYH-GAS }\end{array}$ & $\begin{array}{r}7,389 \\
= \\
=\end{array}$ & $\begin{array}{r}106,552 \\
- \\
-\end{array}$ & $\begin{array}{l}\overline{-} \\
-\end{array}$ & $\begin{array}{r}-18,223 \overline{-} \\
-\end{array}$ & $\begin{array}{r}18,200 \\
-\end{array}$ & $\begin{array}{r}9,240 \\
830 \\
- \\
-\end{array}$ & $\begin{array}{r}45,732 \\
- \\
=\end{array}$ & $\begin{array}{r}-49,488 \\
= \\
-\end{array}$ & $\begin{array}{r}119.425 \\
807 \\
- \\
-\end{array}$ & $\begin{array}{r}13 \% \\
0 \%\end{array}$ \\
\hline $\begin{array}{l}\text { MBT FOEL OSBD } \\
\text { IY TRABSFORLATION }\end{array}$ & 7.389 & $: 06,552$ & - & $-18,223$ & 18.200 & 10,170 & 45,732 & $-49,488$ & 120,232 & 138 \\
\hline $\begin{array}{l}\text { TOTAL G ROSS PLONS } \\
\text { LOSSES } \varepsilon \text { OUISSIOHS }\end{array}$ & $\begin{array}{r}100.698 \\
.0117\end{array}$ & $\begin{array}{r}196.743 \\
-9.996\end{array}$ & $\begin{array}{r}158.74 J \\
-5.025\end{array}$ & $\begin{array}{l}36.070 \\
-4.066\end{array}$ & $\begin{array}{r}18.200 \\
27\end{array}$ & $\begin{array}{r}154.198 \\
583\end{array}$ & $\begin{array}{r}188,727 \\
3,137\end{array}$ & $\begin{array}{r}72.508 \\
5.798\end{array}$ & $\begin{array}{r}908,173 \\
4,577\end{array}$ & $\begin{array}{r}998 \\
1 \%\end{array}$ \\
\hline
\end{tabular}

\section{SOPPLI OF ENERGY}

FOSSIL FORL

HYDROELECTRIC

SUCLEAR

GEO. E SOLAR

$\begin{array}{cc}- & - \\ - & - \\ - & - \\ -2 & -\end{array}$

-
-
-

$\begin{array}{lll}- & - & - \\ - & - & - \\ - & - & - \\ - & - & -\end{array}$

\begin{tabular}{cccc}
- & - & - \\
- & 7.276 & 7.276 & \\
- & - & - \\
\hline- & 7.276 & 7.276
\end{tabular}

\section{HET IHPORTS}

OP BEGIOE

$1 C 6.815$

194.746

$15 \$ .714$

63.780

18,227

154,781

191,864

21,542

905,474

HOTES:

1 TBANSFORGATION LOSS POR 2 TEAHSFORAATION LOSS POR TFA ASFO RUATION LO:SS POB

TEA PSPRATTION LO'SS POR
TEA ASPORAATION LO SS POR

ELECTRICITY GEH. $\quad=70.7 \mathrm{gF}$

PEROLBOS PRODUCES $=4.26$

$\begin{array}{ll}\text { MTURAL GIS } & =0.0 \%\end{array}$ 
REGIONAL EHERGY BALANCE STATEHENT

BY SECTOR AND POEL TYPE

\begin{tabular}{|c|c|c|c|c|c|c|c|c|c|c|}
\hline SECTOR & $\begin{array}{l}\text { DIS TILLATE } \\
\text { OIL }\end{array}$ & $\begin{array}{l}\text { RESIDCALL } \\
\text { OIL }\end{array}$ & $\begin{array}{l}\text { GASOLINE } \\
\text { TALL O }\end{array}$ & $\begin{array}{c}\text { OTHER } \\
\text { HYDRO- } \\
\text { CARBOHS } \\
\text { TS IB 10*\$9 }\end{array}$ & $\begin{array}{c}\text { CRODE } \\
\text { OIL } \\
\text { BTO'S) }\end{array}$ & $\begin{array}{l}\text { MAT URAL } \\
\text { GAS }\end{array}$ & COAL & ELECTBICITY & $\begin{array}{r}\text { SECTOR } \\
\text { TOTAL }\end{array}$ & \\
\hline 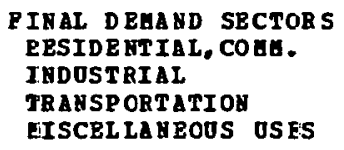 & $\begin{array}{r}55.221 \\
3.714 \\
17.552 \\
1.390\end{array}$ & $\begin{array}{r}21,878 \\
10,033 \\
1,704 \\
29,433\end{array}$ & 183,929 & $\begin{array}{r}23.719 \\
6.859 \\
43.172 \\
970\end{array}$ & $\overline{-}$ & $\begin{array}{r}60,378 \\
10,623 \\
2,197 \\
8,472\end{array}$ & $\begin{array}{r}4,743 \\
10,656 \\
29 \\
-\end{array}$ & $\begin{array}{r}46,884 \\
6,924 \\
82 \\
4,697\end{array}$ & $\begin{array}{r}212,823 \\
48,809 \\
248,665 \\
44,962\end{array}$ & $\begin{array}{r}30 \% \\
7 \% \\
35 \% \\
6 \%\end{array}$ \\
\hline $\begin{array}{l}\text { TCTAL PIBAL } \\
\text { DEHA ND SECTORS }\end{array}$ & 77.877 & 63,048 & 183,929 & 74,720 & - & 81,670 & 15,428 & $58,587$. & 555,259 & $79 x$ \\
\hline $\begin{array}{l}\text { TRANSFORHATIOH } \\
\text { ELECTRICITY GEN. } \\
\text { PETROLEOA PRODOCTS } \\
\text { RATORAL GAS } \\
\text { SYH-GAS }\end{array}$ & $\begin{array}{r}4.889 \\
=\end{array}$ & $\begin{array}{r}112,960 \\
- \\
-\end{array}$ & $\overline{-}$ & - & $\begin{array}{l}- \\
-\end{array}$ & $\begin{array}{r}48 \\
- \\
-\end{array}$ & $\begin{array}{r}101,161 \\
- \\
-\end{array}$ & $\begin{array}{r}-72.054 \\
- \\
-\end{array}$ & $\begin{array}{r}147.004 \\
- \\
-\end{array}$ & $21 \%$ \\
\hline $\begin{array}{l}\text { NET PUEL OSBD } \\
\text { IN TRANSPORHATION }\end{array}$ & 4.889 & 112.950 & - & - & - & 48 & 101,161 & $-72,054$ & 147.004 & $21 x$ \\
\hline $\begin{array}{l}\text { TOTAL GROSS PLORS } \\
\text { LOSSES } \varepsilon \text { OUISSIOHS }\end{array}$ & $\begin{array}{r}82,766 \\
5,028\end{array}$ & $\begin{array}{r}176.008 \\
-1.786\end{array}$ & $\begin{array}{r}183,929 \\
-5,823\end{array}$ & $\begin{array}{l}74,720 \\
-3,530\end{array}$ & - & $\begin{array}{r}81,718 \\
309\end{array}$ & $\begin{array}{r}116,589 \\
1,938\end{array}$ & $\begin{array}{r}58,587 \\
4,685\end{array}$ & $\begin{array}{r}702,263 \\
822\end{array}$ & $\begin{array}{r}100 \% \\
0 \%\end{array}$ \\
\hline
\end{tabular}

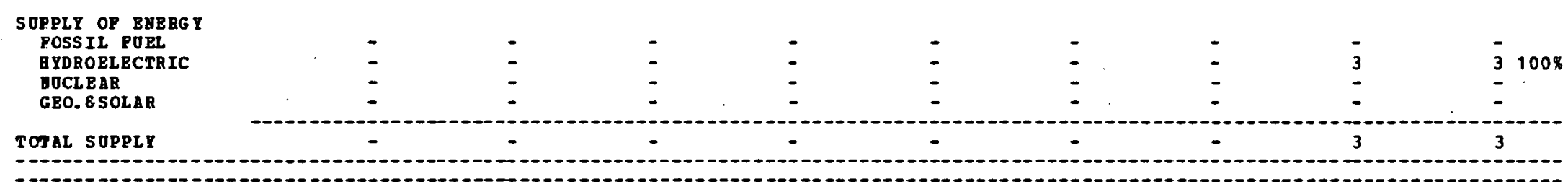

HET IEPORTS
OP REGIOA


REGIONAL ENERGY BALANCE STATEHENT

BY SECTOR AND FOEL TYPE

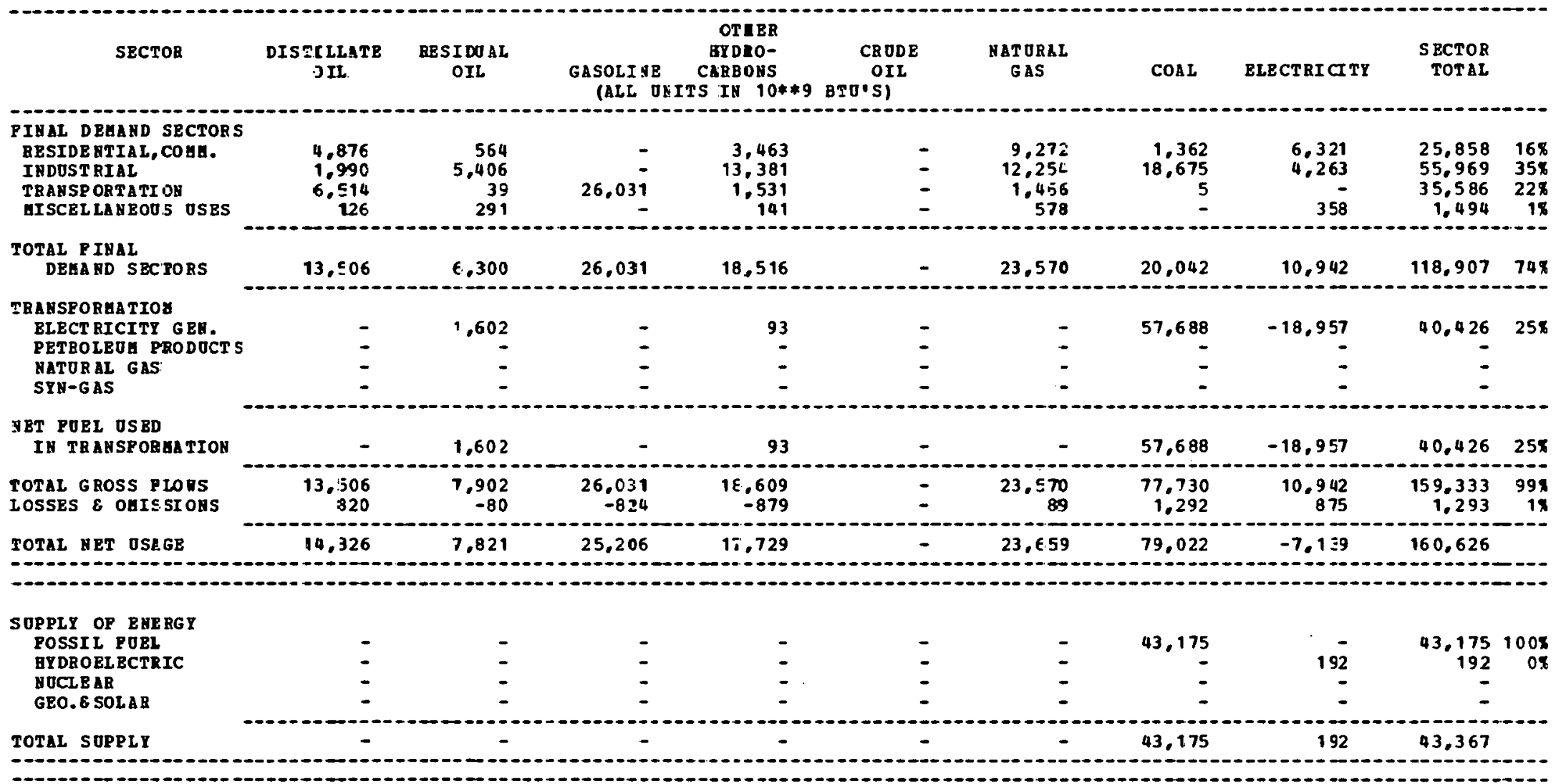

MET IHPORTS

OP BEGION

14.326

7.821

25,206

17,729

23,559

35,847

$-7,331$

117.259

BOTES:

1 TRALS FORHATION LESS POR

TRA ASPO BaATIOY IOSS POR

OY LOSS POB

TRAASPORHATIOU LOSS POR

BLECTRICITY GEN.

PETROLEO I PBODOCTS

NATURAL GAS

STN-G AS

$=58.03 \mathrm{~K}$
$=0.0 \times$
$=0.0 \%$
$=0.0 \%$ 


\begin{tabular}{|c|c|c|c|c|c|c|c|c|c|c|}
\hline & & & $\begin{array}{l}\text { REGIONAL } \\
\text { BY SE }\end{array}$ & $\begin{array}{l}\text { ENERGY BAL } \\
\text { CTOR AND }\end{array}$ & $\begin{array}{l}\text { HCE STAT } \\
\text { OEL TYPE }\end{array}$ & & & & & \\
\hline SECTOR & $\begin{array}{l}\text { DISTILLATE } \\
\text { OIL }\end{array}$ & $\begin{array}{l}\text { RESIDDAL } \\
\text { OIL }\end{array}$ & $\begin{array}{l}\text { GASOL INE } \\
\text { (ALL OHIT }\end{array}$ & $\begin{array}{l}\text { OTRER } \\
\text { ATDRO- } \\
\text { CARBOHS } \\
\text { IS IH 10**9 }\end{array}$ & $\begin{array}{c}\text { CRODE } \\
\text { OIL } \\
\text { BTO'S) }\end{array}$ & $\begin{array}{c}\text { NAT ORAL } \\
\text { GAS } \\
\text { : }\end{array}$ & COAL & ELECT RI CITY & $\begin{array}{l}\text { SECTOR } \\
\text { TOTAL }\end{array}$ & \\
\hline $\begin{array}{l}\text { PIHAL DEMAND SECTORS } \\
\text { RESIDE BTIAL, COAH. } \\
\text { IHDOSTRIAL } \\
\text { TRABSPORTATIOH } \\
\text { GISCELLABEOS OSBS }\end{array}$ & $\begin{array}{r}12.769 \\
4.259 \\
10.617 \\
320\end{array}$ & $\begin{array}{r}1.239 \\
13.472 \\
97 \\
595\end{array}$ & $\begin{array}{r}- \\
\overline{-} \\
56,696\end{array}$ & $\begin{array}{r}8.294 \\
17.319 \\
3.810 \\
330\end{array}$ & 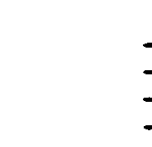 & $\begin{array}{r}13,161 \\
19,927 \\
1,066 \\
737\end{array}$ & $\begin{array}{r}2.553 \\
23,114 \\
11 \\
-\end{array}$ & $\begin{array}{r}13.905 \\
9,761 \\
11 \\
743\end{array}$ & $\begin{array}{r}51.921 \\
87.852 \\
72,308 \\
2,725\end{array}$ & $\begin{array}{r}228 \\
388 \\
31 \% \\
1 \%\end{array}$ \\
\hline $\begin{array}{l}\text { TOTAL PIHAL } \\
\text { DEAA ND SBCTOBS }\end{array}$ & 27,965 & 15.403 & 56,696 & 29.753 & 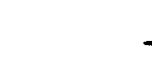 & 34,891 & 25,678 & 24.420 & 214,806 & $92 \%$ \\
\hline $\begin{array}{l}\text { TRANSPORAATION } \\
\text { ELECTRICITY GER. } \\
\text { PETROIEOA PRODOCTS } \\
\text { RATORAL GAS } \\
\text { SYH-GAS }\end{array}$ & $\begin{array}{r}1.069 \\
- \\
-\end{array}$ & $\begin{array}{r}343 \\
-\end{array}$ & : & : & 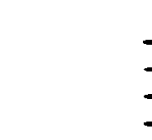 & $\begin{array}{r}1,380 \\
= \\
=\end{array}$ & $\begin{array}{r}23.137 \\
= \\
-\end{array}$ & $\begin{array}{r}-8,366 \\
= \\
=\end{array}$ & $\begin{array}{r}17,563 \\
- \\
-\end{array}$ & $8 \%$ \\
\hline $\begin{array}{l}\text { WET FOEL DSBD } \\
\text { IN TRASSFORGATION }\end{array}$ & 1,069 & 343 & - & - & 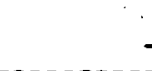 & 1,380 & 23,137 & $-8,366$ & 17.563 & 88 \\
\hline T OTAL, \&ET OSAGE & 30,797 & 15,586 & 54,901 & 28.347 & - & 36,408 & 49,626 & 18.007 & 233.674 & \\
\hline
\end{tabular}

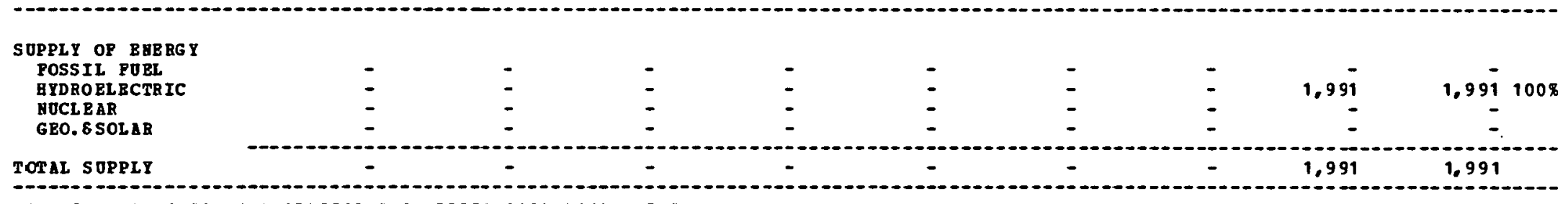

\begin{tabular}{l}
$\begin{array}{l}\text { MET IMPORTS } \\
\text { OP REGIOA }\end{array}$ \\
\hdashline$\quad 30,797$
\end{tabular}


REgIOHAL EJEGGY BALANCE STA TEGEHT

BY SECTOE ABD FOEL TYPE
MALE

\begin{tabular}{|c|c|c|c|c|c|c|c|c|c|}
\hline SECTOR & $\begin{array}{l}\text { DISTILLATE } \\
\text { OIL }\end{array}$ & $\begin{array}{l}\text { BBS I DOAL } \\
\text { OIL }\end{array}$ & $\begin{array}{l}\text { GASOLIEE } \\
\text { (ALl D }\end{array}$ & $\begin{array}{c}\text { OT HER } \\
\text { AYABO- } \\
\text { CABBOAS } \\
\text { TS IE 10**9 }\end{array}$ & $\begin{array}{c}\text { CR ODE } \\
\text { OIL } \\
\text { BTO'S) }\end{array}$ & $\begin{array}{l}\text { MAT OBAL } \\
\text { GAS }\end{array}$ & CO.AL & ELECTRICT TY & $\begin{array}{l}\text { SECTOR } \\
\text { TOTAL }\end{array}$ \\
\hline $\begin{array}{l}\text { PIHAL DBGAND SBCTORS } \\
\text { RESIDE HTIA L, COUA. } \\
\text { INDOSTRIAL } \\
\text { TRANSPORTATI OR } \\
\text { GISCEL LAGBOOS OSBS }\end{array}$ & $\begin{array}{r}15.472 \\
5.864 \\
16.041 \\
387\end{array}$ & $\begin{array}{r}2,003 \\
21,503 \\
3,191 \\
1,014\end{array}$ & 68,419 & $\begin{array}{r}9.863 \\
21.077 \\
.644 \\
378\end{array}$ & - & $\begin{array}{r}17.6 .93 \\
21.818 \\
1.269 \\
1.843\end{array}$ & $\begin{array}{r}3,099 \\
32,170 \\
18 \\
-\end{array}$ & $\begin{array}{r}18.690 \\
10.437 \\
22 \\
1.867\end{array}$ & $\begin{array}{r}66,820 \\
112,869 \\
93,624 \\
5,489\end{array}$ \\
\hline $\begin{array}{l}\text { TOTAL P IHAL } \\
\text { DEHA SD S BCTORS }\end{array}$ & 37.764 & 27.711 & 68,413 & 35,962 & - & 42.613 & $35 . ; 87$ & 31.016 & 278,802 \\
\hline 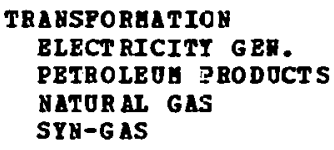 & $\begin{array}{r}958 \\
- \\
-\end{array}$ & $\begin{array}{r}30.739 \\
- \\
-\end{array}$ & $\begin{array}{l}- \\
-\end{array}$ & $\begin{array}{l}- \\
-\end{array}$ & - & $\begin{array}{l}- \\
-\end{array}$ & $\begin{array}{r}12.541 \\
- \\
-\end{array}$ & $\begin{array}{r}-30,057 \\
- \\
-\end{array}$ & $\begin{array}{r}64.181 \\
= \\
-\end{array}$ \\
\hline $\begin{array}{l}\text { MET FUEL OSED } \\
\text { IN TRANSPORMATION }\end{array}$ & 958 & 80.739 & - & - & - & - & 12.541 & $-30,0 \leq 7$ & 64,181 \\
\hline $\begin{array}{l}\text { TOTAL GROSS PLORS } \\
\text { LOSSES } 6 \text { OAISSIOHS }\end{array}$ & $\begin{array}{r}38,722 \\
2,352\end{array}$ & $\begin{array}{r}108,450 \\
-1,100\end{array}$ & $\begin{array}{l}68,419 \\
-2,166\end{array}$ & $\begin{array}{l}\equiv 5,962 \\
-1,699\end{array}$ & - & $\begin{array}{r}42.643 \\
161\end{array}$ & $\begin{array}{r}47 . .328 \\
795\end{array}$ & $\begin{array}{r}31.016 \\
2.480\end{array}$ & $\begin{array}{r}342.983 \\
823\end{array}$ \\
\hline
\end{tabular}

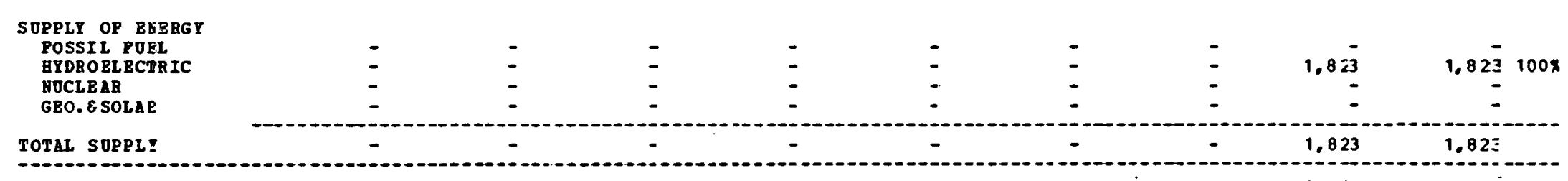

\section{HBT IHPORTS}

OP REGION

41.074

107.349

66,252

34,262

42,804

48.623

1.616

$341.98 \%$

\begin{tabular}{|c|c|c|c|c|c|}
\hline \multicolumn{6}{|c|}{ BS: } \\
\hline 1 & TRA AS PORGAT IOR & joss & FOR & BLECTRICITY GE & .1 \\
\hline & THAES POBALTIOA & & rOR & Th PEODOCL & \\
\hline 4 & TRAYSPORGATIOS & coss & POR & $S M:-G A S$ & 0.0 \\
\hline
\end{tabular}


REGIONAL ENERG BALAHCZ STATEGENT

BY SECTOR AND POEE TYPE

\begin{tabular}{|c|c|c|c|c|c|c|c|c|c|}
\hline SECTOR & $\begin{array}{l}\text { DIS IILLATE } \\
\text { OIL }\end{array}$ & $\begin{array}{l}\text { RESIDOAL } \\
\text { OIL }\end{array}$ & $\begin{array}{l}\text { GASOLINE } \\
\text { (ALL ONI }\end{array}$ & $\begin{array}{l}\text { BYDRO- } \\
\text { CARBONS } \\
\text { TS IN 10*\#9 }\end{array}$ & $\begin{array}{c}\text { CRUDE } \\
\text { OIL } \\
\text { BTO-SI }\end{array}$ & $\begin{array}{l}\text { H ATORAL } \\
\text { GAS }\end{array}$ & COAL & ELECTRICITY & $\begin{array}{l}\text { SECTOR } \\
\text { TOTAL }\end{array}$ \\
\hline $\begin{array}{l}\text { PIHAL DEHAND SECTORS } \\
\text { RESIDE HTIAL, COAA. } \\
\text { IHDOSTRIAL } \\
\text { TRAESP ORTATION } \\
\text { HISCELLANEOOS OSBS }\end{array}$ & $\begin{array}{r}18.432 \\
1.992 \\
12.234 \\
467\end{array}$ & $\begin{array}{r}2.005 \\
7.319 \\
5.5 .996 \\
2.292\end{array}$ & 82.815 & $\begin{array}{r}12.631 \\
6.731 \\
4.894 \\
550\end{array}$ & $=$ & $\begin{array}{r}19.191 \\
7.731 \\
1.547 \\
5.693\end{array}$ & $\begin{array}{r}3,662 \\
9,056 \\
17 \\
-\end{array}$ & $\begin{array}{r}21.076 \\
4.546 \\
19 \\
5.704\end{array}$ & $\begin{array}{r}76,997 \\
37.375 \\
157.522 \\
14.706\end{array}$ \\
\hline $\begin{array}{l}\text { TOTAL FIHAL } \\
\text { DBHA BD SBCTORS }\end{array}$ & 33,125 & 67,612 & 82.815 & 24,806 & - & 34,162 & 12.735 & 31.345 & 286,600 \\
\hline $\begin{array}{l}\text { TRANSPORAATION } \\
\text { ELECTRICITY GEN. } \\
\text { PETROLEOA PRODOCTS } \\
\text { MATORAL GAS } \\
\text { SYH-GAS }\end{array}$ & $\begin{array}{r}1.239 \\
-21.675 \\
-\end{array}$ & $\begin{array}{r}45.780 \\
-6.311 \\
- \\
-\end{array}$ & $-41,423$ & $\begin{array}{r}3,122 \\
-21,095 \\
-\end{array}$ & $\begin{array}{r}89.390 \\
-\end{array}$ & $\begin{array}{r}2,347 \\
4,075 \\
-\end{array}$ & $\begin{array}{r}11.365 \\
= \\
=\end{array}$ & $\begin{array}{r}-18,831 \\
= \\
-\end{array}$ & $\begin{array}{r}45,022 \\
961 \\
- \\
-\end{array}$ \\
\hline $\begin{array}{l}\text { NET POEL OSED } \\
\text { IN TRABS FORGATION }\end{array}$ & $-20,436$ & 37.469 & $-41,423$ & $-17,973$ & 89,390 & 6.422 & 11,365 & $-18,831$ & $\begin{array}{r}45,983 \\
\end{array}$ \\
\hline $\begin{array}{l}\text { TOTAL GROSS PLOWS } \\
\text { LOSSES } \varepsilon \text { OUISSIOHS }\end{array}$ & $\begin{array}{r}34.364 \\
2.087\end{array}$ & $\begin{array}{r}113,392 \\
-1,150\end{array}$ & $\begin{array}{l}82,815 \\
-2,621\end{array}$ & $\begin{array}{l}27,928 \\
-1,319\end{array}$ & $\begin{array}{r}89.390 \\
135\end{array}$ & $\begin{array}{r}40.584 \\
153\end{array}$ & $\begin{array}{r}24,100 \\
400\end{array}$ & $\begin{array}{r}31,345 \\
2,506\end{array}$ & $\begin{array}{r}332,583 \\
192\end{array}$ \\
\hline
\end{tabular}

\section{SOPPLI OP EHERGI}

POSSIL POBL

MTDROLCTRIC

GEO. E SOL AR

$\begin{array}{cc}- & - \\ - & - \\ - & -\end{array}$

$\begin{array}{lll}- & - \\ - & -\end{array}$

- -

-

-

$\begin{array}{ll}- & - \\ - & - \\ - & -\end{array}$

$\frac{-}{-}$

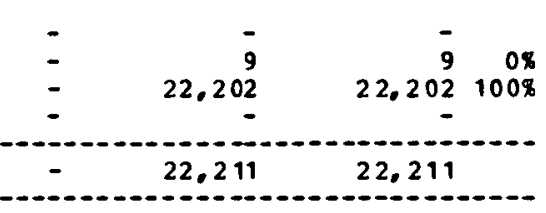

(1)

\section{HET IMPORTS}

14.776

103,930

38.770

5,513

89.525

40.737

24.500

$-7,190$

310,564

yOTES:

\begin{tabular}{|c|c|c|c|c|}
\hline $\begin{array}{l}\text { TAA ASPO RAATION } \\
\text { TRA HS PO RUAT ION }\end{array}$ & $\begin{array}{l}\text { LoSS } \\
\text { LoSS }\end{array}$ & $\begin{array}{l}\text { POR } \\
\text { POR }\end{array}$ & $\begin{array}{l}\text { ELECT RICITY GEN. } \\
\text { PETROLEOH PRODOCTS }\end{array}$ & $\begin{array}{l}=70.518 \\
=1.038\end{array}$ \\
\hline TRANSPORAATION & $\begin{array}{l}\text { LosS } \\
\text { LoSS }\end{array}$ & $\begin{array}{l}\text { POR } \\
\text { POR }\end{array}$ & $\begin{array}{l}\text { MaTOZAL GAS } \\
\text { SWM-5AS }\end{array}$ & $\begin{array}{l}=0.0 \% \\
=0.0 \%\end{array}$ \\
\hline
\end{tabular}


GES ZOHAL EHBRGI BALAYCE STA TEUENT

BY SECTOR AND FOEI TYPE

\begin{tabular}{|c|c|c|c|c|c|c|c|c|c|}
\hline SECTOR & $\begin{array}{c}\text { CISEIILAT3 } \\
\text { OII }\end{array}$ & $\begin{array}{l}\text { RES I DO AL } \\
\text { O IL }\end{array}$ & $\begin{array}{c}\text { GASOLIHE } \\
\text { TALE OR }\end{array}$ & $\begin{array}{l}\text { OTBER } \\
\text { HYDBO- } \\
\text { CARBONS } \\
\text { TS IN 10**9 }\end{array}$ & $\begin{array}{c}\text { CRODE } \\
\text { OIL } \\
\text { BTO'S) }\end{array}$ & $\begin{array}{c}\text { BAT JRAL } \\
\text { JAS }\end{array}$ & COAL & BLECTRI CITY & $\begin{array}{l}\text { SECTOR } \\
\text { TOTAL }\end{array}$ \\
\hline $\begin{array}{l}\text { PIHAL D BHAND SECTORS } \\
\text { RESIDB HTIAL, COAG. } \\
\text { IHDUSTRIAL } \\
\text { TRASSPORTATI OH } \\
\text { GISCEL LAMEOOS OSES }\end{array}$ & $\begin{array}{r}21,378 \\
4,393 \\
9,952 \\
636\end{array}$ & $\begin{array}{r}3,255 \\
10,296 \\
42 \\
1.886\end{array}$ & 114,236 & $\begin{array}{r}26,913 \\
24,526 \\
2,607 \\
2,031\end{array}$ & - & $\begin{array}{r}14.821 \\
21.367 \\
1.941 \\
2.644\end{array}$ & $\begin{array}{r}3.838 \\
11.779 \\
15 \\
-\end{array}$ & $\begin{array}{r}31,184 \\
14,+39 \\
23 \\
1,361\end{array}$ & $\begin{array}{r}101.339 \\
86,530 \\
128,816 \\
8,558\end{array}$ \\
\hline $\begin{array}{l}\text { TOTAL F İAL } \\
\text { DBAA AD SBCTORS }\end{array}$ & 36,359 & 15,479 & 114,236 & 56,077 & - & 40.773 & 15,632 & $46.7 \mathrm{C7}$ & 325,253 \\
\hline $\begin{array}{l}\text { TRABSPORAATIOY } \\
\text { ELECT RICITY GBH. } \\
\text { PBTROLEOH PRODOCTS } \\
\text { HATORAI GAS } \\
\text { SYH-G AS }\end{array}$ & $\begin{array}{r}1,559 \\
= \\
=\end{array}$ & 532 & : & E & $\begin{array}{l}- \\
\end{array}$ & $\begin{array}{r}1.394 \\
=\end{array}$ & $\begin{array}{r}120.771 \\
\vdots \\
-\end{array}$ & $\begin{array}{r}-43,517 \\
-\end{array}$ & $\begin{array}{r}80.739 \\
= \\
=\end{array}$ \\
\hline $\begin{array}{l}\text { NET POBI OSBD } \\
\text { IN TBANSPOB GATION }\end{array}$ & 1.559 & 532 & - & - & - & 1,394 & 120,771 & $-43,517$ & 80.739 \\
\hline $\begin{array}{l}\text { TOTAL GROSS FLORS } \\
\text { LOSSES \& CUISSIONS }\end{array}$ & $\begin{array}{r}37.918 \\
2.303\end{array}$ & $\begin{array}{r}16,011 \\
-162\end{array}$ & $\begin{array}{r}114.236 \\
-3.676\end{array}$ & $\begin{array}{l}56,077 \\
-2,649\end{array}$ & - & $\begin{array}{r}42.167 \\
159\end{array}$ & $\begin{array}{r}135,403 \\
2,267\end{array}$ & $\begin{array}{r}46.707 \\
3.735\end{array}$ & $\begin{array}{r}406.002 \\
2.037\end{array}$ \\
\hline
\end{tabular}

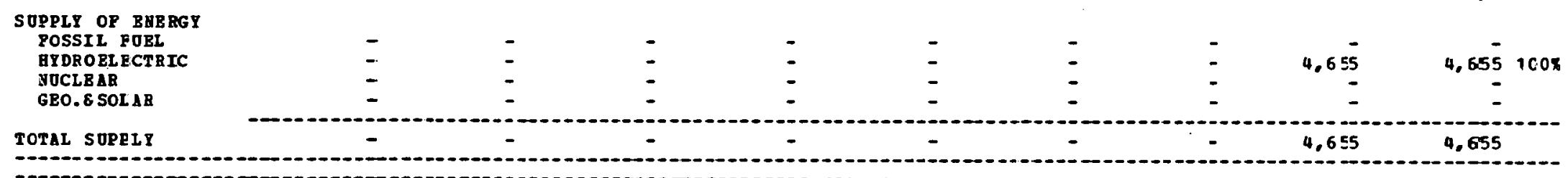

\section{RET IHPORT}

$40.221 \quad 15,848 \quad 110,619$

53,427

c.2. 326

138,670

2.270

$403,3: 84$

MOTES:

\begin{tabular}{|c|c|c|c|}
\hline 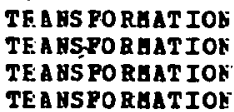 & $\begin{array}{l}\text { LosS } \\
\text { Lo5S } \\
\text { Lo5S } \\
\text { LoSS }\end{array}$ & $\begin{array}{l}\text { POR } \\
\text { POR } \\
\text { POR } \\
\text { POR }\end{array}$ & $\begin{array}{l}\text { BLECTRICITY GEN. } \\
\text { PETROLEOE PRODOCTS } \\
\text { RZTORAL GLS } \\
\text { SYY -GAS }\end{array}$ \\
\hline
\end{tabular}




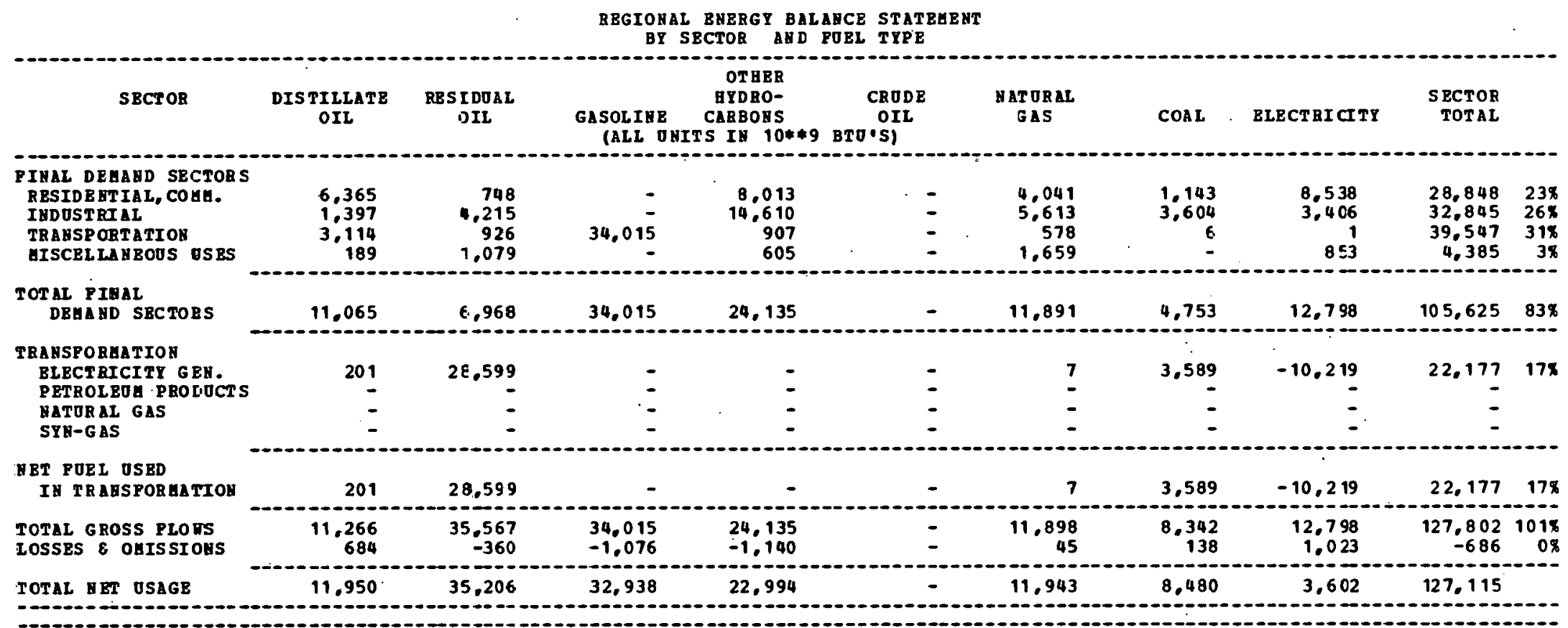

\section{SOPPLT OF EHERG \\ POSS II POBI \\ QYDROBLBCTRIC \\ GBO. 8 SOLAR}

,

TOTAL SUPPLY

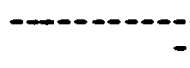

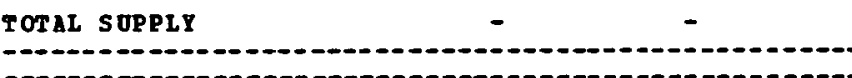

\begin{tabular}{l}
$\begin{array}{l}\text { EBT IHPORTS } \\
\text { OP REGION }\end{array}$ \\
\hline
\end{tabular}


REGIORAL EGERGY BALAHCE STATEAZNT

BY SECTOR. AND POBL TYPE
B

\begin{tabular}{|c|c|c|c|c|c|c|c|c|c|}
\hline SECTOR & $\begin{array}{l}\text { DIS TILIIATE } \\
\text { OIL }\end{array}$ & $\begin{array}{l}\text { RESIDUAL } \\
\text { OIL }\end{array}$ & $\begin{array}{l}\text { GASOLIHE } \\
\text { (ALL }\end{array}$ & $\begin{array}{c}\text { OTABR } \\
\text { AYDRO- } \\
\text { CARBOHS } \\
\text { TS I\$ 10**9 }\end{array}$ & $\begin{array}{c}\text { CRODE } \\
O \subseteq L \\
\text { BTO'SI }\end{array}$ & $\begin{array}{l}\text { HAT ORAL } \\
\text { GAS }\end{array}$ & COAT & ELECTRICITY & $\begin{array}{r}\text { SECTOR } \\
\text { TOTAL }\end{array}$ \\
\hline $\begin{array}{l}\text { PIHAL DEHAND 5BCTOBS } \\
\text { RESIDE ATIAL, COAH. } \\
\text { INDOSTRIAL } \\
\text { TRANSPORTATION } \\
\text { UISCELIAB BOOS OSBS }\end{array}$ & $\begin{array}{r}15.241 \\
4.330 \\
15.275 \\
451\end{array}$ & $\begin{array}{r}2,470 \\
15,384 \\
49 \\
514\end{array}$ & $80,82 \frac{-}{-}$ & $\begin{array}{r}16.770 \\
23.490 \\
6.714 \\
1.401\end{array}$ & $\overline{-}$ & $\begin{array}{r}11.066 \\
27.794 \\
1.378 \\
492\end{array}$ & $\begin{array}{r}2,750 \\
14,860 \\
. \quad 16 \\
-\end{array}$ & $\begin{array}{r}22.535 \\
18.490 \\
12 \\
279\end{array}$ & $\begin{array}{r}72,832 \\
99.848 \\
104,267 \\
3,137\end{array}$ \\
\hline $\begin{array}{l}\text { TOTAL PIAAL } \\
\text { DEHA AD SBCTORS }\end{array}$ & 35,797 & 13.417 & 80,823 & $5.9,375$ & - & 40.730 & $17, \in 26$ & 41.316 & 280,084 \\
\hline $\begin{array}{l}\text { TRANSPORGATIOA } \\
\text { BLECTRICITI GER. } \\
\text { PETROLEUA PRODOCTS } \\
\text { NATORAL GAS } \\
\text { SYH-GAS }\end{array}$ & $\begin{array}{r}1,218 \\
- \\
-\end{array}$ & $\overline{-}$ & $\begin{array}{l}- \\
- \\
-\end{array}$ & $\begin{array}{l}- \\
-\end{array}$ & - & $\begin{array}{r}244 \\
-\end{array}$ & $\begin{array}{r}20,710 \\
= \\
-\end{array}$ & $\begin{array}{r}-7.110 \\
= \\
=\end{array}$ & $\begin{array}{r}15.062 \\
= \\
-\end{array}$ \\
\hline $\begin{array}{l}\text { MBT POEL USED } \\
\text { IY TRABSPCA GATIOH }\end{array}$ & 1,218 & - & - & - & - & 2.14 & 20,710 & $-7,110$ & 15.062 \\
\hline $\begin{array}{l}\text { TOTAL GROSS ?LORS } \\
\text { LOSSES } 8 \text { OUISSIOHS }\end{array}$ & $\begin{array}{r}37.015 \\
2.248\end{array}$ & $\begin{array}{r}13.417 \\
-136\end{array}$ & $\begin{array}{l}80.823 \\
-2.558\end{array}$ & $\begin{array}{l}50,375 \\
-2,380\end{array}$ & $\overline{-}$ & $\begin{array}{r}40.974 \\
155\end{array}$ & $\begin{array}{r}38,336 \\
537\end{array}$ & $\begin{array}{r}41.316 \\
3.304\end{array}$ & $\begin{array}{r}295,146 \\
1,270\end{array}$ \\
\hline
\end{tabular}

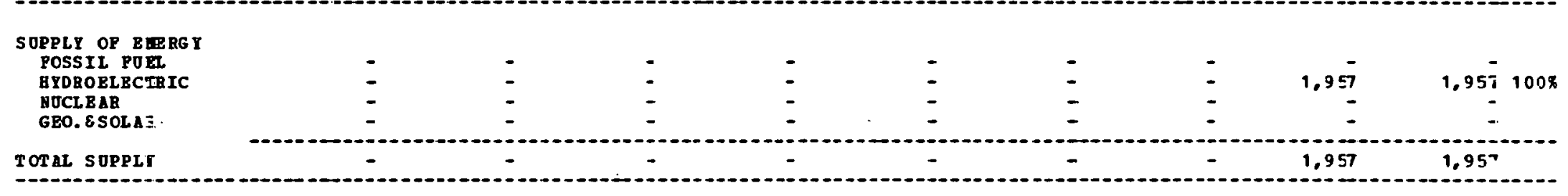

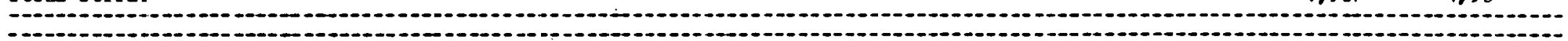

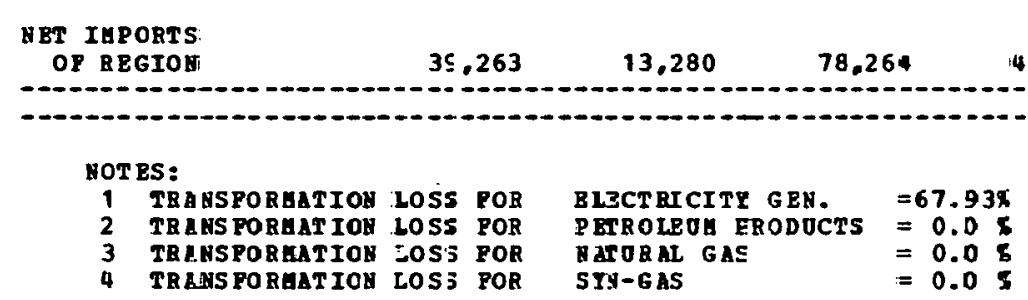


REG IOHAL BNBEGT BALANCE STA TEUENT BY SECTOR AHD FOEL TYPE

\begin{tabular}{|c|c|c|c|c|c|c|c|c|c|c|}
\hline SECTOR & $\begin{array}{l}\text { DISTILLATE } \\
\text { OIL }\end{array}$ & $\begin{array}{l}\text { BESI DOAL } \\
\text { JIL }\end{array}$ & $\begin{array}{l}\text { GASOLIHE } \\
\text { (ALI O }\end{array}$ & $\begin{array}{c}\text { OTHER } \\
\text { HYDRO- } \\
\text { CARBOHS } \\
\text { TS IN 10**9 }\end{array}$ & $\begin{array}{c}\text { CRODE } \\
\text { OIL } \\
\text { BTO'S) }\end{array}$ & $\begin{array}{l}\text { MAT URAL } \\
\text { GAS }\end{array}$ & $\operatorname{COAL}$ & ELBCTRICITY & $\begin{array}{l}\text { SECTOR } \\
\text { TOTAL }\end{array}$ & \\
\hline $\begin{array}{l}\text { PIHAL DRHAND SECTORS } \\
\text { RESIDE HTIAL, COHA. } \\
\text { INDOST RIAL } \\
\text { TRANSP ORTATIOH } \\
\text { GISCEL LA BOOS OSES }\end{array}$ & $\begin{array}{r}18.944 \\
7.092 \\
21.315 \\
582\end{array}$ & $\begin{array}{r}3,130 \\
13,146 \\
74 \\
645\end{array}$ & $\begin{array}{r}- \\
105,911 \\
-\end{array}$ & $\begin{array}{r}24.361 \\
42.104 \\
7.457 \\
1.786\end{array}$ & 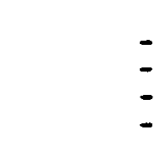 & $\begin{array}{r}14,743 \\
42,011 \\
1,828 \\
724\end{array}$ & $\begin{array}{r}3,628 \\
25,755 \\
26 \\
-\end{array}$ & $\begin{array}{r}29.711 \\
31,946 \\
21 \\
345\end{array}$ & $\begin{array}{r}94.517 \\
167.054 \\
136.632 \\
4.082\end{array}$ & $\begin{array}{r}18 \% \\
318 \\
26 \% \\
1 \%\end{array}$ \\
\hline $\begin{array}{l}\text { TOTAL PIHAL } \\
\text { DEEABD SBCTOBS }\end{array}$ & 47,933 & 21,995 & 105,911 & 75,708 & - & 59,306 & 29,409 & 62,023 & 402,285 & 768 \\
\hline $\begin{array}{l}\text { TRANSPOBHATIOY } \\
\text { ELECT FICITY GEN. } \\
\text { PETROLEOA PRODOCTS } \\
\text { MATURAL GAS } \\
\text { STA-GAS }\end{array}$ & $\begin{array}{r}361 \\
- \\
-\end{array}$ & $\begin{array}{r}5.624 \\
- \\
-\end{array}$ & $\begin{array}{l}- \\
-\end{array}$ & $\begin{array}{l}\overline{-} \\
\overline{-}\end{array}$ & $\overline{-}$ & $\begin{array}{r}6,266 \\
- \\
-\end{array}$ & $\begin{array}{r}172,524 \\
= \\
=\end{array}$ & $\begin{array}{r}-59.679 \\
- \\
-\end{array}$ & $\begin{array}{r}125.096 \\
= \\
=\end{array}$ & $20 x$ \\
\hline $\begin{array}{l}\text { NBT POEL OSED } \\
\text { IA TRAXSFORAATION }\end{array}$ & 361 & 5,624 & - & - & - & 6,266 & 172,524 & -59.679 & 125.096 & $24 \%$ \\
\hline $\begin{array}{l}\text { TOTAL GROSS PLORS } \\
\text { LOSSES E OUISSIOHS }\end{array}$ & $\begin{array}{r}48,294 \\
2.933\end{array}$ & $\begin{array}{r}27.619 \\
-280\end{array}$ & $\begin{array}{r}105,911 \\
-3,353\end{array}$ & $\begin{array}{l}75,708 \\
-3,577\end{array}$ & $\overline{-}$ & $\begin{array}{r}65.572 \\
248\end{array}$ & $\begin{array}{r}201,933 \\
3,357\end{array}$ & $\begin{array}{r}62,023 \\
4,960\end{array}$ & $\begin{array}{r}527,381 \\
4,289\end{array}$ & $\begin{array}{r}998 \\
18\end{array}$ \\
\hline
\end{tabular}

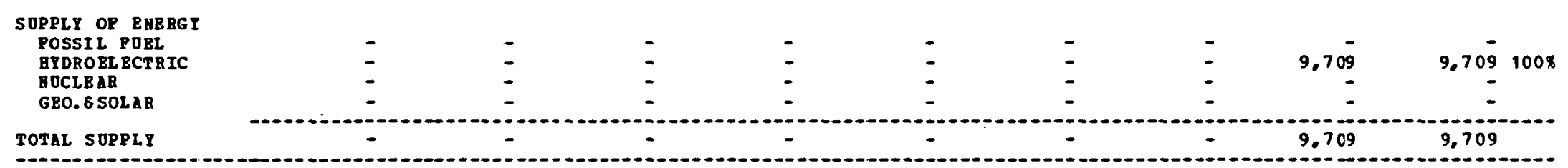

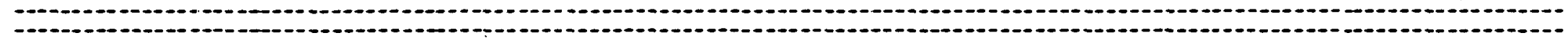

\begin{tabular}{l}
$\begin{array}{l}\text { NET IHPORTS } \\
\text { OP REGIOH }\end{array}$ \\
\hline
\end{tabular}


RB:TIONAL BN3BGY BALANCE STATEHENT

BT SECTOR ABD FOEL TRPE

\begin{tabular}{|c|c|c|c|c|c|c|c|c|c|}
\hline SECTOR & $\begin{array}{c}\text { DESTILLATE } \\
\text { O Il }\end{array}$ & $\begin{array}{l}\text { BISI DOAL } \\
\text { OII }\end{array}$ & $\begin{array}{l}\text { GASOLIHE } \\
\text { (AIL GHI }\end{array}$ & $\begin{array}{l}\text { AYDRO- } \\
\text { CABBOHS } \\
\text { TS IH 10**9 }\end{array}$ & $\begin{array}{c}\text { CRODE } \\
\text { OIL } \\
\text { BTO'S) }\end{array}$ & $\begin{array}{l}\text { MATJRAL } \\
\text { GAS }\end{array}$ & $\operatorname{COAL}$ & ELECTAICITY & $\begin{array}{r}\text { SBCTOR } \\
\text { TOTAL }\end{array}$ \\
\hline 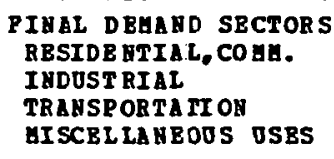 & $\begin{array}{r}5.162 \\
1.895 \\
1.903 \\
154\end{array}$ & $\begin{array}{r}710 \\
5.597 \\
9 \\
222\end{array}$ & 27.587 & $\begin{array}{r}6.500 \\
7.659 \\
384 \\
490\end{array}$ & $\begin{array}{l}- \\
\\
-\end{array}$ & $\begin{array}{r}3.450 \\
7.266 \\
469 \\
245\end{array}$ & $\begin{array}{r}927 \\
6.032 \\
3 \\
- \\
\end{array}$ & $\begin{array}{r}7.321 \\
4.826 \\
4 \\
125 \\
\end{array}$ & $\begin{array}{r}24.070 \\
33.275 \\
30.359 \\
1.235\end{array}$ \\
\hline $\begin{array}{l}\text { TOTAL PIHAL } \\
\text { DEHA KD SBCTORS }\end{array}$ & 9,114 & 6.538 & 27.587 & 15,033 & - & 11.430 & 6,962 & 12,276 & 88,943 \\
\hline $\begin{array}{l}\text { TRABSPORAATIOR } \\
\text { BLECT RICITY GRH. } \\
\text { PETROLEOR PRODOCTS } \\
\text { MATORAL GAS } \\
\text { SYR-GAS }\end{array}$ & $\overline{-}$ & $\begin{array}{r}3,389 \\
- \\
-\end{array}$ & $\overline{-}$ & : & $\overline{-}$ & $\overline{-}$ & $\begin{array}{r}158,256 \\
=\end{array}$ & $\begin{array}{r}-60,851 \\
-\end{array}$ & $\begin{array}{r}100.791 \\
= \\
-\end{array}$ \\
\hline $\begin{array}{l}\text { RBT PUEL OSBD } \\
\text { IH TRANSPOBGATION }\end{array}$ & - & 3,389 & - & - & - & - & 158,256 & $-60,851$ & 100,794 \\
\hline $\begin{array}{l}\text { TOTAL GROSS PLOHS } \\
\text { LOSSES \& OEISSI ONS }\end{array}$ & $\begin{array}{r}9.114 \\
553\end{array}$ & $\begin{array}{r}9,927 \\
-100\end{array}$ & $\begin{array}{r}27.587 \\
-873\end{array}$ & $\begin{array}{r}15.033 \\
-710\end{array}$ & - & $\begin{array}{r}11.430 \\
43\end{array}$ & $\begin{array}{r}165,218 \\
2,747\end{array}$ & $\begin{array}{r}12.276 \\
981\end{array}$ & $\begin{array}{r}189.734 \\
2,647\end{array}$ \\
\hline
\end{tabular}

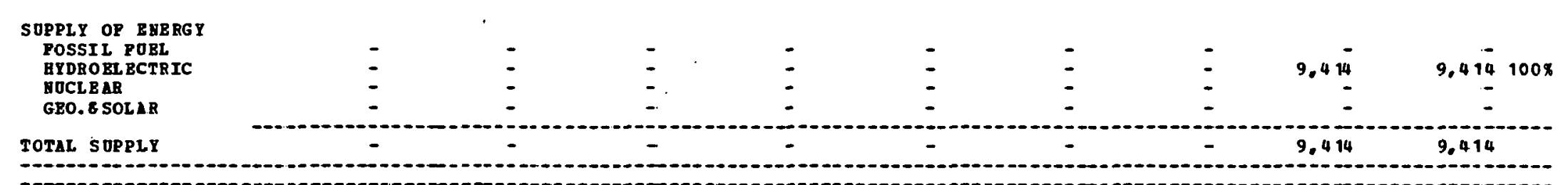

HET IUPORTS

OP RBGIOP

9.667

9.826

26,713

14,322

11.473

167,965

$-57.0 \mathrm{C7}$

182,961

\section{NOT ES:}

\begin{tabular}{|c|c|c|c|c|c|}
\hline 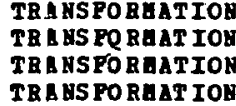 & $\begin{array}{l}\text { loss } \\
\text { loss } \\
\text { LoSs } \\
\text { los }\end{array}$ & & $\begin{array}{l}\text { BLECTRICITI } \\
\text { PETROLEOA ? } \\
\text { HLTORAL GAJ } \\
\text { STI-GAS }\end{array}$ & $\begin{array}{l}\text { GRH. } \\
\text { RODOCTS }\end{array}$ & $\begin{array}{l}=62.365 \\
=0.0 \% \\
=0.05 \\
=0.05\end{array}$ \\
\hline
\end{tabular}


RBGIONAL BNERGY BALAICE STATEGEAT

BI SBCTOR AHD FOBL TYPE

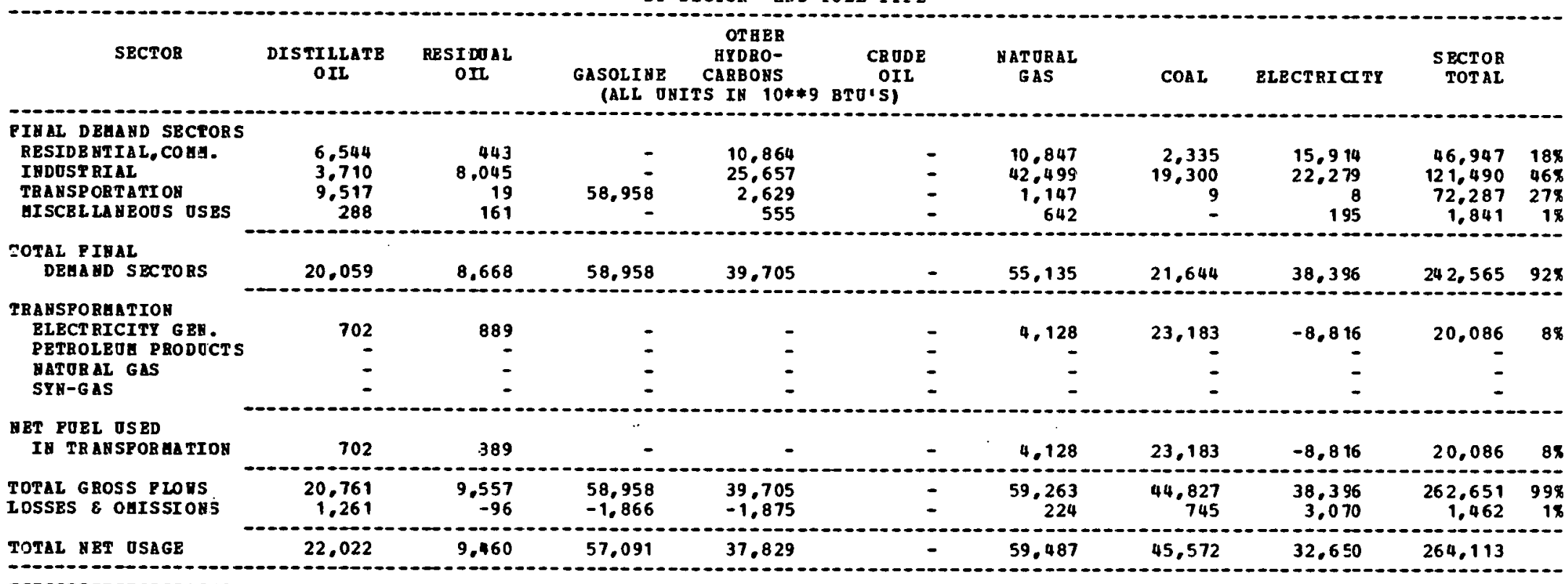

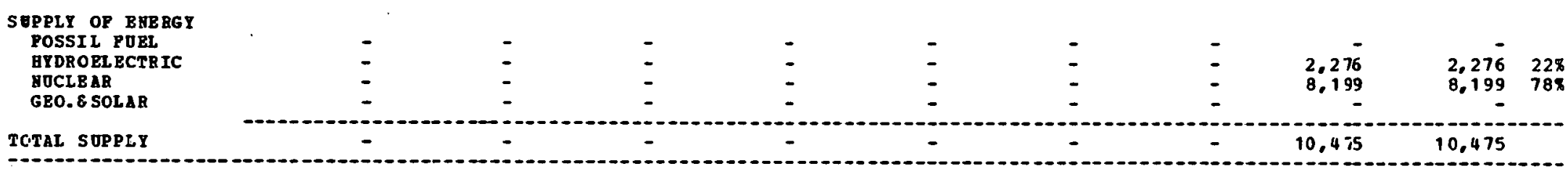

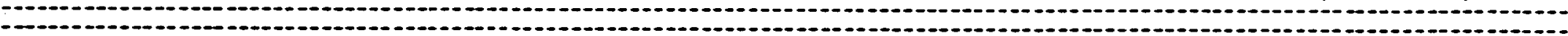

\section{MET IMPOBTS}

DP. REGIOB

22,022

9,460

57,091

37.829

- $\quad 59.487$

45,572

22.175

253.638

HOTES:

TRA BSFORHAM IOR LOSS FOR

TRABSPORAATIOH LOSS POR
TRABSPORAATIOH LOSS POR

TRABSPOBATIOH LOSS POR

BLBCTRICITY GEN.

$\begin{array}{ll} & =69.50 \times \\ \text { ELPCTRICITY GEN } & =0.0 \times\end{array}$

MTORAL GAS

$S$ YN-G AS
$=0.0 \times$

$=0.0 \%$ 
REGIJNLL BNBRGY BALANCE STATEMEYT

BY SECTOR AND POEL TYPE

\begin{tabular}{|c|c|c|c|c|c|c|c|c|c|c|}
\hline SECTOR & $\begin{array}{c}\text { DISTILLATE } \\
\text { OIL }\end{array}$ & $\begin{array}{c}\text { RESI DOAL } \\
\text { CIL }\end{array}$ & $\begin{array}{l}\text { GASOLIN } \\
\text { (ALL }\end{array}$ & $\begin{array}{l}\text { OTAER } \\
\text { EIDZO- } \\
\text { CLBBOHS } \\
\text { TS IS 10**9 }\end{array}$ & $\begin{array}{c}\text { CRODE } \\
\text { OIL } \\
\text { BIO'S) }\end{array}$ & $\begin{array}{l}\text { MATURAL } \\
\text { GAS }\end{array}$ & COAI. & BLECTRICITY & $\begin{array}{l}\text { SECTOR } \\
\text { TOTAL }\end{array}$ & \\
\hline $\begin{array}{l}\text { TOTAL FIRAL } \\
\text { DEHA HD SECTOBS }\end{array}$ & 13,988 & 2,574 & 43,566 & 22,610 & - & 22,568 & 8,570 & 18,706 & 132.682 & $78 x$ \\
\hline $\begin{array}{l}\text { TRANSFORAATICA } \\
\text { BLECTRICITY GBH. } \\
\text { PETROLEOA PRODOCTS } \\
\text { MATURAL GAS } \\
\text { SYH-GAS }\end{array}$ & $\begin{array}{r}2.682 \\
= \\
-\end{array}$ & $\begin{array}{r}294 \\
- \\
-\end{array}$ & :- & $\begin{array}{l}- \\
-\end{array}$ & $\overline{-}$ & $\begin{array}{r}9,598 \\
\vdots \\
-\end{array}$ & $\begin{array}{r}43,279 \\
= \\
=\end{array}$ & $\begin{array}{r}-18,152 \\
- \\
-\end{array}$ & $\begin{array}{r}37.501 \\
= \\
=\end{array}$ & 228 \\
\hline $\begin{array}{l}\text { HET FOEL OSBD } \\
\text { IN TRABSFORGATION }\end{array}$ & 2,682 & 294 & - & - & - & 9,398 & 43,279 & $-18,1 \leq 2$ & 37.501 & 228 \\
\hline $\begin{array}{l}\text { TOTAL GROSS ILORS } \\
\text { LOSSES } 8 \text { OBISSIOZS }\end{array}$ & $\begin{array}{r}16.670 \\
1.012\end{array}$ & $\begin{array}{r}2.868 \\
-29\end{array}$ & $\begin{array}{l}43,566 \\
-1,379\end{array}$ & $\begin{array}{l}22,610 \\
-1,068\end{array}$ & - & $\begin{array}{r}31.366 \\
121\end{array}$ & $\begin{array}{r}51.049 \\
863\end{array}$ & $\begin{array}{r}18.706 \\
1.496\end{array}$ & $\begin{array}{r}170,183 \\
1,016\end{array}$ & $\begin{array}{r}998 \\
18\end{array}$ \\
\hline TOTAL \&ET OSAGE & $17 ; 682$ & 2.838 & 42,186 & 21.541 & - & $32,0: 37$ & 52,812 & $2.0 \leq 0$ & 171.199 & \\
\hline
\end{tabular}

-

SOP PLY OP B REBGY

FOSSII FO ER.

HYDROBLBCTRIC

YOCL.EAR

GEO. \& SOLAE

-
-
-
-

TOTAL SOPPLT

$-$

-
-
-

$\begin{array}{ll}- & = \\ - & =\end{array}$

:-

:-

$\begin{array}{cc}4,517 & 4.5171008 \\ - & - \\ 4,517 & 4,517\end{array}$

\section{NET IUPORTS}

OP BEGION

17,682

2,838

42,186

21,541

32, C 87

52,812

$-2,466$

$166,68=$

\section{NOT BS: \\ 1 TRAFSPORAATION DOSS FOB \\ 2 TRAESPORHATION JOSS POR \\ TRAESPOALATION LOSS FO \\ ELECTRICITY GEN . \\ MAOBal GaS \\ $=0.0 \%$ \\ STHi-G AS \\ $=0.08$
$=0.0 \%$}


REG IOHAL BNERGI BALAECE STA TEHENT

BY SECTOR AND POBL TYPE

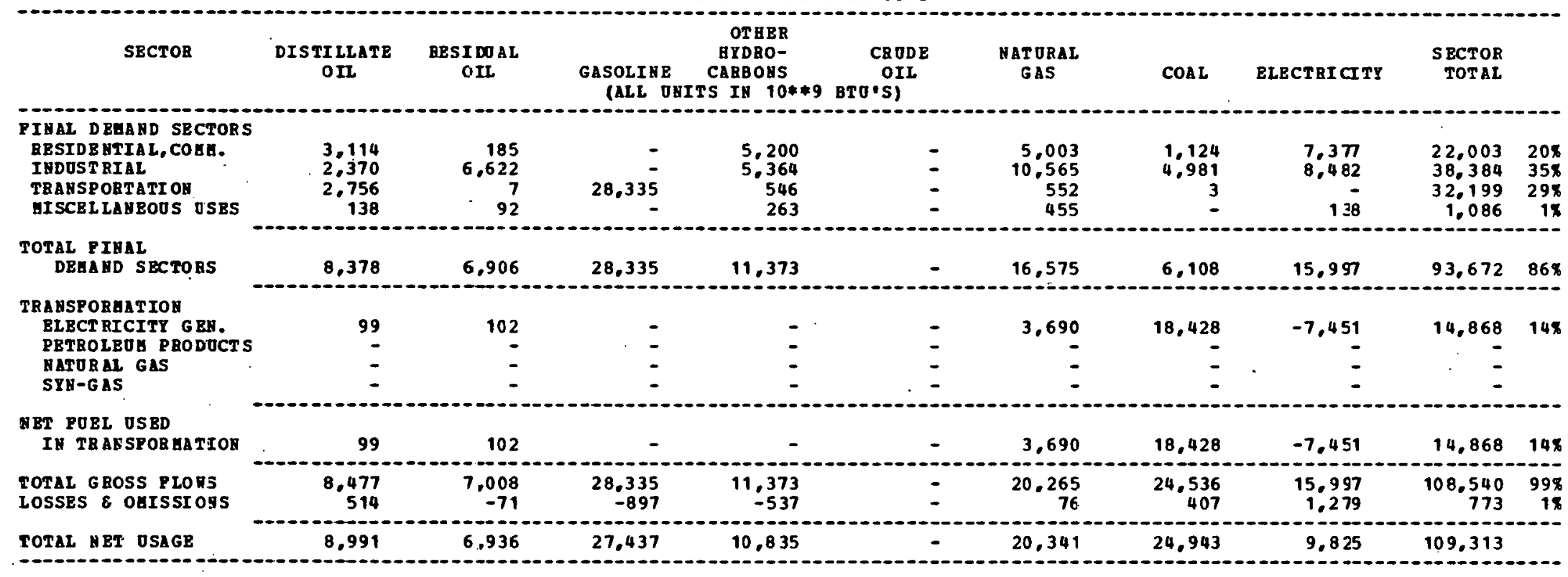

\section{SOPPLY OP BHERG \\ POSSII POBL
GYDROBLBCTRIC \\ GOCL $B$ AB \\ GBO. 8 SOLAB}

TOTAL SOPPLY

-

$\begin{array}{llll}- & - & - & - \\ - & - & - & -\end{array}$

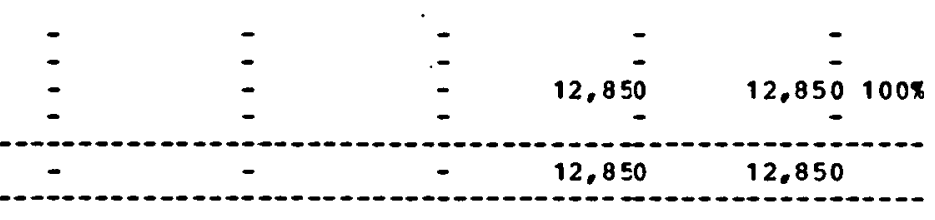

\section{BBT IMPORTS}

- $\quad$

$-$

10,835

20,341

24,943

$-3,024$

96.463

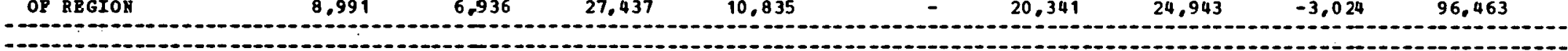

\section{HOTES:}

TRABS FOREAT IOY LOSS POR TBANSFO B MATION LOSS POB

TRABSPQREATION LOSS POR

TBAHSPBBATIOA LOSS POB

BLBCT RICITY GEH.

=66.62\%

PETROLEO PRODOCTS $=0.0 \mathrm{8}$

$\begin{array}{ll}\text { STIORAL GAS } & =0.0 \times \\ \text { SYH-G AS } & =0.0 \times\end{array}$ 
REGTONAL RGERGY BALANCE STATELENT

BY SECTOR: ARD POEL TYPE

\begin{tabular}{|c|c|c|c|c|c|c|c|c|c|c|}
\hline SECTIOR & $\begin{array}{c}\text { DISTILjATE } \\
\text { OIJ }\end{array}$ & $\begin{array}{l}\text { BESI DOAL } \\
\text { DIL }\end{array}$ & $\begin{array}{l}\text { GASOL }=\mathrm{NE} \\
\text { (ALE OHI }\end{array}$ & $\begin{array}{c}\text { OTBER } \\
\text { AYDBO- } \\
\text { CARBONS } \\
\text { TS IS 10**9 }\end{array}$ & $\begin{array}{c}\text { CRODE } \\
\text { OIL } \\
\text { BTO'SI }\end{array}$ & $\begin{array}{l}\text { HAT OE:AL } \\
\text { GAS }\end{array}$ & COAL & ELECTRICITY & $\begin{array}{l}\text { SECTOR } \\
\text { TOTAL }\end{array}$ & \\
\hline $\begin{array}{l}\text { PIRAL D EHAND SECTORS } \\
\text { RESIDE HT IAL, COGH. } \\
\text { IHDOSTRIAL } \\
\text { TRARSPORTATIOH } \\
\text { UISCELIAHBOUS OSBS }\end{array}$ & $\begin{array}{r}3,370 \\
1.050 \\
4.366 \\
149\end{array}$ & $\begin{array}{r}210 \\
3.002 \\
7.754 \\
296\end{array}$ & $30,66 \overline{-}$ & $\begin{array}{r}5.628 \\
.566 \\
.643 \\
285\end{array}$ & $\begin{array}{l}- \\
- \\
-\end{array}$ & $\begin{array}{r}5.510 \\
7.137 \\
598 \\
2.471\end{array}$ & $\begin{array}{r}1,217 \\
2,150 \\
5 \\
-\end{array}$ & $\begin{array}{r}8,103 \\
3,387 \\
5 \\
747\end{array}$ & $\begin{array}{r}24,038 \\
19,292 \\
45,037 \\
3,948\end{array}$ & $\begin{array}{r}178 \\
148 \\
338 \\
38\end{array}$ \\
\hline $\begin{array}{l}\text { TOTAL FIHAL } \\
\text { DEHA ND SEC TORS }\end{array}$ & 8.935 & 11.262 & $30,6.66$ & 10,122 & - & 15,716 & 3,372 & 12.242 & 92,315 & $67 x$ \\
\hline $\begin{array}{l}\text { TRANSFORAATIÖ } \\
\text { ELECTRICITI GBA. } \\
\text { PETROLEOA PRODOCTS } \\
\text { RATURAR GAS } \\
\text { SYY-GAS }\end{array}$ & $\begin{array}{r}140 \\
- \\
-\end{array}$ & $\begin{array}{r}23.474 \\
- \\
-\end{array}$ & - & :- & $\begin{array}{l}- \\
- \\
-\end{array}$ & $\begin{array}{r}6.0+2 \\
= \\
-\end{array}$ & $\begin{array}{r}36,740 \\
- \\
-\end{array}$ & $\begin{array}{r}-20,625 \\
- \\
-\end{array}$ & $\begin{array}{r}45.771 \\
- \\
-\end{array}$ & $33 \%$ \\
\hline $\begin{array}{l}\text { NET FOEL OSED } \\
\text { IH TRAHSPCRAATION }\end{array}$ & 140 & 23,474 & - & - & - & 6,042 & 36,740 & $-20,625$ & 45.771 & $33 \%$ \\
\hline $\begin{array}{l}\text { TOTAL GROSS ?LOMS } \\
\text { LOSSES } 6 \text { OUISSIOAS }\end{array}$ & $\begin{array}{r}9,075 \\
551\end{array}$ & $\begin{array}{r}34,736 \\
-352\end{array}$ & $\begin{array}{r}30,666 \\
-970\end{array}$ & $\begin{array}{r}10.122 \\
-478\end{array}$ & 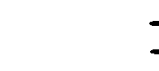 & $\begin{array}{r}21.758 \\
82\end{array}$ & $\begin{array}{r}40,112 \\
666\end{array}$ & $\begin{array}{r}12,242 \\
979\end{array}$ & $\begin{array}{r}138.086 \\
478\end{array}$ & $\begin{array}{r}100 x \\
0 x\end{array}$ \\
\hline TOTAI UET USAGE & 9,626 & 34.383 & 29.595 & 9,643 & & $21, \varepsilon 40$ & 40.778 & -7.403 & 138,564 & \\
\hline
\end{tabular}

\section{SOPPLI OP BIBRGY}

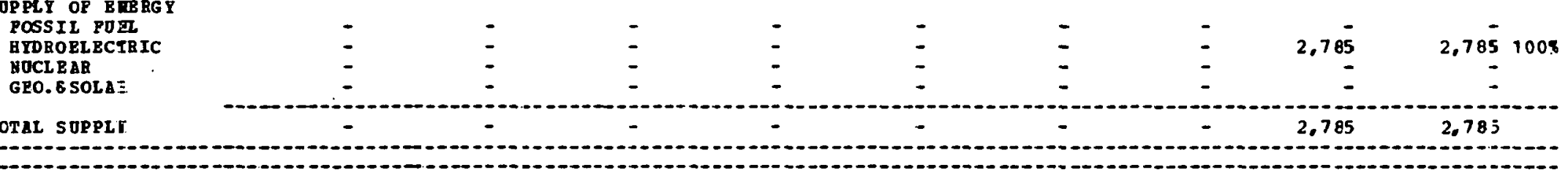

NBT IMPORTS
OP REGIOV

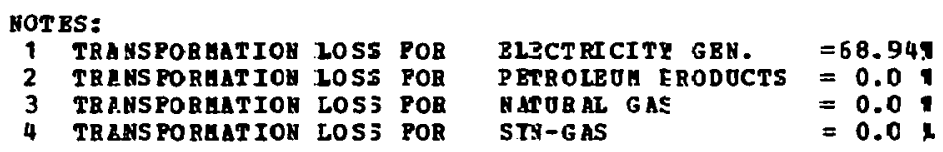


REgIONAL BHERGY BALANCE STATEAENT

BY SECTOR AND POEL TIPE

\begin{tabular}{|c|c|c|c|c|c|c|c|c|c|c|}
\hline SECTOR & $\begin{array}{l}\text { DIS TILLATB } \\
\text { OIL }\end{array}$ & $\begin{array}{l}\text { RBSEDOAL } \\
\text { OIIL }\end{array}$ & $\begin{array}{l}\text { GASOLI HE } \\
\text { (ALL OAI }\end{array}$ & $\begin{array}{c}\text { AYDRO- } \\
\text { CARBOHS } \\
\text { TS IY 10**9 }\end{array}$ & $\begin{array}{c}\text { CRODE } \\
\text { OIL } \\
\text { BTO'S) }\end{array}$ & $\begin{array}{l}\text { NATORAL } \\
\text { GAS }\end{array}$ & COAL & ELECT RI CITY & $\begin{array}{l}\text { SECTOR } \\
\text { TOTAL }\end{array}$ & \\
\hline $\begin{array}{l}\text { PIHAL DEHAHD SECTORS } \\
\text { RESIDE HTIAL, COEL. } \\
\text { IHDOSTRIAL } \\
\text { TRABSPORTATION } \\
\text { GISCELLABBODS OSBS }\end{array}$ & $\begin{array}{r}2,319 \\
1,808 \\
3,521 \\
116\end{array}$ & $\begin{array}{r}625 \\
4,964 \\
75 \\
509\end{array}$ & $\begin{array}{r}- \\
32,565 \\
-\end{array}$ & $\begin{array}{r}5,306 \\
8,039 \\
710 \\
417\end{array}$ & E- & $\begin{array}{r}8.632 \\
15,949 \\
593 \\
1.921\end{array}$ & $\begin{array}{r}723 \\
4.040 \\
4 \\
-\end{array}$ & $\begin{array}{r}7,568 \\
5,860 \\
7 \\
200\end{array}$ & $\begin{array}{r}25,173 \\
40,660 \\
37,475 \\
3,163\end{array}$ & $\begin{array}{r}21 \% \\
34 x \\
31 \% \\
3 x\end{array}$ \\
\hline $\begin{array}{l}\text { TOTAL P IBAL } \\
\text { DEHABD SECTORS }\end{array}$ & 7.764 & 6,173 & 32.565 & 14.472 & - & 27.095 & 4,767 & 13,635 & 106.471 & $88 x$ \\
\hline $\begin{array}{l}\text { TRAHSPORGATIOH } \\
\text { BLECT RICITY GES. } \\
\text { PETROLEOA PRODOCTS } \\
\text { MATORAL GAS } \\
\text { SYR-GAS }\end{array}$ & $\begin{array}{r}4,338 \\
- \\
-\end{array}$ & $\begin{array}{l}8 \\
- \\
-\end{array}$ & $\begin{array}{l}- \\
-\end{array}$ & : & - & $\begin{array}{r}2,375 \\
= \\
-\end{array}$ & $\begin{array}{r}12,974 \\
= \\
-\end{array}$ & $\begin{array}{r}-6,121 \\
- \\
=\end{array}$ & $\begin{array}{r}13,574 \\
= \\
=\end{array}$ & $11 x$ \\
\hline $\begin{array}{l}\text { DEP POEL OSBD } \\
\text { IR TRANSPORAATIOY }\end{array}$ & 4,338 & 8 & - & - & 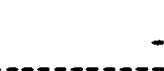 & 2,375 & 12.974 & $-6,121$ & 13.574 & $11 x$ \\
\hline $\begin{array}{l}\text { TOTAL GROSS PLOHS } \\
\text { LOSSES E OUISSIOHS }\end{array}$ & $\begin{array}{r}12.102 \\
735\end{array}$ & $\begin{array}{r}6.181 \\
-62\end{array}$ & $\begin{array}{r}32,565 \\
-1,030\end{array}$ & $\begin{array}{r}14,472 \\
-683\end{array}$ & - & $\begin{array}{r}29.470 \\
111\end{array}$ & $\begin{array}{r}17,741 \\
294\end{array}$ & $\begin{array}{r}13.6 \vdots 5 \\
1.090\end{array}$ & $\begin{array}{r}120.045 \\
454\end{array}$ & $\begin{array}{r}1008 \\
08 \\
\end{array}$ \\
\hline
\end{tabular}

SJPPLI OF ENERGI

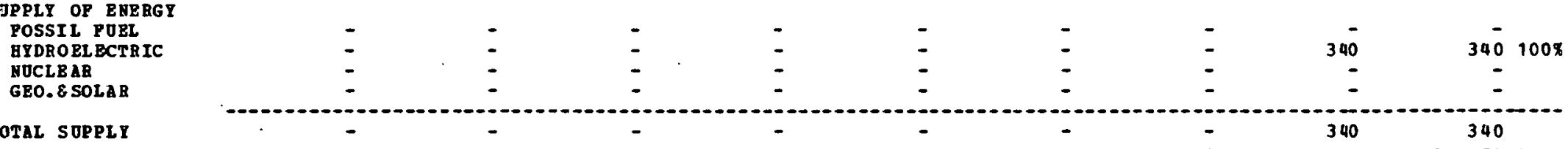

IET IKPORTS

12.837

6.118

31.534

13,788

29.581

18,035

8,264

120,159

NOTES:

TRANS PORAAT IOH LOSS POR TRANSPOBAATIOH LOSS POR TRAHS PORAAT IOI LOSS POR

BLECT RICITY GEN.

$=68.92 \mathrm{x}$

TRAYSPORAATIOH LOSS. PO

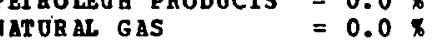

SYH-GAS

$=0.0 \%$ 
REGIOHAL ERBRGT BALANCE STATEHENT

BY SECT JR AND FOEL TYPE

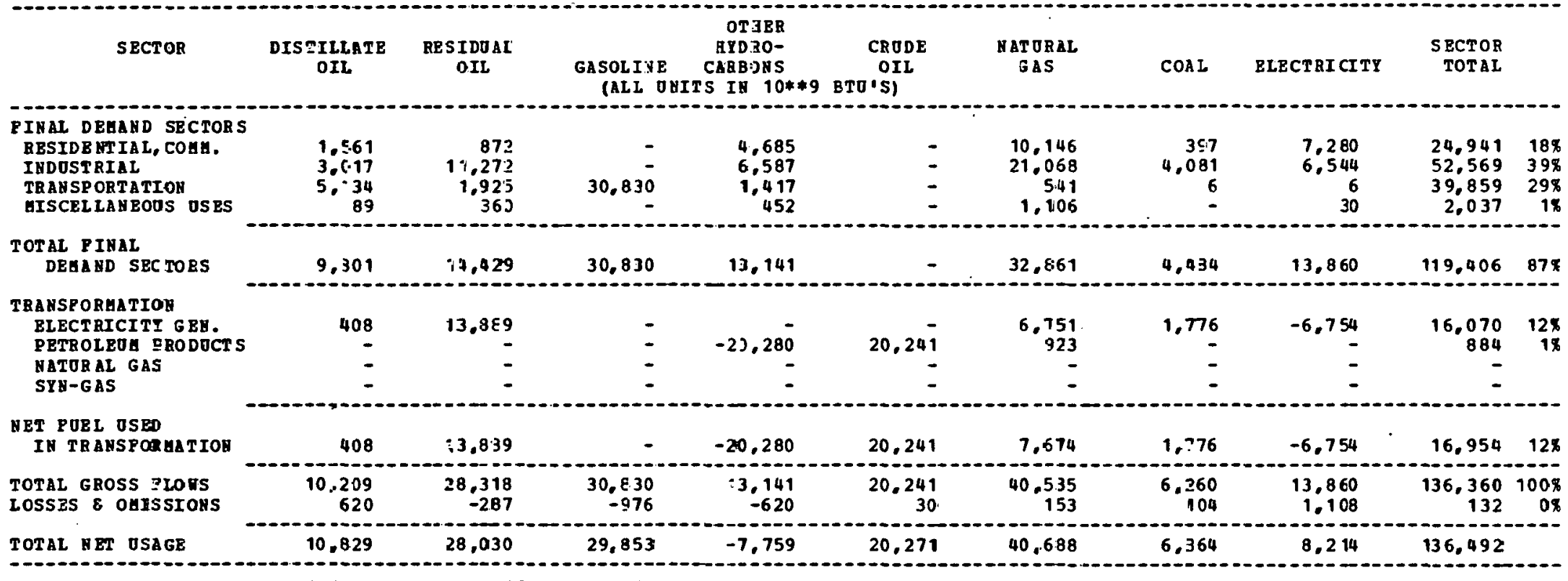

\section{SOPPLY OP EMERG Y \\ POSSIL POEL \\ GYDROELECTRIC}

TOTAL SUPPIT

$\begin{array}{rr}- & - \\ - & - \\ - & -\end{array}$

$\begin{array}{ll}- & - \\ - & -\end{array}$

$-$

$-$

-

-

IMT IMPORT:

OP B BGIOR

1), 829

28,030

$29,8 \leq 3$

20.271

40,68:9

6,364

8. 214

136.492

\section{NOT ES:}
1 TRLNSPOAHATION LOSS POR
TRANS PORUATIOH LOSS POR
TRANSPORMATION LOSS POR
TRANSPORATION LOSS POR
ELBCT RICITI GBN.
$=70.4 \mathrm{~m}$
ATOBAL GA3
SEN-GAS
$=4.188$
$=0.08$
$=0.08$ 
REG IONAL ENBRGY BALANCE STA TEHENT

BY SECTOR AND FOEL TIPE

\begin{tabular}{|c|c|c|c|c|c|c|c|c|c|c|}
\hline SECTOR & $\begin{array}{l}\text { DIS TI LLATE } \\
\text { OIL }\end{array}$ & $\begin{array}{l}\text { RESIDOAL } \\
\text { OII }\end{array}$ & $\begin{array}{l}\text { GASOLINE } \\
\text { (ALL }\end{array}$ & $\begin{array}{l}\text { AYDBO- } \\
\text { CARBOKS } \\
\text { TS IN 10**9 }\end{array}$ & $\begin{array}{c}\text { CR ODE } \\
\text { OIL } \\
\text { BTO'S) }\end{array}$ & $\begin{array}{l}\text { NATORAL } \\
\text { GAS }\end{array}$ & COAL & ELECTRICITI & $\begin{array}{l}\text { SECTOR } \\
\text { TOTAL }\end{array}$ & \\
\hline $\begin{array}{l}\text { PINAL D BHAND SECTORS } \\
\text { RESIDE NTIAL, COBA. } \\
\text { INDOSTRIAL } \\
\text { TRAYSP ORTATI OH } \\
\text { GISCEL LAHEOOS JSBS }\end{array}$ & $\begin{array}{r}3.827 \\
6.790 \\
11.883 \\
342\end{array}$ & $\begin{array}{r}2.076 \\
21.889 \\
4.921 \\
844\end{array}$ & 81,341 & $\begin{array}{r}11.612 \\
10.900 \\
6.432 \\
472\end{array}$ & $\overline{-}$ & $\begin{array}{r}7.934 \\
27.397 \\
692 \\
1.362\end{array}$ & $\begin{array}{r}643 \\
4.163 \\
18 \\
-\end{array}$ & $\begin{array}{r}25,040 \\
9.809 \\
18 \\
1.219\end{array}$ & $\begin{array}{r}51,132 \\
80,948 \\
105,305 \\
4,239\end{array}$ & $\begin{array}{r}16 \% \\
26 \% \\
34 \% \\
1 \%\end{array}$ \\
\hline $\begin{array}{l}\text { TOTAL F IHAL } \\
\text { DEGA KD SBCTORS }\end{array}$ & 22.842 & 29,730 & 81,341 & 29.416 & - & 37,385 & 4,824 & 36,086 & 241,624 & $78 \%$ \\
\hline $\begin{array}{l}\text { TAASSPOBHATIOH } \\
\text { BLECT RICITY GEH. } \\
\text { PETROLEOH PBOIOCTS } \\
\text { NATORAL GAS } \\
\text { STN-GAS }\end{array}$ & $\begin{array}{r}8,628 \\
- \\
-\end{array}$ & $\begin{array}{r}79,002 \\
- \\
-\end{array}$ & $\begin{array}{l}\overline{-} \\
\overline{-}\end{array}$ & $\begin{array}{r}- \\
-4,521 \\
-\end{array}$ & - & $\begin{array}{r}11.853 \\
5.196 \\
-\end{array}$ & $\begin{array}{r}254 \\
- \\
-\end{array}$ & $\begin{array}{r}-30,625 \\
- \\
-\end{array}$ & $\begin{array}{r}69,112 \\
- \\
675 \\
-\end{array}$ & $\begin{array}{r}22 x \\
0 x\end{array}$ \\
\hline $\begin{array}{l}\text { HET POEL OSED } \\
\text { IN TRAESPOR AA TIOH }\end{array}$ & 8,628 & 79,002 & - & -4.521 & 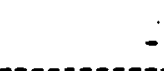 & 17.049 & 254 & $-30,625$ & 69.787 & $22 \pi$ \\
\hline $\begin{array}{l}\text { TOTAL GROSS ELONS } \\
\text { LOSSES } 6 \text { OHISSIOHS }\end{array}$ & $\begin{array}{r}31.470 \\
1.911\end{array}$ & $\begin{array}{r}108,732 \\
-1.103\end{array}$ & $\begin{array}{l}81,341 \\
-2,575\end{array}$ & $\begin{array}{l}29,416 \\
-1,389\end{array}$ & - & $\begin{array}{r}54,434 \\
206\end{array}$ & $\begin{array}{r}5,078 \\
84\end{array}$ & $\begin{array}{r}36.066 \\
2,886\end{array}$ & $\begin{array}{r}311.411 \\
19\end{array}$ & $\begin{array}{r}100 \% \\
0 \%\end{array}$ \\
\hline
\end{tabular}

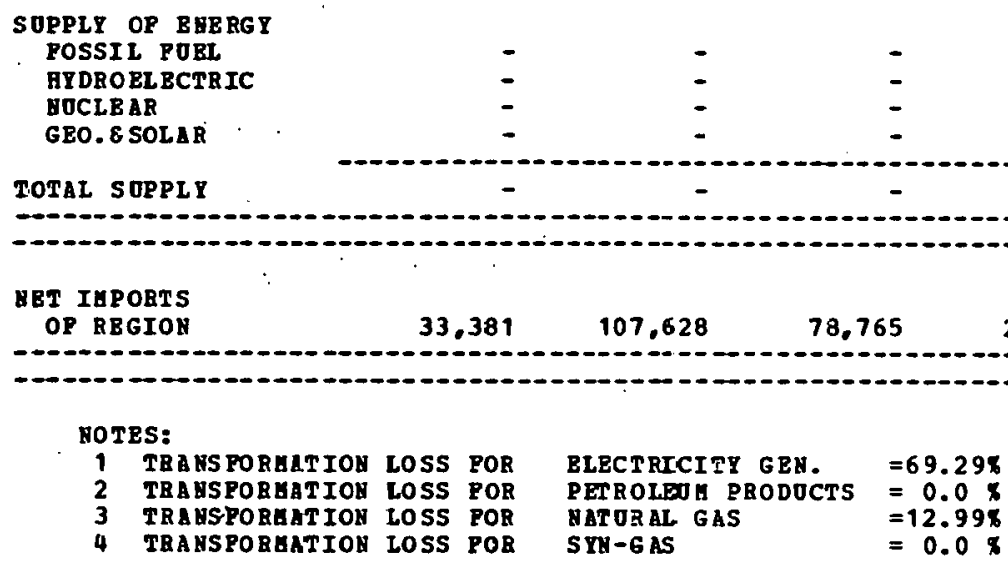


REGFONAL ENERGY BALANCE STATELENT

BY SECT'JR AND POEL TYPE

\begin{tabular}{|c|c|c|c|c|c|c|c|c|c|}
\hline SECTOR & $\begin{array}{l}\text { DIS MILLATE } \\
\text { OIL }\end{array}$ & $\begin{array}{l}\text { RE SI DUAL } \\
\text { OIL }\end{array}$ & $\begin{array}{l}\text { GASOLINE } \\
\text { (ALL O }\end{array}$ & $\begin{array}{l}\text { MYDRO- } \\
\text { CAEBONS } \\
\text { IS IN 10**9 }\end{array}$ & $\begin{array}{c}\text { CRODE } \\
\text { OIL } \\
\text { BTO'S) }\end{array}$ & $\begin{array}{l}\text { MATORBL } \\
\text { GAS }\end{array}$ & COAL & ELECTRICITY & $\begin{array}{r}\text { SECTOR } \\
\text { TOTAL }\end{array}$ \\
\hline 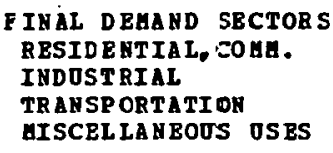 & $\begin{array}{r}3,554 \\
1,694 \\
7,018 \\
533\end{array}$ & $\begin{array}{r}1.914 \\
2.647 \\
164 \\
462\end{array}$ & $\begin{array}{r}- \\
- \\
75,971\end{array}$ & $\begin{array}{r}10.760 \\
3.805 \\
5.611 \\
348\end{array}$ & $\overline{-}$ & $\begin{array}{r}5,050 \\
6,541 \\
555 \\
504\end{array}$ & $\begin{array}{r}559 \\
1,056 \\
12 \\
-\end{array}$ & $\begin{array}{r}24,144 \\
3,759 \\
12 \\
757\end{array}$ & $\begin{array}{r}45,991 \\
19.502 \\
89.743 \\
2.404\end{array}$ \\
\hline $\begin{array}{l}\text { TOTAL PINAL } \\
\text { DEHA AD SEC TOBS }\end{array}$ & 12,999 & 5,187 & 75,971 & $2 c, 524$ & - & $12, \in 60$ & 1,627 & 28,672 & 157,640 \\
\hline $\begin{array}{l}\text { TRANSPORUATION' } \\
\text { ELECTRICITY GEN. } \\
\text { PETROLEUA PRODOCTS } \\
\text { NATORAL GAS } \\
\text { SYH-GAS }\end{array}$ & $\begin{array}{r}279 \\
- \\
-\end{array}$ & $\begin{array}{r}69,120 \\
- \\
-\end{array}$ & 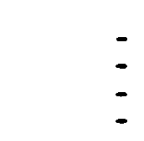 & $\begin{array}{r}616 \\
- \\
-\end{array}$ & - & $\begin{array}{r}42,904 \\
- \\
-\end{array}$ & $\begin{array}{l}- \\
-\end{array}$ & $\begin{array}{r}-35,626 \\
- \\
-\end{array}$ & $\begin{array}{r}77.293 \\
- \\
-\end{array}$ \\
\hline $\begin{array}{l}\text { NET POBL OSE: } \\
\text { IN TRASSPOEAATIOB }\end{array}$ & 279 & E9.120 & - & 616 & - & 42,704 & - & $-35,626$ & 77,293 \\
\hline $\begin{array}{l}\text { TOTAL GROSS PLONS } \\
\text { LOSSES } 8 \text { OUISSIOUS }\end{array}$ & $\begin{array}{r}13.278 \\
806\end{array}$ & $\begin{array}{r}74,307 \\
-754\end{array}$ & $\begin{array}{l}75,971 \\
-2,405\end{array}$ & $\begin{array}{r}21,140 \\
-998\end{array}$ & - & $\begin{array}{r}55,554 \\
210\end{array}$ & $\begin{array}{r}1.627 \\
27\end{array}$ & $\begin{array}{r}28,672 \\
2,293\end{array}$ & $\begin{array}{r}234,933 \\
-820\end{array}$ \\
\hline
\end{tabular}

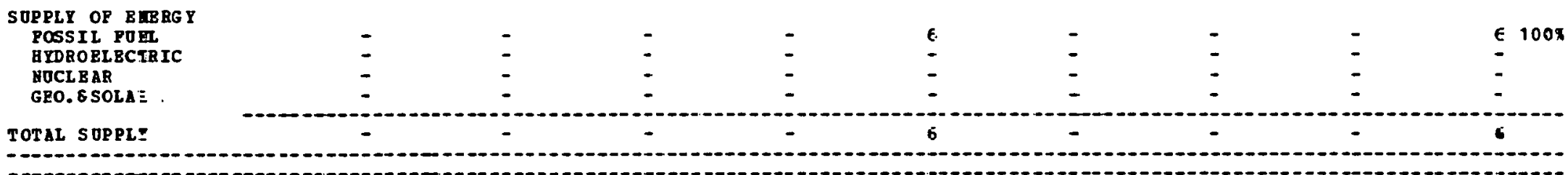

NBT IHPORTS

$14.084 \quad 73,552$

73.565

20,141

$-6 \quad 55, .776$

1,654

234.105

OP REGION

NOTES:
1 TRANS PORHATION LOSS POR TRABSFOREATIOK LOSS POB
RLECTEICITY GEA.
3 TRAHSPOREATIOH LOSS PO
GEN. $=68.459$
TRENSPORAATION LOSS POE
PEROLEOA PRODUCTS $=0.08$
$\begin{array}{lll}\text { SIN }- \text { GS } & =0.0 \\ & =0.0 \mathrm{~W}\end{array}$ 
REGIONAL EHBRGY BALAHCE STATEHEHT

BY SECTOR AHD POEL TYPE

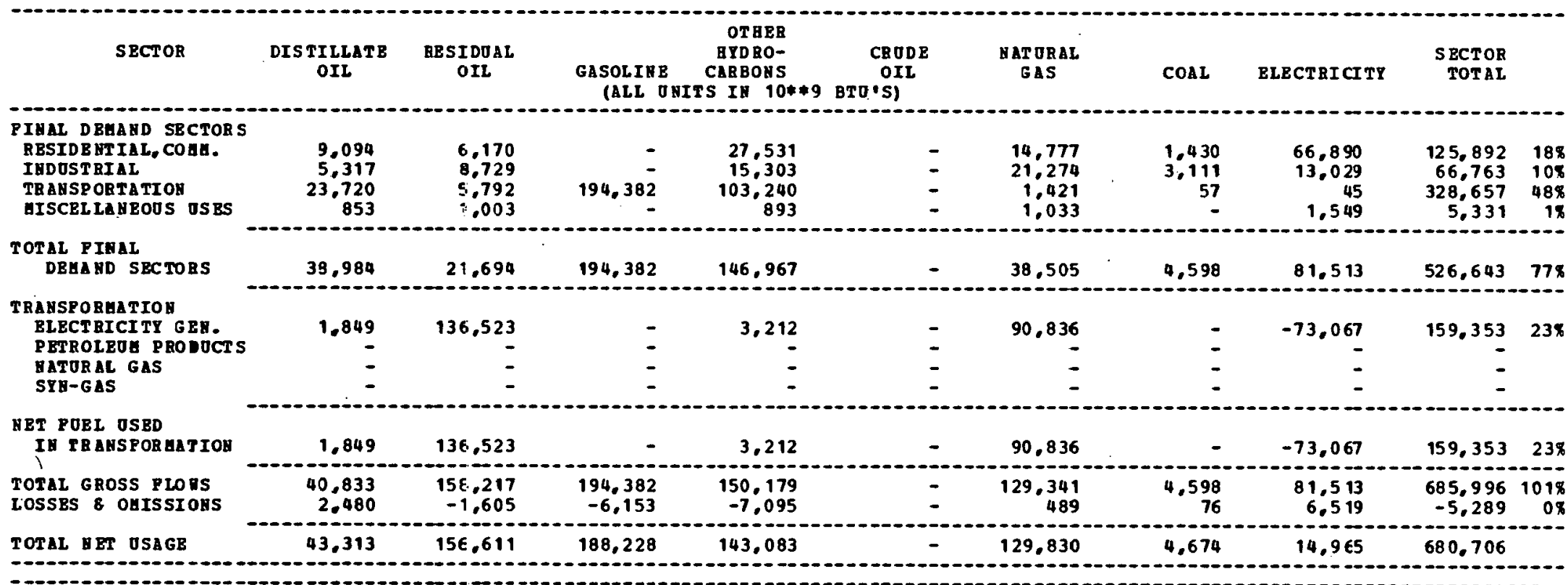

SOPPLY OP BHB BG T

POSSIL PUBL

GYDROBLBCTRIC
NOCLBAR

GBO. ESOLAR

TOTAL SOPPLY

-
-
-
-

-

$=$

\section{3,313}

156.611

188,228

143.083

$-22,655$

129.503

4,674

$-1.009$

641.749

\begin{tabular}{|c|c|c|c|c|c|}
\hline $\begin{array}{l}1 \\
2 \\
3 \\
4\end{array}$ & 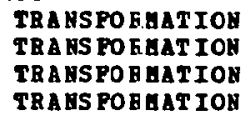 & $\begin{array}{l}\text { LOSS } \\
\text { LOSS } \\
\text { LOSS } \\
\text { LOSS }\end{array}$ & $\begin{array}{l}\text { POR } \\
\text { POR } \\
\text { POR } \\
\text { POR }\end{array}$ & $\begin{array}{l}\text { BLECTRICITY GBN. } \\
\text { PETROLEOE PRODUCTS } \\
\text { NAFORAL GAS } \\
\text { STI-GAS }\end{array}$ & $\begin{array}{l}=68.56 \% \\
=0.0 \% \\
=0.0 \% \\
=0.0 \%\end{array}$ \\
\hline
\end{tabular}


REGIONAL BHBRGI BALANCE STATEHENT

BY SECTOR AND FOBL TYPE

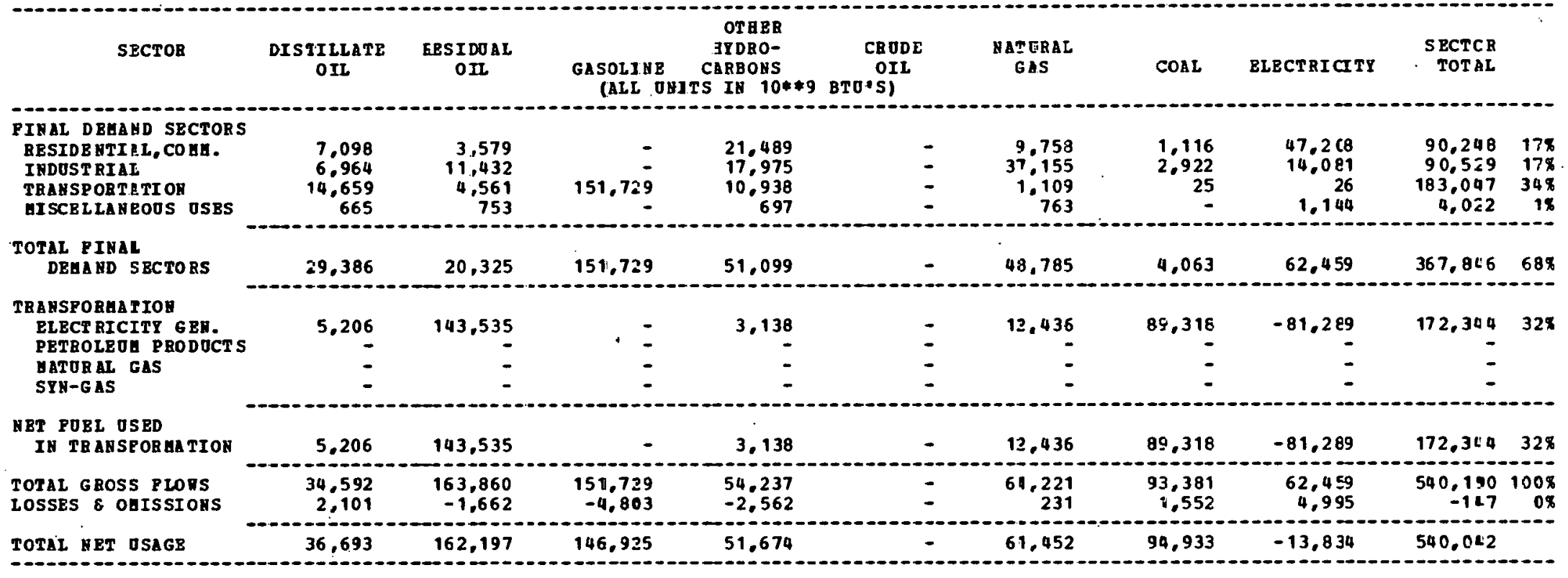

SUPPLY OP EHERGY

BOSSI I PEBL

BYDRO BL BCTRIC

IOCLE AR

GEO.\& SOLAR

TOTAL SOPPIY

$\begin{array}{rr}- & - \\ - & - \\ - & -\end{array}$

-

-

4,130

72

72
$\vdots$
-
72

$4,202100 x$

-.-.-.-

BET IUPORTS

$36,693 \quad 162,197$

146.925

51.674

$-4,130$

61,380

94,933

$-13.834$

535.840

-

MOTES:

1 TBAUS FOBAAT IOH LOSS POR

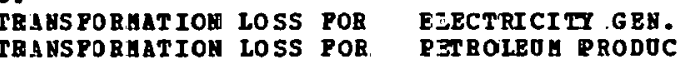

TAAUS PORAAT IOA LOSS POR

TRANSFORAATIOH LOSS POR

G.MPOR.AL GAS

$=67.95 .8$
$=0.08$

STB-GAS

$=0.0 \%$
$=0.0 \times$ 
REGIOHAL BNERGY BALAMCB STATEAENT

IOHAL BNERGY BALANCB STATE
BY SECTOR AND FOEL TYPE

\begin{tabular}{|c|c|c|c|c|c|c|c|c|c|}
\hline SECTOR & $\begin{array}{l}\text { DIS TILLATB } \\
\text { OIL }\end{array}$ & $\begin{array}{l}\text { RESIDOAL } \\
\text { OIL }\end{array}$ & $\begin{array}{l}\text { GASOLINI } \\
\text { IALI }\end{array}$ & $\begin{array}{l}\text { AYDRO- } \\
\text { CARBOHS } \\
\text { TS IH 10**9 }\end{array}$ & $\begin{array}{c}\text { CRODE } \\
\text { OIL } \\
\text { BTU 'S) }\end{array}$ & $\begin{array}{l}\text { NAT ORAL } \\
\text { SAS }\end{array}$ & COAL & ELECTRICITY & $\begin{array}{r}\text { SECTOR } \\
\text { TOTAL }\end{array}$ \\
\hline $\begin{array}{l}\text { FINAL DEHAND SECTORS } \\
\text { RBSIDB BTIAL, COMH. } \\
\text { IHDOSTRIAL } \\
\text { TRAYSPORTATION } \\
\text { UISCELLABEODS ISES }\end{array}$ & $\begin{array}{r}1.244 \\
1.713 \\
2,0.15 \\
117\end{array}$ & $\begin{array}{r}438 \\
5.197 \\
924 \\
350\end{array}$ & 26,583 & $\begin{array}{r}3,766 \\
2,079 \\
679 \\
122\end{array}$ & ! & $\begin{array}{r}1,438 \\
5.506 \\
194 \\
447\end{array}$ & $\begin{array}{r}196 \\
859 \\
4 \\
-\end{array}$ & $\begin{array}{r}7,501 \\
2,034 \\
4 \\
669\end{array}$ & $\begin{array}{r}14.583 \\
18.388 \\
30.403 \\
1.705\end{array}$ \\
\hline $\begin{array}{l}\text { TOTAL PIHAL } \\
\text { DEGAED SECTORS }\end{array}$ & 5,089 & 7.909 & 26,583 & 6.646 & $=$ & 7.585 & 1.059 & 10,208 & 65.079 \\
\hline 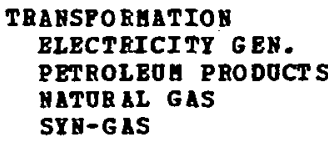 & $\begin{array}{r}727 \\
-\end{array}$ & $\begin{array}{r}3,328 \\
- \\
-\end{array}$ & - & $\begin{array}{r}257 \\
-8,455 \\
- \\
-\end{array}$ & $\begin{array}{r}8.439 \\
-\end{array}$ & $\begin{array}{r}7.407 \\
385 \\
- \\
-\end{array}$ & $\begin{array}{r}25,675 \\
- \\
-\end{array}$ & $\begin{array}{r}-12,036 \\
- \\
-\end{array}$ & $\begin{array}{r}25.358 \\
369 \\
- \\
-\end{array}$ \\
\hline $\begin{array}{l}\text { HET FOEL OSED } \\
\text { IA TRABSFOREATIOH }\end{array}$ & 727 & 3,328 & - & $-8,198$ & 8,439 & 7,792 & 25,675 & $-12,036$ & 25.727 \\
\hline $\begin{array}{l}\text { TOTAL GROSS PLOAS } \\
\text { LOSSES E OHISSIOHS }\end{array}$ & $\begin{array}{r}5.816 \\
353\end{array}$ & $\begin{array}{r}11.237 \\
-114\end{array}$ & $\begin{array}{r}26.583 \\
-841\end{array}$ & $\begin{array}{r}6,903 \\
-326\end{array}$ & $\begin{array}{r}8.439 \\
12\end{array}$ & $\begin{array}{r}15.377 \\
58\end{array}$ & $\begin{array}{r}26.734 \\
444\end{array}$ & $\begin{array}{r}10.208 \\
816\end{array}$ & $\begin{array}{r}90,806 \\
403\end{array}$ \\
\hline
\end{tabular}

SOPPLY OP BHBRG Y

POSSIL POEL

AYDROBL

GBO. SOLA

TOTAL SOPPLY

$\begin{array}{rr}- & - \\ - & - \\ - & -\end{array}$

-

$-$

$-$

$-$

799

$799 \quad 799$

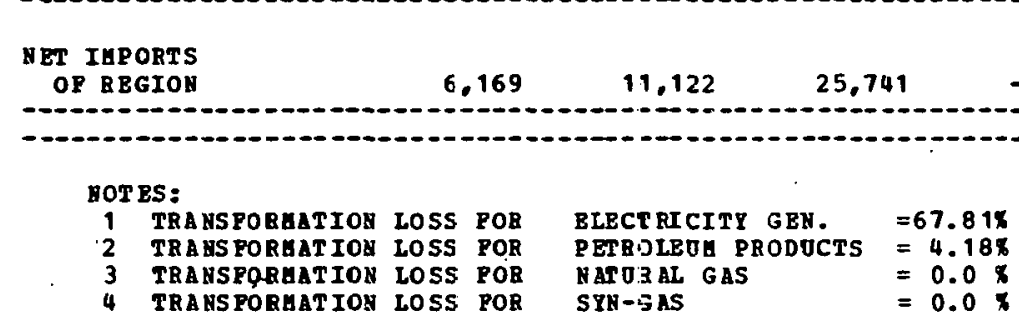




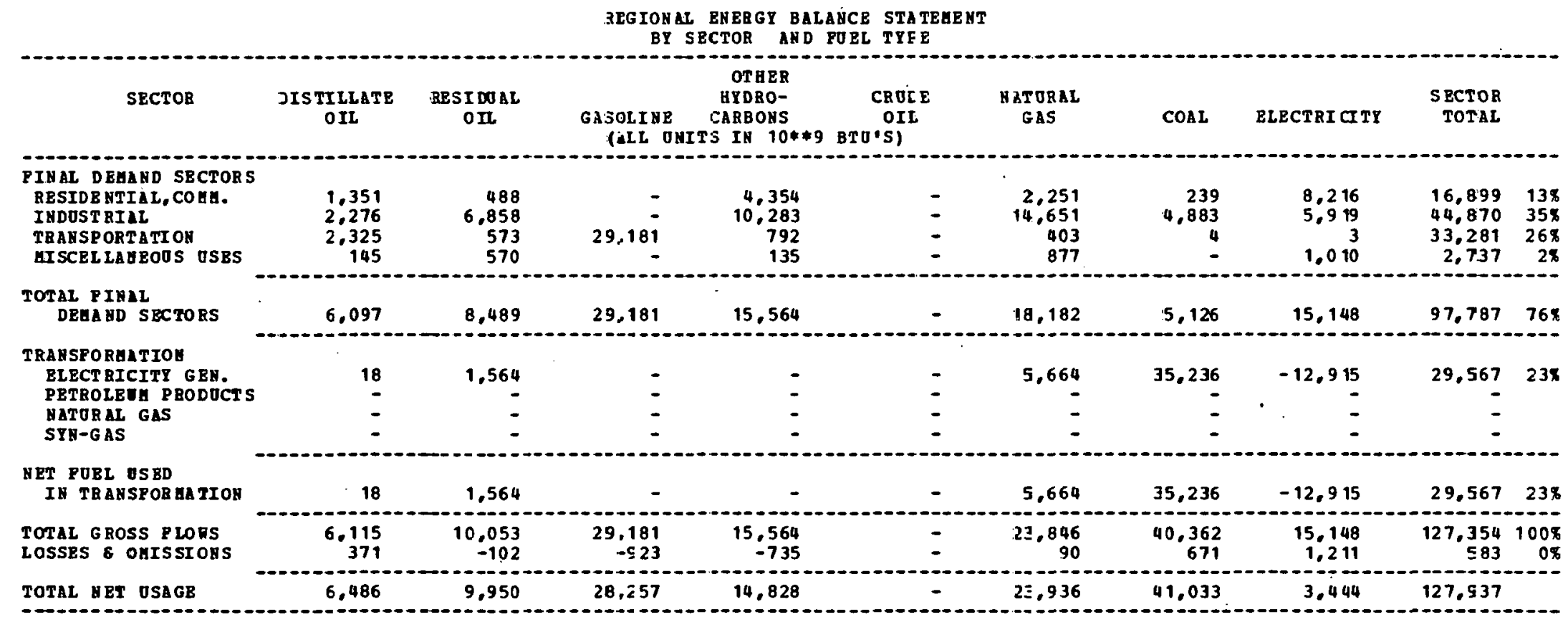

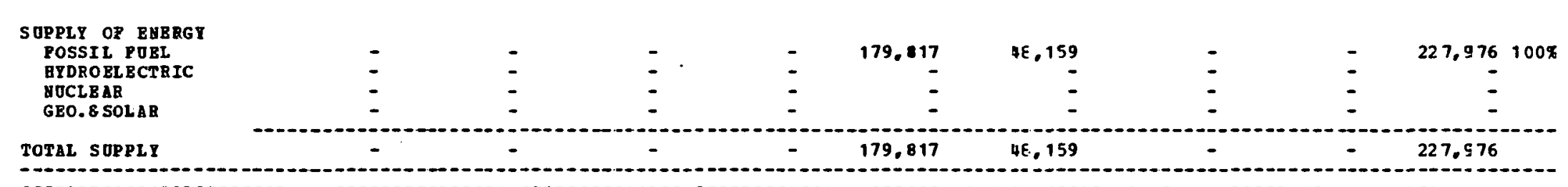

SET IMPORES
OP BBGIOH




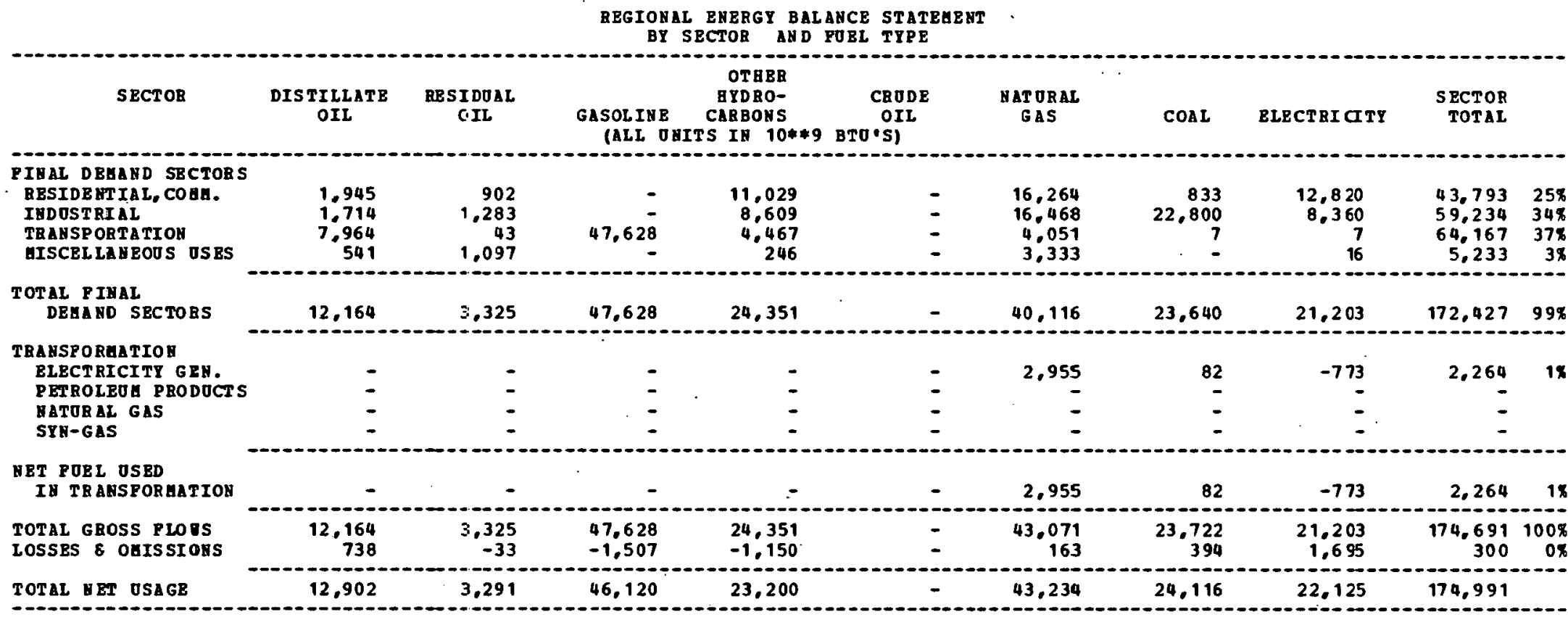

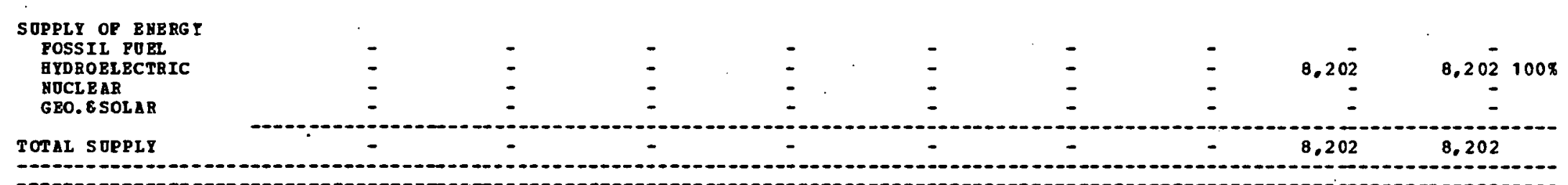

WET IUPORTS
OF REGIOU




\begin{tabular}{|c|c|c|c|c|c|c|c|c|c|c|}
\hline & & & $\begin{array}{r}\text { REGIONAL } \\
\text { BY } 5\end{array}$ & $\begin{array}{l}\text { EHERGY BAL } \\
\text { BCTOB AND }\end{array}$ & $\begin{array}{l}\text { AHCE STATE } \\
\text { FOEL TYPE }\end{array}$ & & & & & \\
\hline SECTOR & $\begin{array}{l}\text { DISTILLATE } \\
\text { OIL }\end{array}$ & $\begin{array}{l}\text { RZSIDOAL } \\
\text { OIL }\end{array}$ & $\begin{array}{l}\text { GASOLINE } \\
\text { (ALL ONI }\end{array}$ & $\begin{array}{c}\text { OTHER } \\
\text { HYDBO- } \\
\text { CARBOHS } \\
\text { TS IH } 10 * 9\end{array}$ & $\begin{array}{c}\text { CRODE } \\
\text { OIL } \\
\text { BTO }{ }^{-S)}\end{array}$ & $\begin{array}{l}\text { NAT URAL } \\
\text { GAS }\end{array}$ & $\operatorname{COAL}$ & ELECTRI CITY & $\begin{array}{l}\text { SECTOR } \\
\text { TOTAL }\end{array}$ & \\
\hline $\begin{array}{l}\text { PINAL DEGAND SECTORS } \\
\text { RESIDENTIAL, COGA. } \\
\text { INDOSTRIAL } \\
\text { TRANSPORTATION } \\
\text { UISCELLANEOUS ESES }\end{array}$ & $\begin{array}{r}1,846 \\
2,540 \\
3,105 \\
106\end{array}$ & $\begin{array}{r}982 \\
4.533 \\
125 \\
502\end{array}$ & $\begin{array}{r}- \\
37,636 \\
-\end{array}$ & $\begin{array}{l}5.687 \\
6.158 \\
1.023 \\
557\end{array}$ & $\begin{array}{l}- \\
-\end{array}$ & $\begin{array}{r}12.217 \\
16.918 \\
658 \\
1.536\end{array}$ & $\begin{array}{r}457 \\
1,808 \\
5 \\
-\end{array}$ & $\begin{array}{r}8.622 \\
4.047 \\
4 \\
35\end{array}$ & $\begin{array}{r}29,811 \\
36,004 \\
42,556 \\
2,736\end{array}$ & $\begin{array}{r}18 \% \\
22 \% \\
26 \% \\
2 \%\end{array}$ \\
\hline $\begin{array}{l}\text { TOTAL PIHAL } \\
\text { DELA SECTOES }\end{array}$ & 7,597 & 6.142 & 37,636 & 13,425 & - & 31,329 & 2.270 & 12,708 & 111.107 & $68 \%$ \\
\hline $\begin{array}{l}\text { TRANSPORHATION } \\
\text { RLECTRICITY GEN. } \\
\text { PETROLEOA PRODOCTS } \\
\text { NATORAL GAS } \\
\text { SYN-GAS }\end{array}$ & $\begin{array}{r}139 \\
-\end{array}$ & $\begin{array}{l}82 \\
- \\
-\end{array}$ & $\overline{-}$ & $\begin{array}{l}\overline{-} \\
\overline{-}\end{array}$ & $\overline{-}$ & $\begin{array}{r}3.866 \\
- \\
-\end{array}$ & $\begin{array}{r}72,760 \\
= \\
=\end{array}$ & $\begin{array}{r}-25,346 \\
= \\
-\end{array}$ & $\begin{array}{r}51.501 \\
= \\
=\end{array}$ & $31 x$ \\
\hline $\begin{array}{l}\text { NET FORL OSBD } \\
\text { IH TRANSPORGATION }\end{array}$ & 139 & 82 & - & - & - & 3,866 & 72,760 & $-25,346$ & 51,501 & $31 \%$ \\
\hline TOTAL NET OSAGE & 8,205 & 6.160 & 36,444 & 12.790 & - & 35,328 & 76.277 & -11.621 & 163.586 & \\
\hline
\end{tabular}

(1)

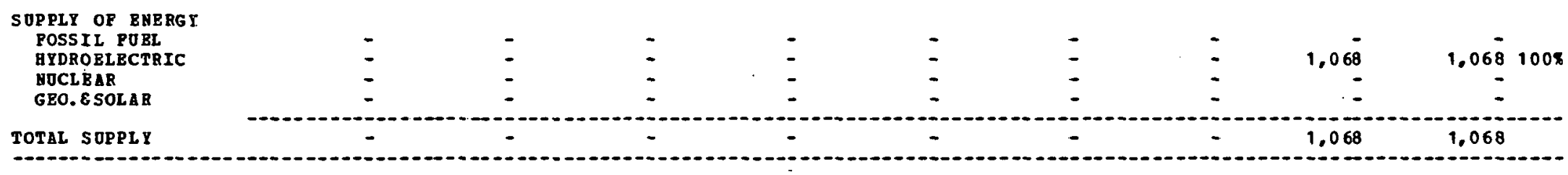

\section{NET IAPORTS}

OP REGIOB

B,205

6.160

36.444

12.790

35,328

76.277

\section{NOT ES:}

TRAYSPORHATION LOSS PO TRA SS POREAT ION LOSS PO

TRANSPORGATION LOSS FOR

TRANS PORAAT ION LOSS POR

BLECTRICITY GEN.

PETROLEUA PRODOCTS $=67.02$

NATUEAL GAS

STN-G AS $\begin{aligned} & =67.028 \\ & =0.08\end{aligned}$

$=0.08$ 
REGIONA: ENERGT BALANCE STATEGEAT

BY SBCTOR AND FOEL TYPE

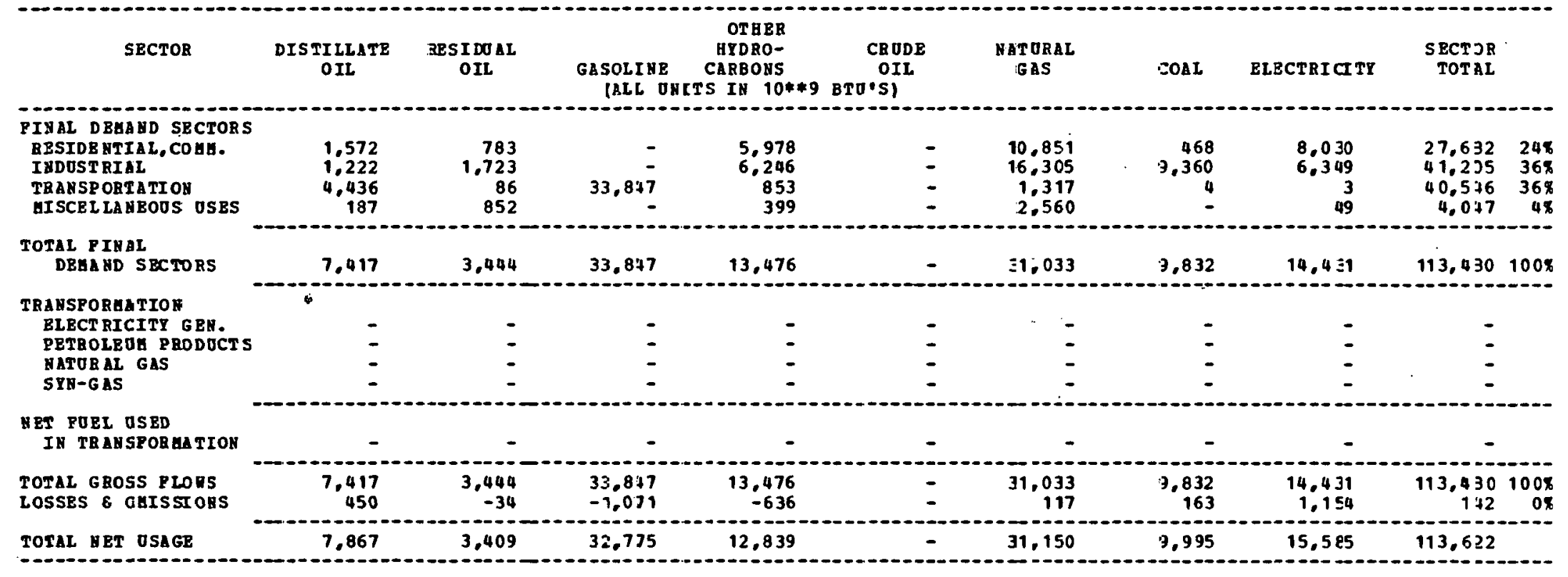

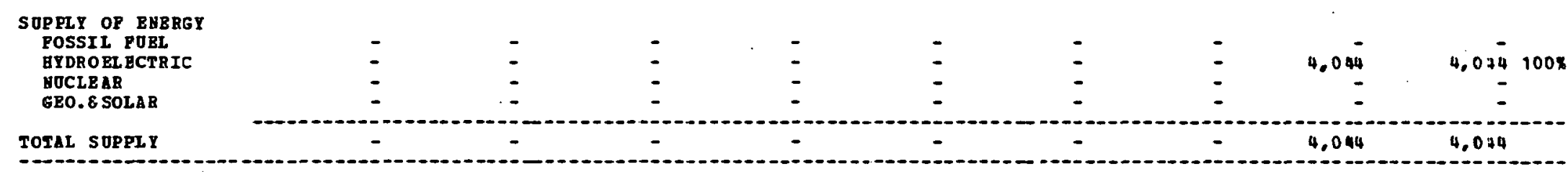

The

\section{VET IUPORTS}

OP BRGION

$7,867 \quad 3,409$

32,775

12,839

$3 \pi, 150$

9,995

11,541

109,578

BOTES:

TRARS FORHAT IOH LOSS FOR

TRARSPB REATIOY LOSS POB

TRA HSFORATION LOSS FOR BATUR

ELECTRICITT GBH.

$=0.0$ 
REGIOHAL EHERGI BALAHCE STATEHENT

BY SECTOR AND FOEL TYPE

\begin{tabular}{|c|c|c|c|c|c|c|c|c|c|c|}
\hline SECTOR & $\begin{array}{l}\text { DISTILLATE } \\
\text { OIL }\end{array}$ & $\begin{array}{l}\text { RESI LOAL } \\
\text { OIL }\end{array}$ & $\begin{array}{l}\text { GASOLINE } \\
\text { (ALL ONI }\end{array}$ & $\begin{array}{l}\text { BYDBO- } \\
\text { CARBOKS } \\
\text { TS IH 10**9 }\end{array}$ & $\begin{array}{c}\text { CRODE } \\
\text { OIL } \\
\text { BTO'S) }\end{array}$ & $\begin{array}{l}\text { MATURAL } \\
\text { GAS }\end{array}$ & COAL & ELECTRI CITY & $\begin{array}{l}\text { SECTOR } \\
\text { TOTAL }\end{array}$ & \\
\hline $\begin{array}{l}\text { PIHAL DEAAAD SBCTORS } \\
\text { RESIDE HTIAL, COAG. } \\
\text { IFDOSTRIAL } \\
\text { TRAYSPORTATION } \\
\text { GISCELLABEOOS OSES }\end{array}$ & $\begin{array}{r}8,962 \\
6,496 \\
46.341 \\
522\end{array}$ & $\begin{array}{r}7.733 \\
16.517 \\
1.332 \\
1.206\end{array}$ & 182.801 & $\begin{array}{r}27,684 \\
33,755 \\
68,448 \\
2,701\end{array}$ & : & $\begin{array}{r}69.020 \\
79.353 \\
3.246 \\
3.419\end{array}$ & $\begin{array}{r}2,224 \\
11,928 \\
52 \\
-\end{array}$ & $\begin{array}{r}49,988 \\
22,1 \in 0 \\
49 \\
77\end{array}$ & $\begin{array}{r}165,611 \\
170,229 \\
302,269 \\
7,925\end{array}$ & $\begin{array}{r}218 \\
22 \% \\
39 \% \\
1 \%\end{array}$ \\
\hline $\begin{array}{l}\text { TOTAL PIHAL } \\
\text { DEGA ND SBC TORS }\end{array}$ & 62.321 & 26,788 & 182,801 & 132,588 & - & 155.038 & 14,204 & 72,294 & 646,034 & $83 x$ \\
\hline $\begin{array}{l}\text { TZA NSPORAATIOH } \\
\text { BLECTRICITY GBH. } \\
\text { PETROLEOA PRODOCTS } \\
\text { EATURAL GAS } \\
\text { SYH-GAS }\end{array}$ & $\begin{array}{r}757 \\
- \\
-\end{array}$ & $\begin{array}{r}2.681 \\
= \\
-\end{array}$ & : & $\begin{array}{r}-4.225 \\
-\end{array}$ & $\begin{array}{r}4.217 \\
-\end{array}$ & $\begin{array}{r}24.498 \\
192 \\
- \\
=\end{array}$ & $\begin{array}{r}167.485 \\
= \\
=\end{array}$ & $\begin{array}{r}-63,517 \\
- \\
-\end{array}$ & $\begin{array}{r}131.904 \\
184 \\
- \\
-\end{array}$ & $\begin{array}{r}17 \% \\
0 \%\end{array}$ \\
\hline 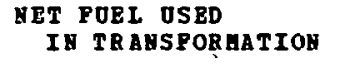 & 757 & 2,681 & - & $-4,225$ & 4.217 & 24,690 & 167,485 & $-63,517$ & 132,088 & $17 \%$ \\
\hline $\begin{array}{l}\text { TOTAL GROSS FLORS } \\
\text { LGSSES \& OAISSIOHS }\end{array}$ & $\begin{array}{r}63.078 \\
3.831\end{array}$ & $\begin{array}{r}29.469 \\
-299\end{array}$ & $\begin{array}{r}182,801 \\
-5,787\end{array}$ & $\begin{array}{r}132,588 \\
-6,264\end{array}$ & $\begin{array}{r}4.217 \\
6\end{array}$ & $\begin{array}{r}179,728 \\
680\end{array}$ & $\begin{array}{r}181,689 \\
3,020\end{array}$ & $\begin{array}{r}72,294 \\
5,781\end{array}$ & 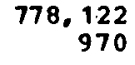 & $\begin{array}{r}100 \% \\
0 \%\end{array}$ \\
\hline
\end{tabular}

122,098

\section{SUPPLY OP ENERG \\ POSSIL PUEL \\ GYDRORLBCTRIC \\ NOCL E AR \\ GEO.ESOLAB}

TOTAL SUPPLY

i

$\overline{-}$

$\begin{array}{ll}- & - \\ - & - \\ - & - \\ - & -\end{array}$

$\begin{array}{ll}- & - \\ - & - \\ - & -\end{array}$

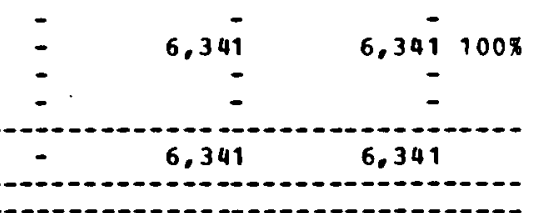

HET IHPORTS

OP.REGIOH

66,909

29.169

177.013

122.098

4.223

180.408

184,709

$8.217 \quad 772.751$

\begin{tabular}{|c|c|c|c|c|c|}
\hline $\begin{array}{l}2 \\
3 \\
3 \\
4\end{array}$ & $\begin{array}{l}\text { TRABSPORGATION } \\
\text { TRA USPORGATION } \\
\text { TRAUSPORAATION } \\
\text { TRAGSPOBEATION }\end{array}$ & $\begin{array}{l}\text { LoSS } \\
\text { LoSS } \\
\text { LosS } \\
\text { LOSS }\end{array}$ & $\begin{array}{l}\text { POR } \\
\text { POR } \\
\text { POR } \\
\text { POB }\end{array}$ & $\begin{array}{l}\text { ELECTRICITY GEN. } \\
\text { PETROLEO P PRODOCTS } \\
\text { NATURAL GAS } \\
\text { SYN-G SS }\end{array}$ & $\begin{array}{l}=67.50 x \\
=4.178 \\
=0.0 x \\
=0.0 x\end{array}$ \\
\hline
\end{tabular}


REGIOHAL 3HESGY BALANCE STATEHENT

BE SEZTOZ AND POEL TYPE

\begin{tabular}{|c|c|c|c|c|c|c|c|c|c|}
\hline SECTOR & $\begin{array}{l}\text { DISTILLATE } \\
\text { OIL }\end{array}$ & $\begin{array}{l}\text { EESIDOAL } \\
\text { O II }\end{array}$ & $\begin{array}{l}\text { GASOL INZ } \\
\text { (ALL JNIT }\end{array}$ & $\begin{array}{l}\text { ATRO- } \\
\text { CAREOHS } \\
\text { IS IN } 10 * * 9\end{array}$ & $\begin{array}{c}\text { CRODE } \\
\text { OIL } \\
\text { BTO'S) }\end{array}$ & $\begin{array}{l}\text { MAT ORAL } \\
\text { GAS }\end{array}$ & COAL & ELECTRICITY & $\begin{array}{l}\text { SECTOR } \\
\text { TOTAL }\end{array}$ \\
\hline $\begin{array}{l}\text { PIHAL DEGAND SECTORS } \\
\text { RESIDERTIAL, COUA. } \\
\text { IHDOSTRIAL } \\
\text { TRABSPORTATIOH } \\
\text { GISCELIABEOOS. OSES }\end{array}$ & $\begin{array}{r}5.273 \\
0.7 \in 2 \\
31.111 \\
1.46 .6\end{array}$ & $\begin{array}{r}2.112 \\
5.913 \\
925 \\
1.545\end{array}$ & 119,415 & $\begin{array}{r}30,126 \\
26,385 \\
6,406 \\
745\end{array}$ & $\overline{-}$ & $\begin{array}{r}41.523 \\
82.953 \\
16.643 \\
3.466\end{array}$ & $\begin{array}{r}1.87 \Xi \\
95,53 E \\
2 \Xi \\
-\end{array}$ & $\begin{array}{r}32.490 \\
37,441 \\
23 \\
99\end{array}$ & $\begin{array}{r}113,397 \\
256,998 \\
174,546 \\
7,261\end{array}$ \\
\hline $\begin{array}{l}\text { TOTAL PINAL } \\
\text { DEGA ND SECTORS }\end{array}$ & 46,552 & 10,495 & 119,415 & 53,662 & - & 144,591 & 97.434 & 70,053 & 552,202 \\
\hline $\begin{array}{l}\text { TRANSPOBAATIOE } \\
\text { BLBCTRICITY GBH. } \\
\text { PETROLEOA PBODUCTS } \\
\text { HATORAL GAS } \\
\text { SYH-GAS }\end{array}$ & $\begin{array}{r}1.220 \\
-1.676 \\
-\end{array}$ & $-32 \overline{-}$ & $\begin{array}{r}-3,701 \\
- \\
-\end{array}$ & $\begin{array}{r}-34,771 \\
-\end{array}$ & $\begin{array}{r}39.79 \overline{-} \\
-\end{array}$ & $\begin{array}{r}854 \\
1.814 \\
- \\
-\end{array}$ & $\begin{array}{r}182,873 \\
- \\
-\end{array}$ & $\begin{array}{r}-62,251 \\
= \\
-\end{array}$ & $\begin{array}{r}122,696 \\
1,142 \\
-\end{array}$ \\
\hline $\begin{array}{l}\text { GET POEL OSED } \\
\text { IH TRANSPORAATION }\end{array}$ & -456 & -322 & $-3,701$ & $-34,771$ & 39.798 & $2,66 \varepsilon$ & 182,873 & $-62,251$ & 123,838 \\
\hline $\begin{array}{l}\text { TOTAL GROSS PLORS } \\
\text { LOSSES } 6 \text { OAISSIOAS }\end{array}$ & $\begin{array}{r}77.772 \\
2.502\end{array}$ & $\begin{array}{r}1 C .495 \\
-106\end{array}$ & $\begin{array}{r}119.415 \\
-3.780\end{array}$ & $\begin{array}{l}63.662 \\
-3.007\end{array}$ & $\begin{array}{r}39,798 \\
60\end{array}$ & $\begin{array}{r}147.259 \\
557\end{array}$ & $\begin{array}{r}280,367 \\
4.660\end{array}$ & $\begin{array}{r}70.053 \\
5.602\end{array}$ & $\begin{array}{r}676.040 \\
6.888\end{array}$ \\
\hline
\end{tabular}

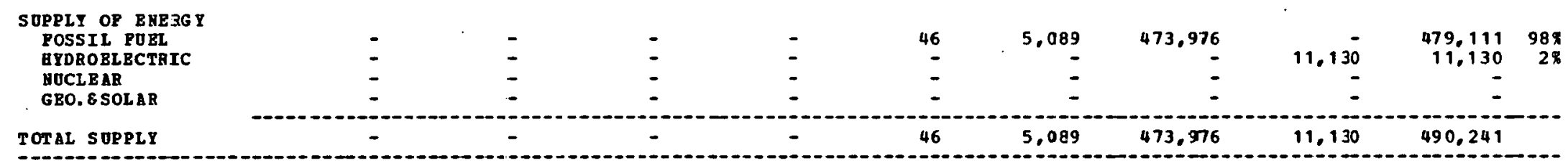

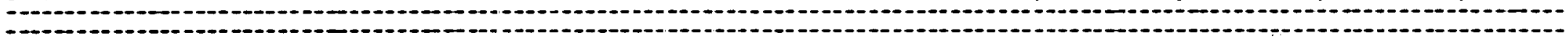

MBT IHPORTS

OP RBGIOH

$\begin{array}{ll}0 & 0\end{array}$

HOT ES:

\begin{tabular}{|c|c|c|c|c|c|}
\hline 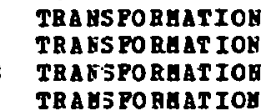 & $\begin{array}{l}\text { Loss } \\
\text { Loss } \\
\text { Loss } \\
\text { Los }\end{array}$ & $\begin{array}{l}\text { POB } \\
\text { FOR } \\
\text { POR } \\
\text { POR }\end{array}$ & $\begin{array}{l}\text { BLECT RIEIT } \\
\text { PETROLBOH } \\
\text { BATERAL GA } \\
\text { STH-GAS }\end{array}$ & $\begin{array}{l}\text { I GEN. } \\
\text { PRODOCTS } \\
\text { IS }\end{array}$ & $\begin{array}{l}=56.348 \\
=2.748 \\
=1.08 \\
=0.08\end{array}$ \\
\hline
\end{tabular}


REGIONAL EHERGY BALANCE STATEMENT BY SECTOR AHD FUEL TYPE

\begin{tabular}{|c|c|c|c|c|c|c|c|c|c|}
\hline SBCTOR & $\begin{array}{l}\text { DISTILLATE } \\
\text { OII }\end{array}$ & $\begin{array}{l}\text { RESI DOAL } \\
\text { OIL }\end{array}$ & $\begin{array}{l}\text { GASOLI HE } \\
\text { (ALL ONI? }\end{array}$ & $\begin{array}{l}\text { AYDBO- } \\
\text { CARBONS } \\
\text { TS IH 10**9 }\end{array}$ & $\begin{array}{l}\text { CRODE } \\
\text { OIL } \\
\text { BTO'SI }\end{array}$ & $\begin{array}{l}\text { MATORAL } \\
\text { GAS }\end{array}$ & COAL & ELECT BI CITY & $\begin{array}{r}\text { SECTOR } \\
\text { TOTAL }\end{array}$ \\
\hline 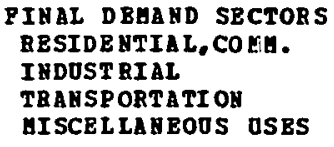 & $\begin{array}{r}5.031 \\
7.443 \\
29.977 \\
884\end{array}$ & $\begin{array}{r}723 \\
2,122 \\
721 \\
332\end{array}$ & 122.851 & $\begin{array}{r}32.783 \\
20.915 \\
12.531 \\
899\end{array}$ & $\begin{array}{l}\overline{-} \\
-\end{array}$ & $\begin{array}{r}44.038 \\
63.033 \\
16.902 \\
3.987\end{array}$ & $\begin{array}{r}3,536 \\
16.783 \\
20 \\
-\end{array}$ & $\begin{array}{r}34.320 \\
30,961 \\
17 \\
202\end{array}$ & $\begin{array}{r}120,431 \\
141,257 \\
183,0.19 \\
6,304\end{array}$ \\
\hline $\begin{array}{l}\text { TOTAL P IHAL } \\
\text { DEAA ND SBCTORS }\end{array}$ & 43.335 & 3.898 & 122,851 & 67,128 & - & 127,960 & 20,339 & 65.500 & 451,011 \\
\hline $\begin{array}{l}\text { TRANSPORHATION } \\
\text { ELECTRICITY GEH. } \\
\text { PETROLEOH PRODDCT S } \\
\text { NATORAL GAS } \\
\text { SYN-GAS }\end{array}$ & $\begin{array}{r}1,777 \\
-10,916 \\
-\end{array}$ & $\begin{array}{r}26.029 \\
-3.483 \\
-\end{array}$ & $\begin{array}{r}-25,001 \\
-\end{array}$ & $\begin{array}{r}-22.610 \\
-\end{array}$ & $\begin{array}{r}60,065 \\
-\end{array}$ & $\begin{array}{r}28,769 \\
2.738 \\
-\end{array}$ & $\begin{array}{r}36,198 \\
= \\
=\end{array}$ & $\begin{array}{r}-30,105 \\
- \\
-\end{array}$ & $\begin{array}{r}62.668 \\
793 \\
-\end{array}$ \\
\hline $\begin{array}{l}\text { DET FOEL OSBD } \\
\text { IN TRABSFORGATION }\end{array}$ & $-9,139$ & 22.546 & $-25,001$ & $-22,610$ & 60,065 & 31,507 & 36,198 & $-30,105$ & 63,461 \\
\hline $\begin{array}{l}\text { TOTAL GROSS FLORS } \\
\text { I.OSSES } 8 \text { OUISSI OES }\end{array}$ & $\begin{array}{r}45.112 \\
2.740\end{array}$ & $\begin{array}{r}29.927 \\
-303\end{array}$ & $\begin{array}{r}122,851 \\
-3,889\end{array}$ & $\begin{array}{l}67,128 \\
-3,171\end{array}$ & $\begin{array}{r}60.065 \\
91\end{array}$ & $\begin{array}{r}159.467 \\
603\end{array}$ & $\begin{array}{r}56,537 \\
940\end{array}$ & $\begin{array}{r}65,500 \\
5,238\end{array}$ & $\begin{array}{r}514.472 \\
2.249\end{array}$ \\
\hline
\end{tabular}

\section{SOPPLY OF ENERGY}

POSSIL POEL

GYDROEL BCTRIC

YOCLB AR

GEO.E SOLAR

-
$\vdots$
-

TOTAL SUPPII

GET IUPORTS

OP BEGION

36,936

26,140

93,960

41,346

60,156

160,070

57,477

40,633

516,721

\begin{tabular}{|c|c|c|c|c|c|}
\hline $\begin{array}{l}2 \\
3\end{array}$ & $\begin{array}{l}\text { TRANS PORAATION } \\
\text { TRA HSPORUATION } \\
\text { TRANS PORAATION } \\
\text { TRANSPORATION }\end{array}$ & $\begin{array}{l}\text { LOSS } \\
\text { LOSS } \\
\text { loSS } \\
\text { LOSS }\end{array}$ & $\begin{array}{l}\text { FOR } \\
\text { POR } \\
\text { POR } \\
\text { POR }\end{array}$ & $\begin{array}{l}\text { ELBCTRICITY GEH. } \\
\text { PETROLEOE PRODOCTS } \\
\text { HATORAL GAS } \\
\text { SIB-GAS }\end{array}$ & $\begin{array}{l}=67.55 \% \\
=1.26 \% \\
=0.0 \\
=0.0\end{array}$ \\
\hline
\end{tabular}


REGICNAL EH3RGY BALALCE STLTBUENT

BY SECTOR AND POEL TYPE

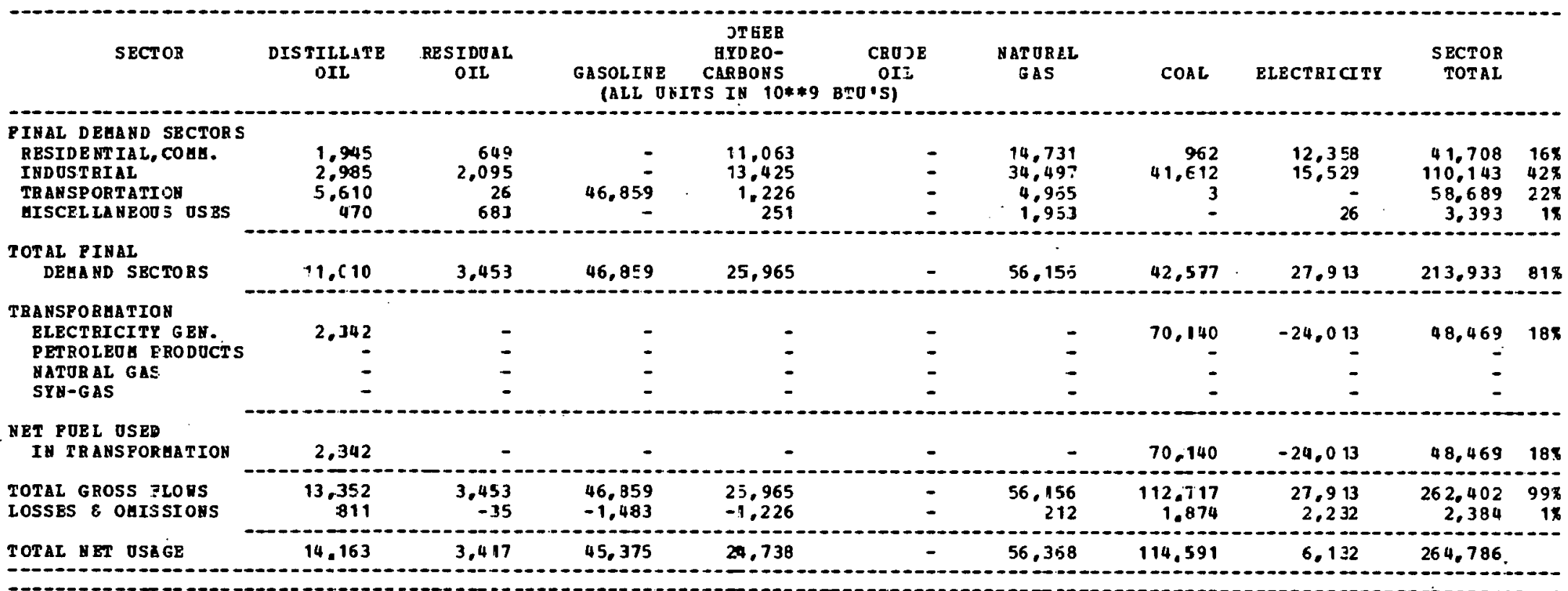

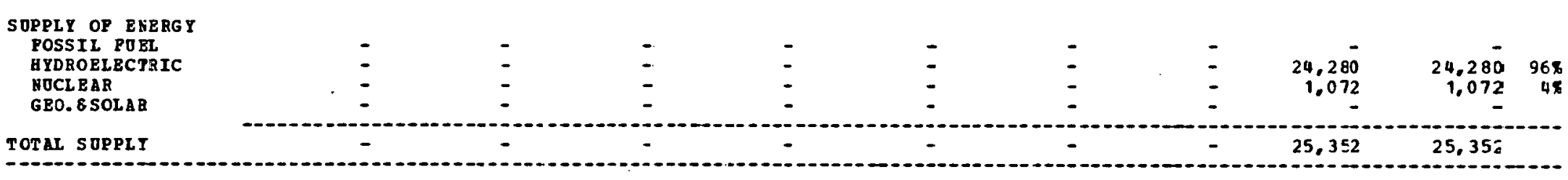

\section{NET IHPORTS}

14,163

3,417

45,375

24.738

56,368

$11 c, 591$

$-19.219$

239,434

$\begin{array}{lll}\text { OP REGION } & 14,163 & 3,417\end{array}$


REGIONAL BHERGI BALAACE STATELENT

BY SBCTOR ABD POEL TYPE

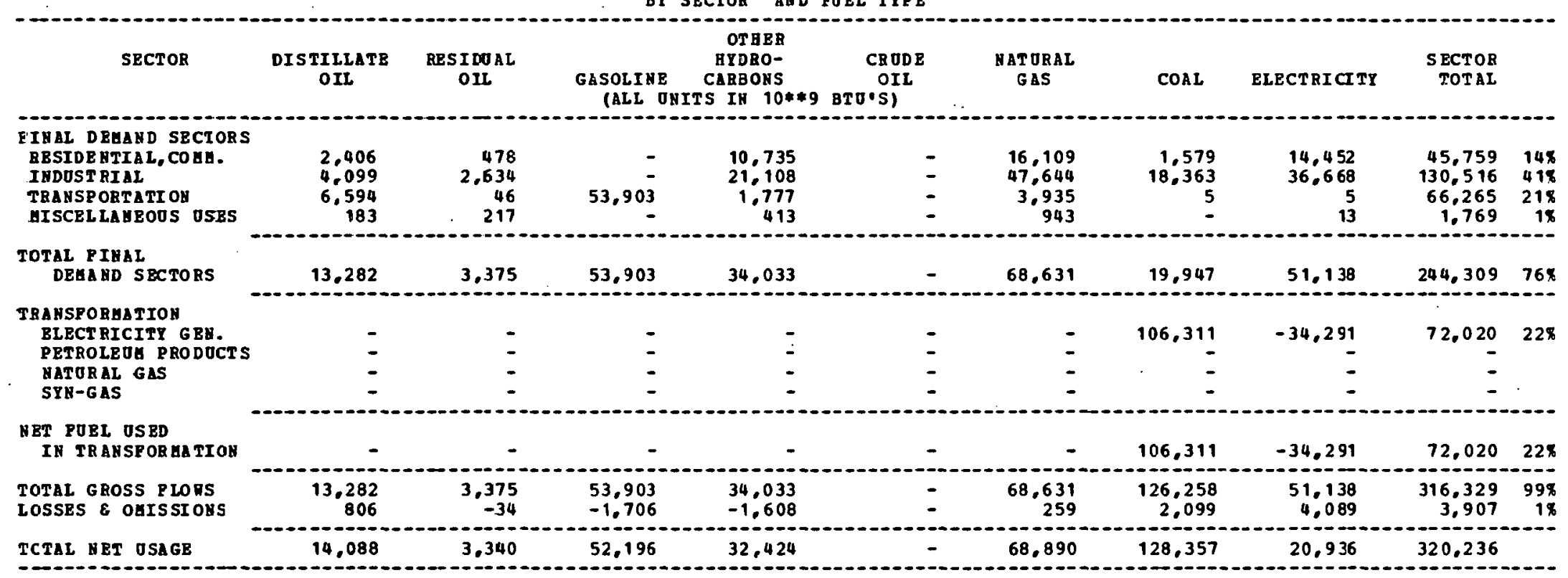

\begin{tabular}{|c|c|c|c|c|c|c|c|c|c|c|}
\hline $\begin{array}{l}\text { S OPPLY OF ENBRGY } \\
\text { POSSIL POEL }\end{array}$ & - & - & - & - & - & - & 44.137 & - & 44,137 & $76 \%$ \\
\hline HYDRO BI ECTRIC & - & - & - & - & - & - & - & 13.795 & 13,795 & 247 \\
\hline NOCLBAR & - & - & - & - & - & - & - & - & 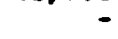 & \\
\hline GEO. E SOLAR & - & - & - & - & - & - & - & - & - & \\
\hline TOTAL SUPPLY & - & - & - & - & - & - & 40,137 & 13,795 & 57.932 & \\
\hline
\end{tabular}

MBT IHPORTS
OF BEGION




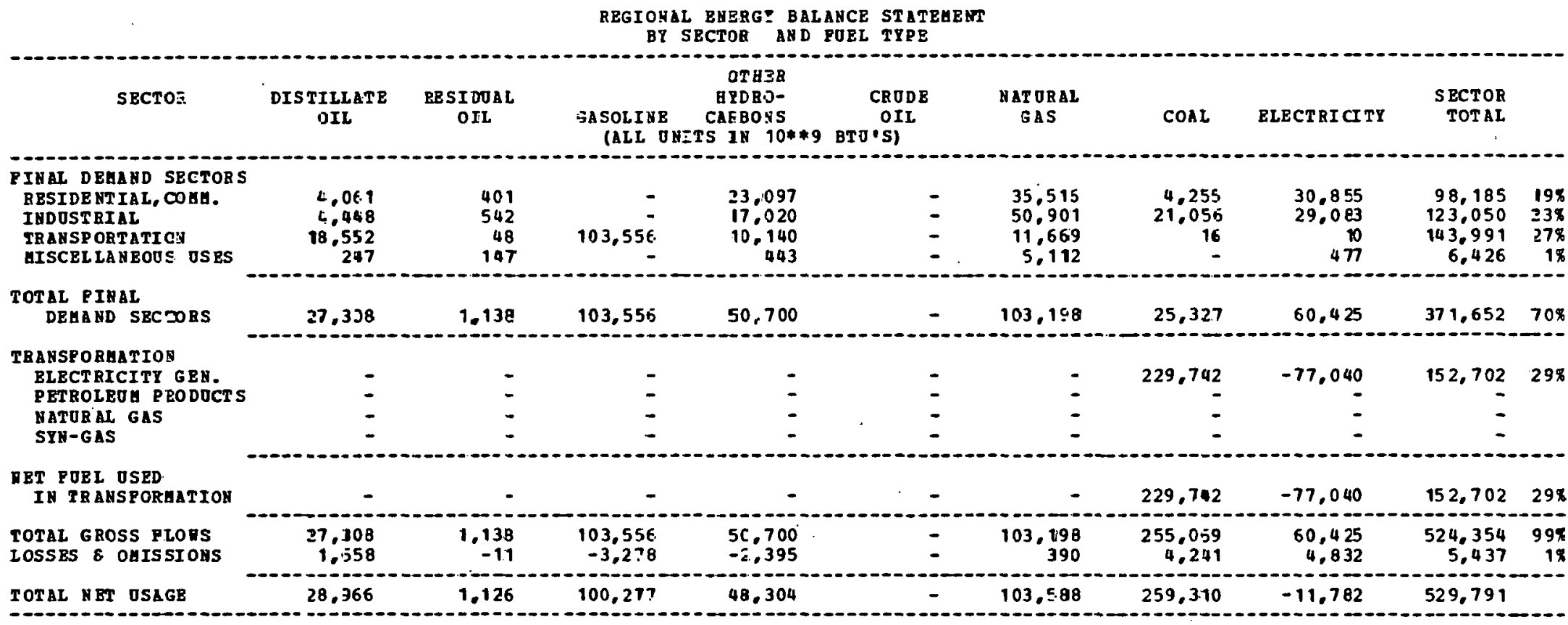

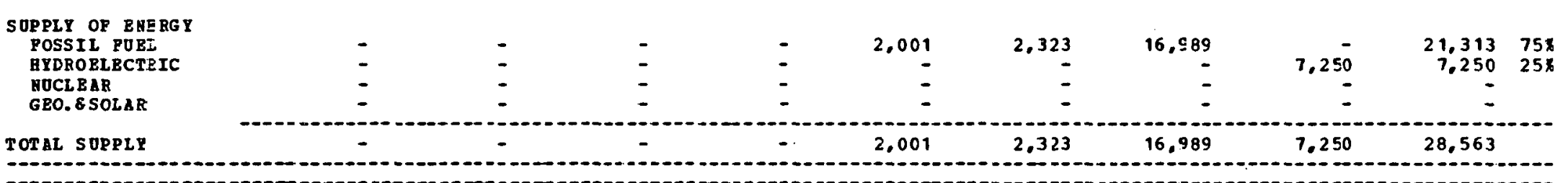

\begin{tabular}{l}
$\begin{array}{l}\text { NBT IHPORTS } \\
\text { OP REGIOH }\end{array}$ \\
\hdashline$\quad 28,966$
\end{tabular}


REGIONAL ENERGI BALANCE STATEUENT

IONAL ENERGE BALANCE STATE
BY SECTOR AHD FORL TYPE

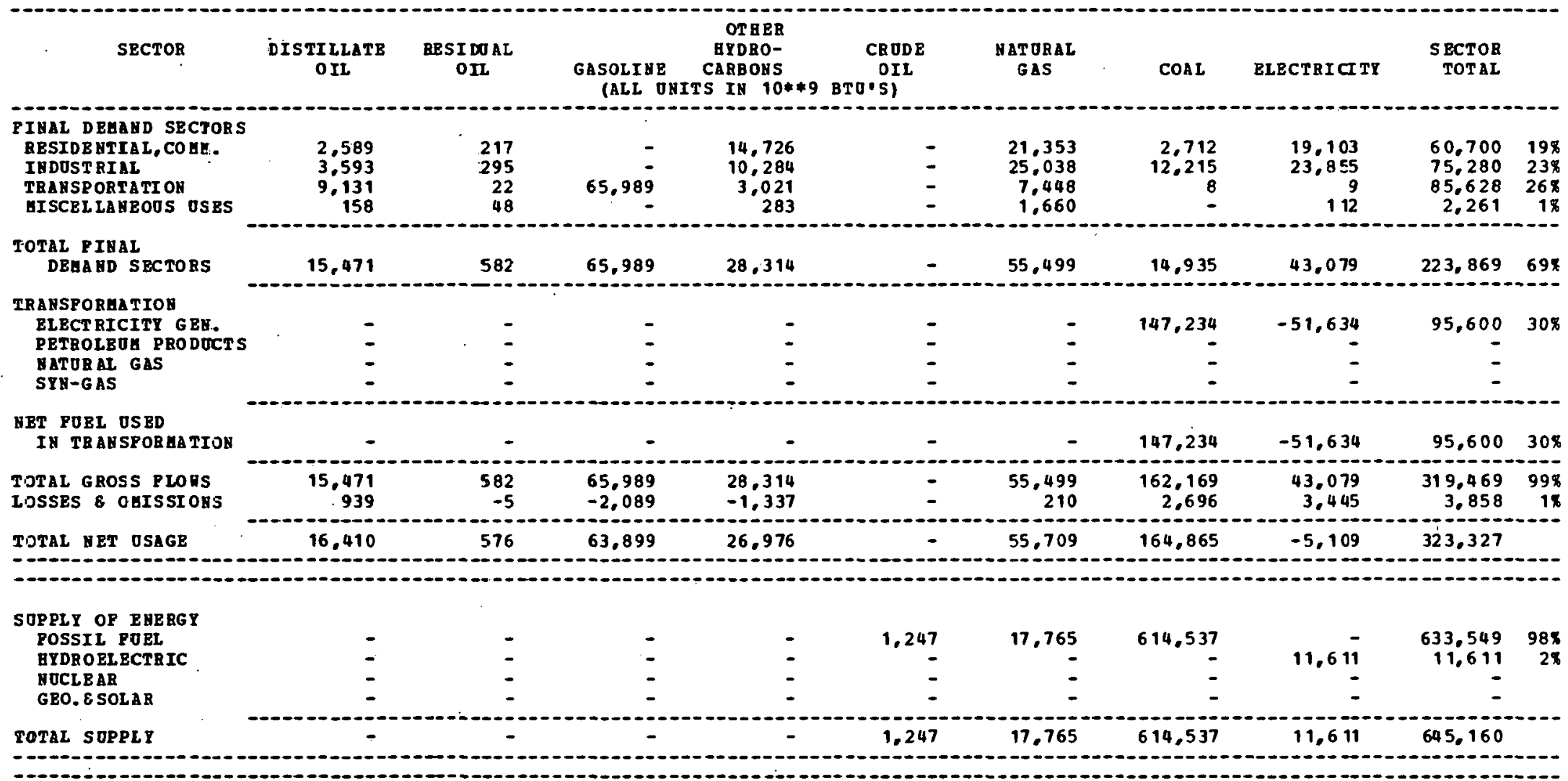

\section{BET IUPORTS}

OP RBGION

16.410

576

63,899

26,976

$-1,247$

37,944

$-449,671$

\section{MOT ES :}

\begin{tabular}{|c|c|c|c|}
\hline $\begin{array}{l}\text { TRABS PQRAAT IOU } \\
\text { TRAASFOREATYON } \\
\text { TRAHS PORAAT ION }\end{array}$ & $\begin{array}{l}\text { Loss } \\
\text { Loss }\end{array}$ & $\begin{array}{l}\text { FOR } \\
\text { POR } \\
\text { FOR }\end{array}$ & $\begin{array}{l}\text { PETROLEJA PRODOCTS } \\
\text { MATORAL GAS } \\
\text { SYN-GAS }\end{array}$ \\
\hline
\end{tabular}


REGIONAL BUERST BALAYCE STATELEUT BI SECEOR AND POEL TYPE

\begin{tabular}{|c|c|c|c|c|c|c|c|c|c|c|}
\hline SECTOR & $\begin{array}{l}\text { DISTILLATE } \\
\text { OIl }\end{array}$ & $\begin{array}{l}\text { RESIDOAL } \\
\text { OIL }\end{array}$ & $\begin{array}{l}\text { GASOLINE } \\
\text { (ALL O }\end{array}$ & $\begin{array}{l}\text { OTHER } \\
\text { HYDBO- } \\
\text { CIRBONS } \\
\text { TS IN 10**9 }\end{array}$ & $\begin{array}{c}\text { CRODE } \\
\text { OIL } \\
\text { BTO'SI }\end{array}$ & $\begin{array}{l}\text { NATORAE } \\
\text { GAS }\end{array}$ & COA $=$ & ELECT:I CITY & $\begin{array}{r}\text { SECTOR } \\
\text { TOTAL }\end{array}$ & \\
\hline $\begin{array}{l}\text { INAL DBHAND SECTORS } \\
\text { RESIDE RTIAL, COUA. } \\
\text { IHDUST RIAL } \\
\text { TRANSPORTATION } \\
\text { GISCELLABOES OSES }\end{array}$ & $\begin{array}{r}5,878 \\
5,702 \\
7,250 \\
181\end{array}$ & $\begin{array}{r}523 \\
2.176 \\
33 \\
298\end{array}$ & $\begin{array}{r}- \\
52,579 \\
-\end{array}$ & $\begin{array}{r}9.157 \\
27 ., 330 \\
1,253 \\
249\end{array}$ & $\overline{-}$ & $\begin{array}{r}16.444 \\
26.149 \\
4.205 \\
8.96\end{array}$ & $\begin{array}{r}2.451 \\
30.410 \\
7 \\
-\end{array}$ & $\begin{array}{r}13.589 \\
29.617 \\
1 \\
344\end{array}$ & $\begin{array}{r}48,042 \\
121.384 \\
65,328 \\
1.968\end{array}$ & $\begin{array}{r}168 \\
408 \\
228 \\
18\end{array}$ \\
\hline $\begin{array}{l}\text { TOTAL F INAL } \\
\text { DELAND SBCTORS }\end{array}$ & 19.011 & $3,0 \equiv 0$ & 52.579 & 37,989 & - & $47,6 \subseteq 4$ & 32,868 & 43,551 & 236,722 & $79 x$ \\
\hline $\begin{array}{l}\text { TRANSPORHATION } \\
\text { ELECTRICITY GEY. } \\
\text { PETROLEOH PRODOCTS } \\
\text { NATURAL GAS } \\
\text { SYN-GAS }\end{array}$ & $\overline{-}$ & $\begin{array}{r}-3 \\
- \\
-\end{array}$ & $\overline{-}$ & $\overline{-}$ & ! & $\begin{array}{l}- \\
\overline{-} \\
-\end{array}$ & $\begin{array}{r}92,914 \\
= \\
-\end{array}$ & $\begin{array}{r}-32.832 \\
= \\
-\end{array}$ & $\begin{array}{r}60,105 \\
= \\
-\end{array}$ & 20.8 \\
\hline $\begin{array}{l}\text { NET POEL OSEJ } \\
\text { IN TR ANSFO3GA TIOU }\end{array}$ & - & 23 & - & - & - & - & 92,914 & $-32,832$ & 60,105 & 208 \\
\hline $\begin{array}{l}\text { TOTAL GROSS ELORS } \\
\text { LOSSBS \& OUISSIONS }\end{array}$ & $\begin{array}{r}19.011 \\
1.154\end{array}$ & $\begin{array}{r}3.053 \\
-30\end{array}$ & $\begin{array}{l}52,579 \\
-1,664\end{array}$ & $\begin{array}{r}31.989 \\
-1.794\end{array}$ & - & $\begin{array}{r}47,5.54 \\
130\end{array}$ & $\begin{array}{r}125,782 \\
2,091\end{array}$ & $\begin{array}{r}43,551 \\
3,483\end{array}$ & $\begin{array}{r}296,827 \\
3,419\end{array}$ & $\begin{array}{r}998 \\
18\end{array}$ \\
\hline TOTAL NET OSAGE & 20,165 & 3.022 & 50,914 & 35,194 & - & 47.874 & 127.373 & 14.202 & 300,246 & \\
\hline
\end{tabular}

\section{SOPPLY OP BIERGY}

GOSSIL POEL

GYDROBLECTRIC

NOCL B AR

GBO. ESOLAE

10

TOTAL SUPPLI

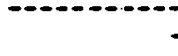

$-$

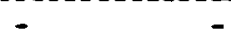

$-$

$-$

5,575

$5,575 \quad 1,266,714$

$2,484 \quad 1,274,773$

\section{NET IHPORTS}

OP BEGION

20,165

$3, \mathrm{C} 22$

50,714

36.194

$42,-, 99-1,138,840$

11.718

$-974.52 \epsilon$

\section{NOTES:}

TRAES PORAATIOA LOSS POB

TRA ESPOREATION LOSS POB

TRAYSFORGATIOH IOSS POB

ELECTRECITY GEN.

MATURAE GAS

TRANSFO RAATION LOSS POR SEH-GAS 
REGIONAL BNERGT BALAHCE STATEHETT

BT SECTOR AHD POEL TYPE

\begin{tabular}{|c|c|c|c|c|c|c|c|c|c|c|}
\hline SECTOR & $\begin{array}{l}\text { DISTILLATB } \\
\text { OIL }\end{array}$ & $\begin{array}{l}\text { RESIDUAL } \\
\text { OEL }\end{array}$ & $\begin{array}{l}\text { GASOL INE } \\
\text { (ALL OHIT }\end{array}$ & $\begin{array}{l}\text { HYDBO- } \\
\text { CABBONS } \\
\text { TS IN 10**9 }\end{array}$ & $\begin{array}{c}\text { CBUDE } \\
\text { OIL } \\
\text { BTO S) }\end{array}$ & $\begin{array}{l}\text { MAT ORAL } \\
\text { JAS }\end{array}$ & COAL & ELECTRI CITY & $\begin{array}{l}\text { SECTOR } \\
\text { TOTAL }\end{array}$ & \\
\hline 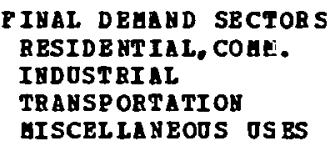 & $\begin{array}{r}5.015 \\
5.651 \\
15.402 \\
191\end{array}$ & $\begin{array}{r}1.161 \\
2.009 \\
3 \\
482\end{array}$ & 77.318 & $\begin{array}{r}11.164 \\
44.507 \\
3.381 \\
376\end{array}$ & $\begin{array}{l}- \\
- \\
-\end{array}$ & $\begin{array}{r}57.526 \\
66.438 \\
12.007 \\
2.911\end{array}$ & $\begin{array}{r}5,025 \\
133,361 \\
19 \\
-\end{array}$ & $\begin{array}{r}18,315 \\
29,890 \\
16 \\
188\end{array}$ & $\begin{array}{r}98,206 \\
281.856 \\
108.146 \\
4.148\end{array}$ & $\begin{array}{l}13 \% \\
38 \% \\
15 \% \\
1 \%\end{array}$ \\
\hline $\begin{array}{l}\text { TOTAL PINAL } \\
\text { DEHA HD SECTOBS }\end{array}$ & 26,259 & 3.655 & 77,318 & 59,428 & - & 138,882 & 138,405 & $48,4 C 9$ & 492.356 & $67 x$ \\
\hline $\begin{array}{l}\text { TRAHSPORGATION } \\
\text { ELECTRICITY GEH. } \\
\text { PETROLEOA PRODOCTS } \\
\text { MATORAL GAS } \\
\text { SYH-GAS }\end{array}$ & $\begin{array}{r}217 \\
-49.044 \\
-\end{array}$ & $\begin{array}{r}1.926 \\
-15.745 \\
=\end{array}$ & $\begin{array}{r}-111.471 \\
-\end{array}$ & $\begin{array}{r}-97,904 \\
-12,101\end{array}$ & $\begin{array}{r}265,485 \\
-\end{array}$ & $\begin{array}{r}50 \\
12,102 \\
13,907 \\
\end{array}$ & $\begin{array}{r}343,572 \\
=\end{array}$ & $\begin{array}{r}-119,300 \\
= \\
=\end{array}$ & $\begin{array}{r}226.465 \\
3.423 \\
1.806\end{array}$ & $\begin{array}{r}31 x \\
0 x \\
0 x\end{array}$ \\
\hline $\begin{array}{l}\text { NET POBL OSBD } \\
\text { IH TRANS PORHATIOA }\end{array}$ & $-48,827$ & $-13,819$ & $-111,471$ & $-110,005$ & 265,485 & 26,059 & 343,572 & $-119,300$ & 231.694 & $32 x$ \\
\hline $\begin{array}{l}\text { TOTAL GROSS PLONS } \\
\text { LOSSES } 8 \text { OAISSIONS }\end{array}$ & $\begin{array}{r}26,476 \\
1,608\end{array}$ & $\begin{array}{r}5.581 \\
-56\end{array}$ & $\begin{array}{l}77.318 \\
-2.447\end{array}$ & $\begin{array}{l}59.428 \\
-2.807\end{array}$ & $\begin{array}{r}265.485 \\
402\end{array}$ & $\begin{array}{r}164.941 \\
624\end{array}$ & $\begin{array}{r}481.977 \\
8.013\end{array}$ & $\begin{array}{r}48,409 \\
3.871\end{array}$ & $\begin{array}{r}724,050 \\
9.208\end{array}$ & $\begin{array}{r}998 \\
18\end{array}$ \\
\hline
\end{tabular}

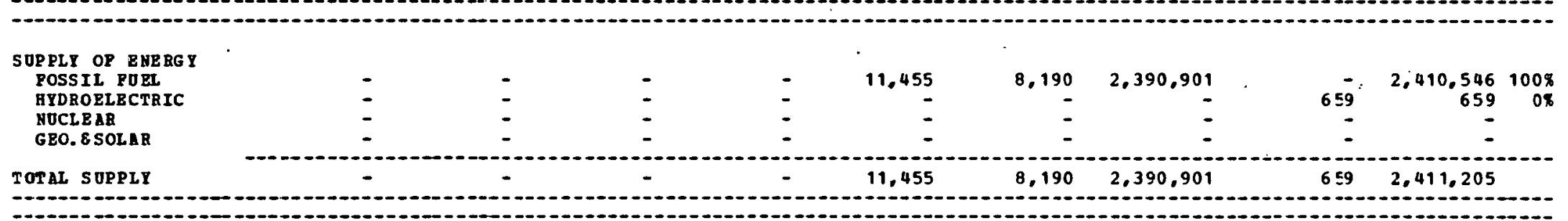

-

BET IMPORTS

$-10,220$

$-36,600$

$-53,384$

254,432

$157,375-1,900,910$

$-67,678-1,677,946$

-

MOT ES:

1 TRANSPORAATION LOSS POR TRANSPORGATION LOSS FOR TRANS PORAATIOH LOSS FOR
TRANS PORHATIOH LOSS FOR

BLECT RI CITY GEN

$\begin{aligned} \text { BLECT RICITY GEN. } & =65.50 \% \\ \text { PETROLEOA PRODOCTS } & =1.234\end{aligned}$

ATOB AL GAS

$S Y S-G A S$ 
REGION LL BNERTI BALANCE STATEGEHT

BY SECTOR AND FU RL TYPE

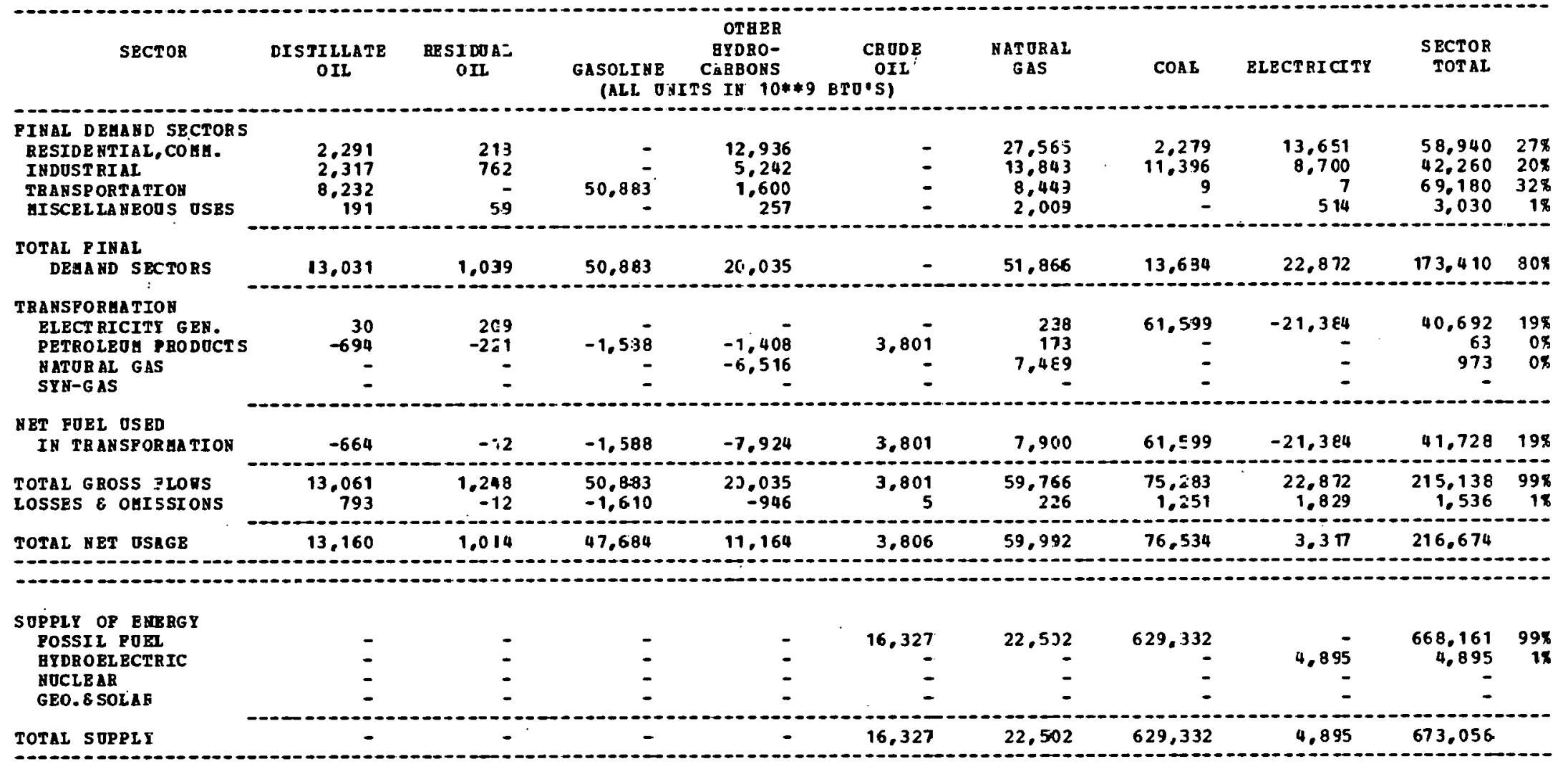

10.0.

\section{HET IHPORTS}

$11,164-12,520$

37,490

$-552,797$

$-1,57 \quad-456,381$

-

\section{NOTBS:}

1 TRA SS PORGATION LOSS POR TRABSPORATION IOSS POE TRABS POREATIOY LOSS POR TRAASPORAATIOA IOSS POB 
REGIONAL ENGRGY BALAHCE STATBAENT

BY SECTOR AND POEL TYPE

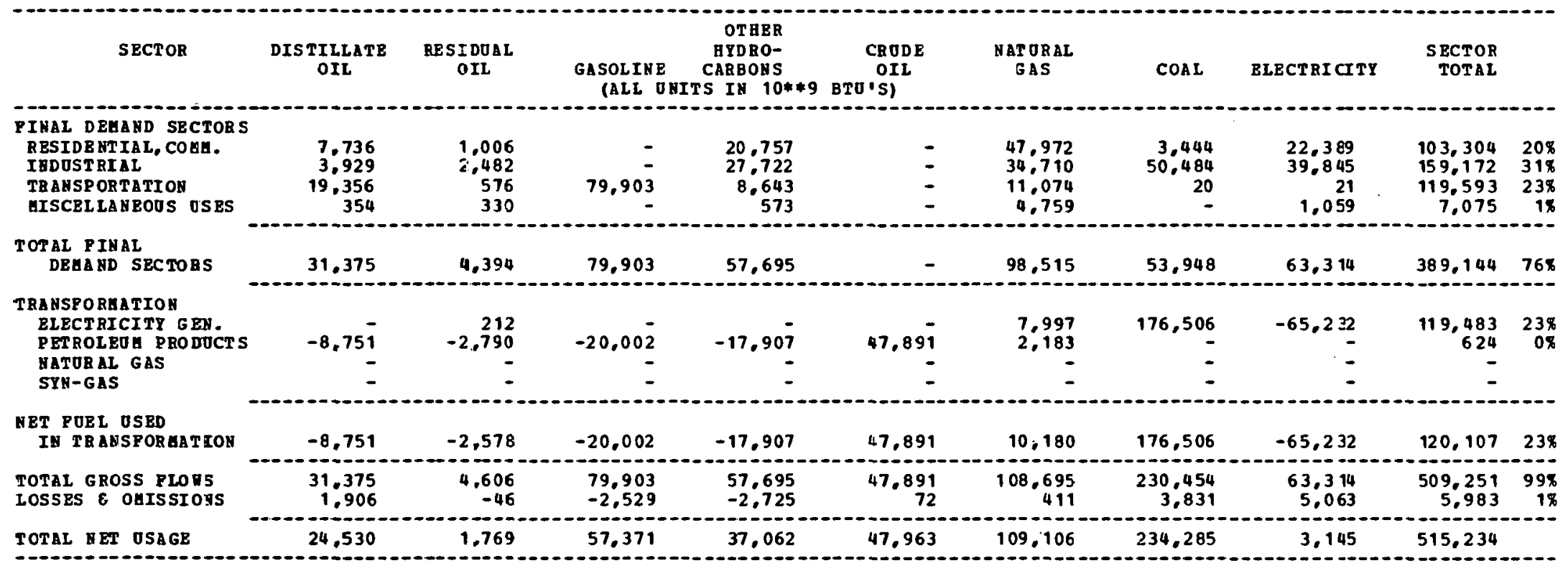

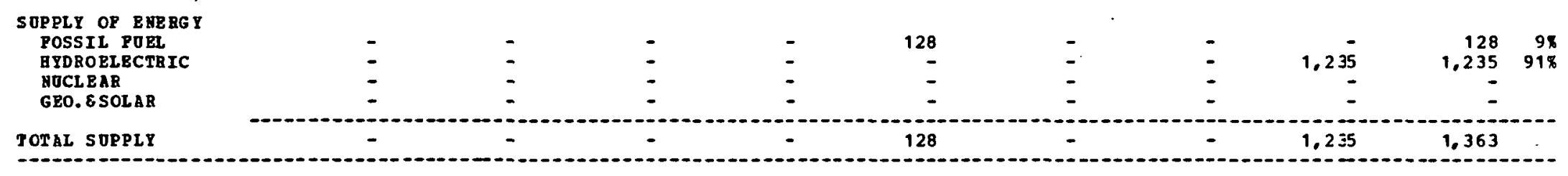

\begin{tabular}{|c|c|c|c|c|c|c|}
\hline \multicolumn{2}{|c|}{$\begin{array}{l}\text { SET IHPORTS } \\
\text { OF REGION }\end{array}$} & \multicolumn{2}{|c|}{24,530} & \multicolumn{3}{|c|}{57.371} \\
\hline พOI & BS: & & & & & \\
\hline $\begin{array}{l}1 \\
2 \\
3\end{array}$ & $\begin{array}{l}\text { TRA NSPORAATIOH } \\
\text { TRANSPORAT IOH } \\
\text { TRANSEORATION }\end{array}$ & $\begin{array}{l}\text { LosS } \\
\text { LosS } \\
\text { LoSS }\end{array}$ & $\begin{array}{l}\text { FOR } \\
\text { POR } \\
\text { POR }\end{array}$ & $\begin{array}{l}\text { ELECT RI CITS } \\
\text { PETROLZOH } \\
\text { NATORAL GAS }\end{array}$ & $\begin{array}{l}\text { GEN } \\
\text { ROD DCTS }\end{array}$ & $\begin{array}{l}=64.69 \% \\
=1.25 \% \\
=0.0 \%\end{array}$ \\
\hline 4 & TR A IS FORHAT ION & LOSS & FOR & STN-GAS & & $=0.0$ \\
\hline
\end{tabular}


REGIONAL 3NERGY BALANCE STA TEYENT

BY SECTCA AHD EOEL TYPE

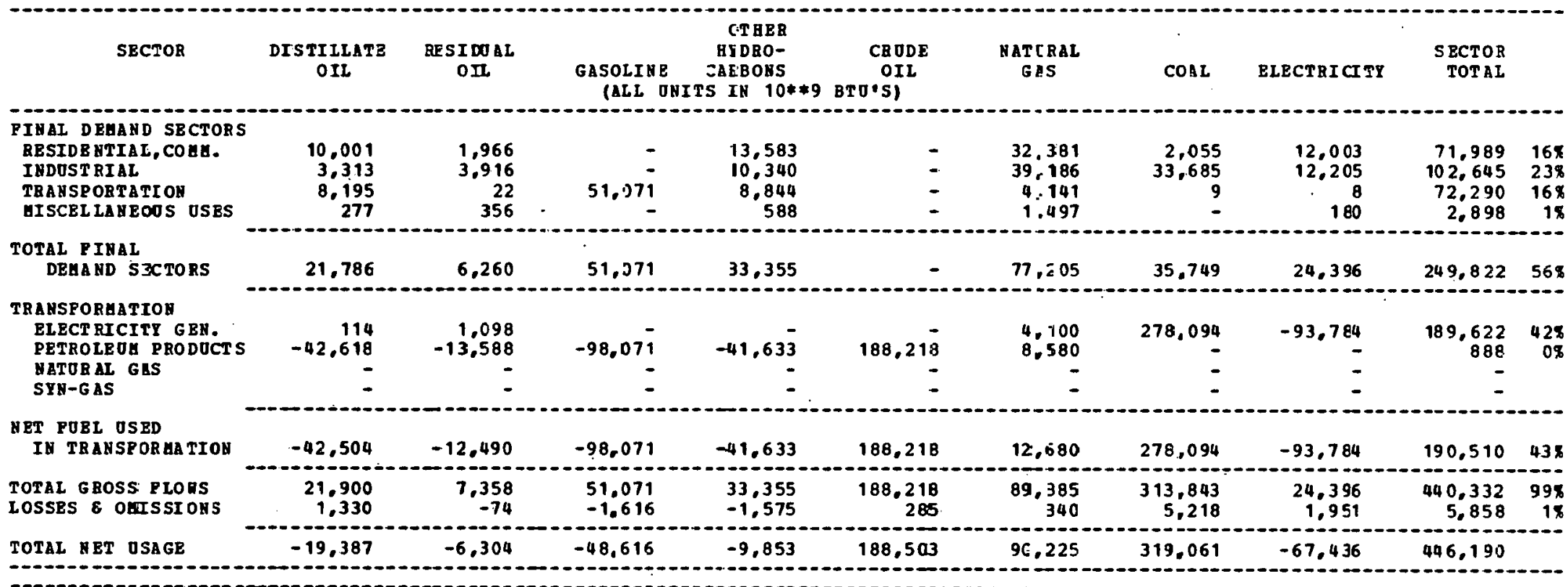

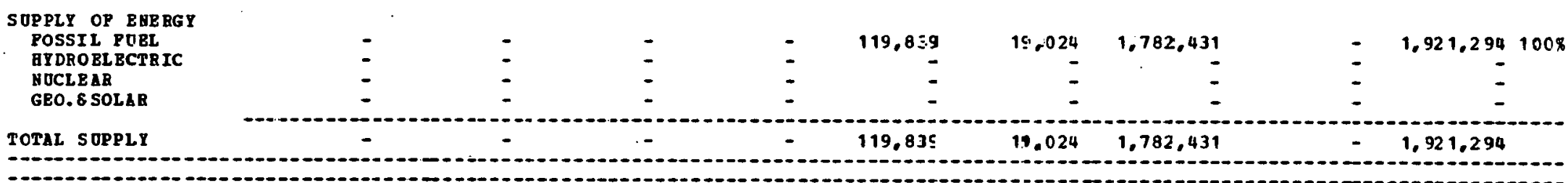

\begin{tabular}{l}
$\begin{array}{l}\text { MBT IHPORTS } \\
\text { OF REGION }\end{array}$ \\
\hdashline$\quad-19,387$
\end{tabular}


REGIONAL EHBRGY BALANCE STATEAENT

BI SECTOR AND POEL TIPE

\begin{tabular}{|c|c|c|c|c|c|c|c|c|c|c|}
\hline SECTOR & $\begin{array}{l}\text { DISTILLATE } \\
\text { OIL. }\end{array}$ & $\begin{array}{l}\text { BESIDOAL } \\
\text { DIL }\end{array}$ & $\begin{array}{l}\text { GASOLINE } \\
\text { (ALL O }\end{array}$ & $\begin{array}{l}\text { AYDBO- } \\
\text { CARBOAS } \\
\text { TS IE 10**9 }\end{array}$ & $\begin{array}{l}\text { CRJDE } \\
\text { OIL } \\
\text { BTO'S) }\end{array}$ & $\begin{array}{l}\text { NaT ORAL } \\
\text { G AS }\end{array}$ & COAL & BLECTRICITY & $\begin{array}{l}\text { SECTOR } \\
\text { TOTAL }\end{array}$ & \\
\hline 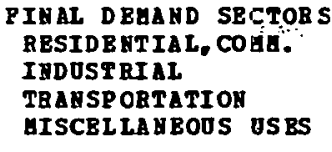 & $\begin{array}{r}4.701 \\
674 \\
2.903 \\
109\end{array}$ & $\begin{array}{r}843 \\
1.573 \\
17 \\
182\end{array}$ & 16,809 & $\begin{array}{r}4.615 \\
4.945 \\
462 \\
251\end{array}$ & $\overline{-}$ & $\begin{array}{r}10,866 \\
9,346 \\
606 \\
557\end{array}$ & $\begin{array}{r}631 \\
12.392 \\
4 \\
-\end{array}$ & $\begin{array}{r}3.498 \\
2.917 \\
- \\
26\end{array}$ & $\begin{array}{r}25,154 \\
31,847 \\
20,801 \\
1,125\end{array}$ & $\begin{array}{r}14 x \\
178 \\
118 \\
18 \\
\end{array}$ \\
\hline $\begin{array}{l}\text { TOTAL PIMAL } \\
\text { DEAA AD SECTOBS }\end{array}$ & 9,387 & 2.615 & 16,809 & 10.273 & - & 21.375 & 13,027 & 6.441 & 78,927 & $43 \%$ \\
\hline $\begin{array}{l}\text { TRABSEORHATIOH } \\
\text { ELECTRICITY GRE. } \\
\text { PETROLEOH PRONOCT S } \\
\text { MATORAL GAS } \\
\text { SYH-GAS }\end{array}$ & $\begin{array}{r}104 \\
-85,146 \\
-\end{array}$ & $\begin{array}{r}1,393 \\
-27,148 \\
- \\
-\end{array}$ & $\begin{array}{r}-195,939 \\
-\end{array}$ & $\begin{array}{r}-74,504 \\
-\end{array}$ & $\begin{array}{r}368,474 \\
-\end{array}$ & $\begin{array}{r}36 \\
16,797 \\
-\end{array}$ & $\begin{array}{r}147.802 \\
= \\
=\end{array}$ & $\begin{array}{r}-50,238 \\
= \\
=\end{array}$ & $\begin{array}{r}99.097 \\
2.534 \\
- \\
-\end{array}$ & $\begin{array}{r}54 \% \\
1 \%\end{array}$ \\
\hline $\begin{array}{l}\text { MBT POBL OSBD } \\
\text { IA TRABSFORGATIOH }\end{array}$ & $-85,042$ & $-25,755$ & $-195,939$ & $-74,504$ & 368,474 & 16.833 & 147,802 & $-50,238$ & 101,631 & $55 \pi$ \\
\hline $\begin{array}{l}\text { TOTAL GROSS PLORS } \\
\text { COSSES } 8 \text { OAISSIOHS }\end{array}$ & $\begin{array}{r}8.491 \\
515\end{array}$ & $\begin{array}{r}4.008 \\
-40\end{array}$ & $\begin{array}{r}16.809 \\
-532\end{array}$ & $\begin{array}{r}10.273 \\
-485\end{array}$ & $\begin{array}{r}368,474 \\
558\end{array}$ & $\begin{array}{r}38,208 \\
144\end{array}$ & $\begin{array}{r}160,829 \\
2,674\end{array}$ & $\begin{array}{r}6.441 \\
515\end{array}$ & $\begin{array}{r}180.558 \\
3.350\end{array}$ & $\begin{array}{r}98 \% \\
2 \%\end{array}$ \\
\hline
\end{tabular}

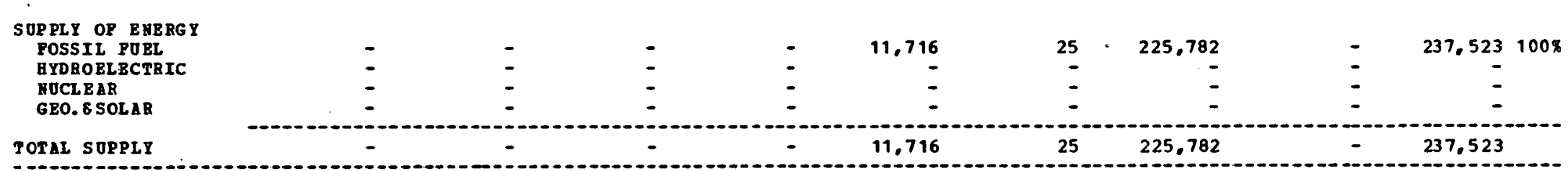

\begin{tabular}{|c|c|c|c|c|c|c|c|c|c|}
\hline OP REGIOB & $-76,139$ & -23.180 & $-179,662$ & -64.716 & 357,316 & 38,327 & -62.2 & -43.2 & $-53,614$ \\
\hline
\end{tabular}

NOT BS:

TRAESPORHATION LOSS FOR

TRA ASPORAATIOH LOSS FOR

TRANSPORGATION LOSS FOR
TBABSPORAATIOA LOSS POR

ELBCTRICITY GEN.

=66.36\%

PETORAOH PROD

$=0.66 \mathrm{x}$

STH-GAS

$=0.0 \mathrm{x}$ 
REG IONAL BHZRGY BALAUCE STATEMENT

BY SECEOR AHD FOEL TYPE

\begin{tabular}{|c|c|c|c|c|c|c|c|c|c|c|}
\hline SECTOR & $\begin{array}{l}\text { DISTILLATB } \\
\text { OI: }\end{array}$ & $\begin{array}{l}\text { BESEDOAL } \\
\text { OIL }\end{array}$ & $\begin{array}{l}\text { GASOLIAE } \\
\text { (ALL O }\end{array}$ & $\begin{array}{c}\text { OTBER } \\
\text { IYDBO- } \\
\text { CABEOHS } \\
\text { TS: IB } 10 * * 9\end{array}$ & $\begin{array}{c}\text { CRODE } \\
\text { OIL } \\
\text { BTO'S) }\end{array}$ & $\begin{array}{l}\text { MAT DRAL } \\
\text { GAS }\end{array}$ & COAL & ELECTRICITY & $\begin{array}{r}\text { SECTOR } \\
\text { TOTAL }\end{array}$ & \\
\hline $\begin{array}{l}\text { PINAL DBHAND SBCTORS } \\
\text { RESIDE HTIAL, COHA. } \\
\text { INDOSTRIAL } \\
\text { TRAHSPORTATION } \\
\text { GISCELIANBOUS OSES }\end{array}$ & $\begin{array}{r}5,752 \\
761 \\
4,036 \\
88\end{array}$ & $\begin{array}{r}2,135 \\
2,120 \\
56 \\
532\end{array}$ & 27.974 & $\begin{array}{r}6.442 \\
8.552 \\
1.583 \\
400\end{array}$ & $\overline{-}$ & $\begin{array}{r}28,007 \\
12,572 \\
964 \\
1.606\end{array}$ & $\begin{array}{r}1,202 \\
7.632 \\
7 \\
-\end{array}$ & $\begin{array}{r}6.939 \\
3.357 \\
21 \\
330\end{array}$ & $\begin{array}{r}50,477 \\
34.994 \\
34,641 \\
2.956\end{array}$ & $\begin{array}{r}26 \% \\
18 \% \\
18 \% \\
2 \%\end{array}$ \\
\hline $\begin{array}{l}\text { TOTAL F IAAL } \\
\text { DBEA BD SBCTOBS }\end{array}$ & 10.637 & 4,843 & 27,974 & 15,977 & - & 43,149 & 8,841 & 10,647 & 123,068 & $64 x$ \\
\hline $\begin{array}{l}\text { TRAHSPOREATIOH } \\
\text { ELBCT RICITI GBH. } \\
\text { PETROLEOH PBODOCTS } \\
\text { MATORAL GAS } \\
\text { SYH-GAS }\end{array}$ & $\begin{array}{r}182 \\
- \\
-\end{array}$ & $\begin{array}{r}7.728 \\
- \\
-\end{array}$ & : & $\begin{array}{l}- \\
-\end{array}$ & $\overline{-}$ & $\begin{array}{r}579 \\
- \\
-\end{array}$ & $\begin{array}{r}87.732 \\
- \\
-\end{array}$ & $\begin{array}{r}-29,397 \\
- \\
-\end{array}$ & $\begin{array}{r}66.794 \\
= \\
=\end{array}$ & $35 \%$ \\
\hline $\begin{array}{l}\text { NET POEL OSED } \\
\text { IU TRAHSPORHATION }\end{array}$ & 182 & 7,728 & - & - & - & 549 & 87,732 & $-29,397$ & 66.794 & $35 \%$ \\
\hline $\begin{array}{l}\text { TOTAL GROSS PLORS } \\
\text { LOSSES } 8 \text { OULSSIONS }\end{array}$ & $\begin{array}{r}10,819 \\
657\end{array}$ & $\begin{array}{r}12.571 \\
-127\end{array}$ & $\begin{array}{r}27.974 \\
-385\end{array}$ & $\begin{array}{r}16,977 \\
-802\end{array}$ & 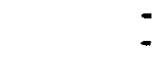 & $\begin{array}{r}43,698 \\
165\end{array}$ & $\begin{array}{r}96,573 \\
1,605\end{array}$ & $\begin{array}{r}10,647 \\
851\end{array}$ & $\begin{array}{r}189,862 \\
1,464\end{array}$ & $\begin{array}{r}99 \% \\
1 \%\end{array}$ \\
\hline
\end{tabular}

SUPPLY OP EIERGY

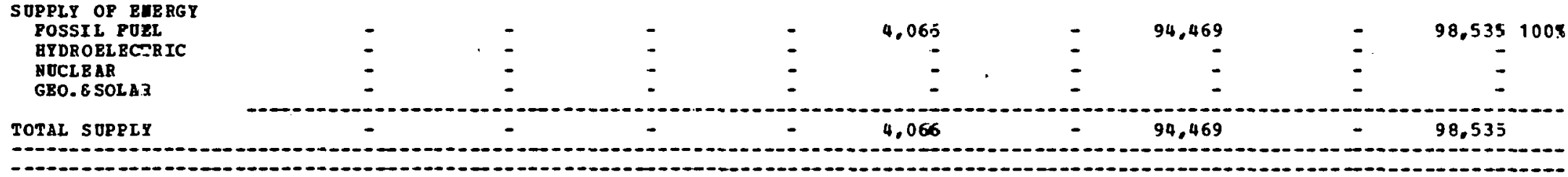

\section{UET IMPORTS}

OP RBGIOZ

11.476

12.443

27,088

16,174

$-4.066$

43.863

3.709

$-17.898$

92,791

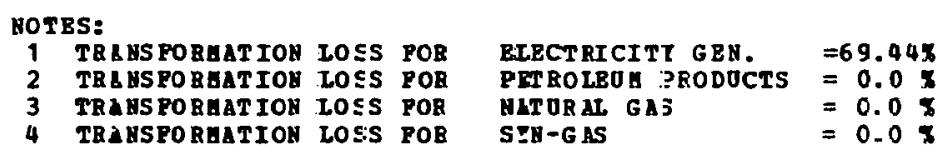


REGIONAL BNEBG BALAHCE STATEAENT

BY SBCTOR AND FORL TYPE

\begin{tabular}{|c|c|c|c|c|c|c|c|c|c|c|}
\hline SECTOR & $\begin{array}{c}\text { DISTrLLaTE } \\
\text { OIl }\end{array}$ & $\begin{array}{l}\text { BESIDJAL } \\
\text { OIC }\end{array}$ & $\begin{array}{l}\text { GASOLINE } \\
\text { (ALL ONIT }\end{array}$ & $\begin{array}{c}\text { OTBER } \\
\text { BYDRO- } \\
\text { CARBOKS } \\
\text { IS IN } 10 * * 9\end{array}$ & $\begin{array}{c}\text { CRUDE } \\
\text { OIL } \\
\text { BTO'SI }\end{array}$ & $\begin{array}{l}\text { RAT URAL } \\
\text { GAS }\end{array}$ & coal & ELECTRI CITY & $\begin{array}{l}\text { SECTOR } \\
\text { TOTAL }\end{array}$ & \\
\hline $\begin{array}{l}\text { PINAL D BAAND SECTORS } \\
\text { RBSIDBRTAL, COAG. } \\
\text { INDOSTRIAL } \\
\text { TRABSPORTATIOH } \\
\text { WISCELLABEOOS OSES }\end{array}$ & $\begin{array}{r}4,535 \\
619 \\
2.700 \\
70\end{array}$ & $\begin{array}{r}1,429 \\
1,393 \\
33 \\
756\end{array}$ & $22,05 \overline{-}$ & $\begin{array}{r}5.079 \\
8.224 \\
662 \\
316\end{array}$ & $\begin{array}{l}- \\
-\end{array}$ & $\begin{array}{r}21.110 \\
10.486 \\
760 \\
2.558\end{array}$ & $\begin{array}{r}948 \\
5.534 \\
4 \\
-\end{array}$ & $\begin{array}{r}5,115 \\
2,608 \\
25 \\
525\end{array}$ & $\begin{array}{r}38,216 \\
29,364 \\
26,240 \\
4,225\end{array}$ & $\begin{array}{r}368 \\
27 \% \\
248 \\
4 \%\end{array}$ \\
\hline $\begin{array}{l}\text { TOTAL PINAL } \\
\text { DEUA AD SECTOAS }\end{array}$ & 7.924 & 4.111 & 22.056 & 14.281 & - & 34.914 & 6,486 & 8.273 & 98,045 & 918 \\
\hline $\begin{array}{l}\text { TRANSPOREATION } \\
\text { ELECTRICITY GEH. } \\
\text { PETROLEUA PRODOCTS } \\
\text { BATORAL GAS } \\
\text { SYH-GAS }\end{array}$ & $\begin{array}{r}140 \\
- \\
-\end{array}$ & $\begin{array}{r}973 \\
- \\
-\end{array}$ & $\overline{-}$ & $-18,409$ & $\overline{-}$ & $\begin{array}{r}421 \\
- \\
21,158 \\
-\end{array}$ & $\begin{array}{r}8,160 \\
\vdots \\
-\end{array}$ & $\begin{array}{r}-3.067 \\
- \\
- \\
-\end{array}$ & $\begin{array}{r}6.627 \\
2.749 \\
-\end{array}$ & $\begin{array}{l}6 \% \\
3 \%\end{array}$ \\
\hline $\begin{array}{l}\text { NET POEL USED } \\
\text { IA TRANSPOR GATION }\end{array}$ & 140 & 973 & - & $-18,409$ & - & 21.579 & 8,160 & $-3,067$ & 9.376 & $9 \%$ \\
\hline $\begin{array}{l}\text { TOTAL GROSS PLONS } \\
\text { LOSSES } \& \text { OHISSIOHS }\end{array}$ & $\begin{array}{r}8.064 \\
489\end{array}$ & $\begin{array}{r}5 . C 84 \\
-51\end{array}$ & $\begin{array}{r}22.056 \\
-698\end{array}$ & $\begin{array}{r}14.281 \\
-674\end{array}$ & - & $\begin{array}{r}56.493 \\
213\end{array}$ & $\begin{array}{r}14.646 \\
243\end{array}$ & $\begin{array}{r}8.273 \\
661\end{array}$ & $\begin{array}{r}107.421 \\
184\end{array}$ & $\begin{array}{r}100 \% \\
0 \%\end{array}$ \\
\hline
\end{tabular}

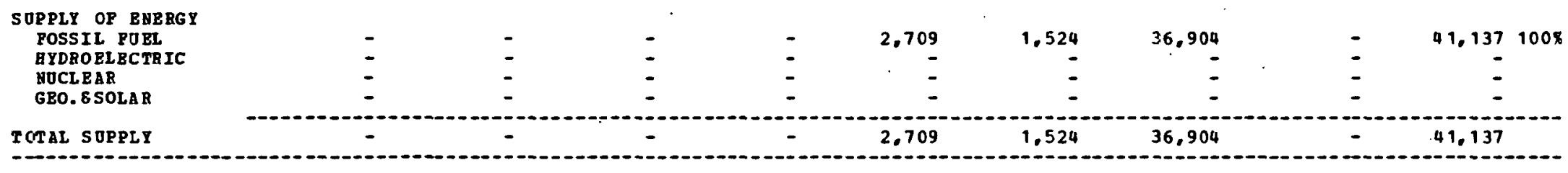

\section{SET IAPORTS}

21,357

$-4,802$

$-2,709$

55,182

$-22,014$

HOTES:

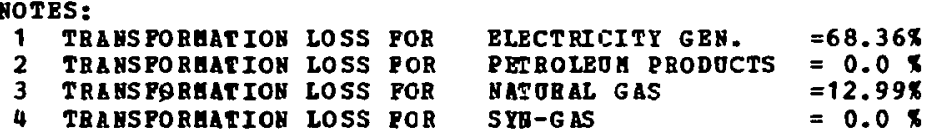


RBGIONAL 3XEEGT BALABCE STATEMERT

$$
\text { BY SECTOE AND FOBL TYPE }
$$

\begin{tabular}{|c|c|c|c|c|c|c|c|c|c|}
\hline SECT JR & $\begin{array}{l}\text { DISTILATE } \\
\text { OIL }\end{array}$ & $\begin{array}{l}\text { RESIDUALL } \\
\text { JIL }\end{array}$ & $\begin{array}{l}\text { GASOLINE } \\
\text { (AL: OHIT }\end{array}$ & $\begin{array}{l}\text { AYDBO- } \\
\text { CARBONS } \\
\text { TS IE 10**9 }\end{array}$ & $\begin{array}{c}\text { CRUDE } \\
\text { OIL } \\
\text { BTO'S) }\end{array}$ & $\begin{array}{l}\text { NAT UEAL } \\
\text { GAS }\end{array}$ & COAL & ELECTRICITY & $\begin{array}{r}\text { SECTOR } \\
\text { TOTAL }\end{array}$ \\
\hline 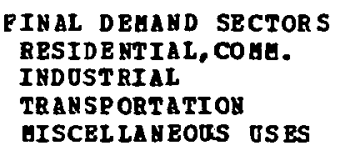 & $\begin{array}{r}4.992 \\
522 \\
1.584 \\
120\end{array}$ & $\begin{array}{r}866 \\
1.352 \\
7 \\
259\end{array}$ & 17.233 & $\begin{array}{r}4.834 \\
5,080 \\
290 \\
259\end{array}$ & 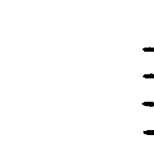 & $\begin{array}{r}10.547 \\
9.456 \\
6 \leq 5 \\
168\end{array}$ & $\begin{array}{r}634 \\
14,906 \\
2 \\
-\end{array}$ & $\begin{array}{r}3,576 \\
2,5 \in 1 \\
- \\
13\end{array}$ & $\begin{array}{r}25,449 \\
34,017 \\
19,841 \\
1,399\end{array}$ \\
\hline $\begin{array}{l}\text { TOTAL PIHAL } \\
\text { DEHA SED SECTORS }\end{array}$ & 7,18 & 2,5014 & 17.233 & 12.463 & - & 21,396 & 15,542 & 6.150 & 80,706 \\
\hline $\begin{array}{l}\text { TRANSPORHATICA } \\
\text { ELECTRICITI GEH. } \\
\text { PETROLBOA PRODOCTS } \\
\text { HATOR AL GAS: } \\
\text { SYH-GAS }\end{array}$ & $\begin{array}{l}93 \\
- \\
-\end{array}$ & - & $\overline{-}$ & : & 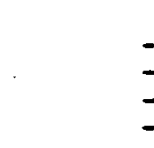 & $\begin{array}{r}247 \\
-\end{array}$ & $\begin{array}{r}3.477 \\
- \\
-\end{array}$ & $\begin{array}{r}-742 \\
- \\
-\end{array}$ & $\begin{array}{r}3.075 \\
- \\
-\end{array}$ \\
\hline $\begin{array}{l}\text { NET FOBL OSBO } \\
\text { IA TRAESPOEMATION }\end{array}$ & 93 & - & - & - & - & 247 & 3,477 & -742 & 3,075 \\
\hline $\begin{array}{l}\text { TOTAL GROSS PLORS } \\
\text { LOSSES } 8 \text { OUISSIOUS }\end{array}$ & $\begin{array}{r}7.511 \\
456\end{array}$ & $\begin{array}{r}2.5: 34 \\
-25\end{array}$ & $\begin{array}{r}17.233 \\
-545\end{array}$ & $\begin{array}{r}10.463 \\
-494\end{array}$ & - & $\begin{array}{r}21,6+3 \\
31\end{array}$ & $\begin{array}{r}19.019 \\
316\end{array}$ & $\begin{array}{r}6,150 \\
491\end{array}$ & $\begin{array}{r}83,781 \\
280\end{array}$ \\
\hline
\end{tabular}

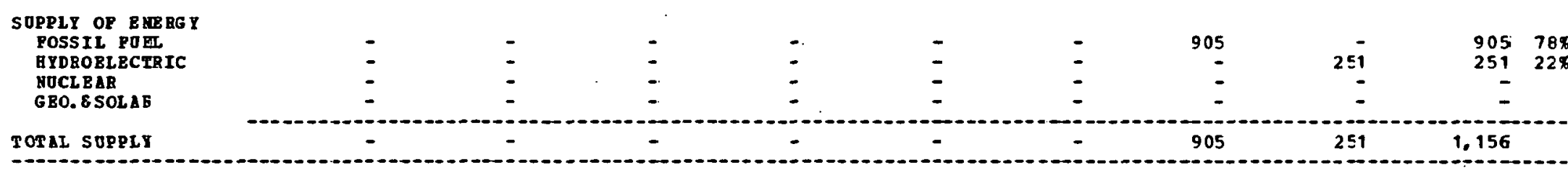

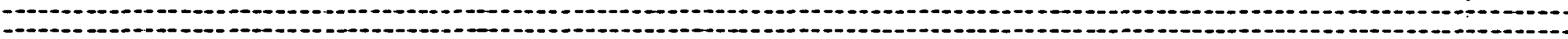

\section{NET IBPORTS \\ OP RBGIOH}

7.967

2.478

16.687

9,968

21.724

18,430

5,648

82,905

\section{NOT ES:}

$$
\begin{aligned}
& \text { TRADSPOBAATION LOSS: FOR ELZCTRICITY GEN. }=80.56 \% \\
& \text { TRARS PORAATIOX LOSS: FOR PEROLEOE PEODOCTS }=0.6 \\
& \text { TRARSPOBAATIOK LOSS FOR HARORAL GAS }
\end{aligned}
$$


REGIONAL BAERT BALAKCE STATEUEXI

BY SECTOR AHD POBL TYPE

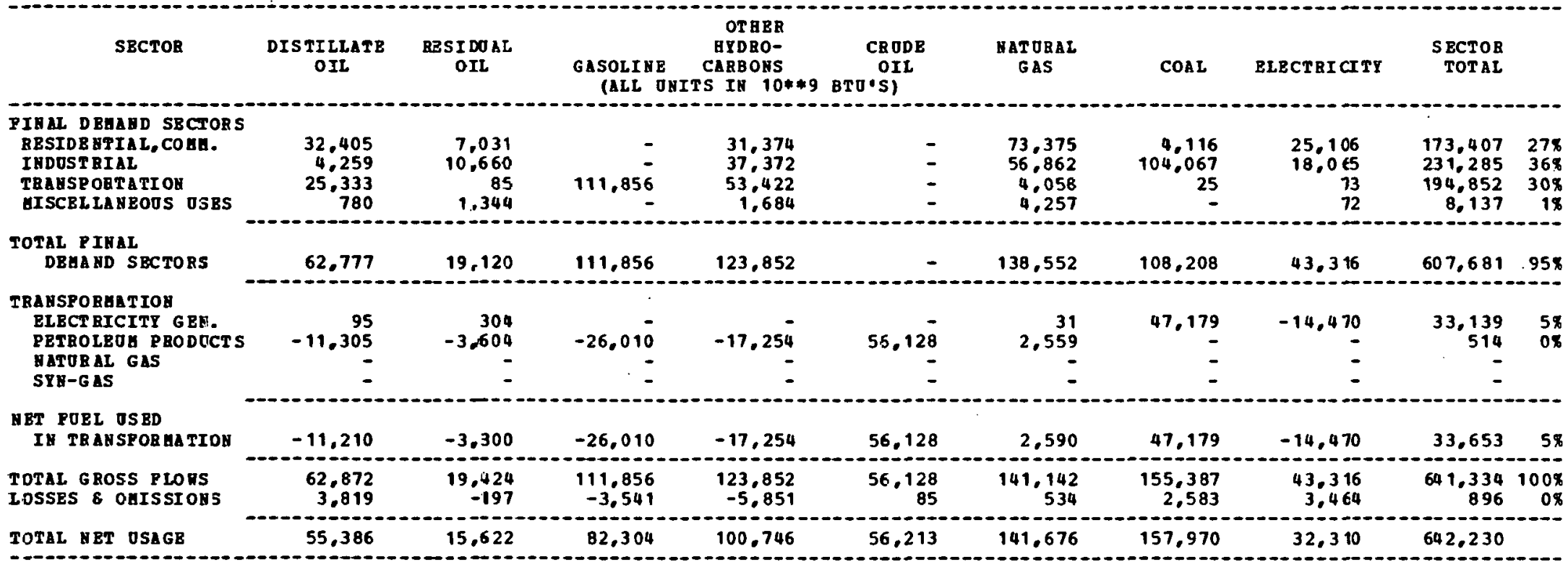

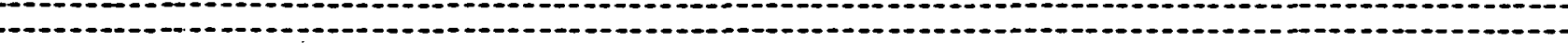

\section{SUPPLY OP RHEBGT}

POSSIL POEL

GYDROBLBCTBIC

NOCLEAB

GEO. 6 SOLA

$\begin{aligned} & - \\ - & - \\ - & -\end{aligned}$

TCTAL SUPPLI

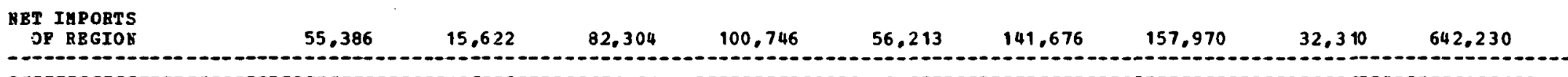

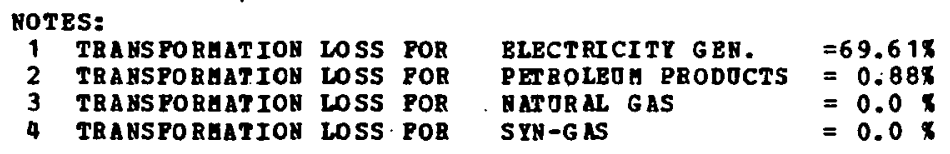


REGIGHAE ENERGY BALAHCE STATEHEYT

E.Y 3 BCTOR AND FOEL TYPE

\begin{tabular}{|c|c|c|c|c|c|c|c|c|c|c|}
\hline SECTCR & $\begin{array}{l}\text { DIS TELLATB } \\
\text { OIL }\end{array}$ & $\begin{array}{l}\text { RESISOAL } \\
\text { OIL }\end{array}$ & $\begin{array}{l}\text { GASOLIYE } \\
\text { TALI ONI }\end{array}$ & $\begin{array}{c}\text { DTEER } \\
\text { BIDEO- } \\
\text { CABBONS } \\
\text { TS IN } 10 * * 9\end{array}$ & $\begin{array}{c}\text { CBUDE } \\
\text { OIL } \\
\text { BTU 'S) }\end{array}$ & $\begin{array}{l}\text { NAT ORAL } \\
\text { JAS }\end{array}$ & COAL & BLECTRICITY & $\begin{array}{r}\text { SECTOR } \\
\text { TOTAL }\end{array}$ & \\
\hline 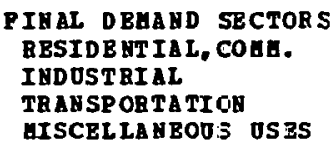 & $\begin{array}{r}10,963 \\
2,149 \\
4.178 \\
264\end{array}$ & $\begin{array}{r}1.814 \\
5.038 \\
15 \\
298\end{array}$ & 37,842 & $\begin{array}{r}10,614 \\
6,244 \\
747 \\
569\end{array}$ & $\begin{array}{l}- \\
- \\
-\end{array}$ & $\begin{array}{r}22,852 \\
31.499 \\
1.373 \\
893\end{array}$ & $\begin{array}{r}1,393 \\
53,493 \\
4 \\
-\end{array}$ & $\begin{array}{r}7.735 \\
7.757 \\
6 \\
15\end{array}$ & $\begin{array}{r}55,371 \\
106,180 \\
44,165 \\
2,039\end{array}$ & $\begin{array}{l}26 \% \\
50 \% \\
21 \% \\
1 \%\end{array}$ \\
\hline $\begin{array}{l}\text { TOTAL FINAL } \\
\text { DBHA ND SBCTORS }\end{array}$ & 17.554 & $\pi, 165$ & 37,842 & 18,174 & $=$ & 56,617 & 54,890 & 15,513 & 207.755 & $98 \%$ \\
\hline $\begin{array}{l}\text { TRANSPOREATIOS } \\
\text { ELECTRICITY GEN. } \\
\text { PETROLBOA ERODOCTS } \\
\text { MATORAL GAS } \\
\text { SYH-GAS }\end{array}$ & $\bar{z}$ & $\begin{array}{l}4 \\
- \\
-\end{array}$ & $\begin{array}{l}\overline{-} \\
\overline{-}\end{array}$ & $\begin{array}{l}\overline{-} \\
\overline{-}\end{array}$ & $\begin{array}{l}- \\
- \\
-\end{array}$ & $\begin{array}{l}- \\
-\end{array}$ & $\begin{array}{r}3,615 \\
: \\
-\end{array}$ & $\begin{array}{r}-1,097 \\
= \\
=\end{array}$ & $\begin{array}{r}2.522 \\
- \\
-\end{array}$ & $1 \%$ \\
\hline $\begin{array}{l}\text { HET POEL OSED } \\
\text { IA TRABSPOBGA TIOH }\end{array}$ & - & 4 & - & - & - & - & 3,615 & $-1,097$ & 2,522 & 18 \\
\hline $\begin{array}{l}\text { TOTAL GROSS PLORS } \\
\text { LOSSES } \& \text { OUISSIORS }\end{array}$ & $\begin{array}{r}17.554 \\
1.066\end{array}$ & $\begin{array}{r}7.169 \\
-72\end{array}$ & $\begin{array}{r}37,842 \\
-1,198\end{array}$ & $\begin{array}{l}1: 3,174 \\
-858\end{array}$ & - & $\begin{array}{r}56.617 \\
274\end{array}$ & $\begin{array}{r}58, \leq 05 \\
5.72\end{array}$ & $\begin{array}{r}15.513 \\
1.240\end{array}$ & $\begin{array}{r}210,277 \\
1,364\end{array}$ & $\begin{array}{r}998 \\
1 \%\end{array}$ \\
\hline
\end{tabular}

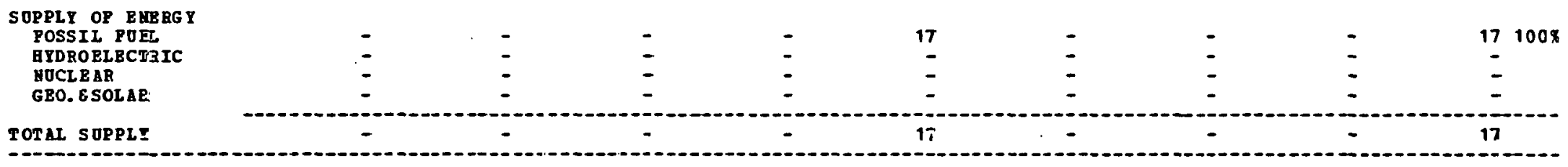

LET IAPOBTS

OP RBGIOH

$1 \varepsilon, 620$

7,096

36,643

17,315

$-17$

56,831

59.477

15,656

211.624

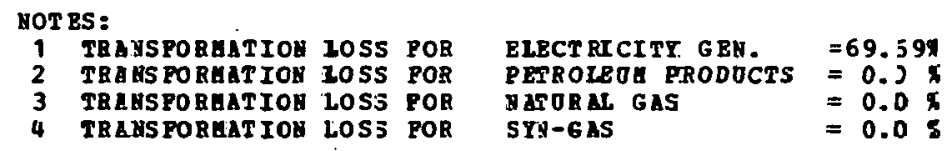


REGIONAL ENERGY BALANCE STATEMENT

BY SECTOR AND FOEL TYPE

\begin{tabular}{|c|c|c|c|c|c|c|c|c|c|c|}
\hline SECTOR & $\begin{array}{l}\text { DISTILLATE } \\
\text { OIL }\end{array}$ & $\begin{array}{l}\text { RESIDOAL } \\
\text { OIL }\end{array}$ & $\begin{array}{l}\text { GASOLINE } \\
\text { (ALL O }\end{array}$ & $\begin{array}{l}\text { HYDRO- } \\
\text { CARBONS } \\
\text { TS IN } 10 * 9\end{array}$ & $\begin{array}{c}\text { CRODE } \\
\text { OIL } \\
\text { BTO'S) }\end{array}$ & $\begin{array}{l}\text { WATORAL } \\
\text { GAS }\end{array}$ & $\operatorname{con} L$ & ELECTRI CITY & $\begin{array}{r}\text { SECTOR } \\
\text { TOTAL }\end{array}$ & \\
\hline $\begin{array}{l}\text { FIHAL DEHAHD SBCTORS } \\
\text { RESIDR RTIAL, COUA. } \\
\text { IBDOSTRIAL } \\
\text { TRABSPOETATI ON } \\
\text { UISCBL LABEOOS OSBS }\end{array}$ & $\begin{array}{r}15.121 \\
5.293 \\
19.606 \\
340\end{array}$ & $\begin{array}{r}899 \\
3.714 \\
125 \\
267\end{array}$ & $\begin{array}{r}\vdots \\
117,992 \\
-\end{array}$ & $\begin{array}{r}23,770 \\
38,136 \\
14.774 \\
636\end{array}$ & : & $\begin{array}{r}102,826 \\
51.649 \\
5,760 \\
3,486\end{array}$ & $\begin{array}{r}5.548 \\
83.571 \\
27 \\
-\end{array}$ & $\begin{array}{r}29.142 \\
31.060 \\
60 \\
505\end{array}$ & $\begin{array}{r}177,306 \\
213,423 \\
158,344 \\
5,234\end{array}$ & $\begin{array}{r}248 \\
29 \% \\
22 \% \\
1 \%\end{array}$ \\
\hline $\begin{array}{l}\text { TOTAL F IBAL } \\
\text { DELABD SBCTORS }\end{array}$ & 40,360 & 5.005 & 117,992 & 77,316 & - & 163.721 & 89,146 & 60,767 & 554,307 & $76 \%$ \\
\hline $\begin{array}{l}\text { TRANSPOREATIO\& } \\
\text { BLECT RICITY GBE. } \\
\text { PETROLBOA PRODOCTS } \\
\text { HATORAL GAS } \\
\text { SYH-GAS }\end{array}$ & $\begin{array}{r}3,772 \\
-19.455 \\
- \\
-\end{array}$ & $\begin{array}{r}1.743 \\
-6.203 \\
- \\
-\end{array}$ & $\begin{array}{r}-44,620 \\
-\end{array}$ & $\begin{array}{r}-11,447 \\
-\end{array}$ & 78,312 & $\begin{array}{r}7.856 \\
3.570 \\
- \\
-\end{array}$ & $\begin{array}{r}241,449 \\
- \\
-\end{array}$ & $\begin{array}{r}-87.484 \\
- \\
-\end{array}$ & $\begin{array}{r}167.336 \\
157 \\
- \\
-\end{array}$ & $\begin{array}{r}23 \% \\
08\end{array}$ \\
\hline $\begin{array}{l}\text { FBT FOEL OSBD } \\
\text { IH TBAESPORAA TION }\end{array}$ & $-15,683$ & $-4,460$ & $-44,620$ & $-11,447$ & 78.312 & 11.426 & 241,449 & -87.484 & 167.493 & $23 \%$ \\
\hline $\begin{array}{l}\text { TOTAL GROSS PLORS } \\
\text { LOSSBS } 6 \text { OUISSI ONS }\end{array}$ & $\begin{array}{r}44.132 \\
2.681\end{array}$ & $\begin{array}{r}6.748 \\
-68\end{array}$ & $\begin{array}{r}117,992 \\
-3,735\end{array}$ & $\begin{array}{l}77,316 \\
-3,653\end{array}$ & $\begin{array}{r}78,312 \\
118\end{array}$ & $\begin{array}{r}175.147 \\
663\end{array}$ & $\begin{array}{r}330,595 \\
5,496\end{array}$ & $\begin{array}{r}60.767 \\
4.859\end{array}$ & $\begin{array}{r}721,800 \\
6,362\end{array}$ & $\begin{array}{r}998 \\
1 \%\end{array}$ \\
\hline
\end{tabular}

(1)

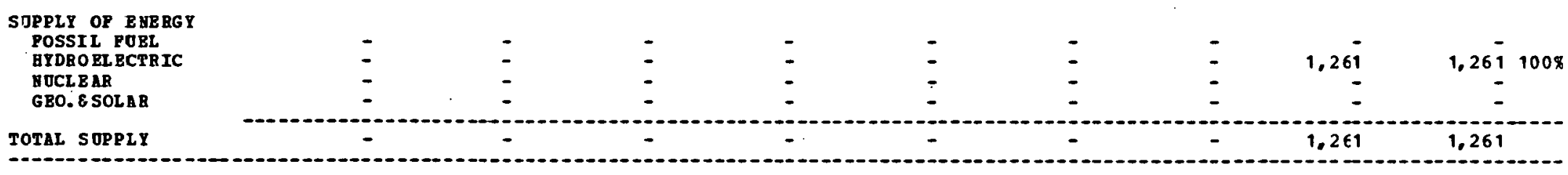

\begin{tabular}{l}
$\begin{array}{l}\text { IET IHPORTS } \\
\text { OP BRGIOH }\end{array}$ \\
\hdashline$\quad 27,358$
\end{tabular}


REGIONAL ENBBGY BALANCE STATBGEHT

BT SECTOR AND FOEL TYPE

\begin{tabular}{|c|c|c|c|c|c|c|c|c|c|c|}
\hline SECTOR & $\begin{array}{c}\text { DISTILLhTE } \\
\text { OII }\end{array}$ & $\begin{array}{l}\text { RESIDUAL } \\
\text { OIL }\end{array}$ & $\begin{array}{l}\text { GaSOLIVE } \\
\text { (ALL O }\end{array}$ & $\begin{array}{l}\text { GYDRO- } \\
\text { CABBCCNS } \\
\text { TS IN 10**9 }\end{array}$ & $\begin{array}{c}\text { CRODE } \\
\text { OIL } \\
\text { BTO'SI }\end{array}$ & $\begin{array}{l}\text { NATURAL } \\
\text { GAS }\end{array}$ & $\cos L$ & ELECTRI CI TY & $\begin{array}{l}\text { SECTOR } \\
\text { TOTAL }\end{array}$ & \\
\hline $\begin{array}{l}\text { PINAL DEGAND SECTORS } \\
\text { RESIDEHTIAL, COAL. } \\
\text { INDUSTRIAL } \\
\text { TRANSPORTATION } \\
\text { GISCEL LAHEOOS OSES }\end{array}$ & $\begin{array}{r}9,567 \\
2.934 \\
7,763 \\
163\end{array}$ & $\begin{array}{r}389 \\
1.697 \\
24 \\
206\end{array}$ & 70.835 & $\begin{array}{r}12,824 \\
17,294 \\
5.292 \\
327\end{array}$ & $\overline{-}$ & $\begin{array}{r}66.545 \\
36,0<7 \\
1.423 \\
4.563\end{array}$ & $\begin{array}{r}3,434 \\
47,277 \\
12 \\
-\end{array}$ & $\begin{array}{r}16.425 \\
15.593 \\
20 \\
605\end{array}$ & $\begin{array}{r}109,184 \\
120,842 \\
85,369 \\
5,858\end{array}$ & $\begin{array}{r}318 \\
348 \\
248 \\
28\end{array}$ \\
\hline $\begin{array}{l}\text { TOTAL FIHAL } \\
\text { DEGA ND SECTORS }\end{array}$ & 20,427 & 2.310 & 70.835 & 35.737 & - & 108,578 & 50,723 & 32.643 & 321,253 & G18 \\
\hline $\begin{array}{l}\text { TRANSPORHATIOY } \\
\text { BLECTRICITY GEH } \\
\text { PETROLBOA PRODOCTS } \\
\text { NATORAL GAS } \\
\text { SYH-G AS }\end{array}$ & $\begin{array}{r}2,5.63 \\
- \\
-\end{array}$ & $\begin{array}{r}67 \\
\div \\
-\end{array}$ & $\begin{array}{l}- \\
-\end{array}$ & $\begin{array}{l}\overline{-} \\
\overline{-}\end{array}$ & $\begin{array}{l}- \\
-\end{array}$ & $\begin{array}{r}327 \\
- \\
-\end{array}$ & $\begin{array}{r}41,117 \\
- \\
-\end{array}$ & $\begin{array}{r}-13,589 \\
= \\
=\end{array}$ & $\begin{array}{r}30.485 \\
= \\
=\end{array}$ & $9 \%$ \\
\hline $\begin{array}{l}\text { BET FOEL OSBD } \\
\text { IN TR AKSFOB AATIOA }\end{array}$ & 2,563 & 67 & - & - & - & 327 & 41,117 & $-13,589$ & 30.485 & $9 x$ \\
\hline $\begin{array}{l}\text { TOTAL GROSS ELORS } \\
\text { LOSSES } \varepsilon \text { OUISSIONS }\end{array}$ & $\begin{array}{r}22.790 \\
1.396\end{array}$ & $\begin{array}{r}2,377 \\
-24\end{array}$ & $\begin{array}{l}70,835 \\
-2,242\end{array}$ & $\begin{array}{l}35,737 \\
-9,688\end{array}$ & - & $\begin{array}{r}108, \leq 05 \\
<12\end{array}$ & $\begin{array}{r}91,840 \\
1,527\end{array}$ & $\begin{array}{r}32,643 \\
2,610\end{array}$ & $\begin{array}{r}351,738 \\
1,991\end{array}$ & $\begin{aligned} 998 \\
1 \%\end{aligned}$ \\
\hline TOTAL NET USLGB & 24,386 & $2,3 \leq 2$ & 68,592 & 34.048 & - & 109,317 & 93,367 & 21,664 & 353,729 & \\
\hline
\end{tabular}

\section{SUPPLY OP ENERGY \\ POSSIL POEI: \\ GYDROEL BCTEIC \\ NOCLB AR \\ GEO. \& SOLAR}

-
$\vdots$
$\vdots$

$\div$

$\therefore \quad$ : $\quad$ :

12

-

-

FET IGPORTS
OP BEGIOR


REGIONAL BHERGY BALANCE STATBAENT

BY SECTOR AND POEL TYPE

\begin{tabular}{|c|c|c|c|c|c|c|c|c|c|}
\hline SBCTOR & $\begin{array}{l}\text { DISTILLATE } \\
\text { OIL }\end{array}$ & $\begin{array}{l}\text { RESI DUAL } \\
\text { OIL }\end{array}$ & $\begin{array}{l}\text { GASOL INE } \\
\text { (ALI ONI }\end{array}$ & $\begin{array}{l}\text { HYDRO- } \\
\text { CARBOHS } \\
\text { TS IA } 10 * * 9\end{array}$ & $\begin{array}{c}\text { CRODE } \\
\text { OIL } \\
\text { BTO SS) }\end{array}$ & $\begin{array}{l}\text { MAT URAL } \\
\text { GAS }\end{array}$ & COAL & ELECTBICITY & $\begin{array}{r}\text { SECTOR } \\
\text { TOTAL }\end{array}$ \\
\hline $\begin{array}{l}\text { PIHAL DBYAND SBCTORS } \\
\text { RESIDE RTIAL, COAG. } \\
\text { IBDOSTRIAL } \\
\text { TRABSPORTATIOA } \\
\text { GISCELLABOOS USBS }\end{array}$ & $\begin{array}{r}14.428 \\
5.737 \\
14.874 \\
252\end{array}$ & $\begin{array}{r}793 \\
3,129 \\
47 \\
329\end{array}$ & 110,335 & $\begin{array}{r}19.469 \\
35.795 \\
7.699 \\
511\end{array}$ & $\begin{array}{l}- \\
-\end{array}$ & $\begin{array}{r}106.397 \\
74.535 \\
3,189 \\
5,457\end{array}$ & $\begin{array}{r}5,516 \\
106,986 \\
25 \\
-\end{array}$ & $\begin{array}{r}26,911 \\
26,361 \\
28 \\
671\end{array}$ & $\begin{array}{r}173,514 \\
252,543 \\
136,197 \\
7,220\end{array}$ \\
\hline $\begin{array}{l}\text { TOTAL PIRAL } \\
\text { DEGABD SBCTORS }\end{array}$ & 35,291 & 4,298 & 110.335 & 63,474 & - & 189,578 & 112.527 & 53.971 & 569.474 \\
\hline $\begin{array}{l}\text { TBAHSPORHATION } \\
\text { ELECTRICITY GBE. } \\
\text { PETROLEOA PRODOCTS } \\
\text { HATORAL GAS } \\
\text { SYB-GAS }\end{array}$ & $\begin{array}{r}1.424 \\
-2.039 \\
-\end{array}$ & $\begin{array}{r}422 \\
-781 \\
- \\
-\end{array}$ & $\begin{array}{r}-3,908 \\
-\end{array}$ & $\begin{array}{r}-1.254 \\
-\end{array}$ & $\begin{array}{r}7.683 \\
-\end{array}$ & $\begin{array}{r}2,449 \\
350 \\
- \\
-\end{array}$ & $\begin{array}{r}144.796 \\
- \\
-\end{array}$ & $\begin{array}{r}-41.594 \\
- \\
-\end{array}$ & $\begin{array}{r}107.497 \\
51 \\
- \\
-\end{array}$ \\
\hline $\begin{array}{l}\text { BET FOEL OSBD } \\
\text { IR TRABSPORAATIOH }\end{array}$ & -615 & -359 & $-3,908$ & $-1,254$ & 7,683 & 2.799 & 144.796 & $-41,594$ & 107,548 \\
\hline $\begin{array}{l}\text { COTAL GROSS PLORS } \\
\text { LOSSES \& OAISSIOYS }\end{array}$ & $\begin{array}{r}36.715 \\
2,230\end{array}$ & $\begin{array}{r}4.720 \\
-47\end{array}$ & $\begin{array}{r}110,335 \\
-3,493\end{array}$ & $\begin{array}{r}63,474 \\
-2,999\end{array}$ & $\begin{array}{r}7.683 \\
11\end{array}$ & $\begin{array}{r}192.377 \\
728\end{array}$ & $\begin{array}{r}257.323 \\
4.278\end{array}$ & $\begin{array}{r}53,971 \\
4,376\end{array}$ & $\begin{array}{r}677.022 \\
5,025\end{array}$ \\
\hline
\end{tabular}

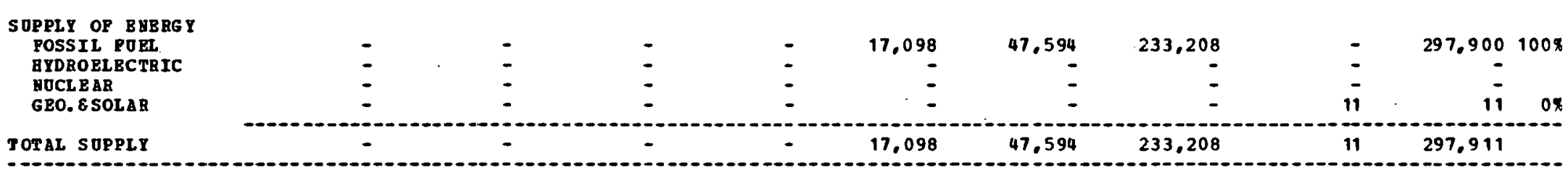

\begin{tabular}{|c|c|c|c|c|c|c|c|c|c|}
\hline OP REGION & 36.906 & 3.891 & 102,933 & 59.220 & -9.403 & 145.511 & 28,393 & $16,6 \varepsilon 2$ & 384,136 \\
\hline
\end{tabular}

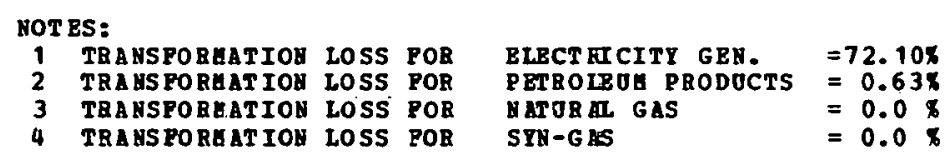


REgIONAL ERG BALARCE STATEGE HT

$$
\text { BY SECTOR AND FOEL TIPE }
$$

\begin{tabular}{|c|c|c|c|c|c|c|c|c|c|c|}
\hline SECEOR & $\begin{array}{l}\text { DISTILLATE } \\
\text { OIL }\end{array}$ & $\begin{array}{l}\text { RESI DJAL } \\
\text { OII }\end{array}$ & $\begin{array}{l}\text { GASOLTHE } \\
\text { (ALI O }\end{array}$ & $\begin{array}{l}\text { OZHER } \\
\text { EYDRO- } \\
\text { CARBOHS } \\
\text { IE IS 10**9 }\end{array}$ & $\begin{array}{l}\text { CR ODE } \\
\text { OIL } \\
\text { BTO.SI }\end{array}$ & $\begin{array}{l}\text { NAT OBAL } \\
\text { GAS }\end{array}$ & $\operatorname{COAL}$ & ELECTRICITY & $\begin{array}{l}\text { SECTOR } \\
\text { TOTAL }\end{array}$ & \\
\hline 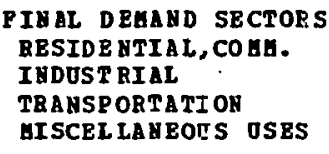 & $\begin{array}{r}1.037 \\
1.150 \\
3.680 \\
38\end{array}$ & $\begin{array}{r}390 \\
403 \\
- \\
202\end{array}$ & $18,72$. & $\begin{array}{r}1.786 \\
14.419 \\
607 \\
90\end{array}$ & $\begin{array}{l}- \\
\overline{-} \\
-\end{array}$ & $\begin{array}{r}14,853 \\
21,294 \\
3,438 \\
1,102\end{array}$ & $\begin{array}{r}1,430 \\
30 \% 405 \\
6 \\
-\end{array}$ & $\begin{array}{r}4,435 \\
4,399 \\
3 \\
8\end{array}$ & $\begin{array}{r}23,931 \\
72,070 \\
26,462 \\
1,440\end{array}$ & $\begin{array}{r}12 \% \\
35 \% \\
13 \% \\
18\end{array}$ \\
\hline $\begin{array}{l}\text { TOTAL PIHAL } \\
\text { DEAA AD SECTORS }\end{array}$ & 5,905 & 995 & 18,728 & 15.902 & - & 40,587 & $31, \varepsilon 41$ & 8,845 & 123,903 & $60 x$ \\
\hline $\begin{array}{l}\text { TRANSFORHATIJH } \\
\text { BLBCT RICITI GEN. } \\
\text { PETROLEOA PRODOCTS } \\
\text { NATORAL GAS } \\
\text { SYH-GAS }\end{array}$ & $\begin{array}{l}- \\
-\end{array}$ & $\begin{array}{r}272 \\
\vdots \\
-\end{array}$ & : & $=$ & $=$ & $\begin{array}{r}351 \\
- \\
-\end{array}$ & $\begin{array}{r}119,578 \\
= \\
-\end{array}$ & $\begin{array}{r}-38,699 \\
- \\
-\end{array}$ & $\begin{array}{r}81.502 \\
- \\
- \\
-\end{array}$ & $39 x$ \\
\hline $\begin{array}{l}\text { MET POEL OS BD } \\
\text { IN TBANSPORATION }\end{array}$ & - & 272 & - & - & - & 351 & 119,578 & $-38,699$ & 81.502 & $39 \%$ \\
\hline $\begin{array}{l}\text { TOTAL GROSS PLORS } \\
\text { LOSSES E OUISSI O\&S }\end{array}$ & $\begin{array}{r}5.905 \\
358\end{array}$ & $\begin{array}{r}1.267 \\
-12\end{array}$ & $\begin{array}{r}18,728 \\
-5.92\end{array}$ & $\begin{array}{r}16,902 \\
-798\end{array}$ & - & $\begin{array}{r}41.038 \\
1.55\end{array}$ & $\begin{array}{r}151,419 \\
2,517\end{array}$ & $\begin{array}{r}8.845 \\
707\end{array}$ & $\begin{array}{r}205,405 \\
2,334\end{array}$ & $\begin{array}{r}99 \% \\
18\end{array}$ \\
\hline
\end{tabular}

\begin{tabular}{|c|c|c|c|c|c|c|c|c|c|c|}
\hline $\begin{array}{l}\text { SOPPIY OP ENBBGY } \\
\text { POSSIL POEI }\end{array}$ & - & - & - & - & .623 & 228,083 & 825,369 & - & $1,058,575$ & 1008 \\
\hline BYDROELBCTRIC & - & - & - & - & - & -200 & - & 911 & 911 & 08 \\
\hline ROCL BAR & - & - & - & - & - & - & - & - & - & \\
\hline GEO.ESOLAE. & - & - & - & - & - & - & - & - & - & \\
\hline TOTAL SUPPLY & - & - & - & - & 4.623 & 228.083 & 825,869 & 911 & $1,059,486$ & \\
\hline
\end{tabular}

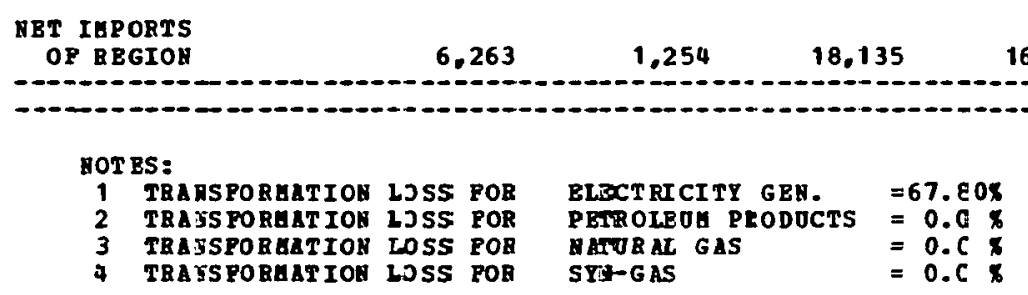


RBGIONAL BHBRY BALABCE STATEAENT

BY SECTOR AND FOEL TYPE

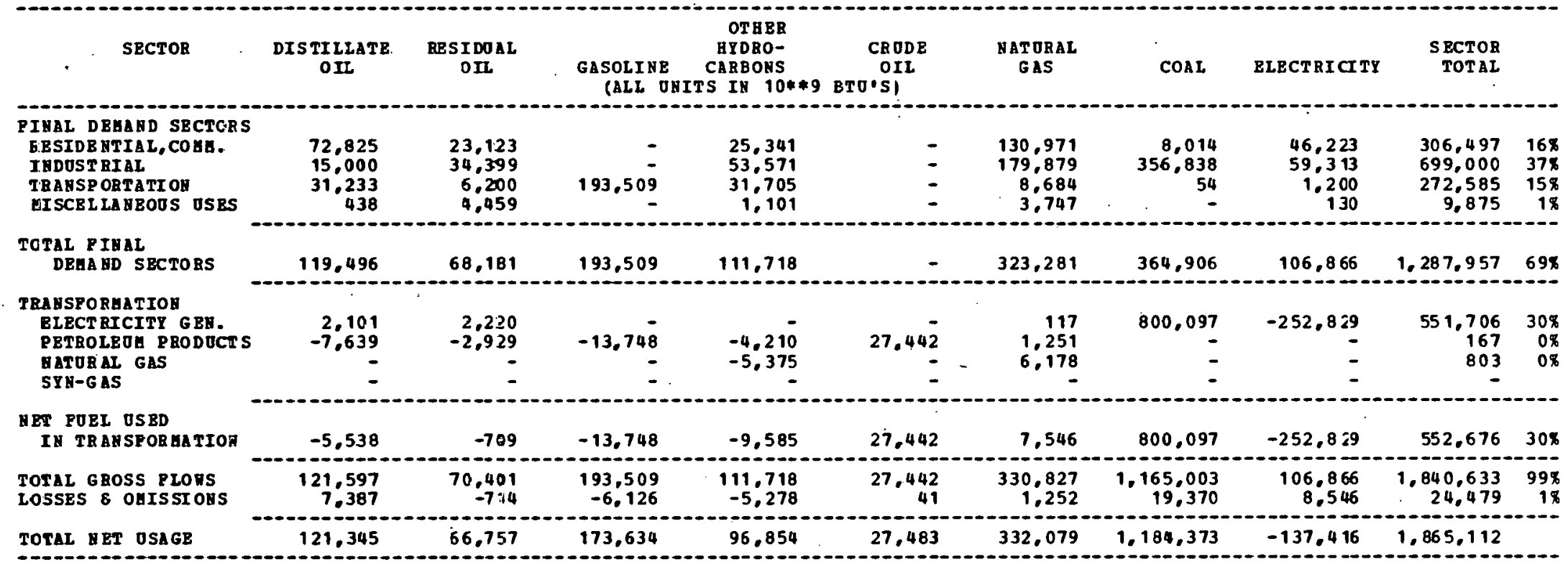

\section{SOPPLY OF EABRGY}

POSSIL POBL

BYDRO BL BCTRIC

UUCLEAR

GEO. 8 SOLAR

-
$\vdots$
-

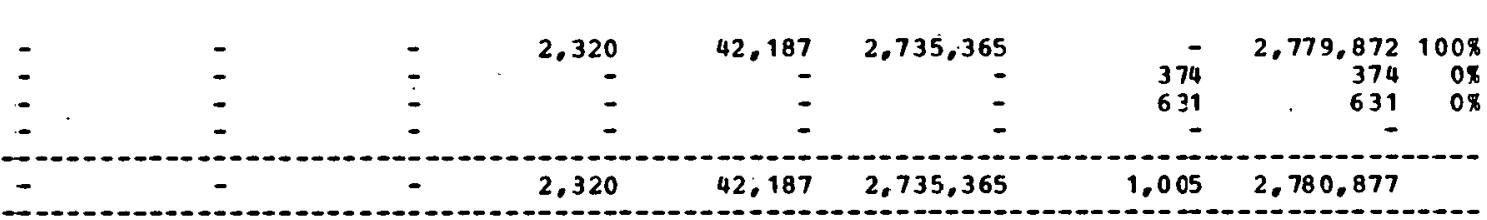

$-$

$-$

66.757

173,634

96,854

25.163

$289,892 \quad-1,550,991$

$-138,421$

$-915,764$

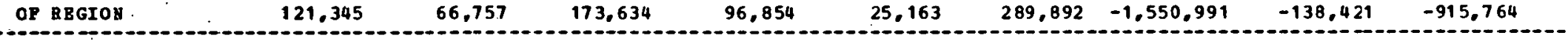

MOTES:

TRABSFORATION LOSS POB TRABSPORAATIOS LOSS POB TRASS PORAATION LOSS POR
TRAASFORATIOH LOSS POB

ELECTRICITI GEN.

ET BOLEO S PBODOCTS $=68.57$

政 $=0.58 \%$

$S Y N=G$ AS 


\begin{tabular}{|c|c|c|c|c|c|c|c|c|c|c|}
\hline & & & $\begin{array}{r}\text { REGIONAL } \\
\text { BT } 5\end{array}$ & $\begin{array}{l}\text { ENERGT BAL } \\
\text { ECTOR AUD }\end{array}$ & $\begin{array}{l}\text { ANCE STATE } \\
\text { POEL TYPE }\end{array}$ & & & & & \\
\hline SECTOA & $\begin{array}{l}\text { DISTILLATE } \\
\text { OIL }\end{array}$ & $\begin{array}{l}\text { BESIDOAL } \\
\text { O IL }\end{array}$ & $\begin{array}{l}\text { GASOLINE } \\
\text { (ALL OHI }\end{array}$ & $\begin{array}{l}\text { CTHER } \\
\text { ASDRJ- } \\
\text { CAEBONS } \\
\text { IS IN } 10 * * 9\end{array}$ & $\begin{array}{c}\text { CRODE } \\
\text { OIl } \\
\text { BTO'SI }\end{array}$ & $\begin{array}{l}\text { MAT URAL } \\
\text { उAS }\end{array}$ & COAL & ELECTRICITY & $\begin{array}{l}\text { SECTOR } \\
\text { TOTAL }\end{array}$ & \\
\hline 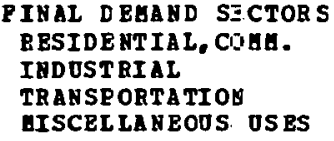 & $\begin{array}{r}3.613 \\
5.3 \varepsilon 2 \\
5.3 E 3 \\
1 C 2\end{array}$ & $\begin{array}{r}1,559 \\
5,339 \\
56 \\
344\end{array}$ & $45,31 \overline{-}$ & $\begin{array}{r}7.563 \\
: 1.196 \\
1,822 \\
223\end{array}$ & $\overline{-}$ & $\begin{array}{r}38,625 \\
60.537 \\
1.123 \\
966\end{array}$ & $\begin{array}{r}2,08 \equiv \\
80,216 \\
9 \\
-\end{array}$ & $\begin{array}{r}10,352 \\
21,142 \\
70 \\
111\end{array}$ & $\begin{array}{r}69.796 \\
183.810 \\
54.777 \\
1.746\end{array}$ & $\begin{array}{r}20 \% \\
54 \% \\
16 \% \\
1 \%\end{array}$ \\
\hline $\begin{array}{l}\text { TOTAL PINAL } \\
\text { DEHAND SBC TORS }\end{array}$ & 21,480 & 7,298 & 45,314 & 20.804 & - & 101,252 & 82,306 & 31,675 & 310,129 & $71 \%$ \\
\hline $\begin{array}{l}\text { TRANSPORUATIOH } \\
\text { RLECTRICITY GEN. } \\
\text { PETROLEOA PEODOCTS } \\
\text { NATORAL GAS } \\
\text { SYH-GAS }\end{array}$ & $\begin{array}{r}387 \\
- \\
-\end{array}$ & $\begin{array}{r}45 \\
- \\
-\end{array}$ & : & $\begin{array}{l}- \\
-\end{array}$ & $\begin{array}{l}- \\
-\end{array}$ & 178 & $\begin{array}{r}39,27.3 \\
= \\
=\end{array}$ & $\begin{array}{r}-12.106 \\
= \\
-\end{array}$ & $\begin{array}{r}27,782 \\
- \\
-\end{array}$ & 8\% \\
\hline $\begin{array}{l}\text { WET FOEL OSED } \\
\text { IN TRANSPORETION }\end{array}$ & 337 & 45 & - & - & - & 1.8 & $39,27 \mathrm{~B}$ & $-12,106$ & 27,782 & $8 x$ \\
\hline $\begin{array}{l}\text { TOTAL GROSS FLORS } \\
\text { LOSSES \& OBISSIONS }\end{array}$ & $\begin{array}{r}21.857 \\
1.328\end{array}$ & $\begin{array}{r}7.343 \\
-74\end{array}$ & $\begin{array}{l}45.314 \\
-1.434\end{array}$ & $\begin{array}{r}20,804 \\
-982\end{array}$ & - & $\begin{array}{r}101.43 C \\
384\end{array}$ & $\begin{array}{r}121.5 .94 \\
2.021\end{array}$ & $\begin{array}{r}31.675 \\
2,533\end{array}$ & $\begin{array}{r}337.911 \\
3,775\end{array}$ & $\begin{array}{l}99 x \\
1 \%\end{array}$ \\
\hline TOTAL & 23,195 & $T, 268$ & 43,877 & 19.821 & - & 101,814 & 123,605 & 22,102 & 341,686 & \\
\hline
\end{tabular}

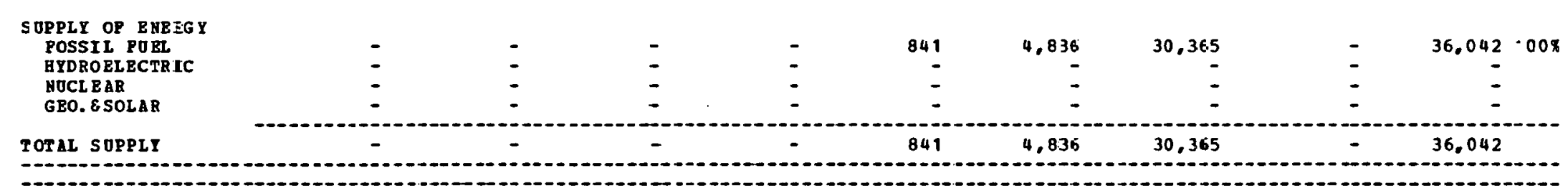

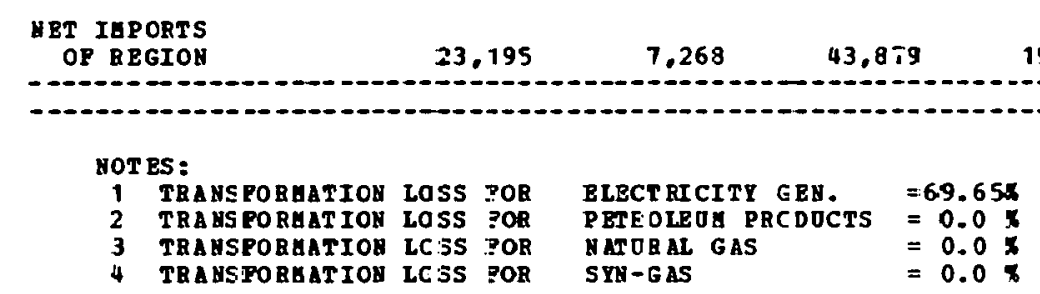


REGIONAL BNBRGY BALANCE STATELEHT

BY SECTOR AND POEL TYPE

\begin{tabular}{|c|c|c|c|c|c|c|c|c|c|c|}
\hline SECTOR & $\begin{array}{l}\text { DISTILLATE } \\
\text { OIL }\end{array}$ & $\begin{array}{l}\text { RESIDOAL } \\
\text { OIL }\end{array}$ & $\begin{array}{l}\text { GaSOLINE } \\
\text { (ALL O }\end{array}$ & $\begin{array}{l}\text { AYDRO- } \\
\text { CARBOHS } \\
\text { IS IM } \quad 10 * \% 9\end{array}$ & $\begin{array}{c}\text { CRODE } \\
\text { OIL } \\
\text { BTO'S) }\end{array}$ & $\begin{array}{l}\text { NATORAL } \\
\text { GAS }\end{array}$ & COAL & ELECTRICI TY & $\begin{array}{r}\text { SECTOR } \\
\text { TOTAL }\end{array}$ & \\
\hline $\begin{array}{l}\text { FIHAL DEHARD SECTORS } \\
\text { RESIDE HTIAL, COHE. } \\
\text { IHDUST RIAL } \\
\text { TRANSP ORTATI ON } \\
\text { GISCELLAHEOOS OSES }\end{array}$ & $\begin{array}{r}34,570 \\
20,979 \\
38,701 \\
589\end{array}$ & $\begin{array}{r}1.574 \\
12.327 \\
354 \\
512 \\
\end{array}$ & 255.956 & $\begin{array}{r}46,341 \\
95,249 \\
25,516 \\
1,182\end{array}$ & : & $\begin{array}{r}247.950 \\
211.544 \\
5.140 \\
7.099\end{array}$ & $\begin{array}{r}12.409 \\
271.716 \\
60 \\
-\end{array}$ & $\begin{array}{r}62,066 \\
91,366 \\
112 \\
942\end{array}$ & $\begin{array}{r}404,910 \\
703,181 \\
325,839 \\
10,324\end{array}$ & $\begin{array}{r}25 \% \\
43 \% \\
20 \% \\
1 \% \\
\end{array}$ \\
\hline $\begin{array}{l}\text { IOTAL PIYAL } \\
\text { DEHA YD SBCTORS }\end{array}$ & 94,839 & 14.767 & 255,956 & 168,288 & - & 471,733 & 284,185 & 154,486 & $1,444,254$ & $88 x$ \\
\hline $\begin{array}{l}\text { TRABSPORGATIOH } \\
\text { BLECT RICITY GEH. } \\
\text { PETBOLEOA PBODOCTS } \\
\text { MATORAL GAS } \\
\text { SYH-GAS }\end{array}$ & $\begin{array}{r}1.414 \\
-29.575 \\
-\end{array}$ & $\begin{array}{r}7.976 \\
-9.430 \\
- \\
-\end{array}$ & $\begin{array}{r}-67.831 \\
-\end{array}$ & $\begin{array}{r}-17,406 \\
-\end{array}$ & 119,049 & $\begin{array}{r}3,542 \\
5,427 \\
-\end{array}$ & $\begin{array}{r}246,504 \\
= \\
-\end{array}$ & $\begin{array}{r}-78.875 \\
- \\
=\end{array}$ & $\begin{array}{r}180,561 \\
234 \\
- \\
-\end{array}$ & $\begin{array}{r}11 \% \\
0 \times\end{array}$ \\
\hline $\begin{array}{l}\text { ERT FOBL OSBD } \\
\text { IB TRANSFOREATION }\end{array}$ & $-28,161$ & $-1,754$ & $-67,831$ & $-17,406$ & 119,049 & 8,969 & 246,504 & $-78,875$ & 180,795 & $11 \%$ \\
\hline $\begin{array}{l}\text { TOTAL G ROSS PLONS } \\
\text { LOSSES } 8 \text { OAISSI OHS }\end{array}$ & $\begin{array}{r}96,253 \\
5.847\end{array}$ & $\begin{array}{r}-22,143 \\
-230\end{array}$ & $\begin{array}{r}255,956 \\
-8,103\end{array}$ & $\begin{array}{r}168,288 \\
-7,951\end{array}$ & $\begin{array}{r}119.049 \\
180\end{array}$ & $\begin{array}{r}480.702 \\
1.820\end{array}$ & $\begin{array}{r}530.689 \\
8.823\end{array}$ & $\begin{array}{r}154,486 \\
12,355\end{array}$ & $\begin{array}{r}1,625,049 \\
12,741\end{array}$ & $\begin{array}{r}99 x \\
1 \%\end{array}$ \\
\hline
\end{tabular}

\begin{tabular}{|c|c|c|c|c|c|c|c|c|c|}
\hline $\begin{array}{l}\text { S OPPLY OF ENEBGY } \\
\text { POSSIL POEL }\end{array}$ & - & - & - & - & 32,573 & 48,083 & 151.413 & - & $232,069100 x$ \\
\hline HYDROBL BCTR IC & - & - & - & - & - & - & - & - & - \\
\hline BOCLEAB & - & - & - & - & - & - & - & - & - \\
\hline GEO. E SOLAR & - & - & - & - & - & - & - & - & - \\
\hline
\end{tabular}

10.0.

\begin{tabular}{l}
$\begin{array}{l}\text { MET IKPORTS } \\
\text { OR'RBGION }\end{array}$ \\
\hline
\end{tabular}

Notes:

TRAAS FORALT IOB LOSS POR TRA PSORHATIOH LOSS POR TRAASPORGATIOH LOSS. POR 
REGIJNAL EAERG BALARCE STATEMENT

BY SECTOR AND POEL TYPE

\begin{tabular}{|c|c|c|c|c|c|c|c|c|c|c|}
\hline SECT OR & $\underset{\text { OII }}{\text { DISTILIATE }}$ & $\begin{array}{c}\text { RBSIDOAL } \\
\text { OIL }\end{array}$ & $\begin{array}{l}\text { GASOLIHE } \\
\text { (ALL ONII }\end{array}$ & $\begin{array}{l}\text { EYDRO- } \\
\text { CLBBONS } \\
\text { S IH 10**9 }\end{array}$ & $\begin{array}{c}\text { CAODE } \\
\text { OIL } \\
\text { BTO'S) }\end{array}$ & $\begin{array}{l}\text { NAT URAL } \\
\text { GAS }\end{array}$ & $\operatorname{coAL}$ & ELECTRICITY & $\begin{array}{r}\text { SECTOR } \\
\text { TOTAL }\end{array}$ & \\
\hline $\begin{array}{l}\text { PIHAL DEHAHD SECTORS } \\
\text { RESIDERTIAL, COBH. } \\
\text { INDOSTRIAL } \\
\text { TRANSPORTATION } \\
\text { GISCELLANEOUS OSRS }\end{array}$ & $\begin{array}{r}2, \quad 20 \\
866 \\
1, \epsilon 37 \\
40\end{array}$ & $\begin{array}{r}81 \\
713 \\
5 \\
34\end{array}$ & 17.178 & $\begin{array}{r}3,110 \\
4.216 \\
448 \\
79\end{array}$ & $\begin{array}{l}- \\
-\end{array}$ & $\begin{array}{r}15.677 \\
8.412 \\
345 \\
433\end{array}$ & $\begin{array}{r}833 \\
9.404 \\
2 \\
-\end{array}$ & $\begin{array}{r}3,820 \\
4,636 \\
57\end{array}$ & $\begin{array}{r}25,844 \\
28,253 \\
19,615 \\
643\end{array}$ & $\begin{array}{l}338 \\
378 \\
258 \\
18\end{array}$ \\
\hline $\begin{array}{l}\text { TOTAL PIHAL } \\
\text { DEBA HD SECTORS }\end{array}$ & 4.863 & 842 & 17,178 & 7.853 & $=$ & 24.867 & 10.239 & 8.513 & 74,355 & $96 x$ \\
\hline $\begin{array}{l}\text { TRANSPOREATIOA } \\
\text { ELECTRICITY GEH. } \\
\text { PETROLEOA ERODOCTS } \\
\text { MATORAL GAS } \\
\text { SYH-GAS }\end{array}$ & $\begin{array}{r}-77, .535 \\
-\end{array}$ & $\begin{array}{r}6.2 \\
-24,753 \\
-\end{array}$ & $\begin{array}{r}-178,056 \\
-\end{array}$ & $\begin{array}{r}-45: .679 \\
-\end{array}$ & $\begin{array}{r}312,505 \\
-\end{array}$ & $\begin{array}{r}14.245 \\
-\end{array}$ & $\begin{array}{r}1,845 \\
- \\
-\end{array}$ & $\begin{array}{r}-389 \\
- \\
-\end{array}$ & $\begin{array}{r}1.518 \\
628 \\
- \\
-\end{array}$ & $\begin{array}{l}2 x \\
1 x\end{array}$ \\
\hline $\begin{array}{l}\text { RBT POEL OSBD } \\
\text { IE TRANSPOEGATION }\end{array}$ & $-77,535$ & -24.691 & $-178,056$ & -45.679 & 312.505 & 14.246 & 1.845 & -389 & 2,146 & $3 x$ \\
\hline $\begin{array}{l}\text { TOTAL GROSS TLORS } \\
\text { LOSSES } 6 \text { OAISSIOHS }\end{array}$ & $\begin{array}{r}4.363 \\
295 \\
\end{array}$ & $\begin{array}{r}904 \\
-9 \\
---9\end{array}$ & $\begin{array}{r}17.178 \\
-543\end{array}$ & $\begin{array}{r}7.853 \\
-371 \\
-\end{array}$ & $\begin{array}{r}312.505 \\
473\end{array}$ & $\begin{array}{r}39.113 \\
148\end{array}$ & $\begin{array}{r}12.084 \\
200\end{array}$ & $\begin{array}{r}8.513 \\
680 \\
-\end{array}$ & $\begin{array}{r}76.501 \\
875 \\
-\end{array}$ & $\begin{array}{r}998 \\
1 \times \\
-\end{array}$ \\
\hline
\end{tabular}

SOPPLY OP BHERGY

POSSIL FU RL.

GYDROBLECTAIC

GOCL BAR

TOTAL SOPPIY

$\begin{array}{cc}- & - \\ - & - \\ - & -\end{array}$

-

HBT IUPORTS

$-72,476$

$-23,858$

$-161.421$

$-33,197$

312,978

39.251

12.284

8,804

77.376

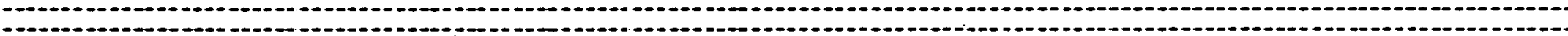

NOT ES :

1 TRAASPOBAATIOE LOSS POR

TRA AS POAAATIOH LOSS POR

TRAESPOBAaTIOR LOSS POR

BLECT RICITY GBN.

TRAGS POBAATION LOSS POR

PBODOCTS $=0.178$

$\begin{array}{ll}\text { SYY-GAS } & =0.0 \% \\ & =0.0 \%\end{array}$ 
REGIONAL ENERGY BALANCE STATEHE HT

BY SECTOR AND FOEL TYPE

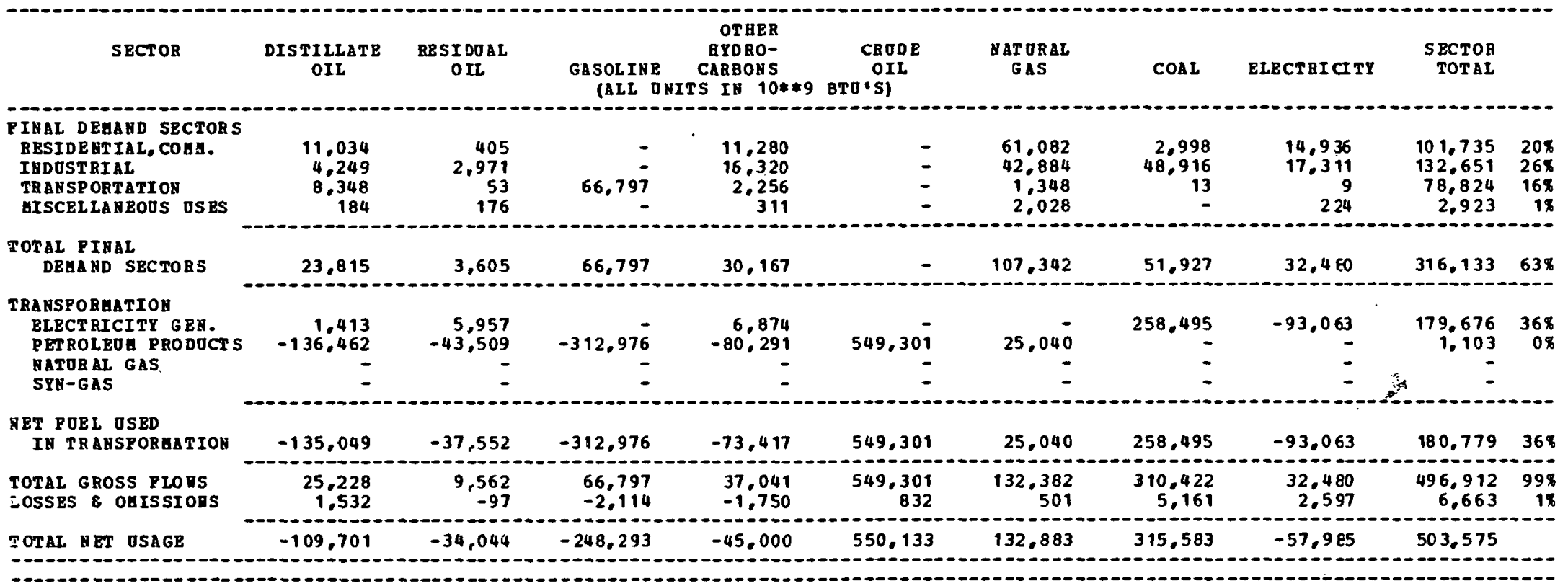

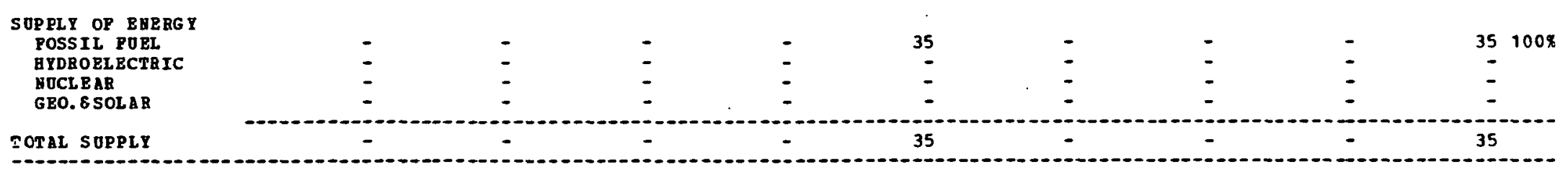

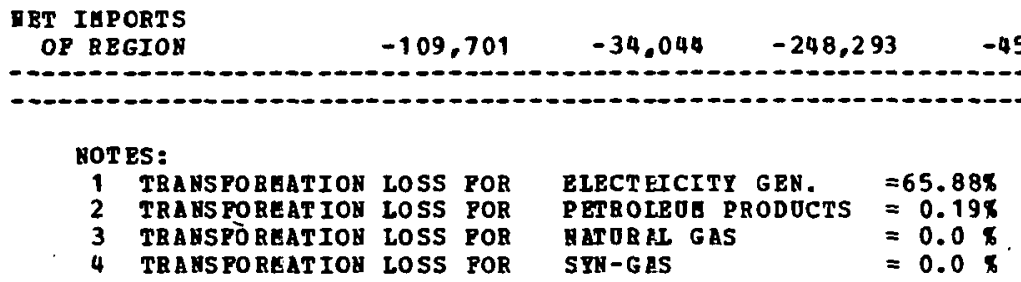




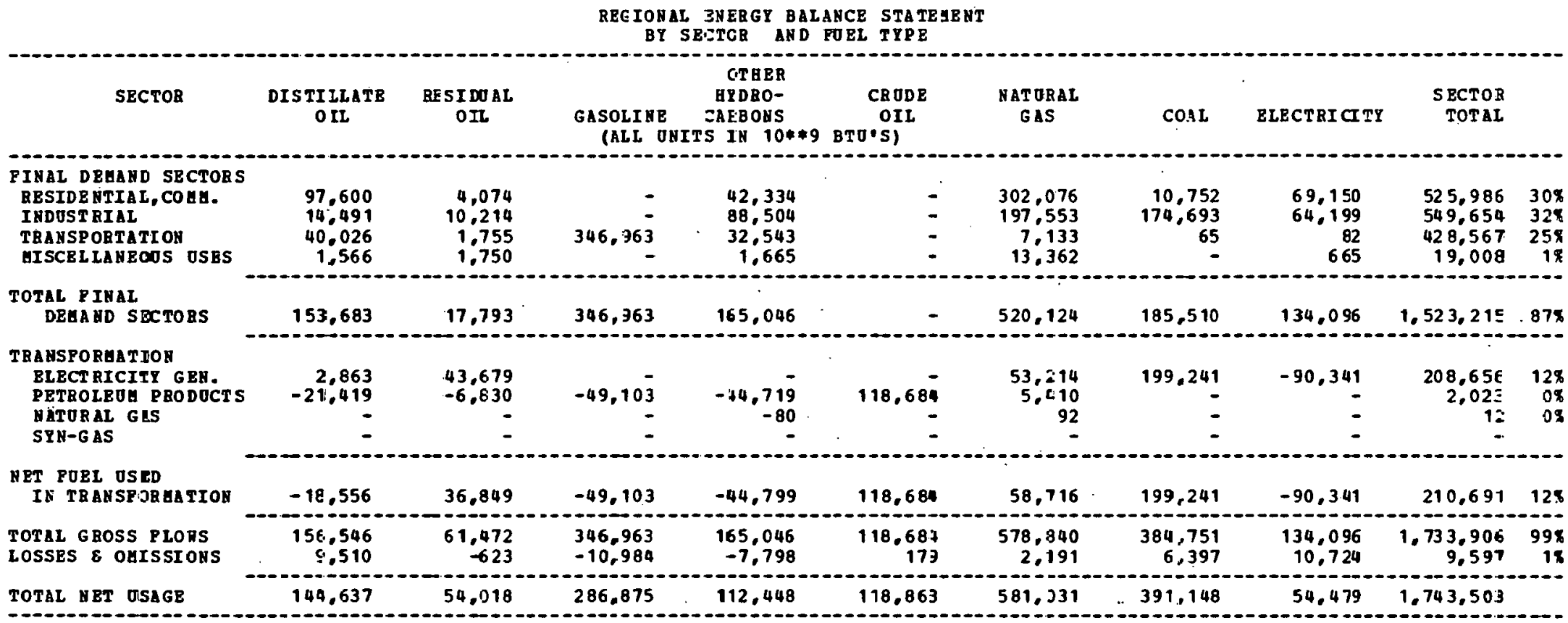

-

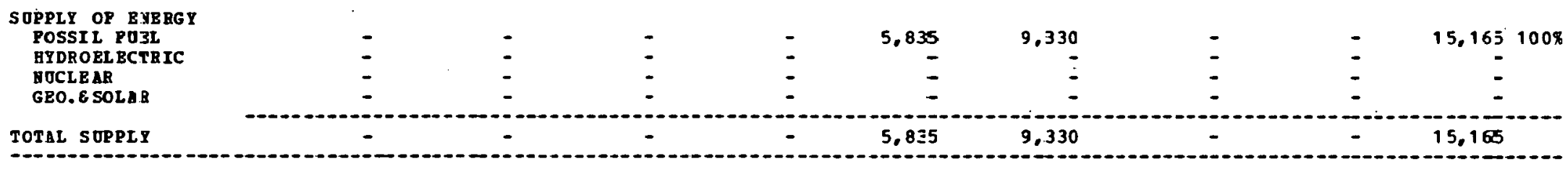

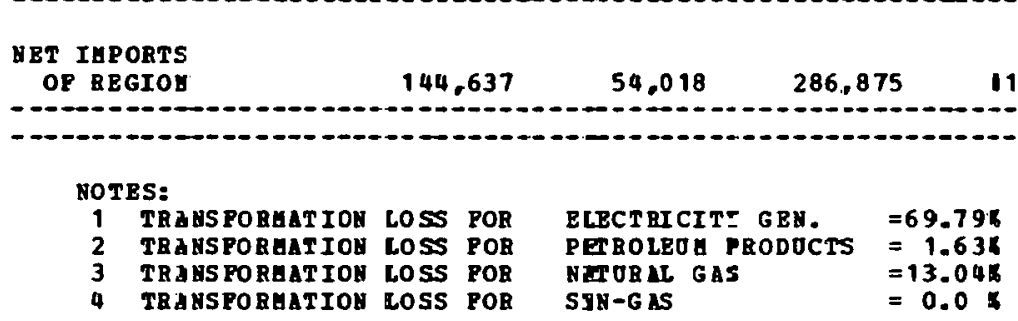




\begin{tabular}{|c|c|c|c|c|c|c|c|c|c|c|}
\hline & & & REGI & $\begin{array}{l}\text { BERRGT BA } \\
\text { CTOR AHD }\end{array}$ & $\begin{array}{l}\text { ANCE STAT } \\
\text { POBL TYPE }\end{array}$ & & & & & \\
\hline SBCTOR & $\begin{array}{l}\text { DISTILLATE } \\
\text { OIL }\end{array}$ & $\begin{array}{l}\text { BBSIDOAL } \\
\text { OIL }\end{array}$ & $\begin{array}{l}\text { GASOLINE } \\
\text { (ALI D }\end{array}$ & $\begin{array}{c}\text { OTBER } \\
\text { HYDRO- } \\
\text { CARBOHS } \\
\text { TS IB 10**9 }\end{array}$ & $\begin{array}{c}\text { CRODE } \\
\text { OIL } \\
\text { BTO'S) }\end{array}$ & $\begin{array}{l}\text { MAT ORAL } \\
\text { GAS }\end{array}$ & COAL & ELECTRICITY & $\begin{array}{l}\text { SECTOR } \\
\text { TOTAL }\end{array}$ & \\
\hline $\begin{array}{l}\text { PIHAL DEHAND SECTORS } \\
\text { RBSIDEHTIAL, COAG. } \\
\text { IRDOSTRIAL } \\
\text { TRABSPORTATIOH } \\
\text { UISCBLLABOOS OSBS }\end{array}$ & $\begin{array}{r}15.724 \\
3.075 \\
3.822 \\
252\end{array}$ & $\begin{array}{r}465 \\
2.423 \\
499 \\
339\end{array}$ & 55,900 & $\begin{array}{r}6,820 \\
23.622 \\
973 \\
268\end{array}$ & $\overline{-}$ & $\begin{array}{r}43,857 \\
28,017 \\
1,149 \\
3,587\end{array}$ & $\begin{array}{r}1,732 \\
26,167 \\
7 \\
-\end{array}$ & $\begin{array}{r}9,806 \\
9,076 \\
2 \\
178\end{array}$ & $\begin{array}{l}78.404 \\
92.380 \\
62.352 \\
4.624\end{array}$ & $\begin{array}{l}268 \\
308 \\
218 \\
28\end{array}$ \\
\hline $\begin{array}{l}\text { TOTAL PIBAL } \\
\text { DEHA GD SBCTOR5 }\end{array}$ & 22.873 & 3,726 & 55,900 & 31,683 & - & 76,610 & 27,906 & 19.062 & 237,760 & $78 \%$ \\
\hline $\begin{array}{l}\text { TEABSPOBAATIOH } \\
\text { BLECTRICITY GBY. } \\
\text { PETROLEOA PRODICTS } \\
\text { MATORAL GAS } \\
\text { SYH-GAS }\end{array}$ & $\begin{array}{r}1.929 \\
-22.006 \\
- \\
-\end{array}$ & $\begin{array}{r}9,220 \\
-7,016 \\
- \\
-\end{array}$ & $\begin{array}{r}-50.450 \\
-\end{array}$ & $\begin{array}{r}-45,946 \\
- \\
-\end{array}$ & 121,938 & $\begin{array}{r}3.511 \\
5.559 \\
-\end{array}$ & $\begin{array}{r}70,746 \\
= \\
-\end{array}$ & $\begin{array}{r}-22.934 \\
= \\
-\end{array}$ & $\begin{array}{r}62,472 \\
2,079 \\
-\end{array}$ & $\begin{array}{r}218 \\
1 \%\end{array}$ \\
\hline $\begin{array}{l}\text { MET POBL OSBD } \\
\text { IH TEAHSPOBGATIOR }\end{array}$ & -20.077 & 2.204 & -50.450 & $-45,946$ & 121,938 & 9,070 & 70,746 & $-22,9 \leq 4$ & 64.551 & 218 \\
\hline $\begin{array}{l}\text { TOTAL GROSS PLONS } \\
\text { LOSSES } 8 \text { OAISSIOUS }\end{array}$ & $\begin{array}{r}24,802 \\
1,506\end{array}$ & $\begin{array}{r}12,946 \\
-131\end{array}$ & $\begin{array}{l}55,900 \\
-1,769\end{array}$ & $\begin{array}{l}31,683 \\
-1,496\end{array}$ & $\begin{array}{r}121,938 \\
184\end{array}$ & $\begin{array}{r}85.680 \\
324\end{array}$ & $\begin{array}{r}98,652 \\
1,640\end{array}$ & $\begin{array}{r}19.062 \\
1.524\end{array}$ & $\begin{array}{r}302,311 \\
1,782\end{array}$ & $\begin{array}{r}998 \\
1 \%\end{array}$ \\
\hline TOTAL BBI & 4.302 & 5,798 & 3,680 & $-15,759$ & 122,122 & 86,004 & 100.292 & $-2,347$ & 304.093 & \\
\hline
\end{tabular}

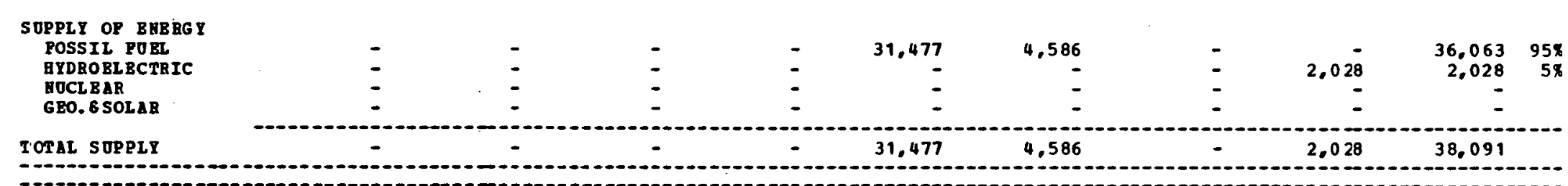

\section{BTT IHPORTS}

4,302

5,798

3,680

90,645

100,292

$-4,375$

266,002

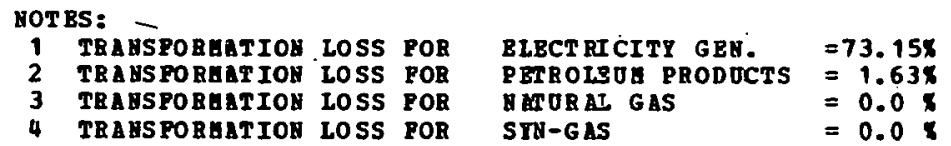


REGIONAL BUIBSI BALARCE STATEGZNT

BY SECTOR AOX FOEL TYPE

\begin{tabular}{|c|c|c|c|c|c|c|c|c|c|c|}
\hline SECTOR & $\begin{array}{c}\text { DISEILLATE } \\
\text { OIb }\end{array}$ & $\begin{array}{l}\text { RESTDOAL } \\
\text { OII }\end{array}$ & $\begin{array}{l}\text { GASOLIHE } \\
\text { (ALL O }\end{array}$ & $\begin{array}{l}\text { MYDBO- } \\
\text { CIRBDSS } \\
\text { TS IN 10**9 }\end{array}$ & $\begin{array}{c}\text { CRODE } \\
\text { OIl } \\
\text { BTO०S) }\end{array}$ & $\begin{array}{l}\text { NAT ORAI } \\
\text { GAS }\end{array}$ & $\cos L$ & ELECTRI CI TY & $\begin{array}{l}\text { SECTOR } \\
\text { TOTAL }\end{array}$ & \\
\hline 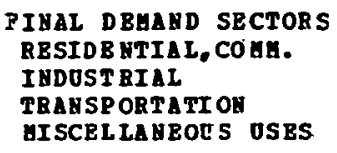 & $\begin{array}{r}21.787 \\
4.068 \\
6.383 \\
350\end{array}$ & $\begin{array}{r}801 \\
2,819 \\
183 \\
379\end{array}$ & $77.45 \overline{-}$ & $\begin{array}{r}9.450 \\
24: .093 \\
2.671 \\
372\end{array}$ & $\begin{array}{l}- \\
-\end{array}$ & $\begin{array}{r}64,717 \\
43,424 \\
1,592 \\
2, \in 80\end{array}$ & $\begin{array}{r}2,430 \\
36,523 \\
11 \\
-\end{array}$ & $\begin{array}{r}14.688 \\
13.309 \\
4 \\
133\end{array}$ & $\begin{array}{r}113,843 \\
124,236 \\
88.797 \\
3.914\end{array}$ & $\begin{array}{r}28 \% \\
318 \\
228 \\
18\end{array}$ \\
\hline $\begin{array}{l}\text { TOTAL PINAL } \\
\text { DEHA ND SBCTORS }\end{array}$ & 33.088 & 4.182 & 77,453 & 36,586 & - & 112,013 & $38,9 \cdot 34$ & 28,134 & 330,790 & $83 \%$ \\
\hline $\begin{array}{l}\text { TRAHSPOREATIOH } \\
\text { BLECT RICITY GEH. } \\
\text { PBTROLEOA PRODOCT S } \\
\text { MATORAL GAS } \\
\text { SYH-GAS }\end{array}$ & $\begin{array}{r}1.304 \\
-2.142 \\
=\end{array}$ & $\begin{array}{r}152 \\
-683 \\
-\end{array}$ & $\begin{array}{r}-4,911 \\
-\end{array}$ & $\begin{array}{r}- \\
-4.472 \\
-.734\end{array}$ & $\begin{array}{r}11,869 \\
-\end{array}$ & $\begin{array}{r}3,223 \\
541 \\
1,953\end{array}$ & $\begin{array}{r}88,427 \\
= \\
=\end{array}$ & $\begin{array}{r}-26.722 \\
- \\
-\end{array}$ & $\begin{array}{r}66.384 \\
202 \\
259 \\
-\end{array}$ & $\begin{array}{r}17 \% \\
08 \\
08\end{array}$ \\
\hline $\begin{array}{l}\text { NET FOBL USBD } \\
\text { IH TBABSPORBA TION }\end{array}$ & -838 & -531 & $-4,911$ & $-6,206$ & 11.869 & 5,757 & 88,427 & $-26,722$ & 66.845 & 178 \\
\hline $\begin{array}{l}\text { TOTAL GROSS FLORS } \\
\text { LOSSES } \& \text { OUISSIOHS }\end{array}$ & $\begin{array}{r}34.392 \\
2.089\end{array}$ & $\begin{array}{r}4.334 \\
-43\end{array}$ & $\begin{array}{l}77,453 \\
-2,452\end{array}$ & $\begin{array}{l}36,586 \\
-1,728\end{array}$ & $\begin{array}{r}11.869 \\
17\end{array}$ & $\begin{array}{r}118,170 \\
447\end{array}$ & $\begin{array}{r}127, \vdots 61 \\
2,117\end{array}$ & $\begin{array}{r}28,134 \\
2,250\end{array}$ & $\begin{array}{r}397.635 \\
2.697\end{array}$ & 998 \\
\hline
\end{tabular}

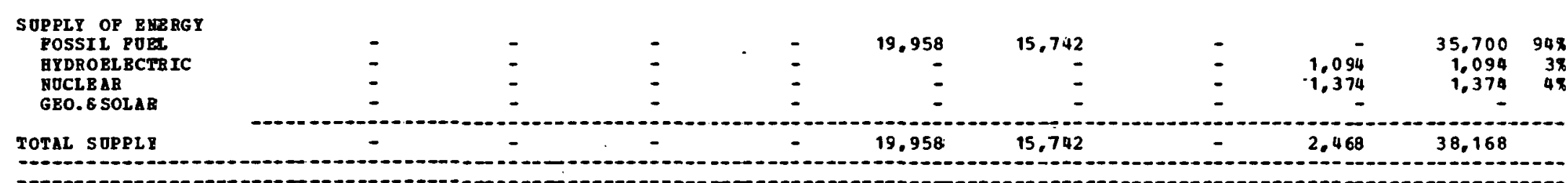

\section{HET IAPORTS}

OP RBGIO\&

34,339

3,607

70,089

28.651

$-8,071$

102.8 .75

129.478

1,194

362,164

HOTBS:

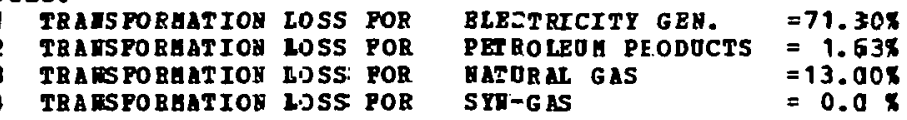


REGIONAL ENERGY BALAHCE STATEMEAT

BY SECTOR AND POEL TYPE

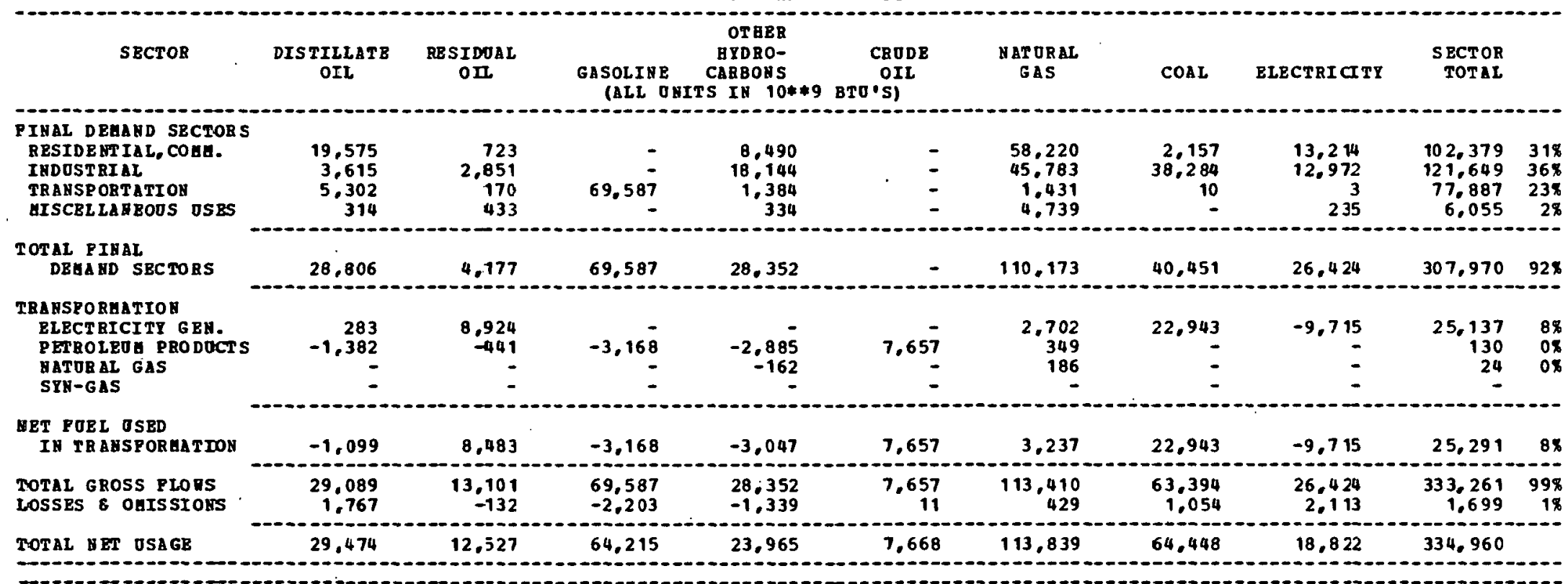

\begin{tabular}{|c|c|c|c|c|c|c|c|c|c|}
\hline $\begin{array}{l}\text { SOPPLY OP EHERG } \\
\text { POSSIL POEL } \\
\text { HYDROELECTRIC } \\
\text { HOCLEAR } \\
\text { GEO.\& SOLAR }\end{array}$ & $\bar{E}$ & $\bar{E}$ & $\begin{array}{l}E \\
E\end{array}$ & E & $\begin{array}{r}26.976 \\
- \\
-\end{array}$ & $\begin{array}{r}18,109 \\
= \\
=\end{array}$ & $\overline{-}$ & 6.600 & $\begin{array}{r}45.085 \\
24 \\
6.600 \\
-\end{array}$ \\
\hline
\end{tabular}

\section{NET IAPORTS}

29.474

12,527

64,215

23,965

$-19.307$

95,730

64,448

12,198

283,251

NOT ES:

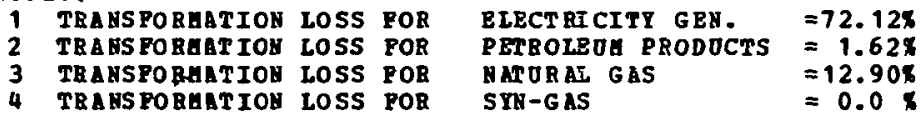


REG IONAL 3NERGY BALANCE STATEHENT BY SBETOR ABD FOBL TYPE

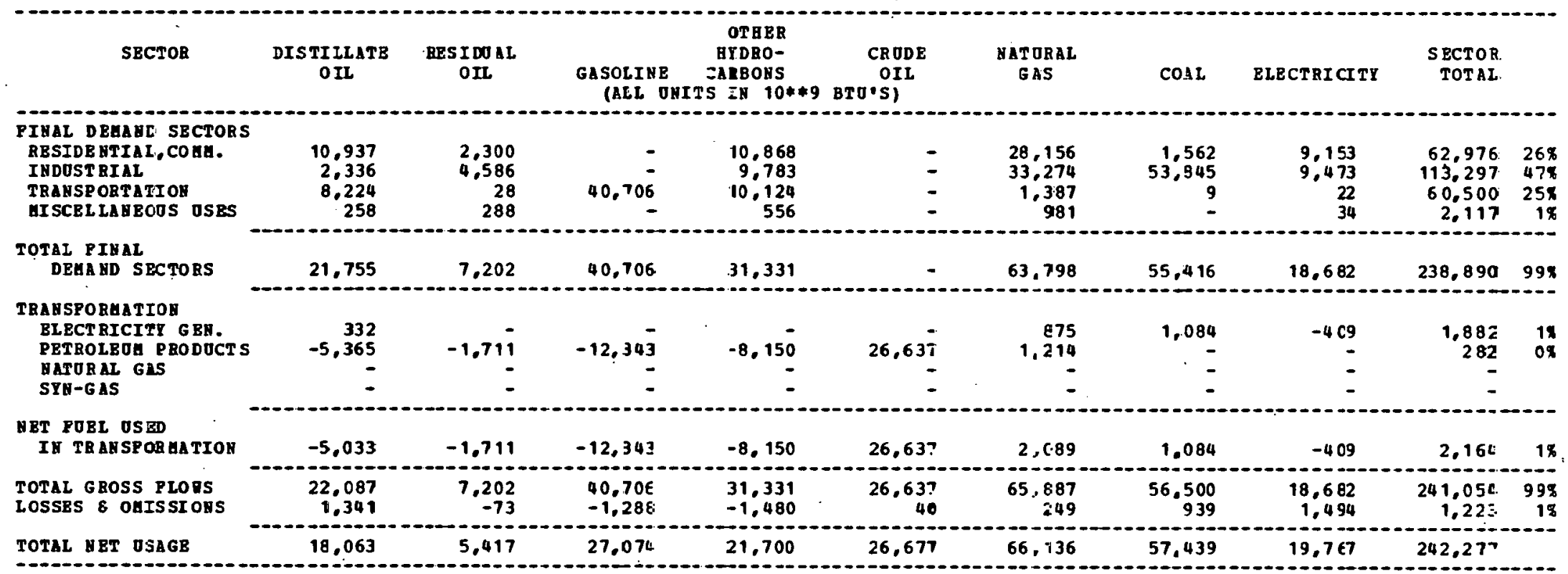

\section{SOPPLY OP ERERGY}

FOSSI 1 POBL

GTDROEL BCTRIC

HOCLBAR
GBO. 8 SOLAB

TOTAL SOPPLY

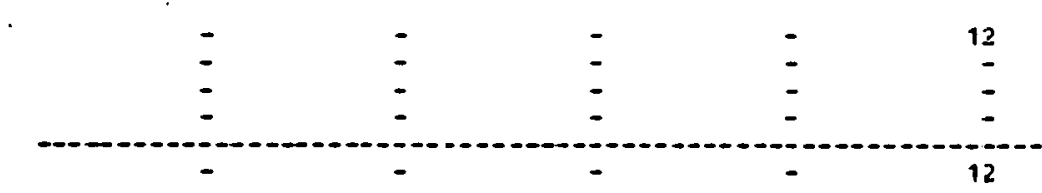

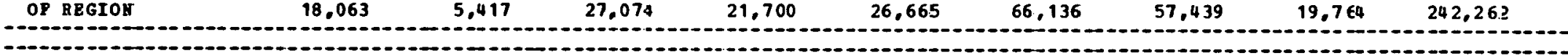

\section{NOTBS:}

TRANS PORAATIOH LOSS POR TRABSPORAATIOH LOSS POR

BI.ECTRICITY GEN.

TRANSPORAATIOS TOSS POB

$=82.158$
$=0.35$

TRABSPORAATIOA HOSS POB

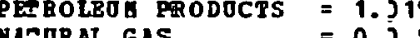

$\begin{array}{lll}\text { SID-GAS } & =0.3 \mathrm{~s}\end{array}$ 
RBGIONAL BHERGY BALANCE STATEGENT

BT SECTOR AND FOBL TYPE

\begin{tabular}{|c|c|c|c|c|c|c|c|c|c|c|}
\hline SECTOR & $\begin{array}{c}\text { DISIILLATE } \\
\text { OIL }\end{array}$ & $\begin{array}{l}\text { RES I DOAL } \\
\text { OII }\end{array}$ & $\begin{array}{l}\text { GASOLIHE } \\
\text { (ALL OHI }\end{array}$ & $\begin{array}{l}\text { OTHER } \\
\text { HYDBO- } \\
\text { CARBOHS } \\
\text { TS IN 10**9 }\end{array}$ & $\begin{array}{c}\text { CR ODE } \\
\text { OIL } \\
\text { BTO'SI }\end{array}$ & $\begin{array}{l}\text { MATUEAL } \\
\text { GAS }\end{array}$ & COAL & ELECTRICI TY & $\begin{array}{l}\text { SECTOR } \\
\text { TOTAL }\end{array}$ & \\
\hline $\begin{array}{l}\text { PIHAL DEGAHD SECTORS } \\
\text { RESIDE RTIAL, COBU. } \\
\text { IHDOST RIAL } \\
\text { TRABSPORTATI ON } \\
\text { GISCELLAHEOS OSES }\end{array}$ & $\begin{array}{r}102,357 \\
28,642 \\
i 09,034 \\
1,712\end{array}$ & $\begin{array}{r}42.194 \\
94.357 \\
1.312 \\
6.163\end{array}$ & 475,324 & $\begin{array}{r}112,218 \\
226,673 \\
102,558 \\
6,837\end{array}$ & $\begin{array}{l}\overline{-} \\
\overline{-}\end{array}$ & $\begin{array}{r}482,677 \\
418,940 \\
16,478 \\
16,833\end{array}$ & $\begin{array}{r}20,088 \\
291.443 \\
149 \\
-\end{array}$ & $\begin{array}{r}126,489 \\
108,106 \\
1,243 \\
3,193\end{array}$ & $\begin{array}{r}886,023 \\
1,168,161 \\
706,098 \\
34,738\end{array}$ & $\begin{array}{r}28 \% \\
37 \% \\
22 \% \\
1 \%\end{array}$ \\
\hline $\begin{array}{l}\text { TOTAL PIBAL } \\
\text { DEHABD SBCTORS }\end{array}$ & 241.745 & 144.026 & 475,324 & 448,286 & - & 934,958 & 311,680 & 239,031 & $2,795,020$ & 898 \\
\hline $\begin{array}{l}\text { TRAHSFOREATIOH } \\
\text { ELECT RICITY GBE. } \\
\text { PETROLBOA PBODOCTS } \\
\text { HATORAL GAS } \\
\text { SYH-G AS }\end{array}$ & $\begin{array}{r}19.157 \\
-391.802 \\
-\end{array}$ & $\begin{array}{r}31,707 \\
-124.922 \\
-\end{array}$ & $\begin{array}{r}-901,524 \overline{-} \\
-\end{array}$ & $\begin{array}{r}-466,332 \\
-\end{array}$ & $\begin{array}{r}1.814 .717 \\
-\end{array}$ & $\begin{array}{r}31,559 \\
82,725 \\
= \\
=\end{array}$ & $\begin{array}{r}373,428 \\
= \\
=\end{array}$ & $\begin{array}{r}-137,593 \\
- \\
-\end{array}$ & $\begin{array}{r}318,258 \\
12,862 \\
=\end{array}$ & $\begin{array}{r}10.8 \\
0.8\end{array}$ \\
\hline $\begin{array}{l}\text { MET POEL OSBD } \\
\text { IB TBANSPORGATION }\end{array}$ & -372.645 & $-93,215$ & $-901,524$ & $-466,332$ & $1,814,717$ & 114,284 & 373,428 & $-137,593$ & 331,120 & $11 x$ \\
\hline $\begin{array}{l}\text { TOTAL GROSS ?LONS } \\
\text { LOSSBS } 8 \text { OHISSIOHS }\end{array}$ & $\begin{array}{r}260,902 \\
15,849\end{array}$ & $\begin{array}{r}175,733 \\
-1.783\end{array}$ & $\begin{array}{l}475,324 \\
-15,048\end{array}$ & $\begin{array}{l}448,286 \\
-21,180\end{array}$ & $\begin{array}{r}1,814,717 \\
2,751\end{array}$ & $\begin{array}{r}1.049,212 \\
3,373\end{array}$ & $\begin{array}{r}685,108 \\
11, \equiv 91\end{array}$ & $\begin{array}{r}239.031 \\
19.116\end{array}$ & $\begin{array}{r}3,126,140 \\
15,069\end{array}$ & $\begin{array}{r}1008 \\
08\end{array}$ \\
\hline
\end{tabular}

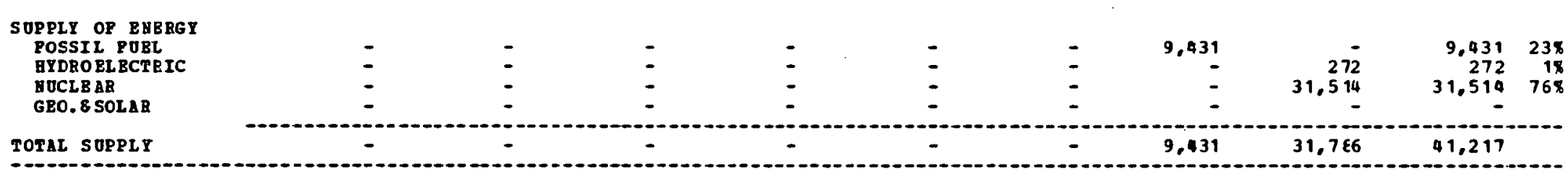

\section{MET IAPORTS}

OP REGION

\section{nOTES:}

\begin{tabular}{|c|c|c|c|c|}
\hline $\begin{array}{l}\text { TRA } \\
\text { TRA } \\
\text { TRA } \\
\text { TRA }\end{array}$ & $\begin{array}{l}\text { Los } \\
\text { Loss } \\
\text { Loss }\end{array}$ & $\begin{array}{l}\text { POR } \\
\text { FOR } \\
\text { POR }\end{array}$ & $\begin{array}{l}\text { BLBCTRICITY GEN. } \\
\text { PBT ROLEOH PBODUCTS } \\
\text { MATORAL GAS } \\
\text { STS-GAS }\end{array}$ & $\begin{array}{l}=59.828 \\
=0.638 \\
=0.0 \\
=0.0\end{array}$ \\
\hline
\end{tabular}


REGIOHAL ENERGY BALANCE STATEHENT

BY SECTOR AND POEL TYPE

\begin{tabular}{|c|c|c|c|c|c|c|c|c|c|c|}
\hline SECTOR & $\begin{array}{l}\text { DISTILLATE } \\
\text { OIL }\end{array}$ & $\begin{array}{l}\text { RESIDOAL } \\
\text { O IL }\end{array}$ & $\begin{array}{l}\text { GASOLINE } \\
\text { (ALL ONI }\end{array}$ & $\begin{array}{l}\text { BYDRO- } \\
\text { CARBO\&S } \\
\text { TS IN } 10 * * 9\end{array}$ & $\begin{array}{c}\text { CRODE } \\
\text { OIL } \\
\text { BTO'S) }\end{array}$ & $\begin{array}{l}\text { NAT ORAL } \\
\text { GAS }\end{array}$ & COAL & BLECTRICITY & $\begin{array}{l}\text { SECTOR } \\
\text { TOTAL }\end{array}$ & \\
\hline $\begin{array}{l}\text { PIHAL DEHAYD SBCTORS } \\
\text { RESIDEHTIAL, COBG. } \\
\text { INDOSTRIAL } \\
\text { TRAHSPORTATIOH } \\
\text { GISCBLLAGEOS OSBS }\end{array}$ & $\begin{array}{r}7.605 \\
950 \\
4.897 \\
117\end{array}$ & $\begin{array}{r}2.852 \\
2.469 \\
59 \\
498\end{array}$ & 36,984 & $\begin{array}{r}8,516 \\
8.113 \\
2,540 \\
529\end{array}$ & $\bar{z}$ & $\begin{array}{r}37.141 \\
14.643 \\
1.274 \\
1.341\end{array}$ & $\begin{array}{r}1,590 \\
7.996 \\
8 \\
-\end{array}$ & $\begin{array}{r}9.235 \\
3.924 \\
28 \\
275\end{array}$ & $\begin{array}{r}66,939 \\
38,095 \\
45,790 \\
2,760\end{array}$ & $\begin{array}{l}308 \\
17 \% \\
20 \% \\
1 \%\end{array}$ \\
\hline $\begin{array}{l}\text { TOTAL PINAL } \\
\text { DBEA AD SBCTORS }\end{array}$ & 13,569 & 5,878 & 36,984 & 19.698 & - & 54,399 & 9,594 & 13,462 & 153,584 & 698 \\
\hline $\begin{array}{l}\text { TRAHSPORHATIOH } \\
\text { ELECT RICITY GER. } \\
\text { PEYROLEOA PRODOCT S } \\
\text { HAYOR AL GAS } \\
\text { SYH-GAS }\end{array}$ & $\begin{array}{r}26 \\
-437 \\
-\end{array}$ & $\begin{array}{r}45 \\
-139 \\
- \\
-\end{array}$ & $\begin{array}{r}-1.005 \\
- \\
-\end{array}$ & -382 & 1.890 & $\begin{array}{r}5.473 \\
86 \\
- \\
-\end{array}$ & $\begin{array}{r}92,370 \\
:-\end{array}$ & $\begin{array}{r}-29.448 \\
- \\
-\end{array}$ & $\begin{array}{r}68,466 \\
13 \\
- \\
-\end{array}$ & $\begin{array}{r}31 \% \\
0 \%\end{array}$ \\
\hline $\begin{array}{l}\text { MBT FOEL OSED } \\
\text { IM TRARSPORAATION }\end{array}$ & -411 & -94 & -1.005 & -382 & 1.890 & 5,559 & 92,370 & $-29,448$ & 68,479 & $31 \%$ \\
\hline $\begin{array}{l}\text { TOTAL GROSS PLORS } \\
\text { LOSSES \& OUISSIOHS }\end{array}$ & $\begin{array}{r}13.595 \\
825\end{array}$ & $\begin{array}{r}5.923 \\
-60\end{array}$ & $\begin{array}{l}36,984 \\
-1,170\end{array}$ & $\begin{array}{r}19.698 \\
-930\end{array}$ & $\begin{array}{r}1.890 \\
2\end{array}$ & $\begin{array}{r}59.958 \\
227\end{array}$ & $\begin{array}{r}101,964 \\
1,695\end{array}$ & $\begin{array}{r}13,4 \in 2 \\
1,076\end{array}$ & $\begin{array}{r}222,063 \\
1,666\end{array}$ & $\begin{array}{r}998 \\
18\end{array}$ \\
\hline
\end{tabular}

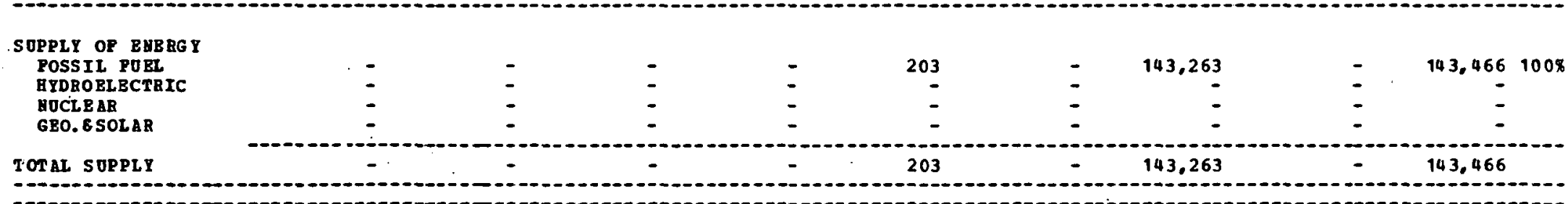

BBT IHPORTS
OR RBGIOB


REG IONAL BNBEGT BALALCE STATEKENT

BY SECTOE AND FURL TYPE

\begin{tabular}{|c|c|c|c|c|c|c|c|c|c|}
\hline SECTOR & $\begin{array}{c}D I S T I L E A T B \\
\text { OIE }\end{array}$ & $\begin{array}{l}\text { RES IDOAL } \\
\text { JIL }\end{array}$ & $\begin{array}{l}\text { GASOL:NE } \\
\text { (AL: ONI? }\end{array}$ & $\begin{array}{l}\text { HYDRO- } \\
\text { CARBONS } \\
\text { TS IL 10**9 }\end{array}$ & $\begin{array}{c}\text { CRODE } \\
\text { OIL } \\
\text { BTO'S) }\end{array}$ & $\begin{array}{l}\text { NATOEAL } \\
\text { GAS }\end{array}$ & $\operatorname{COAL}$ & ELECT RI CITY & $\begin{array}{r}\text { SECTOR } \\
\text { TOTAL }\end{array}$ \\
\hline $\begin{array}{l}\text { PIHAL DEHAND SECTORS } \\
\text { RESIDE NTIAL, COUG. } \\
\text { INDOSTRIAL } \\
\text { TRANSP ORTATI OH } \\
\text { UISCELLA LEOOS OSES }\end{array}$ & $\begin{array}{r}7,208 \\
2,975 \\
5,292 \\
178\end{array}$ & $\begin{array}{r}1.352 \\
1.962 \\
29 \\
34.6\end{array}$ & 40,059 & $\begin{array}{r}9.395 \\
13.560 \\
1.706 \\
636\end{array}$ & $\begin{array}{l}- \\
-\end{array}$ & $\begin{array}{r}32.574 \\
41.266 \\
2.172 \\
1.563\end{array}$ & $\begin{array}{r}1,117 \\
14,199 \\
7 \\
-\end{array}$ & $\begin{array}{r}8,222 \\
8.517 \\
20 \\
218\end{array}$ & $\begin{array}{r}59.908 \\
82.479 \\
49.295 \\
2.941\end{array}$ \\
\hline $\begin{array}{l}\text { TOTAL P IHAL } \\
\text { DEHAND SECTORS }\end{array}$ & 15,653 & 3.729 & 40,069 & 25.297 & - & 77.575 & 15,323 & 16,977 & 194,623 \\
\hline $\begin{array}{l}\text { TRANSPORHATIOH } \\
\text { BLECTRICITY GER. } \\
\text { PETROLEOH PBODOCTS } \\
\text { BATORAL GAS } \\
\text { SYN-GAS }\end{array}$ & $\begin{array}{r}238 \\
-\end{array}$ & $\begin{array}{r}775 \\
- \\
-\end{array}$ & $\begin{array}{l}\overline{-} \\
\overline{-}\end{array}$ & - & $\overline{-}$ & $\begin{array}{r}10,523 \\
= \\
-\end{array}$ & $\begin{array}{r}26,763 \\
- \\
-\end{array}$ & $\begin{array}{r}-10,8 \varepsilon 7 \\
- \\
-\end{array}$ & $\begin{array}{r}27,412 \\
- \\
-\end{array}$ \\
\hline $\begin{array}{l}\text { NET FOEL OSBD } \\
\text { IN TRANSFOAGATIOR }\end{array}$ & 238 & 775 & - & - & - & 10.523 & 26.763 & $-10,887$ & 27.412 \\
\hline $\begin{array}{l}\text { TOTAL GROSS FLOMS } \\
\text { LOSSBS \& OUISSIOHS }\end{array}$ & $\begin{array}{r}15.891 \\
965\end{array}$ & $\begin{array}{r}4.51) 4 \\
-45\end{array}$ & $\begin{array}{l}40,069 \\
-1,268\end{array}$ & $\begin{array}{l}25,297 \\
-1,195\end{array}$ & - & $\begin{array}{r}88,098 \\
333\end{array}$ & $\begin{array}{r}42,086 \\
699\end{array}$ & $\begin{array}{r}16,977 \\
1,357\end{array}$ & $\begin{array}{r}222.035 \\
846\end{array}$ \\
\hline
\end{tabular}

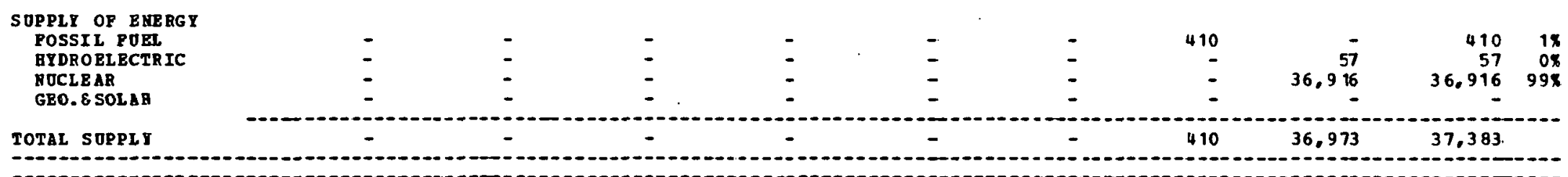

NET IMPOATS

16,856

4,458

38,800

24.101

88,431

42,375

$-29,525$

185,498

\begin{tabular}{|c|c|c|c|c|}
\hline 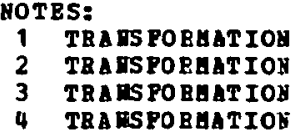 & $\begin{array}{l}\text { LOSS } \\
\text { IDSS } \\
\text { LDSS } \\
\text { LOSS }\end{array}$ & $\begin{array}{l}\text { POR } \\
\text { FOR } \\
\text { POR } \\
\text { POR }\end{array}$ & $\begin{array}{l}\text { BLEETRICITY GEH. } \\
\text { PEEROLEOU PE.ODOCTS } \\
\text { BALORAI. GAS } \\
\text { S YH-GAS }\end{array}$ & $\begin{array}{l}=71.578 \\
=0.0 \% \\
=0.08 \\
=0.08\end{array}$ \\
\hline
\end{tabular}




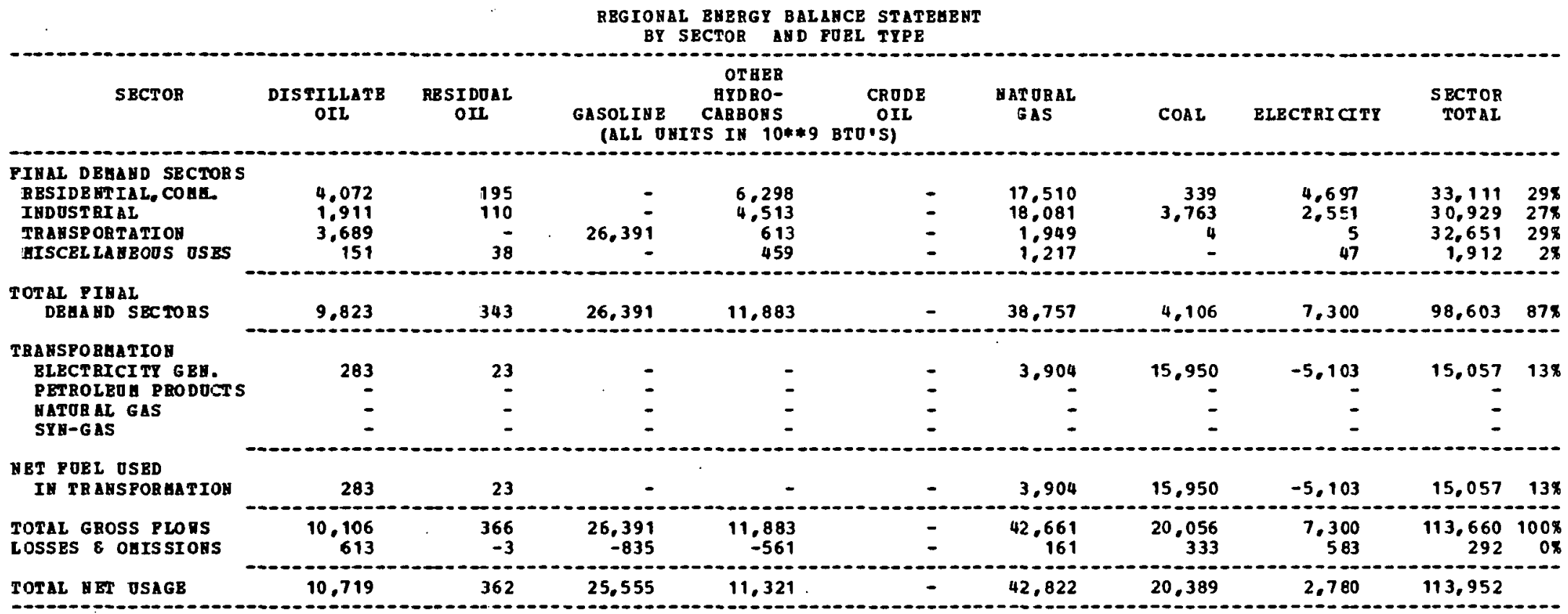

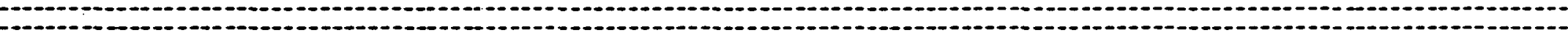

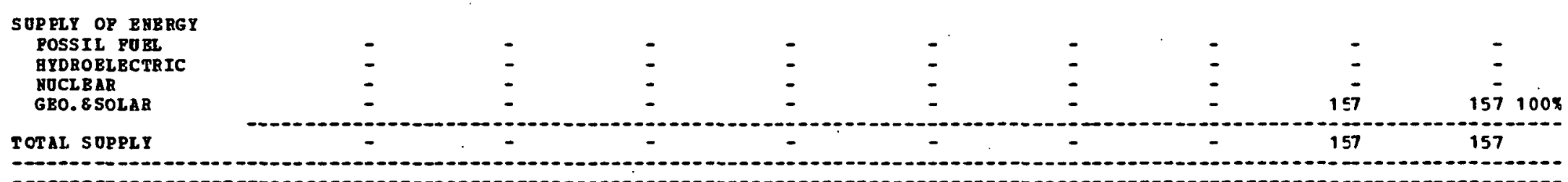

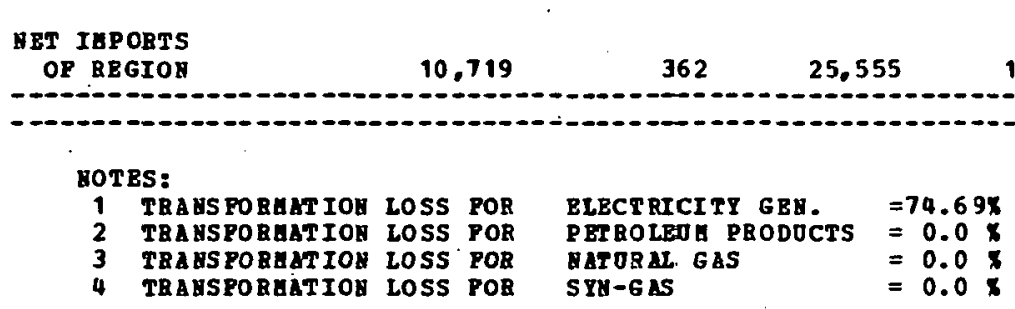


RBGIONAL BNEEGY BALAGCE STATEGENT

BY SBCTOE AHD POEL TYPE

\begin{tabular}{|c|c|c|c|c|c|c|c|c|c|c|}
\hline SECTOR & $\begin{array}{c}\text { DISTILLATE } \\
\text { OIL }\end{array}$ & $\begin{array}{l}\text { RESIDJEL } \\
\text { OII }\end{array}$ & $\begin{array}{l}\text { GASOL CH) } \\
\text { (ALL }\end{array}$ & $\begin{array}{l}\text { HYDRO- } \\
\text { CARBONS } \\
T \leq \text { II 10**9 }\end{array}$ & $\begin{array}{c}\text { CRODE } \\
\text { OIL } \\
\text { BTO०S) }\end{array}$ & $\begin{array}{l}\text { MATORAL } \\
\text { JAS }\end{array}$ & COAL & ELECTRI CITI & $\begin{array}{r}\text { SECTOR } \\
\text { TOTAL }\end{array}$ & \\
\hline 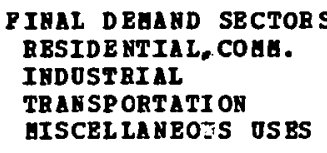 & $\begin{array}{r}4.798 \\
692 \\
2.847 \\
108\end{array}$ & $\begin{array}{r}192 \\
135 \\
10 \\
42\end{array}$ & 22.522 & $\begin{array}{r}5.447 \\
3.336 \\
565 \\
348\end{array}$ & $\overline{-}$ & $\begin{array}{r}13,739 \\
7.199 \\
1,360 \\
509\end{array}$ & $\begin{array}{r}496 \\
1.765 \\
3 \\
-\end{array}$ & $\begin{array}{r}3.879 \\
1.577 \\
34\end{array}$ & $\begin{array}{r}28.551 \\
14.704 \\
27.287 \\
1.141\end{array}$ & $\begin{array}{r}328 \\
16 \% \\
308 \\
18\end{array}$ \\
\hline $\begin{array}{l}\text { TOTAL PIHAL } \\
\text { DBYA SBD SBCTORS }\end{array}$ & 8.445 & 379 & 22,522 & 9.696 & - & 22,987 & 2.264 & 5,490 & 71,683 & $79 \%$ \\
\hline $\begin{array}{l}\text { TRAMSPORHATIOH } \\
\text { BLECTRICITT GBH. } \\
\text { PETROLEOH PRODOCTS } \\
\text { BATORAL GAS } \\
\text { SYH-GAS }\end{array}$ & = & $\begin{array}{l}18 \\
- \\
-\end{array}$ & - & i & $\begin{array}{l}\overline{-} \\
\overline{-}\end{array}$ & $\begin{array}{r}2,743 \\
- \\
-\end{array}$ & $\begin{array}{r}23,169 \\
- \\
-\end{array}$ & $\begin{array}{r}-7.805 \\
- \\
-\end{array}$ & $\begin{array}{r}18,260 \\
- \\
=\end{array}$ & $20 \%$ \\
\hline $\begin{array}{l}\text { HET POEL OSED } \\
\text { IN TEABSPCRAATIOA }\end{array}$ & 135 & 18 & - & $=$ & - & 2.743 & 23,169 & -7.805 & 18,260 & $20 \%$ \\
\hline $\begin{array}{l}\text { TOTAI GROSS PLONS } \\
\text { LOSSES \& OUISSIOAS }\end{array}$ & $\begin{array}{r}8.580 \\
521\end{array}$ & $\begin{array}{r}397 \\
-4\end{array}$ & $\begin{array}{r}22.522 \\
-.713\end{array}$ & $\begin{array}{r}9,696 \\
-458\end{array}$ & $\overline{-}$ & $\begin{array}{r}25.630 \\
97\end{array}$ & $\begin{array}{r}25,+33 \\
422\end{array}$ & $\begin{array}{r}5.490 \\
439\end{array}$ & $\begin{array}{r}89,943 \\
305\end{array}$ & $\begin{array}{r}100 \% \\
0 x\end{array}$ \\
\hline TOTAL HET OSAGE & 9,101 & 392 & 21.808 & 9.237 & - & 25.727 & 25,355 & $-1,875$ & 90,248 & \\
\hline
\end{tabular}

\section{SOPPLY OP EERRG}

POSSIL POE

BYDBOELECIRTC

HOCL BAR

GBO.\& SOLAR

TOTAL SUPPLZ

12

2

Ther

BBT IUPORTS

OP RBGION

9,101

392

21, B08

9,237

25,727

25,855

$-1,891$

$90.23 \pi$

HOTBS:

TRAESPOREATIOH LOSS POR

TRABSFORLATIOH LOSS POB

TRARS POBEATIOH LOSS POR
TRABSPOREATION COSS POR

BLFCTRCITY GEN.

$=70.06 \%$

SE POB YMOAN GAS
SS POR SZI-GAS

$=70.06 \times$
$=0.0 \times$
$=0.08$

$=0.0 \%$
$=0.0 \times$ 


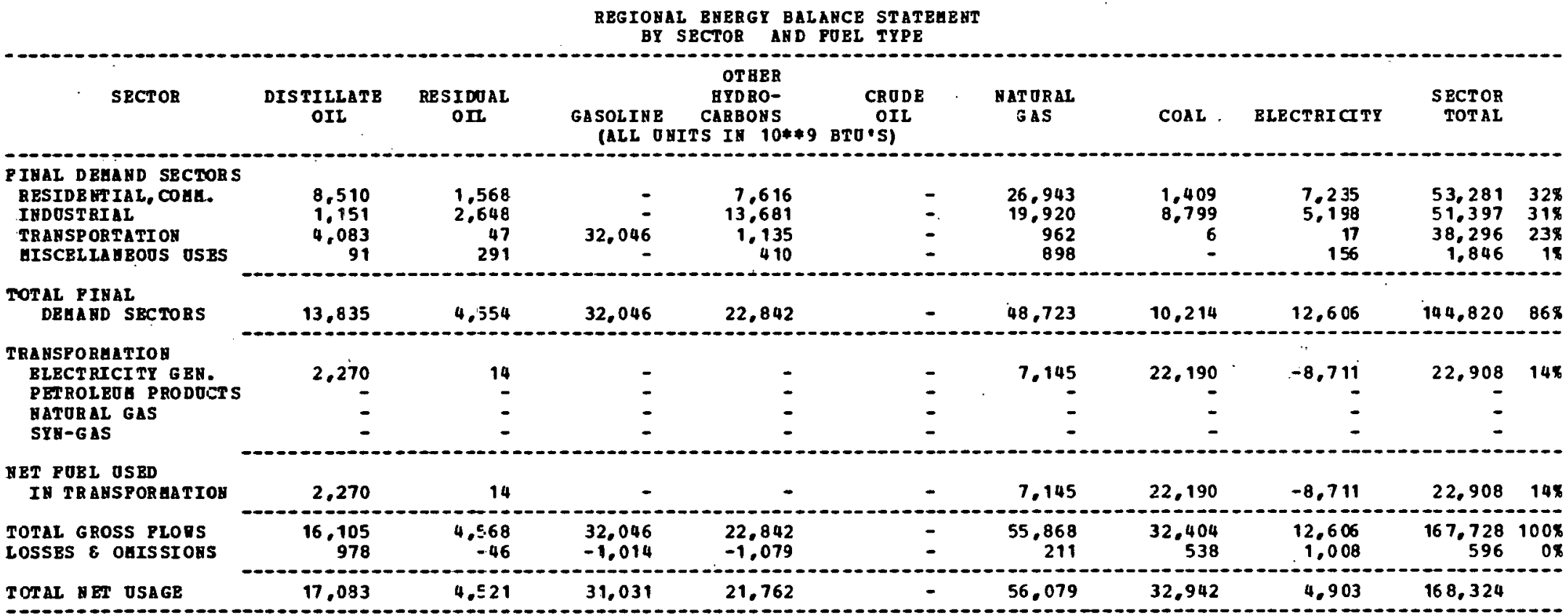

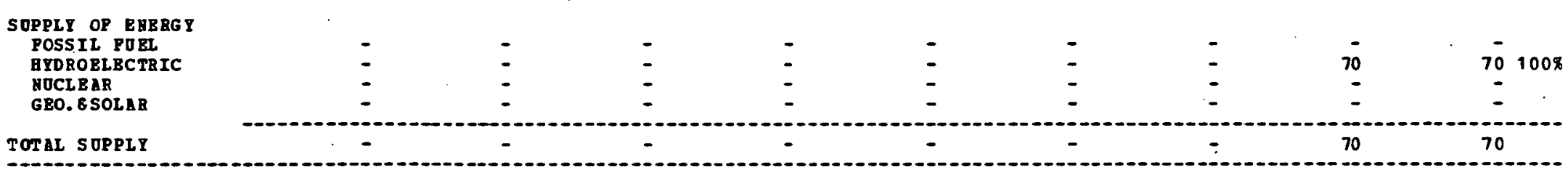

$\begin{aligned} & \text { BET IUPORTS } \\ & \text { OP REGIOH }\end{aligned}$
MOTES:
1 TRABSFORAATIOS LOSS FOR


REG IONAL BNERGI BALANCE STITEUENT

$3 Y$ SECTOR AND POEL TYPE

\begin{tabular}{|c|c|c|c|c|c|c|c|c|c|}
\hline SRCTOR & $\begin{array}{l}\text { DISFILLATE } \\
\text { JIL. }\end{array}$ & $\begin{array}{l}\text { BESIDOAL } \\
\text { DIL }\end{array}$ & $\begin{array}{l}\text { GASOLINE } \\
\text { (ALL OHI }\end{array}$ & $\begin{array}{l}\text { OTIER } \\
\text { HYDAO- } \\
\text { CARBOAS } \\
\text { IS IN } 10 * * 9\end{array}$ & $\begin{array}{c}\text { CRODE } \\
\text { OIL } \\
\text { BTO'S) }\end{array}$ & $\begin{array}{l}\text { NAT ORáL } \\
\text { G AS }\end{array}$ & COAL & ELECTRI CITY & $\begin{array}{r}\text { SECTOR } \\
\text { TOTAL }\end{array}$ \\
\hline $\begin{array}{l}\text { FIALL DEHAND SECTORS } \\
\text { RBSIDEHTIAL, COUA. } \\
\text { INDOSTRIAL } \\
\text { TRAYSPORTATI ON } \\
\text { MISCELLAKEOOS OSBS }\end{array}$ & $\begin{array}{r}11.549 \\
715 \\
2.392 \\
62\end{array}$ & $\begin{array}{r}373 \\
316 \\
41 \\
134\end{array}$ & 28,690 & $\begin{array}{r}7.303 \\
6.997 \\
2.170 \\
269\end{array}$ & $\begin{array}{l}- \\
- \\
-\end{array}$ & $\begin{array}{r}17.074 \\
8.132 \\
570 \\
1.669\end{array}$ & $\begin{array}{r}1,325 \\
2,071 \\
6 \\
-\end{array}$ & $\begin{array}{r}6,350 \\
1.689 \\
3 \\
102\end{array}$ & $\begin{array}{r}43,974 \\
19,970 \\
33,872 \\
2.236\end{array}$ \\
\hline $\begin{array}{l}\text { TOTAL PINAL } \\
\text { DEHA AD SECTORS }\end{array}$ & 14.718 & 864 & 28.690 & 16,739 & - & 27.495 & 3,402 & 8,144 & 100,052 \\
\hline $\begin{array}{l}\text { TRAHSFORUATIOI } \\
\text { BLBCT RICITY GEH. } \\
\text { PETROLEDH PRODOCTS } \\
\text { MATORAL GAS } \\
\text { SYH-GAS }\end{array}$ & $\begin{array}{r}\text { a45 } \\
- \\
-\end{array}$ & $\begin{array}{l}6 \\
- \\
-\end{array}$ & $\begin{array}{l}- \\
-\end{array}$ & $\begin{array}{l}2 \\
- \\
-\end{array}$ & 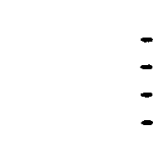 & $\begin{array}{r}7,181 \\
- \\
-\end{array}$ & $\begin{array}{r}4.723 \\
- \\
-\end{array}$ & $\begin{array}{r}-3,204 \\
= \\
-\end{array}$ & $\begin{array}{r}8,953 \\
- \\
-\end{array}$ \\
\hline $\begin{array}{l}\text { N BT FUEL USED } \\
\text { IH TRANSFORAATION }\end{array}$ & 245 & 6 & - & 2 & - & 7,181 & 4.723 & $-3,204$ & 8,953 \\
\hline $\begin{array}{l}\text { TOTAL GROSS FLOHS } \\
\text { ZOSSES } 8 \text { OUIS SI ONS }\end{array}$ & $\begin{array}{r}14.963 \\
.909\end{array}$ & $\begin{array}{r}870 \\
-8\end{array}$ & $\begin{array}{r}28,690 \\
-908\end{array}$ & $\begin{aligned} 16,741 \\
-790\end{aligned}$ & - & $\begin{array}{r}34,676 \\
131\end{array}$ & $\begin{array}{r}8.125 \\
135\end{array}$ & $\begin{array}{r}8,144 \\
651\end{array}$ & $\begin{array}{r}109.005 \\
118\end{array}$ \\
\hline
\end{tabular}

$\begin{aligned} & \text { SOPPLI OR ENERG } \\ & \text { POSSIL PUEL } \\ & \text { MYDROELECTEIC } \\ & \text { YUCLEAR }\end{aligned}$
GEO.ESOLAR

\section{NET IMPORTS}

15,872

861

27,781

15,950

34.8197

8,260

4.349

107,881

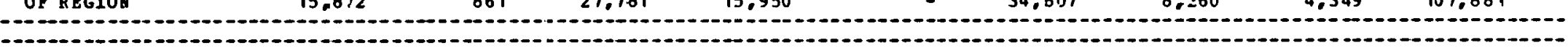

MOT ES:
1 TRANSFORGATIOA LUSS POB
TRANSPORGATION LISS POR
TRANSPORAATION LESS POR
TRALS PORAATIOA LESS POR
ELECT RICITY FEN. $\quad=73.64 \pi$
PETEOLEOH PRJDUCTS $=0.0$
$\begin{array}{ll}\text { AIORAL GAS } & =0.0 \% \\ \text { SYH-GAS } & =0.0 \times\end{array}$ 
REGIONAL ENERGY BALAHCE STATEGENT

BY SBCTOR AND FOEL TYPE

\begin{tabular}{|c|c|c|c|c|c|c|c|c|c|c|}
\hline SECTOR & $\begin{array}{l}\text { DISTILLATE } \\
\text { OII }\end{array}$ & $\begin{array}{l}\text { RES I DOAL } \\
\text { OII }\end{array}$ & $\begin{array}{l}\text { GASOLIBE } \\
\text { (ALL OI }\end{array}$ & $\begin{array}{c}\text { OTHER } \\
\text { GYDBO- } \\
\text { CARBOHS } \\
\text { TS IA 10** }\end{array}$ & $\begin{array}{c}\text { CRODE } \\
\text { OIL } \\
\text { BTO'SI }\end{array}$ & $\begin{array}{l}\text { MATURAL } \\
\text { GAS }\end{array}$ & $\operatorname{COAL}$ & BLECTRICTY & $\begin{array}{l}\text { SECTOR } \\
\text { TOTAL }\end{array}$ & \\
\hline $\begin{array}{l}\text { PIMAL DBLAHD SECTORS } \\
\text { RESIDB HTIAL, COEY. } \\
\text { IHDOST RIAL } \\
\text { TRABSPOBTATIOB } \\
\text { UISCBL LAHBOOS OSBS }\end{array}$ & $\begin{array}{r}51.485 \\
4.812 \\
17.305 \\
277\end{array}$ & $\begin{array}{r}1.738 \\
2.666 \\
203 \\
255\end{array}$ & 127.904 & $\begin{array}{r}32.553 \\
47.021 \\
11.545 \\
1.199\end{array}$ & $\overline{-}$ & $\begin{array}{r}77.185 \\
78.354 \\
2.540 \\
2.841\end{array}$ & $\begin{array}{r}5,908 \\
25,747 \\
28 \\
-\end{array}$ & $\begin{array}{r}28,810 \\
18,382 \\
33 \\
174\end{array}$ & $\begin{array}{r}197,679 \\
176,982 \\
159,558 \\
4,746\end{array}$ & $\begin{array}{r}31 \% \\
28 \% \\
25 \% \\
1 \%\end{array}$ \\
\hline $\begin{array}{l}\text { TOTAL PIBAL } \\
\text { DEHA SECTOBS }\end{array}$ & 73,879 & 4,862 & 127,904 & 92,318 & - & 160,920 & 31,683 & 47,399 & 538,965 & $85 \%$ \\
\hline $\begin{array}{l}\text { TRABSPOBEATIOY } \\
\text { BLBCT RICITY GEH. } \\
\text { PETROLEOA PRODOCTS } \\
\text { HATORAL GAS } \\
\text { SYE-G AS }\end{array}$ & $\begin{array}{r}153 \\
- \\
-\end{array}$ & $\begin{array}{r}2.510 \\
\vdots \\
-\end{array}$ & $\begin{array}{l}5 \\
-\end{array}$ & $\begin{array}{l}- \\
-\end{array}$ & $\overline{-}$ & $\begin{array}{r}8,545 \\
- \\
-\end{array}$ & $\begin{array}{r}124.690 \\
= \\
=\end{array}$ & $\begin{array}{r}-41,410 \\
= \\
=\end{array}$ & $\begin{array}{r}94.488 \\
= \\
=\end{array}$ & $15 \%$ \\
\hline $\begin{array}{l}\text { YET POBL OS BD } \\
\text { IA TRAESPORAATION }\end{array}$ & 153 & 2.510 & - & - & - & 8,545 & 124,690 & $-41,410$ & 94.488 & 158 \\
\hline $\begin{array}{l}\text { TOTAL GROSS PLONS } \\
\text { LOSSES E OHISSIOHS }\end{array}$ & $\begin{array}{r}74.032 \\
4.497\end{array}$ & $\begin{array}{r}7.372 \\
-74\end{array}$ & $\begin{array}{r}127.904 \\
-4,049\end{array}$ & $\begin{array}{l}92,318 \\
-4,361\end{array}$ & - & $\begin{array}{r}169.465 \\
641\end{array}$ & $\begin{array}{r}156,373 \\
2,600\end{array}$ & $\begin{array}{r}47.399 \\
3,790\end{array}$ & $\begin{array}{r}633,453 \\
3,043\end{array}$ & $\begin{array}{r}100 \% \\
0 \%\end{array}$ \\
\hline
\end{tabular}

SUPPLY OP BEERGY

POSSIL PUEL

GYDROBL BCTRIC

HOCLB AR

GEO. 8 SOLAR

-
$\vdots$
$\vdots$
-

TOTAL SOPPLI

$-$

$-$

$-$

\section{A3T IGPOATS}

78.529

7.297

123.854

87.956

170,106

158,973

9.779

636,496

NOTES:

TRAASFORHATIOY LOSS POB

TRA NSPOREATIOH LOSS POR

TRA GS FORALT IOY LOSS POB

BLECTRICITY GEH.

MATORAI GAS

$=0.0 x$

TRABSPOBEATION LOSS POR STI-GE 
RBGIOHAL BIERSY BALANCE STATEHBAT

BY SBCTOH AND POEL TIPE

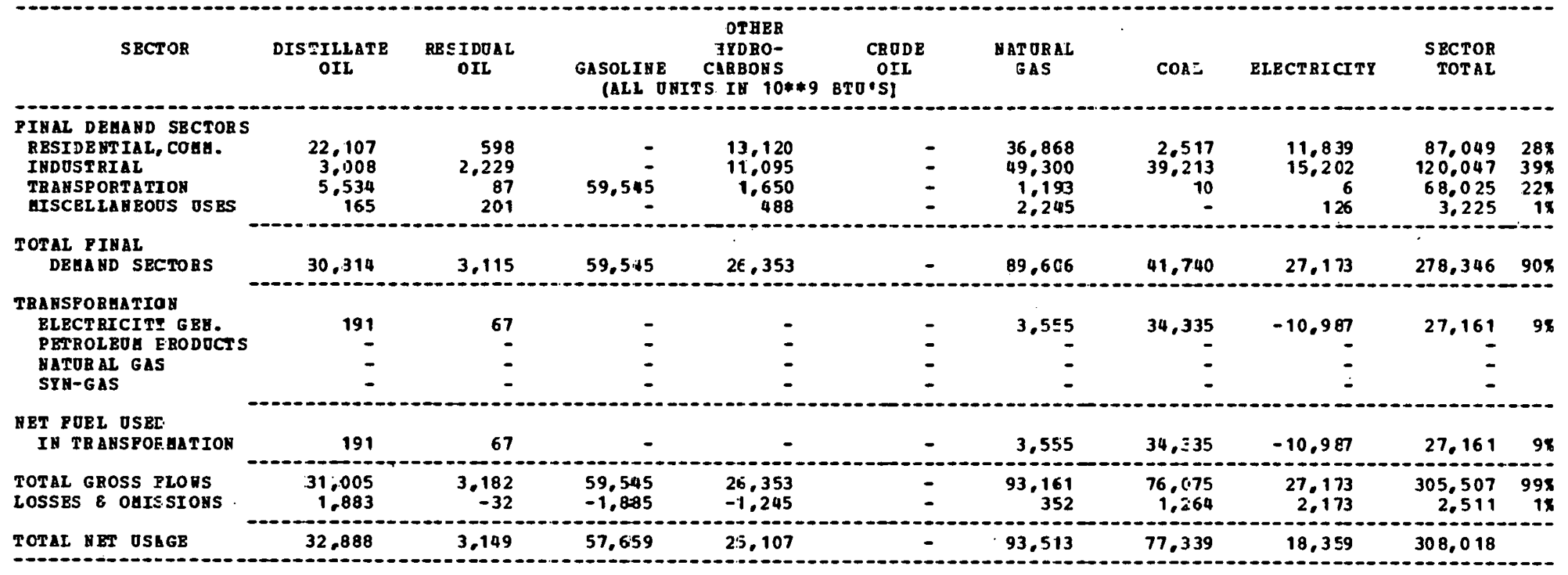

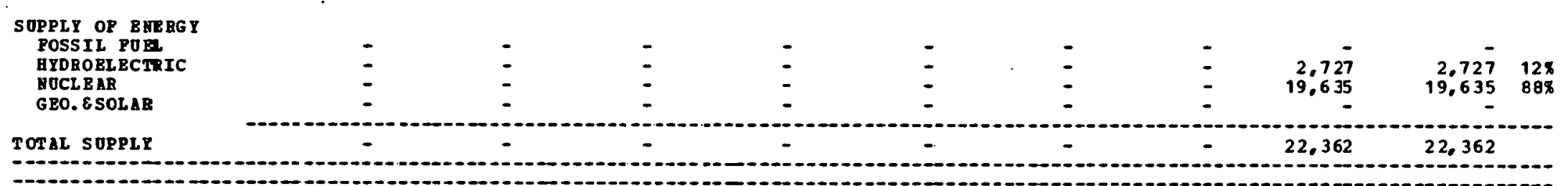

\section{NBT IUPORTS}

OP RBGIOH

32,888

3,149

57,659

25,107

93.513

77.339

$-4.002$

285,656

\section{MOT BS:}
1 TRAESFOAGATIOH LOSS POR TRA IS PO RAAT IOH IOSS FOR TRA WSPOELAT IO TRAES PORAATION LOSS FOR
BLECT HICITY GEN.
$\quad=71.20 x$
AT JBAL GAS
$=0.0 \%$ 


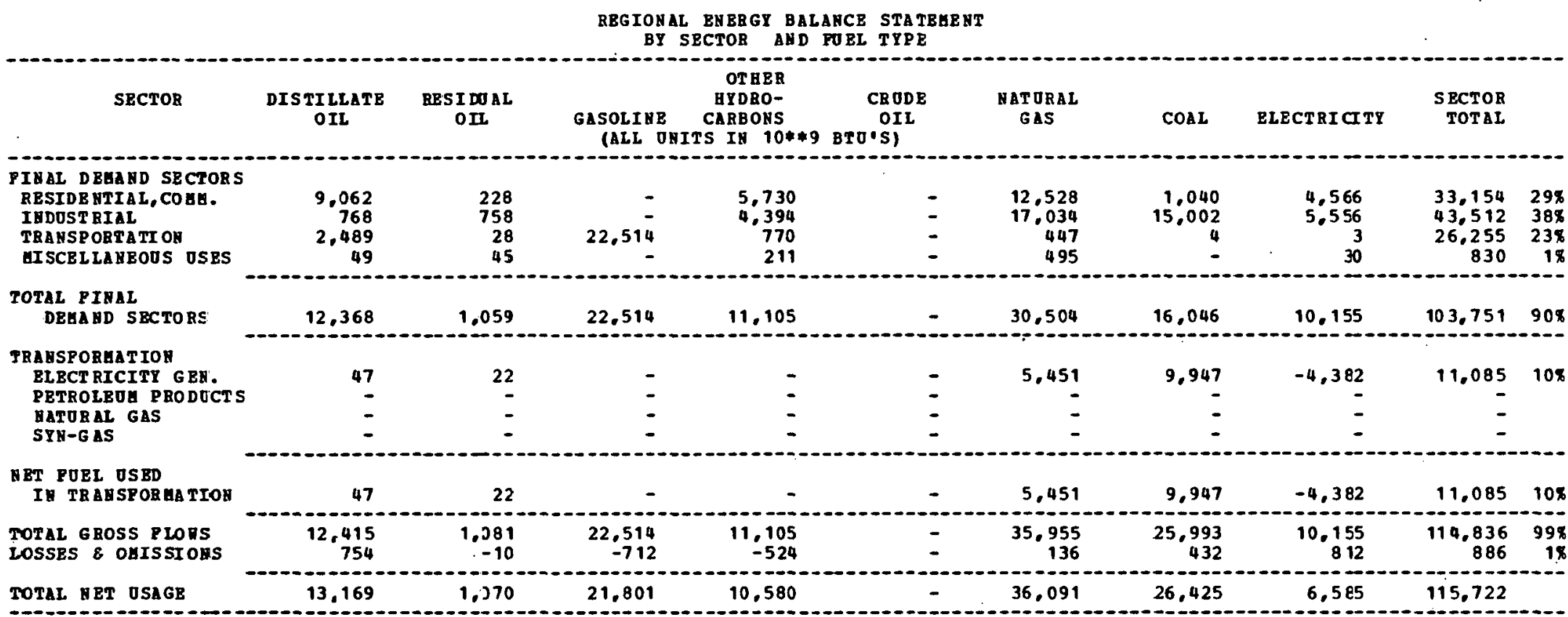

(1)

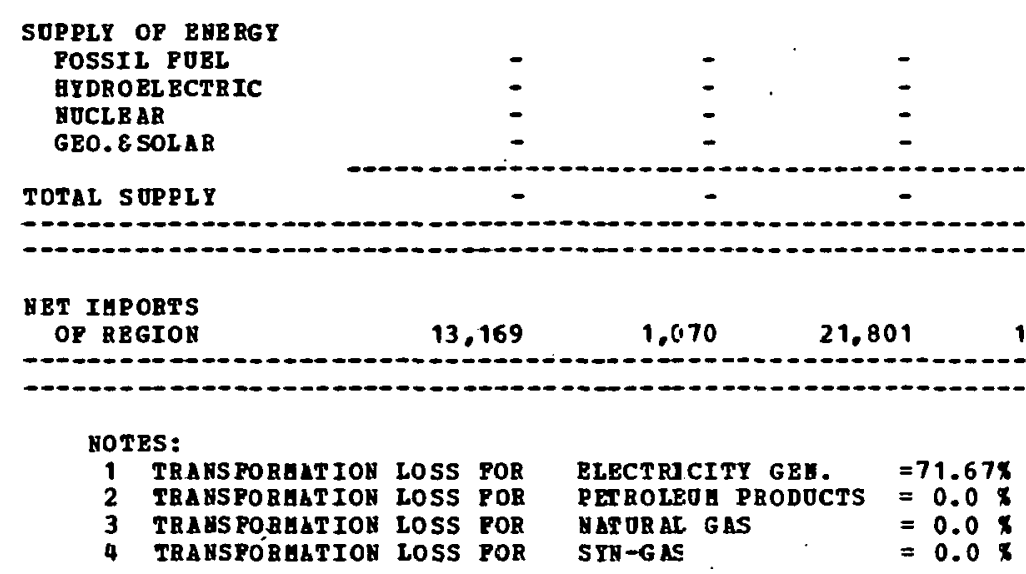


RBGIORAL EHEBGY BALABCE STATEAENT

B: SECTOR AND FOBL TYPE

\begin{tabular}{|c|c|c|c|c|c|c|c|c|c|c|}
\hline SECTOR & $\underset{\text { OIL }}{\text { DISTILLATB }}$ & $\begin{array}{l}\text { RESIDOAL } \\
\text { O II }\end{array}$ & $\begin{array}{l}\text { GaSOLIHZ } \\
\text { (AIL THIT }\end{array}$ & $\begin{array}{r}\text { OTBER } \\
\text { AYDRO- } \\
\text { OABOAS } \\
\text { I IB 10**9 }\end{array}$ & $\begin{array}{c}\text { CRODE } \\
\text { OIL } \\
\text { BTO'S) }\end{array}$ & $\begin{array}{l}\text { VAT ORAL } \\
\text { JAS }\end{array}$ & $\operatorname{COAL}$ & ELECTRICITY & $\begin{array}{r}\text { SBCTOR } \\
\text { TOTAL }\end{array}$ & \\
\hline 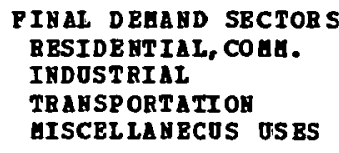 & $\begin{array}{r}10,432 \\
3,283 \\
1.788 \\
94\end{array}$ & $\begin{array}{r}343 \\
6.213 \\
34 \\
107\end{array}$ & 29.174 & $\begin{array}{r}7,975 \\
3,088 \\
419 \\
256\end{array}$ & $\overline{-}$ & $\begin{array}{r}18,072 \\
25,542 \\
602 \\
1,183\end{array}$ & $\begin{array}{r}397 \\
14,500 \\
4 \\
-\end{array}$ & $\begin{array}{r}9,882 \\
9,129 \\
3 \\
7\end{array}$ & $\begin{array}{r}42,601 \\
61,255 \\
32,024 \\
1,717\end{array}$ & $\begin{array}{r}258 \\
368 \\
198 \\
1 \%\end{array}$ \\
\hline $\begin{array}{l}\text { TOTAL PIHAL } \\
\text { DEHA ND SECTOHS }\end{array}$ & 15,597 & 6,697 & 29,174 & 11.738 & - & 45,399 & 14,901 & 14.091 & 137.597 & $80 \pi$ \\
\hline $\begin{array}{l}\text { TRANSPORHATIOH } \\
\text { BLECTRICITY GBH. } \\
\text { PETROLBOA PRODUCTS } \\
\text { HATURAL GAS } \\
\text { SYH-GAS }\end{array}$ & $\begin{array}{r}86 \\
-37,838 \\
-\end{array}$ & $\begin{array}{r}4,259 \\
-13,318 \\
= \\
-\end{array}$ & $\begin{array}{r}-55,52 \overline{0} \\
-\end{array}$ & $\begin{array}{r}-9,531 \\
-\end{array}$ & $105,83 \bar{E}$ & $\begin{array}{r}2,471 \\
10,382 \\
=\end{array}$ & $\begin{array}{r}36,687 \\
- \\
-\end{array}$ & $\begin{array}{r}-11,370 \\
- \\
-\end{array}$ & $\begin{array}{r}32,133 \\
13 \\
= \\
=\end{array}$ & $\begin{array}{r}19 x \\
0 x\end{array}$ \\
\hline $\begin{array}{l}\text { NBT FOBL OSED } \\
\text { IN TR AESPORGATIOA }\end{array}$ & $-37,752$ & $-9,059$ & $-55,32 \mathrm{C}$ & $-9,531$ & 105,838 & $12,8: 53$ & 36.687 & $-11,370$ & $32,14 \epsilon$ & $19 \%$ \\
\hline $\begin{array}{l}\text { TOTAL GROSS FLOES } \\
\text { LOSSES \& OHISSIOHS }\end{array}$ & $\begin{array}{r}15.683 \\
952\end{array}$ & $\begin{array}{r}10.956 \\
-111\end{array}$ & $\begin{array}{r}29.174 \\
-923\end{array}$ & $\begin{array}{r}11.738 \\
-554\end{array}$ & $\begin{array}{r}105,838 \\
160\end{array}$ & $\begin{array}{r}58,252 \\
220\end{array}$ & $\begin{array}{r}51.588 \\
857\end{array}$ & $\begin{array}{r}14.091 \\
1.126\end{array}$ & $\begin{array}{r}169,743 \\
1,729\end{array}$ & $\begin{array}{r}998 \\
18\end{array}$ \\
\hline TOTAL YET OSAGE & $-21,202$ & $-2,473$ & $-27,269$ & 1,652 & 105.998 & 58,472 & 52,445 & 3,847 & 171,472 & \\
\hline
\end{tabular}

SOPPLI OF EUERG

POSSIL POBL

AYDROBLEC'TRIC

MOCLBAR

GBO. GSOLAR

TOTAL SUPPLP

-
-
-

$-$

$-$

$-$

$-\quad 2,248$

$2,248 \quad 2,243$

ger

OP REGIOH

$-21.202$

$-2.473$

$-27,269$

1,652

105,998

58.472

52.445

1.599

169,224

nOT ES:

TRAYSPOBAATIOX LOS5 ROR

TR L WS POREAT IOA LOS: POR

TRatSPORLATIOA LOSS POB

BLECT RICITY GEN.

$\begin{array}{ll}\text { BLCTRTCITY GEH. } & =73.865 \\ \text { PETROLEOA ERODOCTS } & =0.015\end{array}$

ATOBAL GAS

STI-GAS

$=0.0$

$=0.0$ 
REGIOHAL BHERGY BALAHCE STATBEENT

BY SECTOR AUD FUEL TYPE

\begin{tabular}{|c|c|c|c|c|c|c|c|c|c|c|}
\hline SBCTOR & $\begin{array}{c}\text { DISTILLATB } \\
\text { OIL }\end{array}$ & $\begin{array}{c}\text { RESI DOAL } \\
\text { OIL }\end{array}$ & $\begin{array}{l}\text { GASOLIHE } \\
\text { (ALL OHIT }\end{array}$ & $\begin{array}{l}\text { HYDRO- } \\
\text { CARBO\&S } \\
\text { S IB } \quad 10 * * 9\end{array}$ & $\begin{array}{c}\text { CRODE } \\
\text { OII } \\
\text { BTO'S) }\end{array}$ & $\begin{array}{l}\text { NAT DRAL } \\
\text { GAS }\end{array}$ & COAL & ELECTRI CITY & $\begin{array}{l}\text { SECTOR } \\
\text { TOTAL }\end{array}$ & \\
\hline 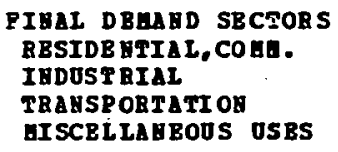 & $\begin{array}{r}5.798 \\
269 \\
1.168 \\
31\end{array}$ & $\begin{array}{r}142 \\
165 \\
17 \\
37\end{array}$ & 14.403 & $\begin{array}{r}3,666 \\
1,341 \\
351 \\
135\end{array}$ & 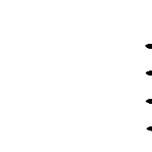 & $\begin{array}{r}7.966 \\
4.035 \\
286 \\
438\end{array}$ & $\begin{array}{r}666 \\
2,159 \\
2 \\
-\end{array}$ & $\begin{array}{r}2,913 \\
1,018 \\
17 \\
27\end{array}$ & $\begin{array}{r}21,151 \\
8,987 \\
16.228 \\
668\end{array}$ & $\begin{array}{r}438 \\
18 \% \\
33 \% \\
18\end{array}$ \\
\hline $\begin{array}{l}\text { TOTAL PIHAL } \\
\text { DEAAGD SBCTOAS }\end{array}$ & 7.266 & 361 & 14.403 & 5.493 & . & 12,725 & 2,827 & 3,959 & 47,034 & $96 \%$ \\
\hline $\begin{array}{l}\text { TRABSPORAATIOZ } \\
\text { BLBCT RICITY GBE. } \\
\text { PETROLBOA PBODOCTS } \\
\text { MATORAL GAS } \\
\text { SYH-GAS }\end{array}$ & $\begin{array}{r}1,919 \\
- \\
-\end{array}$ & $\begin{array}{l}- \\
\overline{-}\end{array}$ & 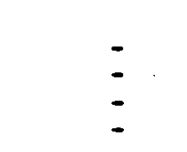 & - & . & $\begin{array}{r}111 \\
- \\
-\end{array}$ & $\begin{array}{l}\bar{z} \\
\bar{z}\end{array}$ & $\begin{array}{r}-5 \mathrm{C7} \\
- \\
-\end{array}$ & $\begin{array}{r}1,523 \\
= \\
-\end{array}$ & 3x \\
\hline $\begin{array}{l}\text { DET FOBL OSBD } \\
\text { IB TR ABSPORLA TIOB }\end{array}$ & 1,919 & - & - & - & 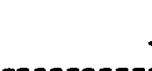 & 111 & - & -507 & 1.523 & $3 x$ \\
\hline $\begin{array}{l}\text { TOTAL GROSS PLORS } \\
\text { LOSSES } 8 \text { OAISSIO\&S }\end{array}$ & $\begin{array}{r}9.185 \\
557\end{array}$ & $\begin{array}{r}361 \\
-3\end{array}$ & $\begin{array}{r}14,403 \\
-455\end{array}$ & $\begin{array}{r}5,493 \\
-259\end{array}$ & . & $\begin{array}{r}12.836 \\
48\end{array}$ & $\begin{array}{r}2.827 \\
47\end{array}$ & $\begin{array}{r}3.959 \\
316\end{array}$ & $\begin{array}{r}48,557 \\
251\end{array}$ & $\begin{array}{r}99 x \\
1 \%\end{array}$ \\
\hline
\end{tabular}

$12,84^{-1}$

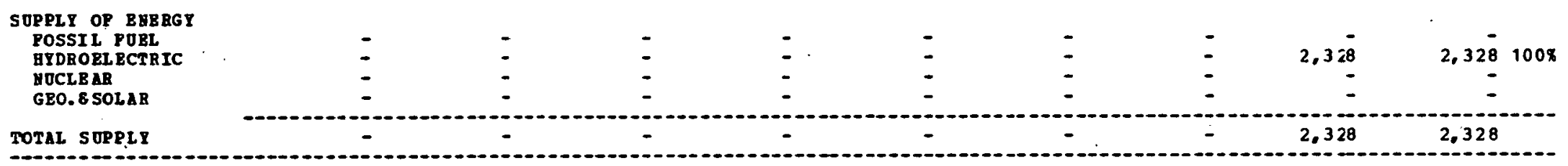

\section{HBT IMPOBTS}

OP RBGIOA

9.742

357

13,947

5,233

12,884

2.874

1,440

46,480

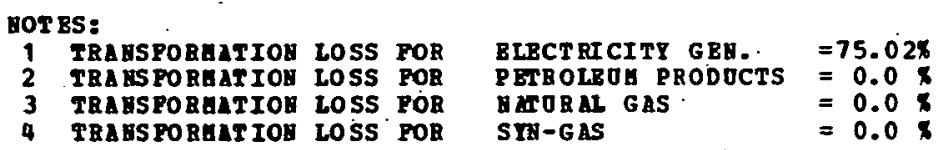


REgIONAL ENERGY BALAHCB STA TEEENT

BY SECTOR ABD POEL TYPE

\begin{tabular}{|c|c|c|c|c|c|c|c|c|c|}
\hline SBC=OR & $\begin{array}{l}\text { DISTILLATE } \\
\text { OIL }\end{array}$ & $\begin{array}{l}\text { RESIIDEL } \\
\text { OII }\end{array}$ & $\begin{array}{l}\text { GASOLINE } \\
\text { (ALL O }\end{array}$ & $\begin{array}{l}\text { BYDRO- } \\
\text { CARBOHS } \\
T \leq \text { II 10**9 }\end{array}$ & $\begin{array}{c}\text { CRODE } \\
\text { OIL } \\
\text { BTOיS) }\end{array}$ & $\begin{array}{l}\text { NATORAL } \\
\text { GAS }\end{array}$ & $\operatorname{COAI}$ & BLECTRICITY & $\begin{array}{l}\text { SECTOB } \\
\text { TOTAL }\end{array}$ \\
\hline $\begin{array}{l}\text { PIHAL DEHAND SECTORS } \\
\text { RESIDE HTIAL, COHA. } \\
\text { IHDOSTRIAL } \\
\text { TRANSPORTATIOH } \\
\text { GISCELLABEOJS OSES }\end{array}$ & $\begin{array}{r}6.819 \\
553 \\
2.093 \\
43\end{array}$ & $\begin{array}{r}188 \\
.818 \\
24 \\
47\end{array}$ & $\begin{array}{r}- \\
17.465 \\
-\end{array}$ & $\begin{array}{r}7,609 \\
3,735 \\
626 \\
162\end{array}$ & - & $\begin{array}{r}10.073 \\
4.831 \\
.351 \\
533\end{array}$ & $\begin{array}{r}723 \\
1, E 56 \\
4 \\
-\end{array}$ & $\begin{array}{r}3,374 \\
1,294 \\
3 \\
34\end{array}$ & $\begin{array}{r}25,726 \\
13,087 \\
20,566 \\
819\end{array}$ \\
\hline $\begin{array}{l}\text { TOTAL PIHAL } \\
\text { DEBA SD SB=TORS }\end{array}$ & 9.508 & 1.077 & 17.465 & 9,132 & - & 15,728 & 2.583 & 4.705 & 60,198 \\
\hline $\begin{array}{l}\text { TRABSPORAATION } \\
\text { BLECT RICITY GEH. } \\
\text { PETROLROA PRODOCTS } \\
\text { HATORAL GAS } \\
\text { SYH-GAS }\end{array}$ & $\begin{array}{r}29 \\
- \\
-\end{array}$ & $\begin{array}{r}2 \geq 4 \\
= \\
-\end{array}$ & $\begin{array}{l}\overline{-} \\
\overline{-}\end{array}$ & : & $\overline{-}$ & $\begin{array}{r}33 \\
- \\
-\end{array}$ & $\begin{array}{r}32,357 \\
= \\
-\end{array}$ & $\begin{array}{r}-9,925 \\
= \\
-\end{array}$ & $\begin{array}{r}22.718 \\
- \\
-\end{array}$ \\
\hline $\begin{array}{l}\text { ABT POEL OSED } \\
\text { IN TRAHSPORGATION }\end{array}$ & 29 & 224 & - & - & - & 33 & 32.357 & $-9,925$ & 22.718 \\
\hline $\begin{array}{l}\text { TOTAL GROSS FLORS } \\
\text { lOSSES } 8 \text { OEISST OHS }\end{array}$ & $\begin{array}{r}9.537 \\
579\end{array}$ & $\begin{array}{r}1,301 \\
-13\end{array}$ & $\begin{array}{r}17.465 \\
-552 \\
\end{array}$ & $\begin{array}{r}9.132 \\
-431\end{array}$ & $\overline{-}$ & $\begin{array}{r}15.761 \\
59\end{array}$ & 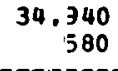 & $\begin{array}{r}4.705 \\
376\end{array}$ & $\begin{array}{r}82.916 \\
598\end{array}$ \\
\hline
\end{tabular}

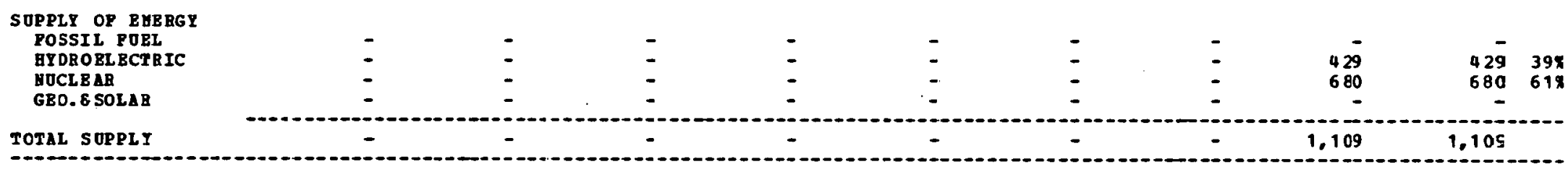

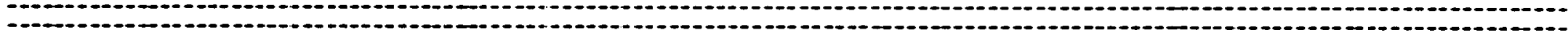

\section{BBT IHPORTS}

OF REGIOH

10.116

$1.287 \quad 16,912$

8,700

15,820

35.520

$-5,9 \leq 2$

82,405

HOTES:

\begin{tabular}{|c|c|c|c|c|}
\hline $\begin{array}{l}\text { TRA GS PORAAT } \\
\text { TRA SSPORAAT } \\
\text { TRA AS FORALT } \\
\text { TRAGSPORAAT }\end{array}$ & 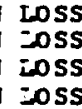 & $\begin{array}{l}\text { POR } \\
\text { POR } \\
\text { POR } \\
\text { POB }\end{array}$ & $\begin{array}{l}\text { ELECIRICITY GEN. } \\
\text { PERTCLEOA PRODOCTS } \\
\text { RAYOE AL GAS } \\
\text { SYH-G AS }\end{array}$ & $\begin{array}{l}=69.608 \\
=0.08 \\
=0.08 \\
=0.0 \mathrm{x}\end{array}$ \\
\hline
\end{tabular}


REGIOHAL BH3RGT BALAHCE STATEHENT

BY SECTOR AHD POBL TYPE

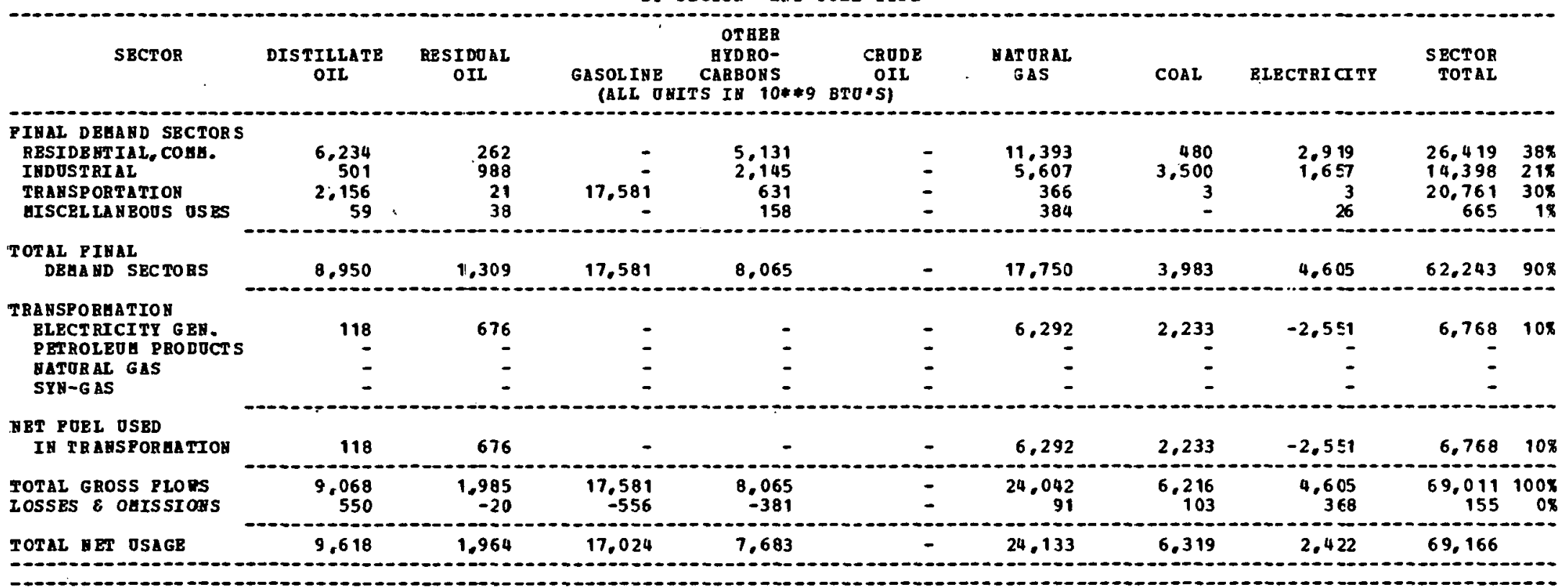

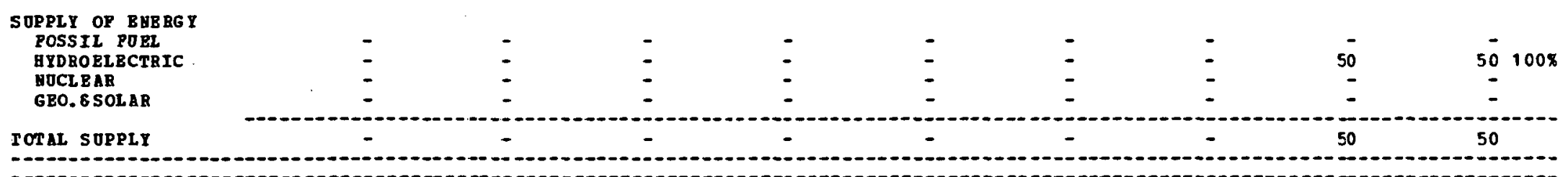

\begin{tabular}{l}
$\begin{array}{l}\text { GET IKPORTS } \\
\text { OR REGIOH }\end{array}$ \\
\hline
\end{tabular}


REGIONAL ENERGT BALANCE STATERBNT

B. SECTOB AKD POEL TYPE

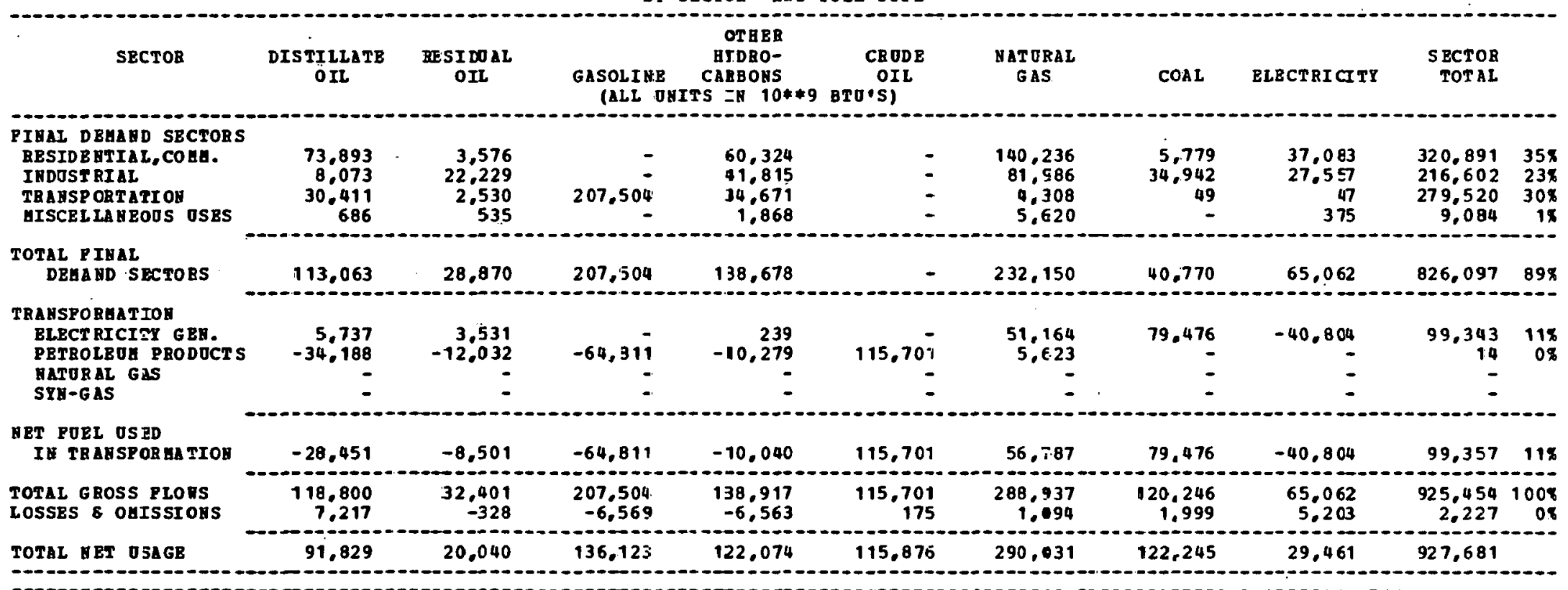

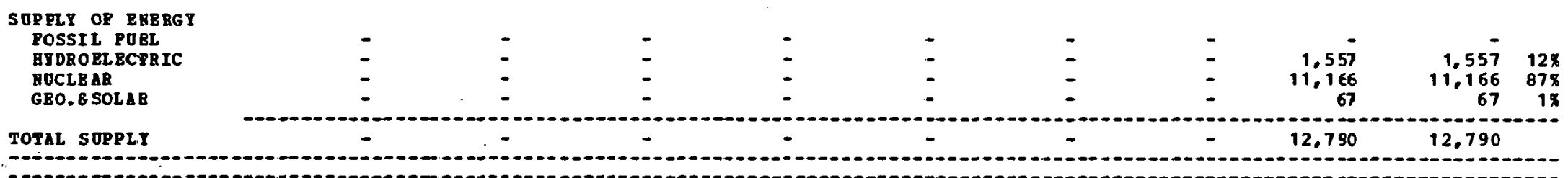

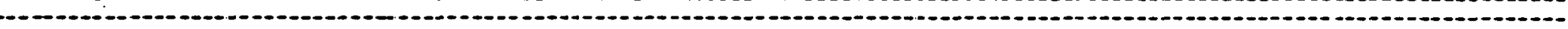

\section{HBT IAPORTS \\ OP RBGIO\&}

91.829

20.040

136.123

122.074

115,876

290,031

122,245

16.671

914.891

noTBS:

TRAMSFORAATIOB cOSS POR
TRABSPOBAATIOB IOSS POB TRABSPOBGATIOH LOSS POB
TRABSFORGATIOH LOSS POR

TRABSFOREATIOH LOSS POR
TRABSPOBAATIOA LOSS POB

$\begin{array}{ll}\text { BLECTRICITY GER. } & =70.388 \\ \text { PEEROLBOS PRODOCTS } & =0.319 \\ \text { BLEORAL GAS } & =0.3 \\ \text { SYM-GAS } & =0.3\end{array}$


REGIONAL BNBRG BALAGCE STATEMEAT

BY SECTOB AND POEL TYPE

\begin{tabular}{|c|c|c|c|c|c|c|c|c|c|}
\hline SBCT OR & $\begin{array}{l}\text { DISTILLATB } \\
\text { OIL }\end{array}$ & $\begin{array}{l}\text { RESI DOAL } \\
\text { OIL }\end{array}$ & $\begin{array}{l}\text { GASOLIHE } \\
\text { (ALL UAI? }\end{array}$ & $\begin{array}{l}\text { HYDRO- } \\
\text { CABBONS } \\
\text { IS IH 10**9 }\end{array}$ & $\begin{array}{c}\text { CRODE } \\
\text { OIL } \\
\text { BTO } 51\end{array}$ & $\begin{array}{l}\text { HAT ORAL } \\
\text { G AS }\end{array}$ & COAL & ELECTRICITY & $\begin{array}{r}\text { SECTOR } \\
\text { TOTAL }\end{array}$ \\
\hline $\begin{array}{l}\text { PIHAL DEHAYD SECTORS } \\
\text { RBSIDB BTIAL, COAB. } \\
\text { INDOSTRIAL } \\
\text { TRANSP ORTATIOH } \\
\text { UISCBLLABEOUS OSBS }\end{array}$ & $\begin{array}{r}4.937 \\
483 \\
2.700 \\
172\end{array}$ & $\begin{array}{r}353 \\
550 \\
6 \\
188\end{array}$ & 18,378 & $\begin{array}{r}5,464 \\
1.089 \\
1.529 \\
96\end{array}$ & 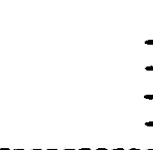 & $\begin{array}{l}7,307 \\
1.735 \\
139 \\
1.107\end{array}$ & $\begin{array}{r}597 \\
2.261 \\
3 \\
-\end{array}$ & $\begin{array}{r}2.946 \\
388 \\
108\end{array}$ & $\begin{array}{r}21,604 \\
6,506 \\
22,755 \\
1,671\end{array}$ \\
\hline $\begin{array}{l}\text { TOTAL FIRAL } \\
\text { DEAA GD SBCTOBS }\end{array}$ & 8,292 & 1,097 & 18,378 & 8,178 & re & 10,288 & 2.861 & 3,442 & 52.536 \\
\hline $\begin{array}{l}\text { PRAHSPORHATIOH } \\
\text { BLECTRICITY GEE. } \\
\text { PETROLEOA PRODOCTS } \\
\text { GATORAL GAS } \\
\text { SYH-GAS }\end{array}$ & $\begin{array}{r}26 \\
- \\
-\end{array}$ & - & ! & - & 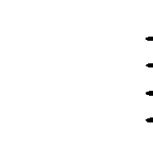 & $\begin{array}{r}154 \\
- \\
-\end{array}$ & $\begin{array}{r}2.448 \\
- \\
-\end{array}$ & $\begin{array}{r}-440 \\
= \\
=\end{array}$ & $\begin{array}{r}2,188 \\
= \\
=\end{array}$ \\
\hline $\begin{array}{l}\text { IBT FOBL OSBD } \\
\text { IB TRAESPORGATIOR }\end{array}$ & 26 & - & - & - & 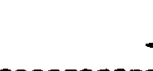 & 154 & 2,448 & -440 & 2,188 \\
\hline $\begin{array}{l}\text { TOTAL GROSS PLORS } \\
\text { IOSSES \& OAISSIOBS }\end{array}$ & $\begin{array}{r}8.318 \\
505\end{array}$ & $\begin{array}{r}1,097 \\
-11\end{array}$ & $\begin{array}{r}18,378 \\
-581\end{array}$ & $\begin{array}{r}8.178 \\
-386\end{array}$ & 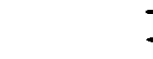 & $\begin{array}{r}10,442 \\
39\end{array}$ & $\begin{array}{r}5,309 \\
88\end{array}$ & $\begin{array}{r}3.442 \\
275\end{array}$ & $\begin{array}{r}54,724 \\
-70\end{array}$ \\
\hline T OTAL YEI OSAGE & 8,823 & 1,085 & 17,796 & 7,791 & 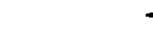 & 10,481 & 5.397 & 3,277 & 54.653 \\
\hline
\end{tabular}

\section{SOPPLY OP BGERG I}

POSSIL POBI

GYDROBLBCTRIC

NOCL B AR

GBO. 8 SOLAB

-

$\begin{array}{lll}- & - \\ - & -\end{array}$

TOTAL SOPPLY

$-$

$-$

$-$

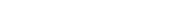

$-40$

8

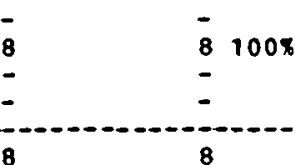

HAT TAPORS

OP RBGIOE

8,823

1.085

17.796

7.791

10.481

5.397

3,269

54,645

\section{MOT ES:}

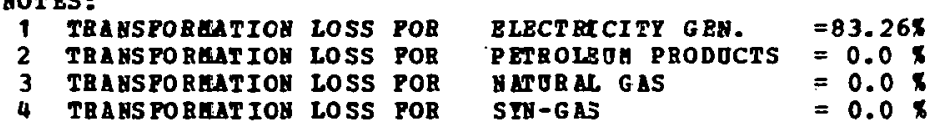


REGEONAZ BNEEGY BALANCE STATEAENT

BY SECTOR AQD FOEL TYPE

\begin{tabular}{|c|c|c|c|c|c|c|c|c|c|c|}
\hline SECTOR & $\begin{array}{l}\text { DIS IILIATE } \\
\text { OII }\end{array}$ & $\begin{array}{c}\text { RES I DOAL } \\
\text { J II }\end{array}$ & $\begin{array}{l}\text { GASOLINE } \\
\text { (ALE EH: }\end{array}$ & $\begin{array}{c}\text { HYDRO- } \\
\text { CARBONS } \\
\text { TS I\$10* 109 }\end{array}$ & $\begin{array}{c}\text { CR ODE } \\
\text { OIL } \\
\text { BTO'S }\end{array}$ & $\begin{array}{l}\text { NATUEAL } \\
\text { GAS }\end{array}$ & $\operatorname{cod} L$ & ELECTRICITY & $\begin{array}{r}\text { SECTOR } \\
\text { TOTAL }\end{array}$ & \\
\hline $\begin{array}{l}\text { PIHAL DEGAND SECTORS } \\
\text { RBSIDENTIAL, COAG. } \\
\text { IHDOSTRIAL } \\
\text { TRANSPORTATIOH } \\
\text { GISCELLANBOUS OSES }\end{array}$ & $\begin{array}{r}3.254 \\
668 \\
2.822 \\
227\end{array}$ & $\begin{array}{r}328 \\
585 \\
8 \\
177\end{array}$ & 15,744 & $\begin{array}{r}4.608 \\
454 \\
307 \\
48\end{array}$ & $\begin{array}{l}\overline{-} \\
-\end{array}$ & $\begin{array}{r}5,210 \\
7,377 \\
6.7 \\
1.007\end{array}$ & $\begin{array}{r}667 \\
806 \\
2 \\
-\end{array}$ & $\begin{array}{r}2.570 \\
352 \\
3 \\
96\end{array}$ & $\begin{array}{r}16,637 \\
10,242 \\
18,953 \\
1.555\end{array}$ & $\begin{array}{r}30 \% \\
19 \% \\
34 \% \\
3 \%\end{array}$ \\
\hline $\begin{array}{l}\text { TOTAL FINAL } \\
\text { DEAABD SBCTORS }\end{array}$ & 6,971 & 1.098 & 15.744 & 3.417 & - & 13,561 & 1,475 & 3.021 & 47,387 & $86 \%$ \\
\hline $\begin{array}{l}\text { TRANSPORGATIOI } \\
\text { ELECTRICITI GRH. } \\
\text { PETROLEOU PRODOCTS } \\
\text { HATORAL GAS } \\
\text { SYH-GAS }\end{array}$ & $\begin{array}{r}1 \\
-2,769 \\
- \\
-\end{array}$ & $\begin{array}{r}3 \\
-851 \\
-\end{array}$ & $\begin{array}{r}-6,65 \bar{z} \\
- \\
-\end{array}$ & $\begin{array}{r}-4.135 \\
-5.739 \\
-\end{array}$ & $\begin{array}{r}13,793 \\
-\end{array}$ & $\begin{array}{r}401 \\
302 \\
6,596 \\
-\end{array}$ & $\begin{array}{r}7,871 \\
= \\
-\end{array}$ & $\begin{array}{r}-1,890 \\
= \\
-\end{array}$ & $\begin{array}{r}6.386 \\
188 \\
857 \\
-\end{array}$ & $\begin{array}{r}12 \% \\
0 \% \\
2 \%\end{array}$ \\
\hline $\begin{array}{l}\text { NET FOEL OSED } \\
\text { IA TRANSFOEAATIOB }\end{array}$ & $-2,768$ & -848 & $-6,652$ & $-9,874$ & 13,793 & 7.799 & 7,871 & $-1,890$ & 7.431 & $14 \%$ \\
\hline $\begin{array}{l}\text { TOTAL GROSS FLONS } \\
\text { LOSSES } E \text { OUISSI OHS }\end{array}$ & $\begin{array}{r}6,972 \\
423\end{array}$ & $\begin{array}{r}1.101 \\
-11\end{array}$ & $\begin{array}{r}15.744 \\
-498\end{array}$ & $\begin{array}{r}5,417 \\
-255\end{array}$ & $\begin{array}{r}13,793 \\
20\end{array}$ & $\begin{array}{r}21,450 \\
31\end{array}$ & $\begin{array}{r}9,346 \\
155\end{array}$ & $\begin{array}{r}3.021 \\
241\end{array}$ & $\begin{array}{r}54,818 \\
157\end{array}$ & $\begin{array}{r}100 \% \\
0 \%\end{array}$ \\
\hline
\end{tabular}

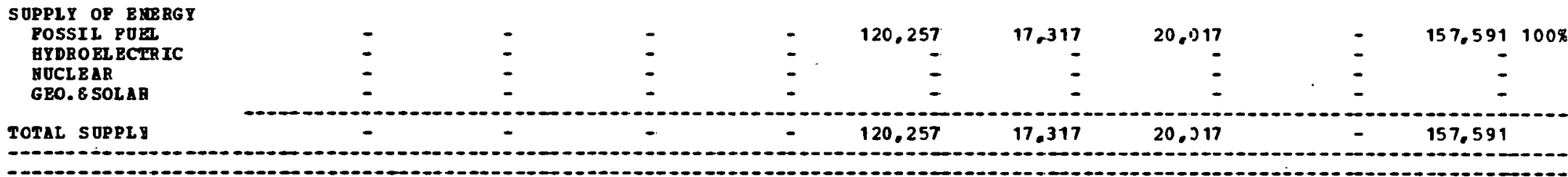

\section{NET IUPORTS}

OP RBGIOH

4.626

$238 \quad 8.593$

$-4.712-106,443$

4.224

$-10,515$

1.372

$-102,615$

\section{MOT BS : \\ 1 TRA LSPOEBATION LOSS POR \\ TRAISPOREATIOH IOSS POR BLECT RICITY GBH. \\ TRAESPOREATIOY LOSS POR \\ PBTZOLEOA PEODOCTS \\ TRADS POBMAT IOH LOSS POB \\ MAT JE AL GAS \\ $=77.86 \%$ \\ SYS-GAS


BEGIONAL RHERGY BALANCE STATEMENT

BY SECTOR AND FOEL TYPE

\begin{tabular}{|c|c|c|c|c|c|c|c|c|c|}
\hline SBCTOR & $\begin{array}{l}\text { DISTILLATB } \\
\text { OIL }\end{array}$ & $\begin{array}{l}\text { BESIDOAL } \\
\text { OIL }\end{array}$ & $\begin{array}{r}\text { GASOLINE } \\
\text { (ALL O }\end{array}$ & $\begin{array}{c}\text { MPDBO- } \\
\text { CARBONS } \\
\text { TS IN 10**9 }\end{array}$ & $\begin{array}{c}\text { CBODE } \\
\text { OIL } \\
\text { BTO०SI }\end{array}$ & $\begin{array}{l}\text { MATORAL } \\
\text { GAS }\end{array}$ & $\operatorname{COAL}$ & BLECTRICITY & $\begin{array}{l}\text { SECTOR } \\
\text { TOTAL }\end{array}$ \\
\hline $\begin{array}{l}\text { PIHAL DEHAND SECTORS } \\
\text { RBSIDBATIAL, COUA. } \\
\text { IHDOSTRIAL } \\
\text { TRAHSPORTATI OH } \\
\text { GISCELLABEOOS OSBS }\end{array}$ & $\begin{array}{r}2.503 \\
1,300 \\
6.282 \\
449\end{array}$ & $\begin{array}{r}342 \\
1,447 \\
104 \\
193\end{array}$ & 19.029 & $\begin{array}{r}4.717 \\
1,104 \\
591 \\
61\end{array}$ & - & $\begin{array}{r}13.340 \\
12.355 \\
546 \\
1.899\end{array}$ & $\begin{array}{r}1,566 \\
2,150 \\
4 \\
-\end{array}$ & $\begin{array}{r}3.743 \\
5.095 \\
61 \\
88\end{array}$ & $\begin{array}{r}26,211 \\
23,451 \\
26,617 \\
2,690\end{array}$ \\
\hline $\begin{array}{l}\text { TOTAL PIYAL } \\
\text { DEAA HD SECTORS }\end{array}$ & 10,534 & 2.086 & 19.029 & 6.473 & - & 28.140 & 3,720 & 8,987 & 78,969 \\
\hline $\begin{array}{l}\text { TRAHSPOBHATIOH } \\
\text { BLECT RICITY GEH. } \\
\text { PETROLEDE PRODDCTS } \\
\text { MATORAL GAS } \\
\text { SYI-GAS }\end{array}$ & $\begin{array}{r}-7.861 \\
-\end{array}$ & $\begin{array}{r}-1,994 \\
-\end{array}$ & $\begin{array}{r}-14,053 \\
-\end{array}$ & $\begin{array}{r}- \\
-2,989 \\
-749 \\
-\end{array}$ & $\begin{array}{r}24.715 \\
-\end{array}$ & $\begin{array}{r}2,199 \\
860 \\
-\end{array}$ & $\begin{array}{l}- \\
-\end{array}$ & $\begin{array}{l}\overline{-} \\
\overline{-}\end{array}$ & $\begin{array}{r}17 \\
111 \\
-\end{array}$ \\
\hline $\begin{array}{l}\text { VET POEL OSBD } \\
\text { IH TR ABSPOBEA TION }\end{array}$ & $-7,861$ & -1.994 & -14.053 & -3.738 & 24.715 & 3.059 & - & - & 128 \\
\hline $\begin{array}{l}\text { TOTAL GROSS PLOIS } \\
\text { LOSSES \& OAISSI OHS }\end{array}$ & $\begin{array}{r}10.534 \\
639\end{array}$ & $\begin{array}{r}2.086 \\
-21\end{array}$ & $\begin{array}{r}19.029 \\
-602\end{array}$ & $\begin{array}{r}6.473 \\
-305\end{array}$ & $\begin{array}{r}24.715 \\
37\end{array}$ & $\begin{array}{r}31.199 \\
118\end{array}$ & $\begin{array}{r}3,720 \\
61\end{array}$ & $\begin{array}{r}8.987 \\
718\end{array}$ & $\begin{array}{r}79.097 \\
646\end{array}$ \\
\hline
\end{tabular}

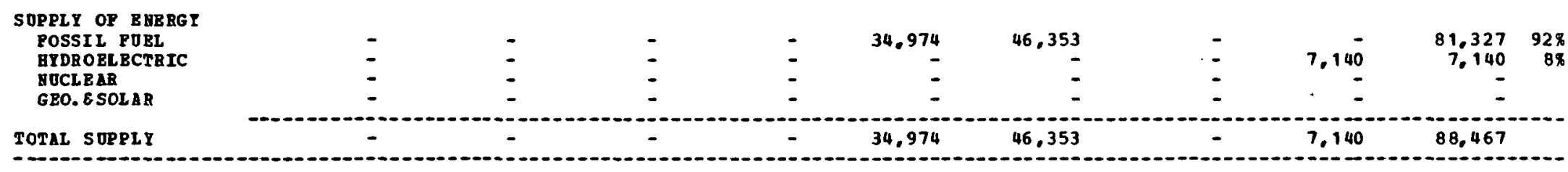

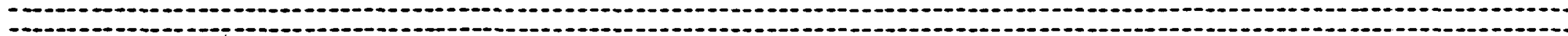

\section{QBT IAPORTS}

OP REGIOI

$70 \quad 4.373$

$2.429 \quad-10,221$

$-15.035$

3.781

2,565

$-8.723$

NoT $\mathrm{BS}$

1. PRABSPORAATIOE LOSS POR TRA BS FORAAT IOY LOSS YOR

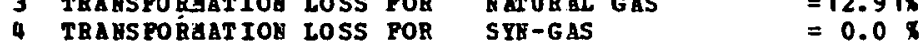

ELECT RICITY GEN. $=0.0$

PETROLEUA PBODUCTS $=0.068$

N ATORAL GAS 
REGIONAL ENERG BALAICE STATEHEAT

BY SBCTOR AND POEL TYPE

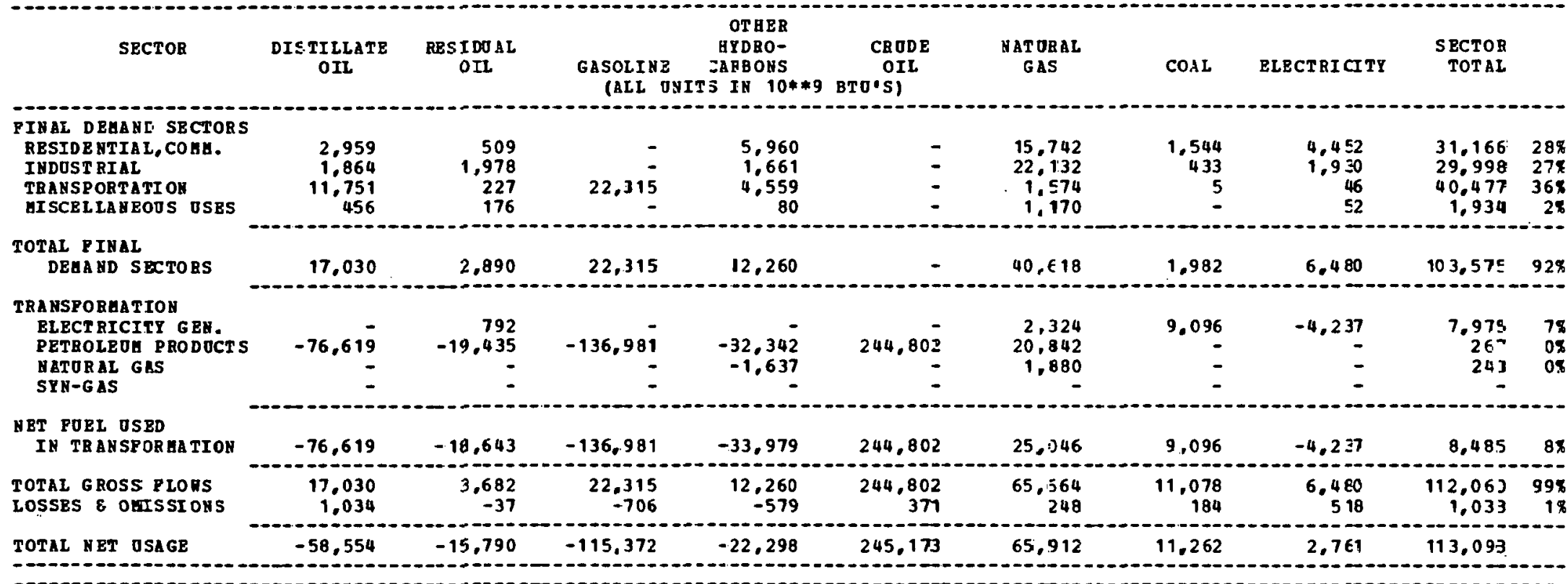

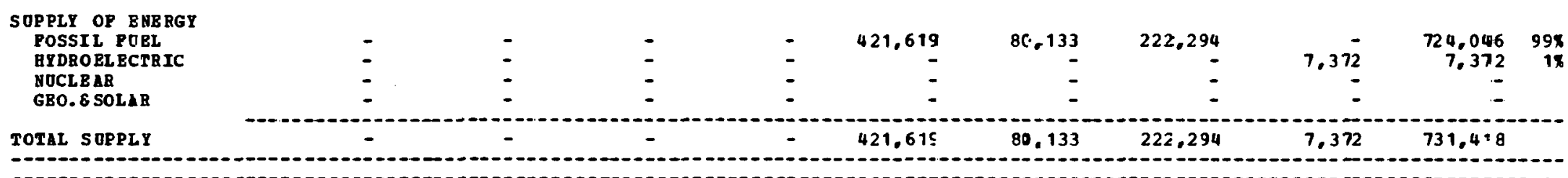

HET IHPORT:

$-58,554 \quad-15,790 \quad-115,372$

$-22,298$

$-176,445$

$-17,220$

$-211,031$

$-4.610$

$-618,324$

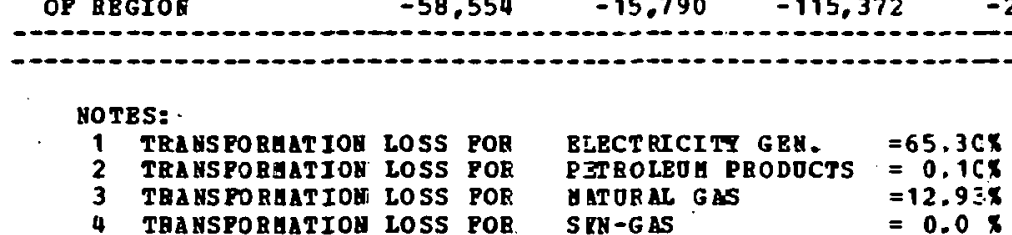




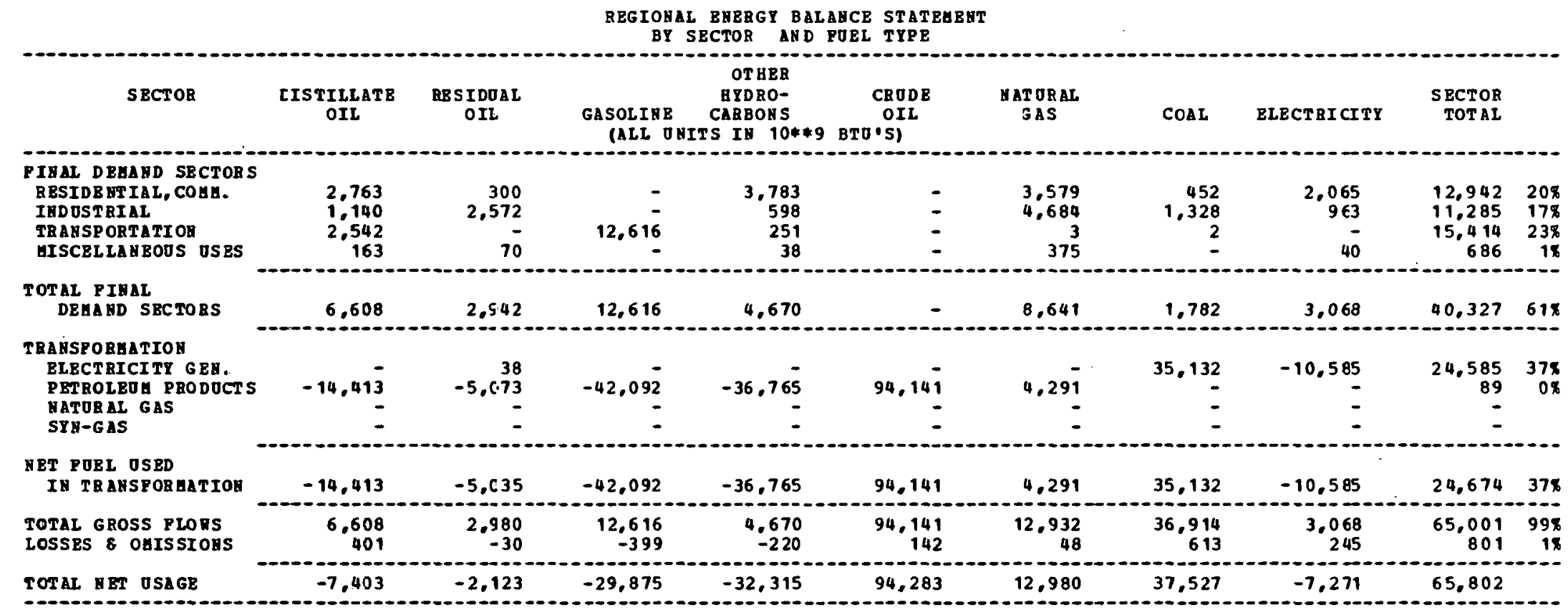

\begin{tabular}{|c|c|c|c|c|c|c|c|c|c|c|}
\hline $\begin{array}{l}\text { SOPPLY OF EHERGY } \\
\text { FOSSIL POEL } \\
\text { HYDROELECTRIC }\end{array}$ & $\overline{-}$ & $\overline{-}$ & - & $\overline{-}$ & 31,848 & 16,156 & 84.436 & $8,130^{-}$ & $\begin{array}{r}132,440 \\
8,130\end{array}$ & $\begin{array}{r}94 x \\
6 \%\end{array}$ \\
\hline ROCL E AR & - & - & - & - & - & - & - & - & - & \\
\hline GEO.E SOL AR & - & - & - & - & - & - & - & - & - & \\
\hline
\end{tabular}

GET IHPORTS
OP REGION


REGIONAL ETERGY BALANCE STATEHZNT

BY SEC:OOB AND FOEL TYPE

\begin{tabular}{|c|c|c|c|c|c|c|c|c|c|c|}
\hline SECTOR & $\begin{array}{l}\text { DISTIILATE } \\
\text { OIL }\end{array}$ & $\begin{array}{l}\text { RES : DOAL } \\
\text { OIL }\end{array}$ & $\begin{array}{l}\text { GASOLIN } \\
\text { (ALI. }\end{array}$ & $\begin{array}{c}\text { OTEER } \\
\text { HYCRO- } \\
\text { CAREONS } \\
\text { TS II 10*\% }\end{array}$ & $\begin{array}{l}\text { CRUDE } \\
\text { OIL } \\
\text { BTO'SI }\end{array}$ & $\begin{array}{l}\text { MATORAI } \\
\text { GAS }\end{array}$ & COAL & ELECTRICITY & $\begin{array}{r}\text { SECTOR } \\
\text { TOTAL }\end{array}$ & \\
\hline 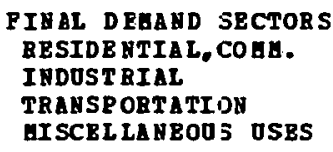 & $\begin{array}{r}7.300 \\
435 \\
5.753 \\
252\end{array}$ & $\begin{array}{r}641 \\
487 \\
12 \\
13.3\end{array}$ & 27,057 & $\begin{array}{r}\varepsilon, 035 \\
1,471 \\
891 \\
142\end{array}$ & $\overline{-}$ & $\begin{array}{r}11.447 \\
1.747 \\
209 \\
857\end{array}$ & $\begin{array}{r}875 \\
2,030 \\
5 \\
-\end{array}$ & $\begin{array}{r}4.619 \\
470 \\
4 \\
79\end{array}$ & $\begin{array}{r}32.917 \\
6.607 \\
33.911 \\
1.463\end{array}$ & $\begin{array}{r}37 \% \\
7 \% \\
38 \% \\
2 \%\end{array}$ \\
\hline $\begin{array}{l}\text { TOTAL FINAL } \\
\text { DEHA AD SBCTORS }\end{array}$ & 13,740 & 1.273 & 27.037 & $1 C, 539$ & - & 14,257 & 2,830 & 5,172 & 74,898 & $85 \pi$ \\
\hline $\begin{array}{l}\text { TBANSPORGATIC. } \\
\text { BLECT RICITY GBN. } \\
\text { PETROLEOA ERODOCTS } \\
\text { HATORAL GAS } \\
\text { SYH-GAS }\end{array}$ & $\begin{array}{r}13 \\
-55,425 \\
-\end{array}$ & $\begin{array}{r}-19.507 \\
-\end{array}$ & $\begin{array}{r}-105,072 \\
-\end{array}$ & $\begin{array}{r}-1 E ., 666 \\
-\end{array}$ & $\begin{array}{r}187,576 \\
-\end{array}$ & 9,117 & $\begin{array}{r}16,359 \\
= \\
-\end{array}$ & $\begin{array}{r}-3,427 \\
- \\
=\end{array}$ & $\begin{array}{r}12,945 \\
23 \\
- \\
-\end{array}$ & $\begin{array}{r}15 \pi \\
0 \pi\end{array}$ \\
\hline $\begin{array}{l}\text { NET POBL OSBD } \\
\text { IH TRANSFOEGATION }\end{array}$ & $-55,412$ & $-13,507$ & -105.072 & -16.666 & 187.576 & 9,117 & 16,359 & $-3,427$ & 12,968 & $15 \%$ \\
\hline $\begin{array}{l}\text { TOTAL GROSS FLORS } \\
\text { LOSSES } 8 \text { OUISSIOHS }\end{array}$ & $\begin{array}{r}13.753 \\
835\end{array}$ & $\begin{array}{r}1.273 \\
-12\end{array}$ & $\begin{array}{r}27.037 \\
-855\end{array}$ & $\begin{array}{r}10.539 \\
-497\end{array}$ & $\begin{array}{r}187.576 \\
284\end{array}$ & $\begin{array}{r}23, \equiv 74 \\
88\end{array}$ & $\begin{array}{r}19,239 \\
3.19\end{array}$ & 5,172 & $\begin{array}{r}87.866 \\
575\end{array}$ & $\begin{array}{r}99 \% \\
1 \%\end{array}$ \\
\hline
\end{tabular}

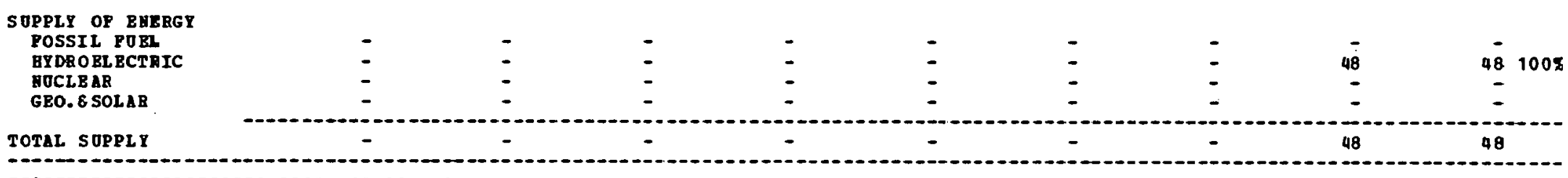

\section{MET IUPORTS}

$-40,836-18,246$

$-78,890$

$-5,624$

187,860

23,462

19,558

2,110

88,393

NOTBS:

\begin{tabular}{|c|c|c|c|c|}
\hline 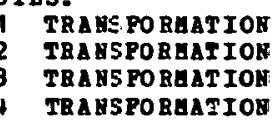 & $\begin{array}{l}\text { Loss } \\
\text { Loss } \\
\text { Loss } \\
\text { Loss }\end{array}$ & $\begin{array}{l}\text { POR } \\
\text { POR } \\
\text { POR } \\
\text { POR }\end{array}$ & $\begin{array}{l}\text { BLECTRICITY GEH. } \\
\text { PETBOLBOA PQJDOCTS } \\
\text { YATERAL GAS } \\
\text { SIR-GAS }\end{array}$ & $\begin{array}{l}=79.07 \% \\
=0.07 \% \\
=0.09 \\
=0.0 \%\end{array}$ \\
\hline
\end{tabular}


RBGIONAL ENBRGT BALABCE STATEMENT

BY SECTOR AND FOBL TYPE

\begin{tabular}{|c|c|c|c|c|c|c|c|c|c|}
\hline SBCTOR & $\begin{array}{l}\text { DISTILLATE } \\
\text { OIL }\end{array}$ & $\begin{array}{l}\text { BESIDOAL } \\
\text { OII }\end{array}$ & $\begin{array}{l}\text { GASOLIUE } \\
\text { (ALL O }\end{array}$ & $\begin{array}{l}\text { HYDRO- } \\
\text { CARBONS } \\
\text { TS IN 10**9 }\end{array}$ & $\begin{array}{c}\text { CRUDE } \\
\text { OIL } \\
\text { BTO'S) }\end{array}$ & $\begin{array}{l}\text { BAT ORAL } \\
\text { GAS }\end{array}$ & COAL & ELECTRICITY & $\begin{array}{r}\text { SECTOR } \\
\text { TOTAL }\end{array}$ \\
\hline 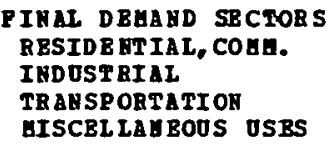 & $\begin{array}{r}2.798 \\
261 \\
1.012 \\
60\end{array}$ & $\begin{array}{r}62 \\
-\end{array}$ & $11.86 \overline{-}$ & $\begin{array}{r}3.552 \\
610 \\
255 \\
96\end{array}$ & $\begin{array}{l}5 \\
\end{array}$ & $\begin{array}{r}3.869 \\
835 \\
- \\
327\end{array}$ & $\begin{array}{r}71 \\
339 \\
1 \\
-\end{array}$ & $\begin{array}{r}1,927 \\
358 \\
20\end{array}$ & $\begin{array}{r}12,217 \\
2,465 \\
13,134 \\
503\end{array}$ \\
\hline $\begin{array}{l}\text { TOTAL PIBAL } \\
\text { DEHA AD SECTORS }\end{array}$ & 4.131 & 62 & 11.866 & 4.513 & - & 5.031 & 411 & 2,305 & 28.319 \\
\hline $\begin{array}{l}\text { TRABSPOREATION } \\
\text { BLECTRICITY GEH. } \\
\text { PETROLBUA PBODOCTS } \\
\text { MATORAL GAS } \\
\text { SYH-GAS }\end{array}$ & $\begin{array}{r}15 \\
- \\
-\end{array}$ & $\begin{array}{l}- \\
-\end{array}$ & $\begin{array}{l}- \\
-\end{array}$ & $\begin{array}{l}\overline{-} \\
\overline{-}\end{array}$ & $\begin{array}{l}- \\
\overline{-} \\
-\end{array}$ & $\begin{array}{r}570 \\
- \\
-\end{array}$ & $\begin{array}{r}275 \\
- \\
-\end{array}$ & $\begin{array}{r}-166 \\
- \\
-\end{array}$ & $\begin{array}{r}694 \\
- \\
-\end{array}$ \\
\hline $\begin{array}{l}\text { HET POBL OSBD } \\
\text { IE TR ANSFORBATION }\end{array}$ & 15 & - & $\overline{-}$ & - & - & 570 & 275 & -166 & 694 \\
\hline $\begin{array}{l}\text { TOTAL GROSS FLOES } \\
\text { LOSSES } 6 \text { OAISSIOHS }\end{array}$ & $\begin{array}{r}4.146 \\
251\end{array}$ & $\begin{array}{l}62 \\
-0\end{array}$ & $\begin{array}{r}11,866 \\
-375\end{array}$ & $\begin{array}{r}4.513 \\
-213\end{array}$ & - & $\begin{array}{r}5.601 \\
21\end{array}$ & $\begin{array}{r}686 \\
11\end{array}$ & $\begin{array}{r}2.305 \\
184\end{array}$ & $\begin{array}{r}29.013 \\
-120\end{array}$ \\
\hline
\end{tabular}

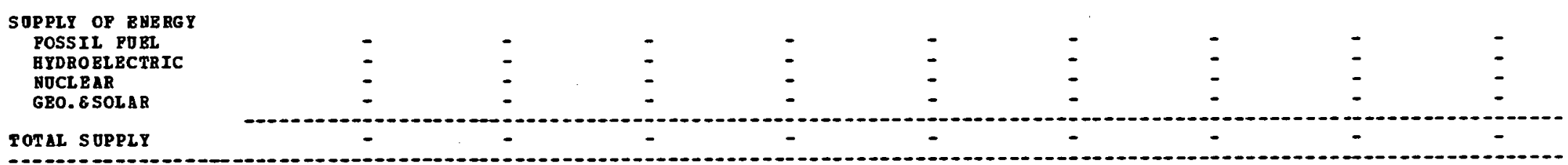

DET IHPORTS

11.490

4.299

5,622

697

2,323

28,892

-

NOTES:
1 TRAHS PORGATION LOSS POR
2 TRABSPOBAATIOB LOSS FOB
TRAUSPORAATIOS LOSS FO
TBABSFORAATIOH LOSS FOB
BLECTRICITI GEN.
PETROLEU PRODOC: $=80.70 \%$
MATOBAL GAS
$=0.0 \%$
$S Y H-G$ AS
$=0.0 \%$
$=0.0 \%$ 
REg IONAL ENERGI BALANCE STATEHENT

3T SECTOR AND FOEL TY?E

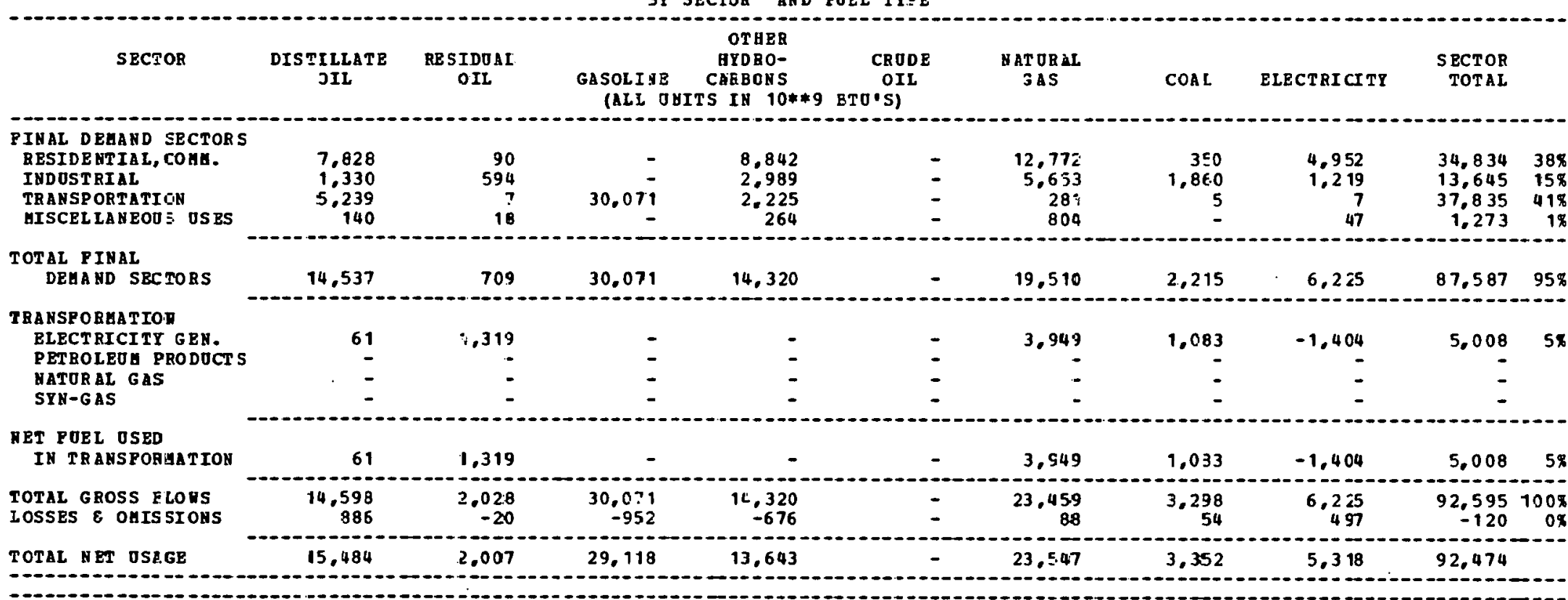

\section{SOPPL. T OP EHERG Y}

FOSSIL POBL

HYDROELECTEIC

NOCLEAR

GEO. ESOLA

-
$\vdots$
$\vdots$
-
-

TOTAL SOPPLY

$-$

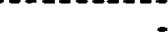

$-$

-

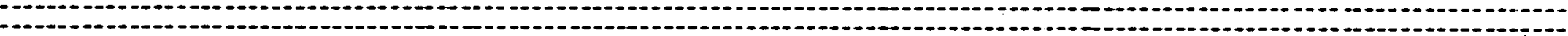

MET IAPORTS

OP REGION

15.484

2.007

29.118

13,643

23,547

3,352

5,318

92,474

HOT ES:

1 TRANSPORGATION LESS POR

TRANS PORAATION L.:SS POR

TRANSPORHATION LESS POR

BLECTRICITt 3EN.

ETE OLE'J $=78.1 \%$

MTORAL GAS

$=0.08$

$\begin{array}{ll}S Y N-G A S & =0.0 \%\end{array}$ 
BEGIONAL BNERGY BALANCE STATEGENT

BY SECTOR AND FOEL TYPE

\begin{tabular}{|c|c|c|c|c|c|c|c|c|c|}
\hline SECTOR & $\begin{array}{l}\text { DISTILLATE } \\
\text { OIL }\end{array}$ & $\begin{array}{l}\text { BESI DOAL } \\
\text { OII. }\end{array}$ & $\begin{array}{l}\text { GaSOLIBE } \\
\text { FALI O }\end{array}$ & $\begin{array}{c}\text { MYDRO- } \\
\text { CARBOHS } \\
\text { TTS IH 10**9 }\end{array}$ & $\begin{array}{c}\text { CRODE } \\
\text { OIL } \\
\text { BTO०SI }\end{array}$ & $\begin{array}{l}\text { MAT URAL } \\
\text { GAS. }\end{array}$ & COAL & BLECTAICITY & $\begin{array}{r}\text { SECTOR } \\
\text { TOTAL }\end{array}$ \\
\hline $\begin{array}{l}\text { PIRAL DBEAHD SECTORS } \\
\text { RESIDERTIAL, COAE. } \\
\text { IHDOSTRIAL } \\
\text { TRABSPORTATIOS } \\
\text { GISCELLAROOS OSBS }\end{array}$ & $\begin{array}{r}4.944 \\
956 \\
2.527 \\
111\end{array}$ & $\begin{array}{r}25 \\
234 \\
48 \\
23\end{array}$ & 21.460 & $\begin{array}{r}6.474 \\
837 \\
561 \\
169\end{array}$ & $\begin{array}{l}\overline{-} \\
\overline{-}\end{array}$ & $\begin{array}{r}7.010 \\
3,998 \\
274 \\
1.391\end{array}$ & $\begin{array}{r}143 \\
80 \\
2 \\
-\end{array}$ & $\begin{array}{r}3.398 \\
546 \\
- \\
82\end{array}$ & $\begin{array}{r}21,994 \\
6,651 \\
24,872 \\
1,776\end{array}$ \\
\hline $\begin{array}{l}\text { TOTAL PIRAL } \\
\text { DEGA BD SBCTORS }\end{array}$ & 8,538 & 330 & 21.460 & 8,041 & - & 12.673 & 225 & 4.026 & 55,293 \\
\hline $\begin{array}{l}\text { ZRARSPORGATIOH } \\
\text { BLECTRICITY GEY. } \\
\text { PETROLBOA PRODOCTS } \\
\text { BATURAL GAS } \\
\text { SYB-GAS }\end{array}$ & $\begin{array}{r}40 \\
-4,830 \\
-\end{array}$ & $\begin{array}{r}8 \\
-1,225 \\
- \\
-\end{array}$ & $\begin{array}{r}-8,645 \\
-\end{array}$ & $\begin{array}{r}- \\
-4.542 \\
-481 \\
-\end{array}$ & $\begin{array}{r}18,576 \\
-\end{array}$ & $\begin{array}{r}11 \\
847 \\
553 \\
-\end{array}$ & $\begin{array}{r}8,618 \\
- \\
-\end{array}$ & $\begin{array}{r}-1,8 \mathrm{e8} \\
- \\
-\end{array}$ & $\begin{array}{r}6.779 \\
181 \\
72 \\
-\end{array}$ \\
\hline $\begin{array}{l}\text { UET FOEL OSBD } \\
\text { IE TRANSF OREATION }\end{array}$ & $-4,790$ & -1.217 & $-8,645$ & -5.023 & 18.576 & 1.401 & 8,618 & $-1,888$ & 7.032 \\
\hline $\begin{array}{l}\text { TOTAL GROSS FLONS } \\
\text { LOSSES } \& \text { OHISSIOYS }\end{array}$ & $\begin{array}{r}8.578 \\
521\end{array}$ & $\begin{array}{r}338 \\
-3\end{array}$ & $\begin{array}{r}21.460 \\
-679\end{array}$ & $\begin{array}{r}8,041 \\
-379\end{array}$ & $\begin{array}{r}18.576 \\
28\end{array}$ & $\begin{array}{r}14.074 \\
53\end{array}$ & $\begin{array}{r}8.843 \\
147\end{array}$ & $\begin{array}{r}4.026 \\
321\end{array}$ & $\begin{array}{r}62,325 \\
8\end{array}$ \\
\hline
\end{tabular}

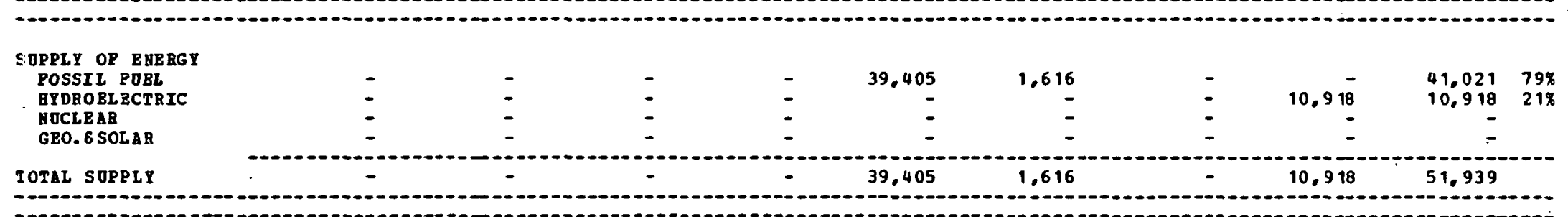

-

\section{MBT IAPOBTS}

OP REGIO\&

4.269

$-390$

12.135

2.638

$-20,800$

12.511

8,990

$-8,458$

10,394

NOT ES:

TRAASPOBEATION LOSS POR TRAHS PORAATIOH LOSS POR TRAASPOBGATIOH LOSS FOR TRA AS PORMAT IOH LOSS YOR 
RBgIOHAL ERERGY BALANCE STATEHENT

BY SECTOR AUD FOEL TYPE

\begin{tabular}{|c|c|c|c|c|c|c|c|c|c|c|}
\hline SECTOR & $\begin{array}{c}\text { DISTILLATB } \\
\text { OII }\end{array}$ & $\begin{array}{l}\text { BRSIDEAL } \\
\text { OII. }\end{array}$ & $\begin{array}{l}\text { GASOLINE } \\
\text { (ALE OHIT }\end{array}$ & $\begin{array}{l}\text { ATDBO- } \\
\text { CABBO\&S } \\
\text { TS IN 10**9 }\end{array}$ & $\begin{array}{c}\text { CRODE } \\
\text { OIL } \\
\text { BTOCSI }\end{array}$ & $\begin{array}{l}\text { MATOBAL } \\
\text { GAS }\end{array}$ & $\operatorname{COAL}$ & ELBCTRICITY & $\begin{array}{r}\text { SECTOE } \\
\text { TOTAI }\end{array}$ & \\
\hline 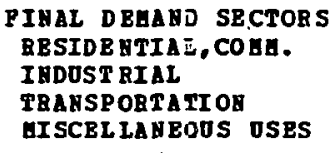 & $\begin{array}{r}995 \\
231 \\
3.024 \\
58\end{array}$ & $\begin{array}{r}104 \\
20 \\
74 \\
31\end{array}$ & 8,283 & $\begin{array}{r}2.043 \\
576 \\
809 \\
72\end{array}$ & $\begin{array}{l}- \\
-\end{array}$ & $\begin{array}{r}5,627 \\
5,5.35 \\
1,126 \\
302\end{array}$ & $\begin{array}{r}71 \\
106 \\
2 \\
-\end{array}$ & $\begin{array}{r}1.754 \\
239 \\
- \\
27\end{array}$ & $\begin{array}{r}10,594 \\
6,707 \\
13,321 \\
496\end{array}$ & $\begin{array}{r}308 \\
198 \\
388 \\
18\end{array}$ \\
\hline $\begin{array}{l}\text { TOTAL PIHAL } \\
\text { DEGA ND SECTORS }\end{array}$ & 4,308 & 229 & 8,286 & 3,500 & - & 12,590 & 179 & 2.020 & $31,11:$ & $89 x$ \\
\hline $\begin{array}{l}\text { THANSFORGAT IOH } \\
\text { ELECT RICI FY GBH. } \\
\text { PETROLEOH PRODOCTS } \\
\text { MATORAL GAS } \\
\text { SYH-GAS }\end{array}$ & $\begin{array}{r}38 \\
-2,499 \\
-\end{array}$ & $\begin{array}{r}41 \\
-245 \\
- \\
-\end{array}$ & $\begin{array}{r}-5,290 \\
-\end{array}$ & $\begin{array}{r}-1.956 \\
-720 \\
-\end{array}$ & 9.628 & $\begin{array}{r}4.319 \\
439 \\
827 \\
-\end{array}$ & $\begin{array}{l}1 \\
- \\
-\end{array}$ & $\begin{array}{r}-1.002 \\
= \\
=\end{array}$ & $\begin{array}{r}3.39 ? \\
707 \\
-\end{array}$ & $\begin{array}{r}10 \% \\
0 \% \\
0 \%\end{array}$ \\
\hline $\begin{array}{l}\text { MET FOEL OSBD } \\
\text { IN TRANSP.JR GA TIOH }\end{array}$ & $-2,461$ & -204 & $-5,290$ & $-2,676$ & 9,623 & 5,585 & 1 & $-1,002$ & $3.58 \mathrm{i}$ & $10 \%$ \\
\hline $\begin{array}{l}\text { TOTAL GROSS FLOYS } \\
\text { LOSSES } 8 \text { OAISSI OHS }\end{array}$ & $\begin{array}{r}4.346 \\
264\end{array}$ & $\begin{array}{r}270 \\
-2\end{array}$ & $\begin{array}{r}8,286 \\
-262\end{array}$ & $\begin{array}{r}3.500 \\
-165\end{array}$ & 9.623 & $\begin{array}{r}18,175 \\
68\end{array}$ & $\begin{array}{r}180 \\
2\end{array}$ & $\begin{array}{r}2.020 \\
161\end{array}$ & $\begin{array}{r}34,693 \\
87\end{array}$ & $\begin{array}{r}1008 \\
0 \%\end{array}$ \\
\hline
\end{tabular}

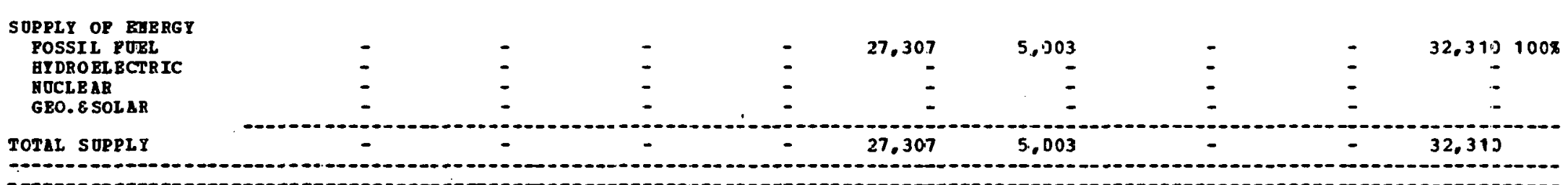

\section{IBT IUPORTS}

OP REGIOH

2.111

$22 \quad 2,733$

$658 \quad-17,664$

13,240

182

1,179

2, 464

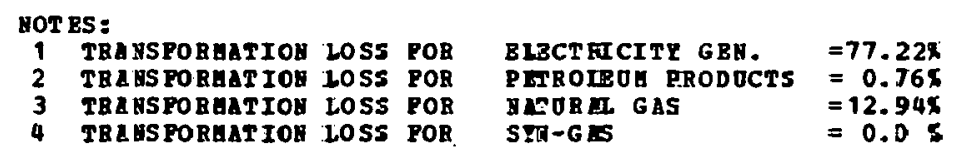


REG IONAL BHERGY BALANCE STATEGBNT

BY SECTOR AHD FOBL TIPE

\begin{tabular}{|c|c|c|c|c|c|c|c|c|c|c|}
\hline SBCTOR & $\begin{array}{l}\text { DISTILLATE } \\
\text { OIL }\end{array}$ & $\begin{array}{c}\text { RESI DIALL } \\
\text { OIJ }\end{array}$ & $\begin{array}{l}\text { GASOLIHE } \\
\text { (ALL O }\end{array}$ & $\begin{array}{l}\text { AYDBO- } \\
\text { CARBONS } \\
\text { TS IN } 10 * * 9\end{array}$ & $\begin{array}{c}\text { CRODE } \\
\text { OIL } \\
\text { BTO'SI }\end{array}$ & $\begin{array}{l}\text { MATURAL } \\
\text { GAS }\end{array}$ & COAL & ELECTRICITY & $\begin{array}{l}\text { SECTOR } \\
\text { TOT AL }\end{array}$ & \\
\hline 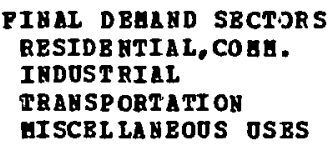 & $\begin{array}{r}2,923 \\
944 \\
4.077 \\
171\end{array}$ & $\begin{array}{r}223 \\
64 \\
121 \\
55\end{array}$ & 24,961 & $\begin{array}{r}5,738 \\
1,816 \\
888 \\
235\end{array}$ & E & $\begin{array}{r}17.132 \\
11.288 \\
2.965 \\
793\end{array}$ & $\begin{array}{r}172 \\
421 \\
4 \\
-\end{array}$ & $\begin{array}{r}5.401 \\
885 \\
- \\
88\end{array}$ & $\begin{array}{r}31,589 \\
15,418 \\
33,016 \\
1,342\end{array}$ & $\begin{array}{r}348 \\
178 \\
358 \\
1 \%\end{array}$ \\
\hline $\begin{array}{l}\text { TOTAL PIHAL } \\
\text { DEHABD SBCTORS }\end{array}$ & 8,115 & 463 & 24,961 & 8,677 & - & 32,178 & 597 & 6.374 & 81,365 & $87 \%$ \\
\hline $\begin{array}{l}\text { TBAHSPORHATION } \\
\text { BLECTRICITY GEH. } \\
\text { PETROLBUA PBODOCTS } \\
\text { MATORAL GAS } \\
\text { SYH-GAS }\end{array}$ & $\begin{array}{r}198 \\
- \\
-\end{array}$ & $\begin{array}{r}645 \\
- \\
-\end{array}$ & : & $\begin{array}{l}- \\
-\end{array}$ & 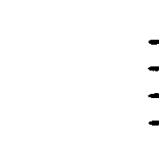 & $\begin{array}{r}14.569 \\
= \\
=\end{array}$ & $\begin{array}{r}382 \\
-\end{array}$ & $\begin{array}{r}-3,940 \\
= \\
=\end{array}$ & $\begin{array}{r}11.854 \\
= \\
-\end{array}$ & $13 \%$ \\
\hline $\begin{array}{l}\text { N3T FOEL OSBD } \\
\text { IA TEARSFORAATIOH }\end{array}$ & 198 & 645 & - & - & - & 14.569 & 382 & $-3,940$ & 11,854 & $13 \%$ \\
\hline $\begin{array}{l}\text { TOTAL GROSS FLOVS } \\
\text { LOSSES } \varepsilon \text { OAISSIORS }\end{array}$ & $\begin{array}{r}8,313 \\
505\end{array}$ & $\begin{array}{r}1.108 \\
-11\end{array}$ & $\begin{array}{r}24.961 \\
-790\end{array}$ & $\begin{array}{r}8,677 \\
-409\end{array}$ & - & $\begin{array}{r}46.747 \\
177\end{array}$ & $\begin{array}{r}979 \\
16\end{array}$ & $\begin{array}{r}6.374 \\
509\end{array}$ & $\begin{array}{r}93.219 \\
-3\end{array}$ & $\begin{array}{r}100 \% \\
0 \%\end{array}$ \\
\hline
\end{tabular}

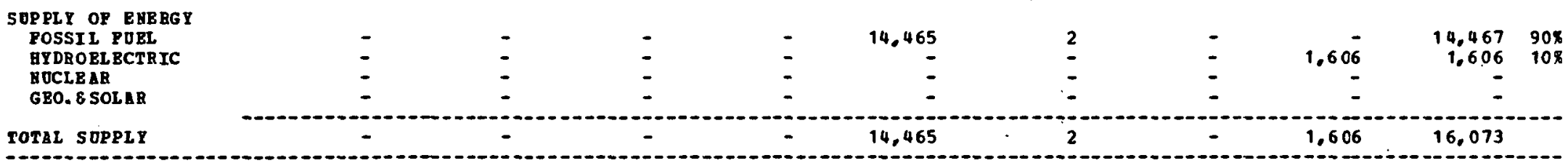

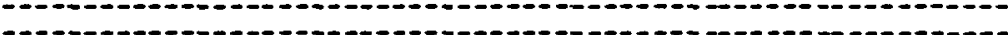

\section{BET ISPORTS}

OP REGIOA

1.096

24.170

$8.267-14,465$

46,922

995

1,337

77,142

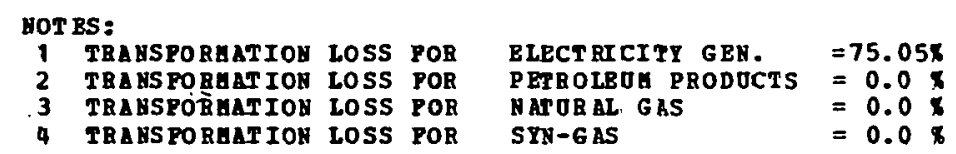


REGIONAL 3NERGY BALANCE STATEAENT

BT SECTCR AND FOEL TYPE

\begin{tabular}{|c|c|c|c|c|c|c|c|c|c|c|}
\hline SECTOR & $\begin{array}{l}\text { DISTILLATB } \\
\text { OIL }\end{array}$ & $\begin{array}{l}\text { RES I DO AL } \\
\text { OII }\end{array}$ & $\begin{array}{l}\text { GASOLINE } \\
\text { (ALL D }\end{array}$ & $\begin{aligned} & \text { GYDRO- } \\
& \text { ZAEBOAS } \\
&=5 \text { IN 10*\% }\end{aligned}$ & $\begin{array}{c}\text { CRODE } \\
\text { OIL } \\
\text { BTO'SI }\end{array}$ & $\begin{array}{l}\text { NATURAL } \\
\text { GAS }\end{array}$ & $\cos x$ & ELECTRICITY & $\begin{array}{r}\text { SECTOR. } \\
\text { TOTAL }\end{array}$ & \\
\hline $\begin{array}{l}\text { PISAL DEHAHE SBCTORS } \\
\text { RBSIDE HTIAL, COHA. } \\
\text { INDOST RIAL } \\
\text { TRANSPORTATIOH } \\
\text { GISCEL LANEODS OSES }\end{array}$ & $\begin{array}{l}5,742 \\
1.797 \\
6.441 \\
216\end{array}$ & $\begin{array}{r}217 \\
111 \\
231 \\
50\end{array}$ & 36,402 & $\begin{array}{r}8,995 \\
3,911 \\
1,296 \\
481\end{array}$ & $\begin{array}{l}- \\
- \\
-\end{array}$ & $\begin{array}{r}21.715 \\
17.019 \\
2.702 \\
1.363\end{array}$ & $\begin{array}{r}354 \\
3,935 \\
6 \\
-\end{array}$ & $\begin{array}{r}6,624 \\
1,970 \\
2 \\
92\end{array}$ & $\begin{array}{r}43.647 \\
28.743 \\
47.080 \\
2.202\end{array}$ & $\begin{array}{r}31 \% \\
20 \% \\
33 \% \\
2 \%\end{array}$ \\
\hline $\begin{array}{l}\text { TOTAL F IHAL } \\
\text { DEHA ND SECTORS }\end{array}$ & 14.196 & 609 & 36,402 & 14.683 & - & 42.799 & 4.295 & 8,688 & $121.67 \bar{a}$ & $85 \pi$ \\
\hline $\begin{array}{l}\text { TRABSPORBATIOH } \\
\text { BLECT RICITY GEH. } \\
\text { PBTROLEOA PBODOCTS } \\
\text { MATORAL GAS } \\
\text { STH-GAS }\end{array}$ & $\begin{array}{r}304 \\
-\end{array}$ & $\begin{array}{r}327 \\
- \\
-\end{array}$ & $\overline{-}$ & : & 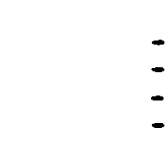 & $\begin{array}{r}8.433 \\
- \\
-\end{array}$ & $\begin{array}{r}20,366 \\
- \\
-\end{array}$ & $\begin{array}{r}-8.661 \\
- \\
-\end{array}$ & $\begin{array}{r}20,76 \subseteq \\
= \\
=\end{array}$ & $15 \%$ \\
\hline $\begin{array}{l}\text { NET POEL OS ZD } \\
\text { IH TRABSFOABA TIOA }\end{array}$ & 304 & 327 & - & - & - & 8,433 & 20,366 & $-8,661$ & 20.769 & $15 \%$ \\
\hline $\begin{array}{l}\text { TOTAL GROSS FLONS } \\
\text { LOSSES } 8 \text { OAISSI OHS }\end{array}$ & $\begin{array}{r}14.500 \\
880\end{array}$ & $\begin{array}{r}936 \\
-9\end{array}$ & $\begin{array}{l}36,4.02 \\
-1,152\end{array}$ & $\begin{array}{r}14.683 \\
-693\end{array}$ & - & $\begin{array}{r}51.232 \\
193\end{array}$ & $\begin{array}{r}24.661 \\
410\end{array}$ & $\begin{array}{r}6,688 \\
694\end{array}$ & $\begin{array}{r}142.44^{\circ} \\
324\end{array}$ & $\begin{array}{r}100 \% \\
0.8\end{array}$ \\
\hline
\end{tabular}

\begin{tabular}{|c|c|c|c|c|c|c|c|c|c|}
\hline $\begin{array}{l}\text { UPLLY OF EABHEY } \\
\text { FOSSI POBL }\end{array}$ & - & - & - & - & - & - & - & . & - \\
\hline GYDBOBLBCTRIC & - & - & - & - & - & - & - & 7.935 & $7.935100 \%$ \\
\hline NOCLEAR & - & - & - & - & - & - & - & - & - \\
\hline GEO. 6 SOLAB & - & & - & - & 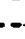 & 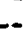 & 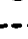 & 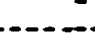 & $\vec{v}$ \\
\hline
\end{tabular}

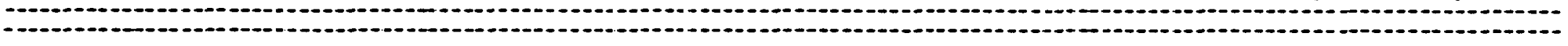

\section{HET IUPORTS}

35,249

13,989

51.425

25,071

$-7,213$

134,830

HOT $\mathrm{ES}:$

1 TRA

TRAHSPORAAT IOH LOSS POR

TRANSPOBAATION LOSS POR

TREASPORGAT TOH LOSJ POR

3L3CTRICITY GBN.

$=70.57 x$

ERTOC $=0.35$

STI-6AS 
REGIONAL BNERGT BALANCE STATEREHT

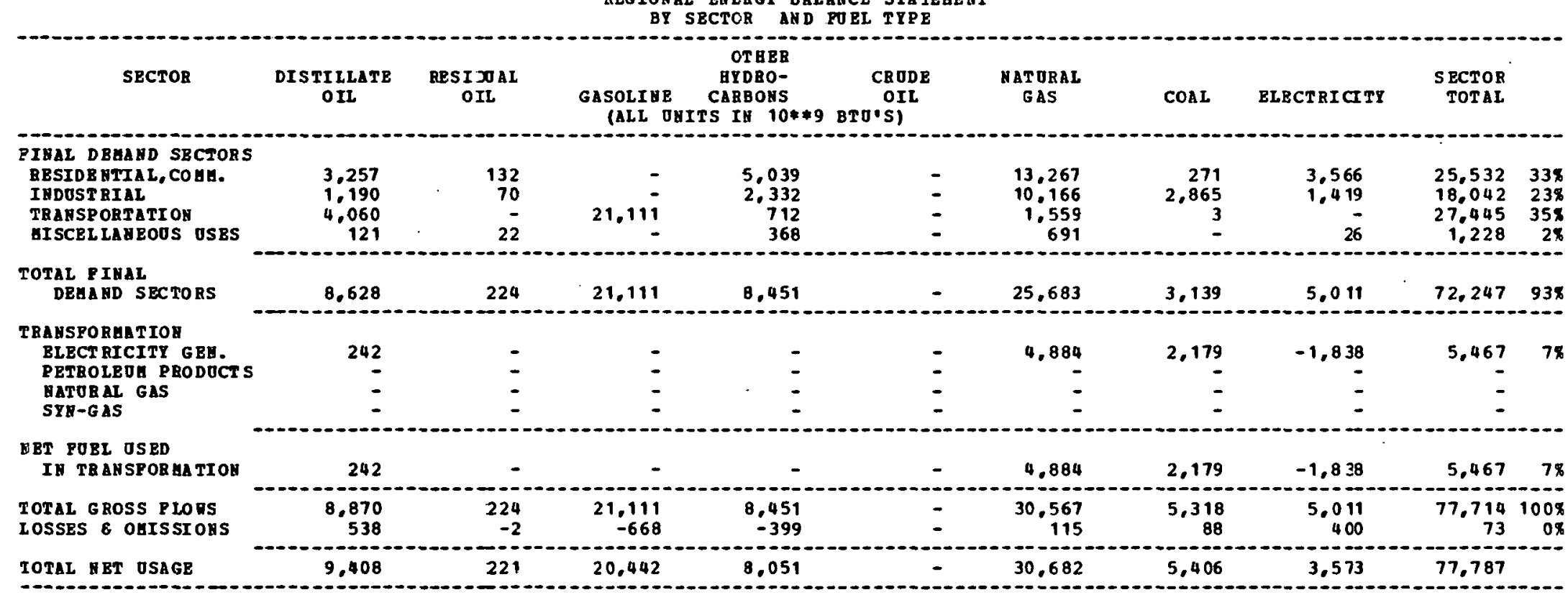

(1)

\section{SOPPLY OP E EERGI}

POSSIL PUEL

GYDROELECTRIC

NOCL.BAB

GEO. \& SOL $\triangle B$

TOTAI SUPPLI

-
$\vdots$
-
-

$\overline{-}$

-
-
-
-

-
-
-
-

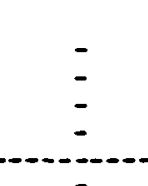

\section{HET IHPORTS \\ OP REGIOH}

9.408

221

20.442

6.051

30,682

5,406

3.573

77.787

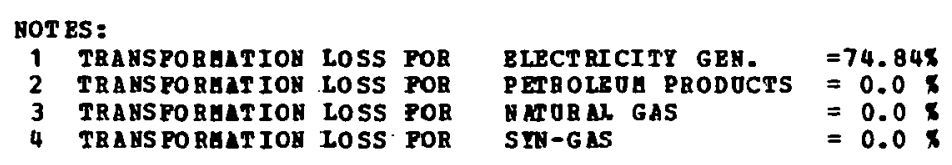


REGIONAL BHERGY BALARCE STA TEHENT

3T SECTOS AND FOEL TYPE
TY

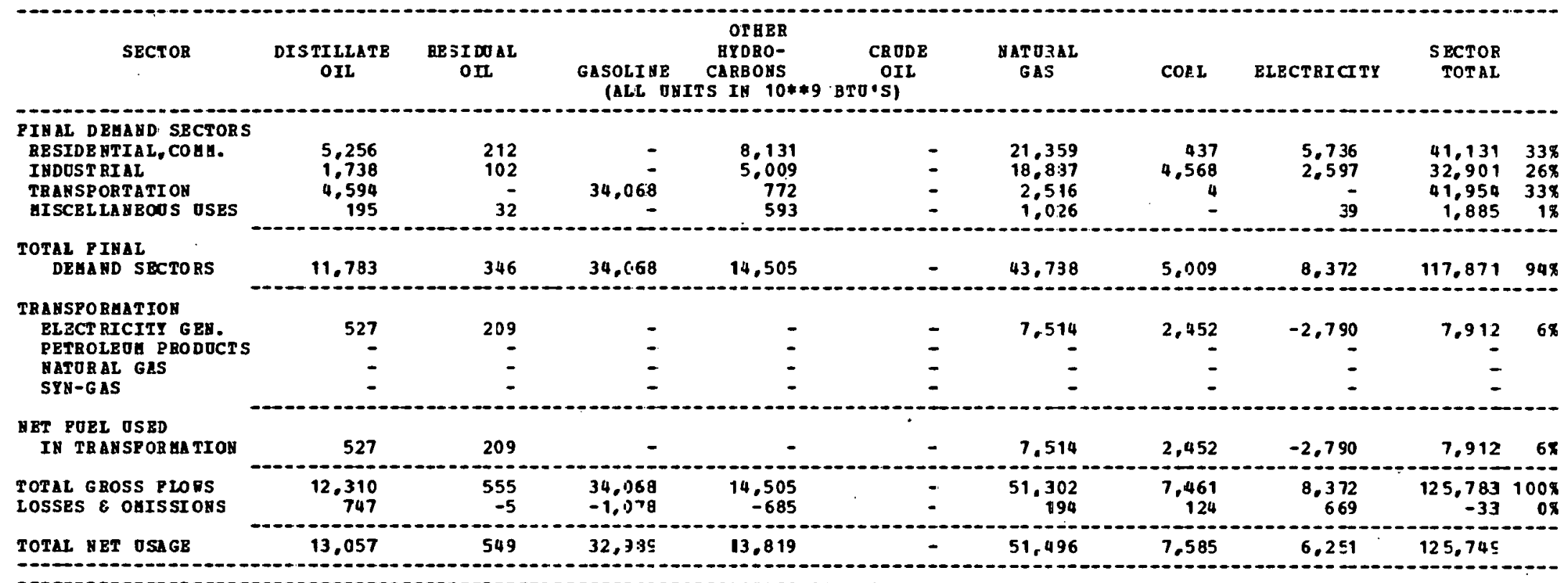

\section{SOPPLY OF BUERG \\ FOSSI L POSL \\ HYDROELBCTRIC \\ NOCLBAR \\ GBO. 8 SOLAE}

TOTAL SOPPLY

-
-
-
-2

-

:

-

$-$

$-$

1

$\begin{array}{lll}- & i & 1008 \\ - & - & \end{array}$

HET IUPORTS

OP REGION

13.057

549

32.989

13,819

51.496

7,585

6,250

125,743

nOT ES:

1 TRAHSPORGATION LOSS POR TRAYSPORAATION LOSS POR
TRAYSPOREATION LOSS POR TRABSFORATIOH LOSS POR

BLECTRICITY GRH.

$\begin{array}{ll}\text { LECTRICITY 6EH. } & =73.93 \mathrm{x} \\ \text { EROLOOA PRODOCTS } & =0.0\end{array}$

SEORAL GAS

SIR-GAS

$=0.0$ 
REg TOHAL BNBBgY BALAHCE STATEGEHT

IONAL ENBBGY BALAHCE STATEAE
BY SECTOR ABD FOBL TYPE

\begin{tabular}{|c|c|c|c|c|c|c|c|c|c|}
\hline SECTOR & $\begin{array}{l}\text { DISTILLATE } \\
\text { OIL }\end{array}$ & $\begin{array}{l}\text { RESI DUAL } \\
\text { OIL }\end{array}$ & $\begin{array}{l}\text { GASOLIHE } \\
\text { (ALL OHI }\end{array}$ & $\begin{array}{c}\text { OTBRR } \\
\text { BYDRO- } \\
\text { CARBONS } \\
\text { TS IN 10**9 }\end{array}$ & $\begin{array}{c}\text { CRODE } \\
\text { OIL } \\
\text { BTO'S) }\end{array}$ & $\begin{array}{l}\text { NaTORAL } \\
\text { GAS }\end{array}$ & $\operatorname{COAL}$ & ELECTRICITY & $\begin{array}{l}\text { SECTOR } \\
\text { TOTAL }\end{array}$ \\
\hline $\begin{array}{l}\text { PINAL D BGAND SECTORS } \\
\text { RBSIDE HTIAL, COBA. } \\
\text { INDUST RIAL } \\
\text { TRANSPORTATION } \\
\text { GISCEL LABEOUS OSBS }\end{array}$ & $\begin{array}{r}9,822 \\
2,313 \\
11.495 \\
364\end{array}$ & $\begin{array}{r}542 \\
135 \\
84\end{array}$ & 63,663 & $\begin{array}{r}15.195 \\
7.772 \\
4.563 \\
1.108\end{array}$ & $\overline{-}$ & $\begin{array}{r}44.507 \\
23.875 \\
4.701 \\
2.646\end{array}$ & $\begin{array}{r}818 \\
5,082 \\
12 \\
-\end{array}$ & $\begin{array}{r}11.922 \\
5.005 \\
22 \\
101\end{array}$ & $\begin{array}{r}82.806 \\
44.182 \\
84.456 \\
4.303\end{array}$ \\
\hline $\begin{array}{l}\text { TOTAL PIHAL } \\
\text { DEGA HD SECTORS }\end{array}$ & 23,994 & 761 & 63,663 & 28,638 & - & 75,729 & 5,912 & 17.050 & 215,747 \\
\hline $\begin{array}{l}\text { TRABSPORHATIOH } \\
\text { BLBCTRICITY GBH. } \\
\text { PETROLEOA PBODOCT } \\
\text { HATORAL GAS } \\
\text { SYH-GAS }\end{array}$ & $\begin{array}{r}340 \\
- \\
-\end{array}$ & $\begin{array}{r}311 \\
-\end{array}$ & $\begin{array}{l}\overline{-} \\
-\end{array}$ & $\begin{array}{l}- \\
-\end{array}$ & 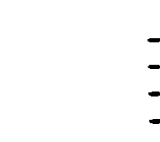 & $\begin{array}{r}25,800 \\
- \\
-\end{array}$ & $\begin{array}{r}21.058 \\
- \\
-\end{array}$ & $\begin{array}{r}-12,262 \\
= \\
=\end{array}$ & $\begin{array}{r}35.247 \\
- \\
=\end{array}$ \\
\hline $\begin{array}{l}\text { MBT POEL OSBD } \\
\text { IN TRANSPOREA TION }\end{array}$ & 340 & 311 & - & - & - & 25,800 & 21.058 & $-12,262$ & 35,247 \\
\hline $\begin{array}{l}\text { TOTAL G ROSS PLORS } \\
\text { LOSSES } 8 \text { OHISSI OHS }\end{array}$ & $\begin{array}{r}24,334 \\
1,478\end{array}$ & $\begin{array}{r}1,072 \\
-10\end{array}$ & $\begin{array}{r}63.663 \\
-2,015\end{array}$ & $\begin{array}{l}28,638 \\
-1,353\end{array}$ & 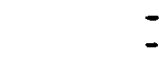 & $\begin{array}{r}101,529 \\
384\end{array}$ & $\begin{array}{r}26,970 \\
448\end{array}$ & $\begin{array}{r}17,050 \\
1,363\end{array}$ & $\begin{array}{r}250,994 \\
295\end{array}$ \\
\hline
\end{tabular}

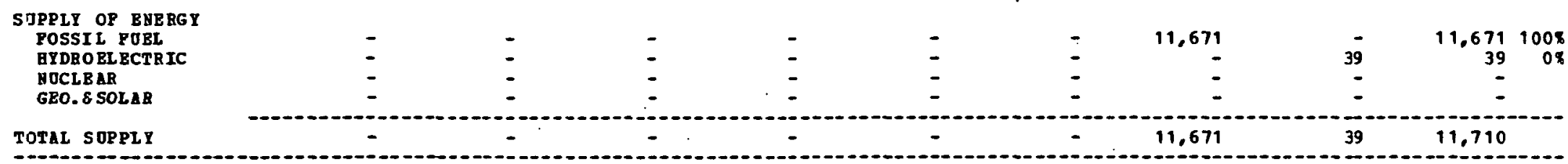

Tor

\section{G3T IHPORTS}

25,812

1,061

61,647

27.284

101,913

15,747

6,112

239,579

-

HOTES:
TRABS PORHAT IOA LOSS POR
TRABSPORAATIOB LOSS POB
TRAAS PORALTION LOSS POR
BLECTRICITY GEB
$=74.19 \%$
TRAASPORALTIOH LOSS POR
NATURAC GAS
$=0.0 \%$
SYH-G AC 
REG IONAL ENERGY BALANCE STATEMENT BY SECTOR AND POEL TYPE

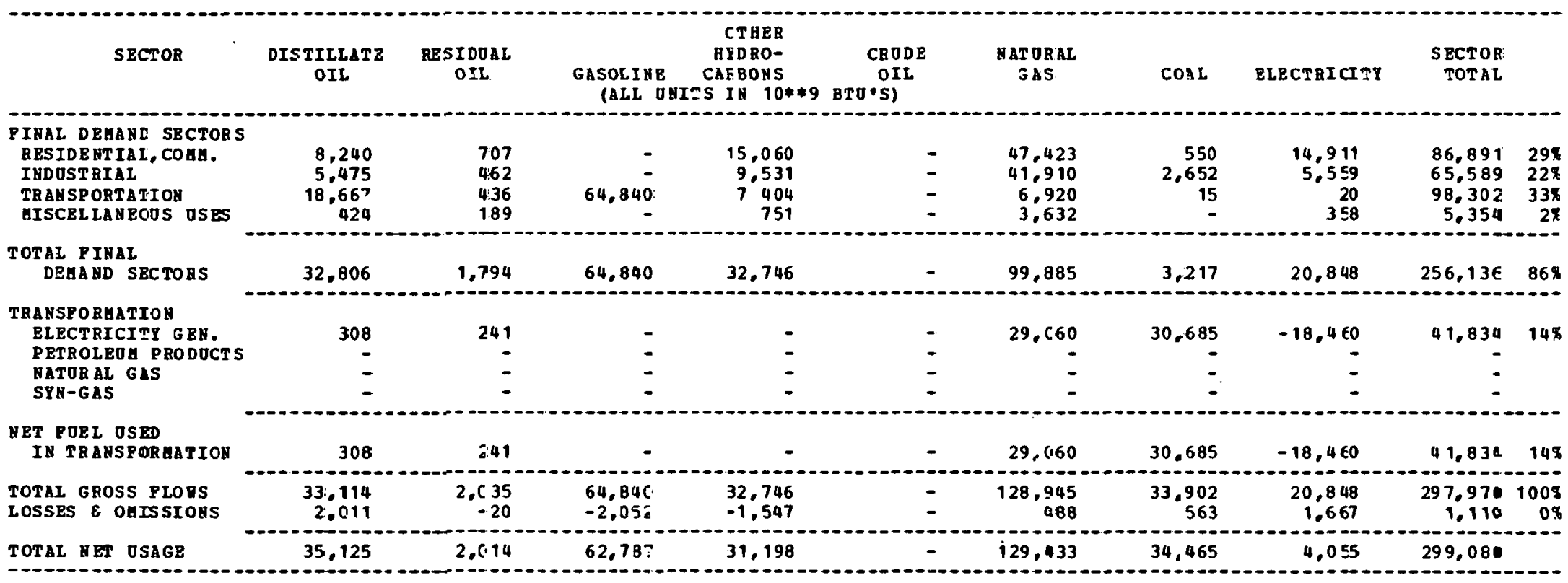

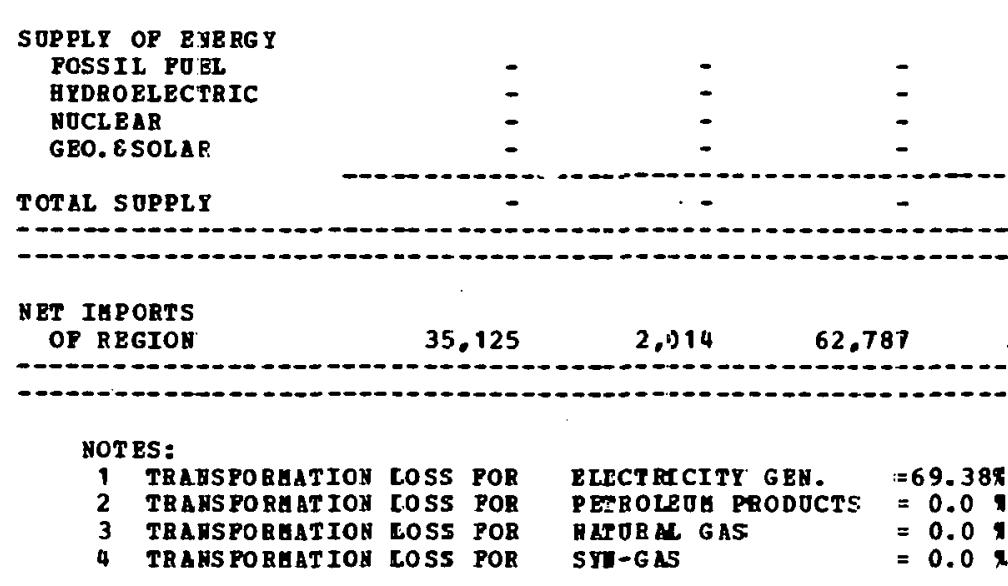




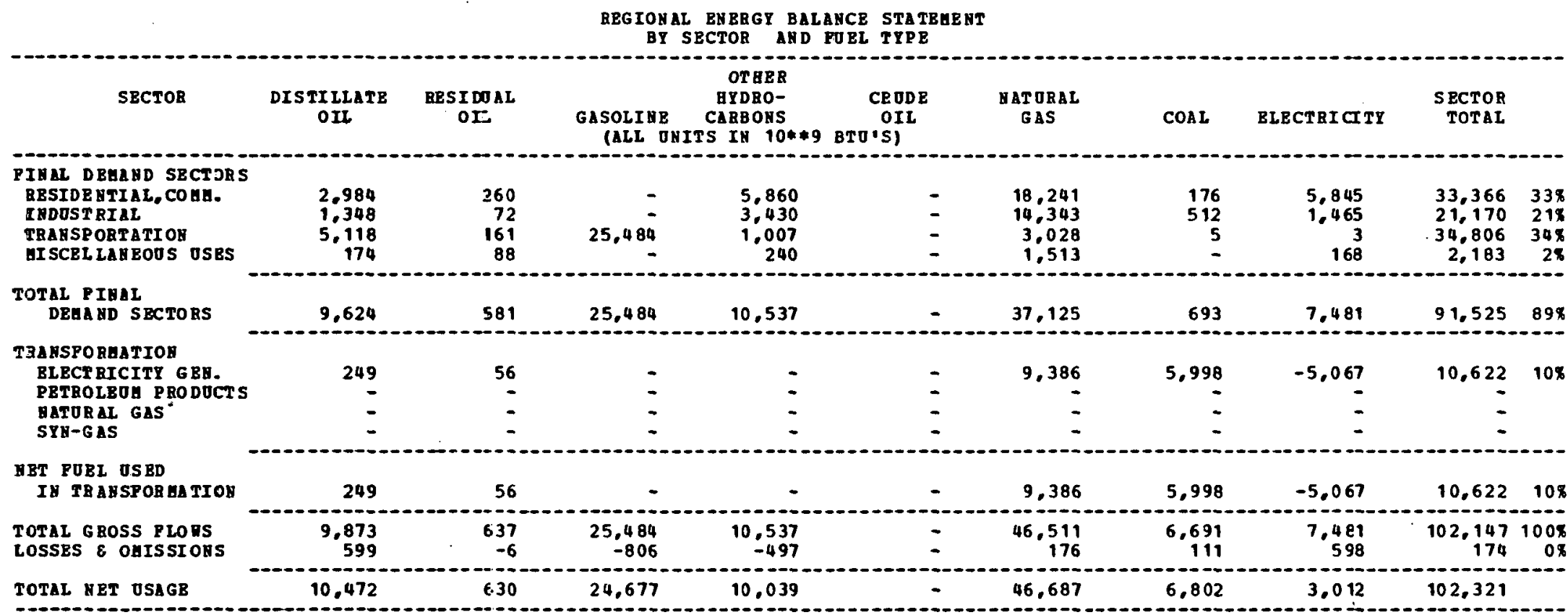

$\begin{array}{ll} & \end{array}$

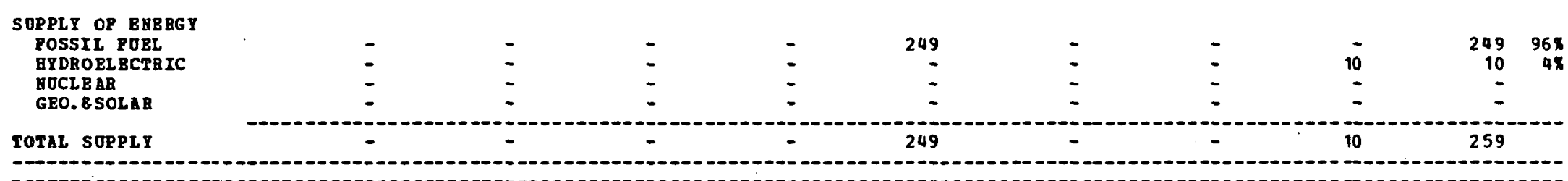

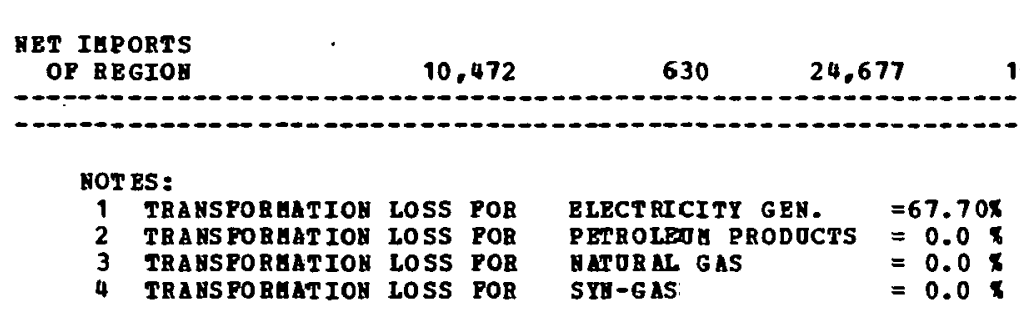


REG IONAL BNERGY BALANCE STATEGENT BI SECTOZ AND FOBL TIPE

\begin{tabular}{|c|c|c|c|c|c|c|c|c|c|}
\hline SBCIOR & $\begin{array}{l}\text { DIS IILLATE } \\
\text { OIL }\end{array}$ & $\begin{array}{l}\text { RES I DD AL } \\
\text { OII }\end{array}$ & $\begin{array}{l}\text { GASOL.THE } \\
\text { (ALI ERIT }\end{array}$ & $\begin{array}{l}\text { HYORO- } \\
\text { CAR:3ONS } \\
\text { S I' 10**9 }\end{array}$ & $\begin{array}{l}\text { CBODE } \\
\text { OIL } \\
\text { BTO'S) }\end{array}$ & $\begin{array}{l}\text { HATOBAL } \\
\text { GAS }\end{array}$ & $\cos L$ & ELECTRICITY & $\begin{array}{l}\text { SECTOR } \\
\text { TOTAL }\end{array}$ \\
\hline $\begin{array}{l}\text { PIMAL DBHA SD SBCTORS } \\
\text { RESIDE HTIAL, COHE. } \\
\text { IHDOST RIAL } \\
\text { TRABSPORTATIOH } \\
\text { GISCEL LAHEODS OSBS }\end{array}$ & $\begin{array}{r}919 \\
2.407 \\
5,708 \\
123\end{array}$ & $\begin{array}{r}94 \\
1.404 \\
63 \\
75\end{array}$ & 27.430 & $\begin{array}{r}5,295 \\
1.037 \\
1.560 \\
244\end{array}$ & : & $\begin{array}{l}21,741 \\
58,101 \\
11,4 \div 0 \\
906\end{array}$ & $\begin{array}{r}290 \\
554 \\
6 \\
-\end{array}$ & $\begin{array}{r}5.743 \\
1.842 \\
13 \\
27\end{array}$ & $\begin{array}{r}35.082 \\
65.745 \\
46.190 \\
1.375\end{array}$ \\
\hline $\begin{array}{l}\text { TOTAL PIBAL } \\
\text { DEGA AD SBCTORS }\end{array}$ & 9,157 & 1.636 & 27,430 & 9.136 & - & 92,158 & 1.250 & 7,625 & 148,392 \\
\hline $\begin{array}{l}\text { TRANSPORHATIOB } \\
\text { BLBCT RICITT GBH. } \\
\text { PBTROLEOA PRODOCTS } \\
\text { BATORAL GAS } \\
\text { SYR-G AS }\end{array}$ & $\begin{array}{r}515 \\
-37.829 \\
- \\
-\end{array}$ & $\begin{array}{r}304 \\
-3.678 \\
- \\
-\end{array}$ & $\begin{array}{r}-78,942- \\
-\end{array}$ & $\begin{array}{r}-30.409 \\
-20,560 \\
-\end{array}$ & $\begin{array}{r}144,910^{-} \\
-\end{array}$ & $\begin{array}{r}19,133 \\
6,506 \\
23,630 \\
-\end{array}$ & $\begin{array}{l}\overline{-} \\
\bar{z}\end{array}$ & $\begin{array}{r}-5,201 \\
- \\
-\end{array}$ & $\begin{array}{r}14.751 \\
658 \\
3.070 \\
-\end{array}$ \\
\hline $\begin{array}{l}\text { YBT POBL OSBD } \\
\text { IN TBAESPOE HA TION }\end{array}$ & $-37,314$ & $-3,374$ & $-78,942$ & $-50,969$ & 144,910 & 49,359 & - & -5.201 & 18.479 \\
\hline $\begin{array}{l}\text { TOTAI GROSS TLONS } \\
\text { LOSSBS \& OUISSIOOSS }\end{array}$ & $\begin{array}{r}9.672 \\
587\end{array}$ & $\begin{array}{r}1,940 \\
-19\end{array}$ & $\begin{array}{r}27.430 \\
-868\end{array}$ & $\begin{array}{r}9.136 \\
-431\end{array}$ & $\begin{array}{r}144,910 \\
219\end{array}$ & $\begin{array}{r}141.527 \\
535\end{array}$ & $\begin{array}{r}1.250 \\
20\end{array}$ & $\begin{array}{r}7.625 \\
609\end{array}$ & $\begin{array}{r}166.871 \\
654\end{array}$ \\
\hline TOTAL AET OSAGB & $-27,569$ & -1.757 & $-52,380$ & $-12,264$ & 145,129 & 142,062 & 1.270 & $3,0 \equiv 3$ & 167,525 \\
\hline
\end{tabular}

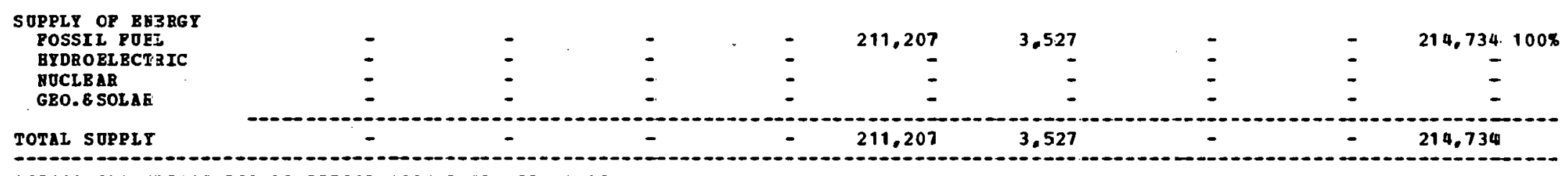

\begin{tabular}{l}
$\begin{array}{l}\text { UBT IMPORTS } \\
\text { OP RBGIOA }\end{array}$ \\
\hline
\end{tabular}


REGIONAL ENERGY BALABCE STATBEEAT

BY SBCTOB AND POBL TYPE

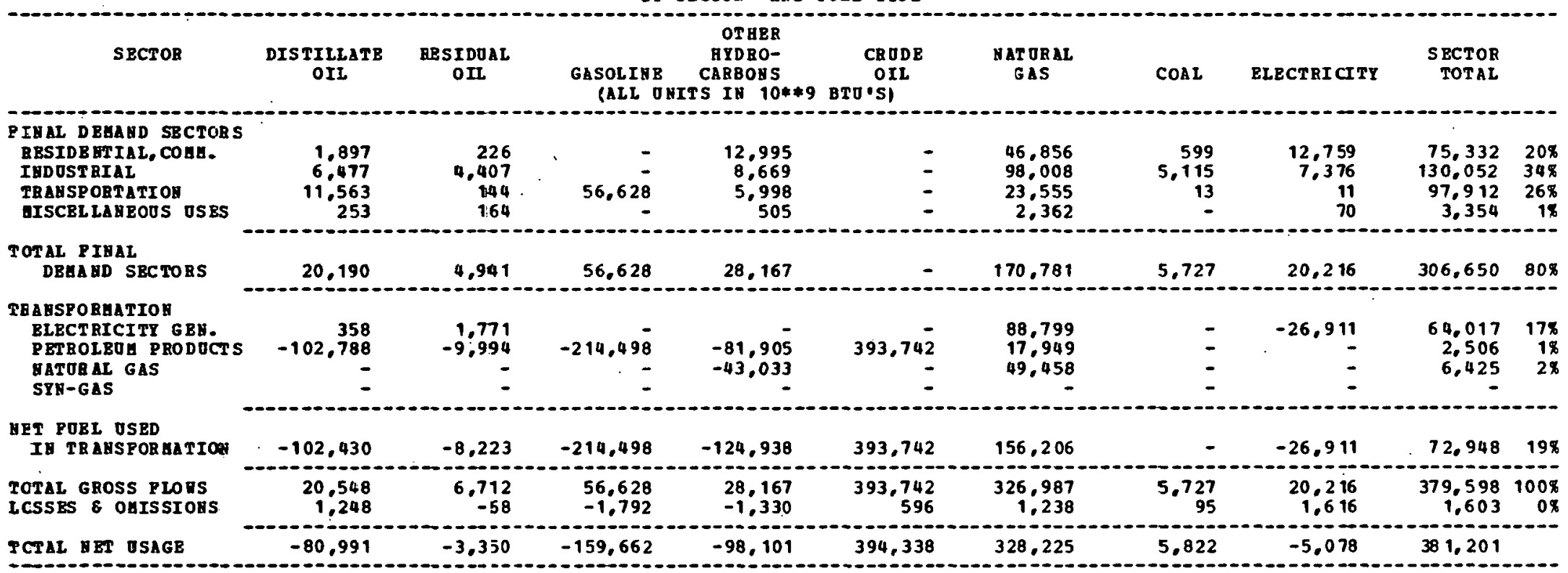

-

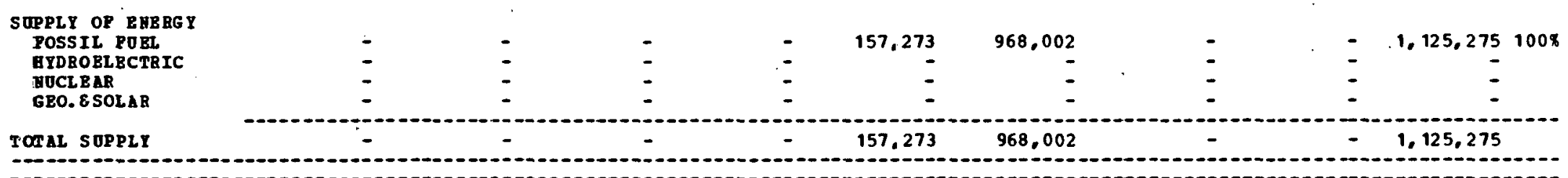

\section{KET IUPORTS}

OF REGIOH

$-80,991$

$-3.350$

$-159,662$

$-98,101$

237,065

$-639,776$

5,822

$-5,078$

$-744,073$

\begin{tabular}{|c|c|c|c|c|c|}
\hline $\begin{array}{l}1 \\
2 \\
3 \\
4\end{array}$ & 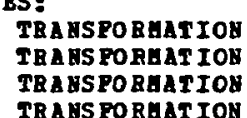 & $\begin{array}{l}\text { LoSS } \\
\text { LosS } \\
\text { LOSS } \\
\text { tosS }\end{array}$ & $\begin{array}{l}\text { POB } \\
\text { FOR } \\
\text { POE } \\
\text { POR }\end{array}$ & $\begin{array}{l}\text { BLECT RTCITY GEN. } \\
\text { PERROLBES PRODOCTS } \\
\text { MAROBA. GAS } \\
\text { SEN-GAS }\end{array}$ & $\begin{array}{l}=70.40 \pi \\
=0.618 \\
=12.998 \\
=0.0\end{array}$ \\
\hline
\end{tabular}


RBGIJNAL BEERGY BALANCE STATEAERT

BT SECTOR AND POEL TYPE

\begin{tabular}{|c|c|c|c|c|c|c|c|c|c|}
\hline SECTOR & $\begin{array}{l}\text { DISTILLATE } \\
\text { OIL }\end{array}$ & $\begin{array}{l}\text { RES I DOAL } \\
\text { C IL }\end{array}$ & $\begin{array}{l}\text { GASOLINE } \\
\text { (ALL OHI }\end{array}$ & $\begin{array}{l}\text { EXDRO- } \\
\text { CARBOHS } \\
\text { TS I* 10**9 }\end{array}$ & $\begin{array}{c}\text { CR UDE } \\
\text { OIL } \\
\text { BTO'SI }\end{array}$ & $\begin{array}{l}\text { HATORAL } \\
\text { GAS }\end{array}$ & COAL & ELECTRI $\simeq$ TY & $\begin{array}{r}\text { SECTOR } \\
\text { TOTAL }\end{array}$ \\
\hline $\begin{array}{l}\text { PIHAL DEHAHD SECTORS } \\
\text { RESIDE GTIAL, COUA. } \\
\text { IMDOST RIAL } \\
\text { TRABSPORTATI ON } \\
\text { GISCBLIANEOOS OSES }\end{array}$ & $\begin{array}{r}12.904 \\
8.834 \\
35.210 \\
575\end{array}$ & $\begin{array}{r}2.191 \\
5.068 \\
203 \\
672\end{array}$ & 173,391 & $\begin{array}{r}43.542 \\
25.552 \\
28.879 \\
1.244\end{array}$ & $\overline{-}$ & $\begin{array}{r}131,2.04 \\
68,025 \\
35,8115 \\
9,593\end{array}$ & $\begin{array}{r}2.671 \\
21.084 \\
46 \\
-\end{array}$ & $\begin{array}{r}35.864 \\
17.300 \\
116 \\
367\end{array}$ & $\begin{array}{r}228.376 \\
145,864 \\
273,661 \\
12,451\end{array}$ \\
\hline $\begin{array}{l}\text { TOTAL PINAL } \\
\text { DEHA ND SECTORS }\end{array}$ & 57,523 & 8.134 & 173,391 & 99.217 & - & 244.639 & $23,8) 1$ & 53,647 & 660,352 \\
\hline $\begin{array}{l}\text { TRANSPORGATIOH } \\
\text { ELECT RICITY GBH. } \\
\text { PETROLEOA PBODOCTS } \\
\text { HATURAL GAS } \\
\text { SYN-GAS }\end{array}$ & $\begin{array}{r}1,685 \\
-96,547 \\
=\end{array}$ & $\begin{array}{r}3.160 \\
-9.383 \\
- \\
-\end{array}$ & $\begin{array}{r}-201,059 \\
-\end{array}$ & $-77,600$ & $\begin{array}{r}369,440^{\circ} \\
-\end{array}$ & $\begin{array}{r}94,152 \\
16,841 \\
=\end{array}$ & $\begin{array}{r}110.612 \\
= \\
=\end{array}$ & $\begin{array}{r}-58.325 \\
= \\
=\end{array}$ & $\begin{array}{r}151,284 \\
1,692 \\
-\end{array}$ \\
\hline $\begin{array}{l}\text { NBT FOEL OSBI } \\
\text { IH TRANSFOE AATIOH }\end{array}$ & $-94,862$ & -5.223 & -201.055 & $-77,600$ & 369.440 & 110.993 & 110,612 & $-58,325$ & 152,976 \\
\hline $\begin{array}{l}\text { TOTAL GROSS ELORS } \\
\text { LOSSES } 8 \text { OMISSIONS }\end{array}$ & $\begin{array}{r}59.208 \\
3.596\end{array}$ & $\begin{array}{r}11.294 \\
-114\end{array}$ & $\begin{array}{r}173,391 \\
-5,439\end{array}$ & $\begin{array}{l}99,217 \\
-0,687\end{array}$ & $\begin{array}{r}369.440 \\
560\end{array}$ & $\begin{array}{r}355,6 ; 32 \\
1,346\end{array}$ & $\begin{array}{r}134.413 \\
2.234\end{array}$ & $\begin{array}{r}53,647 \\
4,290\end{array}$ & $\begin{array}{r}813.328 \\
1.737\end{array}$ \\
\hline
\end{tabular}

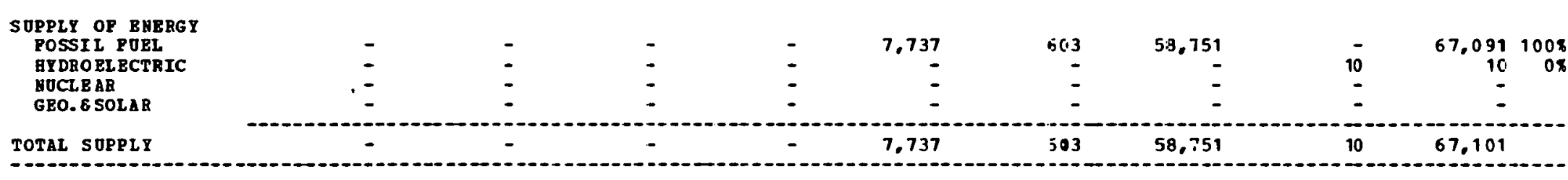

\section{NET IHPORTS}

$-33,742$

1.796

$-33,157$

15,929

362,263

77,896

$-397 \quad 747,964$

HOTES:

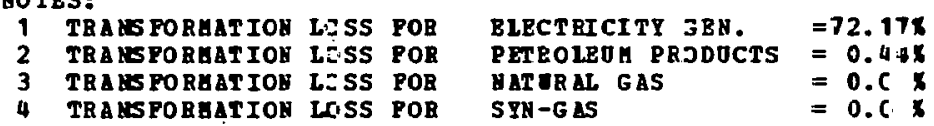


REGIONAL BNERGT BALAHCB STATEGEHT

BY SECTOB AUD FOBL TYPE

\begin{tabular}{|c|c|c|c|c|c|c|c|c|c|}
\hline SECTOR & $\begin{array}{l}\text { DISTILLATE } \\
\text { OIL }\end{array}$ & $\begin{array}{l}\text { RESILOAL } \\
\text { OIL }\end{array}$ & $\begin{array}{l}\text { GASOLIHE } \\
\text { (ALL OHI }\end{array}$ & $\begin{array}{c}\text { OTBBB } \\
\text { BYDRO- } \\
\text { CABBONS } \\
\text { TS IN 10**9 }\end{array}$ & $\begin{array}{c}\text { CRODE } \\
\text { OIl } \\
\text { BTO } S \text { S) }\end{array}$ & $\begin{array}{l}\text { SAT ORAL } \\
\text { G AS }\end{array}$ & COAL & BLBCTBICITY & $\begin{array}{l}\text { SECTOR } \\
\text { TOTAL }\end{array}$ \\
\hline 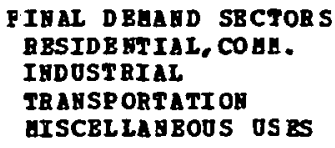 & $\begin{array}{r}3.258 \\
343 \\
2.333 \\
70\end{array}$ & $\begin{array}{r}376 \\
116 \\
6 \\
185\end{array}$ & 29,772 & $\begin{array}{r}8,027 \\
3,190 \\
523 \\
169\end{array}$ & : & $\begin{array}{r}18,738 \\
6,727 \\
817 \\
1.764\end{array}$ & $\begin{array}{r}582 \\
2.768 \\
4 \\
-\end{array}$ & $\begin{array}{r}5.114 \\
1.808 \\
3 \\
102\end{array}$ & $\begin{array}{r}36.095 \\
14.952 \\
33.458 \\
2.290\end{array}$ \\
\hline $\begin{array}{l}\text { TOTAL FIHAL } \\
\text { DEGA AD SBCTOBS }\end{array}$ & 6.004 & 683 & 29,772 & 11,909 & - & 28.046 & 3,354 & 7,027 & 86.795 \\
\hline $\begin{array}{l}\text { TRABSPORAATIOB } \\
\text { RLECTRICITY GBA. } \\
\text { PETROLEOA PRODOCTS } \\
\text { HATORAL GAS } \\
\text { SYH-GAS }\end{array}$ & $\begin{array}{r}272 \\
-\end{array}$ & $\begin{array}{l}- \\
-\end{array}$ & $\begin{array}{l}- \\
-\end{array}$ & : & $\overline{-}$ & $\begin{array}{r}4.429 \\
- \\
-\end{array}$ & $\begin{array}{r}33,924 \\
= \\
-\end{array}$ & $\begin{array}{r}-10.734 \\
- \\
-\end{array}$ & $\begin{array}{r}27.891 \\
= \\
=\end{array}$ \\
\hline $\begin{array}{l}\text { NET POEL OSBD } \\
\text { IB TRAESPOREATIOB }\end{array}$ & 272 & - & - & - & - & 4.429 & 33,924 & -10.734 & 27.891 \\
\hline $\begin{array}{l}\text { TOTAL GROSS PLORS } \\
\text { LOSSES } 8 \text { OAISSIOHS }\end{array}$ & $\begin{array}{r}6.276 \\
381\end{array}$ & $\begin{array}{r}683 \\
-6\end{array}$ & $\begin{array}{r}29.772 \\
-942\end{array}$ & $\begin{array}{r}11.909 \\
-562\end{array}$ & - & $\begin{array}{r}32.475 \\
122\end{array}$ & $\begin{array}{r}37.278 \\
619\end{array}$ & $\begin{array}{r}7.027 \\
561\end{array}$ & $\begin{array}{r}114,686 \\
173\end{array}$ \\
\hline
\end{tabular}

\begin{tabular}{|c|c|c|c|c|c|c|c|c|c|c|}
\hline $\begin{array}{l}\text { S OPPLI OP BAB BGI } \\
\text { FOSS IL POBL } \\
\text { HYDROELECTRIC } \\
\text { HOCLEAR } \\
\text { GBO.\& SOLAR }\end{array}$ & $\begin{array}{l}E \\
\bar{E}\end{array}$ & E & E & $\overline{-}$ & $\bar{E}$ & $\bar{E}$ & $\begin{array}{r}28,776 \\
- \\
-\end{array}$ & $\begin{array}{r}3,500 \\
-\end{array}$ & $\begin{array}{r}28,776 \\
3.500 \\
- \\
-\end{array}$ & $\begin{array}{l}89 \% \\
11 \%\end{array}$ \\
\hline
\end{tabular}

-

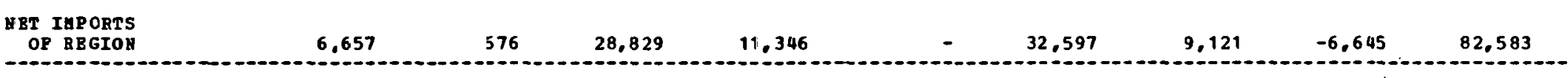

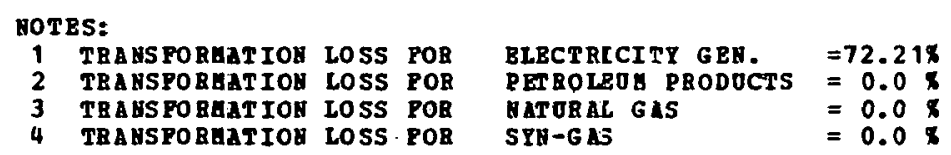


REGIONAL ENERGY BALANCE STATBAENT

BI SECTOR AND FOEL TYPE

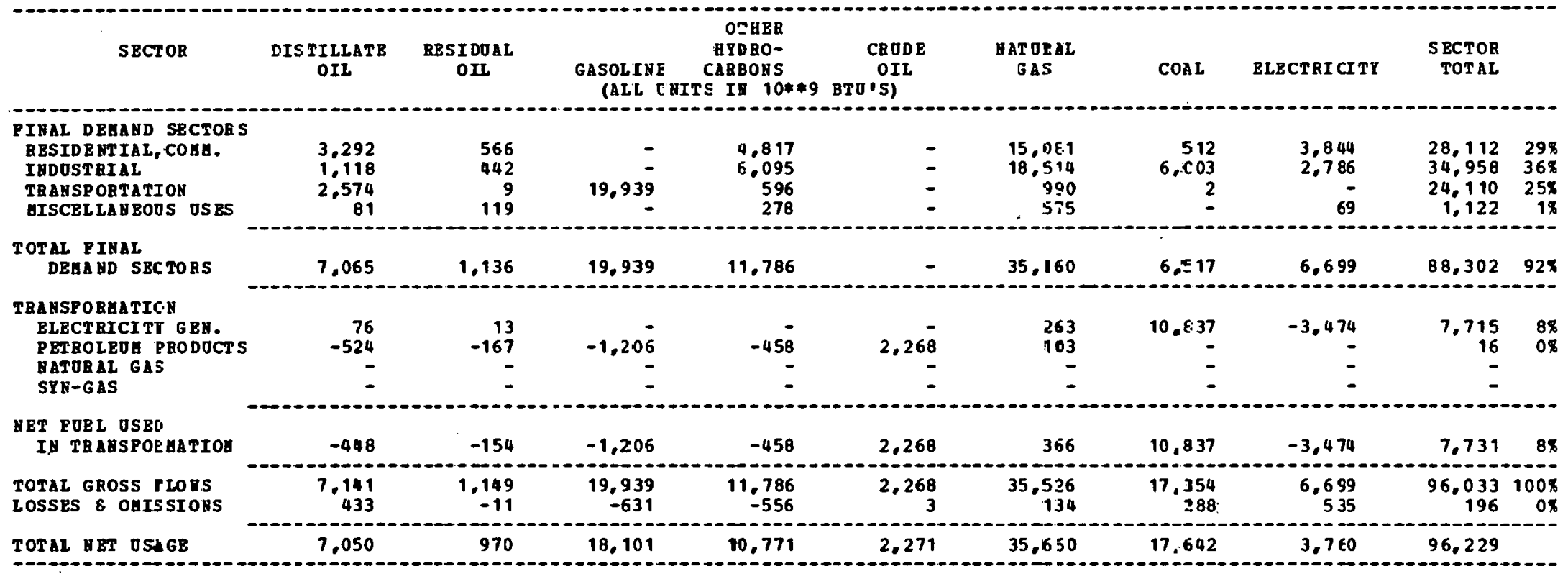

\section{SUP PLI OP BERERY \\ POSSIL POEA \\ GYDROELBCTRIC \\ HOCL E AR \\ GBO. ESOLAB}

TOTAL SUPPLY

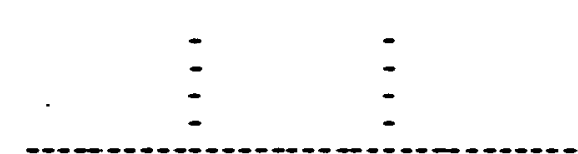

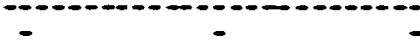

\begin{tabular}{lr}
- & 23 \\
- & - \\
- & - \\
\hline- & 23
\end{tabular}

$\overline{-}$

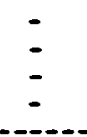

3.022

$\begin{array}{rr}23 & 1 \% \\ 3.022 & 99 \%\end{array}$

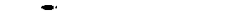

23

$3,022 \quad 3,045$

\section{BET IHPORTS}

7,050

970

18,101

10.771

2.248

35,660

17,642

738

93,184

OP REGION

HOTBS:

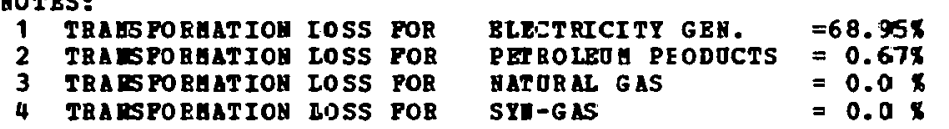


REGIONAL BHERGY BALABCE STATEMEHT

BY SECTOR AID POBL TYPE

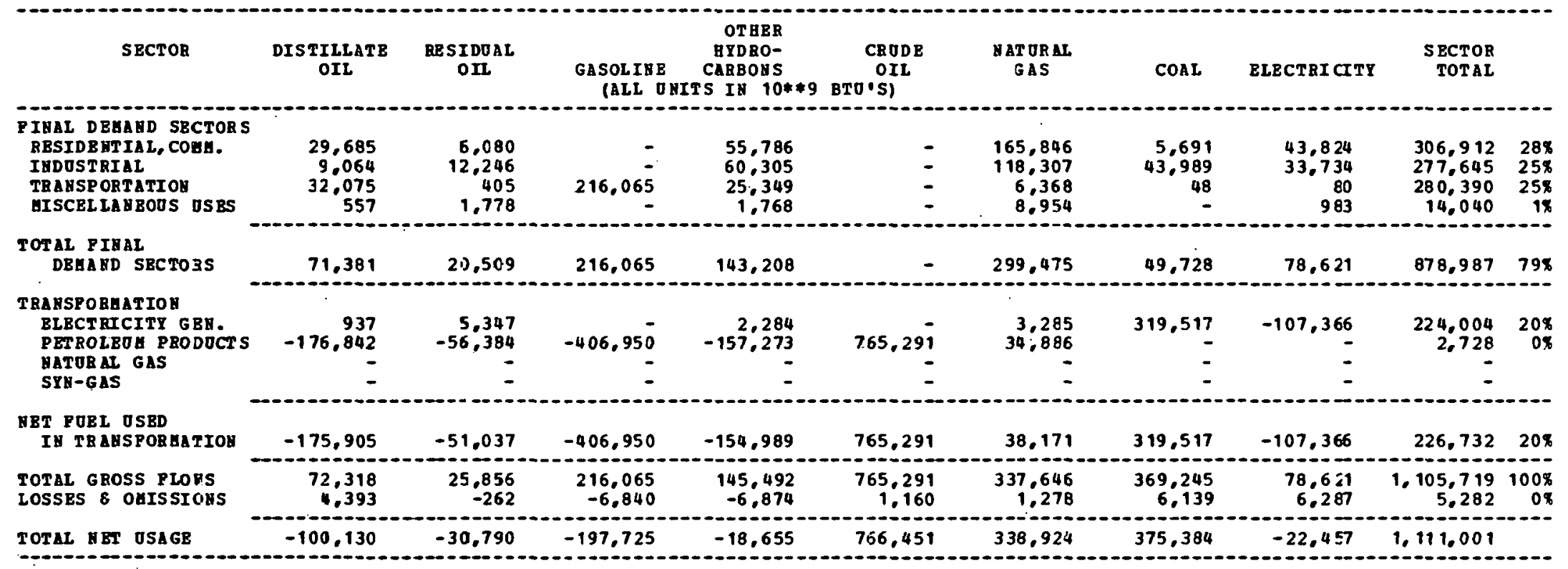

-

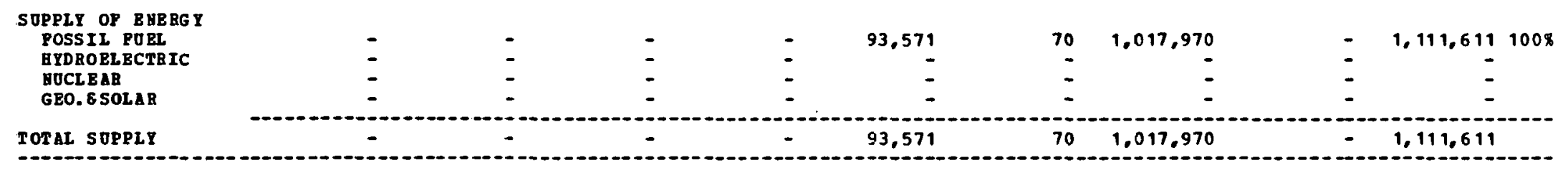

MBT IHPORTS
OP RBGIOS

\begin{tabular}{|c|c|c|c|c|c|}
\hline \multicolumn{6}{|c|}{ ВОT BS: } \\
\hline 1 & 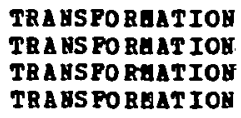 & $\begin{array}{l}\text { LosS } \\
\text { LOSS } \\
\text { LosS } \\
\text { LOSS }\end{array}$ & $\begin{array}{l}\text { POR } \\
\text { POR } \\
\text { POR } \\
\text { POR }\end{array}$ & $\begin{array}{l}\text { BLECT BICITI GEH. } \\
\text { PETROLEOA PRODUCTS } \\
\text { R ATOR LI GAS } \\
\text { SYN-GES }\end{array}$ & $\begin{array}{l}=67.608 \\
=0.348 \\
=0.08 \\
=0.08\end{array}$ \\
\hline
\end{tabular}


RegIONAL BNERG BaLAHCE STATEIENT

BY SECTOR AND FOEL TYPE

\begin{tabular}{|c|c|c|c|c|c|c|c|c|c|c|}
\hline SECTOR & $\begin{array}{l}\text { DISTILLATE } \\
\text { OIL }\end{array}$ & $\begin{array}{l}\text { BES I DO AL } \\
\text { OIL }\end{array}$ & $\begin{array}{l}\text { GASOLINE } \\
\text { (ALL ONIT }\end{array}$ & $\begin{array}{c}\text { CTRER } \\
\text { HYDBO- } \\
\text { ZABBONS } \\
\text { TS IN 10*\$9 }\end{array}$ & $\begin{array}{c}\text { CRODE } \\
\text { OIL } \\
\text { BTO'S) }\end{array}$ & $\begin{array}{l}\text { HATORAL } \\
\text { GAS }\end{array}$ & $\operatorname{COAL}$ & ELECTRICITY & $\begin{array}{l}\text { SECTOP } \\
\text { TOTAL }\end{array}$ & \\
\hline $\begin{array}{l}\text { PIHAI DEGAND SECTORS } \\
\text { RESIDE NTIAL, COHG. } \\
\text { IHDOSTRIAL } \\
\text { TRANSP ORTATL ON } \\
\text { GISCEL LAHEOOS OSES }\end{array}$ & $\begin{array}{r}3.706 \\
2.030 \\
5.362 \\
111\end{array}$ & $\begin{array}{r}441 \\
1.395 \\
28 \\
162\end{array}$ & $39.260^{-}$ & $\begin{array}{r}10,104 \\
9,793 \\
777 \\
245\end{array}$ & 5 & $\begin{array}{r}23.103 \\
17.980 \\
2.952 \\
1.256\end{array}$ & $\begin{array}{r}1,215 \\
14,969 \\
5 \\
-\end{array}$ & $\begin{array}{r}8,198 \\
17.070 \\
2 \\
1.70\end{array}$ & $\begin{array}{r}46,767 \\
63,237 \\
48,386 \\
1,944\end{array}$ & $\begin{array}{r}16 x \\
218 \\
16 \% \\
18\end{array}$ \\
\hline $\begin{array}{l}\text { TOTAL PINAL } \\
\text { DEGA ND S } 3 \text { CTORS }\end{array}$ & 11,209 & 2,026 & 39,260 & 20,919 & - & 45,291 & 16,189 & 25,440 & 160,332 & 548 \\
\hline $\begin{array}{l}\text { TRANSPORHATIOA } \\
\text { ELECTRICITY GEH. } \\
\text { PETROLEUA PRODOCTS } \\
\text { NATORAL GAS } \\
\text { STN-GAS }\end{array}$ & $\begin{array}{r}190 \\
- \\
-\end{array}$ & $\begin{array}{r}253 \\
- \\
-\end{array}$ & : & $\begin{array}{l}- \\
-\end{array}$ & $\stackrel{-}{-}$ & $\begin{array}{r}1,318 \\
= \\
-\end{array}$ & $\begin{array}{r}197,927 \\
- \\
-\end{array}$ & $\begin{array}{r}-68,766 \\
- \\
=\end{array}$ & $\begin{array}{r}130,922 \\
= \\
-\end{array}$ & 448 \\
\hline $\begin{array}{l}\text { NET POEL OSBD } \\
\text { IQ TBANSFORGATION }\end{array}$ & 190 & 253 & - & - & - & $1,31 \varepsilon$ & 197,927 & $-68,766$ & 130,922 & $44 x$ \\
\hline $\begin{array}{l}\text { TOTAL GROSS PLOHS } \\
\text { LOSSES } \& \text { ORISSI OHS }\end{array}$ & $\begin{array}{r}11.399 \\
692\end{array}$ & $\begin{array}{r}2.279 \\
-23\end{array}$ & $\begin{array}{r}39,260 \\
-1,242\end{array}$ & $\begin{array}{r}20,919 \\
-988\end{array}$ & - & 46,609 & $\begin{array}{r}214.116 \\
3.560\end{array}$ & $\begin{array}{r}25.440 \\
2.034\end{array}$ & $\begin{array}{r}291.256 \\
4.209\end{array}$ & $\begin{array}{l}998 \\
18\end{array}$ \\
\hline TOTAL MET OSAGB & 19.091 & 2,255 & 38,017 & 19,930 & - & $4 E, 785$ & 217,676 & -41.251 & 295,465 & \\
\hline
\end{tabular}

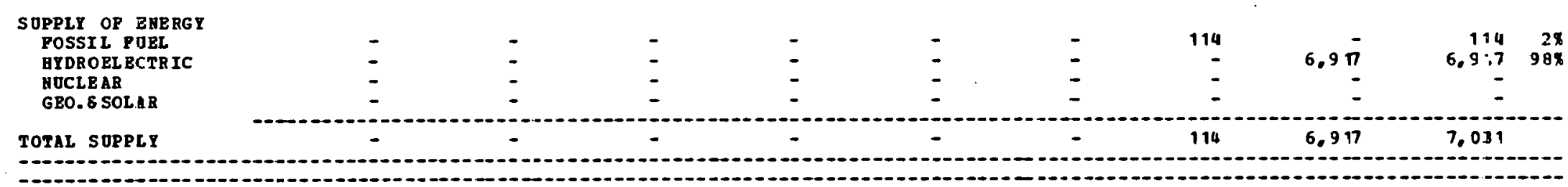

\begin{tabular}{l}
$\begin{array}{l}\text { MET IUPORTS } \\
\text { OP REGIOR }\end{array}$ \\
\hdashline
\end{tabular}


BEG TOHAL EHERGY BALANCE STATEGEHT

BY SECTOR AND FOEL TYPE

\begin{tabular}{|c|c|c|c|c|c|c|c|c|c|c|}
\hline SBCTOR & $\begin{array}{l}\text { DIS II LLATB } \\
\text { OIL }\end{array}$ & $\begin{array}{c}\text { RES I DOAL } \\
\text { JII }\end{array}$ & $\begin{array}{l}\text { GASOLIHE } \\
\text { (ALL OUIT }\end{array}$ & $\begin{array}{l}\text { OTYBO- } \\
\text { CARBOAS } \\
\text { TS IN 10**9 }\end{array}$ & $\begin{array}{c}\text { CRUDE } \\
\text { OII } \\
\text { BTOSI) }\end{array}$ & $\begin{array}{l}\text { NATURAL } \\
\text { GAS }\end{array}$ & COAL & ELECTRICITY & $\begin{array}{r}\text { SECTOR } \\
\text { TOTAL }\end{array}$ & \\
\hline $\begin{array}{l}\text { PIHAL DBHAHD SBCTORS } \\
\text { BBSIDBETIAL, COAH. } \\
\text { IBDOSTRIAL } \\
\text { TRANSPORTATIOA } \\
\text { GISCELLAYEOOS OSBS }\end{array}$ & $\begin{array}{r}5.419 \\
4.459 \\
8.509 \\
283\end{array}$ & $\begin{array}{r}632 \\
2.660 \\
47 \\
246\end{array}$ & $65,74 \overline{-}$ & $\begin{array}{r}17,764 \\
10 \div 258 \\
2,473 \\
467\end{array}$ & $\overline{-}$ & $\begin{array}{r}41.670 \\
45.219 \\
8.458 \\
2.137\end{array}$ & $\begin{array}{r}1.034 \\
7.489 \\
10 \\
-\end{array}$ & $\begin{array}{r}11.818 \\
6.283 \\
5 \\
128\end{array}$ & $\begin{array}{r}78,337 \\
76,368 \\
85,243 \\
3,261\end{array}$ & $\begin{array}{r}298 \\
288 \\
318 \\
18\end{array}$ \\
\hline $\begin{array}{l}\text { TOTAL PIBAL } \\
\text { DBEABD SBCTORS }\end{array}$ & 18.670 & 3,585 & 65.741 & 30,962 & - & 97,484 & 8,533 & 18,234 & 243,209 & 89x \\
\hline $\begin{array}{l}\text { TRANSFORHATIOH } \\
\text { BLECT RICITY GRA. } \\
\text { PBTROLEUA PBODOCTS } \\
\text { YATORLL GAS } \\
\text { SYH-G LS }\end{array}$ & $\begin{array}{r}236 \\
-22.566 \\
-\end{array}$ & $\begin{array}{r}624 \\
-2,194 \\
=\end{array}$ & $\begin{array}{r}-47.090 \\
-\end{array}$ & $\begin{array}{r}-18,136 \\
- \\
-\end{array}$ & $86,440^{-}$ & $\begin{array}{r}24,051 \\
3,940 \\
- \\
-\end{array}$ & $\begin{array}{r}17.491 \\
= \\
=\end{array}$ & $\begin{array}{r}-11,992 \\
- \\
=\end{array}$ & $\begin{array}{r}30.410 \\
394 \\
- \\
-\end{array}$ & $\begin{array}{r}118 \\
0 \%\end{array}$ \\
\hline $\begin{array}{l}\text { RET POEL DSED } \\
\text { IA TEABSFOR TATOR }\end{array}$ & $-22,330$ & -1.570 & -47.090 & $-18,136$ & 86,440 & 27,991 & 17.491 & $-11,992$ & 30,804 & $11 \%$ \\
\hline $\begin{array}{l}\text { TOTAL G BOSS PLORS } \\
\text { LOSSES } 8 \text { OAISSI OHS }\end{array}$ & $\begin{array}{r}18,906 \\
1,148\end{array}$ & $\begin{array}{r}4.209 \\
-42\end{array}$ & $\begin{array}{l}65,741 \\
-2,081\end{array}$ & $\begin{array}{l}30,962 \\
-1,462\end{array}$ & $\begin{array}{r}86,440 \\
131\end{array}$ & $\begin{array}{r}125.475 \\
475\end{array}$ & $\begin{array}{r}26,024 \\
432\end{array}$ & $\begin{array}{r}18,234 \\
1.458\end{array}$ & $\begin{array}{r}274.013 \\
58\end{array}$ & $\begin{array}{r}100 \% \\
0 \%\end{array}$ \\
\hline
\end{tabular}

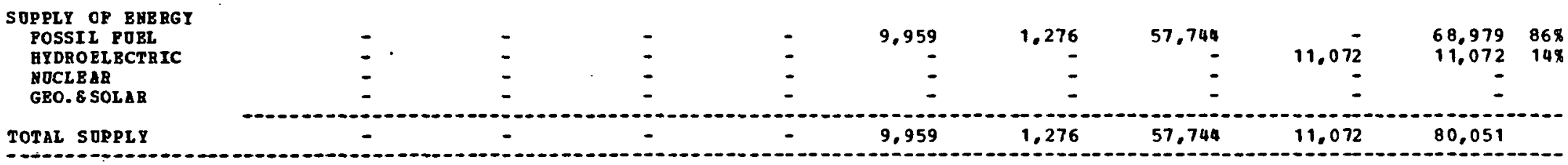

$\begin{array}{ll}10 & \end{array}$

\section{NET IRPORTS}

OF REGIOB

NOTES:

TRABS PORBAT IOH LOSS POR TRAMSPORAATIOH LOSS POR TRA ASPORAATIOH LOSS POR
TRANSPOREATIOH LOSS POR 
REGIONAL ESERGY BALANCE STATEAENT

BY SECTOR AND FOBL TYPE

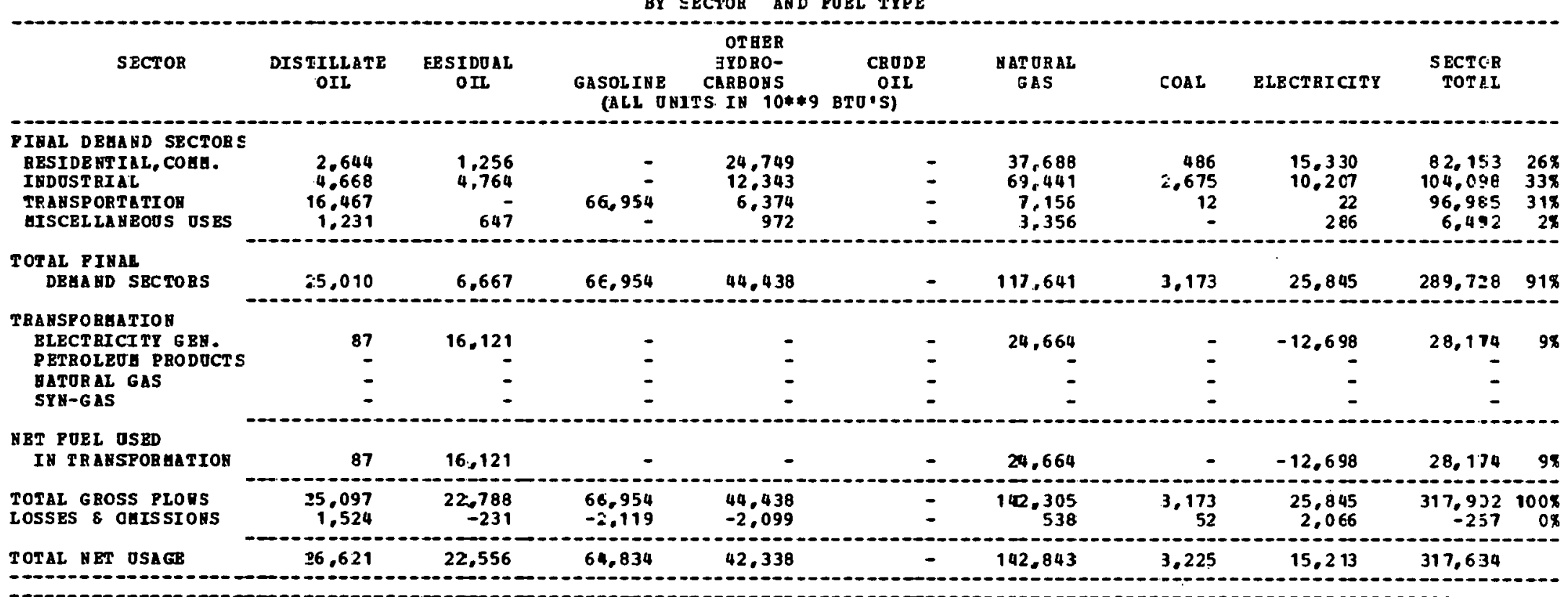

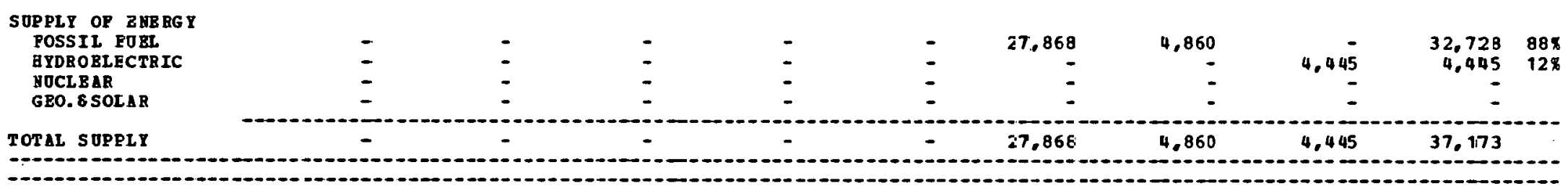

\section{WBT IHPOATS}

OP REGIOU

26,621

22,556

$6+, 834$

42,338

$1 \cdot 4,975$

$-1,634$

10.768

280.461

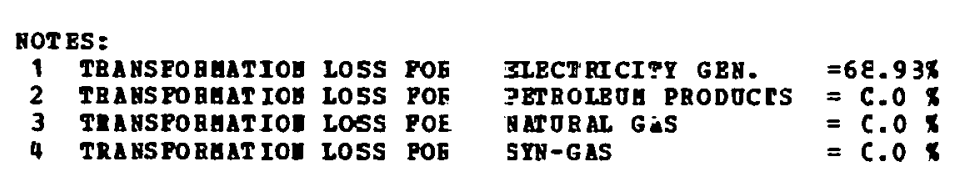


REG IONAL BEREGY BALANCE STATEUENT

BY SBCTOR AND POEL TYPE

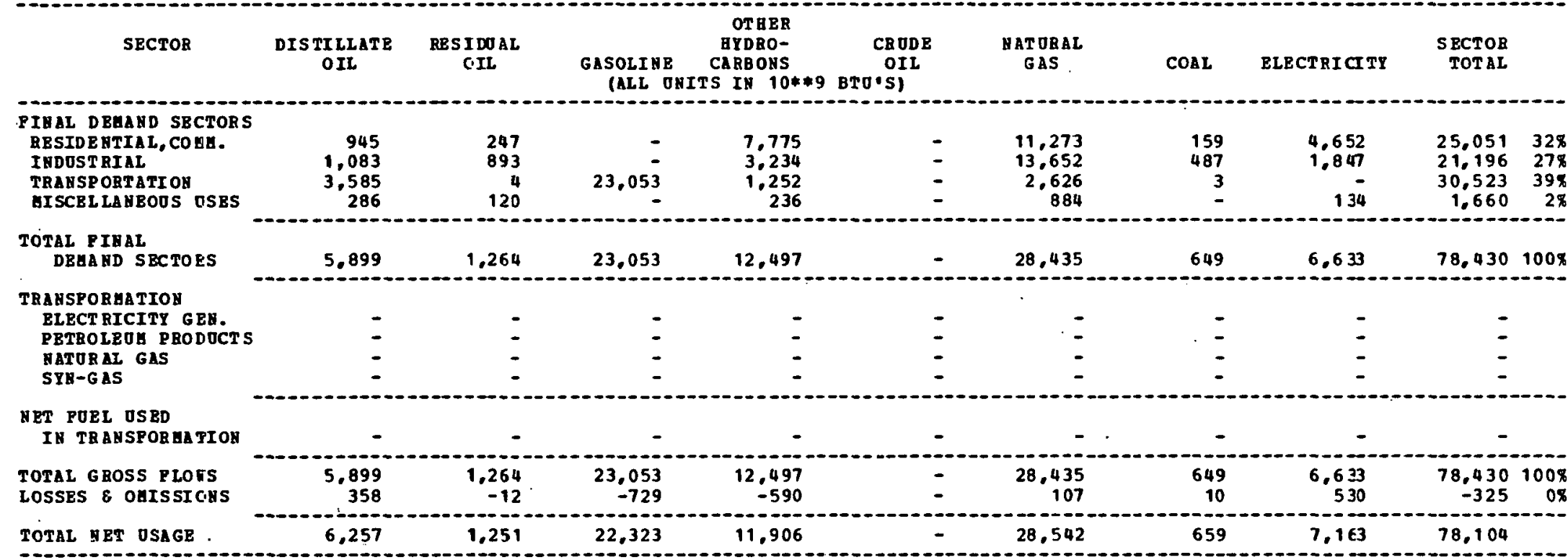

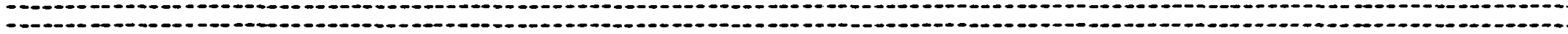

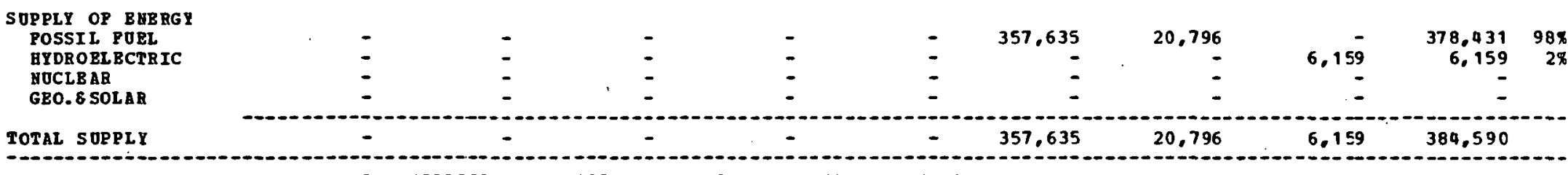

-

BET IUPORTS
OP REGION

\begin{tabular}{|c|c|c|c|c|c|}
\hline \multicolumn{6}{|c|}{ Nor } \\
\hline $\begin{array}{l}1 \\
2\end{array}$ & $\begin{array}{l}\text { TR A US FOBAAT ION } \\
\text { TRABSPOBGATIOB } \\
\text { TRA ASPOBAAT IOU }\end{array}$ & $\begin{array}{l}\text { Loss } \\
\text { Loss } \\
\text { LOSS }\end{array}$ & $\begin{array}{l}\text { POR } \\
\text { POR } \\
\text { POR }\end{array}$ & $\begin{array}{l}\text { BLECTRICITY GEN. } \\
\text { PETROLEOA PBODUCTS } \\
\text { HATORAL GAS }\end{array}$ & $\begin{array}{l}=0.0 \\
=0.0 \\
=0.0\end{array}$ \\
\hline
\end{tabular}


REGIOHAL EHERGY BALAHCE STATEHENT

BY SECTOR AND POEL TIPE

\begin{tabular}{|c|c|c|c|c|c|c|c|c|c|c|}
\hline SECTOR & $\begin{array}{l}\text { DISTILLATE } \\
\text { OIL }\end{array}$ & $\begin{array}{l}\text { RESIDD.SL } \\
\text { OIL }\end{array}$ & $\begin{array}{l}\text { GASOLINE } \\
\text { (AIL O }\end{array}$ & $\begin{array}{c}\text { KYJBO- } \\
\text { CAR JOYS } \\
\text { TS IB 10*\%9 }\end{array}$ & $\begin{array}{c}\text { CRODE } \\
\text { OII } \\
\text { BTO'S) }\end{array}$ & $\begin{array}{l}\text { NATURAL } \\
\text { GAS }\end{array}$ & COAL & ELECT BI CITY & $\begin{array}{l}\text { SECTOR } \\
\text { TOTAL }\end{array}$ & \\
\hline 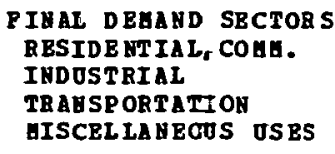 & $\begin{array}{r}3,587 \\
10,149 \\
13,178 \\
541\end{array}$ & $\begin{array}{r}369 \\
5,430 \\
96 \\
145\end{array}$ & 84,250 & $\begin{array}{r}25.659 \\
13.352 \\
11.794 \\
499\end{array}$ & - & $\begin{array}{r}42,163 \\
91,351 \\
10,192 \\
2,152\end{array}$ & $\begin{array}{r}553 \\
3,449 \\
17 \\
-\end{array}$ & $\begin{array}{r}18.433 \\
12.714 \\
14 \\
444\end{array}$ & $\begin{array}{r}90,764 \\
136,445 \\
119,531 \\
3,781\end{array}$ & $\begin{array}{r}22 x \\
33 \% \\
29 \% \\
1 \%\end{array}$ \\
\hline $\begin{array}{l}\text { TOTAL FIMAL } \\
\text { DELABD SECTORS }\end{array}$ & 27,455 & 6.0 .30 & 84.250 & 51,304 & - & 145,858 & 4.019 & 31,605 & 350,521 & $84 \%$ \\
\hline $\begin{array}{l}\text { TRANSPORHATIDH } \\
\text { BLECT RICITY GEN. } \\
\text { PETROLEOH PRODOCTS } \\
\text { HATORAL GAS } \\
\text { SYN-GAS }\end{array}$ & $\begin{array}{r}112 \\
-146,937 \\
-\end{array}$ & $\begin{array}{r}1,37 \\
-14,287 \\
-\end{array}$ & $\begin{array}{r}-306,025 \\
-\end{array}$ & $\begin{array}{r}-141,491 \\
-11,272\end{array}$ & $\begin{array}{r}586,26 \overline{-} \\
\overline{-}\end{array}$ & $\begin{array}{l}89,394 \\
26,725 \\
12,955\end{array}$ & $\begin{array}{l}37 \\
- \\
-\end{array}$ & $\begin{array}{r}-28,137 \\
- \\
-\end{array}$ & $\begin{array}{r}61,5437 \\
4.253 \\
1,683\end{array}$ & $\begin{array}{r}15 \% \\
1 \% \\
0 x\end{array}$ \\
\hline $\begin{array}{l}\text { MET POEL OSED } \\
\text { IA TRANSPOR GATIOA }\end{array}$ & $-146,825$ & $-14,150$ & $-306,1,25$ & $-1 \leq 2,763$ & 586,268 & 129.074 & 37 & $-28,137$ & 67.479 & $16 x$ \\
\hline $\begin{array}{l}\text { TOTAL GROSS FLORS } \\
\text { LOSSES } 8 \text { OYIS SIOAS }\end{array}$ & $\begin{array}{r}27.567 \\
1.674\end{array}$ & $\begin{array}{r}6.167 \\
-62\end{array}$ & $\begin{array}{l}84.250 \\
-2.567\end{array}$ & $\begin{array}{l}5.1,304 \\
-2.424\end{array}$ & $\begin{array}{r}586.268 \\
888\end{array}$ & $\begin{array}{r}274,932 \\
1,041\end{array}$ & $\begin{array}{r}4.056 \\
67\end{array}$ & $\begin{array}{r}31,605 \\
2,527\end{array}$ & $\begin{array}{r}418,005 \\
1,045\end{array}$ & $\begin{array}{r}100 \% \\
0 \%\end{array}$ \\
\hline
\end{tabular}

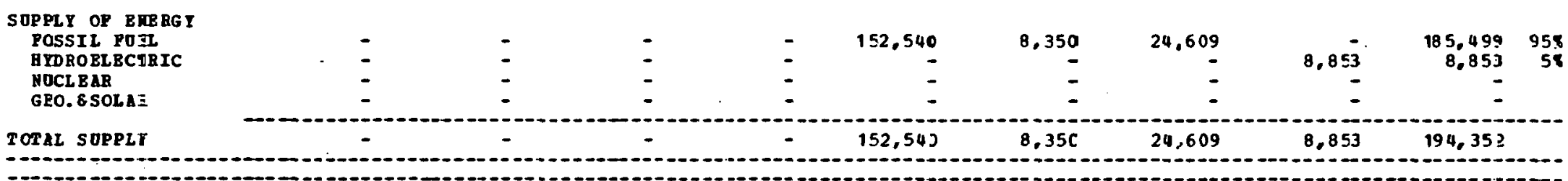

\section{HBT IAPORTS}

OP RBGIOY

$-117,695$

$-8,182$

$-224.442$

$-1) 3,883$

434,615

267.62 I

$-20,485$

\section{HOT BS:}

1 TRAISPORAATION LOSS POB

TRATS PORAATIOA COSS POR
TRAMSPOAGTIOH LOSS POR

TRATSPORAATIOE EOSS POR

BLECT PACITY GEN.

PETROLZOG PEODUCT

MATURM GAS

STY-GAS
$=68.53 \%$.

$=0.59 x$

$=12.39 \%$
$=0.3 \times$ 


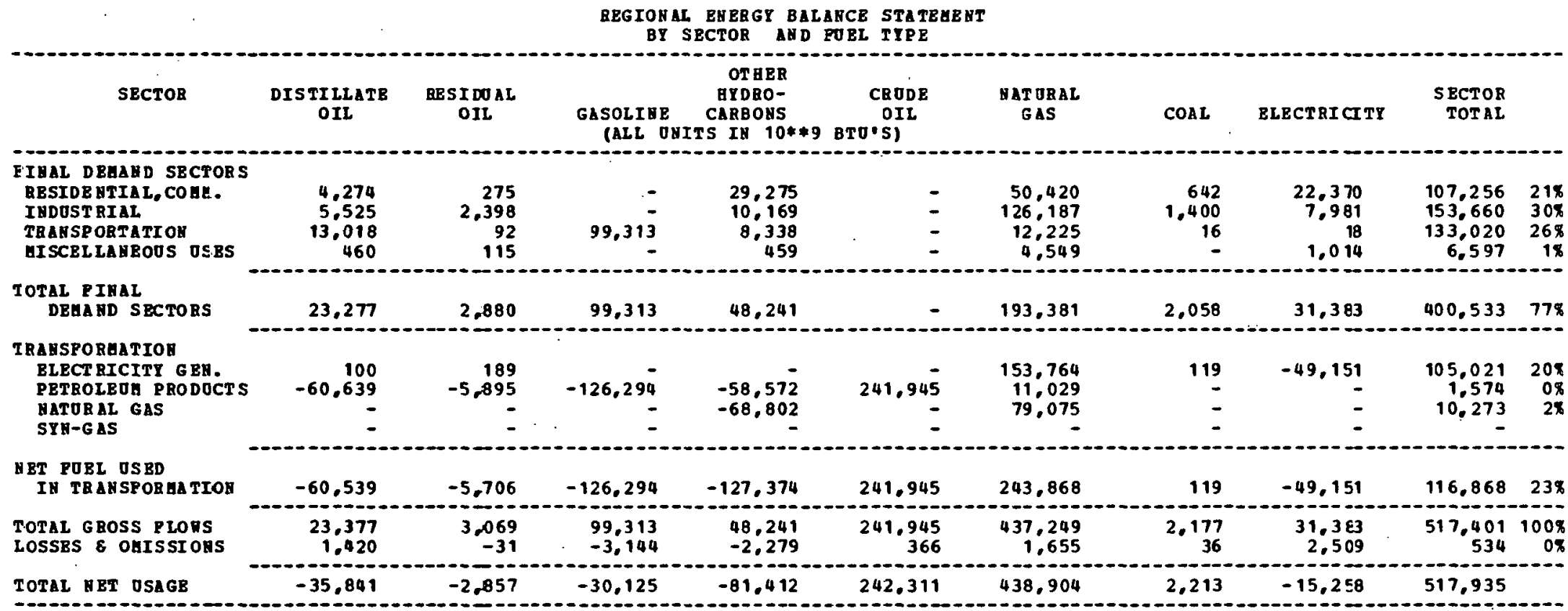

-

\section{SUPPLY OP BHBBG}

POSSIL POBL

BYDROBLBCTRIC

GOCLE AB

GEO. 8 SOLAB

TOTAL SOPPLY

$\begin{array}{rr}- & - \\ - & - \\ - & - \\ - & -\end{array}$

-
-
-
-

\begin{tabular}{rrr}
- & 672.237 & 812.460 \\
$\vdots$ & $\vdots$ & - \\
\hline & - & - \\
\hline & 672.237 & 812.460
\end{tabular}

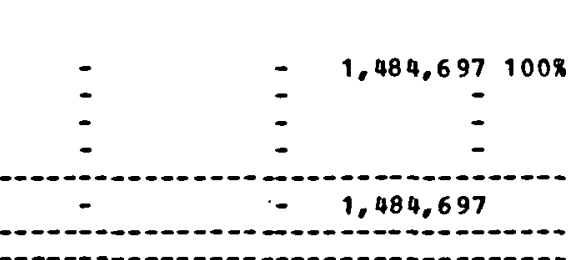

\begin{tabular}{l}
$\begin{array}{l}\text { GET IUPORTS } \\
\text { OP BEGIOA }\end{array}$ \\
\hline
\end{tabular}


REGIONAL ENZRGY BALANCE STATEHENT

EY SECTOB AND POBL TYPE

\begin{tabular}{|c|c|c|c|c|c|c|c|c|c|}
\hline SECIOR & $\begin{array}{l}\text { DIS TILLATE } \\
\text { OIL }\end{array}$ & $\begin{array}{l}\text { BES I DCAL } \\
\text { OIL }\end{array}$ & $\begin{array}{l}\text { GASOLINE } \\
\text { (ALL ONIJ }\end{array}$ & $\begin{array}{l}\text { TTBBR } \\
\text { AYDBO- } \\
\text { CABBOAS } \\
\text { IS IE 10**9 }\end{array}$ & $\begin{array}{c}\text { CRODE } \\
\text { OIL } \\
\text { BTO'S) }\end{array}$ & $\begin{array}{l}\text { MAT DEAL } \\
\text { GAS }\end{array}$ & CCAL & ELECTRICITY & $\begin{array}{l}\text { SECTOR } \\
\text { TOTAL }\end{array}$ \\
\hline 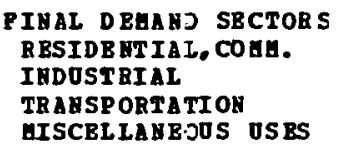 & $\begin{array}{r}1.369 \\
1.729 \\
3.229 \\
222\end{array}$ & $\begin{array}{r}115 \\
727 \\
17 \\
241\end{array}$ & 36,649 & $\begin{array}{r}9.164 \\
13.134 \\
1.912 \\
180\end{array}$ & $\begin{array}{l}- \\
-\end{array}$ & $\begin{array}{r}15,: 15 \\
133,456 \\
4,355 \\
4,944\end{array}$ & $\begin{array}{r}241 \\
232 \\
4 \\
-\end{array}$ & $\begin{array}{r}7,516 \\
2,599 \\
1,116\end{array}$ & $\begin{array}{r}33,520 \\
151,877 \\
46,166 \\
6,703\end{array}$ \\
\hline $\begin{array}{l}\text { TOTAL PINAL } \\
\text { DEHA HD SBCTORS }\end{array}$ & 6.549 & 1.100 & 36.649 & 24,390 & - & 157.870 & 477 & 11,231 & 238.266 \\
\hline $\begin{array}{l}\text { TRANSFORLATIOH } \\
\text { BLECTRICITY GBE. } \\
\text { PETROLEOA PRODDCTS } \\
\text { NATORAL GAS } \\
\text { SIU-GAS }\end{array}$ & $\begin{array}{r}121 \\
-25,682 \\
-\end{array}$ & $\begin{array}{r}469 \\
-2.886 \\
- \\
-\end{array}$ & $\begin{array}{r}-61,818 \\
-\end{array}$ & $\begin{array}{r}-28,340 \\
-4,500 \\
-\end{array}$ & $\begin{array}{r}-\overrightarrow{3} \\
-\end{array}$ & $\begin{array}{r}61.914 \\
5.399 \\
5.173 \\
-\end{array}$ & - & $\begin{array}{r}-20,655 \\
= \\
=\end{array}$ & $\begin{array}{r}41.849 \\
1,101 \\
673 \\
-\end{array}$ \\
\hline $\begin{array}{l}\text { HET POEL OSPD } \\
\text { IU TRANSPORHATIOA }\end{array}$ & $-2 \subseteq, 561$ & $-2,417$ & $-61,818$ & $-.32,840$ & 118,429 & $72, \$ 86$ & - & $-20,655$ & 43,623 \\
\hline $\begin{array}{l}\text { TOTAL GROSS PLORS } \\
\text { LOSSES } \& \text { OIISSIOHS }\end{array}$ & $\begin{array}{r}\epsilon .670 \\
405\end{array}$ & $\begin{array}{r}1.569 \\
-15\end{array}$ & $\begin{array}{r}36,649 \\
-1,160\end{array}$ & $\begin{array}{l}24,390 \\
-1,152\end{array}$ & $\begin{array}{r}118,428 \\
179\end{array}$ & $\begin{array}{r}230,356 \\
372\end{array}$ & $\begin{array}{r}477 \\
7\end{array}$ & $\begin{array}{r}11.2 \exists 1 \\
898\end{array}$ & $\begin{array}{r}281,889 \\
34\end{array}$ \\
\hline TOTAL UET USAGE & $-2 \pi, 606$ & $-1,332$ & $-26,329$ & $-9,602$ & 118,607 & 231.228 & 484 & $-8,525$ & 281.923 \\
\hline
\end{tabular}

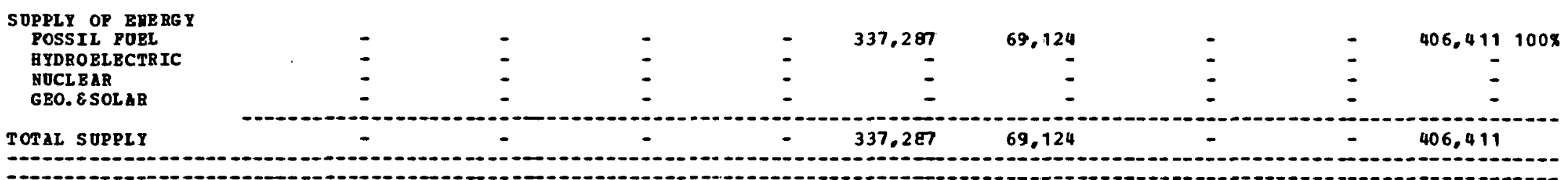

\section{N BT IUPORTS}

OP BBGIOB

$-22.606$

$-1,332$

$-26,329$

$-9,602 \quad-218,679$

162,104

$484 \quad-8,525 \quad-124,487$

NOT BS:

1 TRA HSPOREATIOY LOSS POB

TRE GS FORUAT IOE LOSS POR

3 TRLASPORMATION LOSS POB

TREASPOALATIOH LOSS POR

BL3CT RICITY GBN. $\quad=66.95$

PEDOROA EBODOCTS $=0.395$

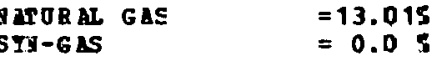


REGIONAL ENEQGY BALANCE STATEHENT

BY SBCTOR AKD. FOBL TIP

\begin{tabular}{|c|c|c|c|c|c|c|c|c|c|c|}
\hline SECTOR & $\begin{array}{l}\text { DIS TI ILATB } \\
\text { OIL }\end{array}$ & $\begin{array}{l}\text { RBSI DO AL } \\
\text { OIL }\end{array}$ & $\begin{array}{l}\text { GASOLIHE } \\
\text { (ALL OHI }\end{array}$ & $\begin{array}{c}\text { OT HER } \\
\text { HYDRO- } \\
\text { CARBOAS } \\
\text { IS IH 10**9 }\end{array}$ & $\begin{array}{c}\text { CRODE } \\
\text { OII } \\
\text { BTO'S) }\end{array}$ & $\begin{array}{l}\text { NATORAL } \\
\text { GAS }\end{array}$ & COAL & ELECTRICITY & $\begin{array}{r}\text { SECTOR } \\
\text { TOTAL }\end{array}$ & \\
\hline $\begin{array}{l}\text { PIHAL DBHABD SBCTORS } \\
\text { RESIDE HTIAL, COAA. } \\
\text { INDOSTRIAL } \\
\text { TRANSP ORTATI ON } \\
\text { GISCELLAHEOOS OSBS }\end{array}$ & $\begin{array}{r}1,146 \\
2,515 \\
8,153 \\
407\end{array}$ & $\begin{array}{r}174 \\
1.478 \\
17 \\
350\end{array}$ & $\begin{array}{r}- \\
-35,600 \\
-\end{array}$ & $\begin{array}{r}7.249 \\
22.815 \\
4.182 \\
188\end{array}$ & $\overline{-}$ & $\begin{array}{r}14.678 \\
135.877 \\
5.615 \\
962\end{array}$ & $\begin{array}{r}237 \\
1.337 \\
6 \\
-\end{array}$ & $\begin{array}{r}7.703 \\
5.718 \\
6 \\
209\end{array}$ & $\begin{array}{r}31.187 \\
169.740 \\
53,579 \\
2.116\end{array}$ & $\begin{array}{r}10 \% \\
568 \\
18 \% \\
1 \%\end{array}$ \\
\hline $\begin{array}{l}\text { TOTAL PIBAL } \\
\text { DEHA SBD SECTORS }\end{array}$ & 12.221 & 2.019 & 35,600 & 34,434 & - & 157,132 & 1,580 & 13.636 & 256.622 & $85 \%$ \\
\hline $\begin{array}{l}\text { TAASSPORGATIOH } \\
\text { BLECT RICITY GBH. } \\
\text { RETROLEOE PBODOCT S } \\
\text { BATORAL GAS } \\
\text { SYH-GAS }\end{array}$ & $\begin{array}{r}116 \\
-69.879 \\
-\end{array}$ & $\begin{array}{r}37 \\
-16,097 \\
-\end{array}$ & -141.218 & $\begin{array}{r}-80,041 \\
-111.822\end{array}$ & $\begin{array}{r}295,969 \\
-\end{array}$ & $\begin{array}{r}34.643 \\
13.492 \\
128.518\end{array}$ & $\begin{array}{l}- \\
\end{array}$ & $\begin{array}{r}-10,182 \\
- \\
-\end{array}$ & $\begin{array}{r}24,614 \\
2,226 \\
16,696\end{array}$ & $\begin{array}{l}88 \\
1 \% \\
6 \%\end{array}$ \\
\hline $\begin{array}{l}\text { MBT FOBI USBD } \\
\text { IN TRAASFOBHATION }\end{array}$ & $-69,763$ & $-16,060$ & $-141,218$ & $-191,863$ & 295,969 & 176.653 & - & $-10,182$ & 43.536 & $14 \%$ \\
\hline $\begin{array}{l}\text { TOTAL GROSS FLOVS } \\
\text { LOSSES } \varepsilon \text { OAISSI OAS }\end{array}$ & $\begin{array}{r}12.337 \\
749\end{array}$ & $\begin{array}{r}2,056 \\
-20\end{array}$ & $\begin{array}{l}35,600 \\
-1,127\end{array}$ & $\begin{array}{r}34,434 \\
-1,626\end{array}$ & $\begin{array}{r}295,969 \\
448\end{array}$ & $\begin{array}{r}333,785 \\
1,263\end{array}$ & $\begin{array}{r}1.580 \\
26\end{array}$ & $\begin{array}{r}13.6=6 \\
1.090\end{array}$ & $\begin{array}{r}300,158 \\
804\end{array}$ & $\begin{array}{r}100 \% \\
0 \%\end{array}$ \\
\hline
\end{tabular}

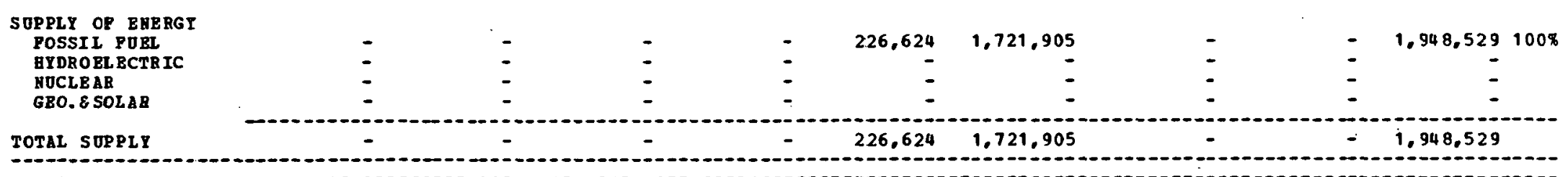

\section{WET IUPOATS}

$\begin{array}{lll}-56,792 & -14,061 & -106,745\end{array}$

$-159,055$

$69,793-1,386,856$

1,606

$4,544 \quad-1,647.566$

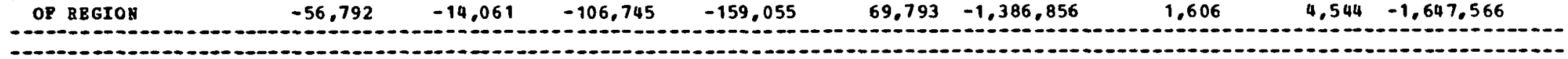

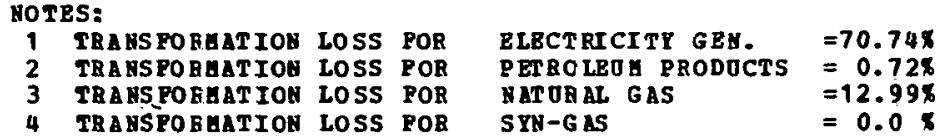


REGIONAL ENBRGY BALANCE STATRUENT

BY SECTOR AND POEL TYPE

\begin{tabular}{|c|c|c|c|c|c|c|c|c|c|c|}
\hline SECTOR & $\underset{\text { OIL }}{\text { DISTIIIATE }}$ & $\begin{array}{l}\text { RESIDUAL } \\
\text { OII }\end{array}$ & $\begin{array}{l}\text { GASOLIXE } \\
\text { (ALL OHI }\end{array}$ & $\begin{array}{l}\text { DTAER } \\
\text { BYDAO- } \\
\text { CABBOHS } \\
\text { TS IH } 10 * \% 9\end{array}$ & $\begin{array}{c}\text { CRODE } \\
\text { OIL } \\
\text { BTO'S) }\end{array}$ & $\begin{array}{l}\text { NATJRAL } \\
\text { G } 45\end{array}$ & $\cdot \operatorname{COAL}$ & ELECTRICITI & $\begin{array}{r}\text { SECTOR } \\
\text { TOTAL }\end{array}$ & \\
\hline $\begin{array}{l}\text { PIHAL DEAANS SECTORS } \\
\text { RESIDB HTIAL, COHA. } \\
\text { INDOSTRIAL } \\
\text { IRANSPORTATIOH } \\
\text { GISCELLAHEOS OSES }\end{array}$ & $\begin{array}{r}821 \\
960 \\
7.146 \\
218 \\
\end{array}$ & $\begin{array}{r}155 \\
342 \\
13 \\
230 \\
\end{array}$ & 27,452 & $\begin{array}{r}5.246 \\
14.683 \\
4.777 \\
145\end{array}$ & 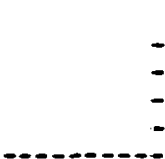 & $\begin{array}{r}10.688 \\
75,556 \\
3.108 \\
549\end{array}$ & $\begin{array}{r}183 \\
557 \\
5 \\
- \\
\end{array}$ & $\begin{array}{r}6,442 \\
2,107 \\
5 \\
130 \\
\end{array}$ & $\begin{array}{r}23,535 \\
94,205 \\
42,506 \\
1,272\end{array}$ & $\begin{array}{r}128 \\
478 \\
218 \\
18\end{array}$ \\
\hline $\begin{array}{l}\text { TOTAL PIHAL } \\
\text { DEAA ND SECTORS }\end{array}$ & $\subseteq .145$ & 740 & 27.452 & 24,851 & - & 89.301 & 745 & 8.684 & 161,518 & $81 \%$ \\
\hline $\begin{array}{l}\text { TRANSPORHATIOH } \\
\text { ELECTRICI TY GEA. } \\
\text { PETROLEOH. PRODOCTS } \\
\text { HATORAL GAS } \\
\text { SYH-GAS }\end{array}$ & $\begin{array}{r}64 \\
- \\
-\end{array}$ & $\begin{array}{r}296 \\
- \\
-\end{array}$ & - & $-10.23 \overline{5}$ & $\begin{array}{l}- \\
-\end{array}$ & $\begin{array}{r}52.701 \\
11.763= \\
=\end{array}$ & : & $\begin{array}{r}-15,659 \\
- \\
-\end{array}$ & $\begin{array}{r}37.602 \\
\overline{-} \\
1.528 \\
-\end{array}$ & $\begin{array}{r}198 \\
18\end{array}$ \\
\hline $\begin{array}{l}\text { NET FOEL OS3D } \\
\text { IM TRAHSFORAATION }\end{array}$ & 64 & 296 & - & $-10,235$ & - & 60,664 & - & $-15,659$ & 39,130 & $20 x$ \\
\hline $\begin{array}{l}\text { TOTAL GROSS FLORS } \\
\text { LOSSES } 8 \text { OULSSIONS }\end{array}$ & $\begin{array}{r}9.209 \\
559\end{array}$ & $\begin{array}{r}1.036 \\
-10\end{array}$ & $\begin{array}{r}27.452 \\
-869\end{array}$ & $\begin{array}{l}24,851 \\
-1.174\end{array}$ & - & $\begin{array}{r}154.565 \\
585\end{array}$ & $\begin{array}{r}745 \\
12\end{array}$ & $\begin{array}{r}8,684 \\
694\end{array}$ & $\begin{array}{r}200,648 \\
-202\end{array}$ & $\begin{array}{r}1008 \\
0 \%\end{array}$ \\
\hline
\end{tabular}

(1)

\begin{tabular}{|c|c|c|c|c|c|c|c|c|c|}
\hline $\begin{array}{l}\text { SUPPLY OP EAR RGY } \\
\text { POSSI I PTEL }\end{array}$ & - & - & - & - & 990,791 & 105.128 & - & - & $1,095,919100 \%$ \\
\hline HYDROELECTRIC & - & - & - & - & - & - & - & - & $100000-100$ \\
\hline BUCL BAR & - & - & - & - & - & - & - & - & - \\
\hline GEO. \& SOLAR & - & - & - & - & - & - & - & - & - \\
\hline TOTAL SOPPLT & - & - & - & - & 990,791 & 105.128 & - & - & $1,095,919$ \\
\hline
\end{tabular}

MBT IUPORTS
OP BEGIOR

\section{NOTES: \\ TREAS FORAAT IOH LOSS POR TREASPORUATIOH LOSS POR TRLuSPOAATION LOSS POB \\ ELECTRICITT GBN . \\ $=70.60 x$ \\ PETROLEOH 2BODOCTS $=0.0$ MTTORAL $G A T$ \\ 4 TRE HSPOBAATIOH IOSS POE STI-G}




\begin{tabular}{|c|c|c|c|c|c|c|c|c|c|c|}
\hline \multicolumn{11}{|c|}{$\begin{array}{l}\text { REGIONAL EHERGY BALANCE STATEAEHT } \\
\text { BY SECTOR AKD FOBL TYPE }\end{array}$} \\
\hline SBCTOR & $\begin{array}{l}\text { DISTILLATE } \\
\text { OIL }\end{array}$ & $\begin{array}{l}\text { RBSIDDAL } \\
\text { OIL. }\end{array}$ & $\begin{array}{l}\text { GASOLIHE } \\
\text { (ALL OHI }\end{array}$ & $\begin{array}{c}\text { OTABR } \\
\text { BYDBO- } \\
\text { CARBO\&S } \\
\text { TS IN 10*\%9 }\end{array}$ & $\begin{array}{l}\text { CRODE } \\
\text { OIL } \\
\text { BTO'S] }\end{array}$ & $\begin{array}{l}\text { NATURAC } \\
\text { GAS }\end{array}$ & COAL & ELECTRICITY & $\begin{array}{l}\text { SECTOR } \\
\text { TOTAL }\end{array}$ & \\
\hline $\begin{array}{l}\text { PIHAL DBGABD SBCTORS } \\
\text { RBSIDERTIAL, COHA. } \\
\text { IRDOSTRIAL } \\
\text { TRAYSPORTATIOH } \\
\text { EISCBLIAYEOOS OSES }\end{array}$ & $\begin{array}{r}768 \\
6.647 \\
6.049 \\
204\end{array}$ & $\begin{array}{r}132 \\
2.792 \\
14 \\
216\end{array}$ & 25,708 & $\begin{array}{r}4.913 \\
29.842 \\
4.260 \\
136\end{array}$ & $\begin{array}{l}\overline{-} \\
-\end{array}$ & $\begin{array}{r}9.775 \\
640.046 \\
2.910 \\
539\end{array}$ & $\begin{array}{r}171 \\
509 \\
5 \\
-\end{array}$ & $\begin{array}{r}5,834 \\
8,8 \leq 9 \\
127\end{array}$ & $\begin{array}{r}21,593 \\
688,695 \\
38,946 \\
1,222\end{array}$ & $\begin{array}{r}38 \\
88 \% \\
5 \% \\
0 \%\end{array}$ \\
\hline $\begin{array}{l}\text { TOTAL FIBAL } \\
\text { DEAA SBD SBCTORS }\end{array}$ & 13,668 & 3,154 & 25,708 & 39,151 & - & 653.270 & 685 & $14,8=0$ & 750,456 & $95 \%$ \\
\hline $\begin{array}{l}\text { TRAYSEOBAATIOY } \\
\text { BLECTRICITY GER. } \\
\text { PETROLEOA PRODUCTS } \\
\text { HATORAL GAS } \\
\text { SFH-GAS }\end{array}$ & -41.006 & $\begin{array}{r}159 \\
-9.446 \\
-\end{array}$ & $\begin{array}{r}-82.868 \\
-\end{array}$ & $\begin{array}{r}-47,406 \\
-122,192\end{array}$ & $\begin{array}{r}173.678 \\
-\end{array}$ & $\begin{array}{r}20.696 \\
7.917 \\
140.438\end{array}$ & ! & $\begin{array}{r}-6,281 \\
\vdots \\
-\end{array}$ & $\begin{array}{r}14.574 \\
1869 \\
18.246 \\
\end{array}$ & $\begin{array}{l}28 \\
0 \% \\
28\end{array}$ \\
\hline $\begin{array}{l}\text { IET POEL OSBD } \\
\text { IY TRABSPOREATIOA }\end{array}$ & $-41,006$ & $-9,237$ & $-82,868$ & $-169,598$ & 173,678 & 169,051 & - & $-6,281$ & 33,689 & 48 \\
\hline $\begin{array}{l}\text { TOTAL GROSS PLOHS } \\
\text { LOSSES } 8 \text { OHISSIOUS }\end{array}$ & $\begin{array}{r}13.668 \\
830\end{array}$ & $\begin{array}{r}3.313 \\
-33\end{array}$ & $\begin{array}{r}25,708 \\
-813\end{array}$ & $\begin{array}{l}39,151 \\
-1,849\end{array}$ & $\begin{array}{r}173.678 \\
263\end{array}$ & $\begin{array}{r}822,321 \\
3,113\end{array}$ & $\begin{array}{r}685 \\
11\end{array}$ & $\begin{array}{r}14,820 \\
1,185\end{array}$ & $\begin{array}{r}784,145 \\
2,706\end{array}$ & $\begin{array}{r}100 \% \\
0 \%\end{array}$ \\
\hline TOTAL HET OSAGE & $-26,507$ & $-6,156$ & -57.973 & $-132,296$ & 173,941 & 825,434 & 696 & 9,724 & 786,851 & \\
\hline
\end{tabular}

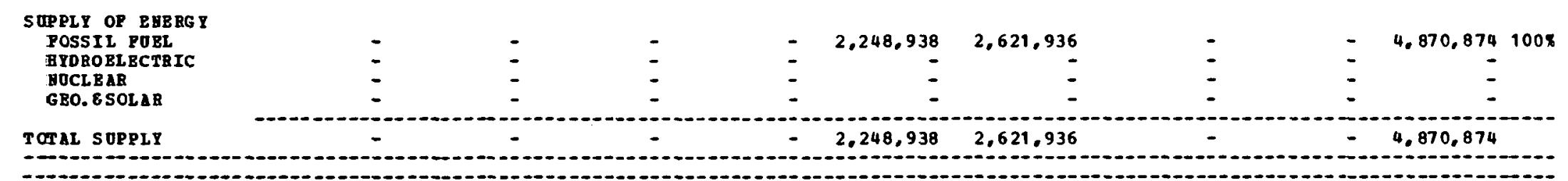

\section{MET IUPORTS}

$-26,507$

$-6,156$

$-57,973$

SOT BS:

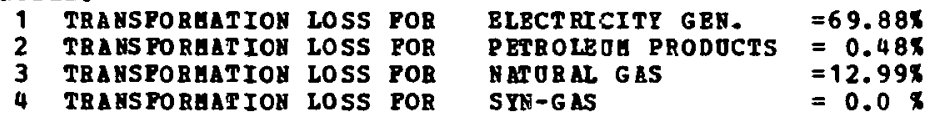


REGIONAL ENEEGY BALANCE STA TEGENT BY SBCTOR ABD FUEL TYPE

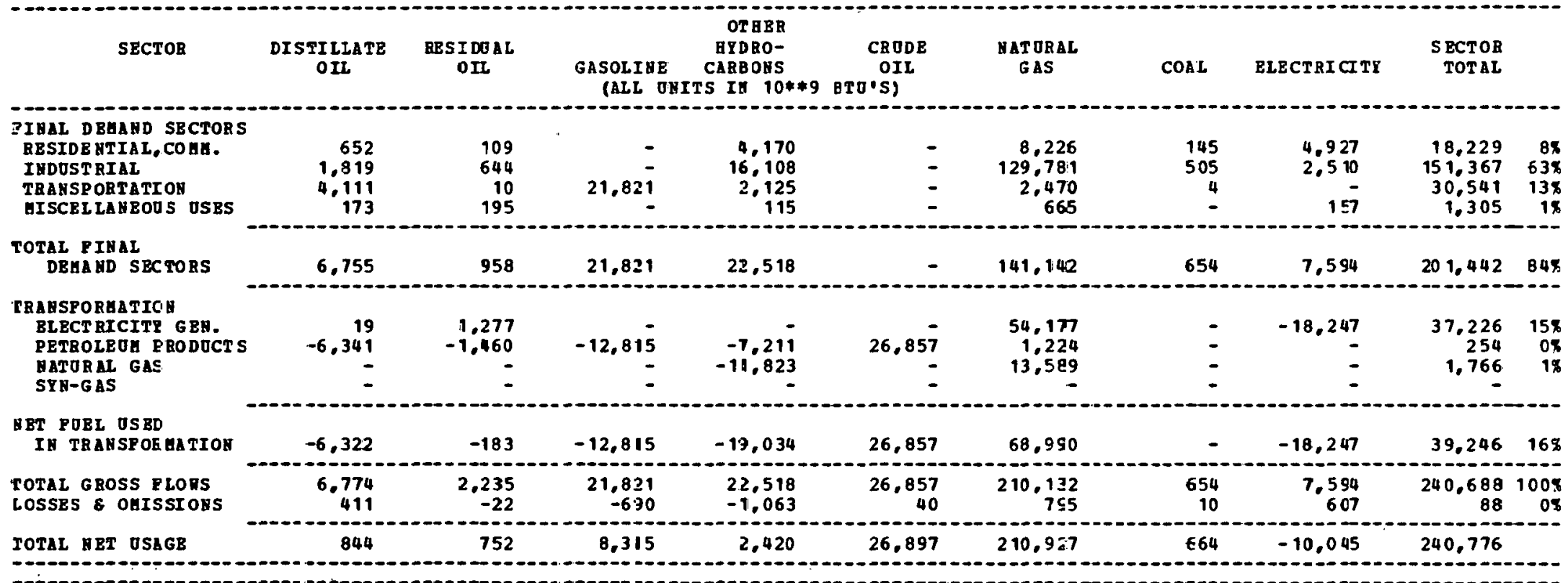

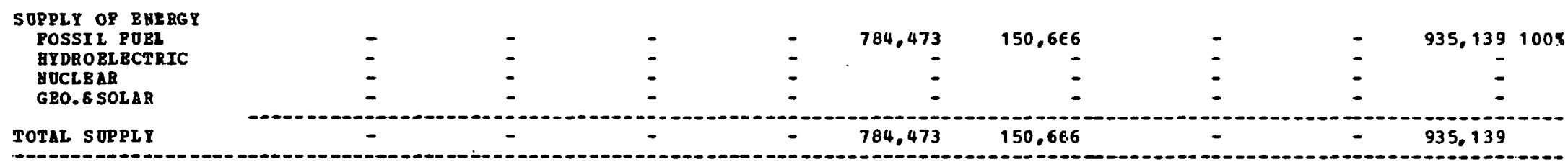

-

\section{MET IAPORTS}

$$
\text { OF BEGIOR }
$$

844

752

8,315

2,420

$-757,575$

60.261

$664 \quad-10.045$

$-694,362$

\section{HOT BS : \\ 1 TRANSPORGATION LOSS POB \\ TRABSPORAATIOH LOSS FOB \\ $\begin{array}{llll}3 & \text { TRABSPOREATION LOSS POF } & \text { NATJRAL GAS } & =13.00 \% \\ 4 & \text { TRABSPORATIOH LOSS POB } & \text { SES-GAS } & =0.0\end{array}$ \\ BLECT RT CITY $C B H$. \\ $=67.11 x$ \\ MaTIRAL GAS}




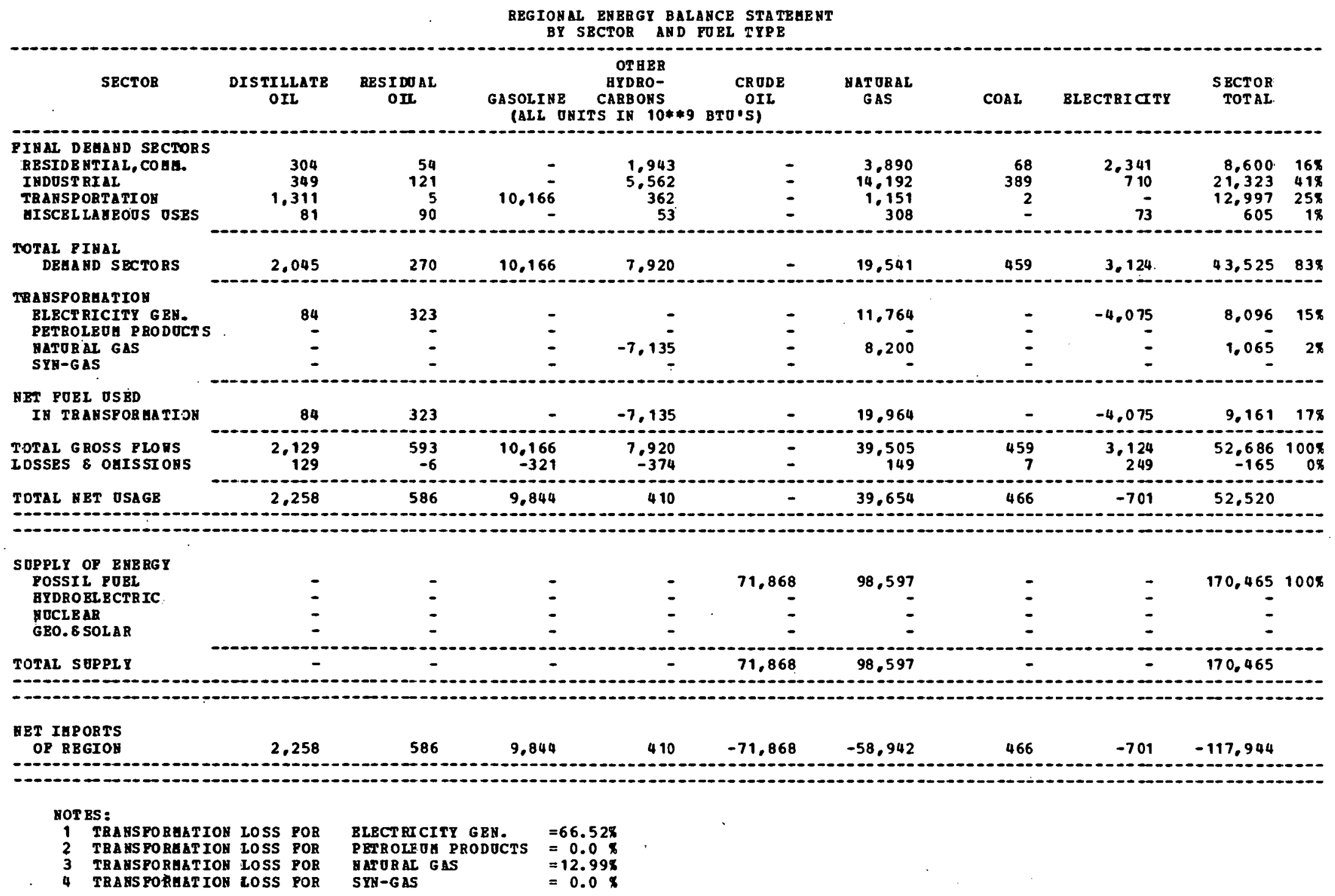


REgIONAL ENERGY BALANCE STATEHE AT

BY S.ECTOR AND FOEL TYPE

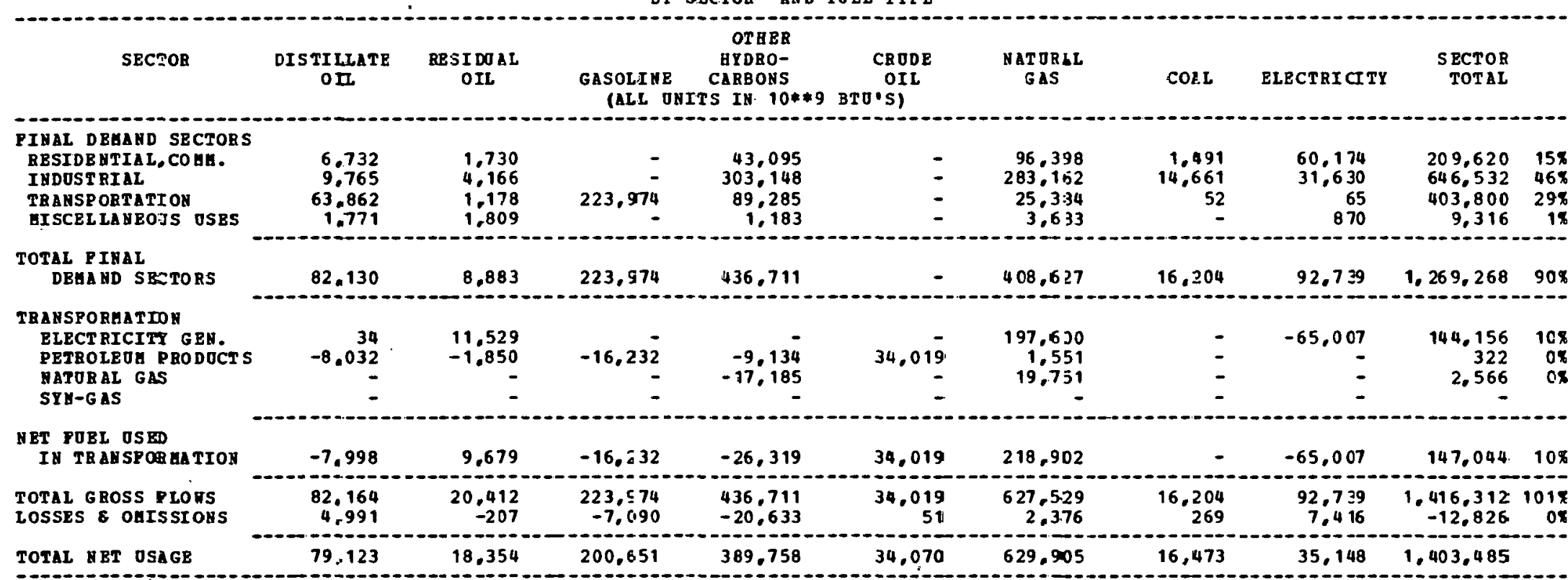

20
-

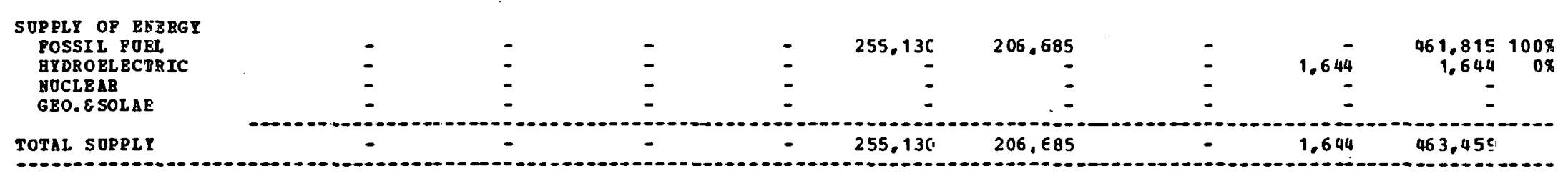

\begin{tabular}{l}
$\begin{array}{l}\text { MET IHPORTS } \\
\text { OF REGION }\end{array}$ \\
\hdashline
\end{tabular}


REGIONAL BNBBGT BALAMCB STATBHENT

BY SBCTOR AXD FOEL IYPE

\begin{tabular}{|c|c|c|c|c|c|c|c|c|c|}
\hline SECTOR & $\begin{array}{l}\text { DIS TILLATE } \\
\text { OIL }\end{array}$ & $\begin{array}{l}\text { RBSIDJAL } \\
\text { OIL }\end{array}$ & $\begin{array}{l}\text { GASOLISB } \\
\text { (ALL O }\end{array}$ & $\begin{array}{c}\text { OTHER } \\
\text { BYDRO- } \\
\text { CARBOHS } \\
\text { TS IB } 10 * \% 9\end{array}$ & $\begin{array}{c}\text { CBODE } \\
\text { OIL } \\
\text { BTO'SI }\end{array}$ & $\begin{array}{l}\text { MAT ORAL } \\
\text { GAS }\end{array}$ & COAL & BLECTRICITY & $\begin{array}{r}\text { SBCTOR } \\
\text { TOTAL }\end{array}$ \\
\hline 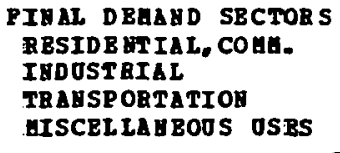 & $\begin{array}{r}1.070 \\
1.257 \\
4.779 \\
285\end{array}$ & $\begin{array}{r}163 \\
396 \\
10 \\
740\end{array}$ & 35,803 & $\begin{array}{r}6.843 \\
24.517 \\
2.371 \\
189\end{array}$ & $\overline{-}$ & $\begin{array}{r}13: 214 \\
17.383 \\
4.053 \\
3.357\end{array}$ & $\begin{array}{r}239 \\
1.080 \\
4 \\
-\end{array}$ & $\begin{array}{r}7.879 \\
2.213 \\
6 \\
794\end{array}$ & $\begin{array}{r}29.408 \\
46,846 \\
47.026 \\
5.065\end{array}$ \\
\hline $\begin{array}{l}\text { TOTAL FIBAL } \\
\text { DEAA AD SBCTOBS }\end{array}$ & 7,391 & 1.209 & 35,803 & 33,920 & - & 38,007 & 1,323 & 10,892 & 128,345 \\
\hline $\begin{array}{l}\text { TRAHSPORALTIOH } \\
\text { BLECTRICITY GEH. } \\
\text { PETROLROA PRODOCTS } \\
\text { YATORAL GAS } \\
\text { SYH-GAS }\end{array}$ & 21 & $\begin{array}{r}595 \\
- \\
-\end{array}$ & : & $-1.47 \overline{-}$ & $\overline{-}$ & $\begin{array}{r}66.289 \\
1.691 \\
-\end{array}$ & $\begin{array}{r}56,237 \\
= \\
-\end{array}$ & $\begin{array}{r}-44,934 \\
= \\
=\end{array}$ & $\begin{array}{r}88,308 \\
220 \\
-\end{array}$ \\
\hline $\begin{array}{l}\text { HBT FOEL OSBD } \\
\text { IH TRAESPORGATIOR }\end{array}$ & 21 & 595 & - & $-1,471$ & - & 67,980 & 66,237 & $-44,934$ & 88,528 \\
\hline $\begin{array}{l}\text { TOTAL GROSS PLOHS } \\
\text { LOSSES E OUISSIOHS }\end{array}$ & $\begin{array}{r}7.412 \\
450\end{array}$ & $\begin{array}{r}1.704 \\
-17\end{array}$ & $\begin{array}{l}35,803 \\
-1,133\end{array}$ & $\begin{array}{l}33,920 \\
-1,602\end{array}$ & - & $\begin{array}{r}105,987 \\
401\end{array}$ & $\begin{array}{r}67.560 \\
1,123\end{array}$ & $\begin{array}{r}10.892 \\
871\end{array}$ & $\begin{array}{r}216,873 \\
92\end{array}$ \\
\hline
\end{tabular}

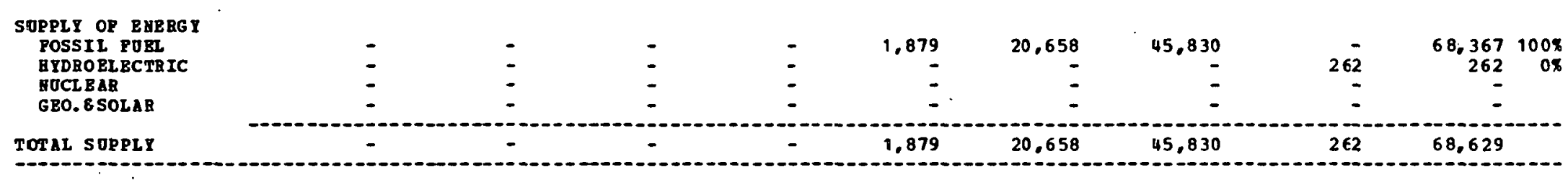

UBT IKPORTS
OP REGIOH


REGIONAL BNERGY BALANCE STATEMENT

BY SECTOR ABD POBL TYPE

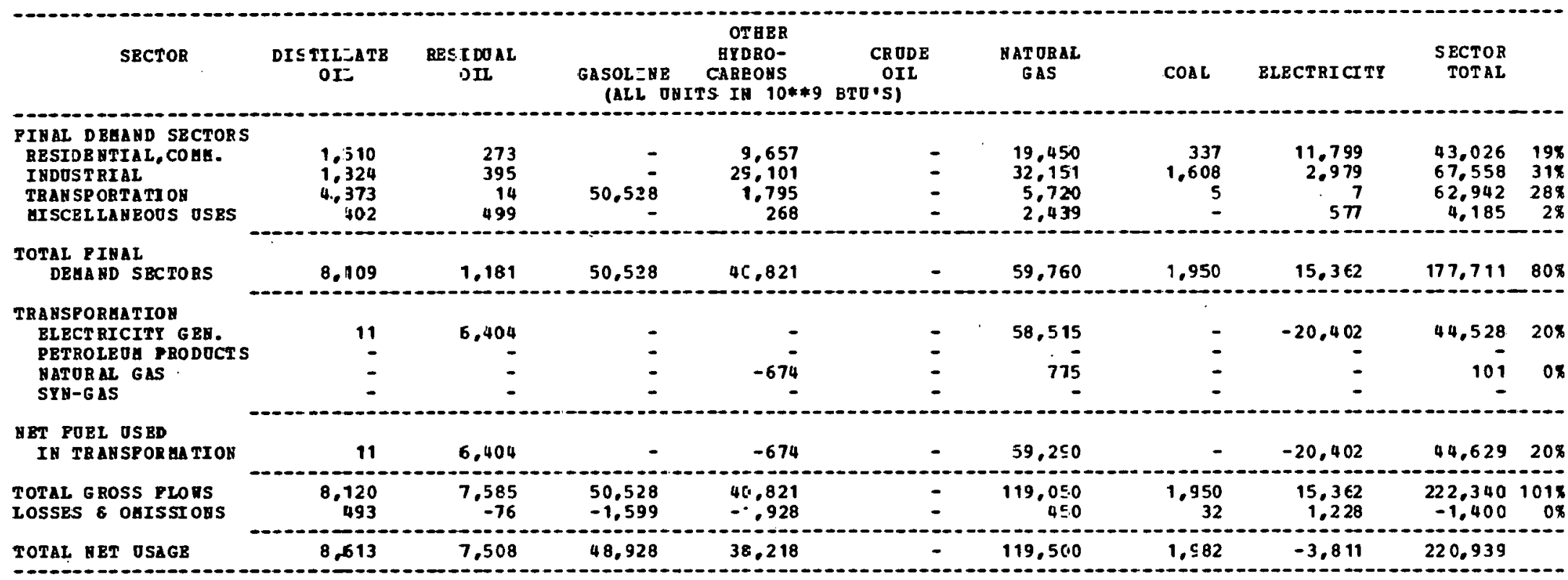

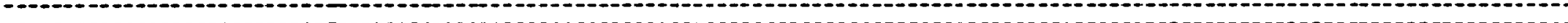

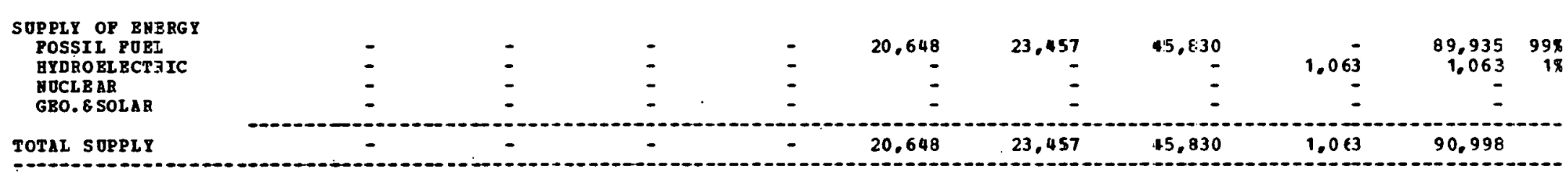

\section{MET IHPORTS}

OP RBGIOH

8,613

7,508

48.928

33,218

$-20,648$

96.043

$-43,847$

$-4,874$

129.941

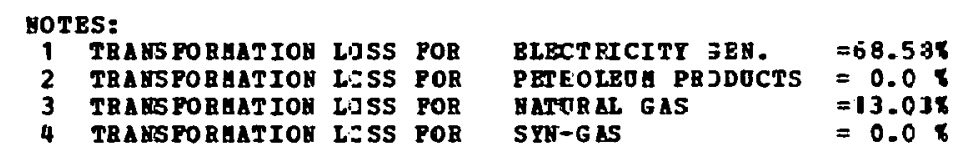


RBGIOHAL EHERGY BALAHCE STATEEENT

BY SBCTOR AND POEL TYPE

\begin{tabular}{|c|c|c|c|c|c|c|c|c|c|c|}
\hline SECTOR & $\begin{array}{l}\text { DIS TILLATE } \\
\text { OIL }\end{array}$ & $\begin{array}{l}\text { RESIDEAL } \\
\text { OII }\end{array}$ & $\begin{array}{l}\text { GASOLINE } \\
\text { (ALL O }\end{array}$ & $\begin{array}{l}\text { BYDRO- } \\
\text { CARBOHS } \\
\text { IS IN 10**9 }\end{array}$ & $\begin{array}{l}\text { CRODE } \\
\text { OIL } \\
\text { BTO'SI }\end{array}$ & $\begin{array}{l}\text { HAT ORAL } \\
\text { GAS }\end{array}$ & COAL & ELECTRI CITY & $\begin{array}{l}\text { SECTOR } \\
\text { TOTAL }\end{array}$ & \\
\hline 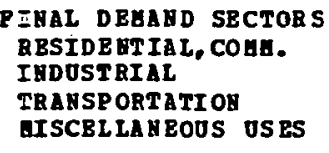 & $\begin{array}{l}1.405 \\
3.886 \\
5,305 \\
374\end{array}$ & $\begin{array}{r}212 \\
1,648 \\
16 \\
385\end{array}$ & 47.009 & $\begin{array}{r}8.984 \\
57.717 \\
2.261 \\
249\end{array}$ & $\begin{array}{l}\overline{-} \\
\overline{-}\end{array}$ & $\begin{array}{r}17.304 \\
190.616 \\
5.322 \\
774\end{array}$ & $\begin{array}{r}313 \\
6.089 \\
6 \\
-\end{array}$ & $\begin{array}{r}10.259 \\
10.786 \\
183\end{array}$ & $\begin{array}{r}38,477 \\
270,742 \\
59,919 \\
1,965\end{array}$ & $\begin{array}{r}98 \\
608 \\
138 \\
08\end{array}$ \\
\hline $\begin{array}{l}\text { TOTAL FIHAL } \\
\text { DEAA AD SECTORS }\end{array}$ & 10,970 & 2,261 & 47,009 & 69,211 & - & 214.016 & 6.408 & 21.228 & 371,103 & $82 \%$ \\
\hline $\begin{array}{l}\text { TEABSPOBAATION } \\
\text { BLECTRICITY GBH. } \\
\text { PETROLEUA PBODOCTS } \\
\text { MATORAL GAS } \\
\text { SYH-GAS }\end{array}$ & $\begin{array}{r}15 \\
-19,658 \\
-\end{array}$ & $\begin{array}{r}1.777 \\
-4.529 \\
- \\
-\end{array}$ & $\begin{array}{r}-39,726 \\
-\end{array}$ & $\begin{array}{r}-22,364 \\
-32,702 \\
-\end{array}$ & $\begin{array}{r}83,258 \\
-\end{array}$ & $\begin{array}{r}109.269 \\
3.795 \\
37.585 \\
-\end{array}$ & $\overline{-}$ & $\begin{array}{r}-35.523 \\
- \\
-\end{array}$ & $\begin{array}{r}75.538 \\
776 \\
4.883 \\
-\end{array}$ & $\begin{array}{r}17 \% \\
0 \% \\
1 \%\end{array}$ \\
\hline $\begin{array}{l}\text { BET POEL OSED } \\
\text { IR TRANSPORAATION }\end{array}$ & $-19,643$ & $-2,752$ & -39.726 & $-55,066$ & 83,258 & 150.649 & - & $-35,523$ & 81,197 & $18 \%$ \\
\hline $\begin{array}{l}\text { TCTAL GROSS PLORS } \\
\text { LCSSES } \& \text { OAIS SIOUS }\end{array}$ & $\begin{array}{r}10,985 \\
667\end{array}$ & $\begin{array}{r}4,038 \\
-40\end{array}$ & $\begin{array}{l}47.009 \\
-1.488\end{array}$ & $\begin{array}{l}69,211 \\
-3,270\end{array}$ & $\begin{array}{r}83,258 \\
126\end{array}$ & $\begin{array}{r}364.665 \\
1.380\end{array}$ & $\begin{array}{r}6.408 \\
106\end{array}$ & $\begin{array}{r}21,228 \\
1,6.97\end{array}$ & $\begin{array}{r}452.300 \\
-820\end{array}$ & $\begin{array}{r}100 \% \\
0 \%\end{array}$ \\
\hline
\end{tabular}

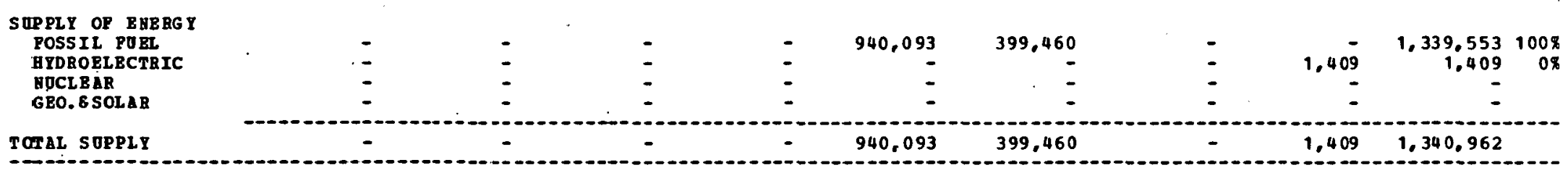

-

I BT IUPORTS
DF RBGIOY

$$
-8,005
$$

$-5.31$

5,794

10.874

$-856,708$

$-33,414$

6,514

BOT ES:

1 TRANSFORAatION LOSS POB

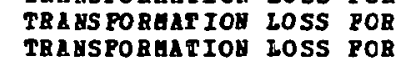

TRABSPORAATIOH LOSS POR
TRANS PORAATIOH LOSS FOR

BLECTRICITI GEN. $\quad=68.01 \%$ 
REGIONAL ENERG.Y BALANCE STATEAEAS

BY SECTOR AHD FOEL TYPE

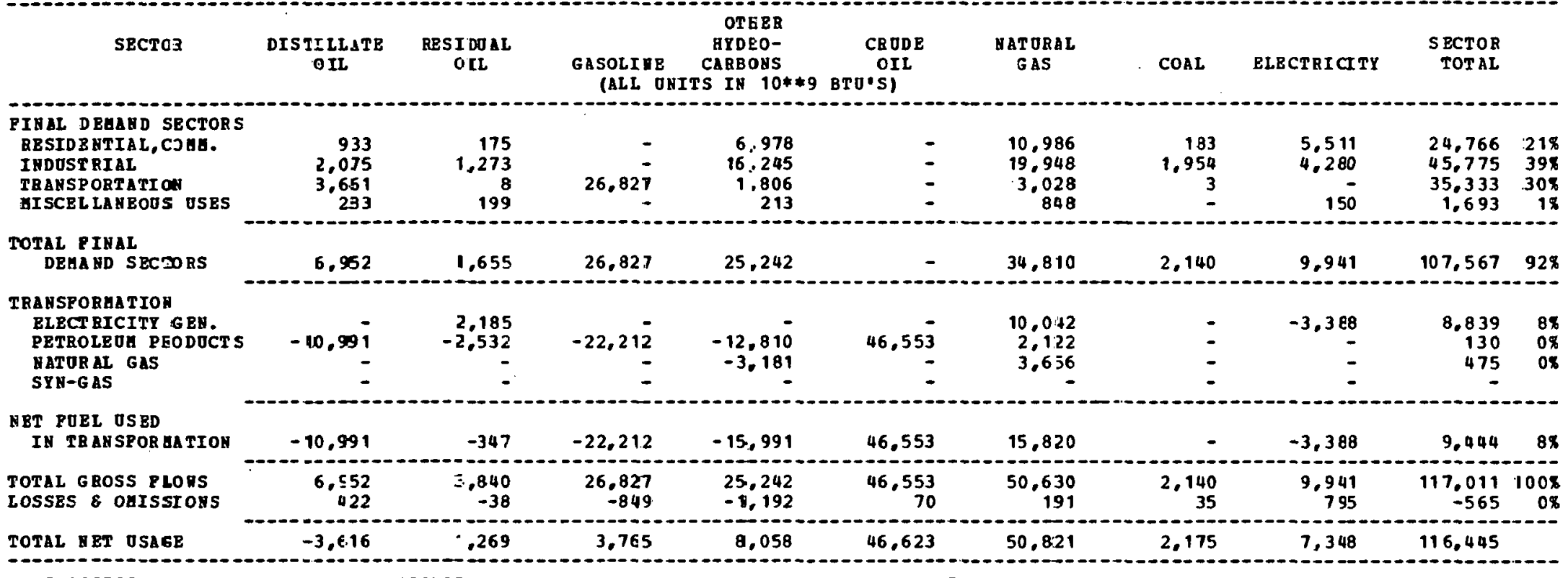

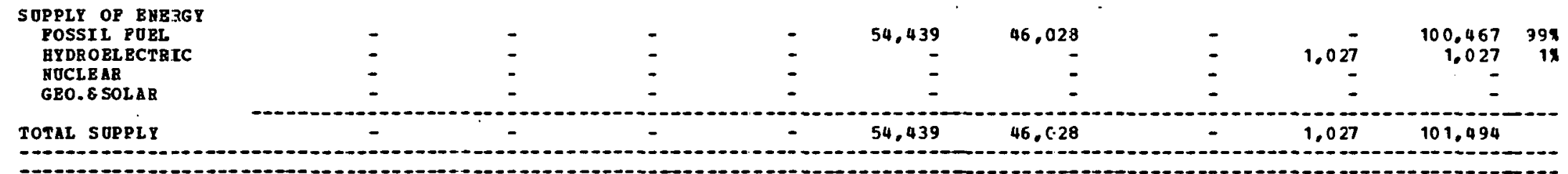

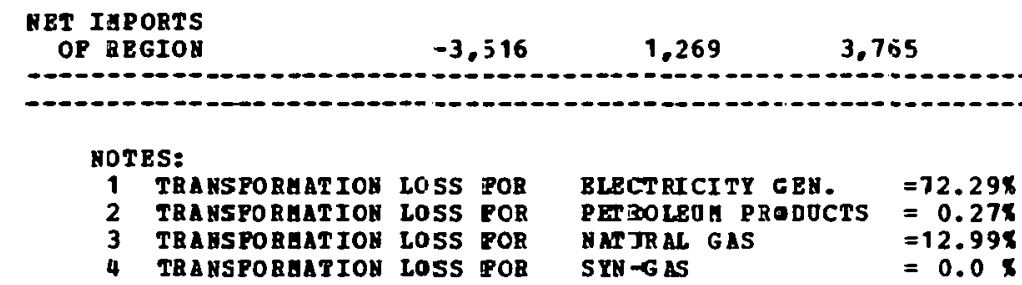


REGIONAL BHERGI BALARCB STATEHEAT

BY SBCTOR AND POBL TYPE

\begin{tabular}{|c|c|c|c|c|c|c|c|c|c|c|}
\hline SECTOR & $\begin{array}{l}\text { DISTILLATB } \\
\text { OIL }\end{array}$ & $\begin{array}{l}\text { RESI DUAL } \\
\text { OIL }\end{array}$ & $\begin{array}{l}\text { GASOLILE } \\
\text { (ALL OHI }\end{array}$ & $\begin{array}{l}\text { AYDBO- } \\
\text { CABBO\&S } \\
\text { TS IH 10**9 }\end{array}$ & $\begin{array}{c}\text { CRODE } \\
\text { OIL } \\
\text { BTU'S) }\end{array}$ & $\begin{array}{l}\text { NAT URAL } \\
\text { G AS }\end{array}$ & $\operatorname{COA} L$ & BLECTRICITY & $\begin{array}{r}\text { SECTOR } \\
\text { TOTAL }\end{array}$ & \\
\hline $\begin{array}{l}\text { TOTAL FIBAL } \\
\text { DEAABD SBCTOBS }\end{array}$ & 11.608 & 1,899 & 27,155 & 13,461 & $=$ & 161,638 & 754 & 12,920 & 229.435 & $93 x$ \\
\hline $\begin{array}{l}\text { TRAYSPOREATIOB } \\
\text { BLECTRICITY GEA. } \\
\text { PETROLEOA PRODUCTS } \\
\text { BATUBAL GAS } \\
\text { SYB-GAS }\end{array}$ & $\begin{array}{r}15 \\
-88,875 \\
-\end{array}$ & $\begin{array}{r}146 \\
-16.991 \\
-\end{array}$ & $\begin{array}{r}-175.071 \\
-\end{array}$ & $\begin{array}{r}-83.850 \\
-8.417 \\
-\end{array}$ & 350,845 & $\begin{array}{r}16,483 \\
15,994 \\
9,674 \\
-\end{array}$ & $\overline{-}$ & $\begin{array}{r}-5,307 \\
= \\
-\end{array}$ & $\begin{array}{r}11,337 \\
2.052 \\
1.257 \\
-\end{array}$ & $\begin{array}{l}58 \\
1 \% \\
1 \%\end{array}$ \\
\hline $\begin{array}{l}\text { ABT POEL USBD } \\
\text { IB TRABSPORAATIOA }\end{array}$ & $-88,860$ & $-16,845$ & $-175,071$ & $-92,267$ & $350: 845$ & 42,151 & - & $-5,307$ & 14.646 & $6 \%$ \\
\hline $\begin{array}{l}\text { TOTAL GROSS PLORS } \\
\text { IOSSES \& OBISSIOHS }\end{array}$ & $\begin{array}{r}11.623 \\
706\end{array}$ & $\begin{array}{r}2.045 \\
-20\end{array}$ & $\begin{array}{r}27,155 \\
-859\end{array}$ & $\begin{array}{r}13,461 \\
-636\end{array}$ & $\begin{array}{r}350.845 \\
531\end{array}$ & $\begin{array}{r}203.789 \\
771\end{array}$ & $\begin{array}{r}754 \\
12\end{array}$ & $\begin{array}{r}12,920 \\
1,033\end{array}$ & $\begin{array}{r}244,081 \\
1,539\end{array}$ & $\begin{array}{r}99 \% \\
17\end{array}$ \\
\hline
\end{tabular}

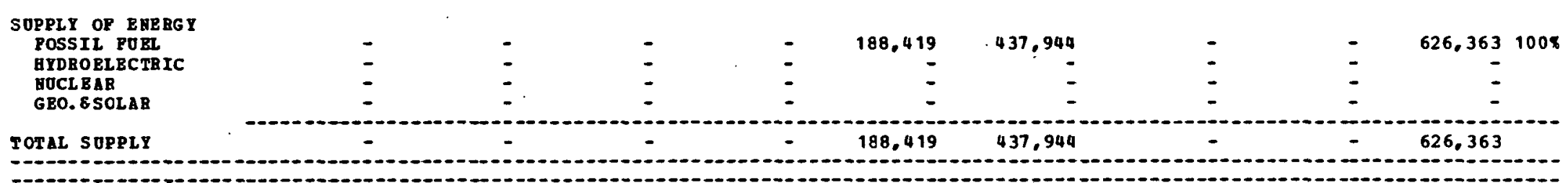

IBT IAPORTS

$-76,545$

$-14,966$

$-\$ 48,775$

$-79,442$

162,957

$-233.383$

766

NOT BS:

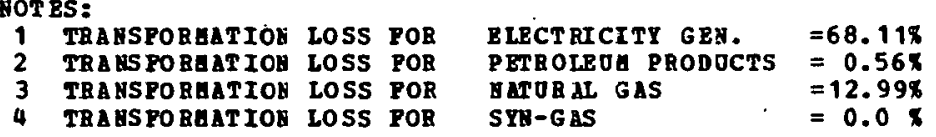




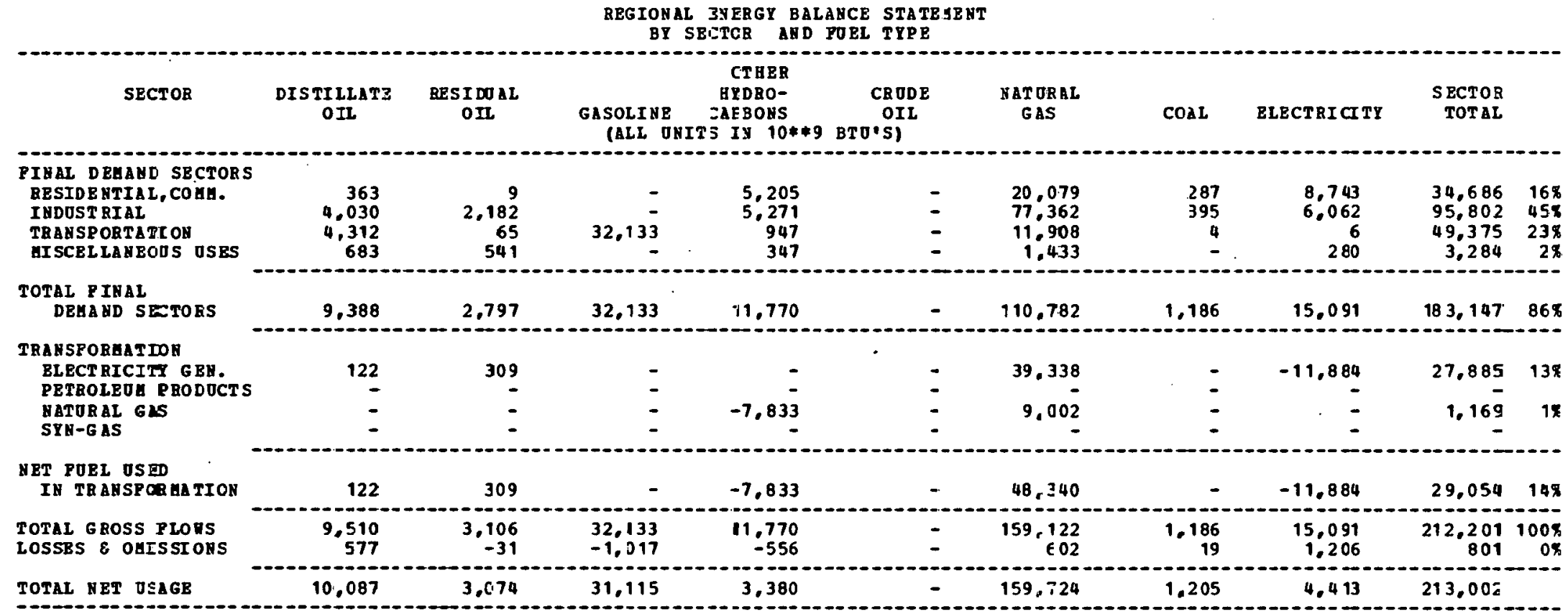

(1)

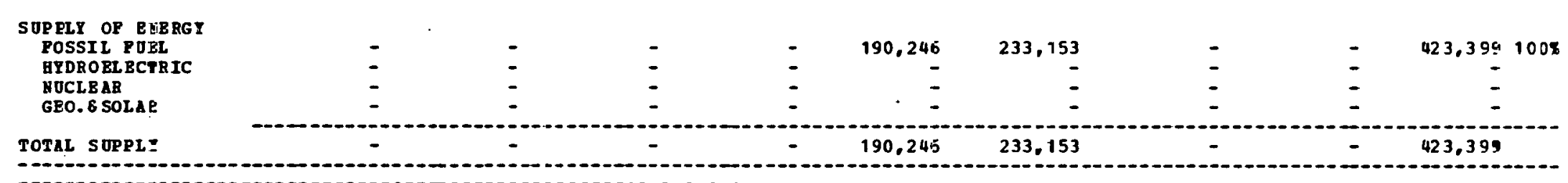

$\begin{aligned} & \text { MET IHPORTS } \\ & \text { OF REGIOH }\end{aligned}$
NOTES:


REGIONAL ENERGT BALANCE STATEAENT

BY SECTOR ARD POEL TYPE

\begin{tabular}{|c|c|c|c|c|c|c|c|c|c|c|}
\hline SECTOR & $\begin{array}{l}\text { DISTILIATE } \\
\text { OIL }\end{array}$ & $\begin{array}{c}\text { BESIDUAL } \\
\text { GIL }\end{array}$ & $\begin{array}{l}\text { GASOLINE } \\
\text { (ALL ONIT }\end{array}$ & $\begin{array}{l}\text { AYDRO- } \\
\text { CARBOIS } \\
\text { IS IB } 10 * * 9\end{array}$ & $\begin{array}{l}\text { CRODE } \\
\text { OIL } \\
\text { BTO'S) }\end{array}$ & $\begin{array}{l}\text { YAT URAL } \\
\text { GAS }\end{array}$ & COAL & ELECTRI CITY & $\begin{array}{r}\text { SECTOR } \\
\text { TOTAL }\end{array}$ & \\
\hline $\begin{array}{l}\text { INAL DEHAYD SECTOR S } \\
\text { RESIDB HTIAL, COHA. } \\
\text { INDUSTRIAL } \\
\text { TRANSPORTATION } \\
\text { GISCELLABOOS USBS }\end{array}$ & $\begin{array}{r}1.754 \\
2.658 \\
9.358 \\
542\end{array}$ & $\begin{array}{r}220 \\
3,465 \\
1,312 \\
118\end{array}$ & 34,718 & $\begin{array}{r}12,307 \\
11,251 \\
3,018 \\
434\end{array}$ & $\begin{array}{l}- \\
-\end{array}$ & $\begin{array}{r}13.471 \\
73.505 \\
9.119 \\
778\end{array}$ & $\begin{array}{r}261 \\
1.349 \\
7 \\
-\end{array}$ & $\begin{array}{r}7.837 \\
7.215 \\
71\end{array}$ & $\begin{array}{r}35,850 \\
99,443 \\
57,532 \\
1,943\end{array}$ & $\begin{array}{r}17 \% \\
48 \% \\
288 \\
\quad 18\end{array}$ \\
\hline $\begin{array}{l}\text { TOTAL P IHAL } \\
\text { DEHA UD SBC TOES }\end{array}$ & 14.312 & 5.115 & 34.718 & 27.010 & - & 96,873 & 1.617 & 15,123 & 194,768 & $94 \%$ \\
\hline $\begin{array}{l}\text { TRANSFORAATIOH } \\
\text { ELBCTRICITY GEA. } \\
\text { PETROLEOE PRODOCTS } \\
\text { BATURAL GAS } \\
\text { SYH-GAS }\end{array}$ & $\begin{array}{r}7 \\
-19,694 \\
- \\
-\end{array}$ & $\begin{array}{r}2.524 \\
-3.760 \\
- \\
-\end{array}$ & $\begin{array}{r}-38,478 \\
-\end{array}$ & $\begin{array}{r}-45.837 \\
-479 \\
-\end{array}$ & $\begin{array}{r}103,281 \\
=\end{array}$ & $\begin{array}{r}12,591 \\
4,70 \mathrm{~B} \\
550 \\
-\end{array}$ & $\begin{array}{r}286 \\
- \\
-\end{array}$ & $\begin{array}{r}-4.249 \\
= \\
=\end{array}$ & $\begin{array}{r}11.159 \\
220 \\
71 \\
-\end{array}$ & $\begin{array}{l}5 \% \\
0 \% \\
0 \%\end{array}$ \\
\hline $\begin{array}{l}\text { HET FOEL OSED } \\
\text { IB TRAESPOREATION }\end{array}$ & $-19,687$ & $-11,236$ & $-38,478$ & $-46,316$ & 103.281 & 17.849 & 286 & $-4,249$ & 11,450 & $6 \%$ \\
\hline $\begin{array}{l}\text { TOTAL GROSS PLOHS } \\
\text { LOSSES } 8 \text { OUISSIOHS }\end{array}$ & $\begin{array}{r}14,319 \\
869\end{array}$ & $\begin{array}{r}7.639 \\
-71\end{array}$ & $\begin{array}{l}34.718 \\
-1.099\end{array}$ & $\begin{array}{l}27,010 \\
-1,276\end{array}$ & $\begin{array}{r}103.281 \\
156\end{array}$ & $\begin{array}{r}114.722 \\
.434\end{array}$ & $\begin{array}{r}1,903 \\
31\end{array}$ & $\begin{array}{r}15.123 \\
1.209\end{array}$ & $\begin{array}{r}206,218 \\
249\end{array}$ & $\begin{array}{r}100 \% \\
0 \%\end{array}$ \\
\hline
\end{tabular}

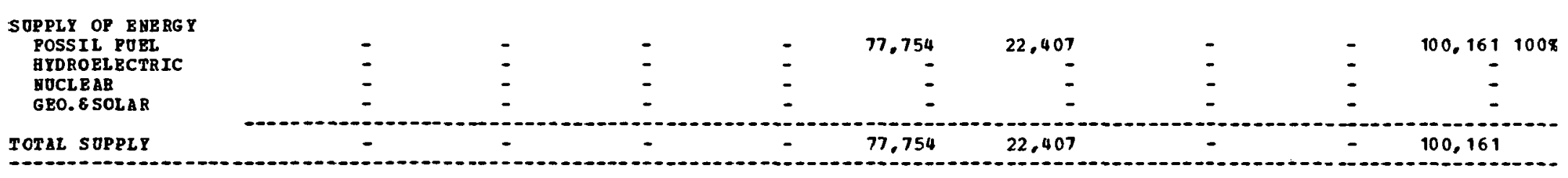

\section{WBT IAPORTS \\ OP BEGIOB}

$-4.505$

3,801

$-4,859$

$-20,582$

25,683

92,749

1,934

12,083

106,306

MOT ES:

1 TBABSPORBATION LOSS POR

TRAMSPOBaAT ION LOSS POB

TBANSPOREATIOH LOSS PO

ELECT RICITY GEN. $\quad=72.42 \times$

AATOR AL G AS

$\begin{array}{ll}\text { ATOR AL GAS } & =12.91 \mathrm{x} \\ S T F-G A S & =0.00\end{array}$

(1)

(1) 
REGIONAL EHERGT BALANCE STATEABNT

BY SECTOB AKD FOBL TYPE

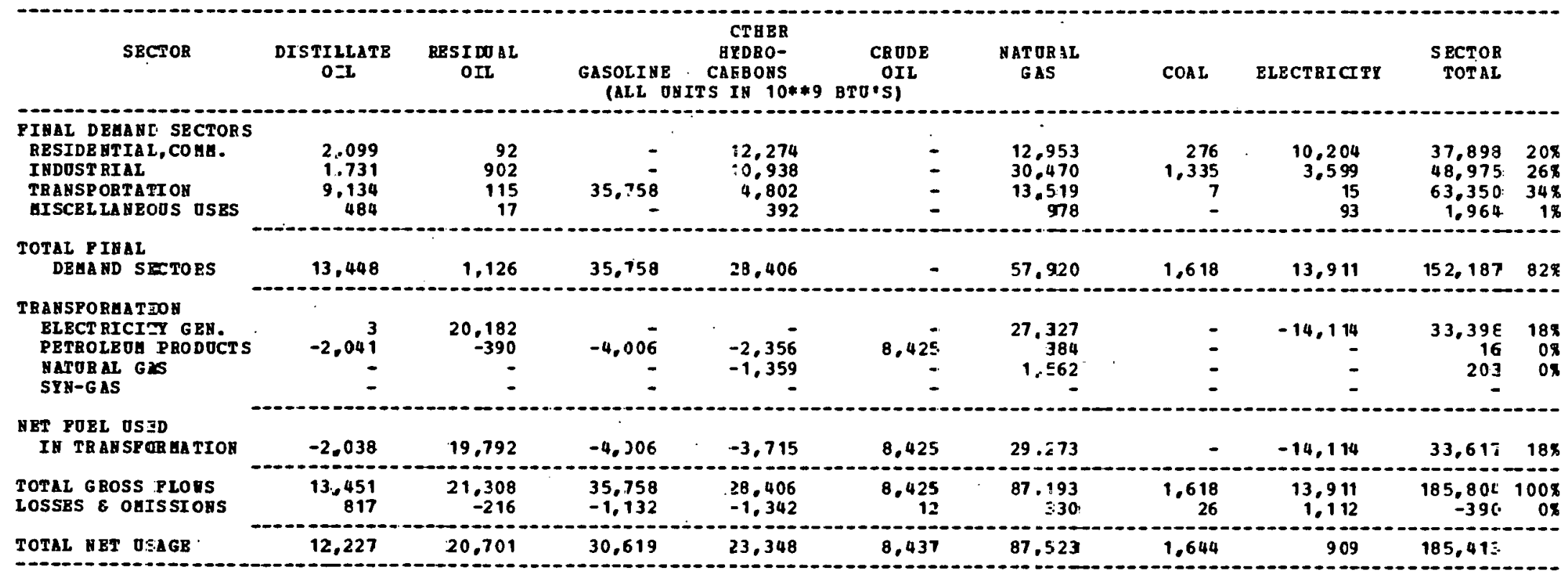

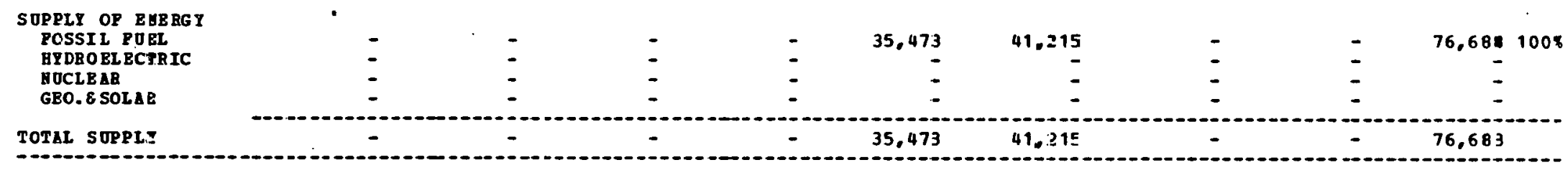

1.227

20,701

30,619

23,348

$-27.035$

46, 308:

1,644

909

108,725

-

HOTES

\begin{tabular}{|c|c|c|c|}
\hline 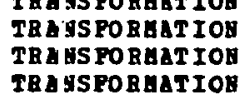 & $\begin{array}{l}105 \\
105 \\
105\end{array}$ & $\begin{array}{l}\text { POR } \\
\text { POR } \\
\text { POR } \\
\text { POB }\end{array}$ & $\begin{array}{l}\text { PEROIBOA PRODOCTS } \\
\text { MLTORAL GAS } \\
\text { SFM-GAS }\end{array}$ \\
\hline
\end{tabular}


REGIOHAL ENBRGT BALAHCE STATEAENT

BT SBCTOR AUD FOBL TYPE

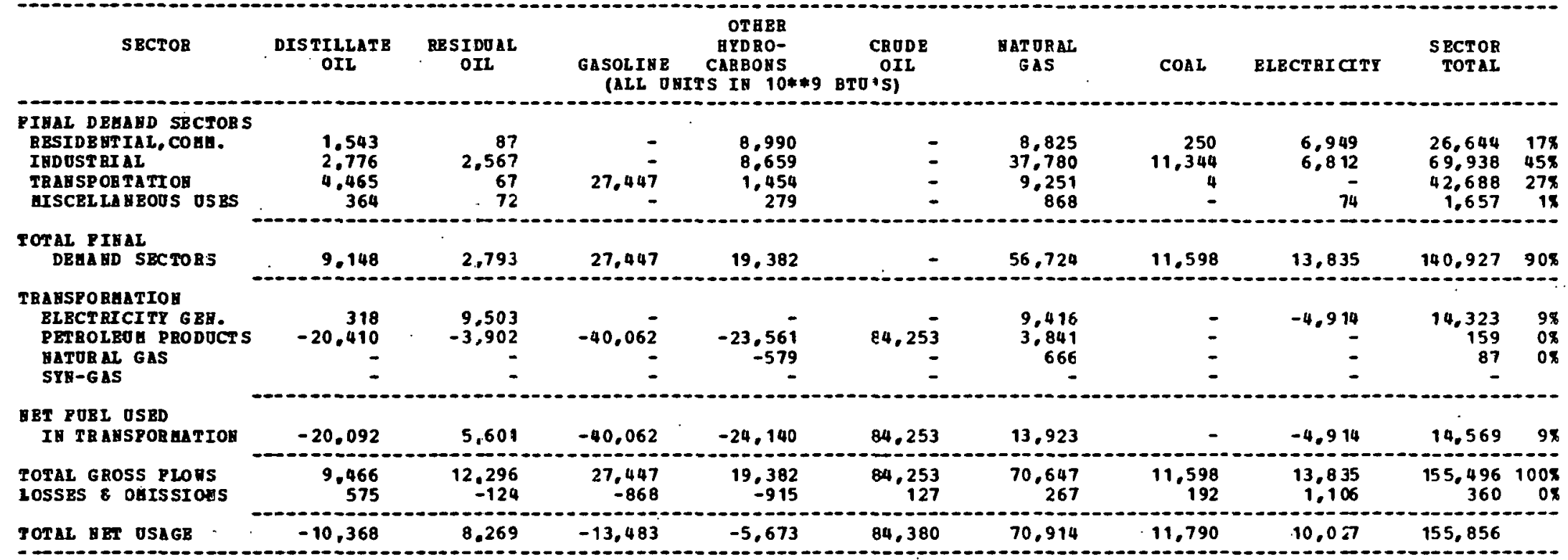

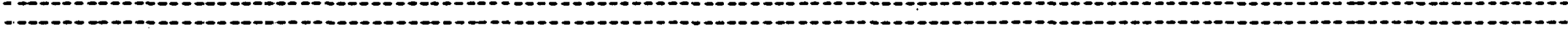

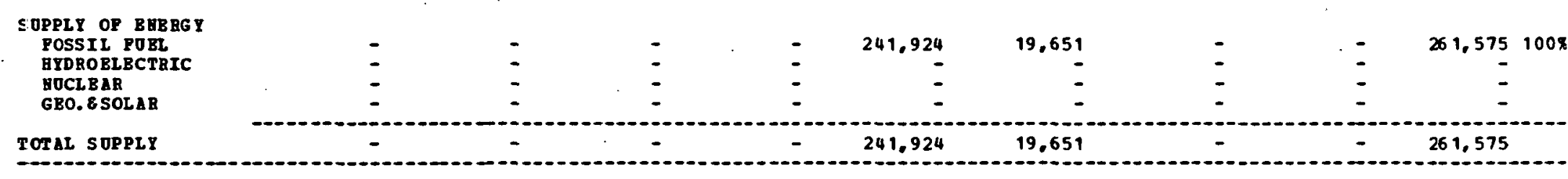

\begin{tabular}{l}
$\begin{array}{l}\text { BET IEPORTS } \\
\text { OR RBGIOB }\end{array}$ \\
\hline SOTES:
\end{tabular}


REGIOHAL BEERGY BALAKCE STATRGENT

3I SBCTOB ARD POEL TYPE

\begin{tabular}{|c|c|c|c|c|c|c|c|c|c|c|}
\hline SECTOR & $\begin{array}{c}\text { DISTI LLATE } \\
\text { JII }\end{array}$ & $\begin{array}{l}\text { BESIDOAL } \\
\text { OIL }\end{array}$ & $\begin{array}{l}\text { GASOLIME } \\
\text { (ALI JLII? }\end{array}$ & $\begin{array}{c}\text { OTJER } \\
\text { HTDBO- } \\
\text { CBRBDNS } \\
\text { TS IN } 10 * * 9\end{array}$ & $\begin{array}{c}\text { CBODE } \\
\text { OIL } \\
\text { BTO'S) }\end{array}$ & $\begin{array}{l}\text { NATORAI } \\
\text { GAS }\end{array}$ & COAL & ELECTRICITY & $\begin{array}{r}\text { SECTOR } \\
\text { TOTAL }\end{array}$ & \\
\hline $\begin{array}{l}\text { PINAL DRGAND SECTORS } \\
\text { RESIDE HTIAL, COHA. } \\
\text { IRDOST RIAL } \\
\text { TRABSPORTATI ON } \\
\text { GISCEL LAYEOUS OSES }\end{array}$ & $\begin{array}{r}2.423 \\
6.046 \\
10,980 \\
622\end{array}$ & $\begin{array}{r}686 \\
7.584 \\
16.154 \\
573\end{array}$ & 51,168 & $\begin{array}{r}13,933 \\
14,349 \\
1,564 \\
372\end{array}$ & - & $\begin{array}{r}17,336 \\
47.63 ! \\
9.82 \\
2,440\end{array}$ & $\begin{array}{r}713 \\
71,3 \geq 4 \\
11 \\
-\end{array}$ & $\begin{array}{r}13,609 \\
21,241 \\
12 \\
134\end{array}$ & $\begin{array}{r}48,700 \\
168,185 \\
89,710 \\
4,141\end{array}$ & $\begin{array}{r}128 \\
418 \\
228 \\
18\end{array}$ \\
\hline $\begin{array}{l}\text { TOTAL PIHAL } \\
\text { DEAA ND SBCIORS }\end{array}$ & $=0,071$ & 24,997 & 51.168 & 30,218 & - & 77.228 & 72,058 & 34,996 & 310,736 & $76 \%$ \\
\hline $\begin{array}{l}\text { TRAKSFORBATIOR } \\
\text { ELECTRICITY GEN. } \\
\text { PETROLEOH PRODOCTS } \\
\text { MATURAL GAS } \\
\text { SYN-GAS }\end{array}$ & $\begin{array}{r}1.089 \\
-109.994 \\
-\end{array}$ & $\begin{array}{r}3.738 \\
-29.031 \\
-\end{array}$ & $\begin{array}{r}-216,17 \overline{-} \\
-\end{array}$ & $\begin{array}{r}-147,198 \\
-311 \\
-31\end{array}$ & $\begin{array}{r}476,357 \\
-\end{array}$ & $\begin{array}{r}12,83.4 \\
21.715 \\
3.57 \\
-\end{array}$ & $\begin{array}{r}114,682 \\
= \\
-\end{array}$ & $\begin{array}{r}-45.232 \\
= \\
=\end{array}$ & $\begin{array}{r}87,111 \\
3,671 \\
46 \\
-\end{array}$ & $\begin{array}{r}21 \% \\
1 \% \\
0 \%\end{array}$ \\
\hline $\begin{array}{l}\text { DET POEL OSBD } \\
\text { IN TRANSPOBSA TIOH }\end{array}$ & $-108,905$ & -17.293 & $-216,178$ & $-147,509$ & 476,357 & $34: 905$ & 114,632 & $-45,232$ & 90,828 & $22 \pi$ \\
\hline $\begin{array}{l}\text { TOTAL GBOSS PLORS } \\
\text { LOSSES } \& \text { OHIS SI ONS }\end{array}$ & $\begin{array}{r}21,160 \\
1.285\end{array}$ & $\begin{array}{r}28.735 \\
-291\end{array}$ & $\begin{array}{r}51,168 \\
-1.619\end{array}$ & $\begin{array}{l}3 C, 218 \\
-1.427\end{array}$ & $\begin{array}{r}476,357 \\
722\end{array}$ & 112,134 & $\begin{array}{r}186,740 \\
3,104\end{array}$ & $\begin{array}{r}34.996 \\
2.798\end{array}$ & $\begin{array}{r}401,564 \\
4,996\end{array}$ & $\begin{array}{r}998 \\
18\end{array}$ \\
\hline TOTAL NET DSAGB & -37.548 & 7,412 & $-166,629$ & $-118,718$ & 477.079 & 112,558 & 189,844 & $-7,4 \geqq 7$ & 406,560 & \\
\hline
\end{tabular}

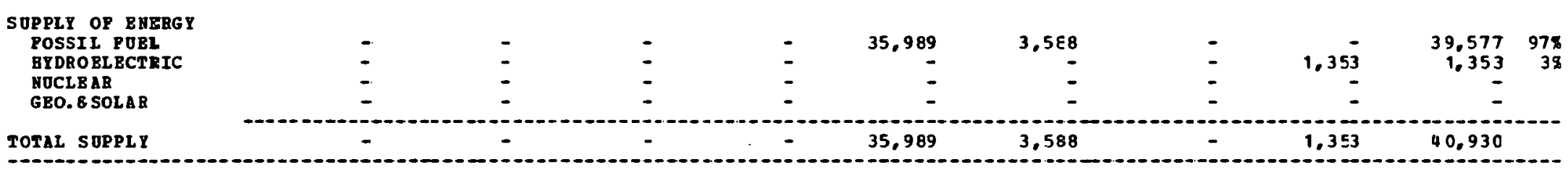

\begin{tabular}{l}
$\begin{array}{l}\text { MBT IHPORTS } \\
\text { OF REGION }\end{array}$ \\
\hline
\end{tabular}


REGIONAL BNERG BALAHCE STATEGENT

BI SECTOR AND POEL TYPE

\begin{tabular}{|c|c|c|c|c|c|c|c|c|c|c|}
\hline SECTOR & $\begin{array}{l}\text { DISTTILIATE } \\
\text { OIL }\end{array}$ & $\begin{array}{l}\text { RESI DDAL } \\
\text { C IL }\end{array}$ & $\begin{array}{l}\text { GASOLIHE } \\
\text { (ALL OHI? }\end{array}$ & $\begin{array}{c}\text { OTHBR } \\
\text { AYDBO- } \\
\text { CARBONS } \\
\text { TS IN 10**9 }\end{array}$ & $\begin{array}{c}\text { CRODE } \\
\text { OIL } \\
\text { BTO'S) }\end{array}$ & $\begin{array}{l}\text { MAT ORAL } \\
\text { GAS }\end{array}$ & COAL & BLECTRI CITY & $\begin{array}{l}\text { SECTCR } \\
\text { TOTAL }\end{array}$ & \\
\hline $\begin{array}{l}\text { PIHAL DEHAND SECTORS } \\
\text { RESIDE HTIAL, COEA. } \\
\text { INDOSTRIAL } \\
\text { TRAESPORTATION } \\
\text { GISCEL LAHEOOS USES }\end{array}$ & $\begin{array}{r}2,287 \\
19,883 \\
54,725 \\
2,708\end{array}$ & $\begin{array}{r}79 \\
8.514 \\
58.445 \\
1.970\end{array}$ & $\begin{array}{r}\overline{-} \\
133,289\end{array}$ & $\begin{array}{r}24.572 \\
38.207 \\
19.050 \\
1.442\end{array}$ & $\begin{array}{l}- \\
-\end{array}$ & $\begin{array}{r}85.832 \\
766.277 \\
49.518 \\
3.626\end{array}$ & $\begin{array}{r}1,170 \\
4,218 \\
46 \\
-\end{array}$ & $\begin{array}{r}40.163 \\
37.954 \\
40 \\
669\end{array}$ & $\begin{array}{r}154,103 \\
875,053 \\
315,113 \\
10,485\end{array}$ & $\begin{array}{r}10 \% \\
55 \% \\
20 \% \\
18\end{array}$ \\
\hline $\begin{array}{l}\text { TOTAL PIBAL } \\
\text { DEHA HD SBCTOBS }\end{array}$ & 79,603 & 69.008 & 133,289 & 83,271 & - & 905.253 & 5,434 & 78.826 & $1,354,684$ & $85 \%$ \\
\hline $\begin{array}{l}\text { TRANSPORHATION } \\
\text { BLECTRICITY GEH. } \\
\text { PETROLEOH PRODOCTS } \\
\text { MATORAL GAS } \\
\text { SYN-GAS }\end{array}$ & $\begin{array}{r}219 \\
-522.670 \\
-\end{array}$ & $\begin{array}{r}36,923 \\
-99,921 \\
=\end{array}$ & $\begin{array}{r}-1,029,591 \\
- \\
-\end{array}$ & $\begin{array}{r}-496.041 \\
-127.991 \\
-\end{array}$ & $\begin{array}{r}2.063 .311 \\
-\end{array}$ & $\begin{array}{r}252.828 \\
94.057 \\
147.102\end{array}$ & $\overline{-}$ & $\begin{array}{r}-97,925 \\
= \\
=\end{array}$ & $\begin{array}{r}192,045 \\
9.145 \\
19.111 \\
-\end{array}$ & $\begin{array}{r}128 \\
18 \\
18\end{array}$ \\
\hline $\begin{array}{l}\text { UET POEL OSED } \\
\text { IN TRANSPOREATIOH }\end{array}$ & $-522,451$ & $-62,998$ & -1.029 .591 & $-624,032$ & 2.063 .311 & 493.987 & - & $-97,925$ & 220.301 & $14 \%$ \\
\hline $\begin{array}{l}\text { TOTAL GROSS PLOMS } \\
\text { IOSSES } 8 \text { OUISSIOHS }\end{array}$ & $\begin{array}{r}79.822 \\
4.849\end{array}$ & $\begin{array}{r}105,931 \\
-1.074\end{array}$ & $\begin{array}{r}133.289 \\
-4.219\end{array}$ & $\begin{array}{l}83,271 \\
-3,934\end{array}$ & $\begin{array}{r}2,063,311 \\
3,128\end{array}$ & $\begin{array}{r}1,399,240 \\
5,298\end{array}$ & $\begin{array}{r}5.434 \\
90\end{array}$ & $\begin{array}{r}78,826 \\
6,304\end{array}$ & $\begin{array}{r}1,574,985 \\
10,441\end{array}$ & $\begin{array}{l}99 \% \\
18\end{array}$ \\
\hline
\end{tabular}

\section{SOPPLY OF ENERGY}

FOSSIL POEL

HYDROBLECTRIC

HOCLBAR

GBO. 8 SOLAR

$\begin{array}{rr}- & - \\ - & \vdots \\ - & -\end{array}$

TOTAL SOPPLY

$-$

-

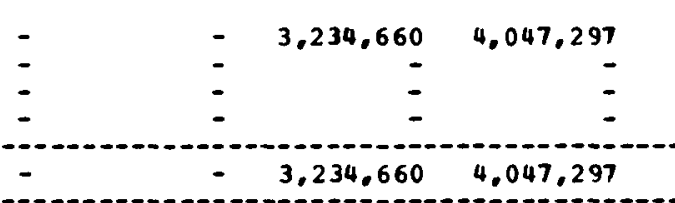

4.935

$-900,521$

$-544,695 \quad-1,168,220 \quad-2,642,758$

5,524

$-12.794-5,696,531$

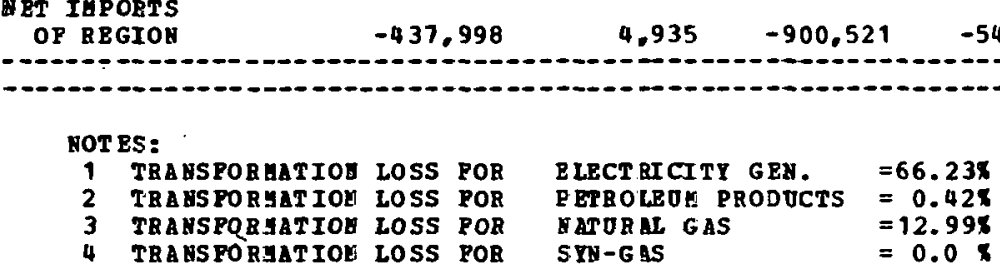


REGIONAL BNERGY BALANCE STATEMENT

BP SECTOR AND FUEL TYPE

\begin{tabular}{|c|c|c|c|c|c|c|c|c|c|c|}
\hline SECTOR & $\begin{array}{l}\text { DISTILLATE } \\
\text { OII }\end{array}$ & $\begin{array}{l}\text { RESI DOAL } \\
\text { OIL }\end{array}$ & $\begin{array}{l}\text { GASOITHE } \\
\quad \text { (AIL OHI }\end{array}$ & $\begin{array}{l}\text { OTHER } \\
\text { EYDBO- } \\
\text { CERBOHS } \\
\text { TS IN 10**9 }\end{array}$ & $\begin{array}{c}\text { CRODE } \\
\text { OIL } \\
\text { BTO०S) }\end{array}$ & $\begin{array}{l}\text { NAT JBAL } \\
\text { G AS }\end{array}$ & $\operatorname{COAL}$ & ELECT RI CITY & $\begin{array}{l}\text { SECTOR } \\
\text { TOTAL }\end{array}$ & \\
\hline $\begin{array}{l}\text { PIHAL DEGAND SECTORS } \\
\text { RESIDERTIAL, COHA. } \\
\text { IHDOSTRIAL } \\
\text { TRANSPORTATIOH } \\
\text { GISCELLAHEOS OSBS }\end{array}$ & $\begin{array}{r}508 \\
11.643 \\
9.159 \\
954\end{array}$ & $\begin{array}{r}12 \\
4.035 \\
6.122 \\
757\end{array}$ & $44 . \varepsilon 68$ & $\begin{array}{r}7.269 \\
10.156 \\
8.977 \\
484\end{array}$ & $\begin{array}{l}- \\
\overline{-} \\
-\end{array}$ & $\begin{array}{r}27.963 \\
543.792 \\
16,628 \\
3.227\end{array}$ & $\begin{array}{r}400 \\
834 \\
9 \\
-\end{array}$ & $\begin{array}{r}12.154 \\
15,484 \\
4 \\
632\end{array}$ & $\begin{array}{r}48,306 \\
585,944 \\
85,767 \\
6.054\end{array}$ & $\begin{array}{r}68 \\
728 \\
118 \\
1 \%\end{array}$ \\
\hline $\begin{array}{l}\text { TOTAL P IHAL } \\
\text { DBHA AD S BCTORS }\end{array}$ & 22,264 & 10,926 & 44,868 & 26,886 & - & 591,610 & 1,243 & 28.274 & 726,071 & 898 \\
\hline $\begin{array}{l}\text { TRAZSPORAATIOH } \\
\text { ELECT RICITY GEH. } \\
\text { PETROLEOE PRODOCTS } \\
\text { NATORAL GAS } \\
\text { SYH-GAS }\end{array}$ & $\begin{array}{r}94 \\
-172,530 \\
-\end{array}$ & $\begin{array}{r}11,448 \\
-32,983 \\
-\end{array}$ & $\begin{array}{r}-339,860 \\
-\end{array}$ & $\begin{array}{r}-162,531 \\
-179,920 \\
-\end{array}$ & 681,084 & $\begin{array}{r}65, .209 \\
31.048 \\
206.786 \\
-\end{array}$ & $\dot{-}$ & $\begin{array}{r}-25,083 \\
= \\
=\end{array}$ & $\begin{array}{r}51,668 \\
4,228 \\
26,866 \\
-\end{array}$ & $\begin{array}{l}6 \% \\
1 \% \\
3 \%\end{array}$ \\
\hline $\begin{array}{l}\text { HET POBL OSBD } \\
\text { IN TRANSEOBGATION }\end{array}$ & $-172,436$ & $-21,535$ & $-339,860$ & $-342,451$ & 681.084 & 303.043 & - & $-25,083$ & $82,76.2$ & $10 \%$ \\
\hline $\begin{array}{l}\text { TOTAL GROSS FLONS } \\
\text { LOSSES \& OUISSIOUS }\end{array}$ & $\begin{array}{r}22,358 \\
1,358\end{array}$ & $\begin{array}{r}22,374 \\
-227\end{array}$ & $\begin{array}{r}44,868 \\
-1 ., 420\end{array}$ & $\begin{array}{l}26,886 \\
-1,270\end{array}$ & $\begin{array}{r}681,084 \\
1,032\end{array}$ & $\begin{array}{r}894,653 \\
3,387\end{array}$ & $\begin{array}{r}1.243 \\
20\end{array}$ & $\begin{array}{r}28.274 \\
2.261\end{array}$ & $\begin{array}{r}808,833 \\
5,142\end{array}$ & $\begin{array}{r}998 \\
18\end{array}$ \\
\hline
\end{tabular}

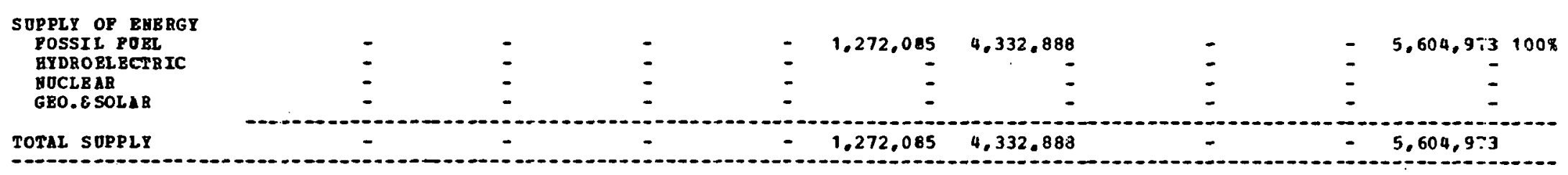

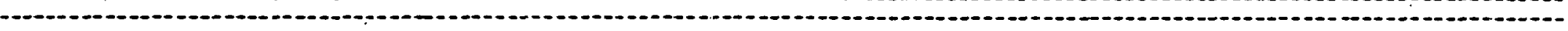

MET IMPORTS
OP REGION

$-148.813$

$-10.836$

$-296,412$

$-316,835$

$-589,968 \quad-3,434.847$

1,263

$5,452 \quad-4,790,997$

NOTBS:

1 TRANS PORAaTIOH LOSS POR

TRAHSPORAATIOA LOSS POR

TRASS PORAATIOA LOSS POO

BLECTBICIT: GEH.

ETRO $=67.326$

TRayspozmatioy cOSS POB

GATUR AL GAS

$=0.59$

S IN - G AS

$=0.0 \mathrm{~K}$ 
REgIONAL RHBRg BALARCE STATEHEHT

BY SECTOR AND POEL TYPE

\begin{tabular}{|c|c|c|c|c|c|c|c|c|c|c|}
\hline SECTOR & $\begin{array}{l}\text { DISTILLATE } \\
\text { OIL }\end{array}$ & $\begin{array}{l}\text { RBSIDOAL } \\
\text { OIL. }\end{array}$ & $\begin{array}{l}\text { GASOLINE } \\
\text { (ALL OHI }\end{array}$ & $\begin{array}{c}\text { OTABR } \\
\text { HYDBO- } \\
\text { CABBOHS } \\
\text { TS IB } 10 * * 9\end{array}$ & $\begin{array}{c}\text { CRODE } \\
\text { OIL } \\
\text { BTO'S) }\end{array}$ & $\begin{array}{l}\text { MAT ORAL } \\
\text { GAS }\end{array}$ & COAL & BLECT RI CITY & $\begin{array}{r}\text { SECTOR } \\
\text { TOTAL }\end{array}$ & \\
\hline $\begin{array}{l}\text { PIAAL DBAAHD SBCTORS } \\
\text { RBSIDBRTIAL, COBA. } \\
\text { IMDOSTRIAL } \\
\text { TRABSPORTATIOH } \\
\text { GISCELLAHEOOS OSBS }\end{array}$ & $\begin{array}{r}951 \\
6.715 \\
7.142 \\
253\end{array}$ & $\begin{array}{r}154 \\
7.511 \\
19.694 \\
255\end{array}$ & $\begin{array}{r}- \\
31.819 \\
-\end{array}$ & $\begin{array}{r}6.080 \\
51.375 \\
1.943 \\
168\end{array}$ & $\begin{array}{l}\overline{-} \\
\overline{-}\end{array}$ & $\begin{array}{r}11.916 \\
93.749 \\
3.602 \\
408\end{array}$ & $\begin{array}{r}212 \\
4.014 \\
8 \\
-\end{array}$ & $\begin{array}{r}7.124 \\
17.961 \\
2 \\
97\end{array}$ & $\begin{array}{r}26,437 \\
181.325 \\
64,210 \\
1,181\end{array}$ & $\begin{array}{r}88 \\
52 \% \\
18 \% \\
0 \%\end{array}$ \\
\hline $\begin{array}{l}\text { TOTAL PIBAL } \\
\text { DEAA SD SECTORS }\end{array}$ & 15,061 & 27,614 & 31,819 & 59.566 & - & 109,675 & 4,234 & 25.184 & 273,153 & $78 \%$ \\
\hline $\begin{array}{l}\text { TRANSPORMATIOH } \\
\text { BLECTRICITY GBH. } \\
\text { PETROLEOA PBODOCTS } \\
\text { MATORAL GAS } \\
\text { STY-CAE }\end{array}$ & $\begin{array}{r}4 \\
-551.975 \\
-\end{array}$ & $\begin{array}{r}573 \\
-127.178 \\
-\end{array}$ & $-1,115,476$ & $\begin{array}{r}-627.815 \\
-7.489\end{array}$ & 2.337 .855 & $\begin{array}{r}75.324 \\
106.573 \\
8.607\end{array}$ & $=$ & $\begin{array}{r}-24,180 \\
=\end{array}$ & $\begin{array}{r}51,721 \\
22,014 \\
1,118\end{array}$ & $\begin{array}{r}15 \% \\
6 \% \\
0 \%\end{array}$ \\
\hline $\begin{array}{l}\text { MET POBI OSBD } \\
\text { IE TRABSFORAATIOA }\end{array}$ & $-551,971$ & $-126,575$ & $-1,115,476$ & $-635,304$ & $2,337,855$ & 190,504 & - & $-24,180$ & 74,853 & $21 \%$ \\
\hline $\begin{array}{l}\text { TOTAL GROSS PLOUS } \\
\text { LOSSES \& OLISSIOUS }\end{array}$ & $\begin{array}{r}15,065 \\
915\end{array}$ & $\begin{array}{r}28.187 \\
-286\end{array}$ & $\begin{array}{r}31,819 \\
-1,007\end{array}$ & $\begin{array}{l}59,566 \\
-2,814\end{array}$ & $\begin{array}{r}2.337 .855 \\
3.544\end{array}$ & $\begin{array}{r}300,179 \\
1,136\end{array}$ & $\begin{array}{r}4.234 \\
70\end{array}$ & $\begin{array}{rl}25,184 \\
2,0 & 14\end{array}$ & $\begin{array}{r}348,006 \\
3.573\end{array}$ & $\begin{array}{r}998 \\
18\end{array}$ \\
\hline
\end{tabular}

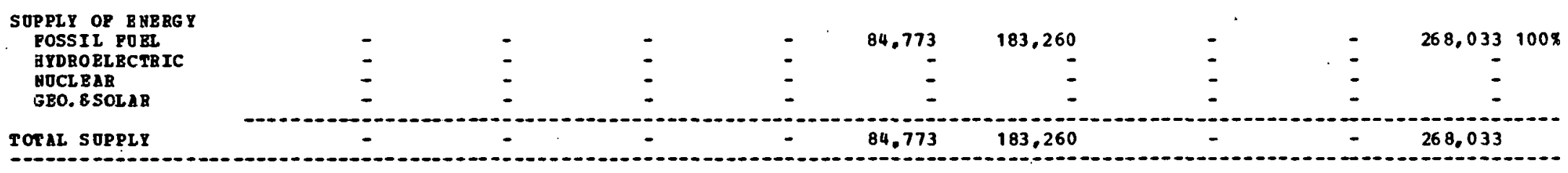

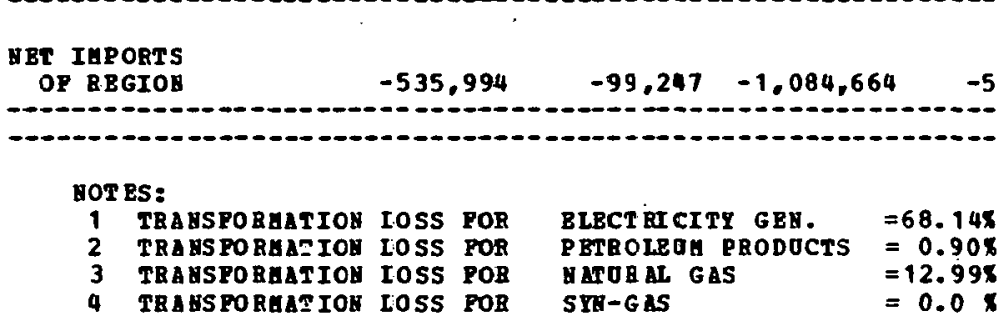


REGIONAL EE BRGI BALAMCE STATEMETT

BY SBCEOR AND POBL TYPE

\begin{tabular}{|c|c|c|c|c|c|c|c|c|c|c|}
\hline SBCT OR & $\begin{array}{c}\text { DISIIIIATE } \\
\text { OII }\end{array}$ & $\begin{array}{l}\text { RESI DOAL } \\
\text { OIL }\end{array}$ & $\begin{array}{l}\text { GASOLIAE } \\
\text { (ALL OHI }\end{array}$ & $\begin{array}{c}\text { OTHEB } \\
\text { BYDRO- } \\
\text { CLRBONS } \\
\text { TS IN 10**9 }\end{array}$ & $\begin{array}{c}\text { CRODE } \\
\text { OIL } \\
\text { BTO'S) }\end{array}$ & $\begin{array}{l}\text { MATORAL } \\
\text { GAS }\end{array}$ & SOAL & ELECTRICITY & $\begin{array}{r}\text { SECTOR } \\
\text { TOTAL }\end{array}$ & \\
\hline $\begin{array}{l}\text { PINAL DBHAND SECTORS } \\
\text { BESIDE ATIAL, COAL. } \\
\text { INDOST RIAL } \\
\text { TRANSPORTATI ON } \\
\text { OISCBLLAHEOUS OSBS }\end{array}$ & $\begin{array}{r}6, C 24 \\
18,-559 \\
57, \approx 04 \\
1, \in 02\end{array}$ & $\begin{array}{r}1.437 \\
11.741 \\
75.671 \\
1.618\end{array}$ & 201,521 & $\begin{array}{r}38,514 \\
273.495 \\
41.069 \\
1.066\end{array}$ & $\overline{-}$ & $\begin{array}{r}84.163 \\
758.363 \\
22,814 \\
2.730\end{array}$ & $\begin{array}{r}1,342 \\
24,614 \\
54 \\
-\end{array}$ & $\begin{array}{r}52.373 \\
61.518 \\
34 \\
646\end{array}$ & $\begin{array}{r}183,853 \\
1.147,990 \\
398,367 \\
7,662\end{array}$ & $\begin{array}{r}9 \% \\
56 \% \\
19 \% \\
0 \%\end{array}$ \\
\hline $\begin{array}{l}\text { TOTAL P IHAL } \\
\text { DEHA BD SBCrORS }\end{array}$ & 83,089 & 90.467 & $201,5 \approx 1$ & 354,144 & - & $868,07)$ & 25.010 & 114.571 & $1,737,872$ & $85 \%$ \\
\hline $\begin{array}{l}\text { TRANSPORHATIOA } \\
\text { ELECT RICITY GEH. } \\
\text { PETROLEOH PPODOCT S } \\
\text { SATORAL GAS } \\
\text { SYN-GAS }\end{array}$ & $\begin{array}{r}2 \\
-691, \in 49 \\
-\end{array}$ & $\begin{array}{r}3,578 \\
-159,323 \\
-\end{array}$ & -1.397 .740 & $\begin{array}{r}-789,086 \\
-145,722\end{array}$ & $\begin{array}{r}2.929,436 \\
=\end{array}$ & $\begin{array}{l}392,633 \\
133,543 \\
167,482\end{array}$ & E & $\begin{array}{r}-135,063 \\
= \\
-\end{array}$ & $\begin{array}{r}261,150 \\
25,178 \\
21,760\end{array}$ & $\begin{array}{r}13 \% \\
1 \% \\
1 \%\end{array}$ \\
\hline $\begin{array}{l}\text { HET FOEL OSBD } \\
\text { IN TRANSPOREATION }\end{array}$ & $-6.91,647$ & $-155,745$ & $-1,397,7<0$ & $-934,808$ & $2,929,436$ & 693,655 & - & $-135,063$ & 308,088 & $15 x$ \\
\hline $\begin{array}{l}\text { TOTAL GROSS FLOHS } \\
\text { LOSSES } \varepsilon \text { OUIS SIOHS }\end{array}$ & $\begin{array}{r}83,091 \\
5,047\end{array}$ & $\begin{array}{r}94.045 \\
-954\end{array}$ & $\begin{array}{r}201.521 \\
-6.379\end{array}$ & $\begin{array}{l}354,144 \\
-16,732\end{array}$ & $\begin{array}{r}2.929 .436 \\
4.441\end{array}$ & $\begin{array}{r}1,561,725 \\
5,913\end{array}$ & $\begin{array}{r}26.010 \\
432\end{array}$ & $\begin{array}{r}114,571 \\
9,162\end{array}$ & $\begin{array}{r}2,045,960 \\
931\end{array}$ & $\begin{array}{r}100 x \\
0 x\end{array}$ \\
\hline
\end{tabular}

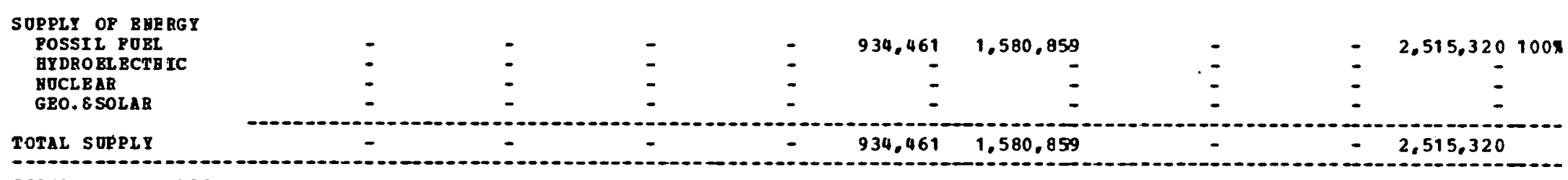

ABT IHPORTS

$-6103.510 \quad-66,232 \quad-1.202,598$

$-597,396 \quad 1,999,416$

$-13,220$

26,442

$-11,329$

$-468,428$

HOTES:
1 TRA PSPOREATION LCSS POR
TRANSTOREATIOH LCSS POR
TRAASFOREATIOA LCSS POB
BLBCTRICITY GEH.
(2) $=65.91 \mathrm{~m}$

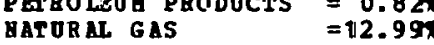
SYH-G AS
$=0.0 \times$ 
REGIOHAL BHERGY BALANCE STATEHENT

BY SECTOR AND FOBL TYPE

\begin{tabular}{|c|c|c|c|c|c|c|c|c|c|c|}
\hline SECTOR & $\begin{array}{l}\text { DISTILLATB } \\
\text { OIL }\end{array}$ & $\begin{array}{l}\text { RESI DOAL } \\
\text { OIZ }\end{array}$ & $\begin{array}{l}\text { GASOLINB } \\
\text { (ALL OAIT }\end{array}$ & $\begin{array}{c}\text { OTAER } \\
\text { MYDRO- } \\
\text { CABBOHS } \\
\text { TS IN 10**9 }\end{array}$ & $\begin{array}{c}\text { CRODE } \\
\text { OIL } \\
\text { BTO'S) }\end{array}$ & $\begin{array}{l}\text { MAT URAL } \\
\text { GAS }\end{array}$ & COAL & BLECTRICITY & $\begin{array}{l}\text { SBCTOR } \\
\text { TOTAL }\end{array}$ & \\
\hline $\begin{array}{l}\text { PIHAL D BEAHD SBCTOBS } \\
\text { RESIDEHTIAL, COHE. } \\
\text { IMDOSTRIAL } \\
\text { TRAYSPORTATIOH } \\
\text { EISCBL LAUBOOS OSBS }\end{array}$ & $\begin{array}{r}3,138 \\
3,462 \\
15,274 \\
835\end{array}$ & $\begin{array}{r}590 \\
1,199 \\
33 \\
1,117\end{array}$ & 104,984 & $\begin{array}{r}20.064 \\
74.949 \\
10.731 \\
555\end{array}$ & $\begin{array}{l}\overline{-} \\
\overline{-}\end{array}$ & $\begin{array}{r}40,858 \\
136,493 \\
11,885 \\
6,606\end{array}$ & $\begin{array}{r}699 \\
4.086 \\
12 \\
-\end{array}$ & $\begin{array}{r}24.788 \\
9.910 \\
24 \\
1.564\end{array}$ & $\begin{array}{r}90,137 \\
230,099 \\
142,943 \\
10,677\end{array}$ & $\begin{array}{r}178 \\
428 \\
268 \\
28\end{array}$ \\
\hline $\begin{array}{l}\text { TOTAL FIRAL } \\
\text { DEHA AD SBCTORS }\end{array}$ & 22.709 & 2.939 & 104,984 & 106.299 & - & 195.842 & 4.797 & $\cdot 36.286$ & 473.856 & $87 \%$ \\
\hline $\begin{array}{l}\text { TBAFSPORHATIOH } \\
\text { ELECTRICITY GEA. } \\
\text { PETROLEOH PRODOCTS } \\
\text { MATORAL GAS } \\
\text { SYH-GAS }\end{array}$ & $\begin{array}{r}21 \\
-2,537 \\
-\end{array}$ & $\begin{array}{r}8,522 \\
-584 \\
=\end{array}$ & $\begin{array}{r}-5.126 \\
-\end{array}$ & $\begin{array}{r}-2,902 \\
-25,887\end{array}$ & $\begin{array}{r}10.743 \\
-\end{array}$ & $\begin{array}{r}94,693 \\
490 \\
29,752 \\
-7\end{array}$ & $\overline{-}$ & $\begin{array}{r}-34,012 \\
= \\
=\end{array}$ & $\begin{array}{r}69,224 \\
84 \\
3,865 \\
-\end{array}$ & $\begin{array}{r}13 \% \\
08 \\
18\end{array}$ \\
\hline $\begin{array}{l}\text { XBT FOBL OSBD } \\
\text { IB TRABSFORAATION }\end{array}$ & -2.516 & 7.938 & $-5,126$ & $-28,789$ & 10.743 & 124,935 & - & $-34,012$ & 73,173 & 138 \\
\hline $\begin{array}{l}\text { TOTAL GROSS PLORS } \\
\text { LOSSES } 6 \text { OHISSIOHS }\end{array}$ & $\begin{array}{r}22,730 \\
1,380\end{array}$ & $\begin{array}{r}11, .661 \\
-\$ 16\end{array}$ & $\begin{array}{r}104,984 \\
-3,323\end{array}$ & $\begin{array}{r}106,299 \\
-5,022\end{array}$ & $\begin{array}{r}10,743 \\
16\end{array}$ & $\begin{array}{r}320,777 \\
1.214\end{array}$ & $\begin{array}{r}4.797 \\
79\end{array}$ & $\begin{array}{r}36.286 \\
2.901\end{array}$ & $\begin{array}{r}547.029 \\
-2.868\end{array}$ & $\begin{array}{r}1018 \\
0 \%\end{array}$ \\
\hline
\end{tabular}

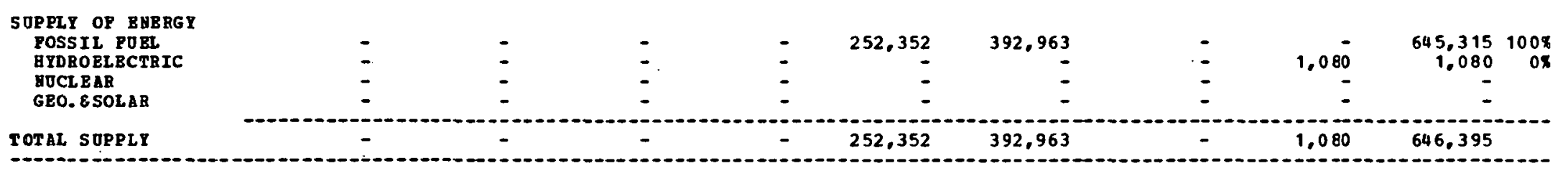

\section{BET IUPORTS}

OP REGION

21,573

10.760

96,534

72.487

$-241.592$

$-70,971$

4,876

4.095

$-102.234$

\section{MOTES:}

TRABS PORHATIOH LOSS POR

TRAASPORAATIOH LOSS POR

TRABS PORAATION LOSS POR

TRAYSPORHATION LOSS POR

BLECTRICITY GEH.

$=67.05 \%$

PEOLOH PRODUCTS $=0.75 \%$

$=0.75 \%$
$=12.99 \%$

$S Y N-G$ AS 
REGIONAL EMER 3 B BALAHCE STATEHEAT

BY SBCSOR AND POBL TYPE

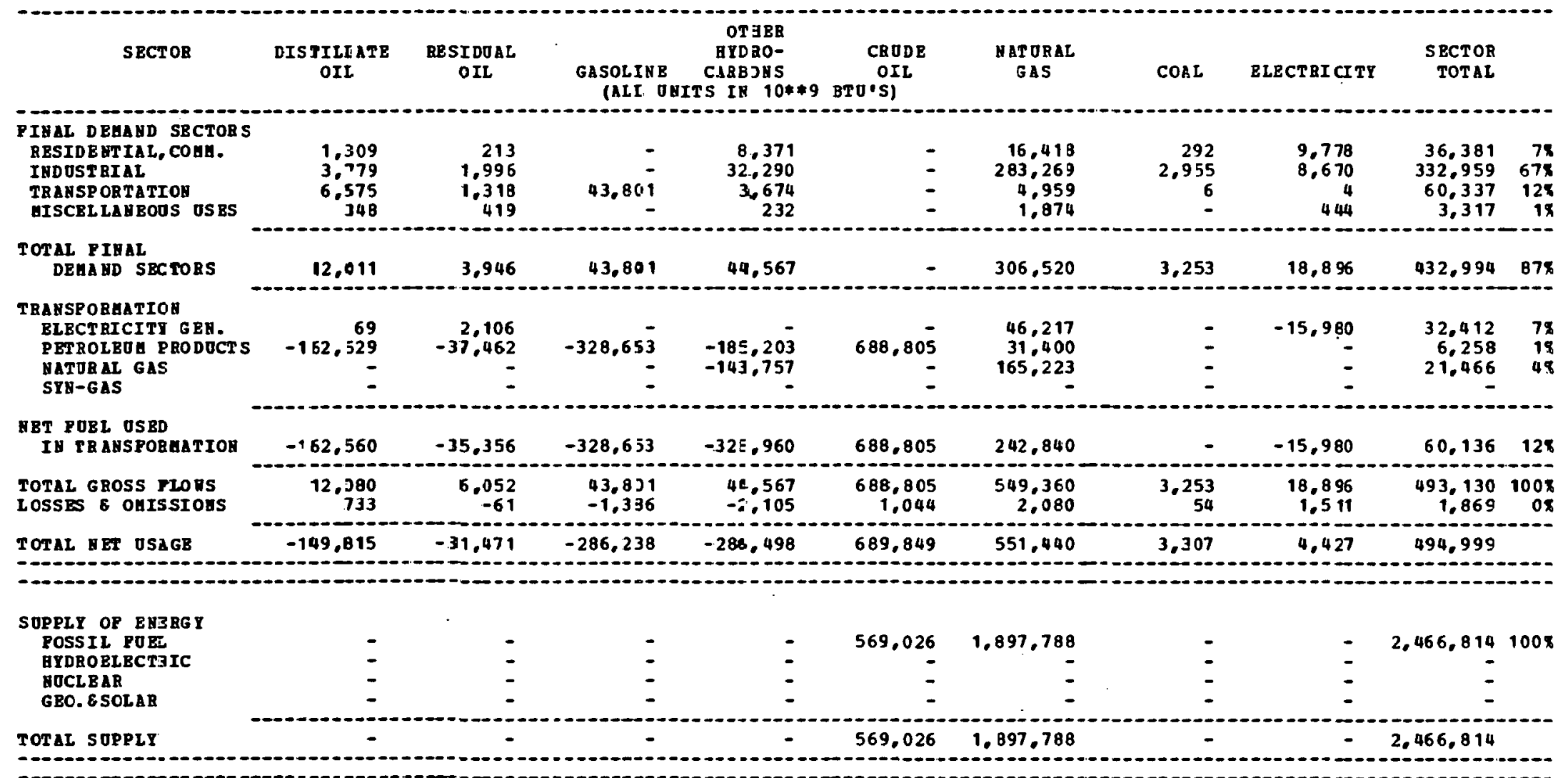

\section{BET IAPORTS}

$-149,815$

$-\$ 1,471$

$-286,238$

$-285,498$

$120,823 \quad-1,346,347$

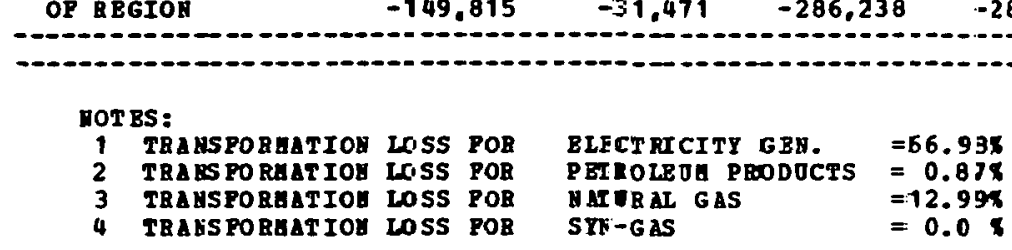


RGGIONAL BNBQG BALAHCE STA TEAE AT

BY SECTOR AND POBL TYPE

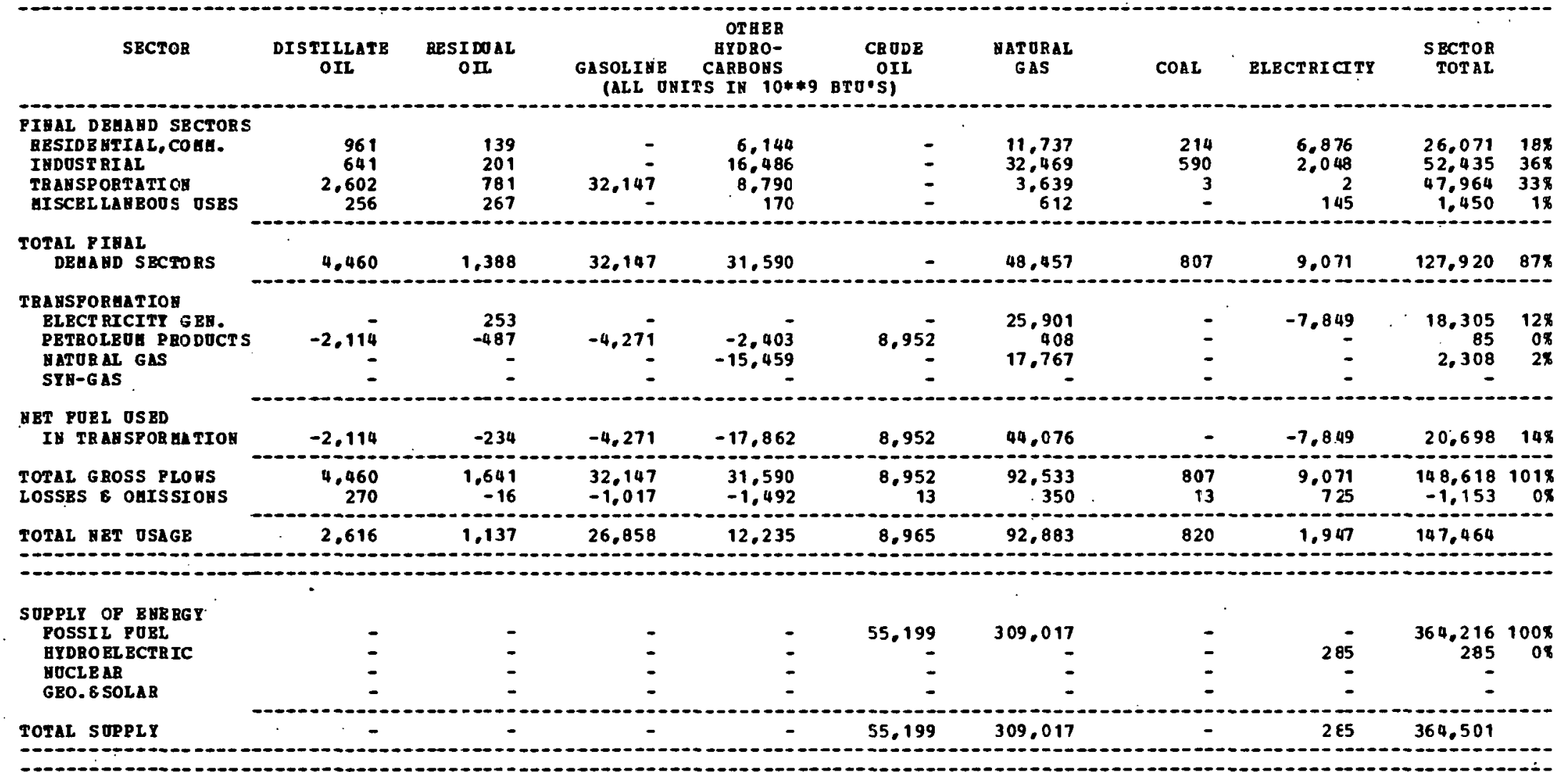

UET IHPORTS
OP RBGIOB


REGISNAL BLERGY BALANCE STATEHEHT

BY S3CTB AND POEL TYPE

\begin{tabular}{|c|c|c|c|c|c|c|c|c|c|c|}
\hline SECTOR & $\begin{array}{c}\text { IESTLLLATE } \\
\text { JIL }\end{array}$ & $\begin{array}{l}\text { RESIDOAL } \\
\text { OII }\end{array}$ & $\begin{array}{l}\text { GASOLINE } \\
\text { (ALL ONIT }\end{array}$ & $\begin{array}{l}\text { AYDRO- } \\
\text { CIRBOWS } \\
\text { TS IN } 10 * * 9\end{array}$ & $\begin{array}{c}\text { CRODE } \\
\text { OIL } \\
\text { BTO } S \text { S) }\end{array}$ & $\begin{array}{l}\text { MAT JRAL } \\
\text { GAS }\end{array}$ & COAL & ELECTRICITY & $\begin{array}{l}\text { SECTOR } \\
\text { TOTAL }\end{array}$ & \\
\hline $\begin{array}{l}\text { PIHAL DEGAMD SECTORS } \\
\text { RESIDE HTIAL, COBA. } \\
\text { INDOSTRIAL } \\
\text { TRANSPORTATIOY } \\
\text { GISCELLANBOOS OSES }\end{array}$ & $\begin{array}{r}1,941 \\
7,850 \\
13,869 \\
966\end{array}$ & $\begin{array}{r}240 \\
2.222 \\
17 \\
803\end{array}$ & $58,26 \overline{0}$ & $\begin{array}{r}11.968 \\
34.461 \\
5.186 \\
316\end{array}$ & $=$ & $\begin{array}{r}25,004 \\
89.233 \\
12.551 \\
3.673\end{array}$ & $\begin{array}{r}386 \\
2.691 \\
11 \\
-\end{array}$ & $\begin{array}{r}11,389 \\
5,848 \\
15 \\
741\end{array}$ & $\begin{array}{r}50,928 \\
142,305 \\
89,909 \\
6,499\end{array}$ & $\begin{array}{r}15 \% \\
428 \\
26 \% \\
28\end{array}$ \\
\hline $\begin{array}{l}\text { TOTAL PIBAL } \\
\text { DEHA SECTORS SECTO }\end{array}$ & 2.4 .626 & 3,282 & 58,260 & 51,931 & - & 130,461 & 3.088 & 17,993 & 289.641 & $35 x$ \\
\hline $\begin{array}{l}\text { TRABSPORBATIOA } \\
\text { ELECTRICITY GBN } \\
\text { PETROLEOA PRODOCTS } \\
\text { MATORAL GAS } \\
\text { SYH-GAS }\end{array}$ & $\begin{array}{r}-46.666 \\
- \\
-\end{array}$ & $\begin{array}{r}2,985 \\
-11,877 \\
=\end{array}$ & $\begin{array}{r}-97,625 \\
-\end{array}$ & $\begin{array}{r}-54,135 \\
-47,540 \\
-\end{array}$ & $\begin{array}{r}201.876 \\
-\end{array}$ & $\begin{array}{r}60,950 \\
9,203 \\
54,638 \\
-\end{array}$ & $\begin{array}{l}\overline{-} \\
\overline{-}\end{array}$ & $\begin{array}{r}-19,297 \\
= \\
-\end{array}$ & $\begin{array}{r}44.695 \\
776 \\
7.098 \\
-\end{array}$ & $\begin{array}{r}13 \% \\
0 \% \\
2 \%\end{array}$ \\
\hline $\begin{array}{l}\text { HBT POEL OSED } \\
\text { IH TRAESTORGATIOH }\end{array}$ & -46.609 & $-8,892$ & -97.625 & $-101,675$ & 201,876 & 124,791 & - & $-19,297$ & 52,569 & 158 \\
\hline $\begin{array}{l}\text { TOTAL GROSS PLONS } \\
\text { LOSSES } 8 \text { OHISSIOHS }\end{array}$ & $\begin{array}{r}.24 .683 \\
1.499\end{array}$ & $\begin{array}{r}6.267 \\
-63\end{array}$ & $\begin{array}{l}58,260 \\
-1,844\end{array}$ & $\begin{array}{l}51,931 \\
-2,453\end{array}$ & $\begin{array}{r}201,876 \\
306\end{array}$ & $\begin{array}{r}255,252 \\
966\end{array}$ & $\begin{array}{r}3,088 \\
51\end{array}$ & $\begin{array}{r}17.993 \\
1.4399\end{array}$ & $\begin{array}{r}342,210 \\
-99\end{array}$ & $\begin{array}{r}100 \% \\
0 \%\end{array}$ \\
\hline TAL AET USAGE & -20.483 & 年 & $-41,209$ & $-52,197$ & 202,182 & $256 ., 218$ & 3,139 & 135 & 342,110 & \\
\hline
\end{tabular}

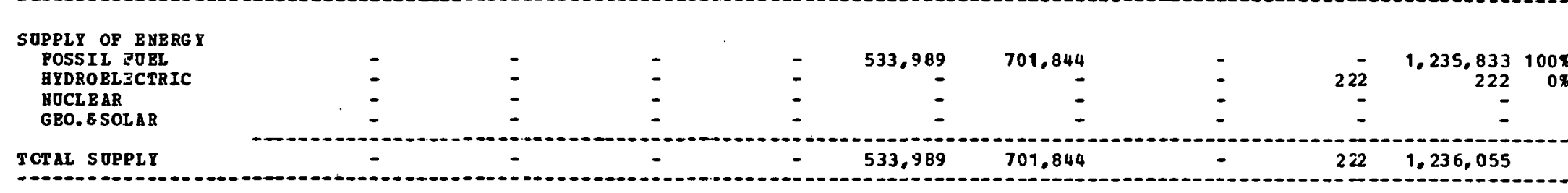

\begin{tabular}{l} 
UBT IUPORTS \\
OP REGIOH \\
\hline
\end{tabular}


REG IONAL BNERGY BALANCE STATEGENT

BI SBCTOR AHD FOEL TIPE

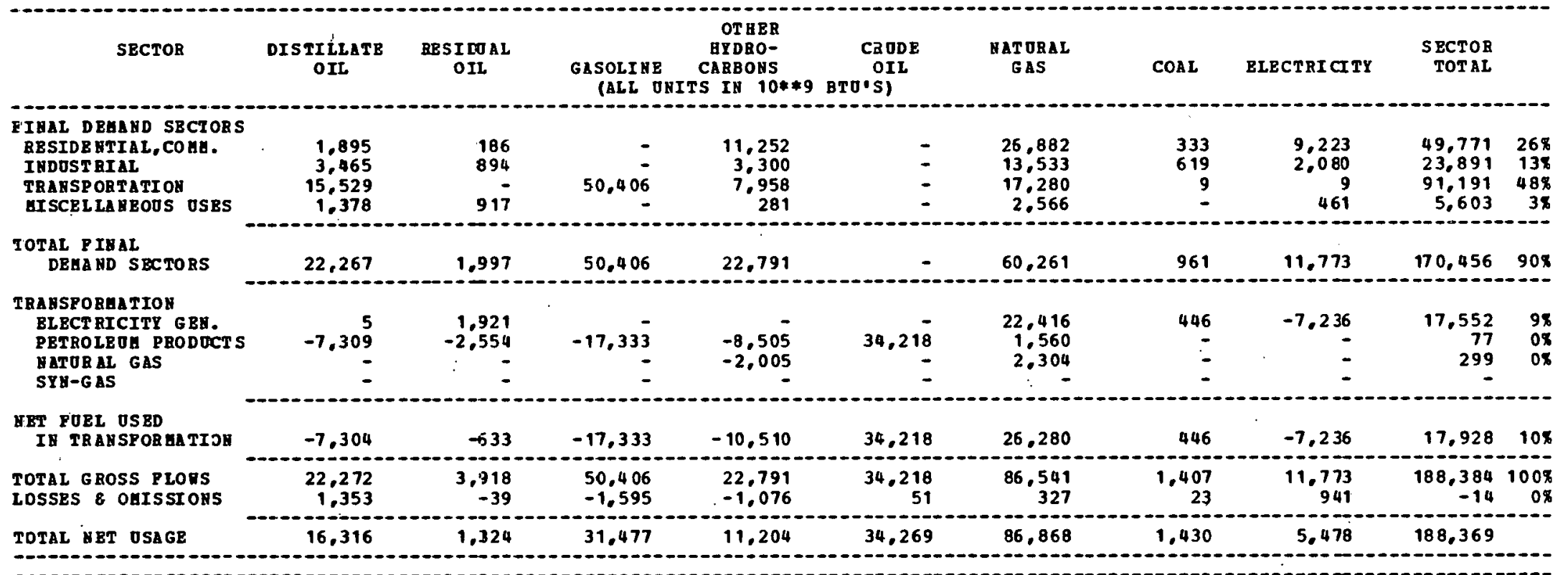

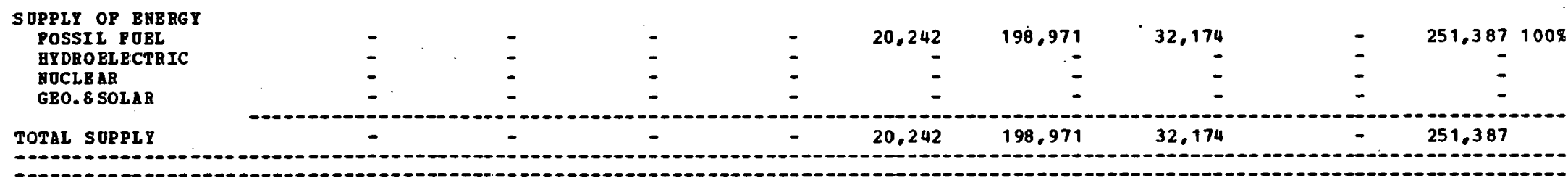

\section{BTT IHPOBTS}

16,316

$1, \$ 24$

31.477

11,204

$14.027 \quad-112,102$

$-30,743$

5,478

$-63,017$

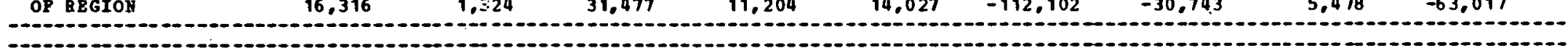

HOTES:

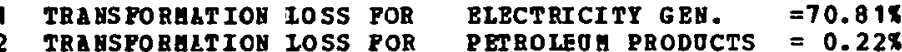

TRAES PORELT ION LOSS FOR

PETROLEOU PRODOCTS $=0.22 \%$

TRABSÉOBARTION ROSS POR

$\begin{array}{ll}\text { MATURAL GAS } & =12.98 \% \\ S Y Y-G \text { AS } & =0.08\end{array}$ 
REGIONAL ENEEG BALANCE STATEMENT

BY SECTOE AND POEL TYPE

\begin{tabular}{|c|c|c|c|c|c|c|c|c|c|c|}
\hline SECTOR & $\begin{array}{l}\text { DISTILIATE } \\
\text { OIL }\end{array}$ & $\begin{array}{l}\text { RESIDOAL } \\
\text { DIL }\end{array}$ & $\begin{array}{l}\text { GASOLZNE } \\
\text { GALE OHI }\end{array}$ & $\begin{array}{l}\text { HYLRO- } \\
\text { CARBOHS } \\
\text { TS IU 10**9 }\end{array}$ & $\begin{array}{c}\text { CRODE } \\
\text { OIL } \\
\text { BTO'SI }\end{array}$ & $\begin{array}{l}\text { MaTORAL } \\
\text { GAS }\end{array}$ & COAL & ELECTRICITY & $\begin{array}{l}\text { SECTOR } \\
\text { TOTAL }\end{array}$ & \\
\hline $\begin{array}{l}\text { PIHAL DEHAND SECTOBS } \\
\text { RESIDE NTIAL, COBH. } \\
\text { INDOSTRIAL } \\
\text { TRARSPORTATION } \\
\text { GISCELLANEOUS OSBS }\end{array}$ & $\begin{array}{r}2,236 \\
1,154 \\
3,447 \\
369\end{array}$ & $\begin{array}{r}503 \\
725 \\
56 \\
535\end{array}$ & 39.253 & $\begin{array}{r}9.590 \\
2.117 \\
2.522 \\
285\end{array}$ & - & $\begin{array}{r}31,938 \\
8.882 \\
5 \subseteq 9 \\
8,6 \equiv 0\end{array}$ & $\begin{array}{r}1,1147 \\
5,777 \\
5 \\
-\end{array}$ & $\begin{array}{r}7,150 \\
1,168 \\
11 \\
768\end{array}$ & $\begin{array}{l}52,564 \\
19,823 \\
45,893 \\
10,587\end{array}$ & $\begin{array}{r}358 \\
138 \\
308 \\
78\end{array}$ \\
\hline $\begin{array}{l}\text { TOTAL PINAL } \\
\text { DEHA ND SBCTOBS }\end{array}$ & 7.206 & 1.819 & 39.253 & 11,514 & - & $50,0<9$ & 6,529 & 9.097 & 128,867 & $85 \%$ \\
\hline $\begin{array}{l}\text { TRALSPOREATIOY } \\
\text { ELECTRICITI GBN. } \\
\text { PETROLEOH PRODOCTS } \\
\text { HATURAL GAS } \\
\text { SYH-GAS }\end{array}$ & $\begin{array}{r}252 \\
- \\
-\end{array}$ & $\begin{array}{r}1,507 \\
- \\
-\end{array}$ & - & : & $\begin{array}{l}- \\
-\end{array}$ & $\begin{array}{r}20.068 \\
= \\
=\end{array}$ & $\begin{array}{r}9,126 \\
- \\
-\end{array}$ & $\begin{array}{r}-7.703 \\
- \\
=\end{array}$ & $\begin{array}{r}23,250 \\
= \\
-\end{array}$ & $15 x$ \\
\hline $\begin{array}{l}\text { NET FOEI OSBD } \\
\text { IN TRAYSPORGATIOH }\end{array}$ & 252 & 1,507 & - & - & - & 20,068 & 9,126 & $-7,703$ & 23,250 & $15 \%$ \\
\hline $\begin{array}{l}\text { TOTAL GROSS FLONS } \\
\text { LOSSES \& OAISSIONS }\end{array}$ & $\begin{array}{r}7.458 \\
453\end{array}$ & $\begin{array}{r}3.326 \\
-33\end{array}$ & $\begin{array}{r}39,253 \\
-1,242\end{array}$ & $\begin{array}{r}.514 \\
-685\end{array}$ & 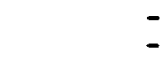 & $\begin{array}{r}70,117 \\
255\end{array}$ & $\begin{array}{r}16,055 \\
266\end{array}$ & $\begin{array}{r}9.097 \\
727\end{array}$ & $\begin{array}{r}152,117 \\
-249\end{array}$ & $\begin{array}{r}100 \% \\
0 \%\end{array}$ \\
\hline
\end{tabular}

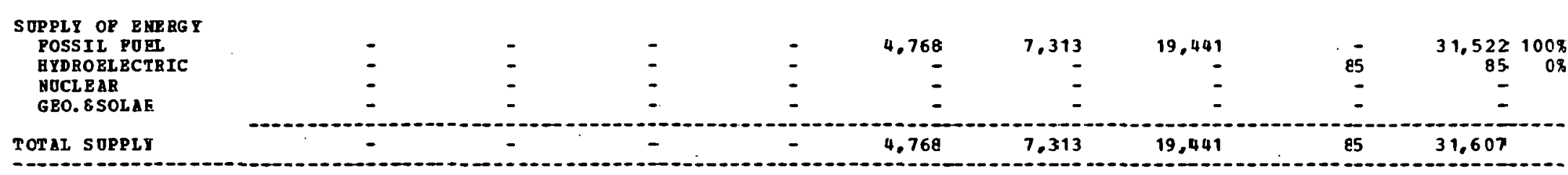

\section{MET IMPORTS \\ OF RBGIOH}

7.911

3.292

$38.91 \mathrm{C}$

13.828

$-4.768$

63,069

$-3,119$

2.036

$120.26 C$

NOT BS:

$$
\begin{aligned}
& \text { TRARSPORAATION LOSS FOR ELXCTRCITR GEN. }=75.11 \% \\
& \text { TR AYS PORATT IOA LOSS FOR PEIROLEUA PLODOCTS }=0 . C \\
& \text { TRA STORATION LJSS FOR MAIVRAL GAS }
\end{aligned}
$$




\begin{tabular}{|c|c|c|c|c|c|c|c|c|c|c|}
\hline & & & $\begin{array}{l}\text { REGIOHAL } \\
\text { BY SE }\end{array}$ & $\begin{array}{l}\text { EXBRGI BALE } \\
\text { CCTOB A\&D }\end{array}$ & $\begin{array}{l}\text { ANCE STATEL } \\
\text { FOEL TYPE }\end{array}$ & & & & & \\
\hline SECTOR & $\begin{array}{l}\text { DIS TILLATE } \\
\text { OIL }\end{array}$ & $\begin{array}{l}\text { aESIDAL } \\
\text { OII. }\end{array}$ & $\begin{array}{l}\text { GASOLI IE } \\
\text { (ALL OHIT }\end{array}$ & $\begin{array}{c}\text { OTBBA } \\
\text { RYDBO- } \\
\text { CARBOHS } \\
\text { IS IN } 10 * * 9\end{array}$ & $\begin{array}{c}\text { CRODE } \\
\text { OIL } \\
\text { BTOSI }\end{array}$ & $\begin{array}{l}\text { MAT ORAL } \\
\text { GAS }\end{array}$ & CoAL & ELECTRICITY & $\begin{array}{r}\text { SECTOR } \\
\text { TOTAL }\end{array}$ & \\
\hline $\begin{array}{l}\text { ZIBAL DEHAHD SBCTORS } \\
\text { RESIDBHTIAL, COHA. } \\
\text { IHDOSTRIAL } \\
\text { TRABSPORTATIOH } \\
\text { UISCELLAEOOS OSBS }\end{array}$ & $\begin{array}{r}6.770 \\
6,457 \\
18.244 \\
1.107\end{array}$ & $\begin{array}{r}2,221 \\
4,180 \\
319 \\
745\end{array}$ & 118,038 & $\begin{array}{r}28,822 \\
18,472 \\
40,424 \\
861\end{array}$ & 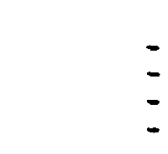 & $\begin{array}{r}110.568 \\
79.803 \\
1.884 \\
8.213\end{array}$ & $\begin{array}{r}3.433 \\
32.146 \\
30 \\
-\end{array}$ & $\begin{array}{r}26.094 \\
7.614 \\
23 \\
731\end{array}$ & $\begin{array}{r}177,908 \\
148,672 \\
178,962 \\
11,657\end{array}$ & $\begin{array}{r}30 \% \\
25 \% \\
30 \% \\
2 \%\end{array}$ \\
\hline $\begin{array}{l}\text { TOTAL PIBAL } \\
\text { DEBA BD SECTORS }\end{array}$ & 32,578 & 7.465 & 118,038 & 88,579 & $=$ & 200,468 & 35,609 & 34.462 & 517,199 & $87 \%$ \\
\hline $\begin{array}{l}\text { TRAYSPOREATIOZ } \\
\text { BLECTRICITY GBE. } \\
\text { PBTROLBOA PBODOCTS } \\
\text { HATOBAL GAS } \\
\text { SYH-G AS }\end{array}$ & $\begin{array}{r}387 \\
-21,022 \\
-\end{array}$ & $\begin{array}{r}2,421 \\
-5,331 \\
-\end{array}$ & $\begin{array}{r}-37,408- \\
-\end{array}$ & $\begin{array}{r}-21.606 \\
-1.827 \\
-\end{array}$ & 82.227 & $\begin{array}{r}36.187 \\
3,748 \\
2,100 \\
-\end{array}$ & $\begin{array}{r}64,940 \\
= \\
=\end{array}$ & $\begin{array}{r}-30,011 \\
= \\
=\end{array}$ & $\begin{array}{r}73,924 \\
608 \\
273 \\
-\end{array}$ & $\begin{array}{r}12 \pi \\
0 \pi \\
0 \pi\end{array}$ \\
\hline $\begin{array}{l}\text { HET FOBL OSBD } \\
\text { IH TBABSPORGA TIOH }\end{array}$ & $-20,635$ & $-2,910$ & $-37,408$ & $-23,433$ & 82,227 & 42.035 & 64,940 & $-30,011$ & 74,805 & 13\% \\
\hline $\begin{array}{l}\text { TOTAL GROSS PLONS } \\
\text { LOSSES \& OBISST OHS }\end{array}$ & $\begin{array}{r}32,965 \\
2,002\end{array}$ & $\begin{array}{r}9,886 \\
-100\end{array}$ & $\begin{array}{r}118,038 \\
-3,736\end{array}$ & $\begin{array}{l}88,579 \\
-4,185\end{array}$ & $\begin{array}{r}82.227 \\
124\end{array}$ & $\begin{array}{r}242.503 \\
918\end{array}$ & $\begin{array}{r}100,549 \\
1,671\end{array}$ & $\begin{array}{r}34.462 \\
2.756\end{array}$ & $\begin{array}{r}592.004 \\
-548\end{array}$ & $\begin{array}{r}1008 \\
0 \%\end{array}$ \\
\hline TOTAL \& BT DSAGB & 13,945 & 4.454 & 76,893 & 60,960 & 82,351 & 243.421 & 102,220 & 7,207 & 591,455 & \\
\hline $\begin{array}{l}\text { SOPPLY OP EAERGY } \\
\text { POSSIL POBL } \\
\text { GYDBOEL BCTRIC } \\
\text { HOCLE AR } \\
\text { GBO.\& SOLAB }\end{array}$ & $\overline{-}$ & $\begin{array}{l}\overline{-} \\
\overline{-}\end{array}$ & $\begin{array}{l}- \\
- \\
-\end{array}$ & $\begin{array}{l}- \\
-\end{array}$ & $\begin{array}{r}74.177 \\
- \\
-\end{array}$ & $\begin{array}{r}40.049 \\
- \\
-\end{array}$ & $\begin{array}{r}11,383 \\
- \\
-\end{array}$ & 2,148 & $\begin{array}{r}125,609 \\
2,148 \\
-\end{array}$ & $\begin{array}{r}98 x \\
2 x\end{array}$ \\
\hline TOTAL SUPPLY & $=$ & - & - & - & 74,177 & 40,049 & 11,383 & 2,148 & 127,757 & \\
\hline $\begin{array}{l}\text { HBT IHPORTS } \\
\text { OP REGION }\end{array}$ & 13,945 & 4.454 & 76,893 & 60,960 & 8,174 & 203,372 & 90.837 & $5,0 \leq 9$ & 463,698 & \\
\hline
\end{tabular}

HOTES:

TEAAS POBAAT IOA LOSS FOB TRABSPOBEATIOY LOSS POB

TBABSPOBAATIOY LOSS POB 
BBGIONAL ENE3GT BALAHCE STATEHENT

BE SECTOB AND POBL TYPE

\begin{tabular}{|c|c|c|c|c|c|c|c|c|c|}
\hline SECTOB & $\begin{array}{c}\text { DIS TILLATE } \\
\text { OIL }\end{array}$ & $\begin{array}{c}\text { RESIDJAL } \\
\text { OIL }\end{array}$ & $\begin{array}{l}\text { GASOLINE } \\
\text { (ALL C HI: }\end{array}$ & $\begin{array}{l}\text { HYORO- } \\
\text { CAR JOHS } \\
\text { IS I I 10**9 }\end{array}$ & $\begin{array}{c}\text { CRDDE } \\
\text { OIL } \\
\text { BTO'S) }\end{array}$ & $\begin{array}{l}\text { MAT ORAL } \\
\text { GAS }\end{array}$ & $\cos L$ & ELECTRICITY & $\begin{array}{r}\text { SECTOR } \\
\text { TOTAL }\end{array}$ \\
\hline 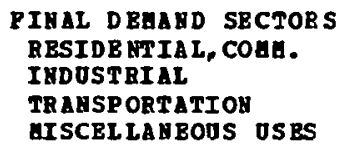 & $\begin{array}{r}1.133 \\
3.935 \\
2.476 \\
267\end{array}$ & $\begin{array}{r}256 \\
2.061 \\
27 \\
169\end{array}$ & 19.647 & $\begin{array}{r}7.584 \\
1.029 \\
609 \\
135\end{array}$ & - & $\begin{array}{r}15.071 \\
51.245 \\
1.812 \\
959\end{array}$ & $\begin{array}{r}512 \\
3,595 \\
3 \\
-\end{array}$ & $\begin{array}{r}3.660 \\
1.776 \\
- \\
101\end{array}$ & $\begin{array}{r}25,216 \\
63.641 \\
24,574 \\
1.631\end{array}$ \\
\hline $\begin{array}{l}\text { TOTAL PIHAL } \\
\text { DEMA AD SBCTOBS }\end{array}$ & 7.811 & 2,513 & 19,647 & 6,357 & - & 69.087 & 4,110 & 5,537 & 115,062 \\
\hline $\begin{array}{l}\text { TRAHSPORHATION } \\
\text { BLECTRICITI GER. } \\
\text { PETROLBOA PRODOCTS } \\
\text { HATORAL.GAS } \\
\text { SY!-GAS }\end{array}$ & $\begin{array}{r}154 \\
-4,203 \\
-\end{array}$ & $\begin{array}{r}18 \\
-1,469 \\
-\end{array}$ & $\begin{array}{r}-9.966 \\
-\end{array}$ & $\begin{array}{r}-4,813 \\
-46,913\end{array}$ & $\begin{array}{r}19.675 \\
-\end{array}$ & $\begin{array}{r}5.900 \\
.397 \\
53.979 \\
-\end{array}$ & $\begin{array}{r}154,591 \\
-\end{array}$ & $\begin{array}{r}-52,383 \\
= \\
-\end{array}$ & $\begin{array}{r}108,280 \\
121 \\
7.006\end{array}$ \\
\hline $\begin{array}{l}\text { MET FOBL OSBD } \\
\text { IH TRAESPOBHATIOY }\end{array}$ & $-4,049$ & $-1,451$ & $-9,966$ & $-51,726$ & 19.675 & 60.796 & 154,591 & $-52,383$ & 115.407 \\
\hline $\begin{array}{l}\text { TOTAL GROSS PLOMS } \\
\text { LOSSES } 8 \text { OIISSIOYS }\end{array}$ & $\begin{array}{r}7.965 \\
483\end{array}$ & $\begin{array}{r}2.531 \\
-25\end{array}$ & $\begin{array}{r}19.647 \\
-622\end{array}$ & $\begin{array}{r}5,357 \\
-300\end{array}$ & $\begin{array}{r}19.675 \\
29\end{array}$ & $\begin{array}{r}129.303 \\
491\end{array}$ & $\begin{array}{r}158,701 \\
2,638\end{array}$ & $\begin{array}{r}5.537 \\
442\end{array}$ & $\begin{array}{r}230,469 \\
3,138\end{array}$ \\
\hline
\end{tabular}

-

SOPPLY OP BHEBG

POSSIL POBL

GYDROBLBCTRIC

NOCL. BAB

GEO. ESOLAR

-
$\vdots$
-
-

$\overline{-}$

$-$

$-\quad 216.857$

$552.149 \quad 285.459$

$\begin{array}{rrr}- & 1,054,465 & 100 x \\ 2,702 & 2.702 & 08\end{array}$

TOTAI SOPPLY

285.459

$2.702 \quad 1.057,167$

\section{gET IHPORTS}

OP RBGIOR

245

1,036

9.5 .58

$-0.5,669$

$-197,152$.

$-421.854$

$-124,119$

$-49,105$

$-823,559$

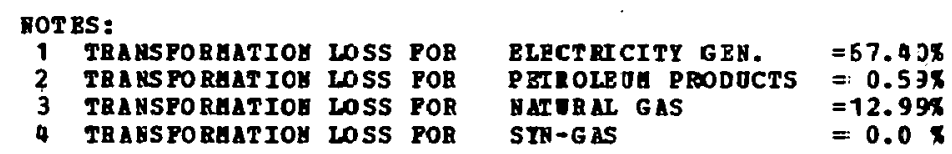

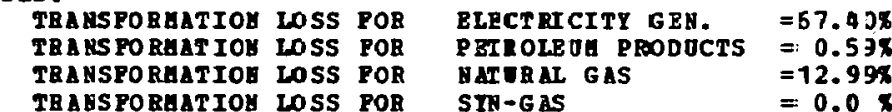


REGIONAL ENERGY BALAKCE STATBUEHT

BY SECTOR ARD TOEL TYPE

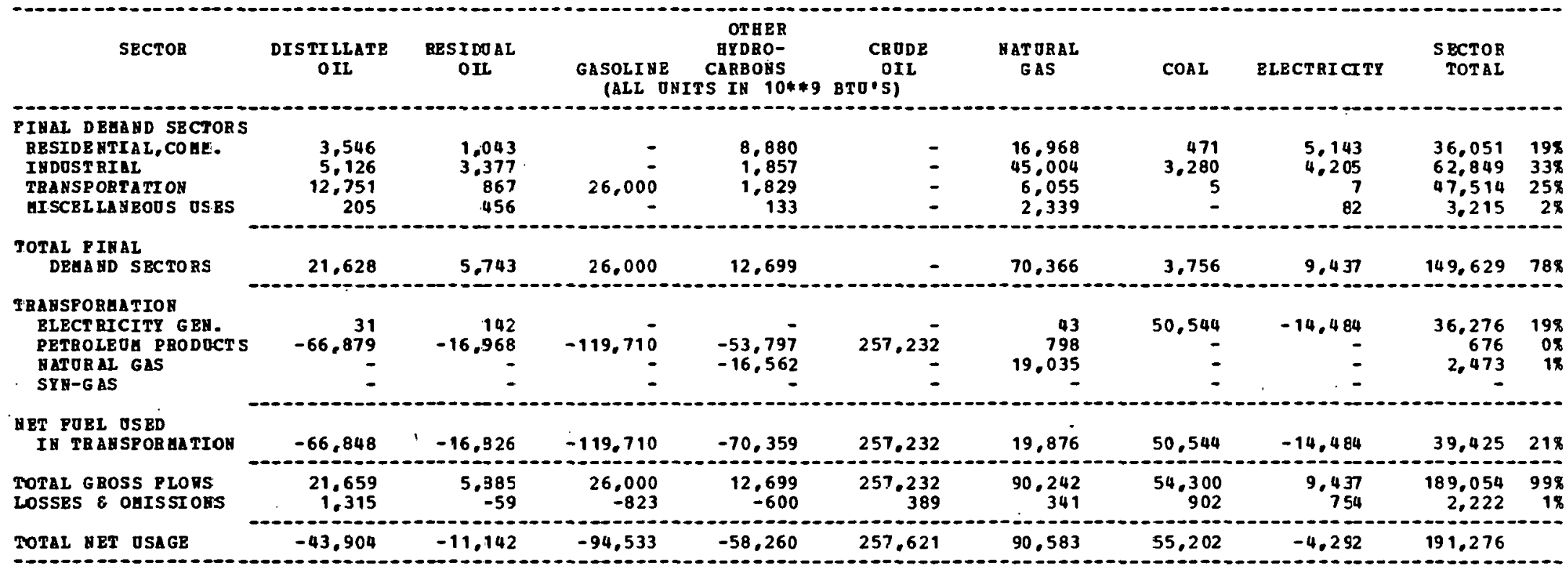

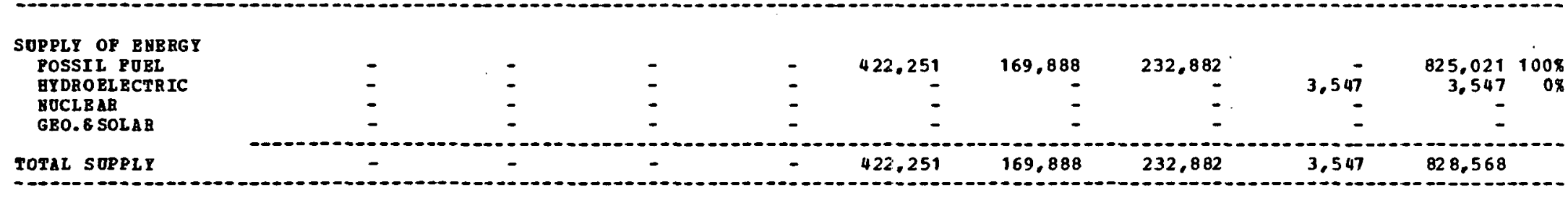

\section{HBT IAPORTS}

OP RBGIOE

$-43,904$

$-11,142$

$-94.533$

$-58,260$

$-164,629$

$-79,304$

$-127,679$

$-7,839$

$-637,291$

BOTBS:

1 TRA TRAASPOBALTIOA LOSS POR TRA

3 TRABS PORHATION LOSS POR

BLECTRICITY GBR. $=71.47 \%$

PETROLEUH PRODUCTS $=0.26 \%$

$\begin{array}{ll}\text { PETRONE G PBODUCTS } & =0.26 \% \\ \text { SATOR AL GAS } & =12.99 \%\end{array}$ 
RESIONAL ENERGY BALANCE STATEEENT

BY SECTOR AUD POEL TYPE

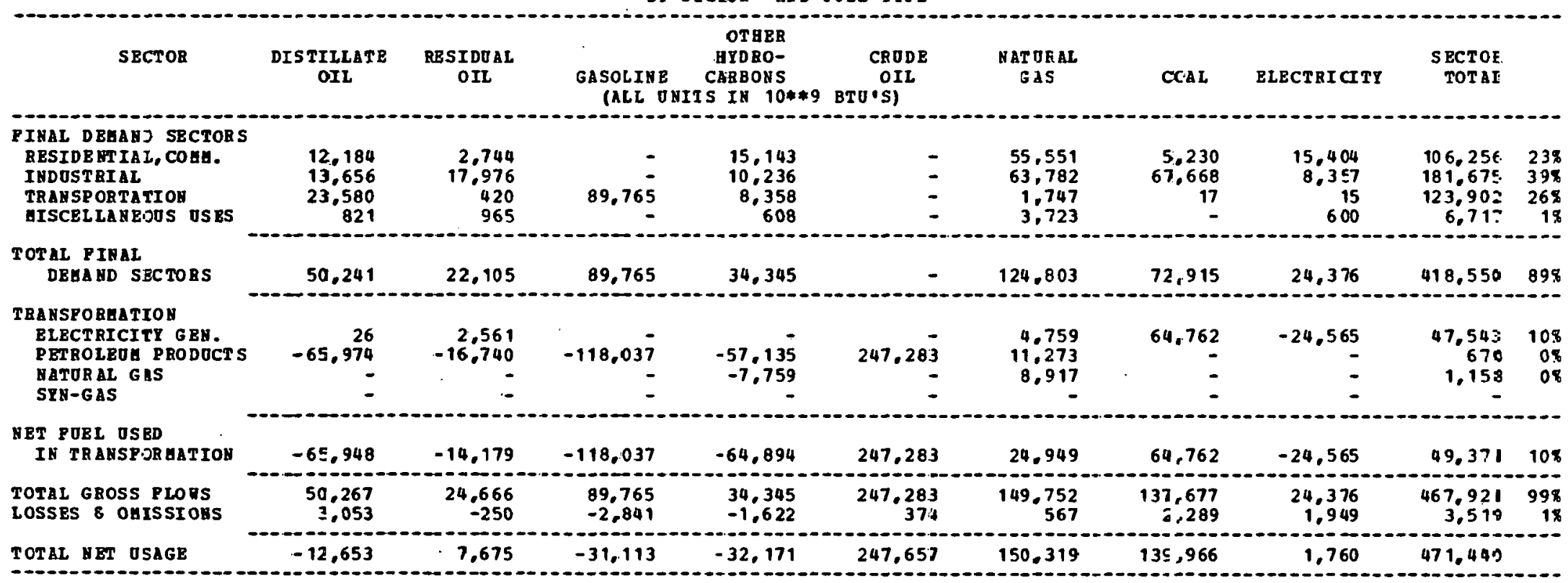

\section{SUPPLY OP BUERG Y}

POSSIL POEL

QYDROELECTRIC

GEO.\& SOLAB

$\begin{aligned}- & - \\ - & - \\ - & -\end{aligned}$

TOTAL SOPPLY

$-$

- $\quad 189.677$

186

\section{MET IUPORTS \\ OP RBGIOH}

$-12.653$

7.675

$-31.113$

$-32.171$

57,980

$-61,345$

$-73,871$

$-2.425$

$-147.925$

NOT $B S$ :

1 TRANS PORAATION LOSS POR

TRAMSPORATIOH LOSS POR

TRANSPOREATIOH COSE PO

ELECT RICITY GEQ. $=65.335$

$\begin{array}{ll}\text { GIUBAL GAS: } & =12.795\end{array}$

BIUBAI GAS

$=12.39$. 
RBG IONAL BHERGI BALABCE STATBAEHT

BY SECTOB AHD POBL TYPE

\begin{tabular}{|c|c|c|c|c|c|c|c|c|c|c|}
\hline SECTOB & $\begin{array}{l}\text { DISTILLATE } \\
\text { OIl }\end{array}$ & $\begin{array}{l}\text { RES I DO AL } \\
\text { OIt }\end{array}$ & $\begin{array}{r}\text { GASOLINB } \\
\text { (ALL O }\end{array}$ & $\begin{array}{l}\text { GYDRO- } \\
\text { CARBOYS } \\
\text { TS IB 10**9 }\end{array}$ & $\begin{array}{c}\text { CRODE } \\
\text { OIL } \\
\text { BTO'S) }\end{array}$ & $\begin{array}{l}\text { BATORAL } \\
\text { GAS }\end{array}$ & $\cos L$ & BLECTRICITY & $\begin{array}{r}\text { SECTOR } \\
\text { TOTAL }\end{array}$ & \\
\hline 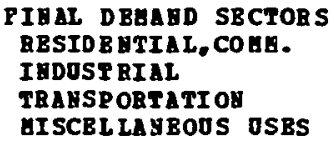 & $\begin{array}{r}6.814 \\
1.959 \\
4.962 \\
498\end{array}$ & $\begin{array}{r}393 \\
67 \\
9 \\
105\end{array}$ & 25,312 & $\begin{array}{r}5,379 \\
2,905 \\
900 \\
250\end{array}$ & - & $\begin{array}{r}8.028 \\
16.402 \\
2.070 \\
558\end{array}$ & $\begin{array}{r}176 \\
650 \\
4 \\
-\end{array}$ & $\begin{array}{r}8.940 \\
7.994 \\
3 \\
104\end{array}$ & $\begin{array}{r}29.730 \\
29.977 \\
33.260 \\
1.515\end{array}$ & $\begin{array}{r}31 \% \\
31 \% \\
35 \% \\
2 \%\end{array}$ \\
\hline $\begin{array}{l}\text { TOTAL PIYAL } \\
\text { DEHABD SBCTOBS }\end{array}$ & 14,233 & 574 & 25,312 & 9.434 & - & 27,058 & 830 & 17,041 & 94,482 & $99 x$ \\
\hline $\begin{array}{l}\text { TRAYSPORAATIOH } \\
\text { BLBCTRICITY GEH. } \\
\text { PETROLBOA PRODUCTS } \\
\text { MATORAL GAS } \\
\text { SYH-GAS }\end{array}$ & $\overline{-}$ & $\begin{array}{l}- \\
-\end{array}$ & $\overline{-}$ & $\begin{array}{l}\overline{-} \\
-\end{array}$ & 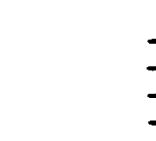 & : & $\overline{-}$ & $\begin{array}{l}- \\
-\end{array}$ & $\begin{array}{l}\overline{-} \\
\overline{-}\end{array}$ & \\
\hline $\begin{array}{l}\text { SBT FOEL OSBD } \\
\text { IB TRABSPOREATIOA }\end{array}$ & - & - & - & - & - & - & - & - & - & \\
\hline $\begin{array}{l}\text { TOTAL GROSS FLONS } \\
\text { LOSSES } 8 \text { OAISSI OHS }\end{array}$ & $\begin{array}{r}14.233 \\
864\end{array}$ & $\begin{array}{r}574 \\
-5\end{array}$ & $\begin{array}{r}25,312 \\
-801\end{array}$ & $\begin{array}{r}9.434 \\
-445\end{array}$ & 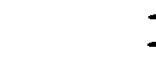 & $\begin{array}{r}27.058 \\
102\end{array}$ & $\begin{array}{r}830 \\
13\end{array}$ & $\begin{array}{r}17.041 \\
1.362\end{array}$ & $\begin{array}{r}94,482 \\
1,090\end{array}$ & $\begin{array}{r}99 x \\
1 \%\end{array}$ \\
\hline
\end{tabular}

\section{SOPPLY OP BABRGY \\ POSSIL POBL \\ GYDROELECTRIC \\ GOCLE AR}

TOTAL SUPPLI

$\begin{array}{rr}- & - \\ - & - \\ - & -\end{array}$

-
-
-
-

-
-
-

$\overline{-}$

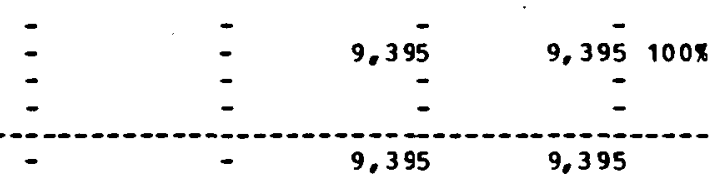

HET IHPORTS
OP REGIOH


REG IONAL ZNBEGP BALABCE STATEGENT

BI '5BCTOE ALD FOEL TYPE

\begin{tabular}{|c|c|c|c|c|c|c|c|c|c|c|}
\hline SECTOR & $\begin{array}{c}\text { DISTILLATE } \\
\text { OIL }\end{array}$ & $\begin{array}{l}\text { BES I DOAL } \\
\text { JIL }\end{array}$ & $\begin{array}{l}\text { GASOLIBE } \\
\text { (ALL O }\end{array}$ & $\begin{array}{l}\text { AYDRO- } \\
\text { CARBOHS } \\
\text { TS IH 10**9 }\end{array}$ & $\begin{array}{c}\text { CR DDE } \\
\text { OIL } \\
\text { BTO'S) }\end{array}$ & $\begin{array}{l}\text { NAT OEAL } \\
\text { GAS }\end{array}$ & $\operatorname{COAL}$ & ELECTRICITI & $\begin{array}{l}\text { SECTOR } \\
\text { TOTAL }\end{array}$ & \\
\hline 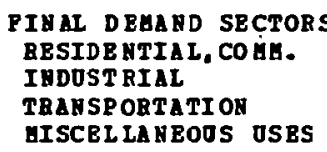 & $\begin{array}{r}2.638 \\
6.347 \\
6.437 \\
474\end{array}$ & $\begin{array}{r}290 \\
5,787 \\
86 \\
140\end{array}$ & $20.05 \overline{-}$ & $\begin{array}{r}4,972 \\
3,356 \\
780 \\
64\end{array}$ & $\overline{-}$ & $\begin{array}{r}13,002 \\
17.238 \\
575 \\
1.067\end{array}$ & $\begin{array}{r}1,6.50 \\
4.648 \\
4 \\
-\end{array}$ & $\begin{array}{r}3.623 \\
10.897 \\
86 \\
49\end{array}$ & $\begin{array}{r}26,175 \\
48.273 \\
28,025 \\
1.794\end{array}$ & $\begin{array}{r}25 \% \\
46 \% \\
27 \% \\
2 x\end{array}$ \\
\hline $\begin{array}{l}\text { TOTAL F IHAL } \\
\text { DEHA AD SBCTORS }\end{array}$ & 15,896 & 6.303 & 20.057 & 9,172 & - & 31,862 & 6.302 & 14,655 & 104,267 & $99 x$ \\
\hline $\begin{array}{l}\text { TRAUSFORGATIOH } \\
\text { BLECTRICITY GBH. } \\
\text { PETROLEOH PRODUCTS } \\
\text { GATORAL GAS } \\
\text { SYH-GAS }\end{array}$ & $\begin{array}{r}152 \\
- \\
-\end{array}$ & $\begin{array}{l}\overline{-} \\
-\end{array}$ & $\begin{array}{l}- \\
-\end{array}$ & $\begin{array}{l}- \\
-\end{array}$ & $\bar{z}$ & $\begin{array}{l}- \\
-\end{array}$ & $\begin{array}{l}- \\
-\end{array}$ & $\begin{array}{r}-38 \\
- \\
-\end{array}$ & $\begin{array}{r}114 \\
- \\
-\end{array}$ & $0 x$ \\
\hline $\begin{array}{l}\text { NET FOBL OSBJ } \\
\text { IE TRAHSPOBGA TIOS }\end{array}$ & 152 & - & - & - & - & - & - & -38 & 114 & $0 x$ \\
\hline $\begin{array}{l}\text { TOTAL G ROSS ?LORS } \\
\text { LOSSES } 8 \text { OUISSIOHS }\end{array}$ & $\begin{array}{r}16.048 \\
974\end{array}$ & $\begin{array}{r}6.303 \\
-63\end{array}$ & $\begin{array}{r}20.057 \\
-6.34\end{array}$ & $\begin{array}{r}9.172 \\
-433\end{array}$ & - & $\begin{array}{r}31.882 \\
120\end{array}$ & $\begin{aligned} 6.302 \\
104\end{aligned}$ & $\begin{array}{r}14,655 \\
1,172\end{array}$ & $\begin{array}{r}104,381 \\
1,240\end{array}$ & $\begin{array}{r}998 \\
18\end{array}$ \\
\hline
\end{tabular}

SOPPLY OP BAERG

POSSIL PUEC

HYDROBLBCTRIC

MOCL BAR

GBO. 8 SOL AR

TOTAL SUPPIY

-
$\vdots$
-
-

-
$\vdots$
-

-

$\begin{array}{ll}- & - \\ - & - \\ - & -\end{array}$

-

-
-
-

11,370

$11.370^{-} 99 x$

$15 \overline{1} 15 \overline{1} 18$

-

\section{HBT IAPORTS
OP RBGIOB}

17,022

6.239

19.422

8.738

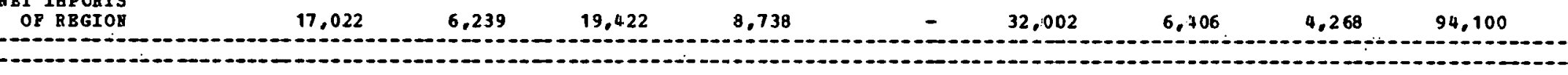

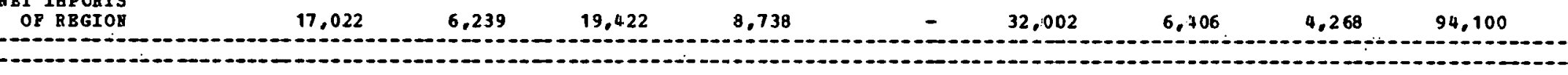

NOT BS:

\begin{tabular}{|c|c|c|c|c|}
\hline 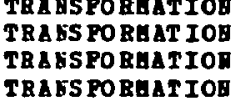 & $\begin{array}{l}\text { Loss } \\
\text { Loss } \\
\text { Loss } \\
\text { Loss }\end{array}$ & $\begin{array}{l}\text { POR } \\
\text { POR } \\
\text { POR } \\
\text { POR }\end{array}$ & $\begin{array}{l}\text { BLFCTRICITY GBN. } \\
\text { PENROLEOA PEODOCTS } \\
\text { YMTORAL GAS } \\
\text { STH-GAS }\end{array}$ & $\begin{array}{l}=7.00 x \\
=0.0 \\
=0.0 \\
=3.0\end{array}$ \\
\hline
\end{tabular}

TRABSPORAATIOR LOSS POR

STri-G AS 


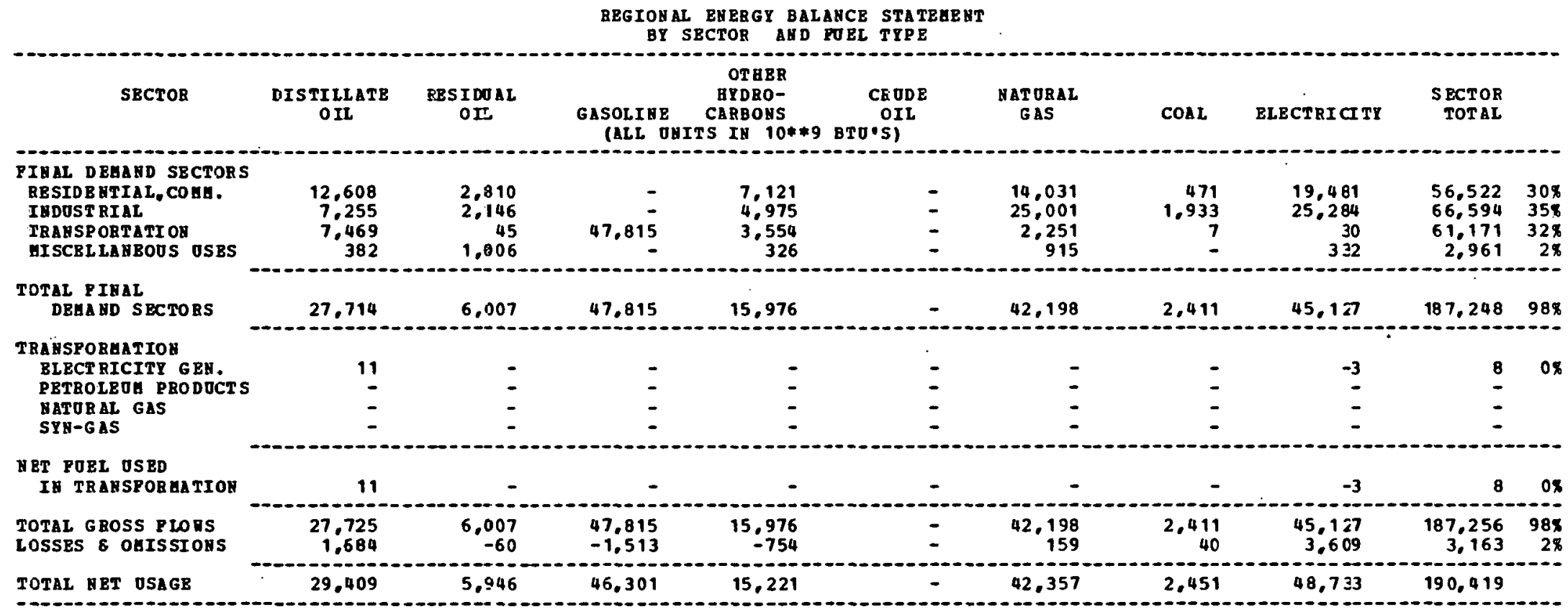

-

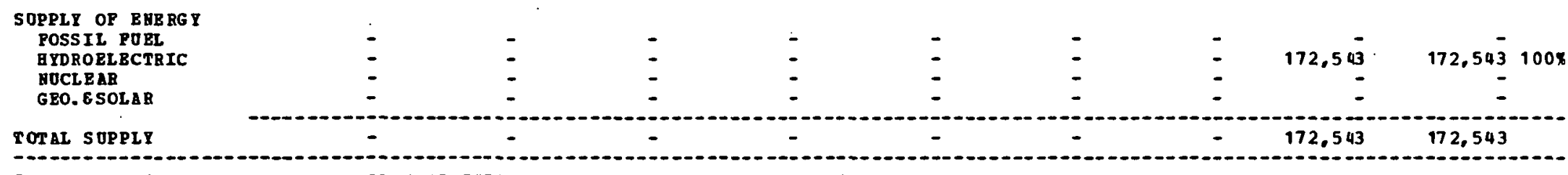

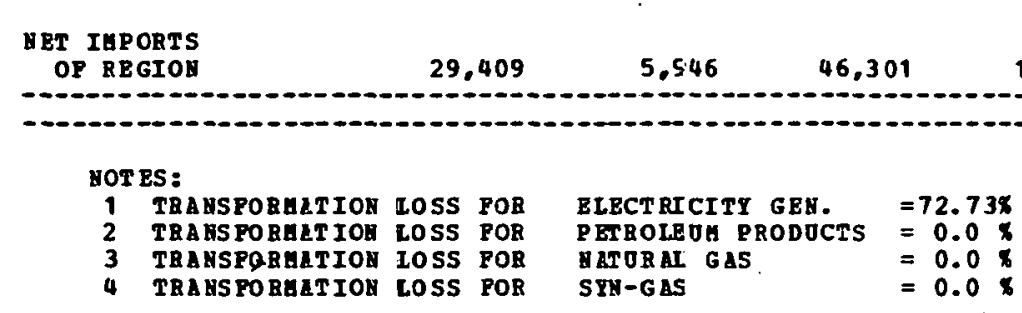




\begin{tabular}{|c|c|c|c|c|c|c|c|c|c|c|}
\hline & & & $\begin{array}{r}\text { REGIONA } \\
\text { BY }\end{array}$ & $\begin{array}{l}\text { BNERGI BAL } \\
\text { E ZTOR AND }\end{array}$ & $\begin{array}{l}\text { AKCE STAT } \\
\text { POEL TYPE }\end{array}$ & & & & & \\
\hline SECIOR & $\begin{array}{c}\text { DIS TILLATE } \\
\text { O=L }\end{array}$ & $\begin{array}{l}\text { BESI DOAL } \\
\text { O IL }\end{array}$ & $\begin{array}{l}\text { GASOLIEB } \\
\text { (ALL. OHI }\end{array}$ & $\begin{array}{c}\text { OTBER } \\
\text { HYDRO- } \\
\text { ZARBOHS } \\
\text { TS IN } 10 * * 9\end{array}$ & $\begin{array}{c}\text { CRODE } \\
\text { OIL } \\
\text { BTO'SI }\end{array}$ & $\begin{array}{l}\text { NATURAL } \\
\text { GAS }\end{array}$ & $\operatorname{COAL}$ & ELECTRICITY & $\begin{array}{l}\text { SECTOR } \\
\text { TOTAL }\end{array}$ & \\
\hline $\begin{array}{l}\text { PIHAL D BHAND SECTORS } \\
\text { RESIDE HTIAL, COUA. } \\
\text { IHDOST RIAL } \\
\text { TRANSPORTATI OU } \\
\text { GISCEL LAYEOTS USES }\end{array}$ & $\begin{array}{r}38.437 \\
12.213 \\
27.457 \\
476\end{array}$ & $\begin{array}{r}12,142 \\
24,355 \\
6,434 \\
4,962\end{array}$ & 147.740 & $\begin{array}{r}18,433 \\
20,832 \\
46,691 \\
819\end{array}$ & $\begin{array}{l}\overline{-} \\
\overline{-}\end{array}$ & $\begin{array}{r}45.475 \\
74.462 \\
5.120 \\
3.133\end{array}$ & $\begin{array}{r}1,637 \\
6,544 \\
36 \\
-\end{array}$ & $\begin{array}{r}66,838 \\
50,624 \\
103 \\
1,458\end{array}$ & $\begin{array}{r}182.962 \\
189.130 \\
233.581 \\
10,850\end{array}$ & $\begin{array}{l}3 \\
3\end{array}$ \\
\hline $\begin{array}{l}\text { TOTAL PIBAL } \\
\text { DEUA ND SECTORS }\end{array}$ & 78,585 & 47.893 & 147,740 & 86,775 & $=$ & 128,190 & 8,317 & 119.023 & 616,523 & \\
\hline $\begin{array}{l}\text { TRANSPOREATIOR } \\
\text { BLECT RICITI GER. } \\
\text { PETROLEOA PRODOCTS } \\
\text { BATORAL GAS } \\
\text { SYB-GAS }\end{array}$ & $\begin{array}{r}164 \\
-714,147 \\
-\end{array}$ & $\begin{array}{r}2.841 \\
-159.589 \\
- \\
-\end{array}$ & $\begin{array}{r}-335,237 \\
-\end{array}$ & -122.948 & 649.055 & 84.218 & $\begin{array}{r}83,664 \\
- \\
-\end{array}$ & $\begin{array}{r}-12,012 \\
- \\
-\end{array}$ & $\begin{array}{r}79.657 \\
1.352 \\
-\end{array}$ & \\
\hline $\begin{array}{l}\text { NET PUBL USED } \\
\text { IN TRAHSECREATION }\end{array}$ & -113.983 & $-156,748$ & $-335,237$ & $-122,948$ & 649,055 & 84.218 & 83,664 & $-12,012$ & 76.009 & \\
\hline $\begin{array}{l}\text { TOTAL GROSS PLONS } \\
\text { LOSSES \& OAISST OHS }\end{array}$ & $\begin{array}{r}78.749 \\
4.783\end{array}$ & $\begin{array}{r}50.734 \\
-514\end{array}$ & $\begin{array}{r}147,740 \\
-4,677\end{array}$ & $\begin{array}{l}86,775 \\
-4,099\end{array}$ & $\begin{array}{r}649.055 \\
984\end{array}$ & $\begin{array}{r}212.408 \\
804\end{array}$ & $\begin{array}{r}91,981 \\
1,529\end{array}$ & $\begin{array}{r}119,023 \\
9,518\end{array}$ & $\begin{array}{r}692,532 \\
8,328\end{array}$ & \\
\hline TOTAL HET OSAGB & -30.614 & $-109,369$ & $-192,174$ & -40.272 & 650,039 & 213.212 & 93,510 & 116,529 & 700,860 & \\
\hline
\end{tabular}

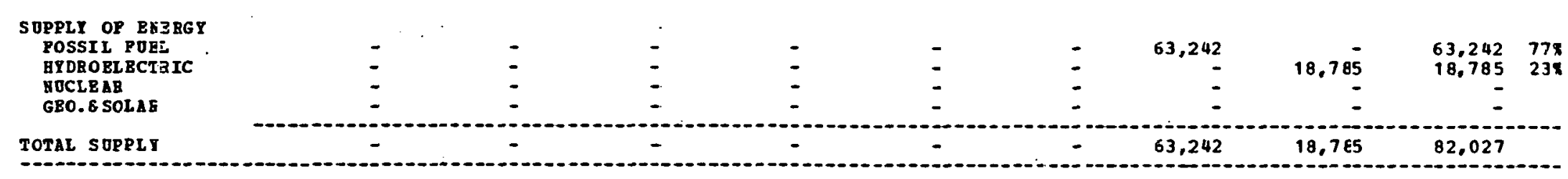

\begin{tabular}{|c|c|c|c|c|c|c|}
\hline \multicolumn{2}{|c|}{$\begin{array}{l}\text { YET IHPOBTS } \\
\text { OP REGIOH }\end{array}$} & -30 & .614 & $-109,369$ & \multicolumn{2}{|c|}{$-192,174$} \\
\hline Ho: & ES: & & & & & \\
\hline $\begin{array}{l}1 \\
2 \\
3 \\
4\end{array}$ & 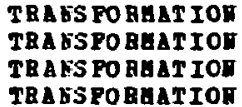 & $\begin{array}{l}\text { Loss } \\
\text { ross } \\
\text { Loss } \\
\text { Loss }\end{array}$ & $\begin{array}{l}\text { POR } \\
\text { POR } \\
\text { POR } \\
\text { POR }\end{array}$ & $\begin{array}{l}\text { BLECTRICIT } \\
\text { PERBOLEOH } \\
\text { MATORAL GL } \\
\text { SYE-G IS }\end{array}$ & $\begin{array}{l}\text { GEH. } \\
\text { SODOCTS }\end{array}$ & $\begin{array}{l}=86.148 \\
=0.188 \\
=0.0 \% \\
=0.08\end{array}$ \\
\hline
\end{tabular}




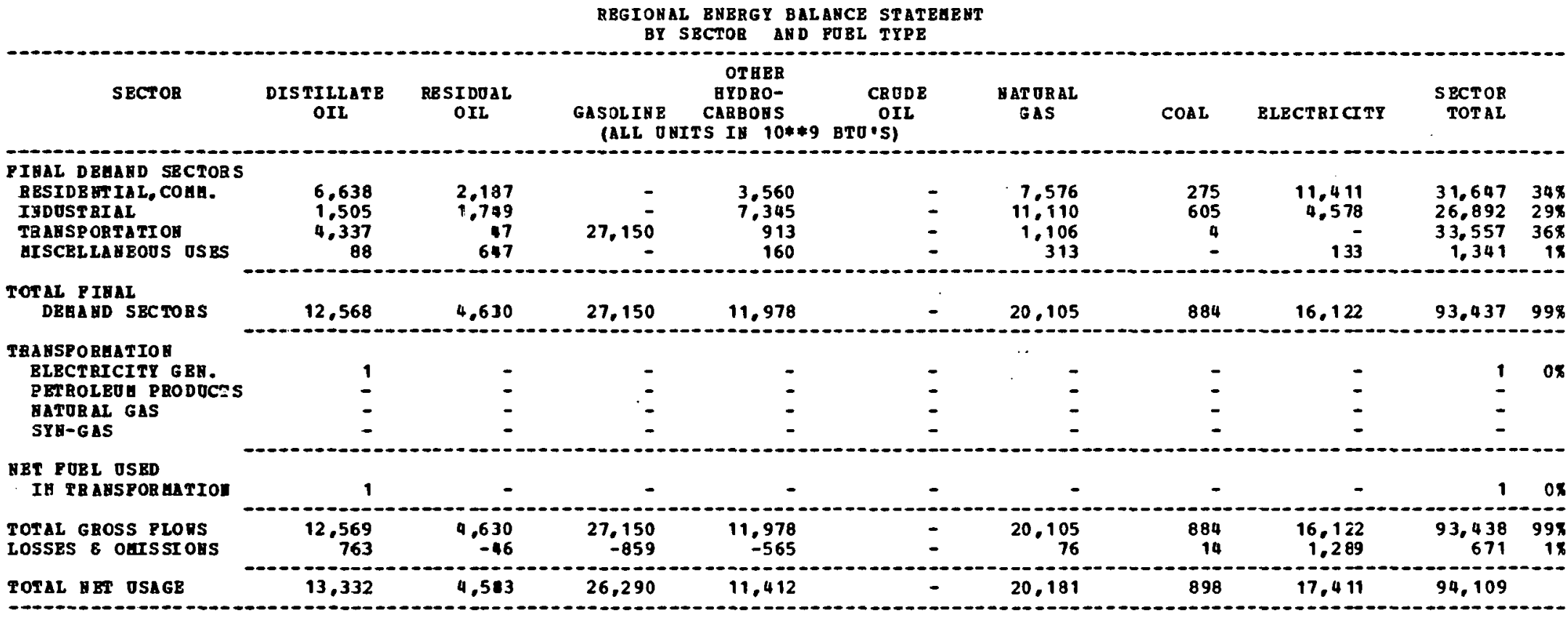

\begin{tabular}{|c|c|c|c|c|c|c|c|c|c|c|}
\hline $\begin{array}{l}\text { SOPPLY OF B BEBGI } \\
\text { POSSIL POBL }\end{array}$ & - & - & - & - & - & - & - & - & - & \\
\hline HYDROBLECTRIC & - & - & - & - & - & - & - & 46,873 & 46,873 & $78 \%$ \\
\hline DOCLB AB & - & - & - & - & - & - & - & 13,319 & 13,319 & $22 \pi$ \\
\hline GBO, E SOLAR & - & - & - & - & - & - & - & - & - & \\
\hline
\end{tabular}

HET IHPORTS
OP RBGIOR


REGIONLL BEERGT BALANCE STATEGEHT BY SBCTOR AND FOEL TYPE

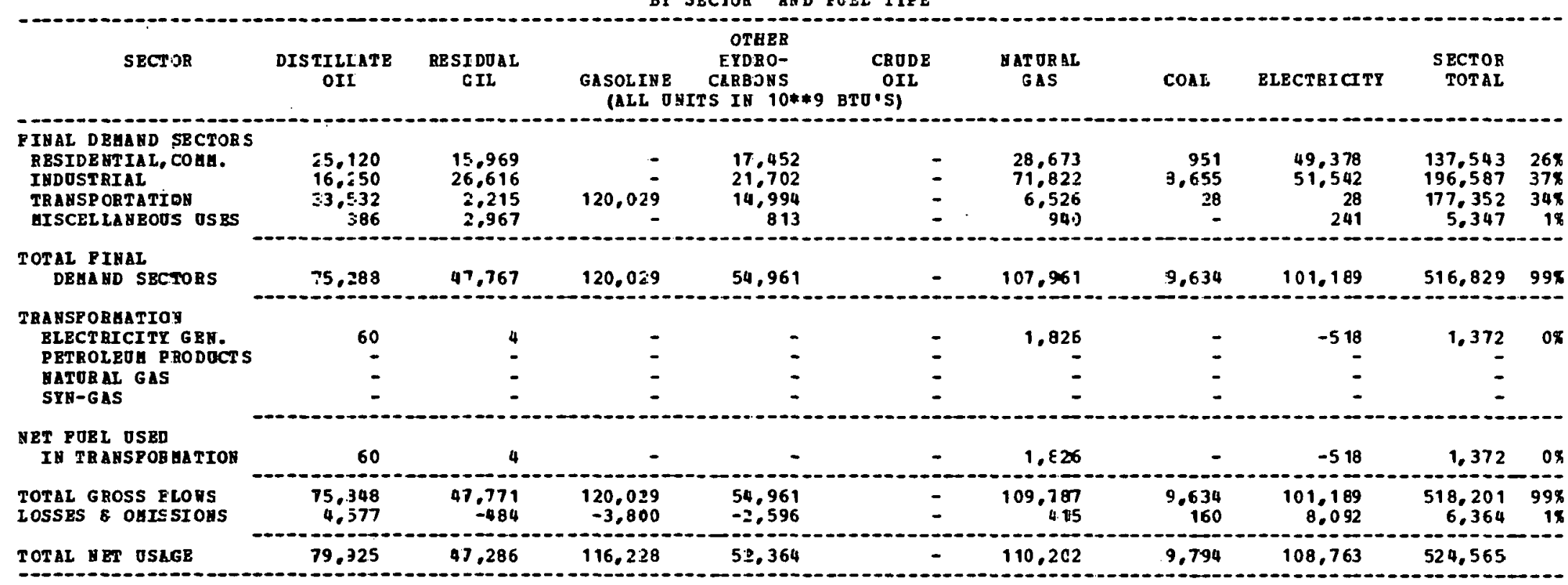

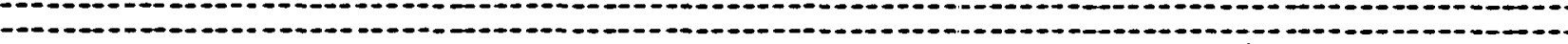

\section{SOPPLY OP BHERGY \\ POSSIL FOBI \\ HYDBOBLECTEIC \\ GOCLEAR \\ GBO. 6 SOLAB}

$\begin{aligned}- & - \\ - & - \\ - & -\end{aligned}$

-

:

-
-
-
-

$79 ; 925$

\subsection{6}

116.228

52,364

$-\quad 110,202$

9,794

25,427

441,229

OR REGIN

\section{NoTBS:}

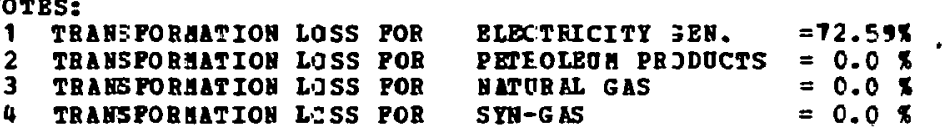


REGIONAL BHEBGI BALARCE STATBHENT

BY SECTOZ AND POBL TYPE

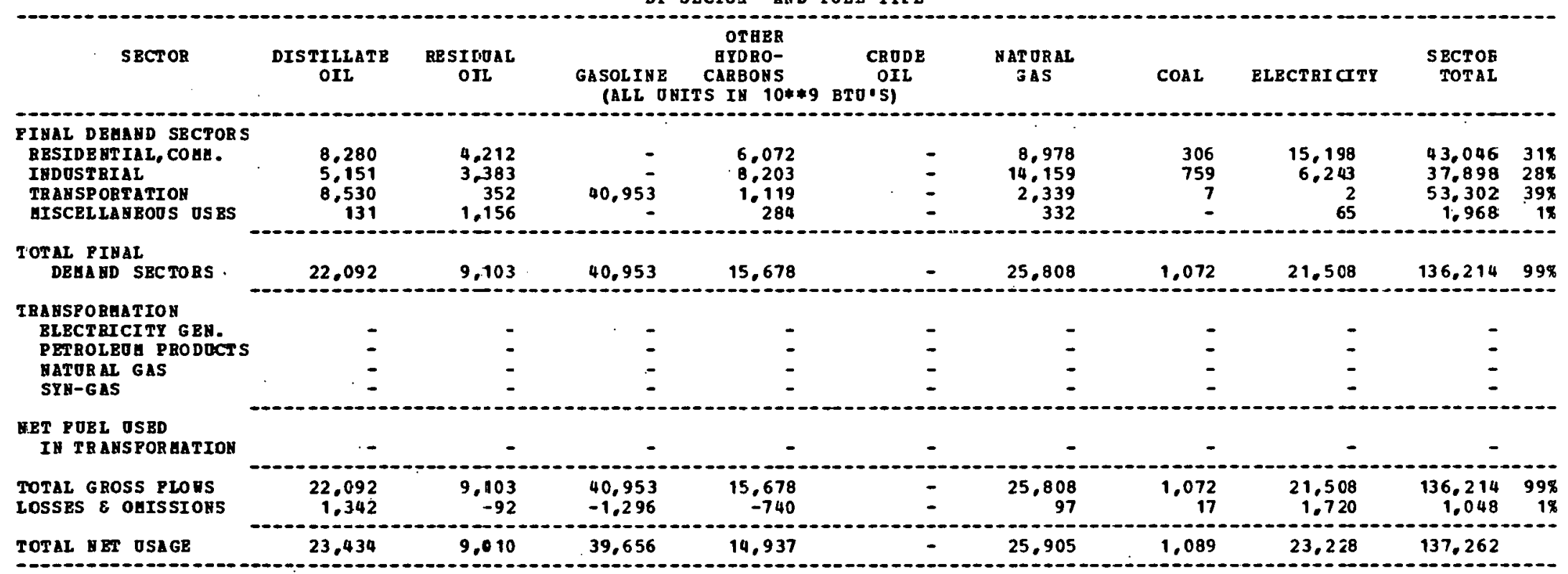

\section{SOPPLY OP BHBEY}

POSSIL PUBL

GYDBOBLBCTRIC

gUCL BAR

GBO. ESOLAB

\begin{tabular}{|c|c|}
\hline - & - \\
\hline & - \\
\hline- & - \\
\hline
\end{tabular}

TOTAL SOPPLY

VBT IIAPORTS

OP REGIOH

23.434

9.010

39.656

14,937

25,905

1.089

14.556

128,590

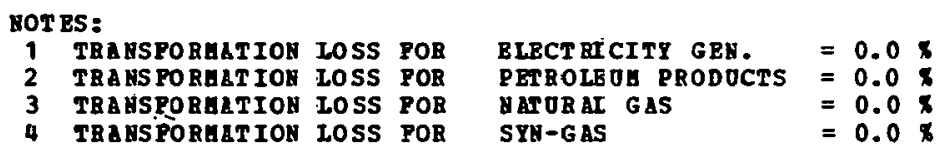


REGIONEL BNERGY BALANCE STATEUENT

3I SECTOR AND POEL TYPE

\begin{tabular}{|c|c|c|c|c|c|c|c|c|c|c|}
\hline SECTOR & $\begin{array}{l}\text { DIS TILLATE } \\
\text { OIL }\end{array}$ & $\begin{array}{l}\text { BESIDOAL } \\
\text { OIL }\end{array}$ & $\begin{array}{l}\text { GaSOLINE } \\
\text { (ALL OHIT }\end{array}$ & $\begin{array}{l}\text { OT JEB } \\
\text { EYD } 30- \\
\text { CERB JNS } \\
\text { TS IH 10**9 }\end{array}$ & $\begin{array}{c}\text { CRODE } \\
\text { OIL } \\
\text { BTO'SI }\end{array}$ & $\begin{array}{l}\text { NAT ORAL } \\
\text { GAS }\end{array}$ & $\operatorname{COAL}$ & ELBCTRICITY & $\begin{array}{l}\text { SECTOR } \\
\text { TOTAL }\end{array}$ & \\
\hline $\begin{array}{l}\text { PINAL DEUAND SECTORS } \\
\text { RESIDE HTIAL, COHA. } \\
\text { INDOSTRIAL } \\
\text { TRANSPORTATIOH } \\
\text { GISCELIAKEOOS OSBS }\end{array}$ & $\begin{array}{r}6.047 \\
1.366 \\
4.255 \\
417\end{array}$ & $\begin{array}{r}583 \\
83 \\
6 \\
198\end{array}$ & 22.758 & $\begin{array}{r}4,630 \\
2,523 \\
1,980 \\
221\end{array}$ & $\begin{array}{l}- \\
-\end{array}$ & $\begin{array}{r}6,978 \\
5,943 \\
1,731 \\
846\end{array}$ & $\begin{array}{r}154 \\
228 \\
4 \\
-\end{array}$ & $\begin{array}{r}8,459 \\
1.393 \\
7 \\
167\end{array}$ & $\begin{array}{r}26.851 \\
11,536 \\
30,741 \\
1,849\end{array}$ & $\begin{array}{r}38 \% \\
16 \% \\
43 \% \\
3 \%\end{array}$ \\
\hline $\begin{array}{l}\text { TOTAL PIHAL } \\
\text { DEAA HD SBCTORS }\end{array}$ & 12,085 & 870 & 22.758 & 9.354 & - & 15,498 & 386 & 10,026 & 70,977 & 998 \\
\hline $\begin{array}{l}\text { TRANSFOREATIO } \\
\text { ELECT RICITY GEH. } \\
\text { PETROLEUA PAODOCT S } \\
\text { IATORAL GAS } \\
\text { SYN-GAS }\end{array}$ & $\bar{z}$ & $\begin{array}{l}- \\
- \\
-\end{array}$ & $\bar{z}$ & $\overline{-}$ & E & $\overline{-}$ & $\bar{z}$ & $\overline{-}$ & $\overline{-}$ & \\
\hline $\begin{array}{l}\text { HET FOEL OSED } \\
\text { IS TRALSFORMATION }\end{array}$ & - & - & - & $\because$ & - & $\overline{-}$ & - & - & - & \\
\hline $\begin{array}{l}\text { TOTAL GROSS PLONS } \\
\text { LOSSES } E \text { OAIS SI ONS }\end{array}$ & $\begin{array}{r}12.085 \\
734\end{array}$ & $\begin{array}{r}870 \\
-8\end{array}$ & $\begin{array}{r}22.758 \\
-720\end{array}$ & $\begin{array}{r}9,354 \\
-441\end{array}$ & - & $\begin{array}{r}15,498 \\
58\end{array}$ & $\begin{array}{r}386 \\
6\end{array}$ & $\begin{array}{r}10,026 \\
801\end{array}$ & $\begin{array}{r}70,977 \\
429\end{array}$ & $\begin{array}{r}99 x \\
18\end{array}$ \\
\hline
\end{tabular}

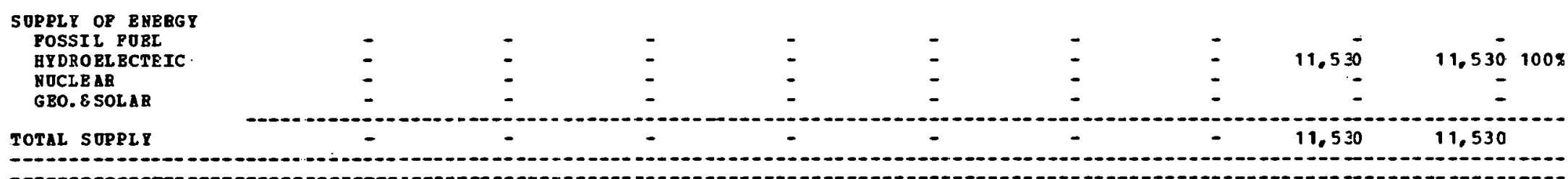

NBT IAPORTS

12.819

861

22,037

9.912

15,556

$\$ 92$

$-702$

59,876

$\begin{array}{ll}0 & 0\end{array}$

NOTES:

\begin{tabular}{|c|c|c|}
\hline $\begin{array}{l}\text { TRAHSPORHATION } \\
\text { TRAHE PORAATIOH } \\
\text { TRANSPORAATIOH } \\
\text { TRABEPORAATIOS }\end{array}$ & $\begin{array}{l}\text { Los: } \\
\text { LOS: } \\
\text { LoS } \\
\text { LOS }\end{array}$ & $\begin{array}{l}50 \\
50 \\
5 \\
50 \\
50\end{array}$ \\
\hline
\end{tabular}




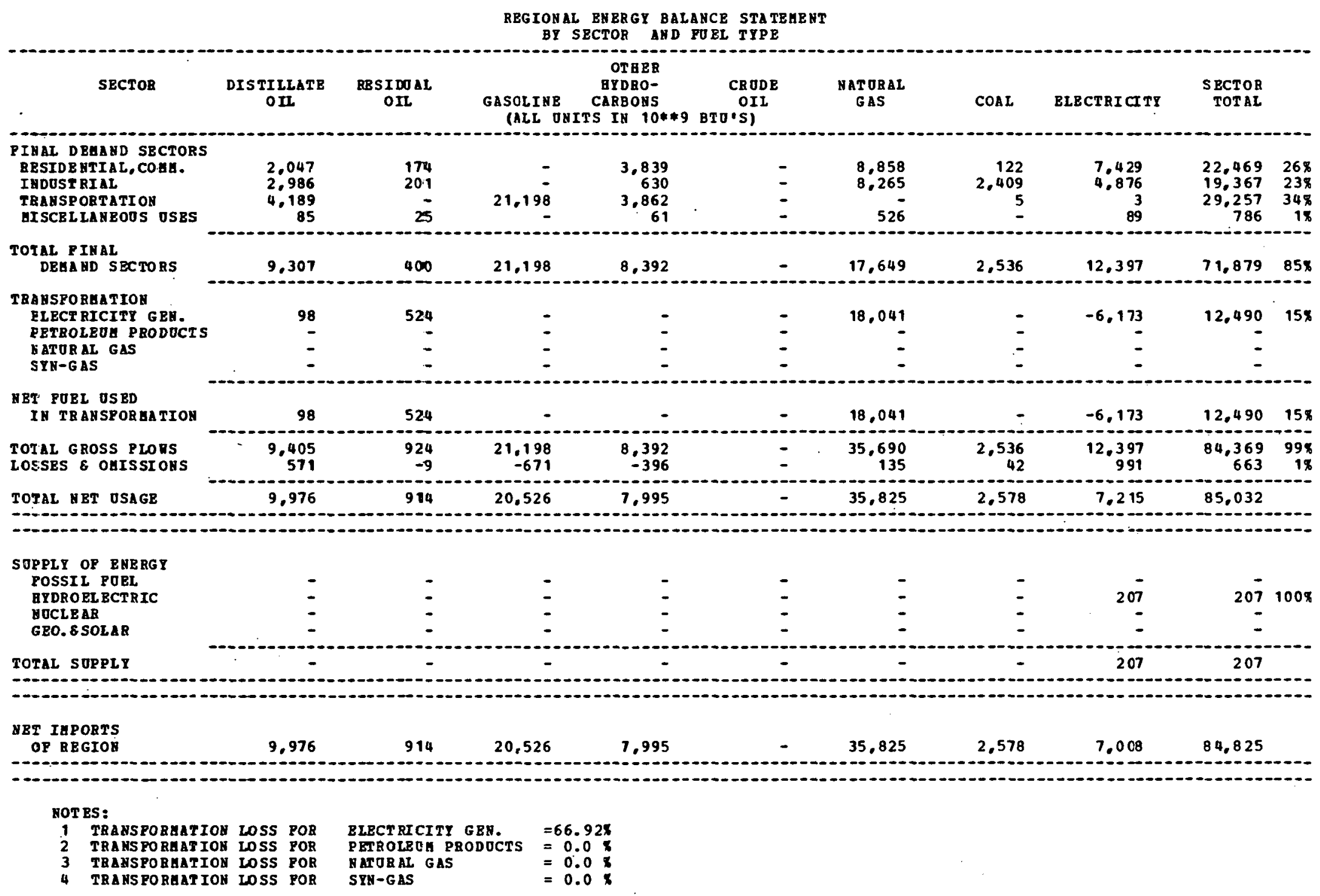




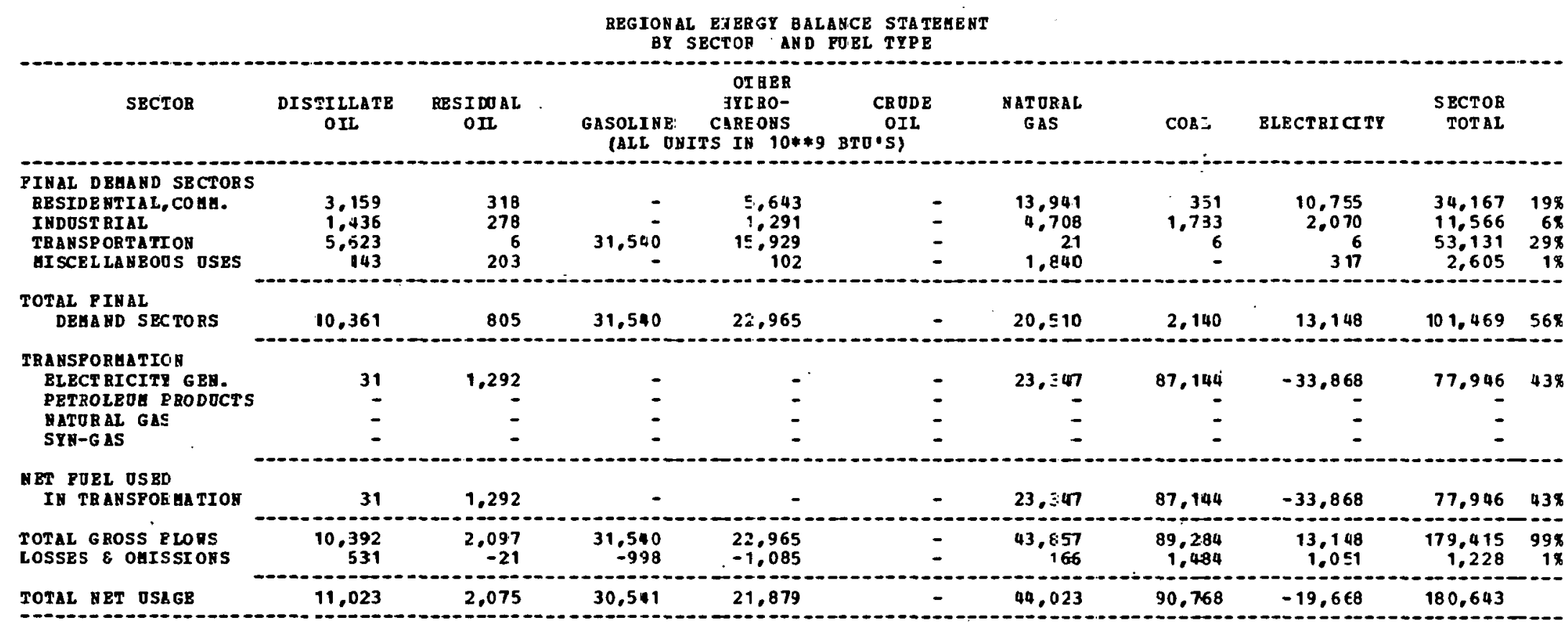

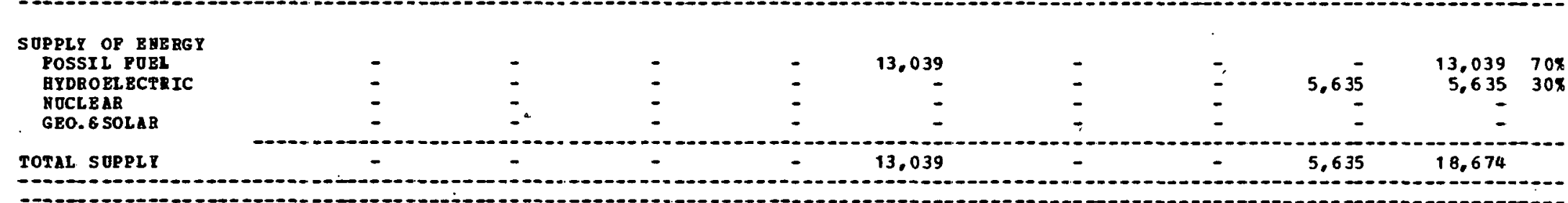

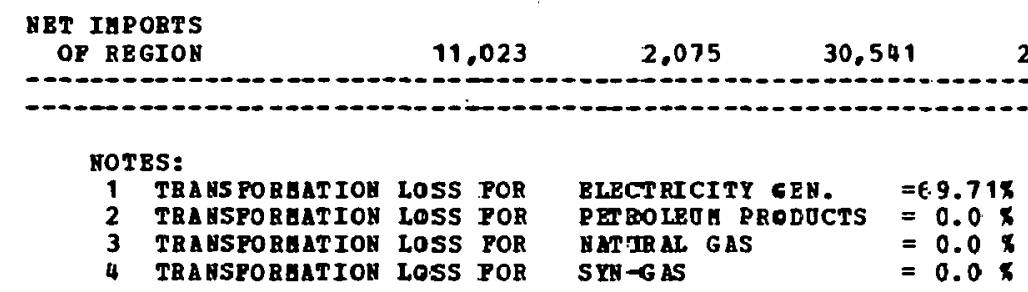


REGIONAL BNERGT BALAHCE STATEHEHT

BY SBCTOR AND POBL TYPE

\begin{tabular}{|c|c|c|c|c|c|c|c|c|c|c|}
\hline SBCTOR & $\begin{array}{l}\text { DISTILLATB } \\
\text { OII }\end{array}$ & $\begin{array}{l}\text { RBSIDOAL } \\
\text { OI }\end{array}$ & $\begin{array}{l}\text { GASOLIHE } \\
\text { (ALL ONIT }\end{array}$ & $\begin{array}{c}\text { OTBBQ } \\
\text { HYDRO- } \\
\text { CARBO\&S } \\
\text { IS IS 10**9 }\end{array}$ & $\begin{array}{c}\text { CRODB } \\
\text { OIL } \\
\text { BTO・S) }\end{array}$ & $\begin{array}{l}\text { NATURAL } \\
\text { GAS }\end{array}$ & COAL & ELECTRICITY & $\begin{array}{r}\text { SECTOR } \\
\text { TOTAL }\end{array}$ & \\
\hline 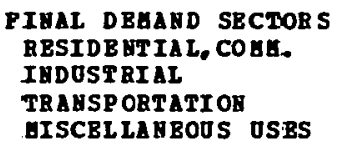 & $\begin{array}{r}3.407 \\
12,875 \\
24.545 \\
444\end{array}$ & $\begin{array}{r}1.13 \overline{-} \\
56 \overline{-}\end{array}$ & 112,119 & $\begin{array}{r}23,549 \\
12,922 \\
16,603 \\
991\end{array}$ & $\begin{array}{l}\overline{-} \\
\overline{-}\end{array}$ & $\begin{array}{r}47.546 \\
44.844 \\
18,143 \\
3,615\end{array}$ & $\begin{array}{r}798 \\
2,067 \\
17 \\
-\end{array}$ & $\begin{array}{r}32.184 \\
13.820 \\
14 \\
725\end{array}$ & $\begin{array}{r}107.484 \\
87.660 \\
171.441 \\
6.441\end{array}$ & $\begin{array}{r}25 \% \\
20 \% \\
40 \% \\
2 \%\end{array}$ \\
\hline $\begin{array}{l}\text { TOTAL PIBAL } \\
\text { DEGABD SBCTORS }\end{array}$ & 41,271 & 1,798 & 112,119 & 54,065 & - & 114,148 & 2,882 & 46,743 & 373.026 & $87 \%$ \\
\hline $\begin{array}{l}\text { TRANSPORGATIOA } \\
\text { ELECTRICITY GEH. } \\
\text { PETROLBOA PBODOCTS } \\
\text { HATORAL GAS } \\
\text { STH-GAS }\end{array}$ & $\begin{array}{r}1.818 \\
- \\
-\end{array}$ & $\begin{array}{r}29.042 \\
= \\
=\end{array}$ & $\begin{array}{r}-16 \overline{-} \\
-\end{array}$ & $\begin{array}{r}-4.043 \\
-\end{array}$ & $\begin{array}{r}4.217 \\
-\end{array}$ & $\begin{array}{r}37.389 \\
192 \\
- \\
-\end{array}$ & $\begin{array}{r}9.770 \\
: \\
-\end{array}$ & $\begin{array}{r}-23,876 \\
= \\
-\end{array}$ & $\begin{array}{r}54.143 \\
198 \\
= \\
-\end{array}$ & $\begin{array}{r}13 \% \\
0 \%\end{array}$ \\
\hline $\begin{array}{l}\text { NET FOBL OSED } \\
\text { IA TBASSPORAATIOE }\end{array}$ & 1,818 & 29,042 & -168 & $-4,043$ & 4.217 & 37.581 & 9.770 & $-23,876$ & 54,341 & $13 \%$ \\
\hline $\begin{array}{l}\text { TOTAL GROSS PLORS } \\
\text { COSSES } 6 \text { OBISSIOHS }\end{array}$ & $\begin{array}{r}43,089 \\
2,617\end{array}$ & $\begin{array}{r}30,840 \\
-312\end{array}$ & $\begin{array}{r}112,119 \\
-3,549\end{array}$ & $\begin{array}{l}54,065 \\
-2,554\end{array}$ & $\begin{array}{r}4.217 \\
6\end{array}$ & $\begin{array}{r}151.729 \\
574\end{array}$ & $\begin{array}{r}12.652 \\
210\end{array}$ & $\begin{array}{r}46.743 \\
3.738\end{array}$ & $\begin{array}{r}427.367 \\
730\end{array}$ & $\begin{array}{r}100 \% \\
0 \%\end{array}$ \\
\hline
\end{tabular}

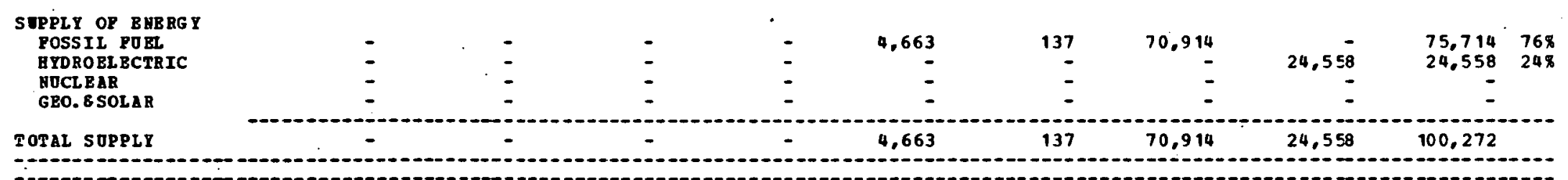

\section{DET IHPORTS}

\section{BOT ES:}

1 TRAUSPORAATION LOSS POR TRA IS PORAATIOH LOSS FOR TRANSPOBHATIOH LOSS POR $\begin{array}{ll}\text { BLECTRIOITY GEX. } & =69.40 \times \\ \text { PETROLEJA PRODOCTS } & =4.49 \%\end{array}$

$\begin{array}{ll}\text { DEROR GAS } & =0.49\end{array}$

$\begin{array}{ll}\text { SWN-GAS } & =0.0 \times\end{array}$ 


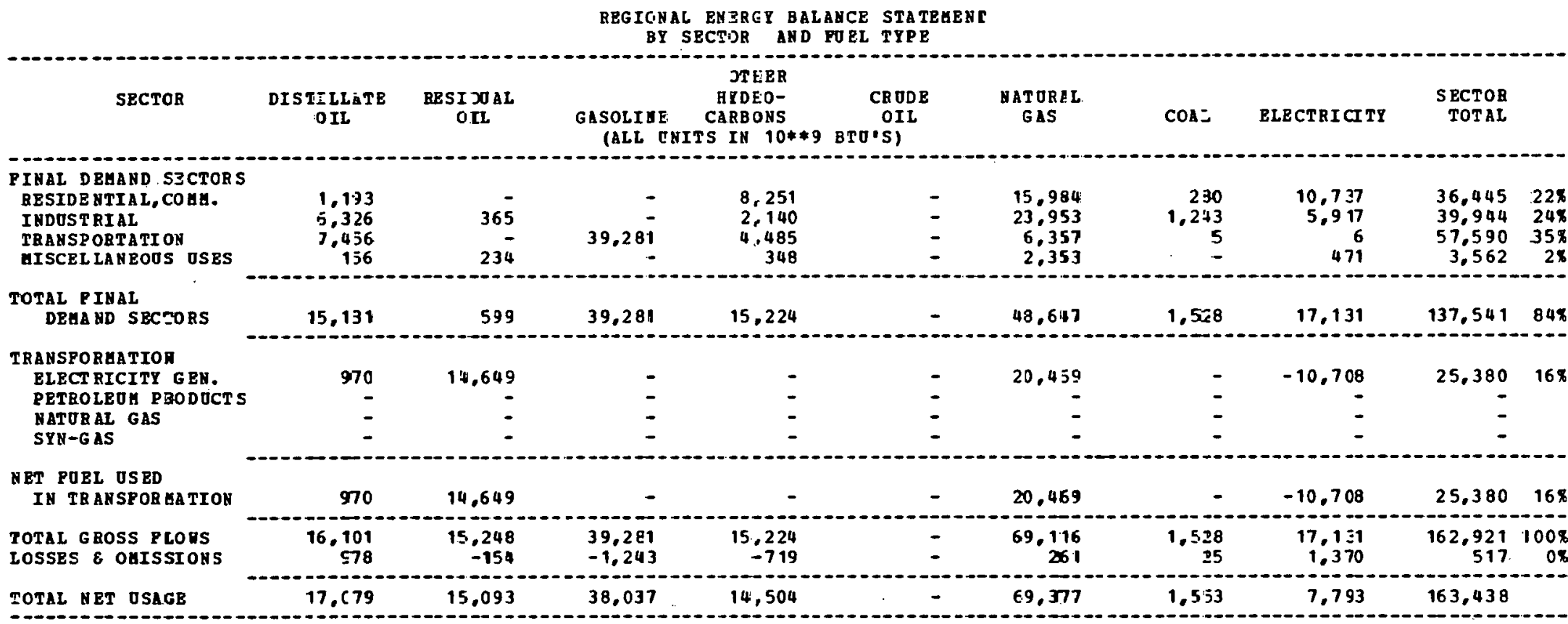

\section{SOPPLY OF EHERGY}

POSSII POEL

HYDROEL ECTA IC

NOCLB AR

GBO. 8 SOLAR

\begin{tabular}{rrr}
- & - & - \\
- & - & - \\
- & - & - \\
\hline- & - & -
\end{tabular}

TOTAL SUPPLY

MET IMPORTS

ET IMPORTS
OP REGION

$17.279 \quad 15.093$

38.037

14.504

69.377

1,553

7.793

163,438

\begin{tabular}{|c|c|c|c|c|c|}
\hline \multicolumn{6}{|c|}{ HOT } \\
\hline 1 & $\begin{array}{l}\text { TRAES POREAT ION } \\
\text { TRA HSPOREATION } \\
\text { TRA HS POREATION }\end{array}$ & $\begin{array}{l}\text { Loss } \\
\text { LosS } \\
\text { LosS }\end{array}$ & $\begin{array}{l}\text { POR } \\
\text { POR } \\
\text { POR }\end{array}$ & $\begin{array}{l}\text { ELECTRICITY GEN. } \\
\text { PER BOLEOA PRODOCTS } \\
\text { MET ORAL GAS }\end{array}$ & $\begin{array}{l}=70.3 \equiv \% \\
=0.0 \% \\
=0.0\end{array}$ \\
\hline 4 & TRA ASFO RHAT ION & & POR & SYN-G AS & $0.0 \%$ \\
\hline
\end{tabular}


REgIONAL BHERGY BALANCE STATEHENT

BY SECTOR AND POEL TYRE

\begin{tabular}{|c|c|c|c|c|c|c|c|c|c|c|}
\hline SECTOR & $\begin{array}{c}\text { DISTILLATB } \\
\text { OIL }\end{array}$ & $\begin{array}{l}\text { RESIDOAL } \\
\text { OIL }\end{array}$ & $\begin{array}{l}\text { GaSOLIN } \\
\text { (ALL }\end{array}$ & $\begin{array}{c}\text { OTAER } \\
\text { BTDBO- } \\
\text { CABBOHS } \\
\text { TS IB 10* }\end{array}$ & $\begin{array}{c}\text { CRODE } \\
\text { OIL } \\
\text { BTO'S! }\end{array}$ & $\begin{array}{l}\text { NAT ORAL } \\
\text { G AS }\end{array}$ & COAL & ELECTRICITY & $\begin{array}{r}\text { SECTOR } \\
\text { TOTAL }\end{array}$ & \\
\hline $\begin{array}{l}\text { PIHAL DEHAHD SECTOBS } \\
\text { RBSIDE HTIAL, COBE. } \\
\text { IHDOSTRIAL } \\
\text { TRAHSPORTATION } \\
\text { GISCELLABEOUS OSBS }\end{array}$ & $\begin{array}{r}1.783 \\
2.159 \\
11.186 \\
200\end{array}$ & $\begin{array}{l}1,263 \\
2.259 \\
5,821 \\
1.031\end{array}$ & 92.915 & $\begin{array}{r}8.153 \\
9.165 \\
17.759 \\
827\end{array}$ & - & $\begin{array}{r}54.860 \\
24.630 \\
1.441 \\
10.720\end{array}$ & $\begin{array}{r}770 \\
2.191 \\
15 \\
-\end{array}$ & $\begin{array}{r}20,746 \\
5,881 \\
45 \\
2.287\end{array}$ & $\begin{array}{r}87.575 \\
46.285 \\
129.182 \\
15,065\end{array}$ & $\begin{array}{r}26 \% \\
14 \% \\
39 \% \\
5 \%\end{array}$ \\
\hline $\begin{array}{l}\text { TOTAL PIBAL } \\
\text { DEUA AD SECTORS }\end{array}$ & 15.328 & 10,374 & 92,915 & 35,904 & - & 91,651 & 2,976 & 28,959 & 278,107 & $84 \%$ \\
\hline $\begin{array}{l}\text { PBARSPOBAATIOH } \\
\text { BLECTRICITY GEY. } \\
\text { PETROLBDA PBODOCTS } \\
\text { MATORAL GAS } \\
\text { SYH-GAS }\end{array}$ & $\begin{array}{r}298 \\
= \\
-\end{array}$ & $\begin{array}{r}51.853 \\
= \\
-\end{array}$ & $\overline{-}$ & $\begin{array}{l}4 \\
- \\
-\end{array}$ & 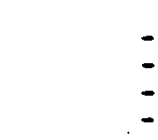 & $\begin{array}{r}28,222 \\
= \\
=\end{array}$ & $\overline{-}$ & $\begin{array}{r}-24.893 \\
= \\
-\end{array}$ & $\begin{array}{r}55,484 \\
= \\
-\end{array}$ & $17 \%$ \\
\hline $\begin{array}{l}\text { FET FOEL OSED } \\
\text { IH TRAESPORAATIOH }\end{array}$ & 298 & 51.853 & - & 4 & $\begin{array}{c}- \\
-\end{array}$ & 28,222 & - & $-24,893$ & 55,484 & $17 \%$ \\
\hline $\begin{array}{l}\text { TOTAL GROSS FLOHS } \\
\text { I OSSES } \varepsilon \text { OLISSIONS }\end{array}$ & $\begin{array}{r}15.626 \\
949\end{array}$ & $\begin{array}{r}62.227 \\
-631 \\
--1 .-0\end{array}$ & $\begin{array}{r}92,915 \\
-2,941\end{array}$ & $\begin{array}{l}35,908 \\
-1,696\end{array}$ & $\overline{-}$ & $\begin{array}{r}119.873 \\
453\end{array}$ & $\begin{array}{r}2,976 \\
49\end{array}$ & $\begin{array}{r}28,959 \\
2,316\end{array}$ & $\begin{array}{r}333,591 \\
-1,500 \\
-\end{array}$ & $\begin{array}{r}1008 \\
0 \%\end{array}$ \\
\hline
\end{tabular}

\section{SUP PLY OF EMERGY}

POSSII POBL

HYDROBLECTRIC

MOCLAR

Grot

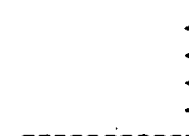

TOTAL SOPPLY

-

$-$

$-$

$-$

\begin{tabular}{lccr}
- & $\overline{3}$ & $\overline{3}$ & 08 \\
- & 7.534 & 7.534 & 1008 \\
\hline & - & - \\
\hline & 7.537 & 7.537
\end{tabular}

GBT IAPORTS

OP REGIOH

16,575

61,595

89,973

34,211

120,326

3,025

$-1,154$

324,553

MOT ES:

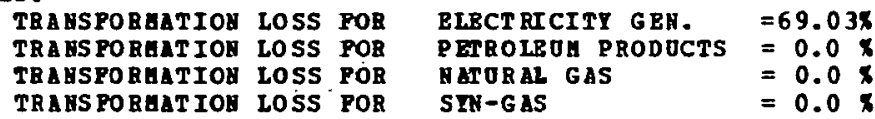


REGIONAL ZNBRGY BALANCE STATELENT

BY SECTOR AND FOEL TYPE

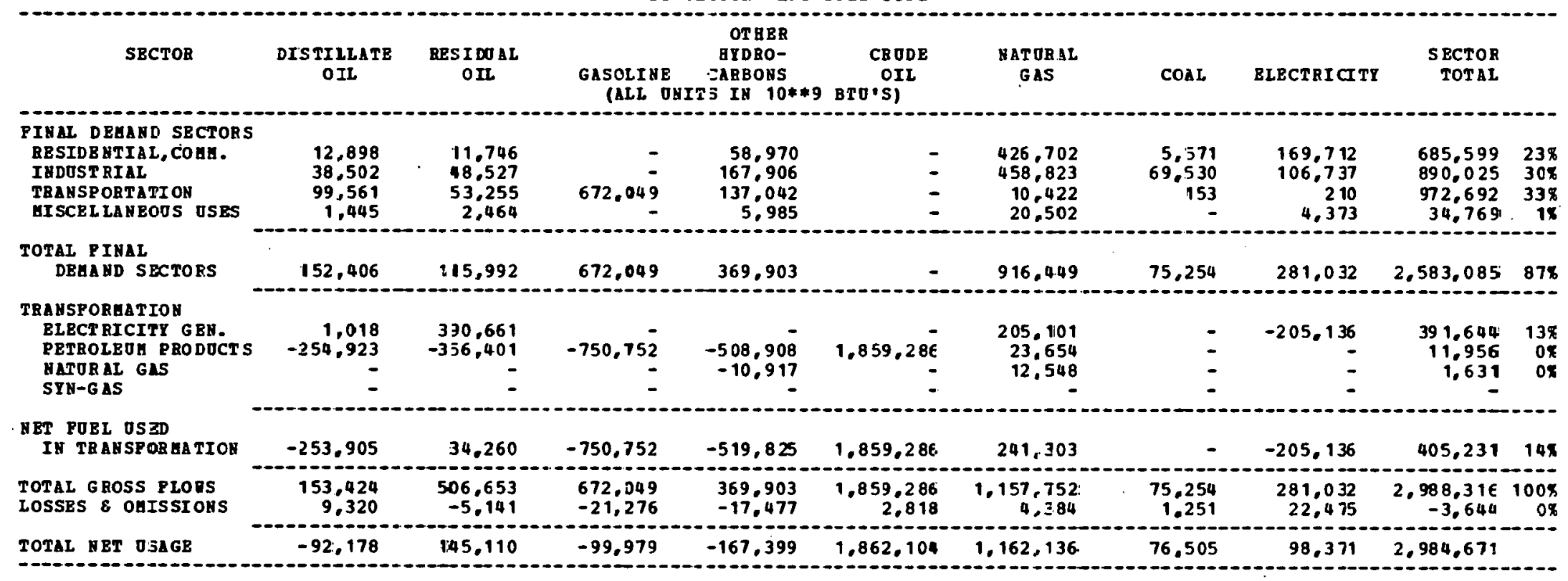

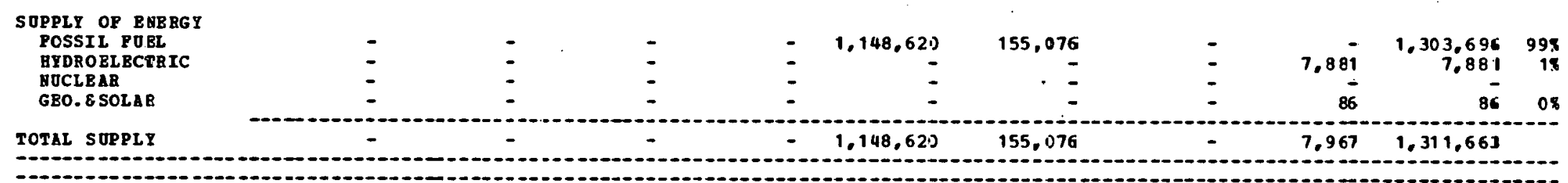

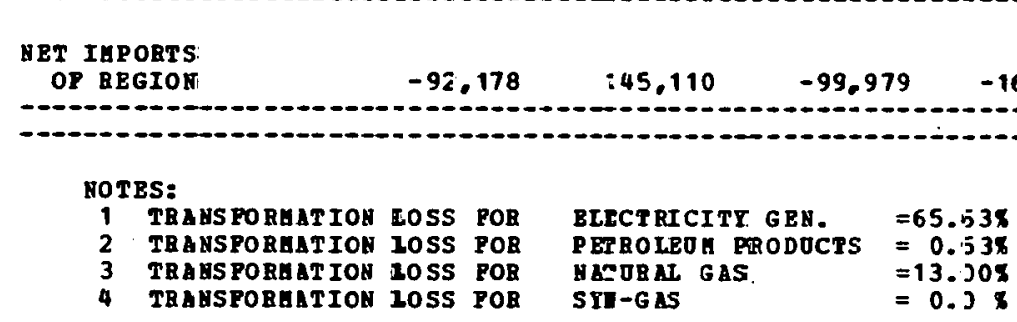




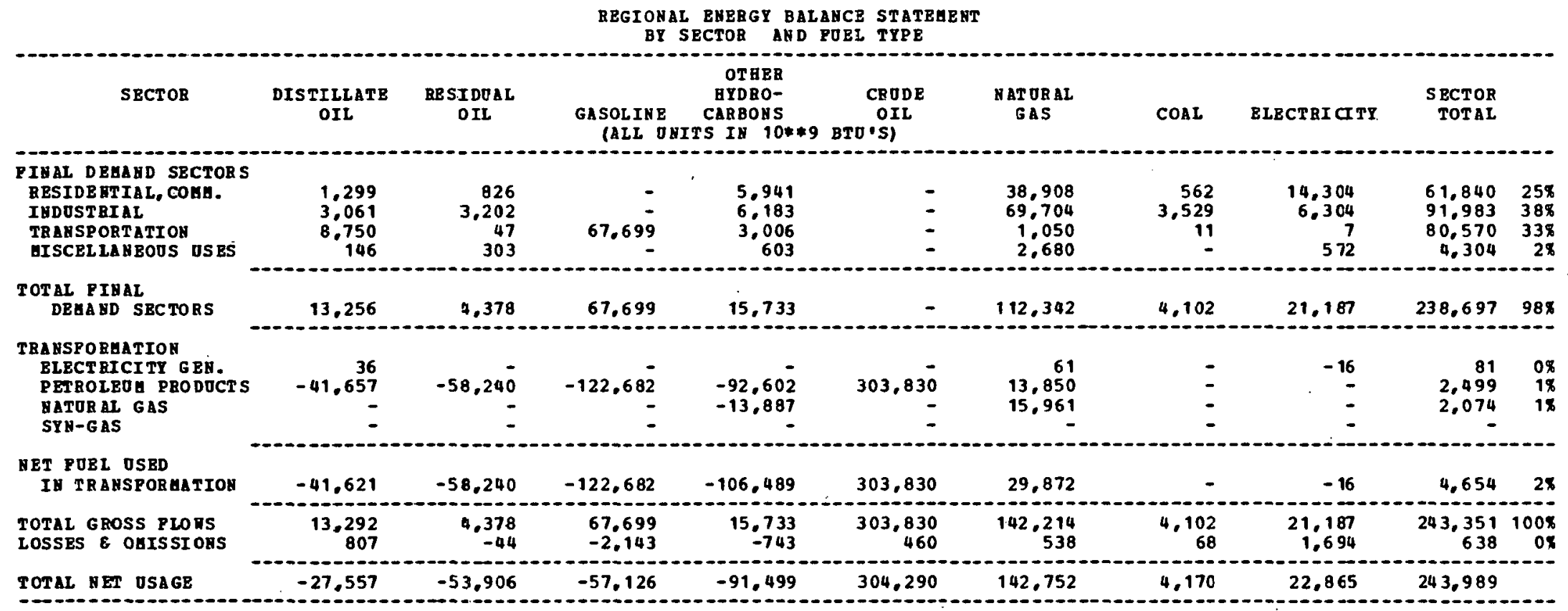

\begin{tabular}{|c|c|c|c|c|c|c|c|c|c|c|}
\hline $\begin{array}{l}\text { SOPPLY OF BHERGI } \\
\text { POSSIL POEL }\end{array}$ & - & - & - & - & 722,877 & 110,145 & - & - & 833,022 & $98 \%$ \\
\hline HYDROBLECTRIC & - & - & - & - & - & - & - & 21,162 & 21,162 & 28 \\
\hline BOCLEAR & - & - & - & - & - & - & - & - & - & \\
\hline GEO. 8 SOLAR & - & - & - & - & - & - & - & - & - & \\
\hline
\end{tabular}

\begin{tabular}{l}
$\begin{array}{l}\text { MET IHPORTS } \\
\text { OP REGIOH }\end{array}$ \\
\hline
\end{tabular}


RBG IONAL ENERGT BALANCE STATELEAT

BY SECTOR AUD FOEL TYPE

\begin{tabular}{|c|c|c|c|c|c|c|c|c|c|c|}
\hline SECTOR & $\begin{array}{l}\text { DISTILLATE } \\
\text { OIL }\end{array}$ & $\begin{array}{l}\text { RESI DO AL } \\
\text { OIL }\end{array}$ & $\begin{array}{l}\text { GASOLIAE } \\
\text { (ALD O }\end{array}$ & $\begin{array}{c}\text { OTHER } \\
\text { GYDRO- } \\
\text { CABBONS } \\
\text { TS IN } 10 * * 9\end{array}$ & $\begin{array}{c}\text { CBODE } \\
\text { OIL } \\
\text { BTO'S) }\end{array}$ & $\begin{array}{l}\text { MATUEAL } \\
\text { GLS }\end{array}$ & $\operatorname{COAL}$ & ELECT RI CITY & $\begin{array}{l}\text { SECTOE } \\
\text { TOTAL }\end{array}$ & \\
\hline $\begin{array}{l}\text { PINAL DRGAND SECTORS } \\
\text { RESIDE NTIAI, COAH. } \\
\text { INDUSTRIAL } \\
\text { TRANSPORTATIOH } \\
\text { GISCEL LAHEDOS OSES }\end{array}$ & $\begin{array}{r}814 \\
1.966 \\
\epsilon .147 \\
91\end{array}$ & $\begin{array}{r}492 \\
2.135 \\
1.399 \\
215\end{array}$ & 42.414 & $\begin{array}{r}3,722 \\
5,822 \\
1,787 \\
378\end{array}$ & $\begin{array}{l}- \\
-\end{array}$ & $\begin{array}{r}24.074 \\
20,585 \\
658 \\
1.981\end{array}$ & $\begin{array}{r}352 \\
4.050 \\
7 \\
-\end{array}$ & $\begin{array}{r}8.781 \\
4.072 \\
7 \\
422\end{array}$ & $\begin{array}{r}38,239 \\
38.636 \\
52.419 \\
3,08\end{array}$ & $\begin{array}{r}298 \\
298 \\
408 \\
28\end{array}$ \\
\hline $\begin{array}{l}\text { TOTAL PIVAL } \\
\text { DEGA ND SBCTORS }\end{array}$ & 5.018 & 4.241 & 42,414 & 11,709 & - & 47.298 & 4,409 & 13,282 & 132,371 & 1008 \\
\hline $\begin{array}{l}\text { TRAYSFO RHATIOH } \\
\text { BLECT RICITY GEN. } \\
\text { PETROLEOH PRODOCTS } \\
\text { RATORAL GAS } \\
\text { SIN-GAS }\end{array}$ & $\begin{array}{l}- \\
\overline{-}\end{array}$ & 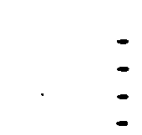 & : & : & $\begin{array}{l}- \\
-\end{array}$ & $\begin{array}{l}\bar{E} \\
\overline{-}\end{array}$ & - & $\bar{E}$ & 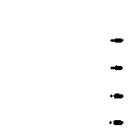 & \\
\hline $\begin{array}{l}\text { VBT POEL US3D } \\
\text { IR TRABSPORGATION }\end{array}$ & - & - & - & - & - & - & - & - & - & \\
\hline $\begin{array}{l}\text { TOTAL GROSS FLO } \\
\text { LOSSES } 8 \text { OAISSIOYS }\end{array}$ & $\begin{array}{r}9,018 \\
547\end{array}$ & $\begin{array}{r}4.241 \\
-43\end{array}$ & $\begin{array}{l}42,414 \\
-1,342\end{array}$ & $\begin{array}{r}11.709 \\
-553\end{array}$ & $=$ & $\begin{array}{r}47.298 \\
179\end{array}$ & $\begin{array}{r}4,409 \\
73\end{array}$ & $\begin{array}{r}13.282 \\
1.062\end{array}$ & $\begin{array}{r}132,377 \\
-76\end{array}$ & $\begin{array}{r}100 \% \\
0 \%\end{array}$ \\
\hline
\end{tabular}

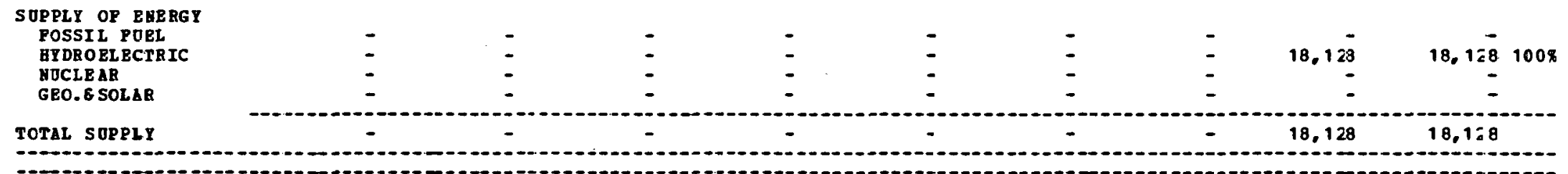

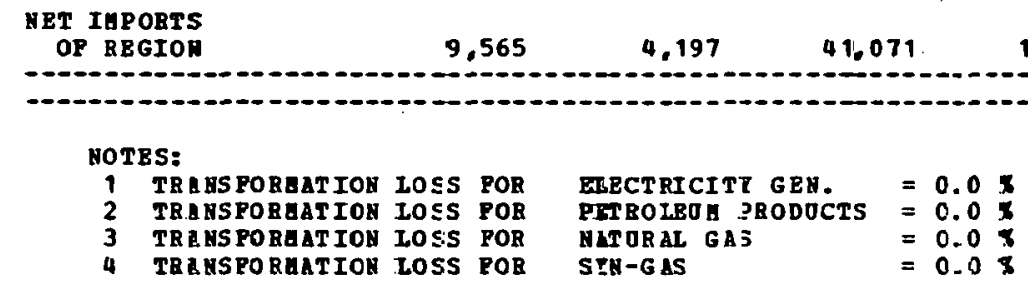


REGIOHAL RHERG BALANCE STATEAEST

BY SBCTOR AND POBL TYPE

\begin{tabular}{|c|c|c|c|c|c|c|c|c|c|c|}
\hline SBCTOR & $\underset{\text { OIL }}{\text { DISTILATE }}$ & $\begin{array}{c}\text { RESIDOAL } \\
\text { OIL }\end{array}$ & $\begin{array}{l}\text { GASOLIAE } \\
\text { (ALI ONIT }\end{array}$ & $\begin{array}{c}\text { OTHER } \\
\text { HYDRO- } \\
\text { CARBOHS } \\
\text { TS I\& } 10 * * 9\end{array}$ & $\begin{array}{c}\text { CRODE } \\
\text { OIL } \\
\text { BTO } S)\end{array}$ & $\begin{array}{l}\text { HAT URAL } \\
\text { GAS }\end{array}$ & COAL & ELECT RI CITY & $\begin{array}{r}\text { SECTOR } \\
\text { TOTAL }\end{array}$ & \\
\hline 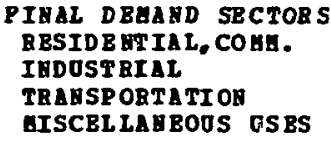 & $\begin{array}{l}1.430 \\
1.659 \\
7.284 \\
160\end{array}$ & $\begin{array}{r}936 \\
1,335 \\
50 \\
580\end{array}$ & 74,528 & $\begin{array}{r}6.539 \\
6.683 \\
2.650 \\
664\end{array}$ & $\bar{z}$ & $\begin{array}{r}43.122 \\
13.481 \\
1.156 \\
5.783\end{array}$ & $\begin{array}{r}618 \\
3.051 \\
12 \\
-\end{array}$ & $\begin{array}{r}15,994 \\
2.935 \\
17 \\
1.234\end{array}$ & $\begin{array}{r}68.639 \\
29.144 \\
85,697 \\
8.421\end{array}$ & $\begin{array}{r}36 \% \\
15 \% \\
45 \% \\
4 \%\end{array}$ \\
\hline DEHABD SECTOBS & 10,533 & 2.901 & 74,528 & 16,536 & - & 63.542 & 3,681 & 20,180 & 191,901 & $100 \%$ \\
\hline
\end{tabular}

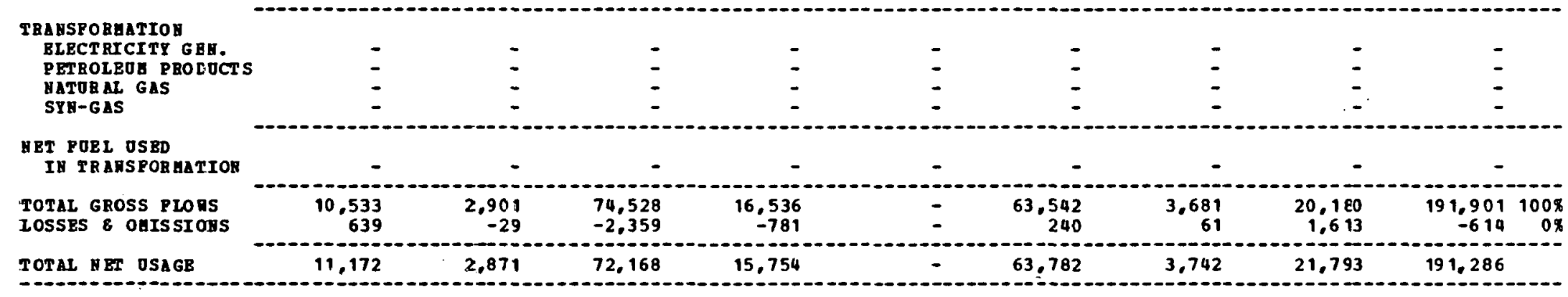

\begin{tabular}{|c|c|c|c|c|c|c|c|c|c|c|}
\hline $\begin{array}{l}\text { SUPPLY OP BEERGY } \\
\text { POSSIL POEL }\end{array}$ & - & $=$ & $\overline{-}$ & - & 139 & 121.585 & - & 42,75 & $\begin{array}{r}121.724 \\
42.756\end{array}$ & $\begin{array}{l}74 x \\
268\end{array}$ \\
\hline YOCLEAB & $=$ & - & - & - & - & - & - & & 42,150 & \\
\hline GBO. E SOLAR & - & - & - & - & - & - & - & & - & \\
\hline
\end{tabular}

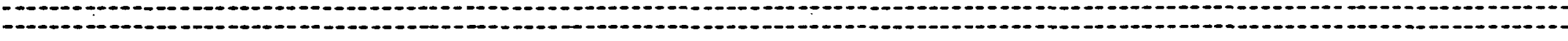

\section{MBT IUPOBTS}

OP REGIOI

11,172

2,871

72,168

15,754

$-139$

$-57,802$

3.742

$-20,962$

26,806

NOT BS :

1 THA SSPORATION LOSS POB

TRABS PORAATIOY LOSS POR
TRA ISPORATION LOSS POR

TRABSPORAATIOH LOSS POR

$\begin{array}{ll} & =0.0 \%\end{array}$

$\begin{array}{ll} & =0.0 \% \\ & =0.0 \%\end{array}$

$\begin{array}{ll}\text { M KTORAL GAS } & =0.0 \% \\ S \text { YS-GIS } & =0.0 \%\end{array}$ 


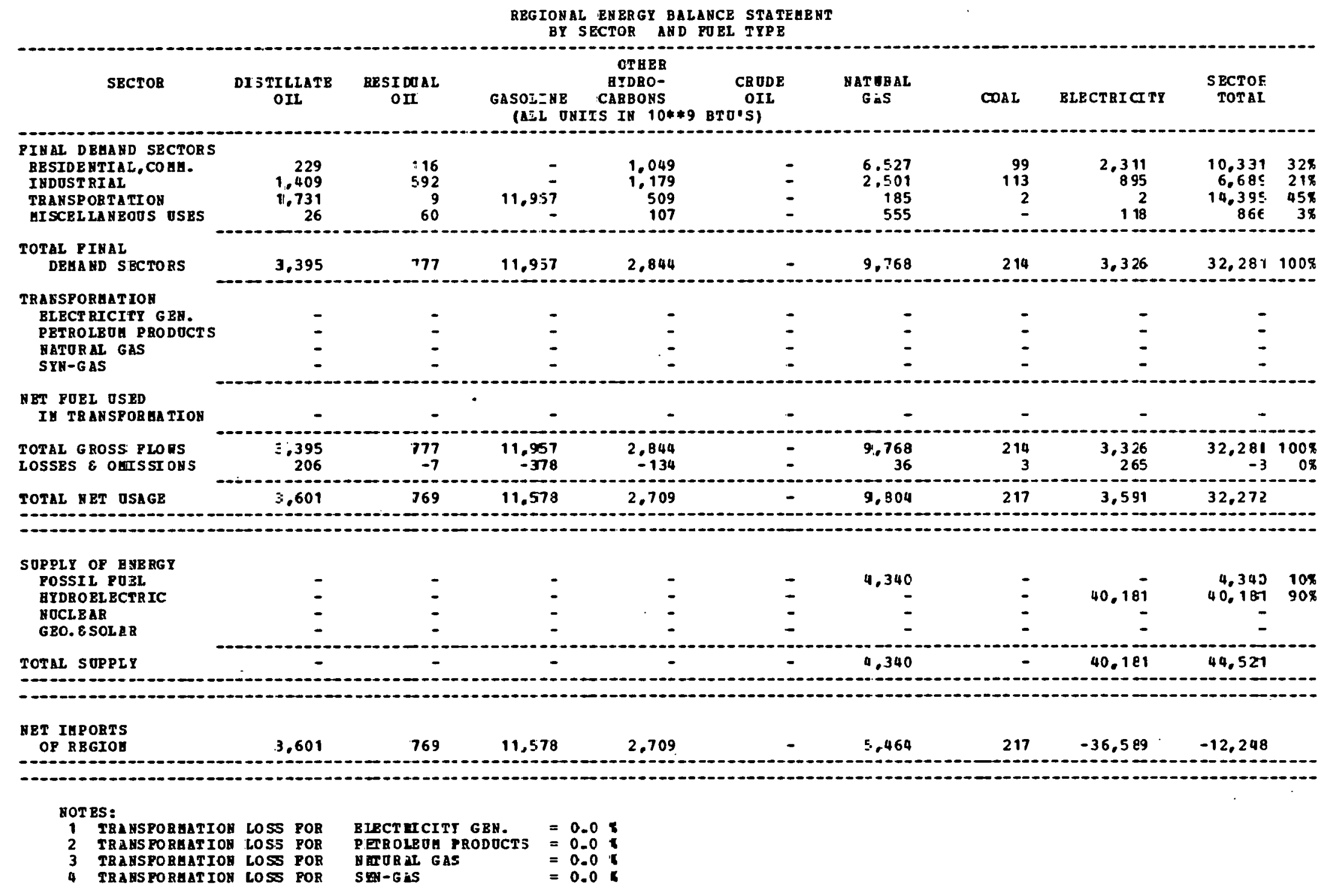


REgIONAL BHERGY BALANCE STATEZENT

\begin{tabular}{|c|c|c|c|c|c|c|c|c|c|c|}
\hline SECTOR & $\begin{array}{l}\text { DISTILLATE } \\
\text { OIL }\end{array}$ & $\begin{array}{l}\text { RESI DOAL } \\
\text { OII }\end{array}$ & $\begin{array}{l}\text { GASOLIBE } \\
\text { (ALL DEI I }\end{array}$ & $\begin{array}{c}\text { OTHER } \\
\text { ATDBO- } \\
\text { CARBOAS } \\
\text { IS IN } 10 * * 9\end{array}$ & $\begin{array}{c}\text { CRODE } \\
\text { OIL } \\
\text { BTO'S) }\end{array}$ & $\begin{array}{l}\text { FATURAL } \\
\text { GAS }\end{array}$ & COAI & ELECTRICITY & $\begin{array}{r}\text { SECTOR } \\
\text { TOTAL }\end{array}$ & \\
\hline 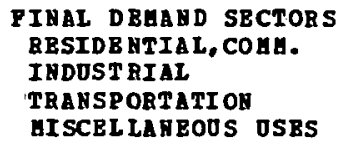 & $\begin{array}{r}154 \\
1.457 \\
1.023 \\
17\end{array}$ & $\begin{array}{r}81 \\
561 \\
1.129 \\
33\end{array}$ & 8.005 & $\begin{array}{r}703 \\
1.225 \\
278 \\
71\end{array}$ & I & $\begin{array}{r}4.407 \\
1.998 \\
124 \\
286\end{array}$ & $\begin{array}{r}67 \\
136 \\
1 \\
-\end{array}$ & $\begin{array}{r}1.567 \\
860 \\
61\end{array}$ & $\begin{array}{r}6,979 \\
6,237 \\
10,560 \\
468\end{array}$ & $\begin{array}{r}268 \\
238 \\
398 \\
28\end{array}$ \\
\hline $\begin{array}{l}\text { TOTAL PIHAL } \\
\text { DEHABD SECTORS }\end{array}$ & 2,651 & 1,804 & 8.005 & 2.277 & - & 6,815 & 204 & 2,488 & 24,244 & $90 \%$ \\
\hline $\begin{array}{l}\text { TRANSPORAATIOH } \\
\text { BLECTRICITY GEA. } \\
\text { PBTROLEOH PRODUCT S } \\
\text { HATORAL GAS } \\
\text { SYH-GAS }\end{array}$ & $\therefore$ & ?2:- & $\begin{array}{l}- \\
-\end{array}$ & $\begin{array}{l}- \\
-\end{array}$ & $\bar{z}$ & $\begin{array}{r}3.456 \\
- \\
-\end{array}$ & $\overline{-}$ & $\begin{array}{r}-931 \\
- \\
-\end{array}$ & $\begin{array}{r}2,597 \\
= \\
=\end{array}$ & $10 \%$ \\
\hline $\begin{array}{l}\text { YBT POEL OSED } \\
\text { IA TRAUSPOREATIOH }\end{array}$ & - & 72 & - & - & - & 3,456 & - & -931 & 2.597 & $10 \%$ \\
\hline $\begin{array}{l}\text { IOTAL GROSS PLORS } \\
\text { LOSSES E OAISSI OHS }\end{array}$ & $\begin{array}{r}2.651 \\
161\end{array}$ & $\begin{array}{r}1,876 \\
-19\end{array}$ & $\begin{array}{r}8.005 \\
-253\end{array}$ & $\begin{array}{r}2.277 \\
-107\end{array}$ & - & $\begin{array}{r}10,271 \\
38\end{array}$ & $\begin{array}{r}204 \\
3\end{array}$ & $\begin{array}{r}2.488 \\
198\end{array}$ & $\begin{array}{r}26.841 \\
22\end{array}$ & $\begin{array}{r}100 \% \\
0 \%\end{array}$ \\
\hline TOTAL HET OSAGE & 2.812 & 1,856 & 7,751 & 2,169 & - & 10,309 & 207 & 1.755 & 26,863 & \\
\hline $\begin{array}{l}\text { SOPPLY OP BHERG } \\
\text { POSSI L PORL } \\
\text { GYDROELECTRIC } \\
\text { NOCLEAR } \\
\text { GEO.E SOLAR }\end{array}$ & $\bar{\vdots}$ & : & $\overline{-}$ & $\begin{array}{l}- \\
-\end{array}$ & 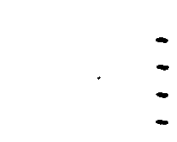 & $\begin{array}{r}3,267 \\
- \\
-\end{array}$ & : & $\begin{array}{r}2,012 \\
1,447 \\
-\end{array}$ & $\begin{array}{r}3,267 \\
2,012 \\
1,447 \\
-.\end{array}$ & $\begin{array}{l}49 \% \\
30 \% \\
22 \%\end{array}$ \\
\hline OTAL SOPPLY & - & - & - & - & - & 3.267 & - & 3,459 & 6,726 & \\
\hline
\end{tabular}

\section{SET IHPORTS}

OP RBGIOY

2.812

1.856

7.751

2,169

7,042

207

$-1.703$

20.137

HOT ES:

1 TRAASPOBUATIOU LOSS POB TRABSFOBAATYON LOSS POR

TRABSPOBGATION LOSS POR

TRABS PORAATIOH LOSS POR

BLECTEICITY GEN.

D $=0.0$

STH-GES

$=0.0 \%$
$=0.0 \%$

$=0.0 \%$ 
REG IOHAL EN BBGY BALANCE STA TEGEAT

BY SECTOR AND FOEL TYPE

\begin{tabular}{|c|c|c|c|c|c|c|c|c|c|c|}
\hline SECTOR & $\begin{array}{c}\text { DESTILLATE } \\
\text { OIL }\end{array}$ & $\begin{array}{l}\text { RES I DU AL } \\
\text { OII }\end{array}$ & $\begin{array}{l}\text { GASOLINE } \\
\text { (ALL DI }\end{array}$ & $\begin{array}{c}\text { OTHER } \\
\text { HYDBO- } \\
\text { CARBONS } \\
\text { TS IH 10* }\end{array}$ & $\begin{array}{c}\text { CBODE } \\
\text { OIL } \\
\text { BTO :S) }\end{array}$ & $\begin{array}{l}\text { NATUBAL } \\
\text { G.AS }\end{array}$ & COAL & ELECTBICITY & $\begin{array}{l}\text { SECTOR } \\
\text { TOTAL }\end{array}$ & . \\
\hline 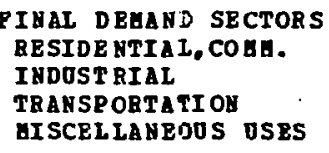 & $\begin{array}{r}6.392 \\
12.393 \\
66.818 \\
716\end{array}$ & $\begin{array}{r}5.615 \\
16.586 \\
67.491 \\
1.739\end{array}$ & 333.034 & $\begin{array}{r}29,223 \\
60,473 \\
122,005 \\
2,965\end{array}$ & $\overline{-}$ & $\begin{array}{r}209,105 \\
141,879 \\
5,165 \\
16,086\end{array}$ & $\begin{array}{r}2,761 \\
28,889 \\
103 \\
-\end{array}$ & $\begin{array}{r}82.691 \\
39.059 \\
171 \\
3.431\end{array}$ & $\begin{array}{r}335,787 \\
299,279 \\
594,787 \\
24,937\end{array}$ & $\begin{array}{r}238 \\
208 \\
408 \\
28\end{array}$ \\
\hline $\begin{array}{l}\text { TOTAL FINAL } \\
\text { DEHA SD SBCTORS }\end{array}$ & 86,319 & 91.431 & 333.034 & 214.666 & - & 372,235 & 31,753 & 125,352 & $1,254,790$ & $85 x$ \\
\hline $\begin{array}{l}\text { TRAESFORGATIOA } \\
\text { ELECT RICITY GEH. } \\
\text { PETROLEOA PRODOCTS } \\
\text { HATORAL GAS } \\
\text { SYH-GAS }\end{array}$ & $\begin{array}{r}113 \\
-161.213 \\
-\end{array}$ & $\begin{array}{r}34,051 \\
-225,388 \\
-\end{array}$ & $\begin{array}{r}-474.774 \\
-\end{array}$ & $\begin{array}{r}50,013 \\
-358,754 \\
-\end{array}$ & $\begin{array}{r}1,175,811 \\
:\end{array}$ & $\begin{array}{r}211.67 \epsilon \\
53.60 C \\
=\end{array}$ & - & $\begin{array}{r}-85,625 \\
= \\
=\end{array}$ & $\begin{array}{r}210,223 \\
9,282 \\
= \\
-\end{array}$ & $\begin{array}{r}148 \\
18\end{array}$ \\
\hline $\begin{array}{l}\text { NET POEL OSED } \\
\text { IN TRANSP JRAATION }\end{array}$ & $-161,100$ & -191.337 & $-474,774$ & $-208,741$ & $1,175,810$ & $265,27 \epsilon$ & - & $-85,625$ & $219,51)$. & $15 \%$ \\
\hline $\begin{array}{l}\text { TOTAL GROSS PLOYS } \\
\text { LOSSES } \& \text { OHESSIOHS }\end{array}$ & $\begin{array}{r}86.432 \\
5.250\end{array}$ & $\begin{array}{r}125.482 \\
-1.273\end{array}$ & $\begin{array}{l}333,034 \\
-10,543\end{array}$ & $\begin{array}{l}264,679 \\
-12,505\end{array}$ & $\begin{array}{r}1,175,811 \\
1,782\end{array}$ & $\begin{array}{r}637,511 \\
2,414\end{array}$ & $\begin{array}{r}31,753 \\
527\end{array}$ & $\begin{array}{r}125,352 \\
10,025\end{array}$ & $\begin{array}{r}1,474,30.3 \\
-4,322\end{array}$ & $\begin{array}{r}100 \% \\
0 \%\end{array}$ \\
\hline
\end{tabular}

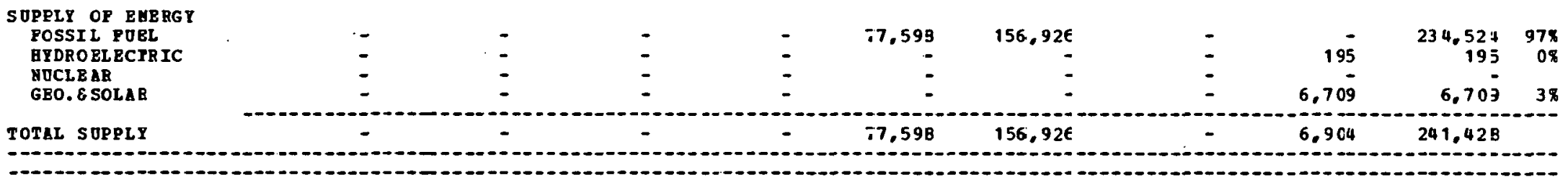

NET ILPORTS

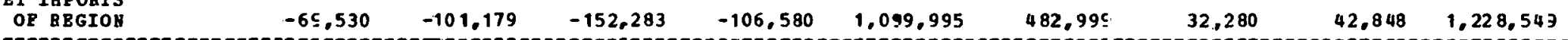

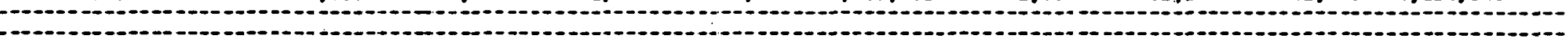

MOTES:

\begin{tabular}{|c|c|c|c|c|}
\hline $\begin{array}{l}\text { TRABS PORE } \\
\text { TRAESFO RAA }\end{array}$ & $\begin{array}{l}\text { Los } \\
\text { Los } \\
\text { cos }\end{array}$ & $\begin{array}{l}\text { FOR } \\
\text { FOR } \\
\text { ZOR } \\
\text { FOR }\end{array}$ & $\begin{array}{l}\text { EIECTRICITI GEN. } \\
\text { PETOLEOH PRODOCTS } \\
\text { MEOKAL GAS } \\
\text { SZI -GAS }\end{array}$ & $\begin{array}{l}=0.75 \% \\
=0.0 \times \\
=0.0 \mathrm{x}\end{array}$ \\
\hline
\end{tabular}




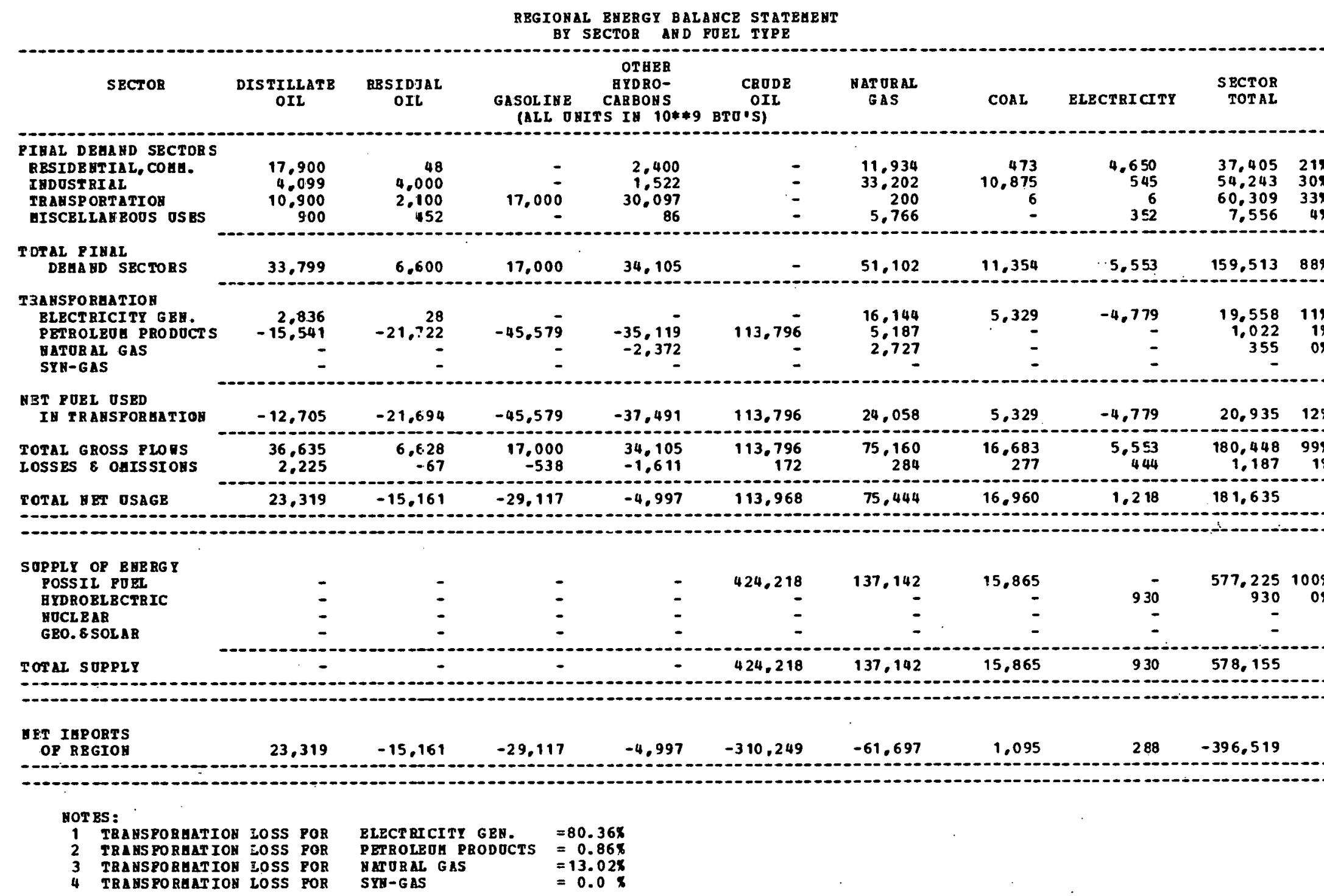


QEG IONAL EHERGT BALANCE STATELENT

BY SECTOB AHD FOEL TYPE

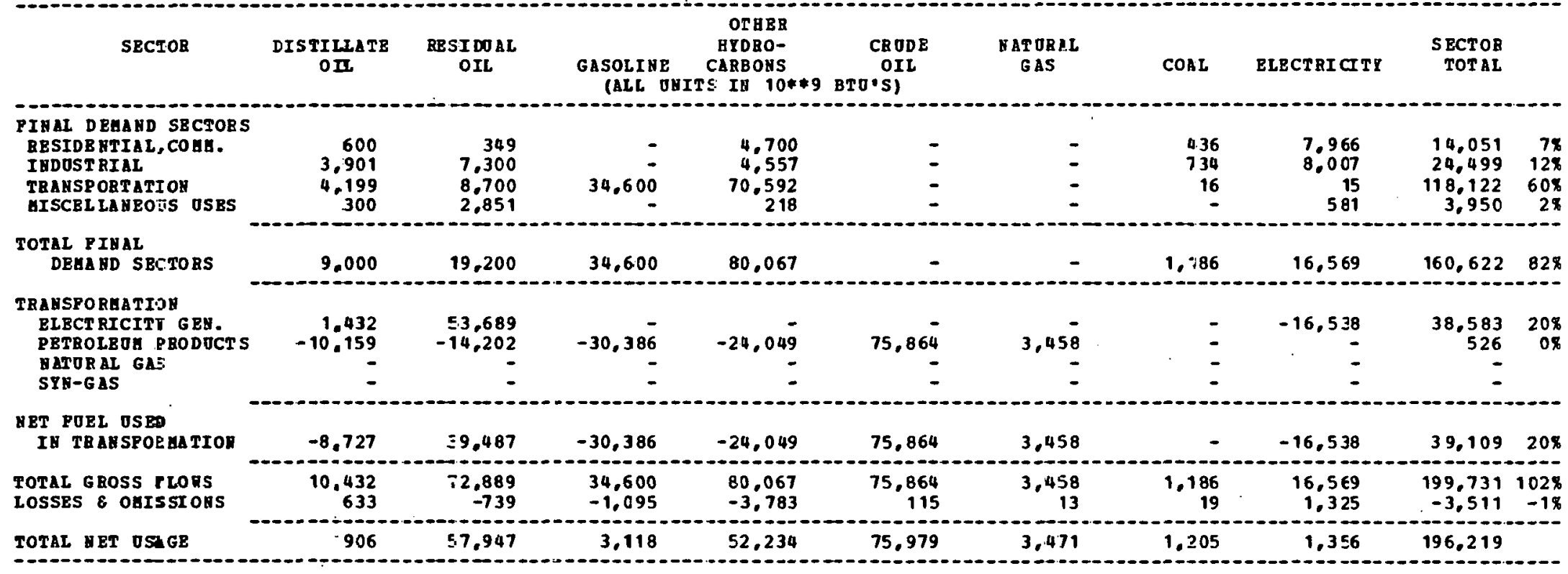

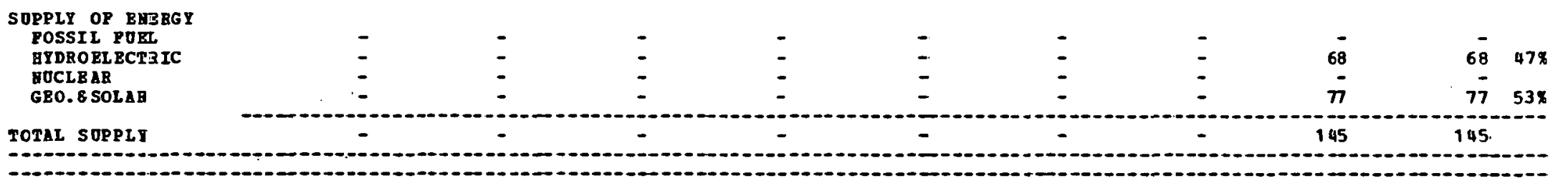

GBT IMPORTS

OF REGION

906

57,947

3. 118

52,234

75.979

3.471

1,205

1,211

196.074

\section{HOTES:}

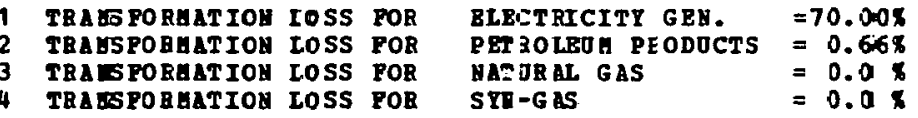


REGIONAL BNERGY BALAYCE STATEHENT

BT SECTOB ABD FOEL TYPE

\begin{tabular}{|c|c|c|c|c|c|c|c|c|c|c|}
\hline SBCTOR & $\begin{array}{l}\text { DISTILLATE } \\
\text { OIL }\end{array}$ & $\begin{array}{l}\text { RESIDOAL } \\
\text { OIL }\end{array}$ & $\begin{array}{l}\text { GASOLIBE } \\
\text { (ALL D }\end{array}$ & $\begin{array}{c}\text { HYORO- } \\
\text { CARBOHS } \\
\text { MITS IH 10**9 }\end{array}$ & $\begin{array}{c}\text { CRODE } \\
\text { OIL } \\
\text { BTO'S) }\end{array}$ & $\begin{array}{l}\text { MAT URAL } \\
\text { GAS }\end{array}$ & COAL & ELECTRICITY & $\begin{array}{r}\text { SECTOR } \\
\text { TOTAL }\end{array}$ & \\
\hline 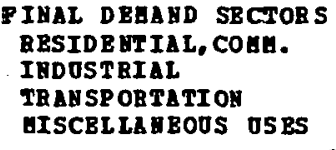 & $\begin{array}{r}3.127,001 \\
802,455 \\
2.159 .099 \\
69.305\end{array}$ & $\begin{array}{r}1,020,388 \\
1,275,558 \\
732.492 \\
245,118\end{array}$ & 13.255 .897 & $\begin{array}{r}2,404,000 \\
3,997,743 \\
2,044,400 \\
105,302\end{array}$ & $=$ & $\begin{array}{r}7.189 .959 \\
10.654 .299 \\
743.604 \\
443.958\end{array}$ & $\begin{array}{r}296,515 \\
4,376,593 \\
2,991 \\
-\end{array}$ & $\begin{array}{r}3,301,138 \\
2,391,149 \\
17,015 \\
84,395\end{array}$ & $\begin{array}{r}17,339,001 \\
23,497,797 \\
18,955,498 \\
948,078\end{array}$ & $\begin{array}{r}24 \% \\
328 \\
268 \\
18\end{array}$ \\
\hline $\begin{array}{l}\text { TOTAL PIBAi } \\
\text { DEAABD SBCTOBS }\end{array}$ & 6.157 .860 & $3,273,556$ & $13.255,897$ & $8,551,445$ & - & 19.031 .820 & $4.676,099$ & $5,793,697$ & $60,740,374$ & $84 \%$ \\
\hline $\begin{array}{l}\text { TRANSPOREATIOH } \\
\text { BLECTRICITT GEY. } \\
\text { PETROLEOE PRODICTS } \\
\text { RATORAL GAS } \\
\text { SYH-GAS }\end{array}$ & $\begin{array}{r}263.019 \\
-5,995,925 \\
-\end{array}$ & $\begin{array}{r}3.219,627 \\
-2,229,350 \\
- \\
-\end{array}$ & $\begin{array}{r}-12,589,071 \\
-\end{array}$ & $\begin{array}{r}78,774 \\
-6,531,169 \\
-1.339,919 \\
-\end{array}$ & $\begin{array}{r}26,316,07 \overline{-} \\
-\end{array}$ & $\begin{array}{r}3.706 .394 \\
1.199 .634 \\
1.539 .992 \\
-\end{array}$ & $\begin{array}{r}8,638,960 \\
= \\
=\end{array}$ & $\begin{array}{r}-4,994,555 \\
= \\
=\end{array}$ & $\begin{array}{r}10,912,219 \\
170.194 \\
200.073\end{array}$ & $\begin{array}{r}15 \% \\
0 \% \\
0 \%\end{array}$ \\
\hline $\begin{array}{l}\text { GBT POBL OSBD } \\
\text { IA TBABSPORGATIOH }\end{array}$ & $-5,732,906$ & 990,277 & $-12,589,071$ & $-7.792,314$ & $26,316,075$ & 6.446 .020 & $8,638,960$ & $-4,994,555$ & $11,282,486$ & $16 x$ \\
\hline $\begin{array}{l}\text { TOTAL GROSS PLORS } \\
\text { LOSSES } 8 \text { OAISSIOHS }\end{array}$ & $\begin{array}{r}6,420,879 \\
390,068\end{array}$ & $\begin{array}{r}6,493,183 \\
-65,890\end{array}$ & $\begin{array}{r}13,255,897 \\
-419,670\end{array}$ & $\begin{array}{r}8,630,219 \\
-407,764\end{array}$ & $\begin{array}{r}26,316,075 \\
39.898\end{array}$ & $\begin{array}{r}25,477,840 \\
96,475\end{array}$ & $\begin{array}{r}13,315,059 \\
221,390\end{array}$ & $\begin{array}{r}5,793,697 \\
463,354\end{array}$ & $\begin{array}{r}72,022,860 \\
317,862\end{array}$ & $\begin{array}{r}100 \% \\
0 \%\end{array}$ \\
\hline
\end{tabular}

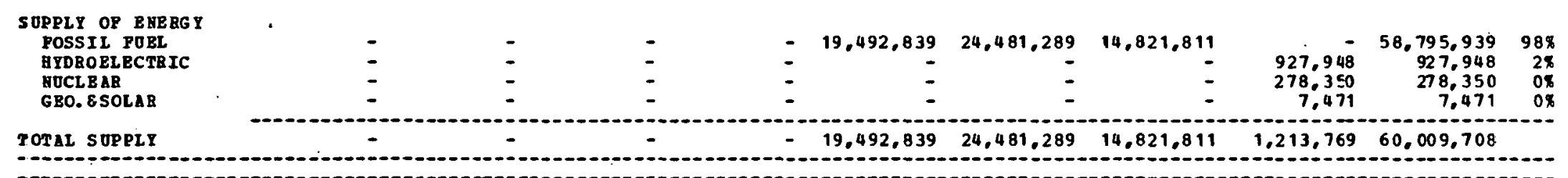

OET IHPORTS
OP RBGIOB


REGIJNLL BNERSY BALANCE STATEEET

GY SBCTOR AND POEL TYPE

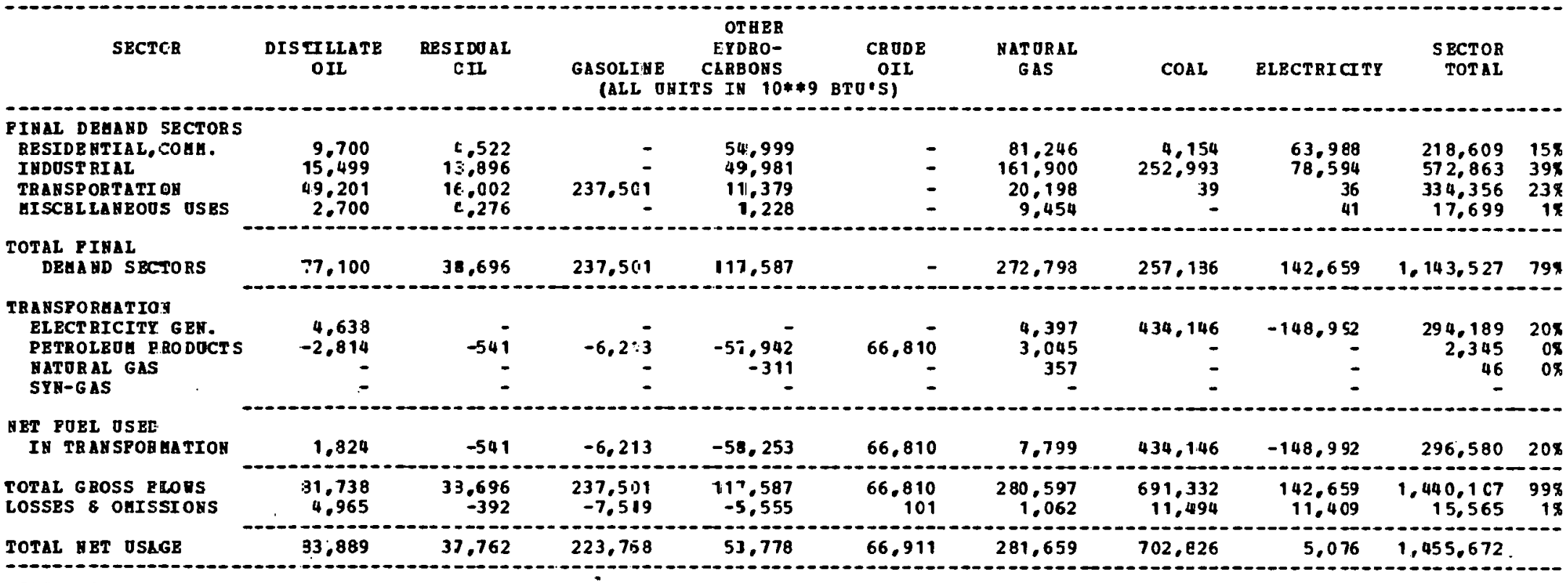

\begin{tabular}{|c|c|c|c|c|c|c|c|c|c|c|}
\hline $\begin{array}{l}\text { POSSI L POBL } \\
\text { HYDROBL BCTRIC } \\
\text { MOCLE AR } \\
\text { GBO. } \text { SOLAB }\end{array}$ & E- & : & $\begin{array}{l}- \\
\overline{-}\end{array}$ & 5 & $\begin{array}{r}67.726 \\
= \\
=\end{array}$ & $\begin{array}{r}14,551 \\
= \\
=\end{array}$ & $\begin{array}{r}495,0131 \\
=\end{array}$ & $\begin{array}{r}40,156 \\
1,072\end{array}$ & $\begin{array}{r}577,306 \\
40,196 \\
1,072\end{array}$ & $\begin{array}{r}938 \\
68 \\
08\end{array}$ \\
\hline
\end{tabular}

NBT IAPORTS
OF REGIOH

83,889

\$7,762

223,768

53,778

$-814$

267,108

207,795

$-36,191$

837,096

\section{NOTBS:}

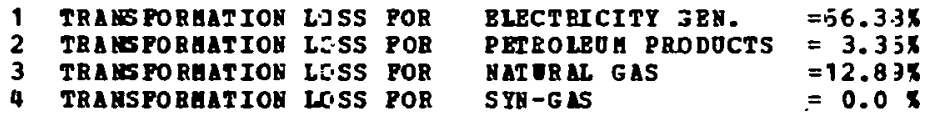


RBGIORAL ENERGT BALANCE STATEHEAT

BY SECTOR AND POBL TYPE

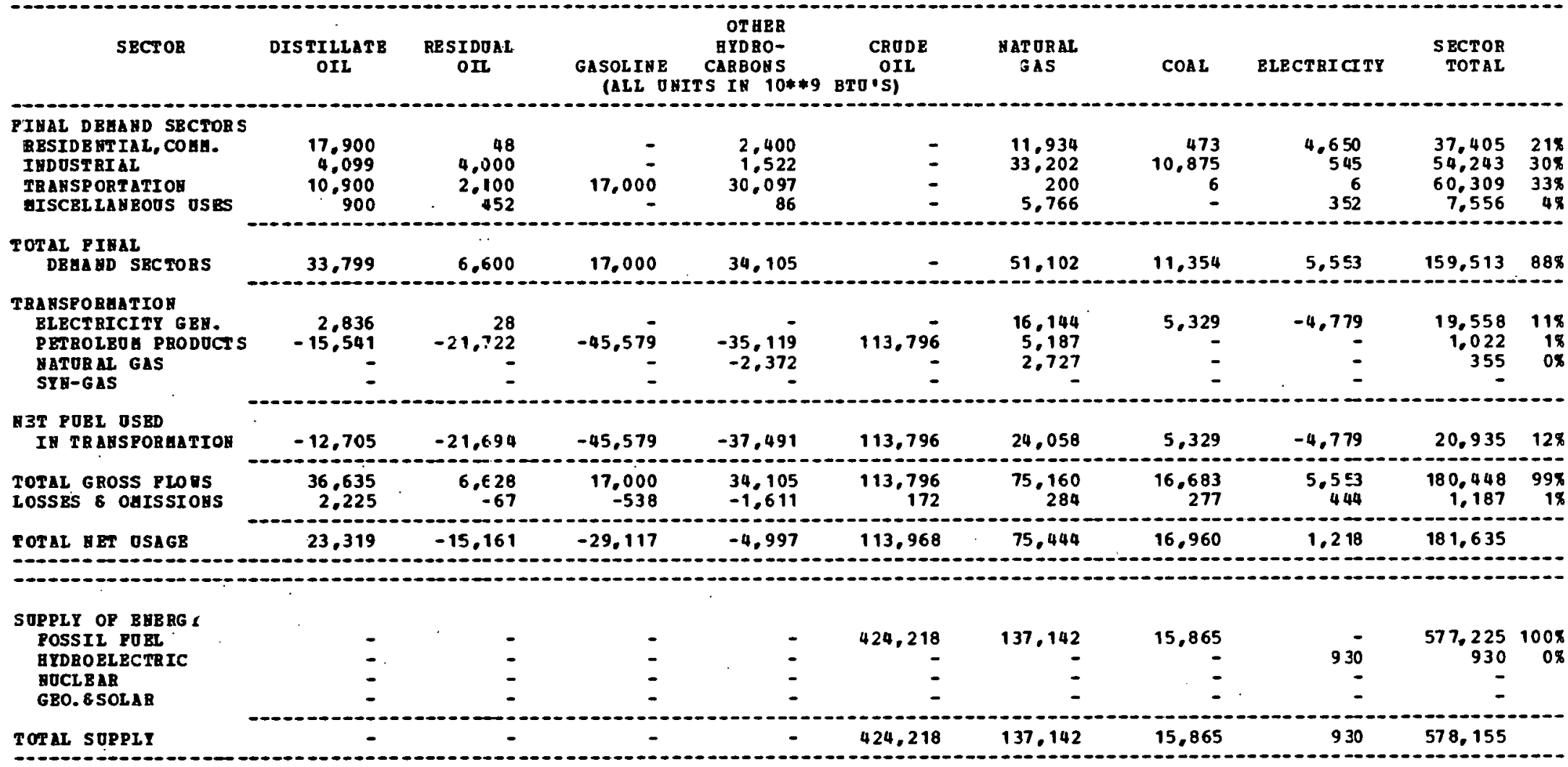

\section{BET IUPOBTS}

53.319

$-4,997$

$-310.249$

$-61,697$

1.095

$288 \quad-396,519$

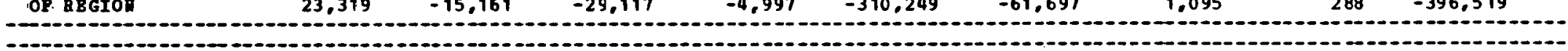

\section{HOT ES:}

1 TRABSPORGATIOM LOSS POB TRAYS FORAATIOY LOSS POR TBABSPORAaTror LOS 4 TBAHS PORAarion lOSS POR $\begin{array}{ll}\text { BLECTRICITY GEN. } & =80.36 \% \\ \text { PETROLEJH PRODUCTS } & =0.86 \%\end{array}$

Buts $=0.86 \%$

$\begin{array}{ll} & =13.02 \%\end{array}$ 
REG ZONAL BNERGY BALANCE STA TEHE HT

BY SECTOR AHD FORL TYPE

\begin{tabular}{|c|c|c|c|c|c|c|c|c|c|c|}
\hline SECTOR & $\begin{array}{l}\text { DIS IILLATB } \\
\text { OII }\end{array}$ & $\begin{array}{l}\text { RRS I DOAL } \\
\text { JII }\end{array}$ & $\begin{array}{l}\text { GASOLIHE } \\
\text { (ALE OHIT }\end{array}$ & $\begin{array}{c}\text { OTHER } \\
\text { AYDRO- } \\
\text { CAR3ONS } \\
\text { S IH 10**9 }\end{array}$ & $\begin{array}{c}\text { CRODE } \\
\text { OIL } \\
\text { BTOיS) }\end{array}$ & $\begin{array}{l}\text { NATUEAL } \\
\text { GAS }\end{array}$ & $\cos 2$ & ELECTRICITE & $\begin{array}{r}\text { SECTOR } \\
\text { TOTAL }\end{array}$ & \\
\hline 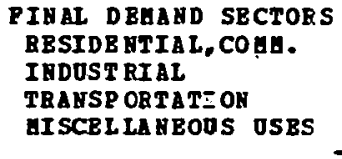 & $\begin{array}{r}4.600 \\
19.201 \\
32.001 \\
600\end{array}$ & $\begin{array}{r}1.497 \\
900 \\
\end{array}$ & 151,400 & $\begin{array}{r}31.800 \\
15,062 \\
21.088 \\
1.339\end{array}$ & $\begin{array}{l}- \\
- \\
-\end{array}$ & $\begin{array}{r}63,530 \\
68,757 \\
24,560 \\
5,968\end{array}$ & $\begin{array}{r}1.078 \\
3.310 \\
22 \\
-\end{array}$ & $\begin{array}{r}42,921 \\
19.737 \\
20 \\
1.196\end{array}$ & $\begin{array}{r}143.929 \\
127,604 \\
229.031 \\
10,003\end{array}$ & $\begin{array}{r}24 \% \\
22 \% \\
398 \\
2 \%\end{array}$ \\
\hline $\begin{array}{l}\text { TOTAL F INAL } \\
\text { DEHA HD SECTORS }\end{array}$ & 56,402 & 2,397 & 151,400 & 69.289 & - & 162,795 & 4.410 & 63.874 & 510,567 & $86 \%$ \\
\hline $\begin{array}{l}\text { TBAHSPORUATION } \\
\text { BLECT RICITT GEM. } \\
\text { PBTROLEOH PRODOCT S } \\
\text { MATORAL GAS } \\
\text { SYH-GAS }\end{array}$ & $\begin{array}{r}2.788 \\
- \\
-\end{array}$ & $\begin{array}{r}13,691 \\
- \\
-\end{array}$ & -168 & $\begin{array}{r}-4.043 \\
-\end{array}$ & $\begin{array}{r}0.217 \\
-\end{array}$ & $\begin{array}{r}57.858 \\
192 \\
- \\
-\end{array}$ & $\begin{array}{r}9.770 \\
- \\
-\end{array}$ & $\begin{array}{r}-34,584 \\
- \\
-\end{array}$ & $\begin{array}{r}79.523 \\
198 \\
- \\
-\end{array}$ & $\begin{array}{r}13.8 \\
0.8\end{array}$ \\
\hline $\begin{array}{l}\text { NET POEL OSED } \\
\text { IN TBABSFOREATIOR }\end{array}$ & 2,788 & 43,691 & -168 & $-4,043$ & 4,217 & 58,350 & 9,770 & $-34,584$ & 79.721 & 138 \\
\hline $\begin{array}{l}\text { TOTAL GROSS RLOHS } \\
\text { LOSSES \& OUISSI OHS }\end{array}$ & $\begin{array}{r}59.190 \\
3.595\end{array}$ & $\begin{array}{r}46.088 \\
-467\end{array}$ & $\begin{array}{r}151,400 \\
-4,793\end{array}$ & $\begin{array}{l}69,289 \\
-3,273\end{array}$ & $\begin{array}{r}4,217 \\
6\end{array}$ & $\begin{array}{r}220,845 \\
836\end{array}$ & 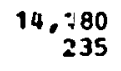 & $\begin{array}{r}63,874 \\
5,108\end{array}$ & $\begin{array}{r}590.288 \\
1.247\end{array}$ & $\begin{array}{r}100 \% \\
0 \%\end{array}$ \\
\hline TOTAL BET OSAGB & 62.785 & $<5,620$ & 146,438 & 61,972 & 4.223 & 221,681 & 14,415 & 34.398 & 591,535 & \\
\hline
\end{tabular}

SOPPLY OP BHERGY

POSSII POEL

EYDROBLBCTRIC

GBO. 8 SOLAE
SOL

$\begin{aligned}- & - \\ - & - \\ - & -\end{aligned}$

$\begin{array}{ll}- & - \\ - & - \\ - & -\end{array}$

4.663

$\begin{array}{r}137 \\ -\quad 70.914 \\ \hline\end{array}$

$\begin{array}{rrr}- & 75.714 & 768 \\ 24.558 & 24.558 & 24 \%\end{array}$

TOTAL SOPPLY

4,663

137

70,314

24,558

100,272

\section{HBT IAPORTS}

OP REGIO\&

62.785

45,620

146,438

61,972

$-439$

221,544

$-56.498$

9.840

491.263

\section{YOTBS:}

\begin{tabular}{|c|c|c|}
\hline Ha AS & & \\
\hline
\end{tabular}


REGIOHAL BHBRG BALANCE STATEHEHT

BY SECTOR AND FOBL TYPE

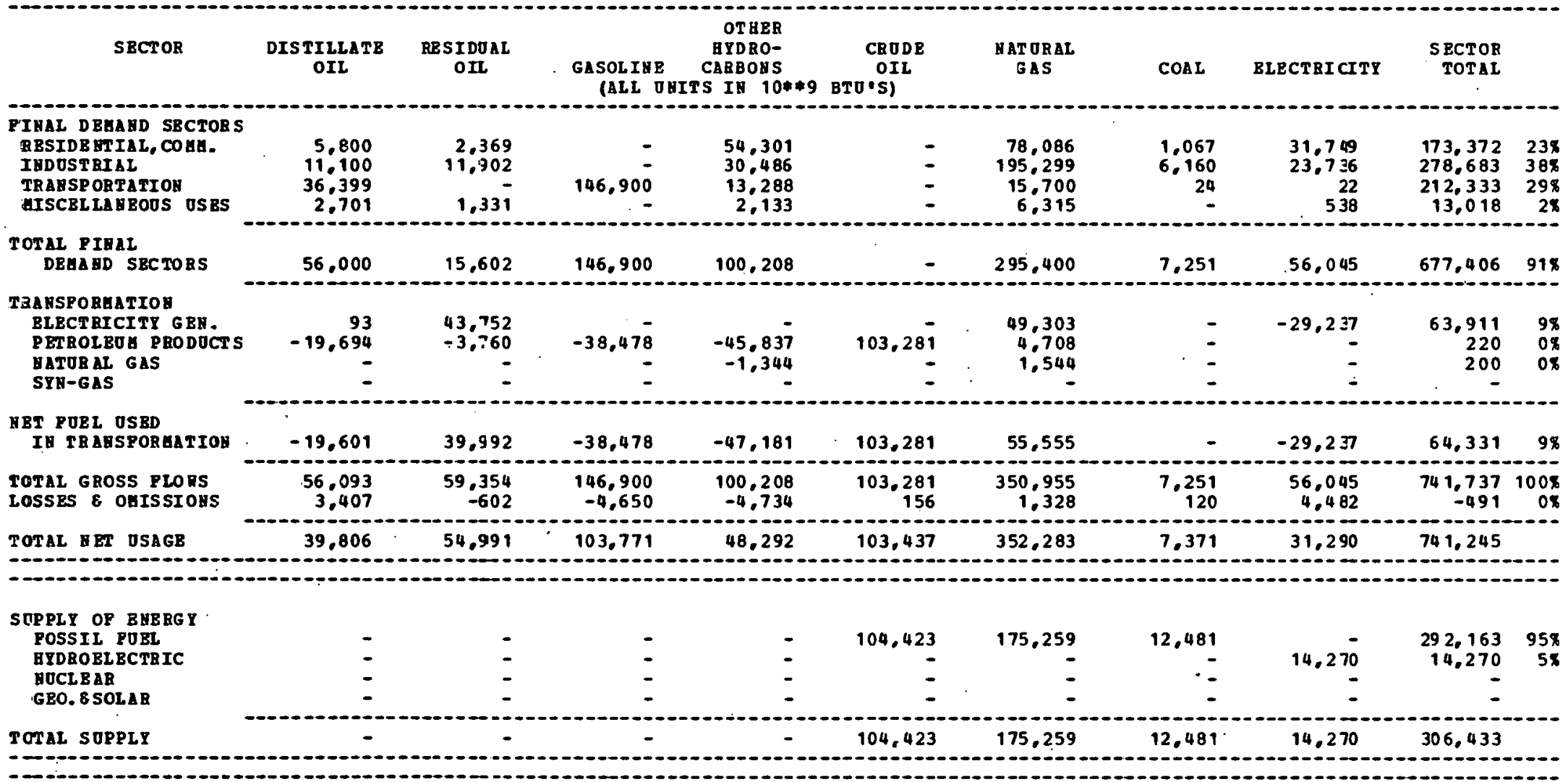

\section{IBT IUPORTS}

OP RBGIOY

39,806

54,991

103.771

48,292

$-985$

177.024

$-5,109$

17,020

434,812

MOTBS :

1 TRAASFORGATION LOSS POR

2 TRABSFORBATIOH LOSS FO

TRABSPORGATION IOSS PO

TRABSPORHATIOA LOSS POR

BLECT RICITY GEN.

$=68.61 x$
$=0.208$

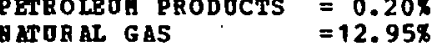

$\begin{array}{ll}\text { SWI-G AS } & =12.95 \% \\ & =0.0 \%\end{array}$ 
REG IONAL ENERGI BALANCE STA TEUERT

BY SECTOR AND EOEL TYPE

\begin{tabular}{|c|c|c|c|c|c|c|c|c|c|c|}
\hline SECTOZ & $\begin{array}{c}\text { DISTILLATE } \\
\text { III }\end{array}$ & $\begin{array}{l}\text { BESI DOAL } \\
\text { OIL }\end{array}$ & $\begin{array}{l}\text { 3ASOLINE } \\
\text { (ALL ONI? }\end{array}$ & 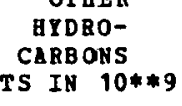 & $\begin{array}{c}\text { CRODE } \\
\text { OIL } \\
\text { BPO'S) }\end{array}$ & $\begin{array}{l}\text { NATORAL } \\
\text { GAS }\end{array}$ & COAL & ELECTRI CITY & $\begin{array}{l}\text { SECTOR } \\
\text { TOTAL }\end{array}$ & \\
\hline $\begin{array}{l}\text { PIHAL DBLAND SBCTORS } \\
\text { RESIDE NTIAL, COAL. } \\
\text { IHDOSTRIAL } \\
\text { TRANSPORTATIOS } \\
\text { MISCELLAHEOOS OSES }\end{array}$ & $\begin{array}{r}24.999 \\
62.606 \\
206.500 \\
2.801\end{array}$ & $\begin{array}{r}21,075 \\
75,197 \\
129,201 \\
6.425\end{array}$ & $1,302,601$ & $\begin{array}{r}114.300 \\
258.636 \\
235.036 \\
11.600\end{array}$ & $\begin{array}{l}- \\
-\end{array}$ & $\begin{array}{r}807,705 \\
733.601 \\
20,201 \\
58,593\end{array}$ & $\begin{array}{r}10,800 \\
111,489 \\
304\end{array}$ & $\begin{array}{r}316,106 \\
166,743 \\
459 \\
12,498\end{array}$ & $\begin{array}{r}1.294 .985 \\
1.408 .272 \\
1.940 .302 \\
91.917\end{array}$ & $\begin{array}{r}248 \\
268 \\
36 \% \\
28\end{array}$ \\
\hline $\begin{array}{l}\text { TOTAL P INAL } \\
\text { DEUA HD SECTORS }\end{array}$ & $252,906^{\circ}$ & 231,898 & $1,302,601$ & 669,572 & - & $1,620,1 C 0$ & 122,593 & 495,806 & $4,735,476$ & 37x \\
\hline $\begin{array}{l}\text { TRANSPORGATIOY } \\
\text { BLECT RICITY GEN. } \\
\text { PETROLEUA PEODOCT S } \\
\text { NATORAL GAS } \\
\text { SYN-GAS }\end{array}$ & $\begin{array}{r}1.465 \\
-457.793 \\
- \\
-\end{array}$ & $\begin{array}{r}476,637 \\
-640,029 \\
- \\
-\end{array}$ & $\begin{array}{r}-1,348,208 \\
-\end{array}$ & $\begin{array}{r}50.017 \\
-960.264 \\
-24.804 \\
-\end{array}$ & $\begin{array}{r}3.338,927 \\
- \\
-\end{array}$ & $\begin{array}{r}448,516 \\
91,104 \\
28,509 \\
-\end{array}$ & $\begin{array}{l}\overline{-} \\
\overline{-}\end{array}$ & $\begin{array}{r}-316,601 \\
= \\
-\end{array}$ & $\begin{array}{r}660,034 \\
23,737 \\
3,705 \\
-\end{array}$ & $\begin{array}{r}128 \\
0 \% \\
0 \%\end{array}$ \\
\hline $\begin{array}{l}\text { SET POEL OSED } \\
\text { IA TRANSP OR WATION }\end{array}$ & $-45 j, 328$ & -163.392 & $-1,348,20.3$ & -935.051 & $3,338,927$ & 568,129 & - & $-316,601$ & 687,476 & $13 \%$ \\
\hline $\begin{array}{l}\text { TOTAL GROSS PLONS } \\
\text { LOSSES } \& \text { OHISSI OHS }\end{array}$ & $\begin{array}{r}294,371 \\
17,883\end{array}$ & $\begin{array}{r}7031.535 \\
-7.18 \mathrm{G}\end{array}$ & $\begin{array}{r}1.302,601 \\
-41,239\end{array}$ & $\begin{array}{l}719,589 \\
-33,999\end{array}$ & $\begin{array}{r}3.338 .927 \\
5,062\end{array}$ & $\begin{array}{r}2,188,225 \\
8.236\end{array}$ & $\begin{aligned} & 122,553 \\
& 2,0 \equiv 8\end{aligned}$ & $\begin{array}{r}495,806 \\
39,6 \leq 2\end{array}$ & $\begin{array}{r}5,422,952 \\
-9,506\end{array}$ & $\begin{array}{r}100 x \\
0 \%\end{array}$ \\
\hline TOTAL WET OSAGE & $-145,538$ & 61,316 & -86.846 & $-299,478$ & $3,343,989$ & $2,196,515$ & $124,6 \leqslant 1$ & 218,857 & 5.413 .445 & \\
\hline
\end{tabular}

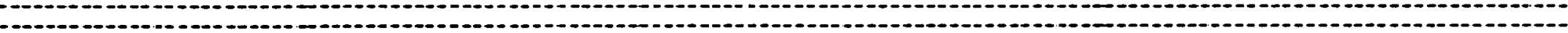

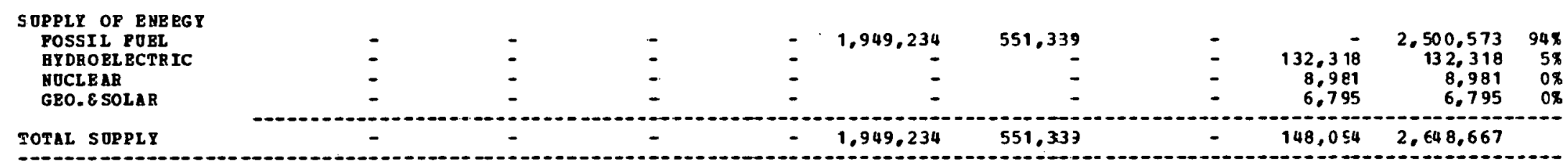

-

\begin{tabular}{|c|c|c|c|c|c|c|c|c|c|}
\hline OP BEGIO\& & $-145,538$ & 61,316 & $-86,8<6$ & $-295,478$ & $1,394,755$ & $1,645.175$ & 124,631 & 70.763 & 2.764 .778 \\
\hline
\end{tabular}

MOTES:

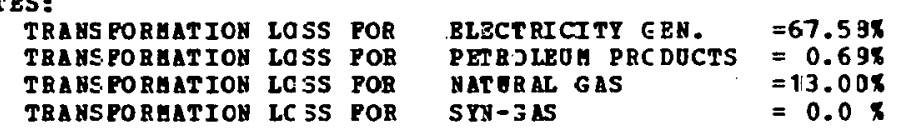


REGIONAL BNEBGY BALARCE STATEGENT

BY SECTOB AND POEL TYPE

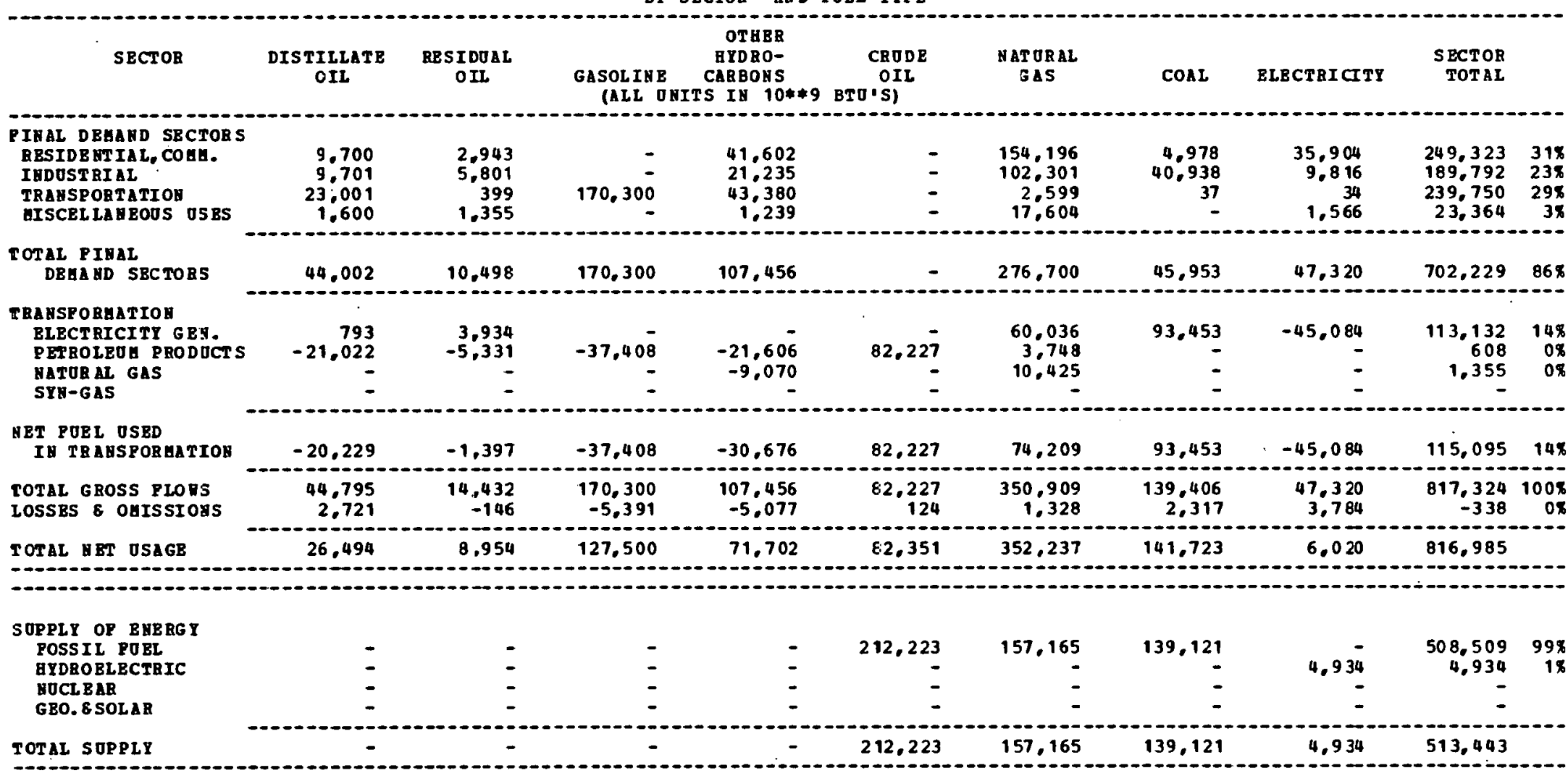

10

\begin{tabular}{|c|c|c|c|c|c|c|c|c|c|}
\hline OP B BGION & 26,494 & 8,954 & 127.500 & 71.702 & $-129,871$ & 195,072 & 2,602 & 1.086 & 303,542 \\
\hline
\end{tabular}

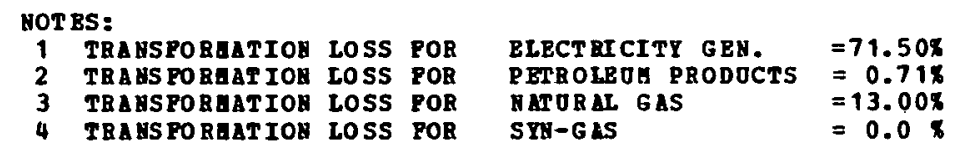


REGIOHAL BHBZGY BALANCE STA TEHENT

BY SECTOR AND FOEL TYPE

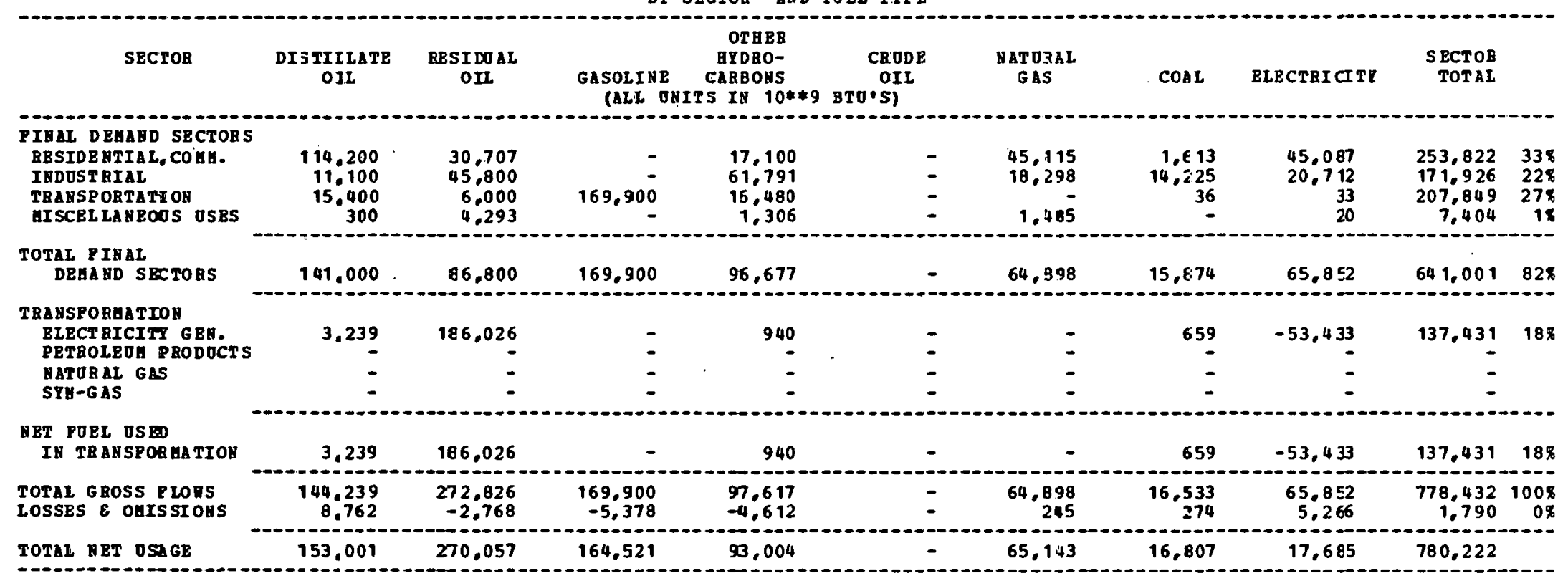

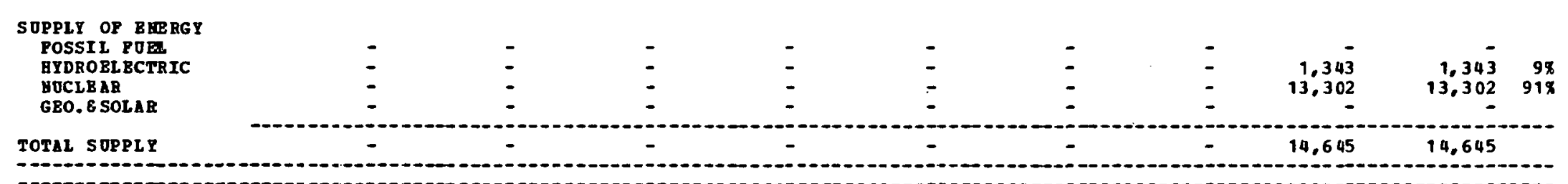

\section{NET IAPORTS}

153.001

270.057

164,521

53, 004

$65,1+3$

16.807

3.040

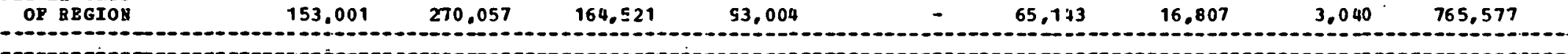

\section{TES:}

TRA HS PORLATIOH LSSS POR TRALSPORAATIOR LOSS POR TRARS PO RHAT IOR LOSS POB

TRA ASPORAATIOY LOSS POR
BLECTRICITY SEN. $\quad=72.0 .2 x$

BREOLPOI PRODOCTS $=0.0$ ATORAL GAS

$=0.0$ 
REGIONAL BHERGY BALANCE STATEMEHT

IONAL BUERGY BALANCE STATEGE
BY SECTOE AND FOEL TYPE

\begin{tabular}{|c|c|c|c|c|c|c|c|c|c|}
\hline SECTOR & $\begin{array}{l}\text { DISTILLATB } \\
\text { OIL }\end{array}$ & $\begin{array}{l}\text { RESIDOAL } \\
\text { OIL }\end{array}$ & $\begin{array}{l}\text { GASOL INE } \\
\text { (ALL OHIT }\end{array}$ & $\begin{array}{l}\text { BYDBO- } \\
\text { CARBOAS } \\
\text { S IB } 10 * * 9\end{array}$ & $\begin{array}{c}\text { CRUDE } \\
\text { OIL } \\
\text { BTO'S) }\end{array}$ & $\begin{array}{l}\text { MaTORAL } \\
\text { GAS }\end{array}$ & COAL & ELECTRICITI & $\begin{array}{l}\text { SECTOR } \\
\text { TOTAL }\end{array}$ \\
\hline $\begin{array}{l}\text { PINAI DBGAMD SBCTORS } \\
\text { RESIDB HTIAL, COBA. } \\
\text { IHDOSTRIAL } \\
\text { TRANSPORTATIOH } \\
\text { UISCBLLAHBODS OSBS }\end{array}$ & $\begin{array}{r}18,600 \\
2.598 \\
2,301 \\
300\end{array}$ & $\begin{array}{r}5.595 \\
23.102 \\
7.800 \\
8.804\end{array}$ & $\begin{array}{r}\overline{-} \\
38,600 \\
-\end{array}$ & $\begin{array}{r}6.800 \\
10.214 \\
1.797 \\
551\end{array}$ & - & $\begin{array}{r}10,270 \\
10,501 \\
531\end{array}$ & $\begin{array}{r}302 \\
2,236 \\
8 \\
-\end{array}$ & $\begin{array}{r}8,999 \\
9,913 \\
7 \\
5\end{array}$ & $\begin{array}{l}50,566 \\
58,564 \\
50,513 \\
10,191\end{array}$ \\
\hline $\begin{array}{l}\text { TOTAL PIHAL } \\
\text { DEEA AD SBCTORS }\end{array}$ & 23,799 & 45.301 & 38.600 & 19,362 & - & 21,302 & 2,546 & 18,924 & 169,834 \\
\hline $\begin{array}{l}\text { TRAYSPOREATION } \\
\text { ELECTRICITY GER. } \\
\text { PETROLBOA PRODUCTS } \\
\text { RATORAL GAS } \\
\text { STH-GAS }\end{array}$ & $\begin{array}{r}1.097 \\
-77.158 \\
-\end{array}$ & $\begin{array}{r}36,149 \\
-29,605 \\
- \\
-\end{array}$ & $\begin{array}{r}-126,975 \\
-\end{array}$ & $\begin{array}{r}7.625 \\
-30.206 \\
= \\
-\end{array}$ & $\begin{array}{r}253.414 \\
-\end{array}$ & $\begin{array}{r}1,060 \\
11,552 \\
-\end{array}$ & $\begin{array}{r}23.618 \\
- \\
=\end{array}$ & $\begin{array}{r}-19,762 \\
= \\
-\end{array}$ & $\begin{array}{r}49,787 \\
1,022 \\
=\end{array}$ \\
\hline $\begin{array}{l}\text { DET FOBL USBD } \\
\text { IH TRABSPOBGATIOH }\end{array}$ & $-76,061$ & 6,544 & $-126,975$ & $-22,581$ & 253.414 & 12,612 & 23,618 & $-19,762$ & 50,809 \\
\hline $\begin{array}{l}\text { TOTAL GROSS PLORS } \\
\text { LOSSES } 8 \text { OUISSIOUS }\end{array}$ & $\begin{array}{r}24.896 \\
1.512\end{array}$ & $\begin{array}{r}81.450 \\
-826\end{array}$ & $\begin{array}{l}38.600 \\
-1,222\end{array}$ & $\begin{array}{l}26,987 \\
-1,275\end{array}$ & $\begin{array}{r}253.414 \\
384\end{array}$ & $\begin{array}{r}33.914 \\
128\end{array}$ & $\begin{array}{r}26,164 \\
435\end{array}$ & $\begin{array}{rl}18,9 & 24 \\
1,513\end{array}$ & $\begin{array}{r}220.643 \\
649\end{array}$ \\
\hline
\end{tabular}

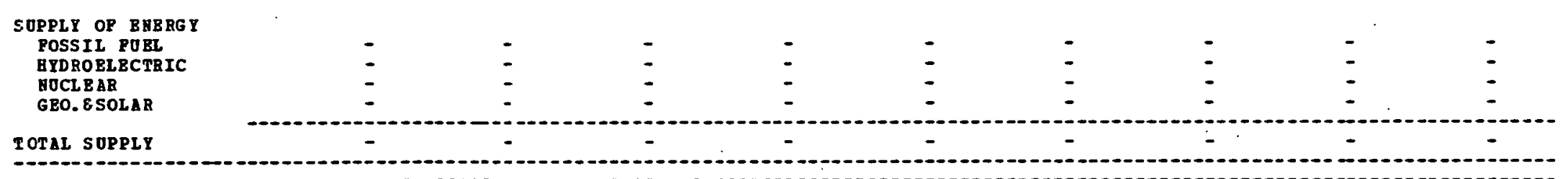

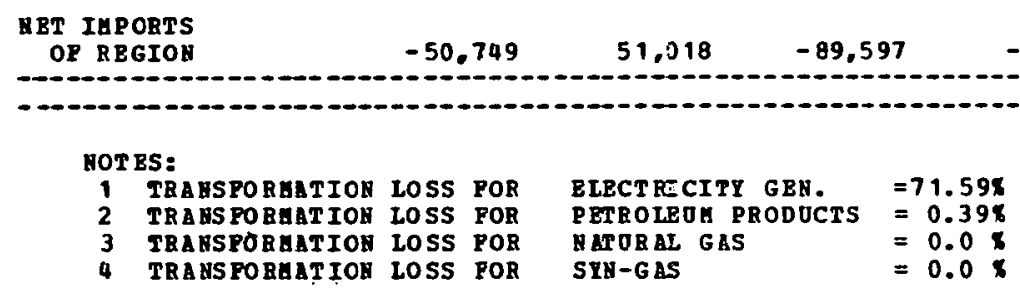


REGIONAL ENERGT BALAGCE STATEGEHT

BY SECTOR AHD FOEL TYPE

\begin{tabular}{|c|c|c|c|c|c|c|c|c|c|c|}
\hline SECTOR & $\begin{array}{l}\text { DISTJLLATE } \\
\text { CIL }\end{array}$ & $\begin{array}{l}\text { BFSIDOAL } \\
\text { OI }\end{array}$ & $\begin{array}{r}\text { GASOLINE } \\
\text { (ALL O }\end{array}$ & $\begin{array}{c}\text { OTHER } \\
\text { HYDBO- } \\
\text { CARBOHS } \\
\text { CS IH } 10 * * 9\end{array}$ & $\begin{array}{c}\text { CRUDE } \\
\text { OIL } \\
\text { BTO'S) }\end{array}$ & $\begin{array}{l}\text { NATURAL } \\
\text { GAS }\end{array}$ & COAL & ELECTRICITY & $\begin{array}{l}\text { SECTOE } \\
\text { TOTAL }\end{array}$ & \\
\hline $\begin{array}{l}\text { PINAL DBHAHD SBCTORS } \\
\text { RESIDE HTIAL, COUA. } \\
\text { IHDUSTRIAL } \\
\text { TRAHSP ORTATI ON } \\
\text { GISCELLAHEOOS USES }\end{array}$ & $\begin{array}{r}13.900 \\
399 \\
3.800 \\
500\end{array}$ & $\begin{array}{r}11,376 \\
799 \\
1,100 \\
23,125\end{array}$ & 31.700 & $\begin{array}{r}100 \\
- \\
-\end{array}$ & $\begin{array}{l}\overline{-} \\
\overline{-}\end{array}$ & $\begin{array}{r}1.094 \\
= \\
228\end{array}$ & $\begin{array}{r}134 \\
- \\
-\end{array}$ & $\begin{array}{r}6,425 \\
1,562 \\
- \\
10\end{array}$ & $\begin{array}{r}33,629 \\
2,960 \\
36,600 \\
24,169\end{array}$ & $\begin{array}{r}26 \% \\
2 \% \\
29 \% \\
19 \%\end{array}$ \\
\hline $\begin{array}{l}\text { TOTAL PIHAI } \\
\text { DEHA HD SBCTORS }\end{array}$ & 13,599 & 37.500 & 31,700 & 100 & - & 1,322 & 134 & 7.997 & 97.352 & $76 \%$ \\
\hline $\begin{array}{l}\text { TRABSPORHATIOH } \\
\text { BLECTRICITY GEN. } \\
\text { PETROLEOE PRODOCTS } \\
\text { NATORAL GAS } \\
\text { STH-GAS }\end{array}$ & $\begin{array}{r}1,418 \\
= \\
=\end{array}$ & $\begin{array}{r}33,617 \\
- \\
-\end{array}$ & $\overline{-}$ & $\begin{array}{l}- \\
-\end{array}$ & 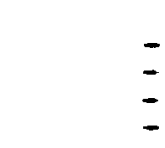 & $\overline{-}$ & $\begin{array}{r}6,835 \\
- \\
-\end{array}$ & $\begin{array}{r}-11,137 \\
- \\
-\end{array}$ & $\begin{array}{r}30.733 \\
- \\
-\end{array}$ & 248 \\
\hline $\begin{array}{l}\text { HET POBL OSED } \\
\text { IN TBAHSEORLATION }\end{array}$ & 1,418 & 33,617 & - & - & - & - & 6,835 & $-11,137$ & 30,733 & 248 \\
\hline $\begin{array}{l}\text { TOTAL GROSS PLONS } \\
\text { LOSSES \& OAISSIOOSS }\end{array}$ & $\begin{array}{l}23,017 \\
1,216\end{array}$ & $\begin{array}{r}71.117 \\
-721\end{array}$ & $\begin{array}{l}31,700 \\
-1,003\end{array}$ & $\begin{array}{r}100 \\
-4\end{array}$ & - & $\begin{array}{r}1,322 \\
5\end{array}$ & $\begin{array}{r}6.969 \\
115\end{array}$ & $\begin{array}{r}7,997 \\
639\end{array}$ & $\begin{array}{r}128.085 \\
246\end{array}$ & $\begin{array}{r}100 \% \\
0 \%\end{array}$ \\
\hline
\end{tabular}

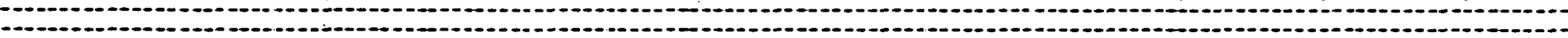

\section{SOPPLY OP EHERG}

POSSIL POEL

HYDROBI ECTRIC

NOCLE AR

GEO.E SOLAB

TOTAL SOPPLY

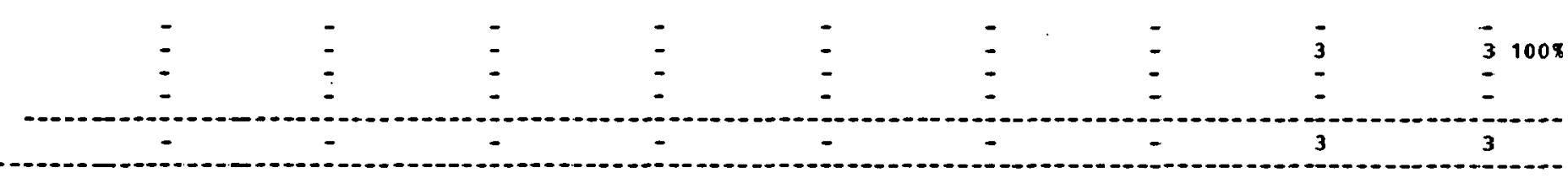

\section{MET IHPORTS}

OF BEGIOY

$21.233 \quad 70.395 \quad 30.696$

95

1,327

7,084

$-2.503$

128,328

\section{NOTES:}

1 TREAS POREAT ION LOSS POR

TRABSPORAATIOA LOSS FOR

TRA US PORAATIOA LOSS POR

TRANSPORAATION LOSS POB
ELBCT 3ICITE GBS.

$=73.40 \mathrm{~K}$
$=0.0 \%$

列 $=0.0 \mathrm{x}$ 


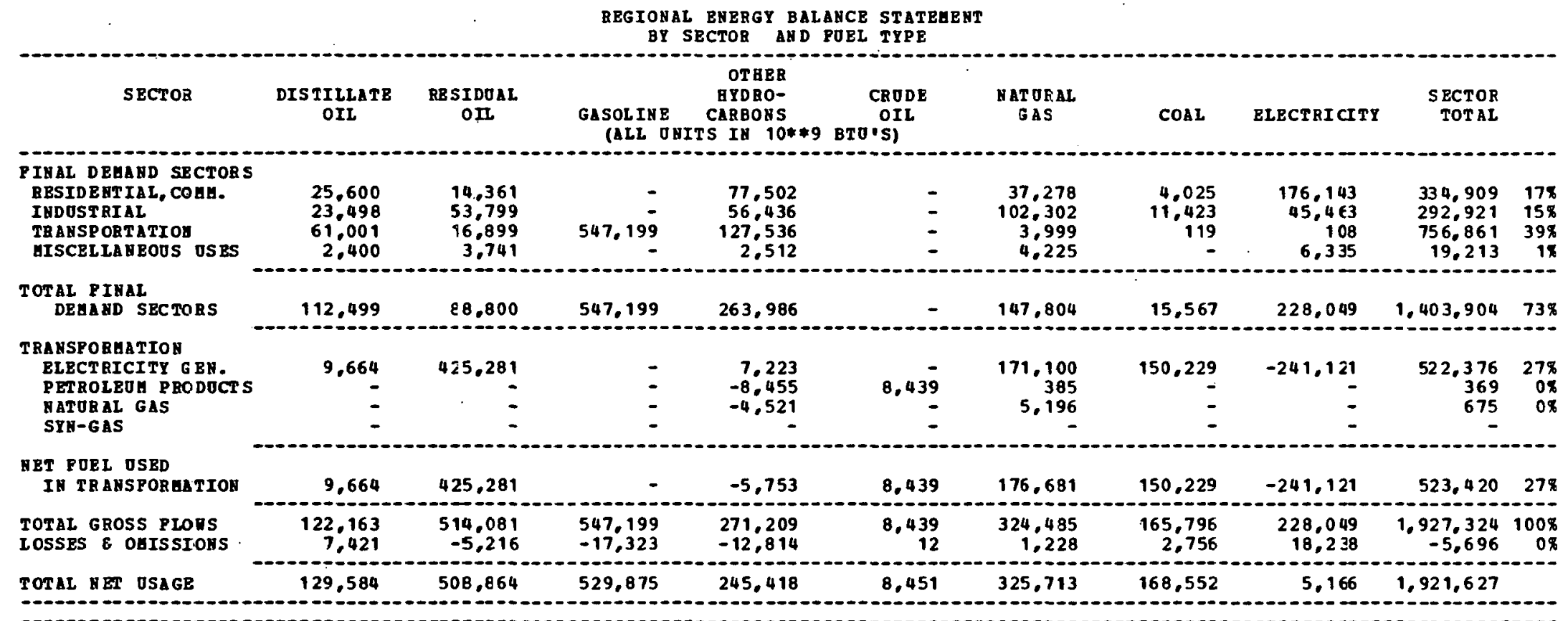

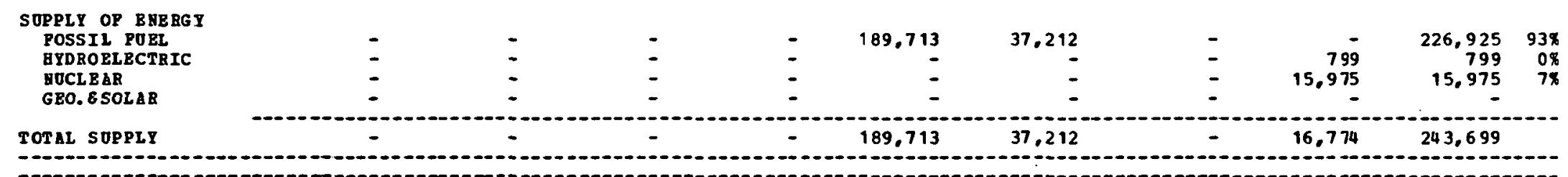

\section{BT IHPORTS}

129,584

508,864

529,875

245,418

$-181,261$

288,501

168,552

$-11,607 \quad 1,677,928$

BOT ES:

\begin{tabular}{|c|c|c|c|c|}
\hline $\begin{array}{l}\text { rov } \\
\text { ION }\end{array}$ & $\begin{array}{l}\text { LoSS } \\
\text { LOSS } \\
\text { LOSS } \\
\text { LOSS }\end{array}$ & & $\begin{array}{l}\text { BLECTRICITY GEN. } \\
\text { PERBOLEOA PRODOCTS } \\
\text { M MTOEAL GAS } \\
\text { SWH-G AS }\end{array}$ & $\begin{array}{l}=68.42 \pi \\
=4.18 \% \\
=12.99 \% \\
=0.0 \times\end{array}$ \\
\hline
\end{tabular}


REGIOHAL ENBRGY BALANCE STA TEGEHT

BY SECTOR AND FOEL TYPE

\begin{tabular}{|c|c|c|c|c|c|c|c|c|c|c|}
\hline SECTOR & $\begin{array}{c}\text { DISTIILATE } \\
\text { OIL }\end{array}$ & $\begin{array}{c}\text { BBS I DO AL } \\
\text { OIL }\end{array}$ & $\begin{array}{r}\text { GASOLI HE } \\
\text { (ALL O }\end{array}$ & $\begin{array}{c}\text { OTHEB } \\
\text { HYDBO- } \\
\text { CABBONS } \\
\text { TS IN } 10 * * 9\end{array}$ & $\begin{array}{c}\text { CRODE } \\
\text { OIL } \\
\text { BTO'S) }\end{array}$ & $\begin{array}{c}\text { NATURAL } \\
\text { GAS }\end{array}$ & $\operatorname{cosL}$ & ELECTRI CITY & $\begin{array}{r}\text { SECTOR } \\
\text { TOTAL }\end{array}$ & \\
\hline 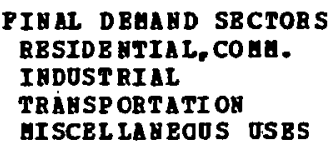 & $\begin{array}{r}17.400 \\
17.002 \\
65.500 \\
999\end{array}$ & $\begin{array}{r}12.247 \\
45.701 \\
3.638 \\
3.756\end{array}$ & 354.701 & $\begin{array}{r}53.601 \\
69.662 \\
73.060 \\
5.256\end{array}$ & $\begin{array}{l}\overline{-} \\
\overline{-}\end{array}$ & $\begin{array}{r}124.903 \\
173,696 \\
6,201 \\
11.296\end{array}$ & $\begin{array}{r}4,308 \\
25,025 \\
75 \\
-\end{array}$ & $\begin{array}{r}89.333 \\
48,732 \\
69 \\
257\end{array}$ & $\begin{array}{r}301.792 \\
379.818 \\
503.304 \\
21.564\end{array}$ & $\begin{array}{r}21 \% \\
26 \% \\
35 \% \\
2 x\end{array}$ \\
\hline $\begin{array}{l}\text { TOTAL PIBAL } \\
\text { DEHABD SBCTOAS }\end{array}$ & 100,901 & 65.402 & 354.701 & 201,579 & - & 316,096 & 29,408 & 138,391 & $1,206,478$ & $84 \%$ \\
\hline $\begin{array}{l}\text { TRAHSPORHATIOH } \\
\text { BLECTRICITY GBH. } \\
\text { PETROLEOA PBODOCTS } \\
\text { HATOR AL GAS } \\
\text { SYH-GAS }\end{array}$ & $\begin{array}{r}15,013 \\
- \\
-\end{array}$ & $\begin{array}{r}24.466 \\
= \\
=\end{array}$ & $\begin{array}{l}\overline{-} \\
\overline{-}\end{array}$ & $\begin{array}{r}-24.505 \\
-\end{array}$ & $\begin{array}{r}24,45 \overline{-} \\
- \\
-\end{array}$ & $\begin{array}{r}35.115 \\
1.115 \\
-\end{array}$ & $\begin{array}{r}255,579 \\
- \\
-\end{array}$ & $\begin{array}{r}-105.669 \\
- \\
-\end{array}$ & $\begin{array}{r}224.504 \\
1.068 \\
=\end{array}$ & $\begin{array}{r}16 \% \\
0 \times\end{array}$ \\
\hline $\begin{array}{l}\text { HET FOBL OS 3D } \\
\text { IN TEAYSPOREATION }\end{array}$ & 15,013 & 24.466 & - & -24.505 & 24,458 & 36,230 & 255,579 & $-105,669$ & $225.57 \bar{z}$ & $16 \%$ \\
\hline $\begin{array}{l}\text { TOTAI GROSS PLOES } \\
\text { LOSSES } 8 \text { OAISSI OHS }\end{array}$ & $\begin{array}{r}115,914 \\
7,041\end{array}$ & $\begin{array}{r}89,868 \\
-911\end{array}$ & $\begin{array}{l}354,701 \\
-11.229\end{array}$ & $\begin{array}{r}201,579 \\
-9,524\end{array}$ & $\begin{array}{r}24.458 \\
37\end{array}$ & $\begin{array}{r}352,326 \\
1,334\end{array}$ & $\begin{array}{r}284.987 \\
4.738\end{array}$ & $\begin{array}{r}138,391 \\
11,067\end{array}$ & $\begin{array}{r}1.432 .056 \\
2.55 \vdots\end{array}$ & $\begin{array}{r}100 \% \\
08\end{array}$ \\
\hline
\end{tabular}

0
0

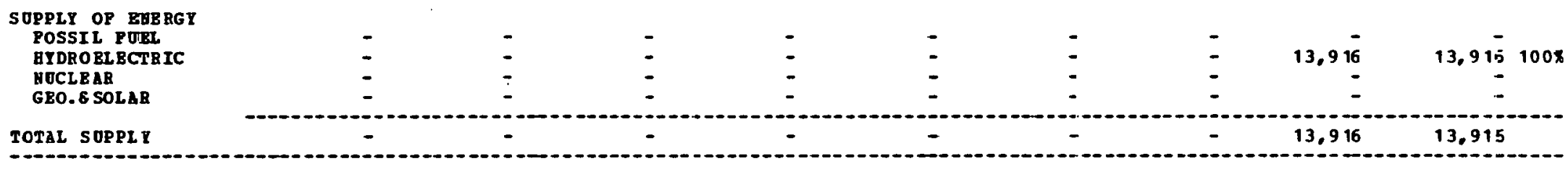

\begin{tabular}{|c|c|c|c|c|c|c|c|c|c|}
\hline $\begin{array}{l}\text { T IAPOATS } \\
\text { OP RBIOE }\end{array}$ & 122.955 & 88,956 & 343,471 & 167,549 & 24.495 & 353,660 & 289.725 & 29.873 & $1,420,687$ \\
\hline
\end{tabular}

\section{MOTBS:}

1 TRABSFORAATIOH IOSS POR TRABSPOBAATIOH LOSS POR

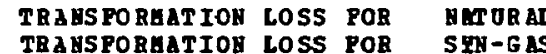

BIECTRICITI GEH

$=68.00 x$ 
REGIONAL RHERGY BALAHCE STATEAENT

BY SECTOR AND POEL TYPE

\begin{tabular}{|c|c|c|c|c|c|c|c|c|c|c|}
\hline SBCTOR & $\begin{array}{l}\text { DISTILLATE } \\
\text { OIL }\end{array}$ & $\begin{array}{l}\text { RESIDOAL } \\
\text { OIL }\end{array}$ & $\begin{array}{l}\text { GASOLINE } \\
\text { (ALI. ONI? }\end{array}$ & $\begin{array}{c}\text { OTBER } \\
\text { BYDBO- } \\
\text { CABBONS } \\
\text { TS IN 10**9 }\end{array}$ & $\begin{array}{c}\text { CRODE } \\
\text { OIL } \\
\text { BTO'S) }\end{array}$ & $\begin{array}{l}\text { HAT URAL } \\
\text { GAS }\end{array}$ & COAL & ELECTRICITY & $\begin{array}{r}\text { SECTOR } \\
\text { TOTAL }\end{array}$ & \\
\hline $\begin{array}{l}\text { F IHAL DEGAND SBCTOBS } \\
\text { RESIDERTIAL, COHA. } \\
\text { IBDOSTRIAL } \\
\text { TRANSPOETATION } \\
\text { GISCEL LABEOUS OSES }\end{array}$ & $\begin{array}{r}600 \\
3.901 \\
4.199 \\
300\end{array}$ & $\begin{array}{r}349 \\
7.300 \\
8.700 \\
2.851\end{array}$ & 34,600 & $\begin{array}{r}4.700 \\
4.557 \\
70.592 \\
218\end{array}$ & $\overline{-}$ & $\overline{-}$ & $\begin{array}{r}436 \\
734 \\
16 \\
-\end{array}$ & $\begin{array}{r}7,966 \\
8,007 \\
15 \\
5 \mathrm{E1}\end{array}$ & $\begin{array}{r}14,051 \\
24,499 \\
118,122 \\
3,950\end{array}$ & $\begin{array}{r}7 \% \\
128 \\
608 \\
28\end{array}$ \\
\hline $\begin{array}{l}\text { TOTAL PIMAL } \\
\text { DEBABD SECTORS }\end{array}$ & 9,000 & 19,200 & 34,600 & 80.067 & - & - & 1,186 & 16,569 & 160,622 & $82 x$ \\
\hline $\begin{array}{l}\text { TRARSFOREATIOH } \\
\text { ELBCTRICITY GBA. } \\
\text { PETROLBOA PRODUCTS } \\
\text { MATURAI GAS } \\
\text { SYH-GAS }\end{array}$ & $\begin{array}{r}1,432 \\
-10,159 \\
-\end{array}$ & $\begin{array}{r}53,689 \\
-14,202 \\
- \\
-\end{array}$ & $\begin{array}{r}-30,386 \\
-\end{array}$ & $\begin{array}{r}-24,049 \\
-\end{array}$ & $\begin{array}{r}75.864 \\
-\end{array}$ & $\begin{array}{r}3.45 \overline{8} \\
-\end{array}$ & $\bar{z}$ & $\begin{array}{r}-16,538 \\
= \\
-\end{array}$ & $\begin{array}{r}38.583 \\
526 \\
- \\
-\end{array}$ & $\begin{array}{r}20 \% \\
0 \%\end{array}$ \\
\hline $\begin{array}{l}\text { SET POEL OSED } \\
\text { IS TRABSFORGATIOH }\end{array}$ & $-8,727$ & 39,487 & $-30,386$ & -24.049 & 75.864 & 3.458 & - & $-16,538$ & 39,109 & $20 \%$ \\
\hline $\begin{array}{l}\text { TOTAL GROSS PLORS } \\
\text { LOSSES } 8 \text { OUISSIOMS }\end{array}$ & $\begin{array}{r}10.432 \\
633 \\
\end{array}$ & $\begin{array}{r}72,889 \\
-739\end{array}$ & $\begin{array}{r}34.600 \\
-1.095\end{array}$ & $\begin{array}{l}80,067 \\
-3,783\end{array}$ & $\begin{array}{r}75.864 \\
115\end{array}$ & $\begin{array}{r}3.458 \\
13\end{array}$ & $\begin{array}{r}1,186 \\
19\end{array}$ & $\begin{array}{r}16.569 \\
1.325\end{array}$ & $\begin{array}{r}199,731 \\
-3,511\end{array}$ & $\begin{array}{r}1028 \\
-18\end{array}$ \\
\hline
\end{tabular}

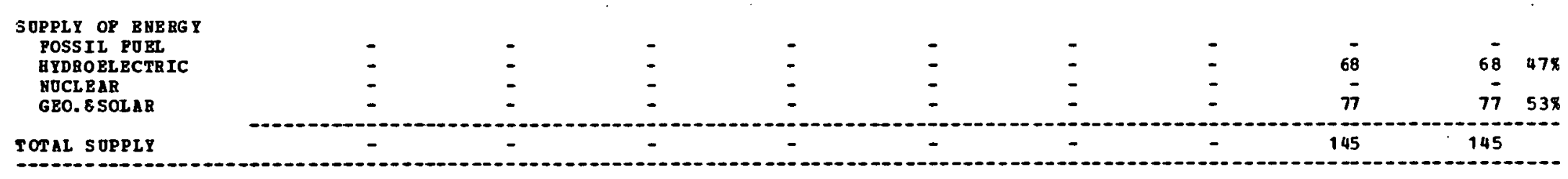

YET IUPORTS
OP REGIO\&


RBFIONAL ENBRGT BALANCE STATEUENT

BY SECTOR AND POEL TYPE

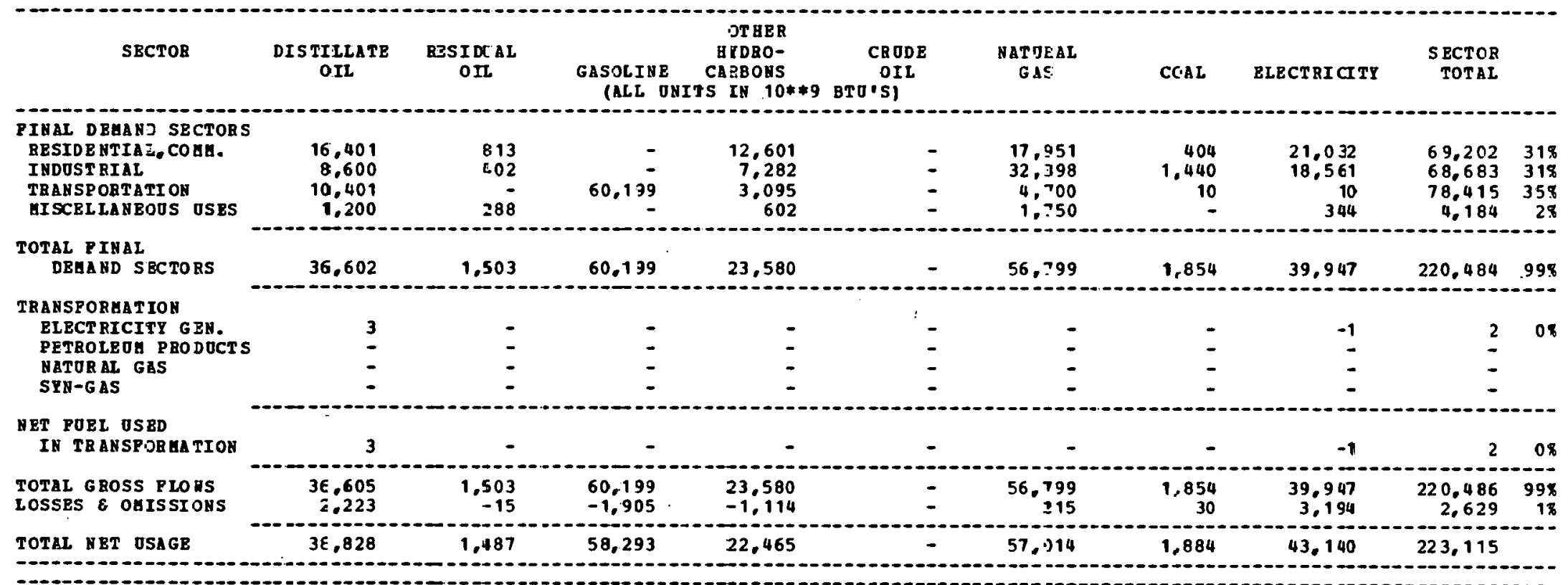

\section{SOPPLI OP EUERG}

POSSIL PORL

GYDROBLBCI

GBO ESOLAR

GEO. ESOLAR

-
-
-

$\begin{array}{ll}- & - \\ - & - \\ - & -\end{array}$

-

-

NET IUPORTS
OP REGIOU

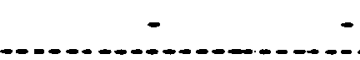

-

38,828 58.293

22.465

57.014

1,884

15,198

195,173

HOT ES:
TRABSPORHATION LOSS POR TRATSPORAATION L.OSS POR
TRABSFO BMATIOB COSS POR
BLECT RACITY GER.
$=66.57 \%$
PETOLZO PRODUCTS $=0.1)$
TTORA GAS
$=0.3$. 
3RGIONAL ENERGI BALANCE STA TEGENT

BY SECTOR AND FOBL TYPE

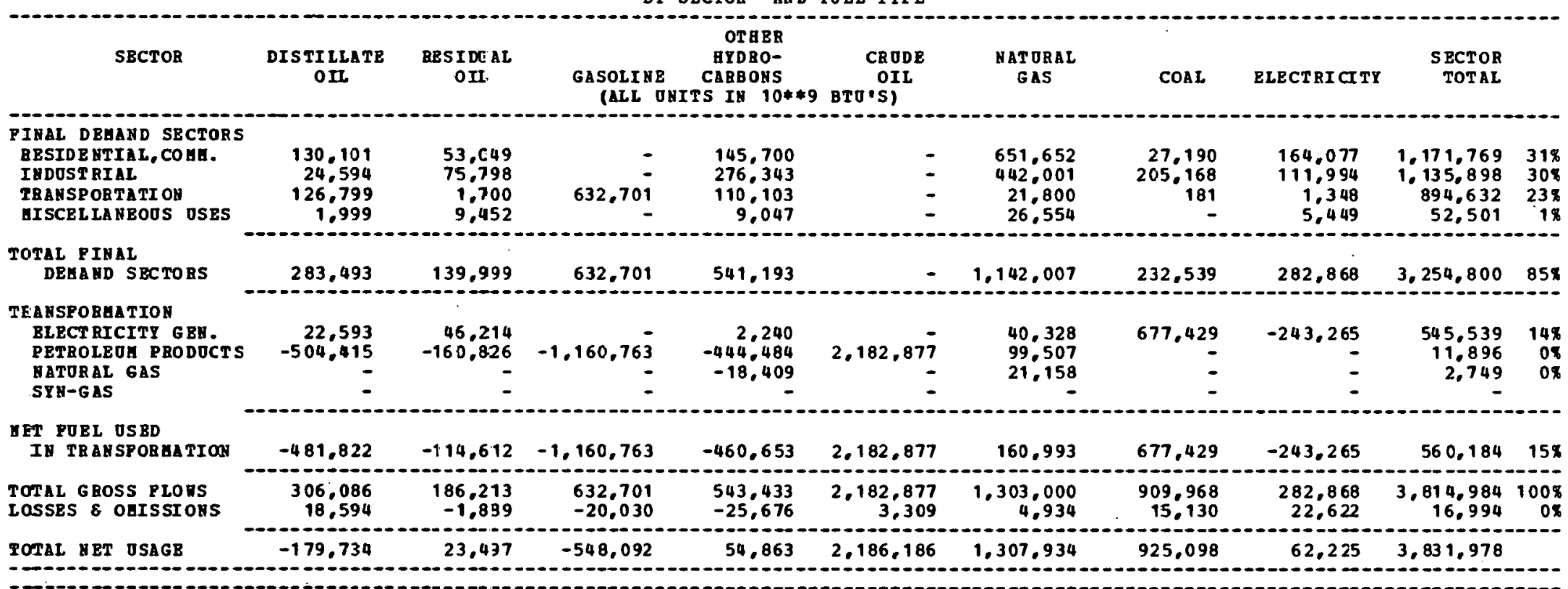

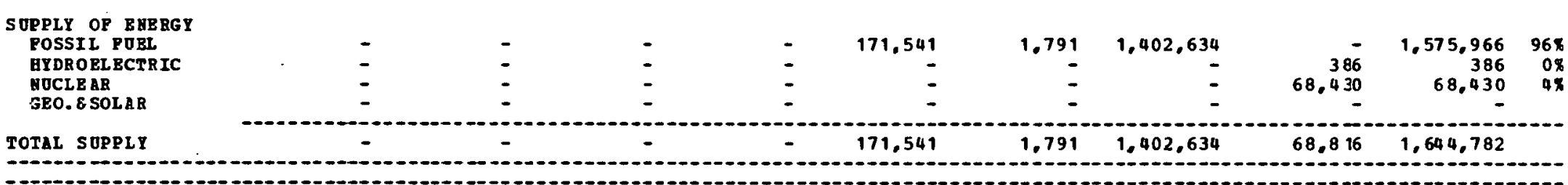

\section{NBT IUPORTS}

OP REGTOH

$-179,734$

23,497

$-548.092$

54,863

$2,014,645$

$-477,535$

$-6,590 \quad 2,187,196$

NOTES:
1 TRABS PORAATIOH LOSS POR TRAXSPOBAATIOH LOSS POR TRAHS PORAATION LOSS POA
BLECTRICITY GBN.
$\quad=69.16$
PETOLEO PRODOCTS $=0.52 \mathrm{x}$
MATORAL GAS $\quad=12.99 \%$ 
BEGIOHAL FJERGY BALANCE STATEGEAT

BY SECTOR AND POBL TYPE

\begin{tabular}{|c|c|c|c|c|c|c|c|c|c|c|}
\hline SBCTOR & $\begin{array}{c}\text { DIS?ILLATE } \\
\text { OIL }\end{array}$ & $\begin{array}{l}\text { RES IDOAL } \\
\text { OIi }\end{array}$ & $\begin{array}{l}\text { GASOL IHE } \\
\text { (ALL OHI }\end{array}$ & $\begin{array}{l}\text { JYDRO- } \\
\text { CARBOHS } \\
\text { TS: IN 10**9 }\end{array}$ & $\begin{array}{c}\text { CRODE } \\
\text { OIL } \\
\text { BTO'SI }\end{array}$ & $\begin{array}{l}\text { WATOBAL } \\
\text { GAS }\end{array}$ & COAL & ELECTRI CITY & $\begin{array}{r}\text { SECTOR } \\
\text { TOTAL. }\end{array}$ & \\
\hline 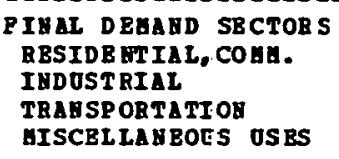 & $\begin{array}{r}103,801 \\
24,503 \\
65,800 \\
2,498\end{array}$ & $\begin{array}{r}13.859 \\
6.9 .902 \\
798 \\
3.541\end{array}$ & 358,299 & $\begin{array}{r}100,500 \\
104,517 \\
76,466 \\
5.394\end{array}$ & : & $\begin{array}{r}225,736 \\
297,398 \\
12,599 \\
10,965\end{array}$ & $\begin{array}{r}13,186 \\
460,656 \\
68 \\
-\end{array}$ & $\begin{array}{r}76.848 \\
80.014 \\
153 \\
185\end{array}$ & $\begin{array}{r}539,930 \\
1.037,090 \\
514,583 \\
22,583\end{array}$ & $\begin{array}{l}218 \\
418 \\
20 \% \\
1 \%\end{array}$ \\
\hline $\begin{array}{l}\text { TOT AL FIAAL } \\
\text { DEHA HD SBC TORS }\end{array}$ & 196,102 & 97,100 & 358,299 & 286,877 & - & 547,098 & 473,910 & $157,2 \mathrm{CO}$ & $2,114,186$ & $83 \%$ \\
\hline $\begin{array}{l}\text { TRAYSPORHATIOA } \\
\text { BLBCTRICITY GBE. } \\
\text { PETROLEOA PRODOCTS } \\
\text { MATORAL GAS } \\
\text { SYH-GAS }\end{array}$ & $\begin{array}{r}753 \\
-239,524 \\
-\end{array}$ & $\begin{array}{r}4,960 \\
-66,837 \\
-\end{array}$ & $\begin{array}{r}-482,2: 35 \\
-\end{array}$ & $-321,502$ & $\begin{array}{r}1.040,746 \\
-\end{array}$ & $\begin{array}{r}10.459 \\
47.443 \\
- \\
-\end{array}$ & $\begin{array}{r}571.253 \\
= \\
=\end{array}$ & $\begin{array}{r}-195,827 \\
= \\
-\end{array}$ & $\begin{array}{r}391,598 \\
7.941 \\
=\end{array}$ & $\begin{array}{r}15 \% \\
0 \%\end{array}$ \\
\hline $\begin{array}{l}\text { WET POEL OSBD } \\
\text { IB TRABSPOBGATION }\end{array}$ & $-208,371$ & $-61,877$ & $-482,235$ & $-321,502$ & 1.040 .746 & 57.902 & 571.253 & $-195,827$ & 399,539 & 16.8 \\
\hline $\begin{array}{l}\text { TOTAL GROSS TLONS } \\
\text { LOSSES } 8 \text { OUISSIOUS }\end{array}$ & $\begin{array}{r}197,455 \\
11.995\end{array}$ & $\begin{array}{r}99,060 \\
-1,005 \\
\end{array}$ & $\begin{array}{l}358,239 \\
-11,343\end{array}$ & $\begin{array}{l}286,877 \\
-13,554\end{array}$ & $\begin{array}{r}1.040 .746 \\
1.577\end{array}$ & $\begin{array}{r}605,000 \\
2,200\end{array}$ & $\begin{array}{r}1.045 .163 \\
17.378\end{array}$ & $\begin{array}{r}157,200 \\
12,572\end{array}$ & $\begin{array}{r}2.513 .725 \\
19.911\end{array}$ & $\begin{array}{r}998 \\
18\end{array}$ \\
\hline
\end{tabular}

\begin{tabular}{|c|c|c|c|c|c|c|c|c|c|}
\hline $\begin{array}{l}\text { SUPPLY OP B HBEG } \\
\text { POSSIL POBL } \\
\text { BYDROBIECTRIC } \\
\text { MOCLBAR } \\
\text { GEO. ESOLAR }\end{array}$ & : & $\overline{-}$ & ! & $\overline{-}$ & $\begin{array}{r}30,809 \\
= \\
=\end{array}$ & $\begin{array}{r}302 \\
-\end{array}$ & $\begin{array}{r}585, \varepsilon 46 \\
- \\
-\end{array}$ & $\begin{array}{r}- \\
1.638 \\
-\end{array}$ & $\begin{array}{r}616,957 \\
1,638 \\
-\end{array}$ \\
\hline
\end{tabular}

\section{MET IAPORTS}

$-173$

31.217

$-135,329$

$-48,179 \quad 1,011,514$

NOT BS:

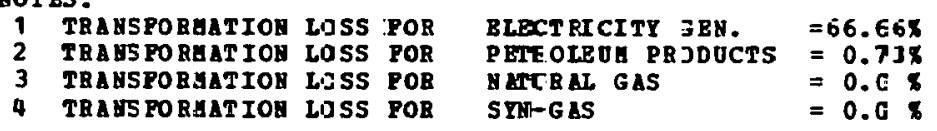


REGIONAL EARRG BALANCE STATEHENT

BY SECTOR AND POEL TYPE

\begin{tabular}{|c|c|c|c|c|c|c|c|c|c|}
\hline SECTOR & $\begin{array}{l}\text { DISTILLATE } \\
\text { OIl }\end{array}$ & $\begin{array}{l}\text { RESI DOAL } \\
\text { O IL }\end{array}$ & $\begin{array}{l}\text { GASOLIHE } \\
\text { (ALL ONII }\end{array}$ & $\begin{array}{l}\text { HYDBO- } \\
\text { CARBO\&S } \\
\text { TS IH 10**9 }\end{array}$ & $\begin{array}{c}\text { CR ODE } \\
\text { OIL } \\
\text { BTO'SI }\end{array}$ & $\begin{array}{l}\text { MAT ORAL } \\
\text { GAS }\end{array}$ & COAL & ELẸCTRI CITY & $\begin{array}{l}\text { SECTOR } \\
\text { TOT AL }\end{array}$ \\
\hline 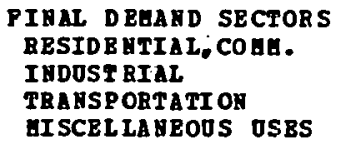 & $\begin{array}{r}35,100 \\
13.096 \\
37.001 \\
1.302\end{array}$ & $\begin{array}{r}1.642 \\
798 \\
- \\
255\end{array}$ & $227.500^{-}$ & $\begin{array}{r}54.298 \\
36.929 \\
8.880 \\
3.960\end{array}$ & $\overline{-}$ & $\begin{array}{r}149.725 \\
139.200 \\
16.800 \\
8.173\end{array}$ & $\begin{array}{r}2,921 \\
35,680 \\
35 \\
-\end{array}$ & $\begin{array}{r}40,156 \\
22,999 \\
32 \\
313\end{array}$ & $\begin{array}{r}283,842 \\
248,702 \\
290,248 \\
14,003\end{array}$ \\
\hline $\begin{array}{l}\text { TOTAL PINAL } \\
\text { DEMA AD SBCTORS }\end{array}$ & 86,499 & 2,695 & 227,500 & 104,067 & - & 313,898 & 38,636 & 63,500 & 836.795 \\
\hline $\begin{array}{l}\text { PRABSPORUATIOH } \\
\text { BLECT RICITY GEN. } \\
\text { PBTROLEOA PRODOCTS } \\
\text { HATURAL GAS } \\
\text { STY-GAS }\end{array}$ & $\begin{array}{r}1.532 \\
= \\
-\end{array}$ & $\begin{array}{r}917 \\
- \\
-\end{array}$ & - & $\begin{array}{l}- \\
-\end{array}$ & $\overline{-}$ & $\begin{array}{r}63,133 \\
- \\
-\end{array}$ & $\begin{array}{r}110,498 \\
= \\
=\end{array}$ & $\begin{array}{r}-48,426 \\
= \\
=\end{array}$ & $\begin{array}{r}127.654 \\
= \\
=\end{array}$ \\
\hline $\begin{array}{l}\text { BET PUEL OSBD } \\
\text { IY TRANSPOR MA TION }\end{array}$ & 1,532 & $\begin{array}{r}317 \\
-\end{array}$ & $\overline{-}$ & $=$ & - & 63,133 & 110,498 & $-48,426$ & 127,654 \\
\hline $\begin{array}{l}\text { TOTAL GROSS PLONS } \\
\text { LOSSES } 8 \text { OAISSIOHS }\end{array}$ & $\begin{array}{r}88.031 \\
5.347\end{array}$ & $\begin{array}{r}3.512 \\
-36\end{array}$ & $\begin{array}{r}227,500 \\
-7,202\end{array}$ & $\begin{array}{r}104,067 \\
-4,916\end{array}$ & $\overline{-}$ & $\begin{array}{r}377.031 \\
1.427\end{array}$ & $\begin{array}{r}149.134 \\
2.479\end{array}$ & $\begin{array}{r}63.500 \\
5.078\end{array}$ & $\begin{array}{r}964.449 \\
2.177\end{array}$ \\
\hline
\end{tabular}

\section{SOPPLY OP BHBRG}

POSSIL FOBL

GYDROELBCTRIC

YUCLEAR

GRO. 8 SOL AB

TOTAL SUPPLT

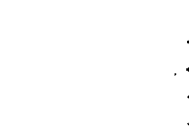

SPI

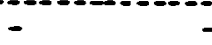

$-$

-

$11,671 \quad 3,235$

3,235

BOT BS : 
REGIONAL REERGI BALANCE STATEHENT

IY SECTOR ASD POBL TYPE

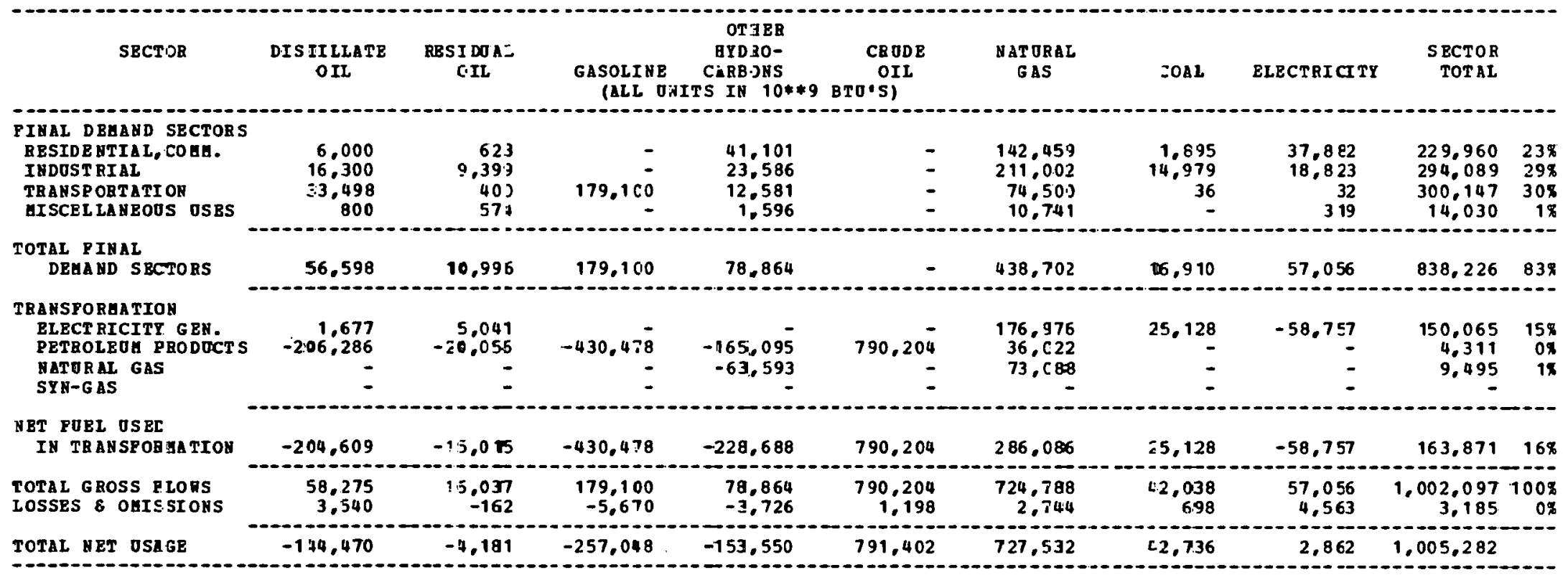

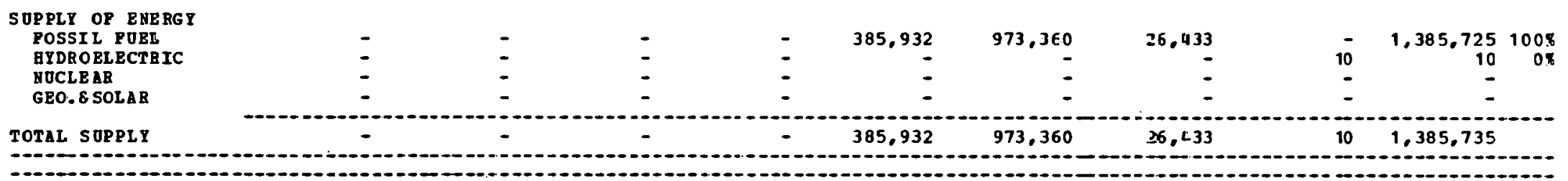

\section{NBT IUPORTS \\ OP REGIOH}

$-744.470$

$-4,181 \quad-257,048$

$-15], 550$

405,470

$-245,327$

16,303

$2,852 \quad-380,452$

NOTES:

1 TRAAS FORHATIOH LOSS POR

IRANSPOAaTION LOSS POQ

TRAMSPORAATION LOSS POR

ELECTRTCITY SEN.
PETEOLEO PB JDOCTS

HATCRAL GAS

$=71.86 x$

SYN-G ES 
BEGIOHAL BHERG BALAHCE STATEGET

OY SECTOR ALD PORL TYPE

\begin{tabular}{|c|c|c|c|c|c|c|c|c|c|c|}
\hline S BCTOR & $\begin{array}{l}\text { DISTILLATE } \\
\text { OIL }\end{array}$ & $\underset{\text { OIE }}{\operatorname{BBSIDOAL}}$ & $\begin{array}{l}\text { GASOLINE } \\
\text { (ALL O }\end{array}$ & $\begin{array}{c}\text { OTHER } \\
\text { BYDRO- } \\
\text { CARBO\&S } \\
\text { IS IX } 10 * * 9\end{array}$ & $\begin{array}{l}\text { CRODB } \\
\text { OIL } \\
\text { BTO'SI }\end{array}$ & $\begin{array}{l}\text { MAT ORAL } \\
\text { GAS }\end{array}$ & COAL & ELECTRICITY & $\begin{array}{r}\text { SECTOR } \\
\text { TOTAL }\end{array}$ & \\
\hline $\begin{array}{l}\text { PIKAL DBHAND SECTORS } \\
\text { BESIDE HTIAL, COBH. } \\
\text { IUDOSTRIAL } \\
\text { TRANSPORTATIOH } \\
\text { UISCBLLABBOOS OSBS }\end{array}$ & $\begin{array}{r}9.601 \\
11.697 \\
38,600 \\
799\end{array}$ & $\begin{array}{r}1.002 \\
4.800 \\
- \\
299\end{array}$ & 213,199 & $\begin{array}{r}54.202 \\
43.384 \\
17.079 \\
1.078\end{array}$ & $\overline{-}$ & $\begin{array}{r}118,505 \\
87,102 \\
35,401 \\
10,197\end{array}$ & $\begin{array}{r}9,553 \\
83,929 \\
40 \\
-\end{array}$ & $\begin{array}{r}58,782 \\
87.241 \\
37 \\
2,612\end{array}$ & $\begin{array}{r}251,645 \\
318,153 \\
304,356 \\
14,985\end{array}$ & $\begin{array}{l}20 \% \\
26 \% \\
25 \% \\
1 \%\end{array}$ \\
\hline $\begin{array}{l}\text { TOTAL PIHAL } \\
\text { DEAA BD SECTOAS }\end{array}$ & 60.697 & 6,101 & 213,199 & $115, .743$ & - & 251,205 & 93,522 & 148,672 & 889,139 & 728 \\
\hline $\begin{array}{l}\text { TBABSPOBAATIOA } \\
\text { BLBCTRICITY GBH. } \\
\text { PETROLEUA PRODUCTS } \\
\text { AATORAL GAS } \\
\text { SYY-GAS }\end{array}$ & $\begin{array}{r}30 \\
-56.776 \\
-\end{array}$ & $\begin{array}{r}904 \\
-18,100 \\
-\end{array}$ & $\begin{array}{r}-129.778 \\
-\end{array}$ & $\begin{array}{r}-116,166 \\
-11.274 \\
-\end{array}$ & $\begin{array}{r}310,723 \\
- \\
-\end{array}$ & $\begin{array}{r}8,235 \\
14,164 \\
12.957 \\
-\end{array}$ & $\begin{array}{r}473,754 \\
= \\
-\end{array}$ & $\begin{array}{r}-160,946 \\
= \\
=\end{array}$ & $\begin{array}{r}321,977 \\
4,067 \\
1,683 \\
-\end{array}$ & $\begin{array}{r}268 \\
08 \\
08\end{array}$ \\
\hline $\begin{array}{l}\text { NET POEL OSED } \\
\text { IY TRABSPOREATIOH }\end{array}$ & -56.746 & $-17 . \$ 96$ & $-129,778$ & $-127,440$ & 310.723 & 35,356 & 473,754 & $-160,946$ & 327,727 & $27 \%$ \\
\hline $\begin{array}{l}\text { TOTAL GROSS FLOIS } \\
\text { LOSSES } 8 \text { OAISSIOUS }\end{array}$ & $\begin{array}{r}60,727 \\
3,689\end{array}$ & $\begin{array}{r}7.005 \\
-71\end{array}$ & $\begin{array}{r}213,199 \\
-6,749\end{array}$ & $\begin{array}{r}115,743 \\
-5,468\end{array}$ & $\begin{array}{r}310.723 \\
471\end{array}$ & $\begin{array}{r}286.561 \\
1.085\end{array}$ & $\begin{array}{r}567.276 \\
9.432\end{array}$ & $\begin{array}{r}148.672 \\
11.890\end{array}$ & $\begin{array}{r}1,216,866 \\
14,278\end{array}$ & $\begin{array}{r}998 \\
18\end{array}$ \\
\hline
\end{tabular}

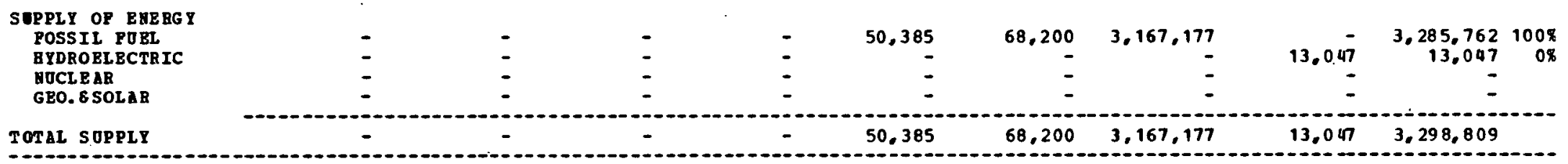

(1)

\section{IET IHPORTS}

7.640

76.671

$-17,165$

260,809

$219,446 \quad-2,590,468$

$-13,430 \quad-2,067,664$

(1)

NOT ES:

1 TBAYSPORMATIOA LOSS POB

TRA HS PORHAT IOB LOSS POR

TRANSPORAATIOH LOSS POB

RLECTRICITY GEN.

$=66.67 \%$

TRAMS PORAATION LOSS POR

NATORAL GAS

$=1.25 \%$

SW-GAS

$=12.99 \pi$
$=0.0 \%$ 
REGIONAL BHERG BALANCE STLTEHEHT

BP SBCTOR AND POEL TYPE

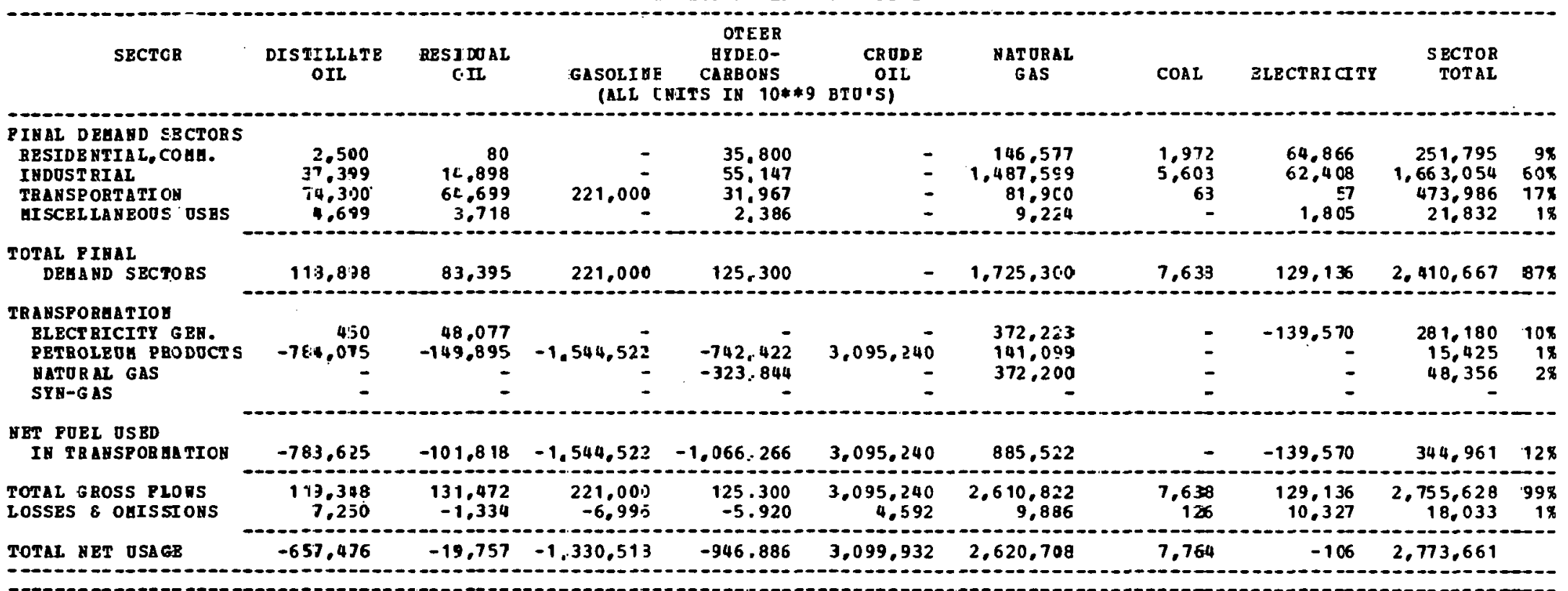

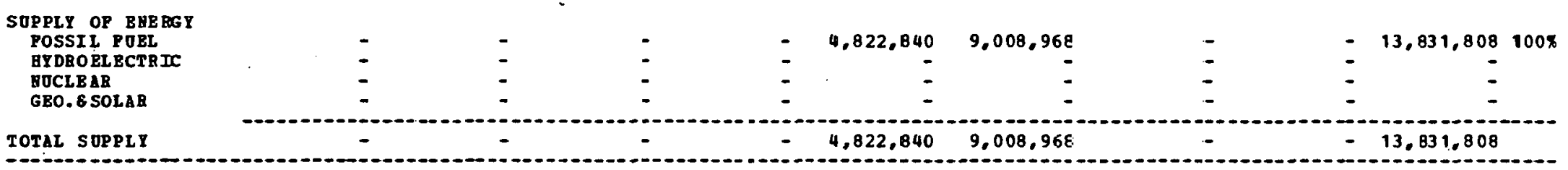

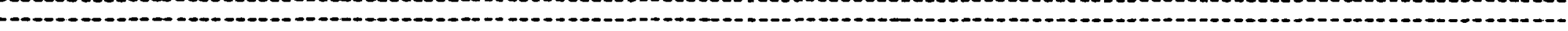

\section{HET IHPORTS}

MOTES:

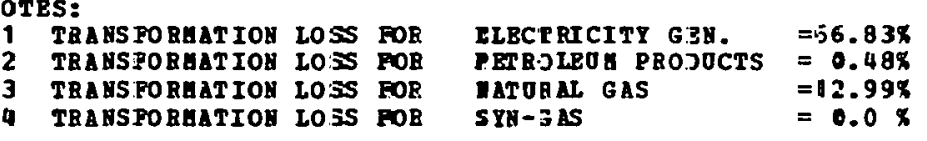


QBGIONAL ENERGT BALANCE STATEGENT

BY SBCTOB ABD POEL TYPE

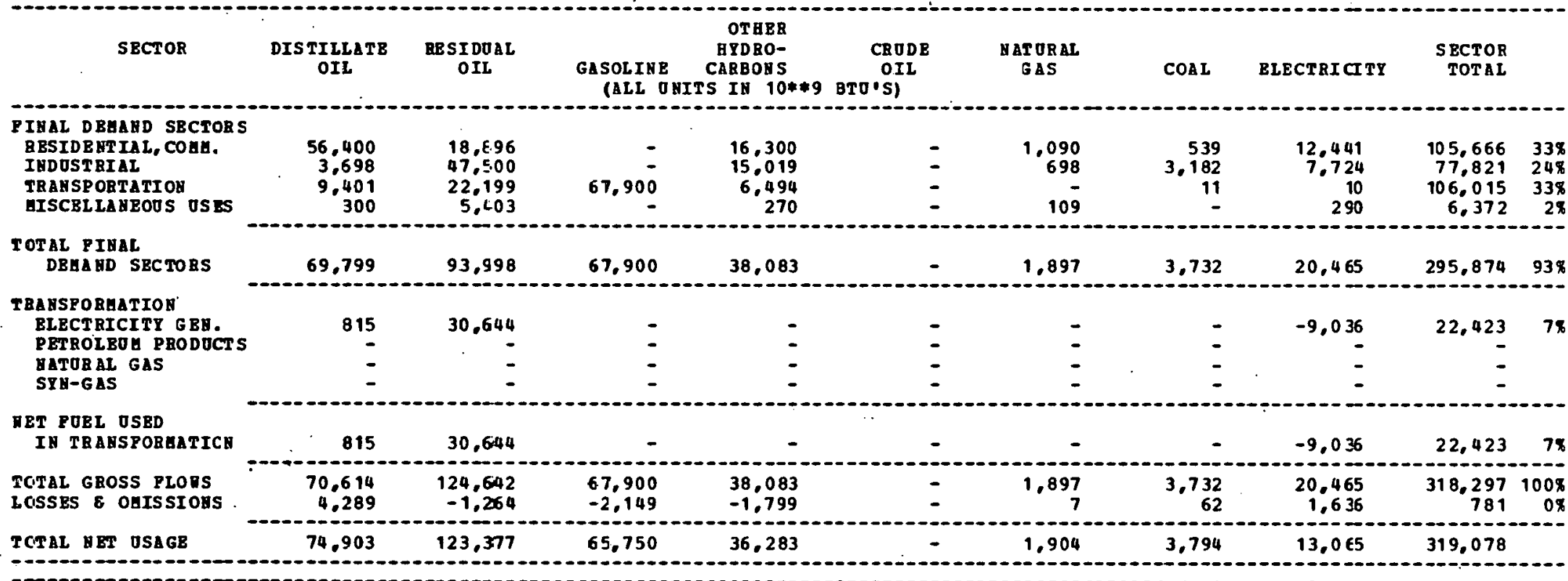

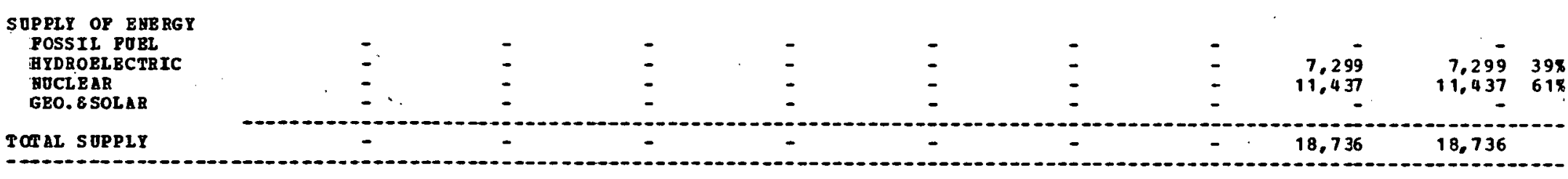

HET IKPORTS

$74.903 \quad 123,377$

65,750

36.283

1,904

3,794

$-5.670$

300,342

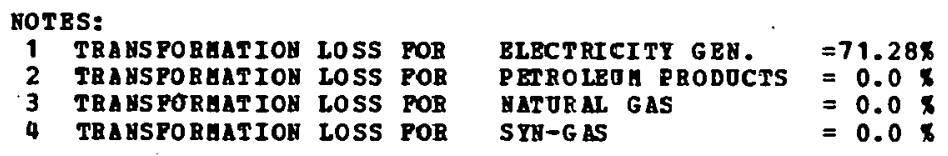

4 TRAYSPOBAATIOH LOSS POB

MATURAL GAS

$=0.0 \%$ 
REGI JUEL ENERGY BALANCE STATEUEAT

BY SECTOR AND POEL TYPE

\begin{tabular}{|c|c|c|c|c|c|c|c|c|c|c|}
\hline SECTOR & $\begin{array}{c}\text { DIS FILLATE } \\
\text { OIL }\end{array}$ & $\begin{array}{c}\text { BESIDOAL } \\
\text { DIL }\end{array}$ & $\begin{array}{l}\text { GASOLIRE } \\
\text { (ALL JUIT }\end{array}$ & $\begin{array}{l}\text { OT JBR } \\
\text { AYDZO- } \\
\text { CARBOHS } \\
\text { TS IR 10**9 }\end{array}$ & $\begin{array}{c}\text { CRODE } \\
\text { OIL } \\
\text { BTO'S) }\end{array}$ & $\begin{array}{l}\text { NAT OR.AL } \\
\text { GAS }\end{array}$ & $\operatorname{COAL}$ & BLECTRI CITY & $\begin{array}{r}\text { SECTOR } \\
\text { TOTAL }\end{array}$ & \\
\hline $\begin{array}{l}\text { PINAL DEGAND SECTORS } \\
\text { RESIDE HTIAL, COAB. } \\
\text { INDOST RIAL } \\
\text { TRANSPORTATI ON } \\
\text { GISCEL LANEOOE OSZS }\end{array}$ & $\begin{array}{l}72.800 \\
18,101 \\
30.497 \\
1.400\end{array}$ & $\begin{array}{r}25,517 \\
47,003 \\
25,500 \\
3,384\end{array}$ & $232.201 \overline{-}$ & $\begin{array}{r}38.399 \\
40.059 \\
22.762 \\
1.662\end{array}$ & - & $\begin{array}{r}122,871 \\
66.732 \\
2,439 \\
8,136\end{array}$ & $\begin{array}{r}3,790 \\
161,863 \\
71 \\
-\end{array}$ & $\begin{array}{r}65,021 \\
29.688 \\
700 \\
30\end{array}$ & $\begin{array}{r}328,398 \\
363,416 \\
314.132 \\
20,582\end{array}$ & $\begin{array}{r}278 \\
30 \% \\
268 \\
28\end{array}$ \\
\hline $\begin{array}{l}\text { COTAL PIHAL } \\
\text { DEGA HD SBCTORS }\end{array}$ & 122.798 & 107.404 & 232.201 & $-02,882$ & - & 200.090 & 165,724 & 95,439 & $1.026,528$ & $84 \%$ \\
\hline $\begin{array}{l}\text { TRANSFORHATIOB } \\
\text { BLECT RICITY GER. } \\
\text { PBTROLEOA PEODOCTS } \\
\text { HATORAL GAS } \\
\text { SYH-GAS }\end{array}$ & $\begin{array}{r}8,302 \\
- \\
-\end{array}$ & $\begin{array}{r}152.724 \\
- \\
-\end{array}$ & $\begin{array}{l}- \\
-\end{array}$ & $\begin{array}{r}-18,223 \\
- \\
-\end{array}$ & 18,200 & $\begin{array}{r}9.056 \\
830 \\
-\end{array}$ & $\begin{array}{r}97,683 \\
:-\end{array}$ & $\begin{array}{r}-85,089 \\
- \\
-\end{array}$ & $\begin{array}{r}182.676 \\
807 \\
- \\
-\end{array}$ & $\begin{array}{r}15 \% \\
0 \%\end{array}$ \\
\hline $\begin{array}{l}\text { BET POBL USBD } \\
\text { IN TRAHSFOREATION }\end{array}$ & 8,302 & 152.724 & - & $-18 ;, 223$ & 18,200 & 9.886 & $97.6: 33$ & $-85,089$ & 183.483 & $15 \%$ \\
\hline $\begin{array}{l}\text { TOTAL GROSS PLONS } \\
\text { LOSSES } 8 \text { OHISSIONS }\end{array}$ & $\begin{array}{r}131,100 \\
7,964\end{array}$ & $\begin{array}{r}260.128 \\
-2.639\end{array}$ & $\begin{array}{r}232.2 C 1 \\
-7,3 \leq 1\end{array}$ & $\begin{array}{r}102,882 \\
-4,861\end{array}$ & $\begin{array}{r}18.200 \\
27\end{array}$ & $\begin{array}{r}209,966 \\
795\end{array}$ & $\begin{array}{r}263,4,37 \\
4,379\end{array}$ & $\begin{array}{r}95,439 \\
7,632\end{array}$ & $\begin{array}{r}1,210,011 \\
5,947\end{array}$ & $\begin{array}{r}100 \% \\
0 \%\end{array}$ \\
\hline
\end{tabular}

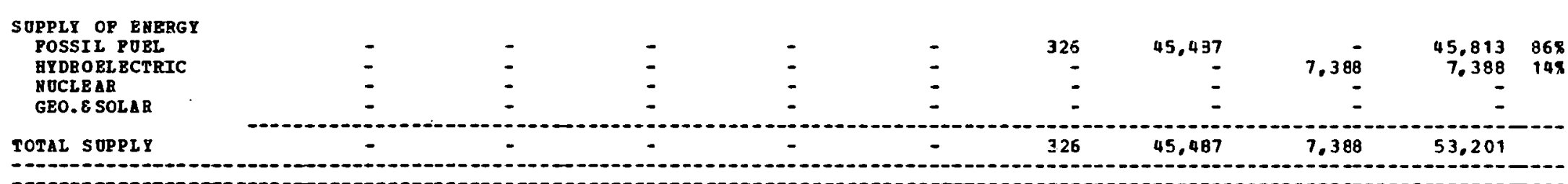

\section{HET IAPORTS}

$139.064 \quad 25.7 .488 \quad 224.849$

70,797

18,227

$210,4.35$

222,299

$10,594 \quad 1,162,757$

MOTBS:

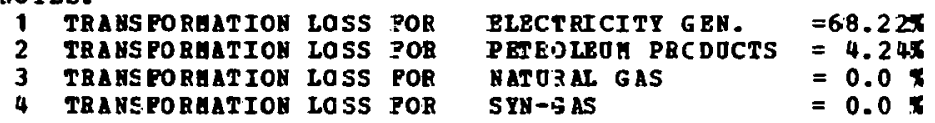


REGIONAL BHERG BALANCE STATEABNT

BY SECTOB AHD FOEL TYPE

\begin{tabular}{|c|c|c|c|c|c|c|c|c|c|c|}
\hline SBCT OR & $\begin{array}{l}\text { DISTILLATE } \\
\text { OIL }\end{array}$ & $\begin{array}{l}\text { BESIDDAL } \\
\text { OIL }\end{array}$ & $\begin{array}{l}\text { GASOL IHE } \\
\text { (ALL OHI }\end{array}$ & $\begin{array}{l}\text { GYDRO- } \\
\text { CARBOHS } \\
\text { TS IH 10**9 }\end{array}$ & $\begin{array}{c}\text { CRODE } \\
\text { OIL } \\
\text { BTO'S) }\end{array}$ & $\begin{array}{l}\text { SAT ORAL } \\
\text { GAS }\end{array}$ & COAL & BLECTRICITY & $\begin{array}{r}\text { SECTOR } \\
\text { TOTAL }\end{array}$ & \\
\hline $\begin{array}{l}\text { FIHAL DEHAED SECTORS } \\
\text { RBSIDBEPIAL, COAY. } \\
\text { IHDOSTRIAL } \\
\text { TRABSPORTATIOH } \\
\text { UISCEL LABEOOS OSBS }\end{array}$ & $\begin{array}{r}325.700 \\
9.203 \\
25.599 \\
300\end{array}$ & $\begin{array}{r}169,473 \\
50,399 \\
17,500 \\
23,728\end{array}$ & 295,300 & $\begin{array}{r}32.700 \\
88.030 \\
54.157 \\
2.125\end{array}$ & $\overline{-}$ & $\begin{array}{r}121.815 \\
31,901 \\
600 \\
4.085\end{array}$ & $\begin{array}{r}3,650 \\
20.564 \\
79 \\
-\end{array}$ & $\begin{array}{r}71,396 \\
31,250 \\
1 \in 2 \\
169\end{array}$ & $\begin{array}{r}724,734 \\
231.347 \\
393,397 \\
30,407\end{array}$ & $\begin{array}{r}46 \% \\
15 \% \\
25 \% \\
2 \%\end{array}$ \\
\hline $\begin{array}{l}\text { TOTAL PIHAL } \\
\text { DBAA BD SBCTOBS }\end{array}$ & 360,802 & 261,100 & 295,300 & 177,012 & - & 158,401 & 24,293 & 102,977 & 1.379 .885$. & $87 \%$ \\
\hline $\begin{array}{l}\text { TRANSPOREATIOH } \\
\text { BLECTRICITY GEH. } \\
\text { PETROLBOE PRODOCTS } \\
\text { VATORAL GAS } \\
\text { SYH-GAS }\end{array}$ & $\begin{array}{r}5,192 \\
- \\
-\end{array}$ & $\begin{array}{r}282,651 \\
= \\
-\end{array}$ & - & $\begin{array}{r}355 \\
- \\
-\end{array}$ & : & $\begin{array}{r}5,456 \\
- \\
-\end{array}$ & $\begin{array}{r}340 \\
- \\
-\end{array}$ & $\begin{array}{r}-95,479 \\
- \\
-\end{array}$ & $\begin{array}{r}198,515 \\
= \\
=\end{array}$ & $13 \%$ \\
\hline $\begin{array}{l}\text { MET POEL OSED } \\
\text { IN TRABSFORHATIOA }\end{array}$ & 5,192 & 282,651 & - & 355 & - & 5,456 & 340 & -95.479 & 198,515 & 138 \\
\hline $\begin{array}{l}\text { TOTAL GROSS PLORS } \\
\text { IOSSES } 8 \text { OAISSIOYS }\end{array}$ & $\begin{array}{r}365,994 \\
22,234\end{array}$ & $\begin{array}{r}543,751 \\
-5,517\end{array}$ & $\begin{array}{r}295,300 \\
-9,348\end{array}$ & $\begin{array}{r}177,367 \\
-8,380\end{array}$ & - & $\begin{array}{r}163,857 \\
620\end{array}$ & $\begin{array}{r}24,633 \\
409\end{array}$ & $\begin{array}{r}102,977 \\
8,235\end{array}$ & $\begin{array}{r}1,578,400 \\
8,252\end{array}$ & $\begin{array}{r}99 \% \\
1 \%\end{array}$ \\
\hline
\end{tabular}

\section{SOPPLI OP EUERG}

POSSII POEL

HYDROELBCTRIC

HOCL BAR

GEO. ESOLAR

$\begin{array}{rrrr}- & - & - \\ - & - & - \\ - & - & -\end{array}$

ZOTAL SOPPLY

$-$

$-$

$-$

388,228

538,233

285,951

168,986

164,477

25,042

$-4,120$

NOT BS:

1 TRANSPOBAATIOH LOSS POB

$=67.52 \pi$
$=0.0$

$\begin{array}{lll}4 & \text { TRANSPORAATION LOSS POR NATURAL GAS } & =0.0 \% \\ & & =0.0 \%\end{array}$ 
BRE IONAL ZNEBGT BALANCE STATELENT

BY SEZTOR AND FOBL TYPE

\begin{tabular}{|c|c|c|c|c|c|c|c|c|c|c|}
\hline SECTOR & $\begin{array}{l}\text { DISTIELATB } \\
\text { OIL }\end{array}$ & $\begin{array}{l}\text { BESI DOAL } \\
\text { OII }\end{array}$ & $\begin{array}{l}\text { GASOLIFE } \\
\text { (ALL O }\end{array}$ & $\begin{array}{l}\text { OTHER } \\
\text { HYDRO- } \\
\text { CARBOHS } \\
S \text { IY 10**9 }\end{array}$ & $\begin{array}{l}\text { CBODE } \\
\text { OIL } \\
\text { BTU'S) }\end{array}$ & $\underset{\text { GAS }}{\operatorname{MATORAL}}$ & $\cos L$ & BLECTRICITI & $\begin{array}{l}\text { SECTOR } \\
\text { TOTAL }\end{array}$ & \\
\hline 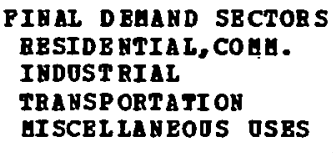 & $\begin{array}{r}168,300 \\
28,396 \\
59,199 \\
2,700\end{array}$ & $\begin{array}{r}6,439 \\
20,496 \\
2,700 \\
3,160\end{array}$ & 598.301 & $\begin{array}{r}72,997 \\
162,533 \\
38,445 \\
2,871\end{array}$ & $\begin{array}{l}\bar{z} \\
\overline{-}\end{array}$ & $\begin{array}{r}506.194 \\
349.404 \\
12.301 \\
26.6 .08\end{array}$ & $\begin{array}{r}18,541 \\
302.055 \\
99 \\
-\end{array}$ & $\begin{array}{r}115,166 \\
109,272 \\
91 \\
1,321\end{array}$ & $\begin{array}{r}887,637 \\
972,156 \\
711,136 \\
36,660\end{array}$ & $\begin{array}{r}29 \% \\
32 \% \\
23 \% \\
1 \%\end{array}$ \\
\hline $\begin{array}{l}\text { TOTAL PIBAL } \\
\text { DEUABD SECTORS }\end{array}$ & 258.595 & 32,795 & 598,301 & 276,846 & - & 894.507 & 320,695 & 225.850 & $2,607,589$ & $85 \pi$ \\
\hline $\begin{array}{l}\text { TRAHSPORUATION } \\
\text { BLECT RICITY GEH. } \\
\text { PETROLBOA PRODOCTS } \\
\text { MATORAL GAS } \\
\text { SYH-G AS }\end{array}$ & $\begin{array}{r}7.863 \\
-46.949 \\
-\end{array}$ & $\begin{array}{r}66,933 \\
-14,970 \\
- \\
-\end{array}$ & $\begin{array}{r}-107.532 \\
-\end{array}$ & $\begin{array}{r}-98,022 \\
-1,976\end{array}$ & $260,14 \bar{E}$ & $\begin{array}{r}63.701 \\
11.859 \\
2.271 \\
-\end{array}$ & $\begin{array}{r}499,570 \\
- \\
-\end{array}$ & $\begin{array}{r}-190,796 \\
= \\
-\end{array}$ & $\begin{array}{r}447.271 \\
4.434 \\
295 \\
-\end{array}$ & $\begin{array}{r}15 \% \\
0 \% \\
0 \%\end{array}$ \\
\hline $\begin{array}{l}\text { HBT POEL OSED } \\
\text { IA TRABSPOQ TIOA TION }\end{array}$ & $-39,086$ & 51,963 & $-107,532$ & $-99,998$ & 260,148 & 77,831 & 499.570 & $-190,796$ & 452,000 & $15 \pi$ \\
\hline $\begin{array}{l}\text { TOTAL GROSS PLOUS } \\
\text { LOSSES } 8 \text { OUISSIOHS }\end{array}$ & $\begin{array}{r}266,458 \\
16,187\end{array}$ & $\begin{array}{l}99,728 \\
-1,011\end{array}$ & $\begin{array}{l}598,301 \\
-18,741\end{array}$ & $\begin{array}{l}276,846 \\
-13,080\end{array}$ & $\begin{array}{r}260.148 \\
394\end{array}$ & $\begin{array}{r}972,338 \\
3,681\end{array}$ & $\begin{array}{r}820,265 \\
13,638\end{array}$ & $\begin{array}{r}225,850 \\
18,062\end{array}$ & $\begin{array}{r}3.059,589 \\
18,930\end{array}$ & $\begin{array}{r}998 \\
1 \%\end{array}$ \\
\hline TOTAL NET & 235.696 & 83.746 & 471,727 & 163,767 & 260,542 & 976,0191 & 833,903 & 53,116 & $3,078,519$ & \\
\hline
\end{tabular}

-

\begin{tabular}{|c|c|c|c|c|c|c|c|c|c|c|}
\hline 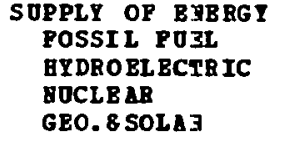 & : & $\overline{-}$ & : & $\overline{-}$ & $\begin{array}{r}84,275 \\
= \\
=\end{array}$ & $\begin{array}{r}47.767 \\
= \\
-\end{array}$ & : & $\begin{array}{r}5.590 \\
7.974\end{array}$ & $\begin{array}{r}132,042 \\
5,590 \\
7.974 \\
-\end{array}$ & $\begin{array}{r}91 \% \\
48 \\
5 \%\end{array}$ \\
\hline TOTAL SOPPLY & - & - & - & - & 84,275 & 47.767 & - & 13,564 & 145,606 & \\
\hline
\end{tabular}

\section{NET IEPORTS}

OP REGIOY

83,746

471.727

163.767

176,267

928.252

833,903

$39,552 \quad 2,932,913$

NOTES:

1 TRAES PORHAT ICH LOSS FOR TRAUSPOBGATIOU LOSS POB TRAYSPORAATICH LOSS POB TRAESPOREATIOA LOSS POB

BLECTRICITY GEN.

$\begin{array}{ll} & =70.10 x \\ \text { GEN. } & =1.63 x\end{array}$

YMOBAL GAS

YYi-G AS
$=12.93 \mathrm{x}$ 
REGIONAL EHERGY BALAXCE STATBAENT

BY SECTOB ARD FORL TYPE

\begin{tabular}{|c|c|c|c|c|c|c|c|c|c|c|}
\hline SECTOR & $\begin{array}{l}\text { DIS TILLATE } \\
\text { OIL }\end{array}$ & $\begin{array}{l}\text { BESIJOAL } \\
\text { OIL }\end{array}$ & $\begin{array}{l}\text { GASOLIYR } \\
\text { (ALL ONIT }\end{array}$ & $\begin{array}{l}\text { BYDBO- } \\
\text { CARBOAS } \\
\text { CS IS 10**9 }\end{array}$ & $\begin{array}{c}\text { CRODE } \\
\text { OIL } \\
\text { BTO'S) }\end{array}$ & $\begin{array}{l}\text { YAT JRAL } \\
\text { GAS }\end{array}$ & $\operatorname{COAL}$ & ELECTRICITY & $\begin{array}{r}\text { SBCTOR } \\
\text { TOTAL }\end{array}$ & \\
\hline $\begin{array}{l}\text { PIHAL DBMAHD SECTORS } \\
\text { BESIDE HTIAL, COBA. } \\
\text { IHDOSTRIAL } \\
\text { TRANSPORTATIOH } \\
\text { GISCELIAHEOOS OSES }\end{array}$ & $\begin{array}{r}95,499 \\
12.102 \\
37.101 \\
901\end{array}$ & $\begin{array}{r}4,379 \\
30,697 \\
2,600 \\
721\end{array}$ & 269.300 & $\begin{array}{r}78,600 \\
49,327 \\
34,268 \\
2.421\end{array}$ & $\overline{-}$ & $\begin{array}{r}179,103 \\
114,301 \\
5,601 \\
7,595\end{array}$ & $\begin{array}{r}7,344 \\
54,439 \\
59 \\
-\end{array}$ & $\begin{array}{r}46.688 \\
38.550 \\
54 \\
509\end{array}$ & $\begin{array}{r}411,613 \\
299,416 \\
348,983 \\
12,147\end{array}$ & $\begin{array}{l}348 \\
248 \\
298 \\
1 \%\end{array}$ \\
\hline $\begin{array}{l}\text { TOTAL PIBAL } \\
\text { DEGA KD SBCTORS }\end{array}$ & 145,603 & 38,397 & 269,300 & 164,616 & - & 306,600 & 61,842 & 85.801 & $1,072,159$ & $88 \pi$ \\
\hline $\begin{array}{l}\text { TRANSPORAATIOH } \\
\text { BLECTRICITY GER. } \\
\text { PETROLEOA PRODOCTS } \\
\text { MATORAI GAS } \\
\text { SYH-GAS }\end{array}$ & $\begin{array}{r}5,903 \\
-101.786 \\
=\end{array}$ & $\begin{array}{r}6,207 \\
-35,823 \\
-\end{array}$ & $\begin{array}{r}-192.960^{\circ} \\
-\end{array}$ & $\begin{array}{r}239 \\
-30.605 \\
-\end{array}$ & 344.474 & $\begin{array}{r}58,709 \\
16,742 \\
=\end{array}$ & $\begin{array}{r}132,163 \\
- \\
-\end{array}$ & $\begin{array}{r}-56,456 \\
=\end{array}$ & $\begin{array}{r}146,765 \\
42 \\
- \\
-\end{array}$ & $\begin{array}{r}128 \\
0 \%\end{array}$ \\
\hline $\begin{array}{l}\text { SBT FOEL OSBD } \\
\text { IN TRABSFORAATIOH }\end{array}$ & $-95,883$ & -29.616 & $-192,960$ & $-30 \cdot 366$ & 344,474 & 75,451 & 132,163 & $-56,456$ & 146,807 & $12 \%$ \\
\hline $\begin{array}{l}\text { TOTAL GROSS FLONS } \\
\text { EOSSES } 8 \text { OAISSIORS }\end{array}$ & $\begin{array}{r}151.506 \\
9.203\end{array}$ & $\begin{array}{r}44.604 \\
-452\end{array}$ & $\begin{array}{r}269,300 \\
-8,525\end{array}$ & $\begin{array}{r}164,855 \\
-7,789\end{array}$ & $\begin{array}{r}344.474 \\
522\end{array}$ & $\begin{array}{r}382,051 \\
1,446\end{array}$ & $\begin{array}{r}194,005 \\
3,225\end{array}$ & $\begin{array}{r}85,801 \\
6.861\end{array}$ & $\begin{array}{r}1,218,966 \\
4,493\end{array}$ & $\begin{array}{r}1008 \\
08\end{array}$ \\
\hline
\end{tabular}

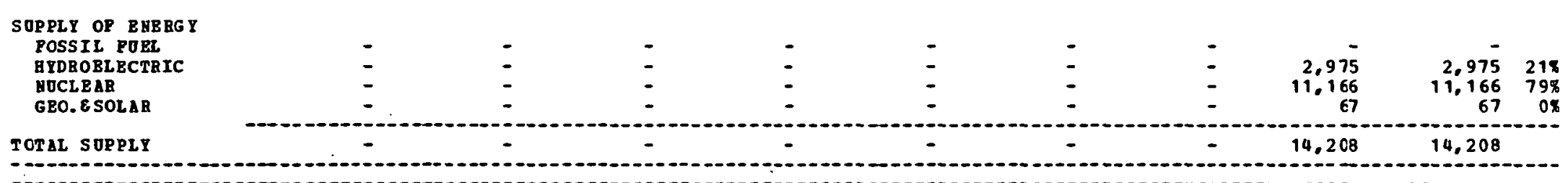

\begin{tabular}{l}
$\begin{array}{l}\text { GET IHPORTS } \\
\text { OP REGIOH }\end{array}$ \\
\hdashline$\quad 58,923$
\end{tabular}


REGIOHAL FNERGY BALAHCE STATEUENT

BE SECTOR AND FOEL TYPE

\begin{tabular}{|c|c|c|c|c|c|c|c|c|c|c|}
\hline SECTOR & $\begin{array}{c}\text { DI:STILLATE } \\
\text { OJL }\end{array}$ & $\begin{array}{c}\text { RE } \leq I D O A L \\
\text { OIL }\end{array}$ & $\begin{array}{l}\text { GASOLIHE } \\
\text { (ALL ONIS }\end{array}$ & $\begin{array}{l}\text { BYDBO- } \\
\text { CARBONS } \\
\text { TS IN 10**9 }\end{array}$ & $\begin{array}{c}\text { CEODE } \\
\text { CIL } \\
\text { BTO } S \text { SI }\end{array}$ & $\begin{array}{l}\text { NAT OR.LL } \\
\text { GAS }\end{array}$ & COAL & ELECTRI CITY & $\begin{array}{r}\text { SECTOR } \\
\text { TOTAL }\end{array}$ & \\
\hline $\begin{array}{l}\text { PINAL DEHAND SECTORS } \\
\text { RESIDERTIAL, COAG. } \\
\text { IRDOSTRIAL } \\
\text { TRANSPORTATION } \\
\text { UISCELLANEOS OSBS }\end{array}$ & $\begin{array}{r}9.100 \\
11.593 \\
29.000 \\
2,100\end{array}$ & $\begin{array}{r}292 \\
8,796 \\
2,699 \\
103\end{array}$ & 154.999 & $\begin{array}{r}53.200 \\
47.702 \\
10.991 \\
1.698\end{array}$ & - & $\begin{array}{r}50,433 \\
138,133 \\
58.538 \\
5.898\end{array}$ & $\begin{array}{r}1,197 \\
6,670 \\
24 \\
-\end{array}$ & $\begin{array}{r}39.642 \\
22.996 \\
21 \\
559\end{array}$ & $\begin{array}{r}153,834 \\
235,860 \\
256,332 \\
10,358\end{array}$ & $\begin{array}{r}218 \\
318 \\
348 \\
18\end{array}$ \\
\hline $\begin{array}{l}\text { TOTAL F IRAL } \\
\text { DEGA AD SECTORS }\end{array}$ & 51.793 & 11,890 & 154,099 & 113,591 & - & 253,002 & 7.391 & 63,218 & 656,384 & $88 \%$ \\
\hline $\begin{array}{l}\text { TRANSFORMATIOB } \\
\text { ELECTRICITY GEH. } \\
\text { PETROLEU PRODUCT S } \\
\text { HATORAL GAS } \\
\text { SYH-GAS }\end{array}$ & $\begin{array}{r}387 \\
-131,307 \\
-\end{array}$ & $\begin{array}{r}36,309 \\
-25,104 \\
-\end{array}$ & $\begin{array}{r}-257.734 \\
-\end{array}$ & $\begin{array}{r}-149,944 \\
-2,255 \\
-\end{array}$ & 542.023 & $\begin{array}{r}61,489 \\
24.709 \\
2.592 \\
-\end{array}$ & $\begin{array}{r}28.787 \\
= \\
=\end{array}$ & $\begin{array}{r}-37,610 \\
- \\
-\end{array}$ & $\begin{array}{r}89,362 \\
2,643 \\
337 \\
-\end{array}$ & $\begin{array}{r}128 \\
08 \\
08\end{array}$ \\
\hline $\begin{array}{l}\text { NET FOEL OSED } \\
\text { IN TRANSFOZBATION }\end{array}$ & $-i 30,920$ & 11,205 & -257.734 & $-1 \leq 2,199$ & 542.023 & 88,790 & 28.787 & $-37,610$ & 92,342 & 128 \\
\hline $\begin{array}{l}\text { TOTAL GROSS ?LONS } \\
\text { LOSSES E OUISSIOHS }\end{array}$ & $\begin{array}{r}52.180 \\
3.169\end{array}$ & $\begin{array}{r}18,199 \\
-489\end{array}$ & $\begin{array}{r}154,999 \\
-4,907\end{array}$ & $\begin{array}{r}1.3 .591 \\
-5,366\end{array}$ & $\begin{array}{r}542.023 \\
821\end{array}$ & $\begin{array}{r}341.792 \\
1.294\end{array}$ & $\begin{array}{r}36.578 \\
609\end{array}$ & $\begin{array}{r}63.218 \\
5.055\end{array}$ & $\begin{array}{r}748,726 \\
188\end{array}$ & $\begin{array}{r}100 \% \\
0 \%\end{array}$ \\
\hline
\end{tabular}

-

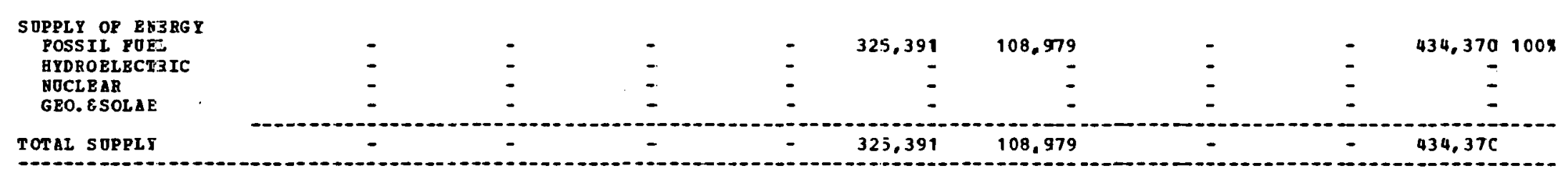

\begin{tabular}{l}
$\begin{array}{l}\text { MET IHPORTS } \\
\text { OP BEGIOB }\end{array}$ \\
\hdashline$-75,957$
\end{tabular}




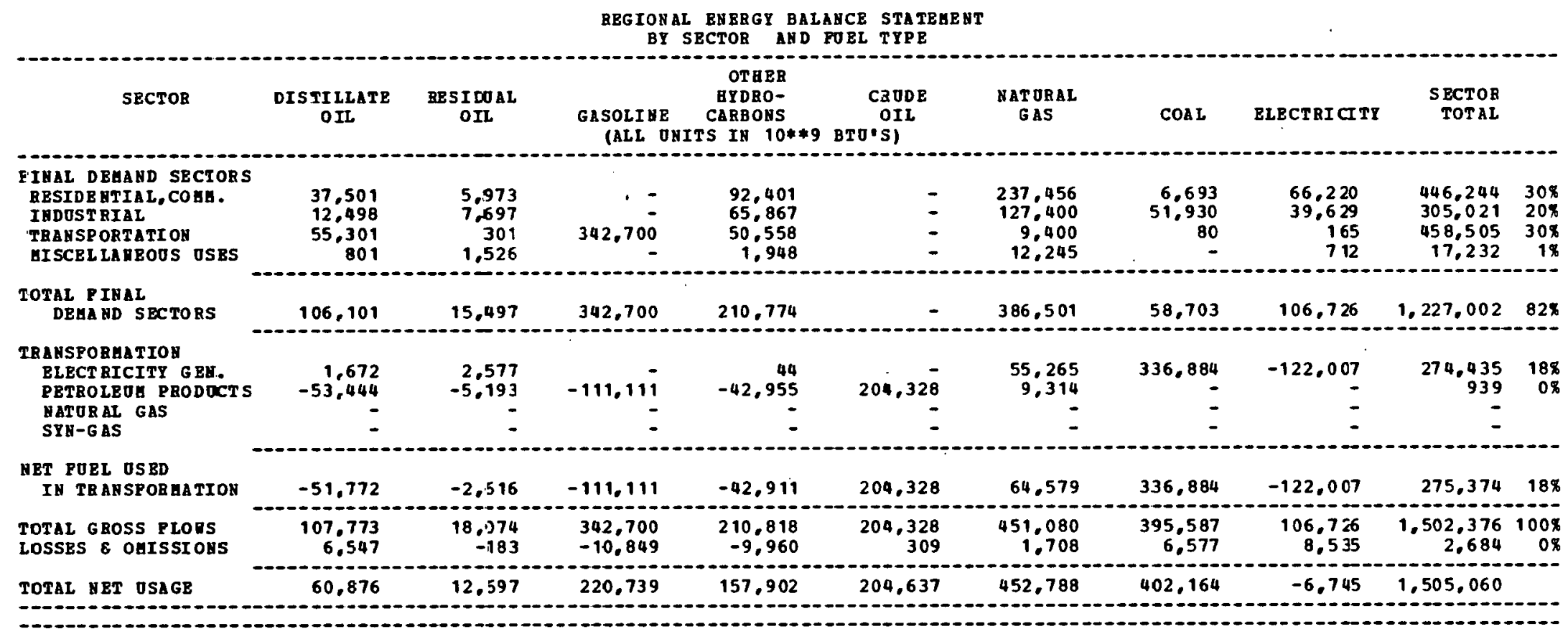

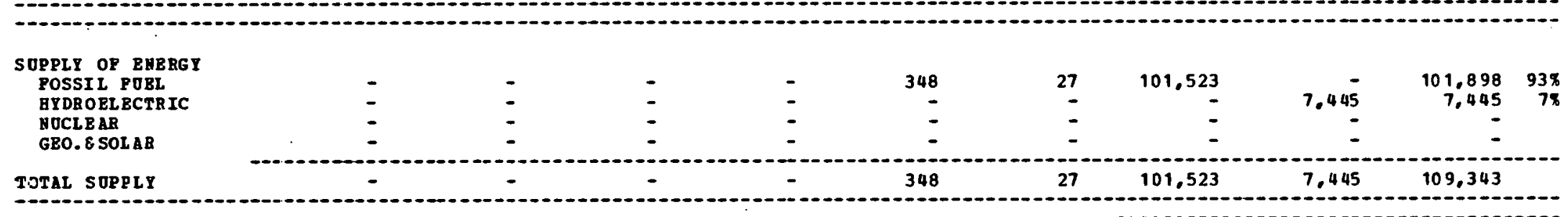

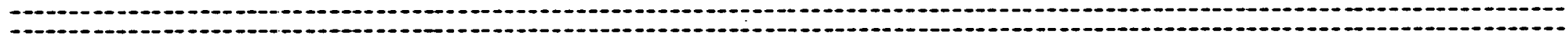

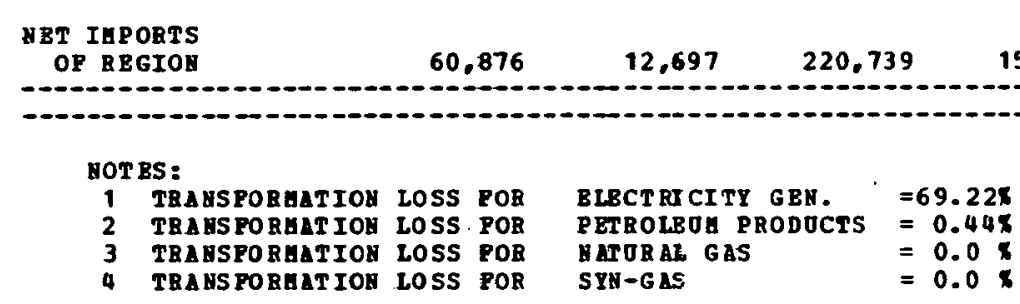


REGIJN LL ENERIY BALANCE STATEGEAT

BY SBCTOR AND FOBL TYPE

\begin{tabular}{|c|c|c|c|c|c|c|c|c|c|}
\hline SECTCR & $\begin{array}{c}\text { DISTILLATB } \\
\text { OIL }\end{array}$ & $\begin{array}{c}\text { BES I DOAL } \\
\text { OIL }\end{array}$ & $\begin{array}{l}\text { GASOLINE } \\
\text { (ALL'O }\end{array}$ & $\begin{array}{l}\text { EYDRO- } \\
\text { CLRBONS } \\
\text { IS IN } 10 * 0\end{array}$ & $\begin{array}{c}\text { CRUDE } \\
\text { OIL } \\
\text { BTOSI }\end{array}$ & $\begin{array}{l}\text { NAT ORAL } \\
\text { GAS }\end{array}$ & $\cos \mathrm{L}$ & ELECTRICITI & $\begin{array}{l}\text { SECTOR } \\
\text { TOTAL }\end{array}$ \\
\hline $\begin{array}{l}\text { FIHAL DEGAHD SECTORS } \\
\text { RBSIDBETIAL, COAH. } \\
\text { IHDOSTRIAL } \\
\text { TRABSPORTATI OR } \\
\text { UISCEL LANBOOS OSBS }\end{array}$ & $\begin{array}{r}7.799 \\
8.496 \\
21.701 \\
1.401\end{array}$ & $\begin{array}{r}1.024 \\
8.498 \\
300 \\
474\end{array}$ & 59.299 & $\begin{array}{r}14.701 \\
5.855 \\
5.294 \\
189\end{array}$ & $\begin{array}{l}\overline{-} \\
\overline{-}\end{array}$ & $\begin{array}{r}40.934 \\
42.000 \\
1.700 \\
4.065\end{array}$ & $\begin{array}{r}4.879 \\
7.035 \\
12 \\
-\end{array}$ & $\begin{array}{r}11.453 \\
17.379 \\
193 \\
188\end{array}$ & $\begin{array}{r}80,790 \\
89,263 \\
88,499 \\
6,317\end{array}$ \\
\hline $\begin{array}{l}\text { TOTAL PIBAL } \\
\text { DEGA SD SECIORS }\end{array}$ & $\$ 9,397$ & 10.296 & 59.299 & 26,039 & - & $88,69 \cdot 7$ & 11,926 & 29.213 & 264,869 \\
\hline $\begin{array}{l}\text { TRABSPORHATIOA } \\
\text { BLECTRICITY GBH. } \\
\text { PETROLEOA PRODOCT } \\
\text { BATORAL GAS } \\
\text { SYH-GAS }\end{array}$ & $\begin{array}{r}152 \\
-80,182 \\
- \\
-\end{array}$ & $\begin{array}{r}792 \\
-20.339 \\
-\end{array}$ & $\begin{array}{r}-143,3 \equiv 9= \\
=\end{array}$ & $\begin{array}{r}-3 C .476 \\
-1.209 \\
-\end{array}$ & $\begin{array}{r}252.096 \\
-\end{array}$ & $\begin{array}{r}2.363 \\
22.423 \\
1.388 \\
-\end{array}$ & $\begin{array}{r}13,8.33 \\
- \\
-\end{array}$ & $\begin{array}{r}-5,365 \\
= \\
=\end{array}$ & $\begin{array}{r}11.781 \\
180 \\
179 \\
-\end{array}$ \\
\hline $\begin{array}{l}\text { RET FOEL OSBD } \\
\text { IE TRAYSPORHATIOH }\end{array}$ & -80.030 & $-19,547$ & $-143,339$ & $-3 \circ .685$ & 252,096 & 26.177 & 13,833 & $-5,365$ & 12,140 \\
\hline $\begin{array}{l}\text { TOTAL GROSS FiOHS } \\
\text { LOSSES \& OHISIIOHS }\end{array}$ & $\begin{array}{r}39.549 \\
2.402\end{array}$ & $\begin{array}{r}11.088 \\
-112\end{array}$ & $\begin{array}{l}59.299 \\
-1,877\end{array}$ & $\begin{array}{l}26.039 \\
-\because .230\end{array}$ & $\begin{array}{r}252,096 \\
382\end{array}$ & $\begin{array}{r}114.876 \\
434\end{array}$ & $\begin{array}{r}25,759 \\
428\end{array}$ & $\begin{array}{r}29,213 \\
2,336\end{array}$ & $\begin{array}{r}277,009 \\
2,764\end{array}$ \\
\hline
\end{tabular}

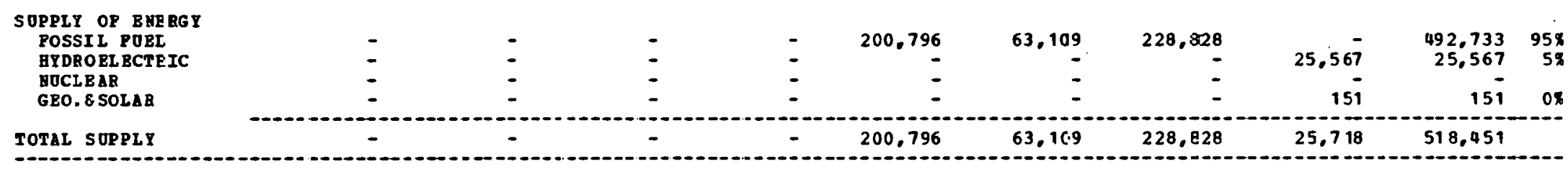

\begin{tabular}{|c|c|c|c|c|c|c|c|c|c|}
\hline $\begin{array}{l}\text { BT IUPORTS } \\
\text { OF REGIOR }\end{array}$ & $-38,230$ & $-9,363$ & $-85,917$ & $-5,876$ & 51,682 & 52,201 & $-202, \varepsilon 40$ & 466 & -238.677 \\
\hline
\end{tabular}

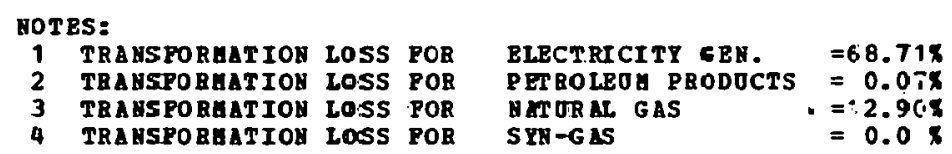


REGIONAL BNERGI BALANCE STATBEEHT

BY SECTOQ AND FOEL TYPE

\begin{tabular}{|c|c|c|c|c|c|c|c|c|c|}
\hline SECTOR & $\begin{array}{l}\text { DISTILLATE } \\
\text { OIL }\end{array}$ & $\begin{array}{l}\text { RESIDOAL } \\
\text { OIL }\end{array}$ & $\begin{array}{l}\text { GASOLINE } \\
\text { (ALL ONIT }\end{array}$ & $\begin{array}{c}\text { OTBER } \\
\text { HYDBO- } \\
\text { CARBONS } \\
\text { TS IN 10* }\end{array}$ & $\begin{array}{c}\text { CRODE } \\
\text { OIL } \\
\text { BTO०S) }\end{array}$ & $\begin{array}{l}\text { NAT URAL } \\
\text { GAS }\end{array}$ & COAL & RLECTRICITY & $\begin{array}{l}\text { SECTOR } \\
\text { TOTAL }\end{array}$ \\
\hline 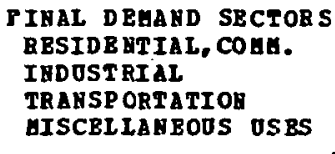 & $\begin{array}{r}13.700 \\
7.801 \\
29.102 \\
799\end{array}$ & $\begin{array}{r}1.239 \\
598 \\
1.000 \\
361\end{array}$ & 116.999 & $\begin{array}{r}26.900 \\
15.023 \\
9.887 \\
1.103\end{array}$ & ! & $\begin{array}{r}84.851 \\
72,798 \\
13.900 \\
6.049\end{array}$ & $\begin{array}{r}807 \\
2,756 \\
25 \\
-\end{array}$ & $\begin{array}{r}27.253 \\
7.543 \\
23 \\
670\end{array}$ & $\begin{array}{r}154,750 \\
106,519 \\
170,936 \\
8,982\end{array}$ \\
\hline $\begin{array}{l}\text { TOTAL PIHAL } \\
\text { DEHABD SBC TORS }\end{array}$ & 51,402 & 3,198 & 116,999 & 52,913 & - & 177,598 & 3,588 & $35,4 \in 9$ & 441,187 \\
\hline $\begin{array}{l}\text { TRAHSPO RHATIOB } \\
\text { BLECTRICITY GBE. } \\
\text { PETROLEOA PRODUCTS } \\
\text { NATURAL GAS } \\
\text { SYH-GAS }\end{array}$ & $\begin{array}{r}1.040 \\
-2.499 \\
- \\
-\end{array}$ & $\begin{array}{r}961 \\
-245 \\
-\end{array}$ & $\begin{array}{r}-5,290 \\
-\end{array}$ & $\begin{array}{r}-1,956 \\
-720 \\
-\end{array}$ & 9.628 & $\begin{array}{r}55.266 \\
439 \\
827 \\
-\end{array}$ & $\begin{array}{r}31.019 \\
- \\
-\end{array}$ & $\begin{array}{r}-25,984 \\
=\end{array}$ & $\begin{array}{r}62,302 \\
77 \\
107 \\
-\end{array}$ \\
\hline $\begin{array}{l}\text { DBT POEL OSED } \\
\text { IH TRANSFOREATIOR }\end{array}$ & $-1,459$ & 716 & $-5,290$ & -2.676 & 9,628 & 56,532 & 31,019 & $-25,984$ & 62,486 \\
\hline $\begin{array}{l}\text { TOTAL GROSS PLORS } \\
\text { I.OSSES } 8 \text { O\&ISSIOHS }\end{array}$ & $\begin{array}{r}52,442 \\
3,185\end{array}$ & $\begin{array}{r}4.159 \\
-42\end{array}$ & $\begin{array}{r}116,999 \\
-3,704\end{array}$ & $\begin{array}{l}52,913 \\
-2,500\end{array}$ & $\begin{array}{r}9,628 \\
14\end{array}$ & $\begin{array}{r}234.130 \\
886\end{array}$ & $\begin{array}{r}34,607 \\
575\end{array}$ & $\begin{array}{r}35,489 \\
2.838\end{array}$ & $\begin{array}{r}503,673 \\
1,254\end{array}$ \\
\hline
\end{tabular}

\begin{tabular}{|c|c|c|c|c|c|c|c|c|c|}
\hline $\begin{array}{l}\text { SOPPLY OP EREBGY } \\
\text { POSSIL POER } \\
\text { HYDROBLBCTEIC } \\
\text { HOCLEAR } \\
\text { GEO.ESOLAR }\end{array}$ & I & $\bar{z}$ & $\begin{array}{l}- \\
\overline{-}\end{array}$ & : & $\begin{array}{r}41,992 \\
= \\
=\end{array}$ & $\begin{array}{r}5,005 \\
- \\
-\end{array}$ & $\overline{-}$ & $\begin{array}{l}4,652 \\
2,046 \\
-\end{array}$ & $\begin{array}{r}46,997 \\
4,652 \\
2,046 \\
-\end{array}$ \\
\hline
\end{tabular}

-

$\begin{aligned} & \text { BET IMPORTS } \\ & \text { OF REGIOH }\end{aligned}$
0


REGION AL EXERGY BALANCE STATEAENT

BT SECTOR ABD FUEL TYPE

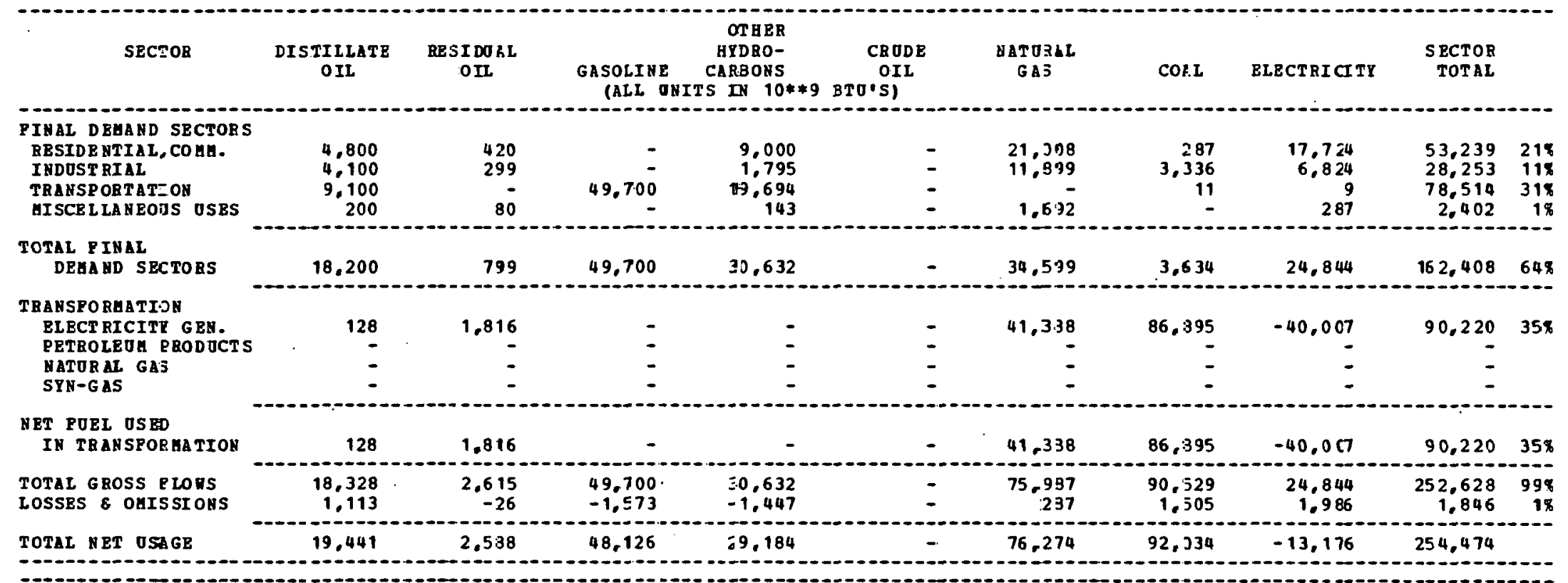

SOPPLY OP ERERG

POSSIL POER

HYDRORLECTRIC

NOCLBAR

GEO.E SOLAR

TOTAL SOPPLI

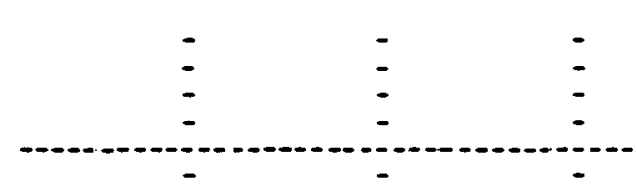

$\begin{array}{lcc}- & - & 557 \\ - & - & - \\ - & - & -\end{array}$

.

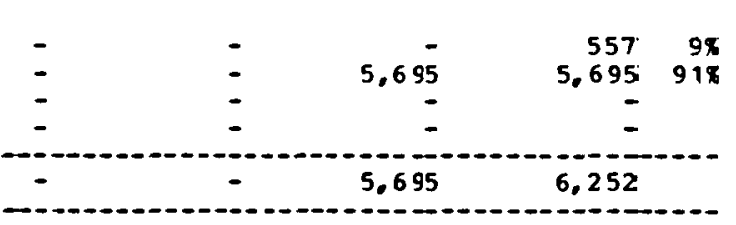

\section{NET IMPORTS}

OP REGIOH

19,441

2.588

48,126

29,184

$-557$

76,274

92,034

$-18,871$

248,222

\section{NOT ES: \\ 1 TRANSPORHATIOY LOSS FOB \\ TRALS PORAATIOY LOSS POR \\ 4 TRassonarion LOSS POR \\ PETROLEO AFODOCTS $=0.0$ \% \\ $S T R-G A S$ \\ $=0.0$}


REGIOYAL EMERGY BALANCE STATEAENT

BY SECTOR AKD FOEL TYPE

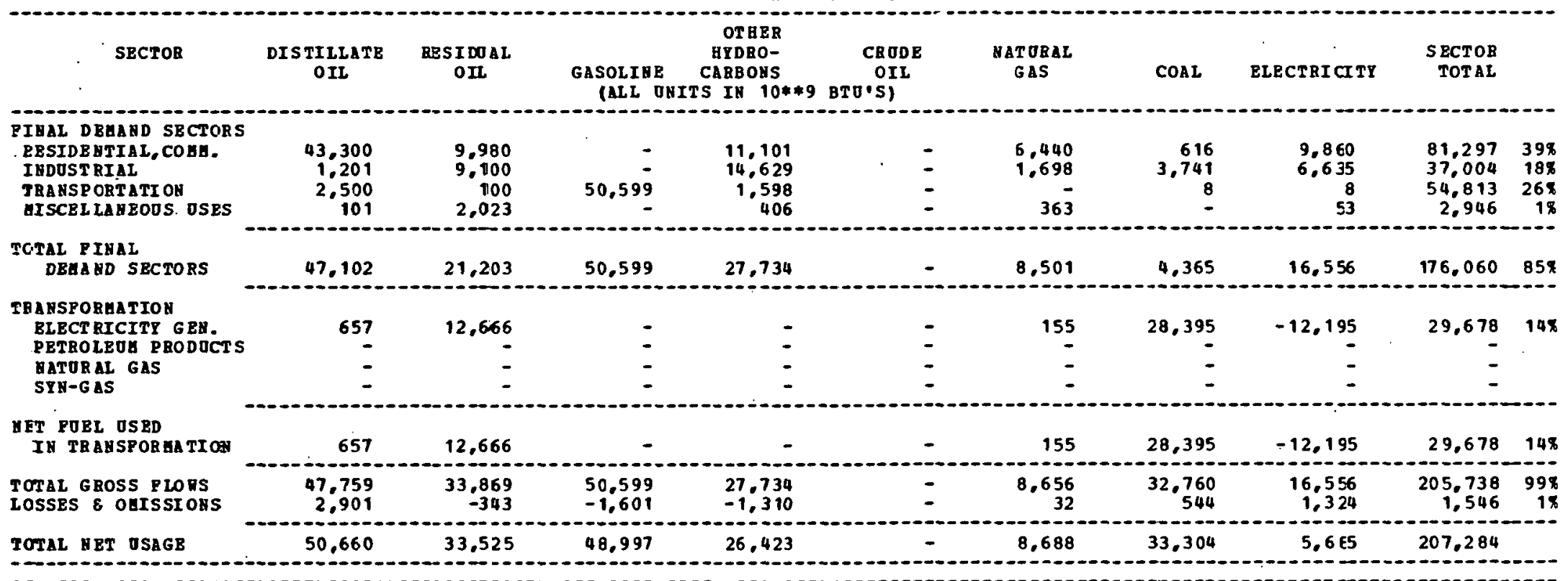

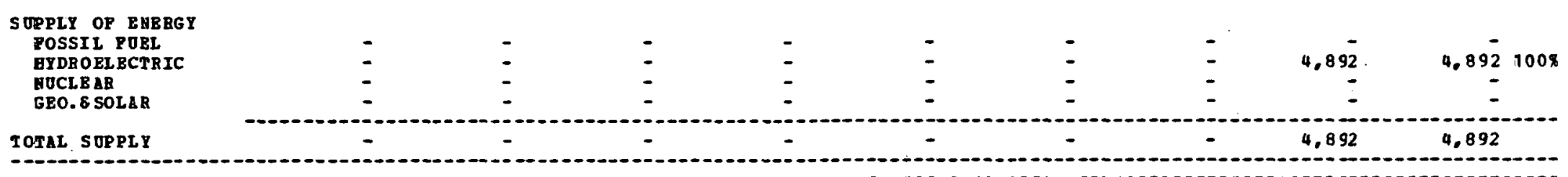

\section{BBT IHPOETS}

50,660

33.525

48,997

26,423

8,688

33,304

793

202,392

\section{NOTBS:}

1 TRA HORUAT IOH LOSS POR

TRA NSPORAATIOH LOSS FO

TRA ISPOBAATI LOSS POB

BIECTRTCITT GEY.

$=70.88 \%$
$=0.0 \%$

MATORAL GAS

SYH $-G$ AS 
REgIOHAL 3REEgT BALANCE STATEGENT

OP SECTOE AND POEL TIPE

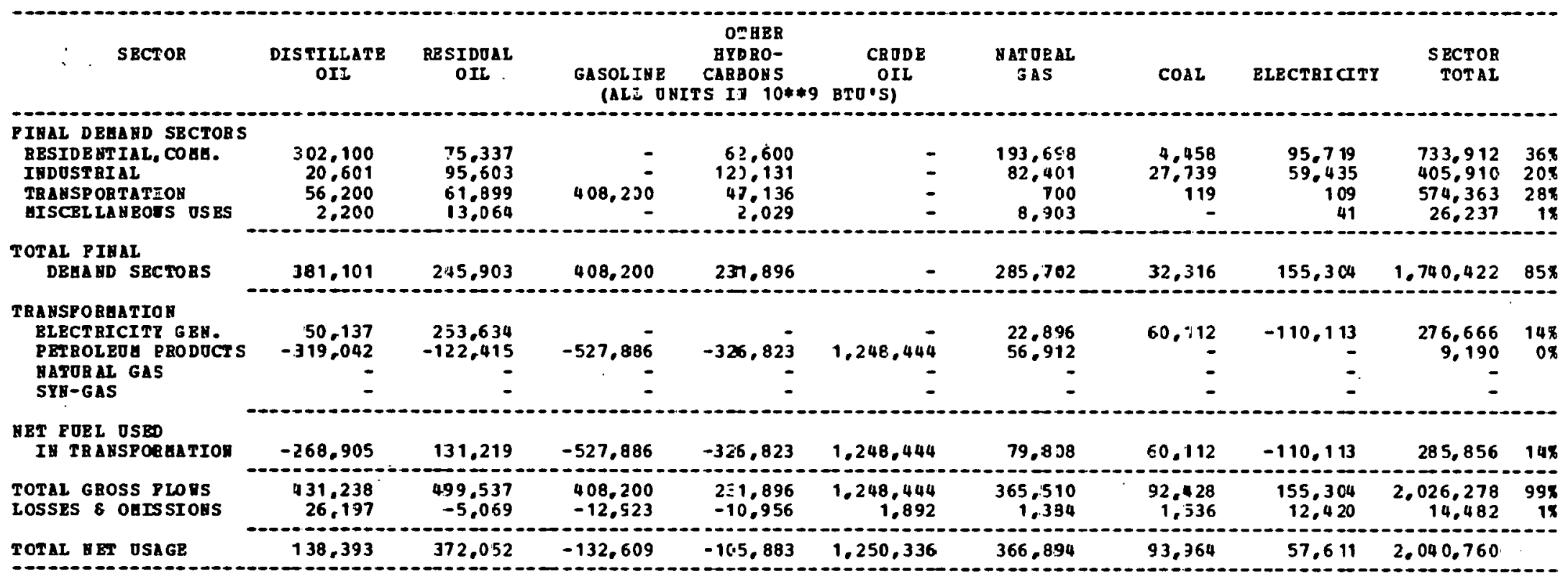

0
-

\section{SOPPLI OF BEBRGY}

FOSSIL POEI

AYDROELBCTRIC

GOCLEAR

GBO.8 SOLAR

$\begin{array}{rll}- & - & - \\ - & - & - \\ - & - & -\end{array}$

E

$\begin{array}{ll}- & - \\ - & - \\ - & -\end{array}$

-

\begin{tabular}{lccc}
- & - & $\overline{1}$ & 16 \\
- & 12,248 & 12,248 & $0 \%$ \\
- & - & - & $100 \%$ \\
\hline- & 12,264 & 12,264 &
\end{tabular}

TOTAL SOPPL:

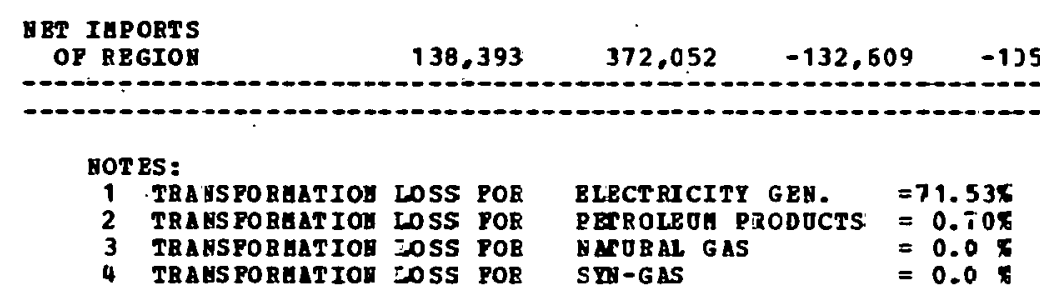




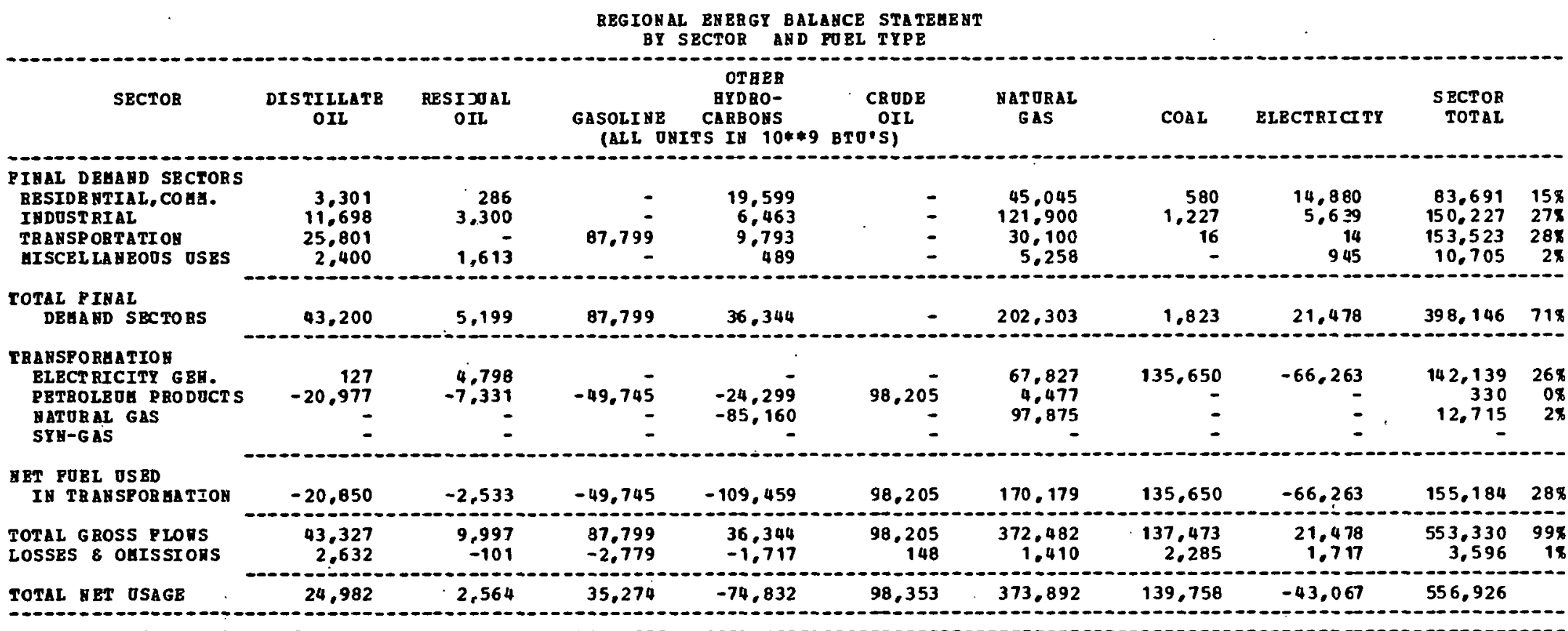

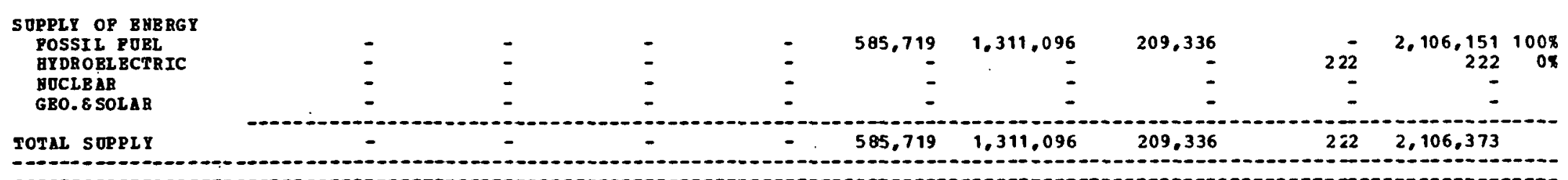

\section{ABT IUPOBTS}

24,982

2,564

35,274

$-74,832$

$-487,365$

$-937,203$

$-69,577$
MOTES:
1 TRABSFORGATIOH LOSS FOR TRABSFOBATIOA LOSS POB TRABS FOBAATIOH LOSS POA
$\begin{array}{ll}\text { ELECTRICITY GEN. } & =68.20 \times \\ \text { PERROLROY PRODOCTS } & =0.32\end{array}$

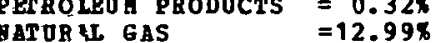
TRABSPORAATION LOSS POR
SYN-G QS
$=0.0 \%$ 
REGIONAL ENERGY BALANCE STATEGENT

BY SECTOR AND POEL TYPE

\begin{tabular}{|c|c|c|c|c|c|c|c|c|c|c|}
\hline SECTOR & $\begin{array}{c}\text { DISFILLATE } \\
\text { OI }\end{array}$ & $\begin{array}{l}\text { RESIDOAL } \\
\text { OIL }\end{array}$ & $\begin{array}{l}\text { GASOLIRE } \\
\text { (ALL ONI }\end{array}$ & $\begin{array}{l}\text { RYDRO- } \\
\text { CABBONS } \\
\text { TS IH 10**9 }\end{array}$ & $\begin{array}{c}\text { CRODE } \\
\text { OIL } \\
\text { BTO'S) }\end{array}$ & $\begin{array}{l}\text { NAT URAL } \\
\text { BAS }\end{array}$ & COAL & BLECT BI CITY & $\begin{array}{l}\text { SECTOR } \\
\text { TOTAL }\end{array}$ & \\
\hline $\begin{array}{l}\text { PINAI DEYAND SECTORS } \\
\text { RESIDE HTIAL, COAG. } \\
\text { IHDOSTRIAL } \\
\text { TRANSPORTATION } \\
\text { GISCELIANEOOS OSBS }\end{array}$ & $\begin{array}{r}5+75,799 \\
27,504 \\
57,599 \\
3,201\end{array}$ & $\begin{array}{r}331,903 \\
64.003 \\
108,200 \\
43.295\end{array}$ & 787,599 & $\begin{array}{r}98,400 \\
225,709 \\
235,336 \\
5,588\end{array}$ & $\begin{array}{l}- \\
-\end{array}$ & $\begin{array}{r}481.635 \\
143,803 \\
3,100 \\
13.865\end{array}$ & $\begin{array}{r}11.175 \\
257,090 \\
303 \\
-\end{array}$ & $\begin{array}{r}215.529 \\
106,500 \\
9.012 \\
8.538\end{array}$ & $\begin{array}{r}1,684,441 \\
824,609 \\
1,211,149 \\
74,487\end{array}$ & $\begin{array}{r}398 \\
198 \\
288 \\
28\end{array}$ \\
\hline $\begin{array}{l}\text { TOTAL PIHAL } \\
\text { DEGA HD SBCTOBS }\end{array}$ & $644, .103$ & 547.401 & 787,539 & 565,033 & - & 642.403 & $268, \leq 68$ & 339.579 & $3,794,686$ & 878 \\
\hline $\begin{array}{l}\text { TRANSPORHATION } \\
\text { BLECTRICITI GEY. } \\
\text { PETROLEOA PRODUCTS } \\
\text { NATORAL GAS } \\
\text { SYH-GAS }\end{array}$ & $\begin{array}{r}58,383 \\
-54,802 \\
-\end{array}$ & $\begin{array}{r}519.718 \\
-21.030 \\
- \\
-\end{array}$ & $\begin{array}{r}-92,01 \overline{-} \\
-\end{array}$ & $\begin{array}{r}-52,748 \\
-\end{array}$ & $\begin{array}{r}212,750 \\
-\end{array}$ & $\begin{array}{r}71,012 \\
9,698 \\
- \\
-\end{array}$ & $\begin{array}{r}141,743 \\
= \\
=\end{array}$ & $\begin{array}{r}-232,5 \varepsilon 1 \\
- \\
-\end{array}$ & $\begin{array}{r}558,275 \\
1,850 \\
- \\
-\end{array}$ & $\begin{array}{r}138 \\
08\end{array}$ \\
\hline $\begin{array}{l}\text { NET FOEL OSED } \\
\text { IN TBASSPOZGATIOY }\end{array}$ & 3.581 & 498,688 & $-92,018$ & $-52,748$ & 212.750 & 80,710 & 141,743 & $-232,5 \varepsilon 1$ & 560,125 & 138 \\
\hline $\begin{array}{l}\text { TOTAL GROSS RLONS } \\
\text { LOSSES } 8 \text { OHISSIOHS }\end{array}$ & $\begin{array}{r}702.486 \\
42.675\end{array}$ & $\begin{array}{r}1.067 .119 \\
-10,828\end{array}$ & $\begin{array}{l}787,599 \\
-24,934\end{array}$ & $\begin{array}{l}565,033 \\
-26,696\end{array}$ & $\begin{array}{r}212,750 \\
322\end{array}$ & $\begin{array}{r}723,113 \\
2,738\end{array}$ & $\begin{array}{r}410.311 \\
6,822\end{array}$ & $\begin{array}{r}339,579 \\
27,158\end{array}$ & $\begin{array}{r}4,354,811 \\
17,256\end{array}$ & $\begin{array}{r}100 \% \\
0 \%\end{array}$ \\
\hline
\end{tabular}

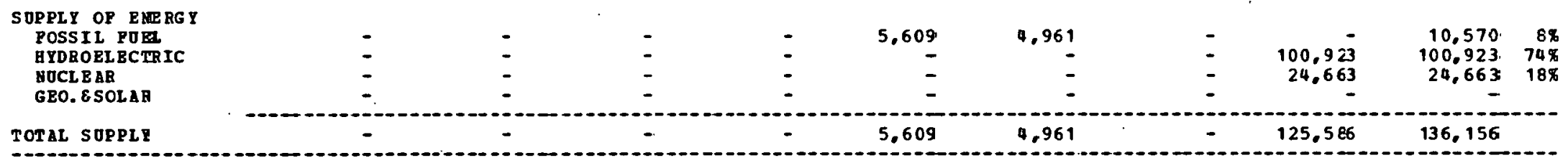

\begin{tabular}{|c|c|c|c|c|c|c|c|c|c|}
\hline OP REGIOH & 690,359 & $1,035,260$ & 670,646 & 485,588 & 207.463 & 720.890 & 417.133 & 8,570 & $4.235,911$ \\
\hline
\end{tabular}

\section{NOT ES :}

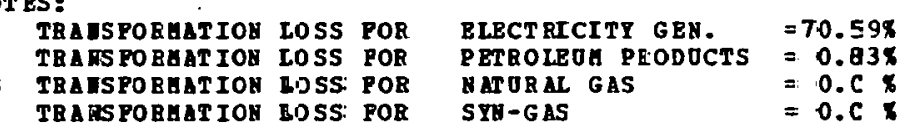

TRAISPOBATION DOSS: POR WATORAL GAS 
REG IONAL ENERGT BALANCE STATEHENT

BY SECTOR AND POEL TYPE

\begin{tabular}{|c|c|c|c|c|c|c|c|c|c|c|}
\hline SECTOR & $\begin{array}{c}\text { DISTILLATE } \\
\text { OIL }\end{array}$ & $\begin{array}{l}\text { BBSIDOAL } \\
\text { OII }\end{array}$ & $\begin{array}{l}\text { GASOLINE } \\
\text { (ALL OI }\end{array}$ & $\begin{array}{l}\text { GYDRO- } \\
\text { CARBONS } \\
\text { TS IN } 10 * * 9\end{array}$ & $\begin{array}{l}\text { CR ODE } \\
\text { OIL } \\
\text { BTO'SI }\end{array}$ & $\begin{array}{l}\text { MATOBAL } \\
\text { GAS }\end{array}$ & COAL & BLECTRICITY & $\begin{array}{l}\text { SECTOR } \\
\text { TOTAL }\end{array}$ & \\
\hline 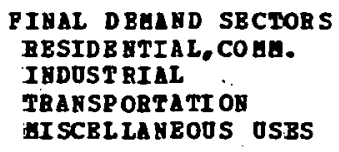 & $\begin{array}{l}67.199 \\
18.799 \\
51.100 \\
2.000\end{array}$ & $\begin{array}{r}10.403 \\
46.202 \\
1.598 \\
4.396\end{array}$ & 359,100 & $\begin{array}{r}84,600 \\
109,733 \\
17,963 \\
6,383\end{array}$ & $\begin{array}{l}\overline{-} \\
\overline{-}\end{array}$ & $\begin{array}{r}46.873 \\
97.396 \\
6.102 \\
5.725\end{array}$ & $\begin{array}{r}12,065 \\
58,588 \\
66 \\
-\end{array}$ & $\begin{array}{r}98,475 \\
68,636 \\
61 \\
2,945\end{array}$ & $\begin{array}{r}319,615 \\
399,354 \\
435,490 \\
21,449\end{array}$ & $\begin{array}{r}218 \\
268 \\
28 \% \\
18\end{array}$ \\
\hline $\begin{array}{l}\text { TOTAL PINAL } \\
\text { DEUA AD S BCTORS }\end{array}$ & 139,098 & 62.099 & 359,100 & 218,679 & - & 156.096 & 70,719 & 170.117 & $1.175,908$ & $77 x$ \\
\hline $\begin{array}{l}\text { TRAMSPORGATIOH } \\
\text { BLECT RICITY GEH. } \\
\text { PETROLEOA PRODOCTS } \\
\text { MATORAL GAS } \\
\text { STN-GAS }\end{array}$ & $\begin{array}{r}3,339 \\
- \\
-\end{array}$ & $\begin{array}{r}38,144 \\
- \\
-\end{array}$ & $\begin{array}{l}- \\
-\end{array}$ & $\begin{array}{l}- \\
-\end{array}$ & $\begin{array}{l}- \\
\overline{-}\end{array}$ & $\begin{array}{r}7,911 \\
= \\
-\end{array}$ & $\begin{array}{r}475,850 \\
= \\
=\end{array}$ & $\begin{array}{r}-181,376 \\
= \\
=\end{array}$ & $\begin{array}{r}34,868 \\
= \\
-\end{array}$ & $22 \%$ \\
\hline $\begin{array}{l}\text { IST POEL OSBD } \\
\text { IN TRABSPOREATIOR }\end{array}$ & 3.339 & 38,144 & - & - & - & 7.911 & 475.850 & $-181 \cdot 376$ & 343,868 & $22 \%$ \\
\hline $\begin{array}{l}\text { TOTAL GROSS FLORS } \\
\text { LOSSES \& ORISSI OHS }\end{array}$ & $\begin{array}{r}142.437 \\
8.653\end{array}$ & $\begin{array}{r}100.243 \\
-1.017\end{array}$ & $\begin{array}{l}359,100 \\
-11,368\end{array}$ & $\begin{array}{l}218,679 \\
-10,332\end{array}$ & - & $\begin{array}{r}164.007 \\
621\end{array}$ & $\begin{array}{r}546,569 \\
9,087\end{array}$ & $\begin{array}{r}170,177 \\
13.605\end{array}$ & $\begin{array}{r}1,519,776 \\
9,248\end{array}$ & $\begin{array}{r}99 \% \\
1 \%\end{array}$ \\
\hline
\end{tabular}

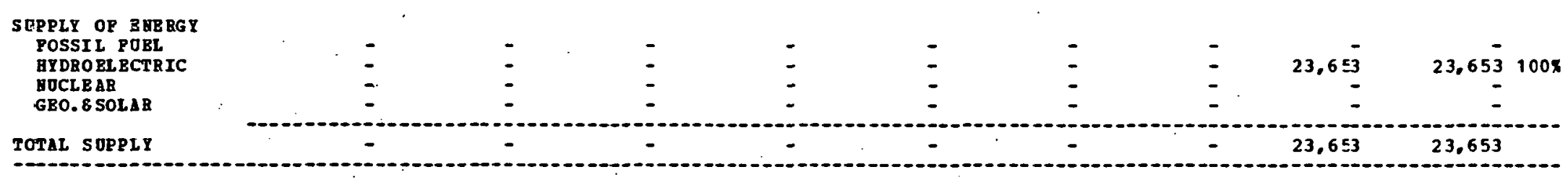

-

\section{HBT IUPORTS}

OP RBGIOE

151,090

99.225

347,731

208,346

164,628

555,656

$-21,306$

HOTBS:

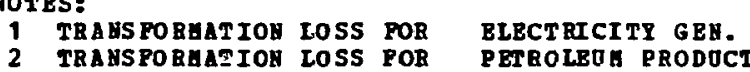

TRAYSPORAAEIOH LOSS PO

THABSPORGATIOA LOSS POA 
REGICHAL EUBREY BALAGCE STATEHENT

ET SECTOB AND POSL TYPE

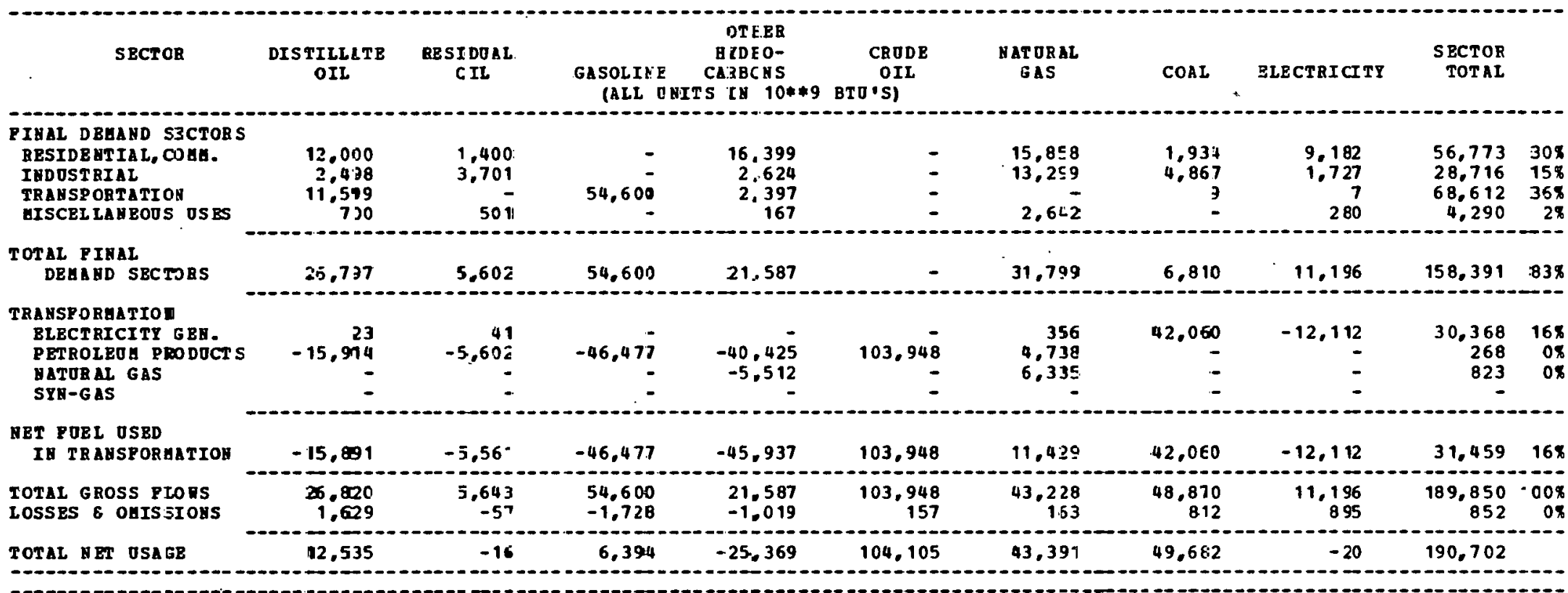

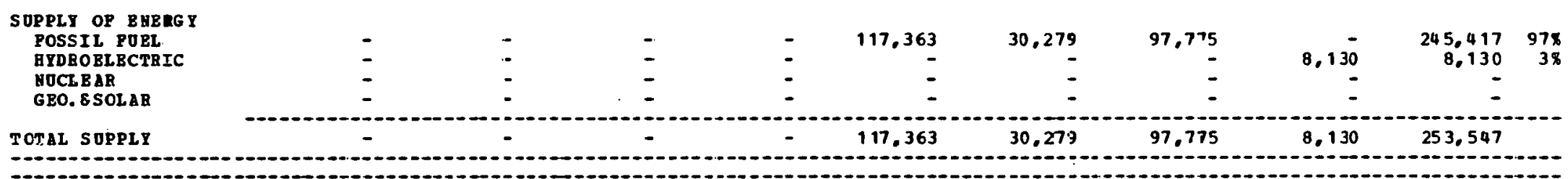

HET IHPORTS

OP REGIO

12,535

$-16$

6,394

$-2 \subseteq .369$

$-13,257$

13,112

$-48,092$

$-8,150$

$-62.844$

\section{NOT ES :}

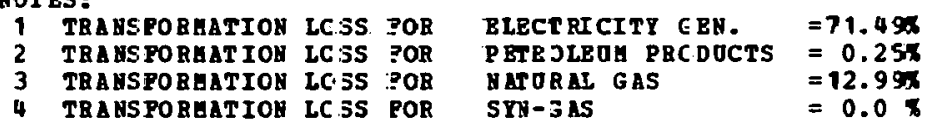


RBG IOHAL EHERGY BALANCE STATEHENT

BY SECTOR AND POEL TYPE

\begin{tabular}{|c|c|c|c|c|c|c|c|c|c|c|}
\hline SECTOR & $\begin{array}{c}\text { DISTILLATE } \\
\text { OIL }\end{array}$ & $\begin{array}{l}\text { RESIDOAL } \\
\text { OIL }\end{array}$ & $\begin{array}{l}\text { GASOLINE } \\
\text { (ALL OHI }\end{array}$ & $\begin{array}{c}\text { OTHER } \\
\text { GYDBO- } \\
\text { CARBONS } \\
\text { TS IN } 10 * * 9\end{array}$ & $\begin{array}{c}\text { CRODE } \\
\text { OIL } \\
\text { BTO'S) }\end{array}$ & $\begin{array}{c}\text { NATORAL } \\
\text { GAS }\end{array}$ & COAL & ELECTRICITY & $\begin{array}{r}\text { SBCTOR } \\
\text { TOTAL }\end{array}$ & \\
\hline $\begin{array}{l}\text { PINAL DEGAND SBCTORS } \\
\text { RESIDBHTIAL, COAH. } \\
\text { INDOSTRIAL } \\
\text { TRANSPORTATIOH } \\
\text { UISCBLLAHEOOS OS3S }\end{array}$ & $\begin{array}{l}88.100 \\
46.697 \\
92.601 \\
1.501\end{array}$ & $\begin{array}{r}3.882 \\
27.701 \\
600 \\
1.419\end{array}$ & 652,301 & $\begin{array}{r}118,098 \\
204.266 \\
51,723 \\
3,014\end{array}$ & $\overline{-}$ & $\begin{array}{r}626,150 \\
461,403 \\
13,101 \\
23,052\end{array}$ & $\begin{array}{r}31,626 \\
603,363 \\
144 \\
-\end{array}$ & $\begin{array}{r}156,099 \\
203,521 \\
224 \\
3.058\end{array}$ & $\begin{array}{r}1,023,955 \\
1,546,951 \\
810,694 \\
32,044\end{array}$ & $\begin{array}{r}258 \\
37 \% \\
20 \% \\
1 \%\end{array}$ \\
\hline $\begin{array}{l}\text { TOTAL FIRAL } \\
\text { DEHA HD SBCTORS }\end{array}$ & 228,899 & $33,6,02$ & 652,301 & 377,101 & - & $1,123,706$ & 635.133 & 362,902 & $3,413.644$ & $82 \pi$ \\
\hline $\begin{array}{l}\text { TRANSPORAATIOH } \\
\text { BLECTRICITY GBH, } \\
\text { PETROLEUH PRODOCT S } \\
\text { NATURAL GAS } \\
\text { SYH-GAS }\end{array}$ & $\begin{array}{r}9,948 \\
-263,127 \\
-\end{array}$ & $\begin{array}{r}11.444 \\
-83.895 \\
-\end{array}$ & $\begin{array}{r}-603,483 \\
-\end{array}$ & $\begin{array}{r}6.874 \\
-154.823 \\
-\end{array}$ & $\begin{array}{r}1.059 .167 \\
-\end{array}$ & $\begin{array}{r}15,314 \\
48,283 \\
-\end{array}$ & $\begin{array}{r}985.024 \\
= \\
-\end{array}$ & $\begin{array}{r}-327,785 \\
- \\
-\end{array}$ & $\begin{array}{r}700,819 \\
2,122 \\
- \\
-\end{array}$ & $\begin{array}{r}17 \% \\
0 \%\end{array}$ \\
\hline $\begin{array}{l}\text { HET FOEL OSED } \\
\text { IN TRABSPOBEATIOH }\end{array}$ & $-253,179$ & $-72,451$ & $-603,483$ & $-147,949$ & $1,059,167$ & 63,597 & 985.024 & $-327,785$ & 702,941 & $17 \%$ \\
\hline $\begin{array}{l}\text { TOTAL GROSS PLORS } \\
\text { LOSSES } 6 \text { OBISSIOHS }\end{array}$ & $\begin{array}{r}238,847 \\
14,509\end{array}$ & $\begin{array}{r}45.046 \\
-457\end{array}$ & $\begin{array}{l}652,301 \\
-20,651\end{array}$ & $\begin{array}{l}383,975 \\
-18,142\end{array}$ & $\begin{array}{r}1.059,167 \\
1.605\end{array}$ & $\begin{array}{r}1,187,303 \\
4,495\end{array}$ & $\begin{array}{r}1,620,157 \\
26,938\end{array}$ & $\begin{array}{r}362,902 \\
29.023\end{array}$ & $\begin{array}{r}4,116,585 \\
\quad 37,322\end{array}$ & $\begin{array}{r}99 \% \\
1 \%\end{array}$ \\
\hline
\end{tabular}

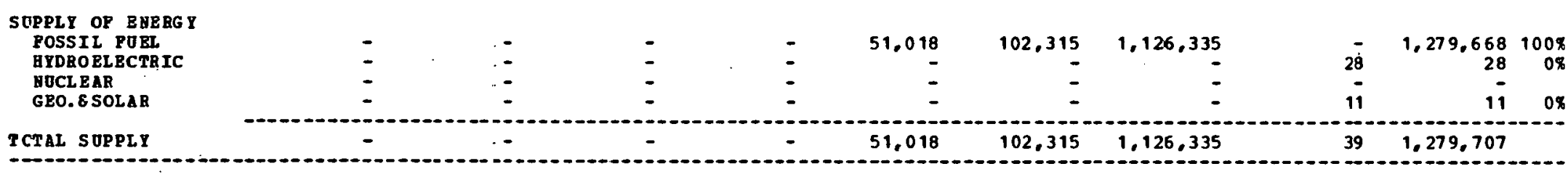

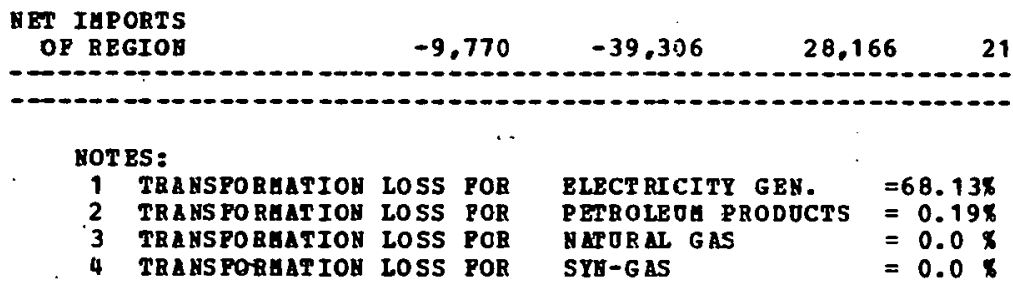


REGIONAL BNEBGT BALANCE STATEGENT

BY SECTOR AUD FOEL TYPE

\begin{tabular}{|c|c|c|c|c|c|c|c|c|c|}
\hline SECTOR & $\begin{array}{l}\text { DISTILLATE } \\
\text { DIL }\end{array}$ & $\begin{array}{l}\text { RESI DO AL } \\
\text { OIL }\end{array}$ & $\begin{array}{l}\text { GASOLIHE } \\
\text { (ALL OI }\end{array}$ & $\begin{array}{l}\text { GYDRO- } \\
\text { CARBONS } \\
\text { TS IN 10**9 }\end{array}$ & $\begin{array}{l}\text { CR ODE } \\
\text { OIL } \\
\text { BTU.S) }\end{array}$ & $\begin{array}{l}\text { NATUREL } \\
\text { GAS }\end{array}$ & COAL & ELECTRICITY & $\begin{array}{l}\text { SECTOR } \\
\text { TOTAL }\end{array}$ \\
\hline $\begin{array}{l}\text { PINAL DEGAND SECTORS } \\
\text { RESIDE HTIAL, COAB. } \\
\text { INDUSTRIAL } \\
\text { TRANSPORTATI OW } \\
\text { GISCEL LAHEOOS USES }\end{array}$ & $\begin{array}{r}3.351 \\
15.876 \\
23.200 \\
1.050\end{array}$ & $\begin{array}{r}527 \\
8.000 \\
200 \\
272\end{array}$ & 216,101 & $\begin{array}{r}63,702 \\
24,759 \\
19,582 \\
999\end{array}$ & $\begin{array}{l}- \\
-\end{array}$ & $\begin{array}{r}105,169 \\
234,101 \\
26,603 \\
11,331\end{array}$ & $\begin{array}{r}1,398 \\
4,593 \\
34 \\
-\end{array}$ & $\begin{array}{r}46,319 \\
21,279 \\
32 \\
2,523\end{array}$ & $\begin{array}{r}226,416 \\
309,628 \\
285,752 \\
16,125\end{array}$ \\
\hline $\begin{array}{l}\text { TOTAL PIHAL } \\
\text { DERA RD SECTORS }\end{array}$ & 50,397 & 0,999 & 216,101 & 109.042 & - & 377,204 & 6,025 & 70,153 & 837.921 \\
\hline $\begin{array}{l}\text { TRANSPORHATIOY } \\
\text { RLECT RICITY GEN. } \\
\text { PETROLEOU PEODOCTS } \\
\text { MATORAL GAS } \\
\text { STH-GAS }\end{array}$ & $\begin{array}{r}286 \\
-237.258 \\
-\end{array}$ & $\begin{array}{r}461 \\
-23,068 \\
- \\
-\end{array}$ & $\begin{array}{r}-494,137 \\
-\end{array}$ & $\begin{array}{l}-228,403 \\
-102,723\end{array}$ & $\begin{array}{r}946,641 \\
-\end{array}$ & $\begin{array}{r}276,155 \\
43,153 \\
118,052 \\
\end{array}$ & $\begin{array}{r}5.2 \\
- \\
-\end{array}$ & $\begin{array}{r}-88,262 \\
- \\
-\end{array}$ & $\begin{array}{r}188,702 \\
6,928 \\
15,339 \\
-\end{array}$ \\
\hline $\begin{array}{l}\text { WET POEL OSED } \\
\text { IN TRANSPORHATION }\end{array}$ & $-236,972$ & $-22,607$ & -494.137 & $-331,126$ & 946,641 & 437.380 & 52 & $-88,262$ & 210,969 \\
\hline $\begin{array}{l}\text { TOTAL GROSS PLORS } \\
\text { LOSSES } 8 \text { OUISSION5 }\end{array}$ & $\begin{array}{r}50,683 \\
3,078\end{array}$ & $\begin{array}{r}5.460 \\
-95\end{array}$ & $\begin{array}{r}216,101 \\
-6,841\end{array}$ & $\begin{array}{r}109.042 \\
-5,152\end{array}$ & $\begin{array}{r}946.641 \\
1.435\end{array}$ & $\begin{array}{r}814.584 \\
3.084\end{array}$ & $\begin{array}{r}6.077 \\
101\end{array}$ & $\begin{array}{r}70,153 \\
5.610\end{array}$ & $\begin{array}{r}1,048,890 \\
1.220\end{array}$ \\
\hline TOTAL AET OSAGE & $-163,696$ & -13.703 & $-284,877$ & -227.236 & 948.076 & $817 ; 66: 3$ & 6.178 & $-12,498$ & 1.050 .110 \\
\hline
\end{tabular}

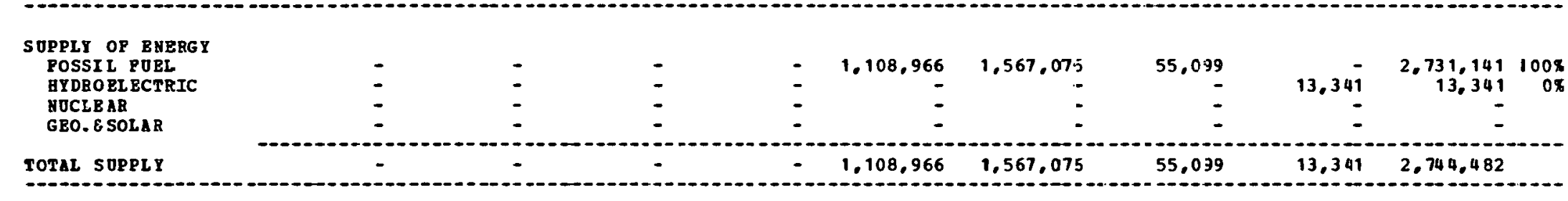

\section{YBT IAPORTS}

OP REGIÓN

$-183.496$

$-13,703$

$-284.877$

$-22 \pi, 236$

$-160,889$

$-749.407$

$-48,920$

$-25,839 \quad-1,694,371$

\begin{tabular}{|c|c|c|c|c|c|}
\hline & & & & & \\
\hline $\begin{array}{l}1 \\
2 \\
3 \\
4\end{array}$ & $\begin{array}{l}\text { TRAAS PORHAT ION } \\
\text { TRANSPOBAATION } \\
\text { TRANSPORAATIOA } \\
\text { TRABSPOREATIOS }\end{array}$ & $\begin{array}{l}\text { LOSS } \\
\text { LasS } \\
\text { Lass } \\
\text { LCSS }\end{array}$ & $\begin{array}{l}\text { POR } \\
\text { TOR } \\
\text { ?OR } \\
\text { ?OR }\end{array}$ & $\begin{array}{l}\text { BLECTRICITY GEH. } \\
\text { PETROLEOK PBCDOCTS } \\
\text { MATUBAL GAS } \\
\text { SYH-SAS }\end{array}$ & $\begin{array}{l}=68.13 \% \\
=0.70 \% \\
=12.978 \\
=0.08\end{array}$ \\
\hline
\end{tabular}


REGIONAL BNERGY BALAHCE STATBHENT

BY SECTOR AND POEL TYPE

\begin{tabular}{|c|c|c|c|c|c|c|c|c|c|c|}
\hline SECTOR & $\begin{array}{l}\text { DISTILLATE } \\
\text { OIL }\end{array}$ & $\begin{array}{l}\text { RB SIDOAL } \\
\text { OIL }\end{array}$ & $\begin{array}{l}\text { GASOLINE } \\
\text { (ALL ONIT }\end{array}$ & $\begin{array}{c}\text { OTBEB } \\
\text { BTDRO- } \\
\text { CARBOAS } \\
\text { IS IN } 10 * * 9\end{array}$ & $\begin{array}{c}\text { CRODE } \\
\text { OIL } \\
\text { BTO'SI }\end{array}$ & $\begin{array}{l}\text { MAT ORAL } \\
\text { GAS }\end{array}$ & $\operatorname{COAL}$ & BLECTRI CITY & $\begin{array}{l}\text { SECTOR } \\
\text { TOTAL }\end{array}$ & \\
\hline $\begin{array}{l}\text { PINAL D EHAND SECEORS } \\
\text { RESIDE HTIAL, COBA. } \\
\text { INDOSTRIAL } \\
\text { TRANSPORTATIOH } \\
\text { GISCEL LAYEOOS OSES }\end{array}$ & $\begin{array}{r}31.500 \\
17.499 \\
42.300 \\
500\end{array}$ & $\begin{array}{r}20,390 \\
20,401 \\
1,800 \\
4,012\end{array}$ & 155.800 & $\begin{array}{r}23.101 \\
27.439 \\
16.083 \\
1.082\end{array}$ & - & $\begin{array}{r}35.567 \\
61.201 \\
8.899 \\
1.134\end{array}$ & $\begin{array}{r}1.165 \\
6.033 \\
34 \\
-\end{array}$ & $\begin{array}{r}62,093 \\
40,753 \\
30 \\
223\end{array}$ & $\begin{array}{r}173,816 \\
17,3,326 \\
224,946 \\
6,951\end{array}$ & $\begin{array}{r}30 \% \\
30 \% \\
38 \% \\
1 \%\end{array}$ \\
\hline $\begin{array}{l}\text { TOTAL PIHAL } \\
\text { DEAA HD SECTOBS }\end{array}$ & 91,799 & 46.603 & 155,800 & 67.705 & - & 106.801 & 7,232 & 103,099 & 579.039 & $99 \%$ \\
\hline $\begin{array}{l}\text { TRAYSPOREATION } \\
\text { BLECTRICITY GEY. } \\
\text { PETROLEOA PRODUCTS } \\
\text { NATORAL GAS } \\
\text { STH-GAS }\end{array}$ & $\begin{array}{l}60 \\
- \\
-\end{array}$ & $\begin{array}{l}4 \\
-\end{array}$ & $\overline{-}$ & $\overline{-}$ & $\overline{-}$ & $\begin{array}{r}1,826 \\
=\end{array}$ & $\begin{array}{l}\overline{-} \\
\overline{-}\end{array}$ & $\begin{array}{r}-518 \\
\vdots \\
-\end{array}$ & $\begin{array}{r}1,372 \\
= \\
=\end{array}$ & $0 \%$ \\
\hline $\begin{array}{l}\text { DBT POBL OSBD } \\
\text { IA TR ABSPOREATIOH }\end{array}$ & 60 & 4 & - & - & - & 1.826 & - & -518 & 1,372 & $0 \%$ \\
\hline $\begin{array}{l}\text { TOTAL GROSS FLORS } \\
\text { LOSSES } 8 \text { OUISSIOBS }\end{array}$ & $\begin{array}{r}91,859 \\
5,580\end{array}$ & $\begin{array}{r}46.607 \\
-472\end{array}$ & $\begin{array}{r}155,800 \\
-4,932\end{array}$ & $\begin{array}{l}67,705 \\
-3,198\end{array}$ & 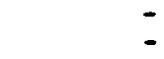 & $\begin{array}{r}108,627 \\
411\end{array}$ & $\begin{array}{r}7.232 \\
120\end{array}$ & $\begin{array}{r}103,099 \\
8,245\end{array}$ & $\begin{array}{r}580,411 \\
5,753\end{array}$ & $\begin{array}{r}99 \% \\
1 \%\end{array}$ \\
\hline
\end{tabular}

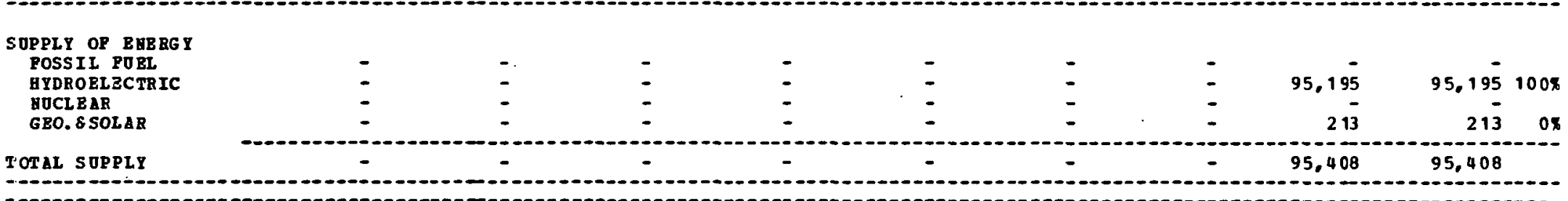

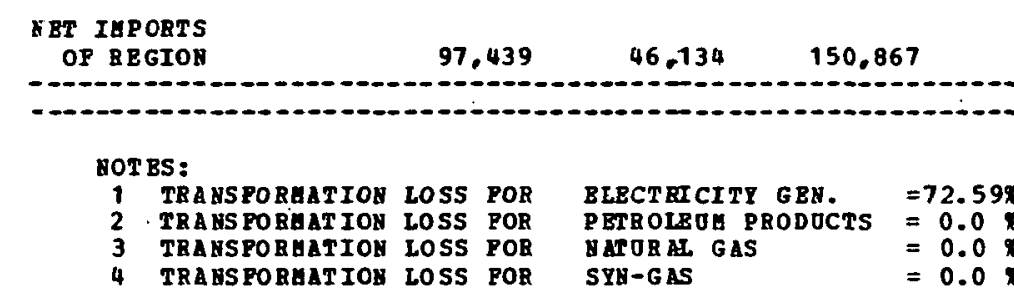


REG IOHAL BHERGY BALAYCE STA TBAENT

BY SECTOR ABD POEL TYPE

\begin{tabular}{|c|c|c|c|c|c|c|c|c|c|c|}
\hline SECTOR & $\begin{array}{c}\text { DISTILLATE } \\
\text { OIL }\end{array}$ & $\begin{array}{l}\text { EBS I DO AL } \\
\text { O IL }\end{array}$ & $\begin{array}{l}\text { GASOLISE } \\
\text { (ALL O }\end{array}$ & $\begin{array}{c}\text { HYDBO- } \\
\text { CARBONS } \\
\text { TS IN 10**9 }\end{array}$ & $\begin{array}{c}\text { CR ODE } \\
\text { OII } \\
\text { BTO'SI }\end{array}$ & $\begin{array}{l}\text { MATORAL } \\
\text { GAS }\end{array}$ & $\cos L$ & ELECTRICITY & $\begin{array}{r}\text { SECTOR } \\
\text { TOTAL }\end{array}$ & \\
\hline $\begin{array}{l}\text { PIHAL DBEAHD SECTORS } \\
\text { RESIDERTIAL.COAG. } \\
\text { INDOSTRIAL - } \\
\text { TRANSPORTATI OH- } \\
\text { GISCELLABOAS OSES }\end{array}$ & $\begin{array}{r}257,200 \\
35,000 \\
95.399 \\
1.300\end{array}$ & $\begin{array}{r}87.5 .18 \\
117.0100 \\
40.0 .00 \\
18.634\end{array}$ & $609.10 \%$ & $\begin{array}{r}78,202 \\
209,877 \\
70,508 \\
3,519\end{array}$ & $\overline{-}$ & $\begin{array}{r}403,493 \\
379,108 \\
22,900 \\
13,9.10 \\
-\end{array}$ & $\begin{array}{r}23,842 \\
816,022 \\
: 73 \\
- \\
-\end{array}$ & $\begin{array}{r}148.295 \\
155.746 \\
3.069 \\
389 \\
\end{array}$ & $\begin{array}{r}998,550 \\
1,712,853 \\
841.150 \\
37.802 \\
\end{array}$ & $\begin{array}{r}238 \\
398 \\
198 \\
18\end{array}$ \\
\hline $\begin{array}{l}\text { TOTAL PIMAL } \\
\text { DEHA HD SETTORS }\end{array}$ & 388,899 & $263,3 \cdot 22$ & 609,101 & 362,106 & $\therefore$ & 819,411 & 840,037 & 307,499 & $3,590,355$ & 82x \\
\hline $\begin{array}{l}\text { TRAESPORAATIOH } \\
\text { BLECPRICITT GEH. } \\
\text { PETROLEOA PRODUCT S } \\
\text { MAPURAL GAS } \\
\text { SYE-GAS }\end{array}$ & $\begin{array}{r}20,889 \\
-275,910 \\
-\end{array}$ & $\begin{array}{r}111.133 \\
-114.229 \\
-\end{array}$ & $-629,272$ & $\begin{array}{r}\therefore 187,010 \\
-123 \\
-123\end{array}$ & $\begin{array}{r}\vdots .279,260 \\
\div-\end{array}$ & $\begin{array}{r}2,530 \\
58,317 \\
1+1 \\
-\end{array}$ & $\begin{array}{r}934.036 \\
= \\
=\end{array}$ & $\begin{array}{r}-327.619 \\
- \\
-\end{array}$ & $\begin{array}{r}741,089 \\
1.156 \\
18 \\
-\end{array}$ & $\begin{array}{r}17 x \\
0 x \\
08\end{array}$ \\
\hline $\begin{array}{l}\text { NET FOEL DSED } \\
\text { IH. TR ANSPOCEATION }\end{array}$ & $-755,021$ & $-33,036$ & $-629,272$ & $-187,133$ & $1,279,260$ & 61,048 & 934.036 & $-327,619$ & 742,263 & $17 x$ \\
\hline $\begin{array}{l}\text { TOTAL GROSS PLONS } \\
\text { LOSSES E OMISSIOHS }\end{array}$ & $\begin{array}{r}09,788 \\
24.894\end{array}$ & $\begin{array}{r}374,495 \\
-3,800\end{array}$ & $\begin{array}{l}609,101 \\
-19,283\end{array}$ & $\begin{array}{l}362,106 \\
-17,108\end{array}$ & $\begin{array}{r}1.279,260 \\
1,939\end{array}$ & $\begin{array}{r}880,459 \\
3,333\end{array}$ & $\begin{array}{r}1,774,1073 \\
29.497\end{array}$ & $\begin{array}{r}307.499 \\
24.592\end{array}$ & $\begin{array}{r}4,332,618 \\
44,065\end{array}$ & $\begin{array}{r}998 \\
1 \%\end{array}$ \\
\hline $\begin{array}{l}\text { SOPPLY OP BN3RGY } \\
\text { POSSI I POEI } \\
\text { GYDROBLECTZIC } \\
\text { AOCLEAR } \\
\text { GEO.8 SOLAE. }\end{array}$ & $\overline{-}$ & $=$ & $\bar{z}$ & : & $\begin{array}{r}18,687 \\
= \\
-\end{array}$ & $\begin{array}{r}85.816 \\
= \\
-\end{array}$ & $\begin{array}{r}2,223,960 \\
\vdots \\
-\end{array}$ & $\begin{array}{r}7.484 \\
1.242 \\
-\end{array}$ & $\begin{array}{r}2,328,463 \\
7,484 \\
1,242 \\
\end{array}$ & $\begin{array}{r}100 \% \\
0 \% \\
0 \%\end{array}$ \\
\hline TOTAL SOPPIJ & - & - & - & - & 18,687 & 85,816 & $2,223,960$ & 8,726 & $2,337,189$ & \\
\hline
\end{tabular}

\section{NBI IUPORTS}

OP RBGIOH

58,772

226,465

$-39,454$

$157,864 \quad 1,262.512$

797.976

$-420,389$

$-4,253 \quad 2,039,494$.

HOTBS:

1 TRA GS PORAATIOY LOSS POR TRA ESPOBAATION ROSS FOR

TRAISFORMATIOS LOSS POR

BLECTRICI TY GEY.

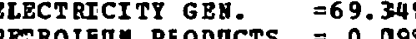

B.

MACORAI GAS 
REGIONAL ENERGY BALAHCE STATELENT

BP SECTOR AND FOEL TYPE

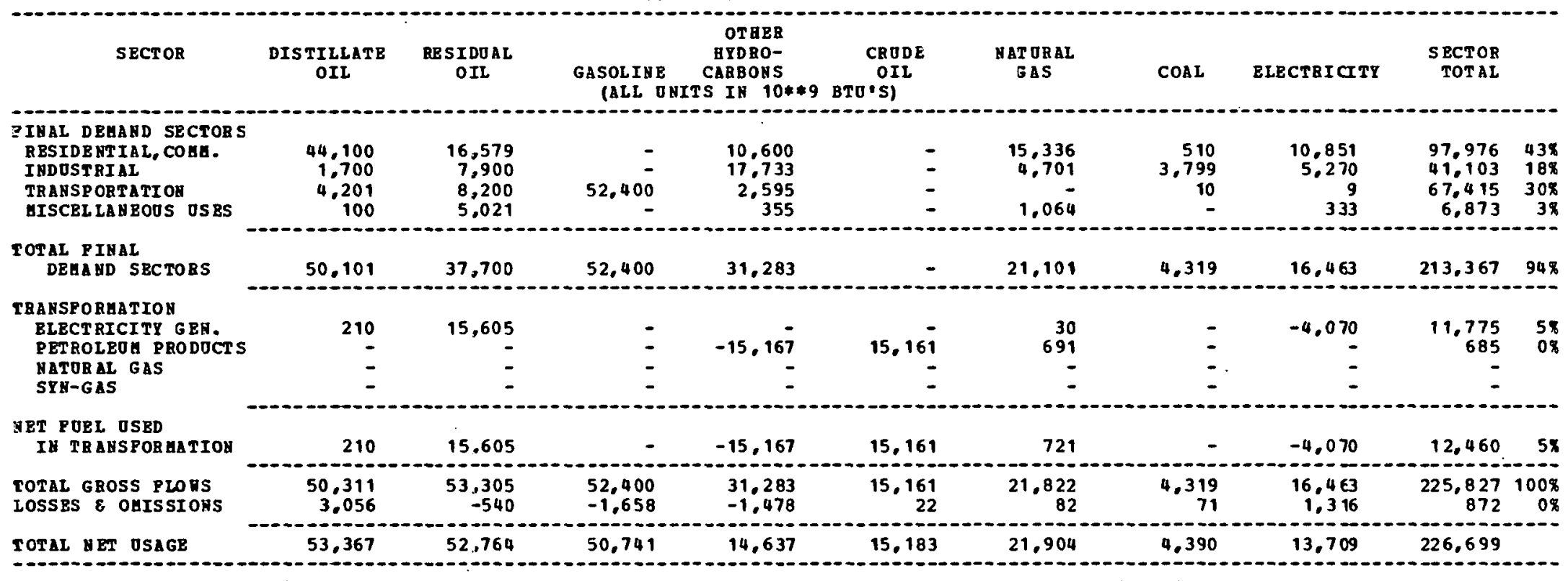

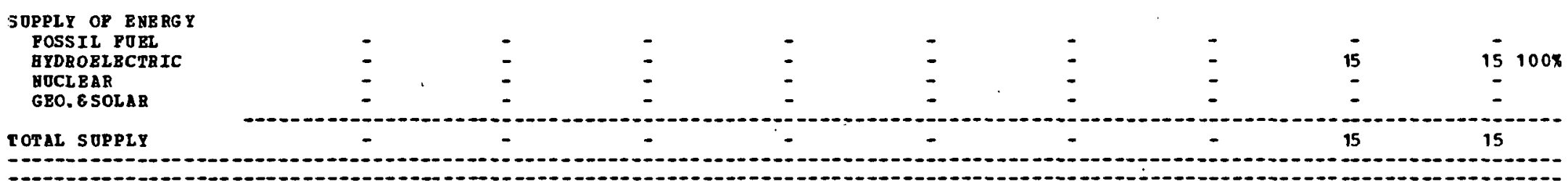

\begin{tabular}{l}
$\begin{array}{l}\text { MBT IMPORTS } \\
\text { OP REGIOR }\end{array}$ \\
\hline
\end{tabular}


REGIONAL BAEBGY BALANCE STATELENT

BY SZCTOB AND FOEL TYPE

\begin{tabular}{|c|c|c|c|c|c|c|c|c|c|c|}
\hline SECTOR & $\begin{array}{c}\text { DISTELLATB } \\
\text { OII }\end{array}$ & $\begin{array}{l}\text { FESI DOAL } \\
\text { OII }\end{array}$ & $\begin{array}{l}\text { GASOLIHE } \\
\quad \text { (ALL OS }\end{array}$ & $\begin{array}{l}\text { AYDRO- } \\
\text { CARBOSS } \\
\text { rS IH 10** }\end{array}$ & $\begin{array}{c}\text { CRODE } \\
\text { OII } \\
\text { BTO'S) }\end{array}$ & $\begin{array}{l}\text { MATUZ3AL } \\
\text { GAS }\end{array}$ & $\operatorname{COAL}$ & ELECTRICITY & $\begin{array}{l}\text { SECTO.3 } \\
\text { TOTAZ }\end{array}$ & \\
\hline $\begin{array}{l}\text { FIHAL DEHAND SBCTORS } \\
\text { RESIDE NTIAL, COAH. } \\
\text { INDOST RIAL. } \\
\text { TRANSPORTATIOH } \\
\text { EISCEL LAKEODS OSES }\end{array}$ & $\begin{array}{r}20.299 \\
10.303 \\
25.401 \\
.999\end{array}$ & $\begin{array}{r}1.271 \\
24.695 \\
7.800 \\
931\end{array}$ & 184.699 & $\begin{array}{r}33.899 \\
53.948 \\
7.084 \\
1.715\end{array}$ & $\begin{array}{l}\overline{-} \\
-\end{array}$ & $\begin{array}{r}33.240 \\
85: 303 \\
3.500 \\
6.361\end{array}$ & $\begin{array}{r}7,330 \\
39,719 \\
27 \\
-\end{array}$ & $\begin{array}{r}48,828 \\
48,747 \\
25 \\
1,925\end{array}$ & $\begin{array}{r}144.867 \\
262.415 \\
228,635 \\
11.831\end{array}$ & $\begin{array}{r}198 \\
34 x \\
29 \% \\
28\end{array}$ \\
\hline $\begin{array}{l}\text { TOTAL P IHAL } \\
\text { DEHA AD SECTORS }\end{array}$ & 56.902 & 34,697 & 184.699 & 96,646 & - & 128,204 & 47,076 & 99.525 & 647.747 & 83\% \\
\hline $\begin{array}{l}\text { TRANSPORGATION } \\
\text { BLECT RICITY GEH. } \\
\text { PETROLEOA PRODOCTS } \\
\text { NATORAL GAS } \\
\text { SYN-GAS }\end{array}$ & $\begin{array}{r}3,855 \\
= \\
-\end{array}$ & $\begin{array}{r}24,767 \\
- \\
-\end{array}$ & $\begin{array}{l}- \\
-\end{array}$ & $\begin{array}{l}- \\
-\end{array}$ & $\begin{array}{l}\text { - } \\
\text { - }\end{array}$ & $\begin{array}{r}25,533 \\
- \\
-\end{array}$ & $\begin{array}{r}134,604 \\
= \\
=\end{array}$ & $\begin{array}{r}-60,156 \\
- \\
-\end{array}$ & $\begin{array}{r}128,703 \\
= \\
-\end{array}$ & $16 x$ \\
\hline $\begin{array}{l}\text { MET POEL USED } \\
\text { IN TBANSFORGATIOH }\end{array}$ & 3.855 & 24.767 & - & - & - & 25,533 & 134,604 & $-60,156$ & 128,703 & $16 \%$ \\
\hline $\begin{array}{l}\text { TOTAL GROSS PLONS } \\
\text { LOSSES \& OUISSIONS }\end{array}$ & $\begin{array}{r}61,757 \\
3,690\end{array}$ & $\begin{array}{r}59,464 \\
-603\end{array}$ & $\begin{array}{r}184.699 \\
-5.847\end{array}$ & $\begin{array}{l}96.646 \\
-4.566\end{array}$ & - & $\begin{array}{r}153:, 337 \\
582\end{array}$ & $\begin{array}{r}181,680 \\
3,020\end{array}$ & $\begin{array}{r}99,525 \\
7.959\end{array}$ & $\begin{array}{r}776,452 \\
4,235\end{array}$ & $\begin{array}{r}99 \% \\
1 \%\end{array}$ \\
\hline TOTAL NET OSAGE & 64.447 & 58,860 & 178.851 & 92.079 & - & 154,419 & 184,700 & 47,328 & 780.639 & \\
\hline
\end{tabular}

SUPPIY OP ENERGY

POSSIL FUBL

BYDROEL ECTE

GEO.\& SOLAB

-
$\vdots$
-
-

-

-
-
-

-

=

-

\begin{tabular}{lrrr}
- & 12,490 & 12,495 & $37 \%$ \\
- & 21,049 & 21,049 & $63 \%$ \\
\hline & - & - & -
\end{tabular}

NET IMPORTS
OF REGIOH


QBGIONAL BAERG BALANCE STATEHBNT

BY SECTOR AND POEL TYPE

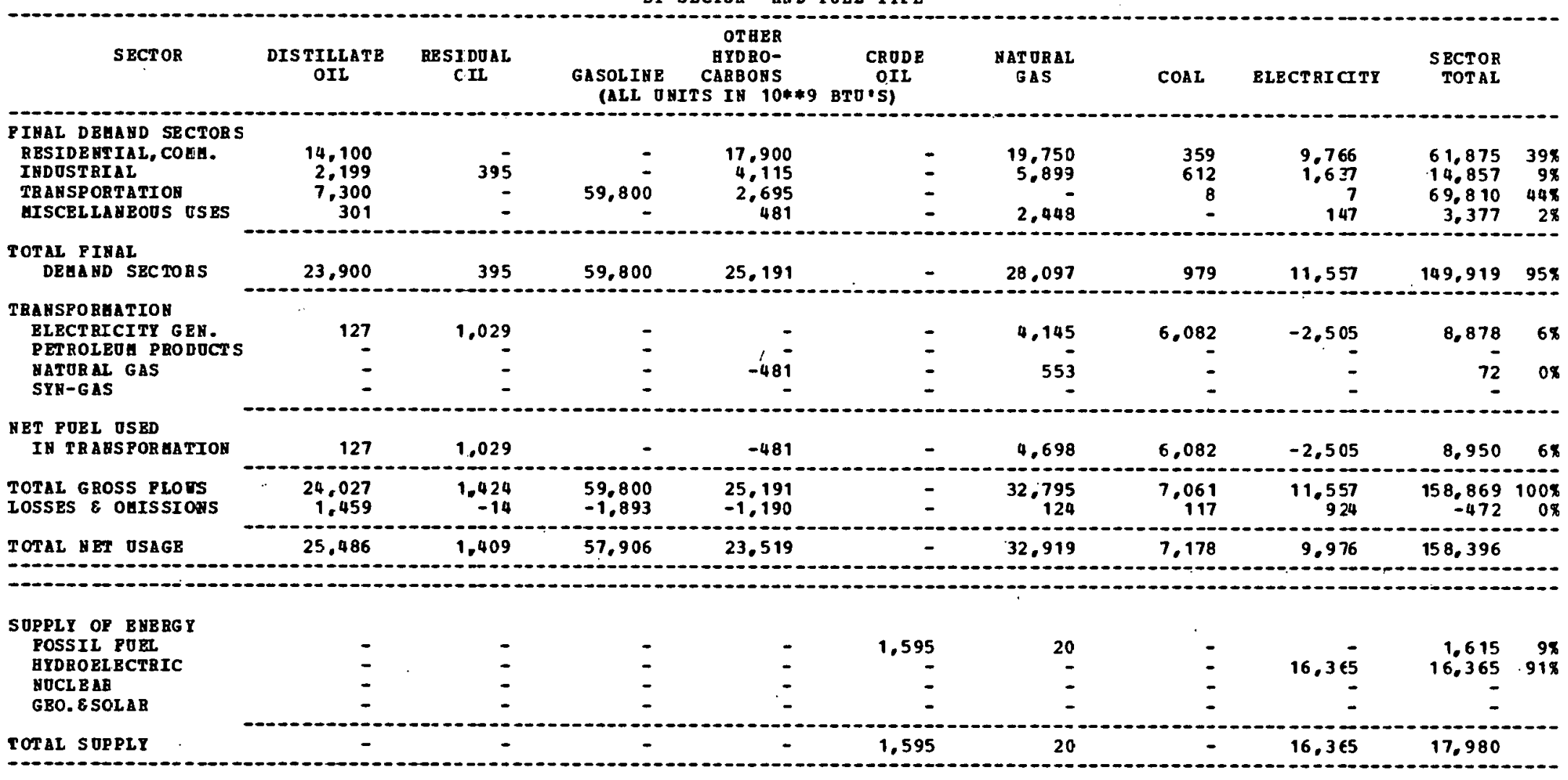

XET IAPORTS

OP REGIOH

25,486

1.409

57,906

23,519

$-1.595$

32,899

7,178

$-6,388$

140.416

MOT ES:

1 TRANSPORAATION LOSS POR
2 TRAHS PORATION LOSS POR

TRANSPORAATIOA LOSS POR

TRANS PORAAT IOA LOSS POB

ELECT EICITY GEY. $=77.99 \%$

DTROLEUA PRODUCTS $=0.0 \%$

$\begin{array}{ll}S Y Y-G L S & =13.02 \% \\ & =0.0 \%\end{array}$ 
REGIOHAL ENERGT BALAHCE STATBHE HT

BT SECTOR AND FOEL TYPE

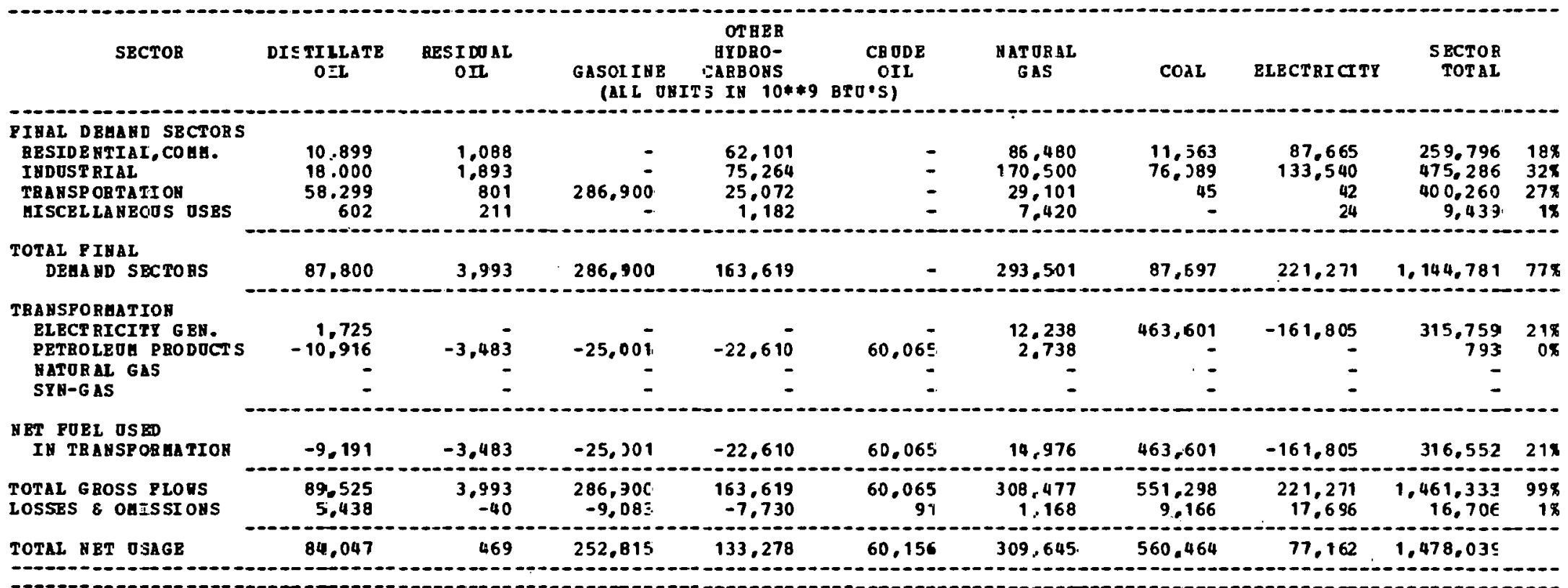

\begin{tabular}{|c|c|c|c|c|c|c|c|c|c|c|}
\hline $\begin{array}{l}\text { SOPPLY OP E EBRGI } \\
\text { POSSII POBE }\end{array}$ & - & - & - & - & 1.189 & 34 & 215.337 & - & & 858 \\
\hline GYDROBL BCTRIC & - & - & - & - & 1.00 & 34 & 215,317 & 38,694 & $\begin{array}{r}210,560 \\
38,692\end{array}$ & $15 \%$ \\
\hline BUCL B AR & - & - & - & - & - & - & - & - & - & \\
\hline GBO. 8 SOL $\triangle \mathrm{B}$ & - & - & - & - & - & - & - & - & - & \\
\hline
\end{tabular}

-10
0

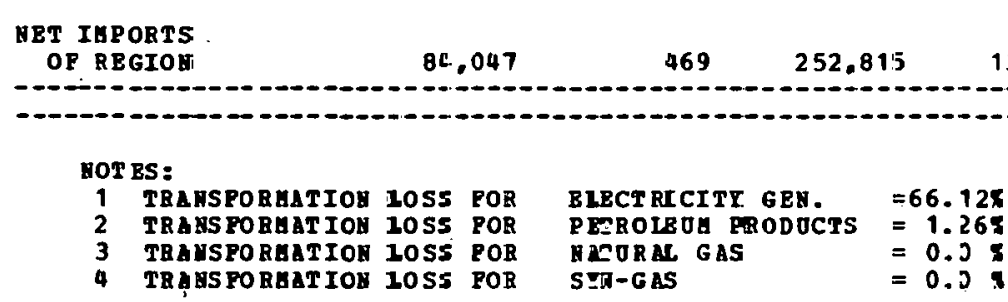


REG IONAL EHBRGI BALANCE STA TEGEHT

BI SECTOR AHD POEL TYPE

\begin{tabular}{|c|c|c|c|c|c|c|c|c|c|c|}
\hline SECTOR & $\begin{array}{l}\text { DISTILLATB } \\
\text { OIl }\end{array}$ & $\begin{array}{l}\text { BESI DOAL } \\
\text { OIL }\end{array}$ & $\begin{array}{l}\text { GASOLINE } \\
\text { (ALI O }\end{array}$ & 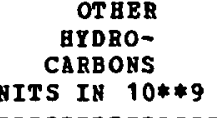 & $\begin{array}{c}\text { CRODE } \\
\text { OIL } \\
\text { BTO'SI }\end{array}$ & $\begin{array}{c}\text { NAT ORAL } \\
\text { GAS }\end{array}$ & COAL. & ELECTRICITY & $\begin{array}{r}\text { SECTOR } \\
\text { TOTAL }\end{array}$ & \\
\hline $\begin{array}{l}\text { PIHAL D EBAMD SBCTORS } \\
\text { RBSIDB ATIAL, COBA. } \\
\text { INDUST RIAL } \\
\text { TRABSPORTATIOH } \\
\text { GISCEL LAHEOOS OSBS }\end{array}$ & $\begin{array}{r}28,198 \\
64,188 \\
203,800 \\
7,500\end{array}$ & $\begin{array}{r}5.830 \\
35.995 \\
98.800 \\
8.369\end{array}$ & 943.400 & $\begin{array}{r}180,296 \\
1,009,483 \\
183,703 \\
4,990\end{array}$ & $\begin{array}{l}- \\
-\end{array}$ & $\begin{array}{r}377,118 \\
2,954,490 \\
106,800 \\
27,778\end{array}$ & $\begin{array}{r}6.284 \\
66.678 \\
181 \\
-\end{array}$ & $\begin{array}{r}230.649 \\
174.280 \\
165 \\
6.576\end{array}$ & $\begin{array}{r}828,375 \\
4,305,114 \\
1,536,849 \\
55,213\end{array}$ & $\begin{array}{l}11 \% \\
55 \% \\
20 \% \\
18\end{array}$ \\
\hline $\begin{array}{l}\text { TOTAL PINAL } \\
\text { DEHA BD SBCTORS }\end{array}$ & 303.686 & 148,994 & 943.400 & $1,378,472$ & - & $3,466,186$ & 73.143 & 411.670 & $6,725,551$ & $86 \%$ \\
\hline $\begin{array}{l}\text { TRANSPORGATIOH } \\
\text { BLECT RICITY GEA. } \\
\text { PETROLEUA PRODOCTS } \\
\text { HATORAL GAS } \\
\text { SYH-GAS }\end{array}$ & $\begin{array}{r}442 \\
-1,604,012 \\
-\end{array}$ & $\begin{array}{r}38,953 \\
-369,487 \\
-\end{array}$ & -3.241 .516 & $\begin{array}{r}-1,829,529 \\
-637.146 \\
-\end{array}$ & $\begin{array}{r}6,793,689 \\
-\end{array}$ & $\begin{array}{r}1,290,836 \\
309,695 \\
732,285 \\
-\end{array}$ & $\begin{array}{r}66.237 \\
= \\
=\end{array}$ & $\begin{array}{r}-453,611 \\
= \\
-\end{array}$ & $\begin{array}{r}942,857 \\
58,840 \\
95,139 \\
-\end{array}$ & $\begin{array}{r}128 \\
18 \\
18\end{array}$ \\
\hline $\begin{array}{l}\text { WBT POEL OSBD } \\
\text { IN TRABSPORAATION }\end{array}$ & -1.603 .570 & -330.534 & -3.241 .516 & -2.466 .675 & 6.793 .689 & 2.332 .816 & 66.237 & $-453,611$ & $1,096,836$ & $14 \%$ \\
\hline $\begin{array}{l}\text { TOTAL GROSS PLOHS } \\
\text { LOSSES } \& \text { OUISSIOHS }\end{array}$ & $\begin{array}{r}304.128 \\
18.475\end{array}$ & $\begin{array}{r}187.947 \\
-1.907\end{array}$ & $\begin{array}{l}943,400 \\
-29,867\end{array}$ & $\begin{array}{r}1,378,472 \\
-65,130\end{array}$ & $\begin{array}{r}6,793,689 \\
10.299\end{array}$ & $\begin{array}{r}5,799,002 \\
21.958\end{array}$ & $\begin{array}{r}139,380 \\
2,317\end{array}$ & $\begin{array}{r}411.670 \\
32.923\end{array}$ & $\begin{array}{r}7,822,387 \\
-10,929 \\
-\end{array}$ & $\begin{array}{r}1008 \\
08\end{array}$ \\
\hline
\end{tabular}

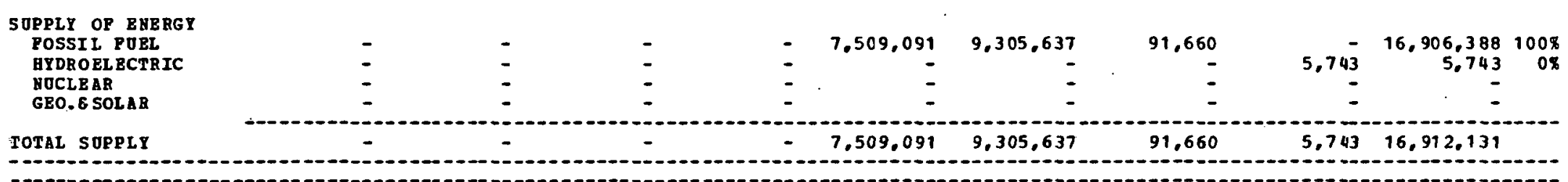

\section{SET IHPORTS}

$-183,447$

$-2,327,983$

$-1,153,333$

$-705.102$

50.037

$-14,760 \quad-9,100,673$

HOTBS:

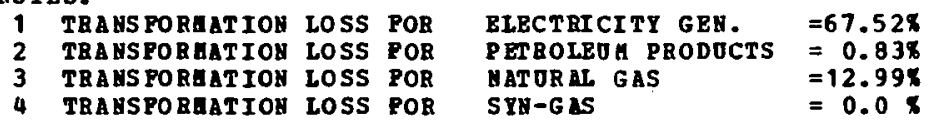


REGIONAL RNERGT BALAMCE STATELSAT

BY SBCTOB AND POEL TYPE

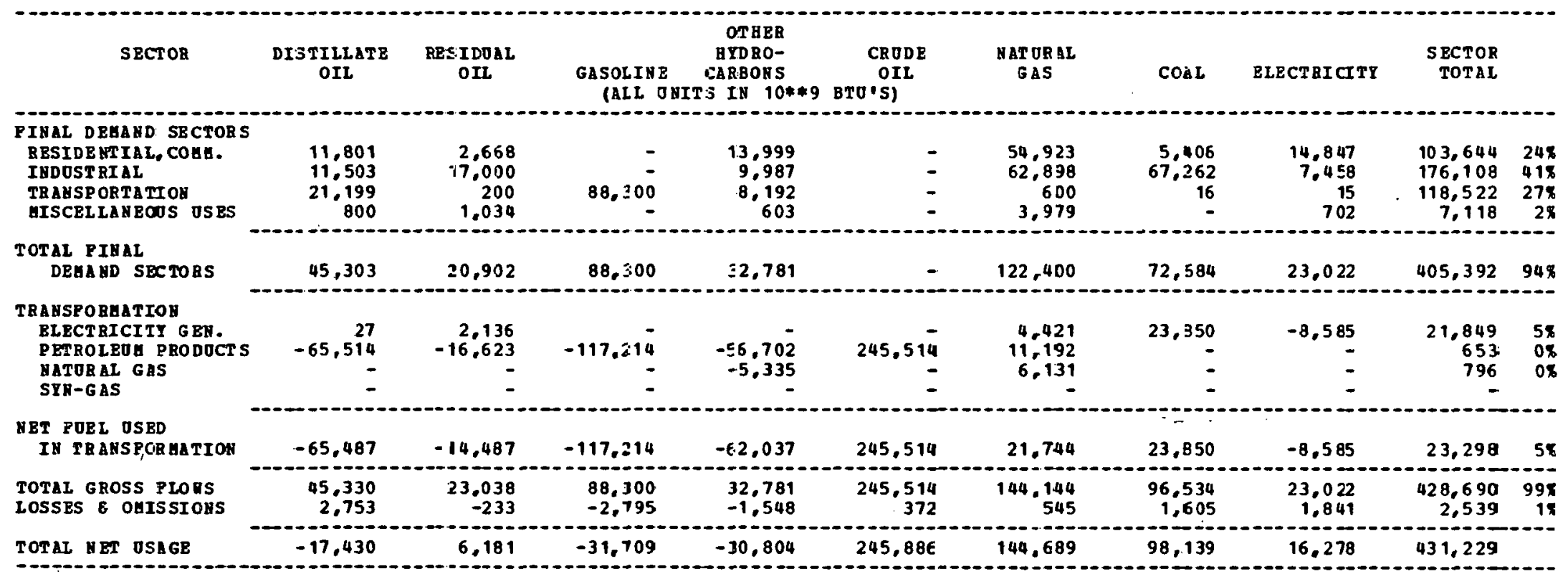

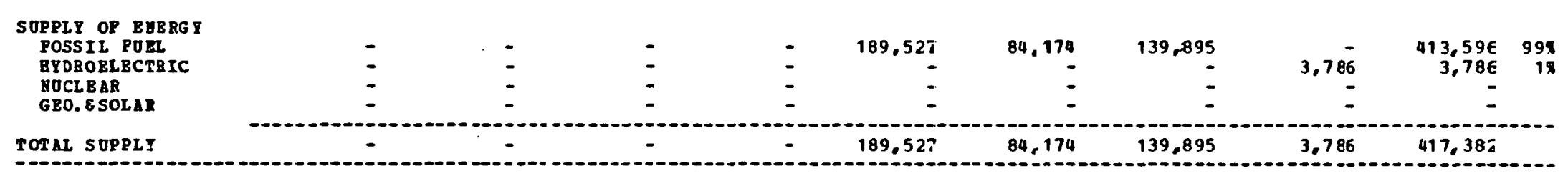

UET IMPORTS
OF REGIOB


REGIONAL BHERGY BALANCE STATEGEHT

BI SECTOR AND FOBL TYPE

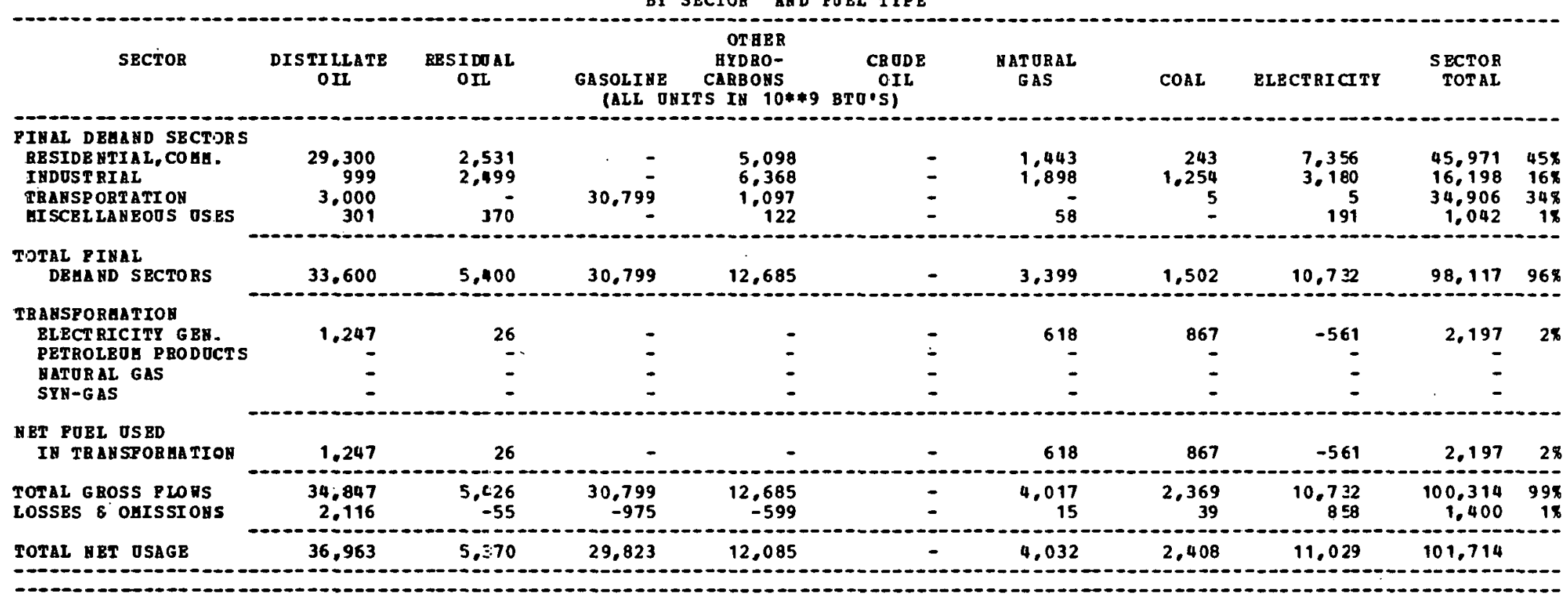

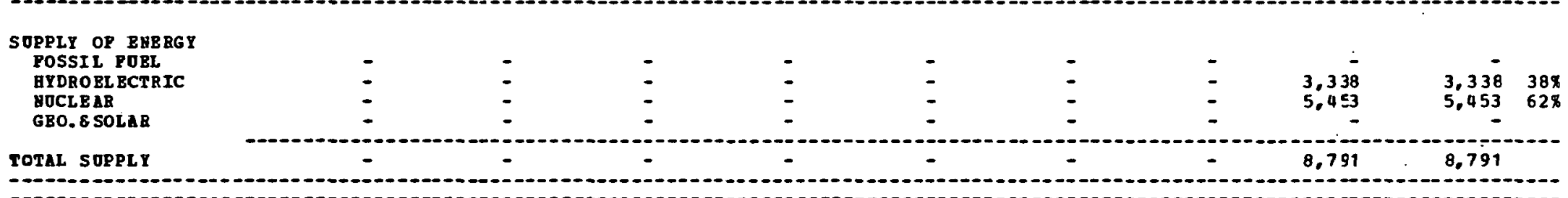

\section{AET IHPORTS}

36,963

5,370

29,823

12,085

4,032

2,408

2,238

92,923

\section{MOTES:}

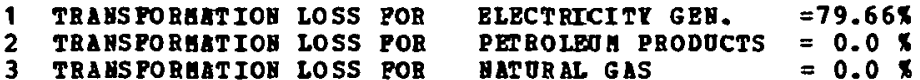

$\begin{array}{ll}\text { TRAESPORAITION LOSS POR SYB-GAS } & =0.0\end{array}$ 
REGIOHAL ENEBGT BALAHCE STATELBHT

BI SECTOB AUD POEL TIPE

\begin{tabular}{|c|c|c|c|c|c|c|c|c|c|c|}
\hline SECTOR & $\begin{array}{l}\text { DISIILLATE } \\
\text { OIL }\end{array}$ & $\begin{array}{l}\text { BESIDUAL } \\
\text { OII }\end{array}$ & $\begin{array}{l}\text { GASOLIH } \\
\text { (AIL }\end{array}$ & $\begin{array}{c}\text { OTBRR } \\
\text { BYDRO- } \\
\text { CARBOAS } \\
\text { IE IB 10** }\end{array}$ & $\begin{array}{c}\text { CBODE } \\
\text { OIL } \\
\text { BTO'S) }\end{array}$ & $\begin{array}{l}\text { MAT URHL } \\
\text { S AS }\end{array}$ & COAL & BLECTRICITY & $\begin{array}{l}\text { SECTOR } \\
\text { TOTAL }\end{array}$ & \\
\hline 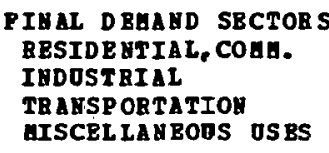 & $\begin{array}{l}72.001 \\
17.696 \\
56.498 \\
1.800\end{array}$ & $\begin{array}{r}7.846 \\
51.101 \\
59.500 \\
6.255\end{array}$ & 318,401 & $\begin{array}{r}45,899 \\
58,007 \\
56,962 \\
1,761\end{array}$ & :- & $\begin{array}{r}77,486 \\
60.795 \\
5,999 \\
13,6=3\end{array}$ & $\begin{array}{r}14,424 \\
78,552 \\
68 \\
-\end{array}$ & $\begin{array}{r}81.355 \\
31.173 \\
62 \\
13.787\end{array}$ & $\begin{array}{r}299.011 \\
297.324 \\
497.490 \\
37.216\end{array}$ & $\begin{array}{r}228 \\
228 \\
378 \\
38\end{array}$ \\
\hline $\begin{array}{l}\text { TOTAL RIRAL } \\
\text { DEHA KD SBCTORS }\end{array}$ & 147.995 & 124.702 & 318,401 & 162,629 & - & 157,893 & $93, C 44$ & 126.377 & $1,131,041$ & $85 \%$ \\
\hline $\begin{array}{l}\text { TRABSPOBGATIOB } \\
\text { ELECTRICITT GBN. } \\
\text { PETROLEOE PRODDCTS } \\
\text { HATURAL GAS } \\
\text { STH-GAS }\end{array}$ & $\begin{array}{r}5.471 \\
-21.675 \\
-\end{array}$ & $\begin{array}{r}157.936 \\
-8.311 \\
- \\
-\end{array}$ & $\begin{array}{r}-41.423 \\
-\end{array}$ & $\begin{array}{r}3,122 \\
-21,095 \\
=\end{array}$ & 89.390 & $\begin{array}{r}3,786 \\
4,075 \\
- \\
-\end{array}$ & $\begin{array}{r}122,589 \\
- \\
-\end{array}$ & $\begin{array}{r}-94,109 \\
- \\
-\end{array}$ & $\begin{array}{r}198.795 \\
961 \\
-\end{array}$ & $\begin{array}{r}158 \\
0 \%\end{array}$ \\
\hline $\begin{array}{l}\text { MET FOEL OSBD } \\
\text { IA TBABSPOAGATIOA }\end{array}$ & -16.204 & 149,625 & $-41,423$ & $-17,973$ & 89,390 & 7,861 & 122,589 & $-94,109$ & 199.756 & $15 \%$ \\
\hline $\begin{array}{l}\text { TOTAL GROSS ELOUS } \\
\text { LOSSBS } \& \text { OHISSIOHS }\end{array}$ & $\begin{array}{r}153,466 \\
9,323\end{array}$ & $\begin{array}{r}282,638 \\
-2,868\end{array}$ & $\begin{array}{l}318,401 \\
-10,080\end{array}$ & $\begin{array}{r}165,751 \\
-7,831\end{array}$ & $\begin{array}{r}89,390 \\
135\end{array}$ & $\begin{array}{r}165,754 \\
627\end{array}$ & $\begin{array}{r}215,633 \\
3,585\end{array}$ & $\begin{array}{r}126,377 \\
10,107\end{array}$ & $\begin{array}{r}1,330,797 \\
2,998\end{array}$ & $\begin{array}{r}100 \% \\
0 \%\end{array}$ \\
\hline
\end{tabular}

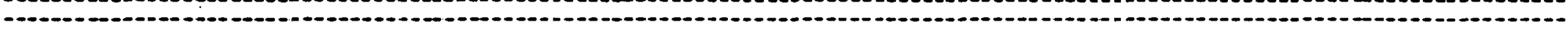

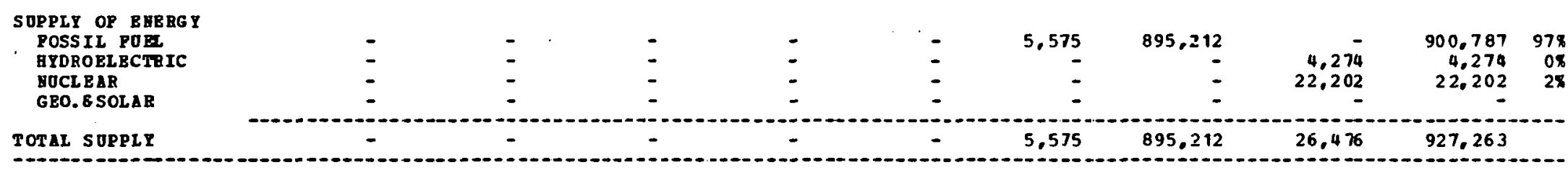

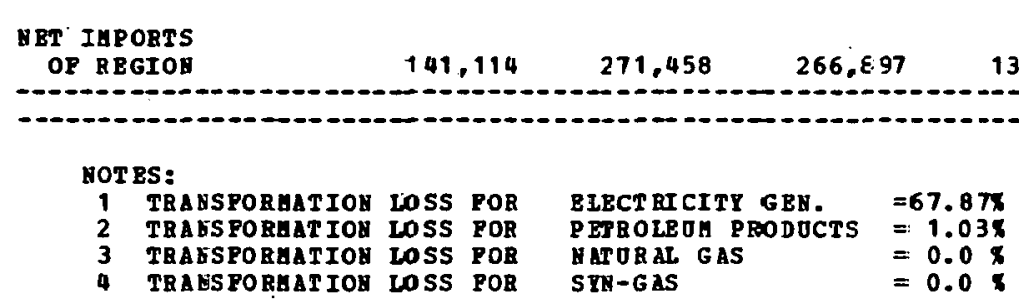


REGIOHAL BHEBGY BALAKCE STATEAERT

$$
\text { BY SECTOR AND FOBL TYPE }
$$

\begin{tabular}{|c|c|c|c|c|c|c|c|c|c|}
\hline SECTOR & $\begin{array}{c}\text { DISTILLATE } \\
\text { OIL }\end{array}$ & $\begin{array}{l}\text { RESIDJAL } \\
\text { OII }\end{array}$ & $\begin{array}{l}\text { GASOLIHE } \\
\text { (ALL OHI }\end{array}$ & $\begin{array}{c}\text { OTBER } \\
\text { BYDBO- } \\
\text { CARBOHS } \\
\text { TS IN } 10 * * 9\end{array}$ & $\begin{array}{l}\text { CR ODE } \\
\text { OIL } \\
\text { BTO'S) }\end{array}$ & $\begin{array}{l}\text { MAT ORAL } \\
\text { GAS }\end{array}$ & $\operatorname{COAL}$ & ELECT RICITI & $\begin{array}{l}\text { S BCTOR } \\
\text { TOTAL }\end{array}$ \\
\hline $\begin{array}{l}\text { TOTAL PINAL } \\
\text { DEHA UD SBCTORS }\end{array}$ & 114,398 & 68,597 & 216.400 & 112,926 & - & 203,001 & 14.446 & 187,179 & 917,047 \\
\hline $\begin{array}{l}\text { TRAHSPORAATION } \\
\text { BLECT RICITY GBH. } \\
\text { PETROLEOA PRODOCT S } \\
\text { GATURAI GAS } \\
\text { SYH-GAS }\end{array}$ & $\begin{array}{r}173 \\
-114,147 \\
-\end{array}$ & $\begin{array}{r}2,841 \\
-159,589 \\
-\end{array}$ & $\begin{array}{r}-335,237 \\
-\end{array}$ & $-122,948$ & 649.055 & $\begin{array}{r}84.218 \\
- \\
-\end{array}$ & $\begin{array}{r}83.664 \\
- \\
-\end{array}$ & $\begin{array}{r}-12.014 \\
= \\
=\end{array}$ & $\begin{array}{r}74.664 \\
1.352 \\
- \\
-\end{array}$ \\
\hline $\begin{array}{l}\text { MET POEL -OSED } \\
\text { IA TBANSFORGA TIOY }\end{array}$ & $-113,974$ & $-156,748$ & $-335,237$ & $-122,948$ & 649,055 & 84.218 & 83,664 & $-12,014$ & 76.016 \\
\hline $\begin{array}{l}\text { TOTAL GROSS PLOHS } \\
\text { LOSSES } 8 \text { OUISSIONS }\end{array}$ & $\begin{array}{r}114,571 \\
6,960\end{array}$ & $\begin{array}{r}T 1,538 \\
-725\end{array}$ & $\begin{array}{r}216.400 \\
-6,851\end{array}$ & $\begin{array}{r}112,926 \\
-5,335\end{array}$ & $\begin{array}{r}649.055 \\
984\end{array}$ & $\begin{array}{r}287,219 \\
1.087\end{array}$ & $\begin{array}{r}98,110 \\
1,631\end{array}$ & $\begin{array}{r}187.179 \\
14.969\end{array}$ & $\begin{array}{r}993.063 \\
12.720\end{array}$ \\
\hline TOTAL AET OSAGB & 7.384 & $-88, .776$ & $-125,688$ & $-15,357$ & 650.039 & 288,306 & 99,741 & 190,134 & $.005,783$ \\
\hline
\end{tabular}

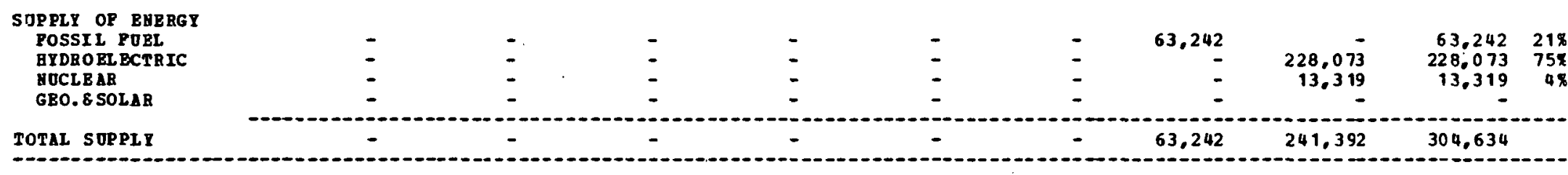

-

\section{HET IAPORTS}

7,38

$-88,776-125,688$

$-15,357$

650,039

288,306

36.499

$-51.257$

701,149

-

HOTBS:

$$
\begin{aligned}
& \begin{array}{llll}
\text { TRANS PORALTION LOSS POR } & \text { ELECTRICITY GEN. } & =86.14 \times \\
\text { TRANSPORARION LOSS POB } & \text { PEROLEOH PRODOCTS } & =0.18 \pi
\end{array} \\
& \begin{array}{lll}
\text { TRABSPORGLTIOH LOSS POR } & \text { YATORAL GAS } & =0.0 \\
\text { TRABSPOBHATIOR LOSS POB } & \text { SYH-G AS } & =0.0
\end{array}
\end{aligned}
$$


REGIOAAL ERBRG BALABCE STATEGEAT

BI SECTOZ AND POBL TYPE

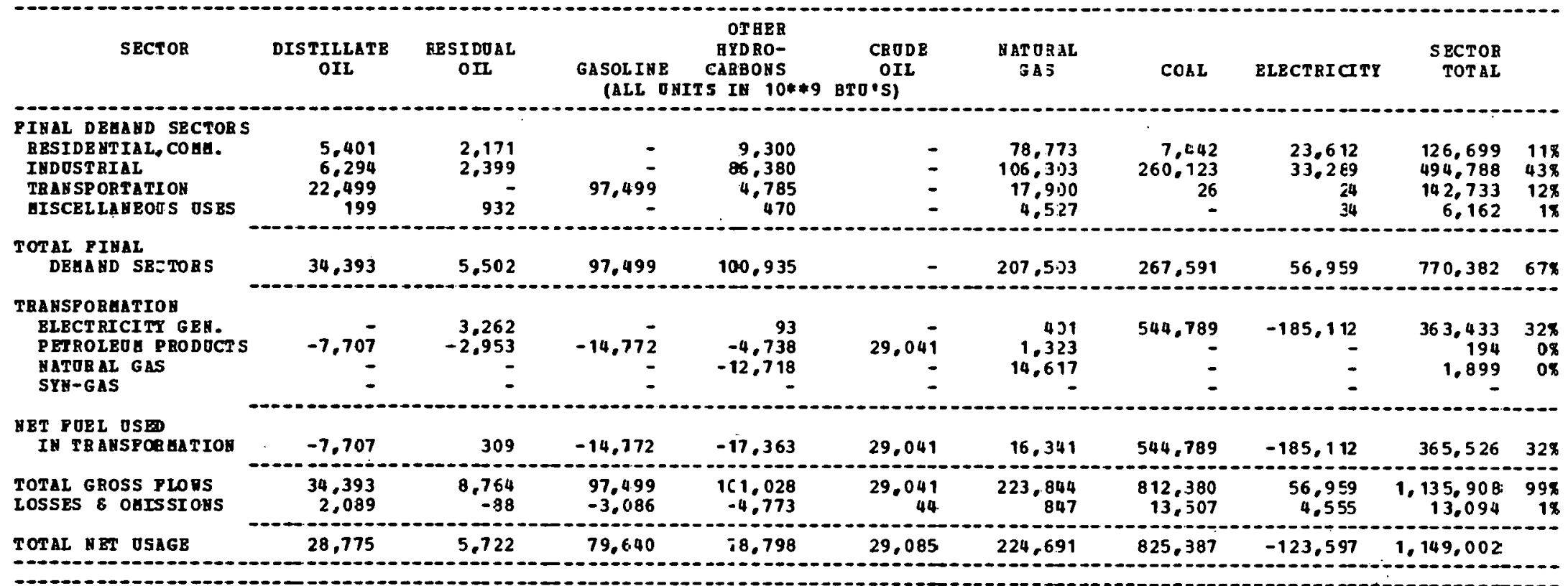

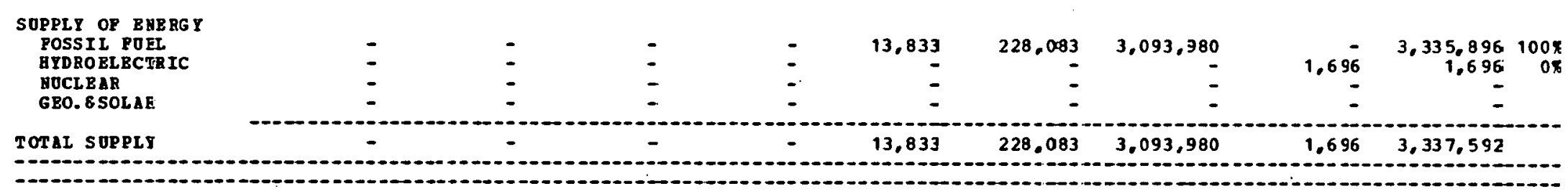

\section{BET IHPORTS
OP REGIOH}

28,775

5,722

79,640

78,798

15,252

$-3,391-2,268,092$

$-125,293-2,188,589$

HOT BS:

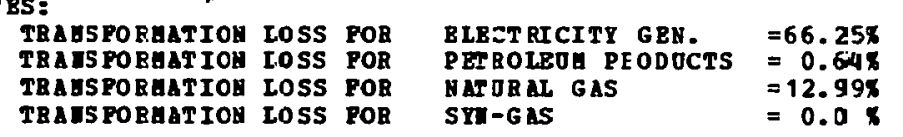

2 TRAIS PORAATIOH LOSS POR
TRAGSPORAATIOH LOSS POR

TBAIS PORAAT IOH LOSS POB

aringas

$=0.0 \%$ 


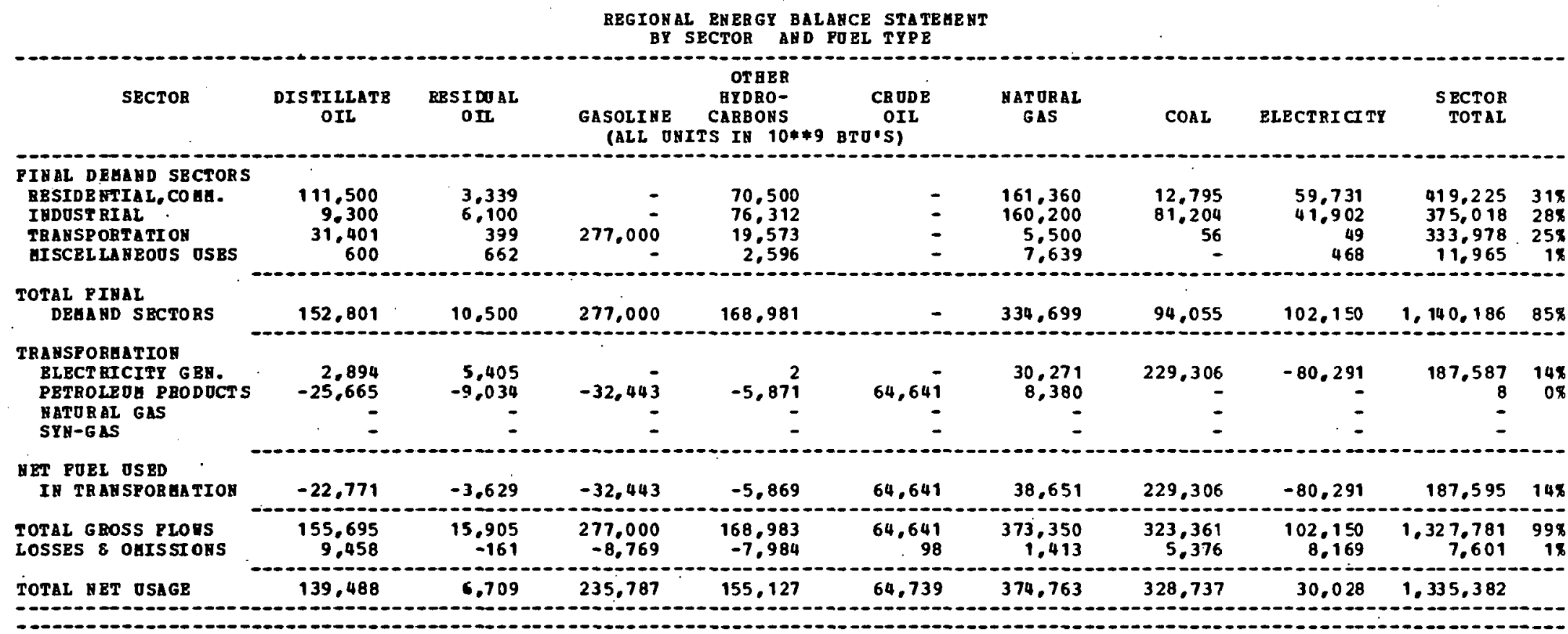

\begin{tabular}{|c|c|c|c|c|c|c|c|c|c|}
\hline $\begin{array}{l}\text { POSSI I FOBL } \\
\text { GYDROELECTRIC } \\
\text { QOCLEAB } \\
\text { GBO.E SOLAR. }\end{array}$ & $\overline{-}$ & $\overline{-}$ & - & E & : & $\begin{array}{l}- \\
-\end{array}$ & $=$ & $\begin{array}{r}7,045 \\
20,315\end{array}$ & $\begin{array}{r}7,04 \overline{5} \\
20,315\end{array}$ \\
\hline TOTAL SUPPLY & - & - & - & - & - & - & - & 27,360 & 27,360 \\
\hline
\end{tabular}

\section{MET IAPORTS}

OP RBGION

139,488

6,709

235,787

155,127

64.739

374,763

\section{HOTES:}

\begin{tabular}{|c|c|c|c|}
\hline 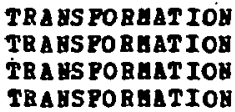 & $\begin{array}{l}\text { LOSS } \\
\text { LOSS } \\
\text { LOSS } \\
\text { LOSS }\end{array}$ & $\begin{array}{l}\text { POR } \\
\text { POR } \\
\text { POR } \\
\text { POR }\end{array}$ & $\begin{array}{l}\text { BLECPRICITY GEH. } \\
\text { PETROLRDA PBODOCTS } \\
\text { MATORAL GAS } \\
\text { SYH-GAS }\end{array}$ \\
\hline
\end{tabular}


REGICNAL BHERGY BALAHCE STATEGEAT

EY SECTOR AND PORL TYPE

\begin{tabular}{|c|c|c|c|c|c|c|c|c|c|c|}
\hline SECTOR & $\begin{array}{l}\text { DISTILLATE } \\
\text { OIL }\end{array}$ & $\begin{array}{l}\text { RESIDOAL } \\
\text { OIL }\end{array}$ & $\begin{array}{l}\text { GASOFINE } \\
\text { (ILE ONIT }\end{array}$ & $\begin{array}{c}\text { OT BER } \\
\text { ATDRO- } \\
\text { CARBOHS } \\
\text { IS IS } 10 * \% 9\end{array}$ & $\begin{array}{c}\text { CRODE } \\
\text { OIL } \\
\text { BTO'S) }\end{array}$ & $\begin{array}{l}\text { NaTOEAL } \\
\text { GSS }\end{array}$ & CCAL & ELECTRI CITY & $\begin{array}{l}\text { SECTOE } \\
\text { TOTAI }\end{array}$ & \\
\hline 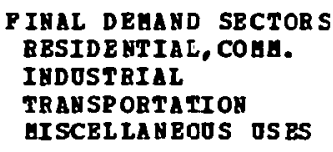 & $\begin{array}{r}5.200 \\
9.300 \\
20.800 \\
300\end{array}$ & $\begin{array}{r}1.466 \\
5,900 \\
1.301 \\
637\end{array}$ & $38,30 \bar{c}$ & $\begin{array}{r}13.001 \\
2.781 \\
3.096 \\
192\end{array}$ & $\begin{array}{l}\overline{-} \\
\overline{-}\end{array}$ & $\begin{array}{r}24,403 \\
68,199 \\
8,000 \\
3,198\end{array}$ & $\begin{array}{r}685 \\
5.344 \\
7 \\
-\end{array}$ & $\begin{array}{r}7,347 \\
6.004 \\
7 \\
111\end{array}$ & $\begin{array}{l}52,10 z \\
97,52 \varepsilon \\
72,111 \\
4,43 \varepsilon\end{array}$ & $\begin{array}{r}178 \\
338 \\
248 \\
18\end{array}$ \\
\hline $\begin{array}{l}\text { TOTAL P INAL } \\
\text { DEEA AD SBC TOBS }\end{array}$ & 35,600 & 9,304 & 38,000 & 19,070 & - & 104,700 & 6.036 & $13,4 \in 9$ & 226,175 & $75 \pi$ \\
\hline $\begin{array}{l}\text { TRANSFOREATIOA } \\
\text { ELECTRICITY GER. } \\
\text { PETROLEOA PRODOCTS } \\
\text { HATURAL GAS } \\
\text { SPH-GAS }\end{array}$ & $\begin{array}{r}31 \\
-77,735 \\
-\end{array}$ & $\begin{array}{r}567 \\
-19.722 \\
- \\
-\end{array}$ & $-139.140^{\circ}$ & $\begin{array}{r}-54.102 \\
-25,019 \\
-\end{array}$ & $\begin{array}{r}298,984 \\
-\end{array}$ & $\begin{array}{r}381 \\
2,702 \\
28,754 \\
-\end{array}$ & $\begin{array}{r}95,595 \\
- \\
-\end{array}$ & $\begin{array}{r}-31,354 \\
- \\
-\end{array}$ & $\begin{array}{r}65.225 \\
98 \% \\
3.735 \\
\end{array}$ & $\begin{aligned} 22 \pi \\
08 \\
18\end{aligned}$ \\
\hline $\begin{array}{l}\text { NET FOEL OSBD } \\
\text { IY TRAHSPOBGATIOH }\end{array}$ & $-77,704$ & -19.155 & $-139,140$ & $-39,121$ & 298,984 & 31,837 & 95.595 & $-31,354$ & 69.942 & $23 \%$ \\
\hline $\begin{array}{l}\text { TOTAL GROSS PLORS } \\
\text { LOSSES E OAISSIOHS }\end{array}$ & $\begin{array}{r}35,631 \\
2,164\end{array}$ & $\begin{array}{r}9.871 \\
-100\end{array}$ & $\begin{array}{r}38,000 \\
-1,203\end{array}$ & $\begin{array}{r}19.070 \\
-901\end{array}$ & $\begin{array}{r}298,984 \\
453\end{array}$ & $\begin{array}{r}136 ; 537 \\
517\end{array}$ & $\begin{array}{r}101.631 \\
1.689\end{array}$ & $\begin{array}{r}13.469 \\
1.07\end{array}$ & $\begin{array}{r}296.12^{-} \\
3.69=\end{array}$ & $\begin{array}{r}998 \\
18\end{array}$ \\
\hline TOTAL NET OSAGE & $-39,939$ & $-9,951$ & $-102,343$ & $-70,952$ & 299.437 & 137.054 & 103,320 & $-16,807$ & 299,818 & \\
\hline
\end{tabular}

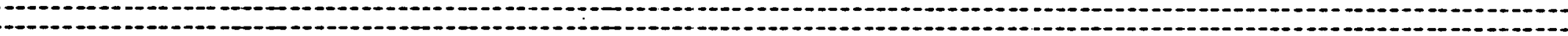

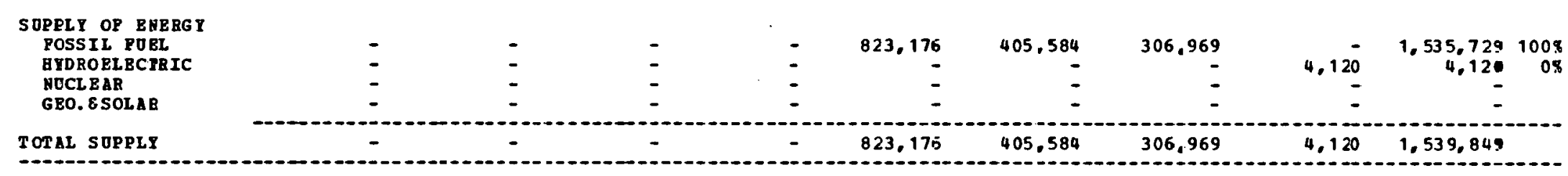

\begin{tabular}{l}
$\begin{array}{l}\text { HET IHPORTS } \\
\text { OP REGIOA }\end{array}$ \\
\hline MOTES:
\end{tabular}


REG IONAL ENEBGX BALANCB STA TEAENT

BY SECTOR AND FOEL TYPE

\begin{tabular}{|c|c|c|c|c|c|c|c|c|c|c|}
\hline SECTOR & $\begin{array}{l}\text { DISTILLATE } \\
\text { OIL }\end{array}$ & $\begin{array}{l}\text { RBSIDC AL } \\
\text { OIL }\end{array}$ & $\begin{array}{l}\text { GASOLINE } \\
\text { (ALL D }\end{array}$ & $\begin{array}{c}\text { HYDRO- } \\
\text { CABBONS } \\
\text { INITS IN 10** }\end{array}$ & $\begin{array}{r}\text { CRODE } \\
\text { OIL } \\
9 \text { BTO'SI }\end{array}$ & $\begin{array}{l}\text { MATORAL } \\
\text { GAS }\end{array}$ & COAL & ELECTRICITY & $\begin{array}{l}\text { SECTOR } \\
\text { TOTAL }\end{array}$ & \\
\hline 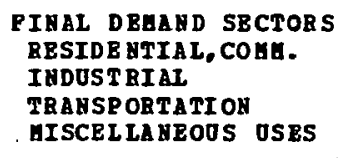 & $\begin{array}{r}3.127 .001 \\
802,455 \\
2.159 .099 \\
.69 .305\end{array}$ & $\begin{array}{r}1,020,388 \\
1,275,558 \\
732,492 \\
245,118\end{array}$ & 13.255 .897 & $\begin{array}{r}2,404,000 \\
3,997,743 \\
2,044,400 \\
105,302\end{array}$ & - & $\begin{array}{r}7,189,959 \\
10,654,299 \\
743,604 \\
443,958\end{array}$ & $\begin{array}{r}296,515 \\
4,376,593 \\
2,991 \\
-\end{array}$ & $\begin{array}{r}3,301,138 \\
2,391,149 \\
17.015 \\
84.395\end{array}$ & $\begin{array}{r}17,339,001 \\
23,497,797 \\
18,955,498 \\
948,078\end{array}$ & $\begin{array}{r}248 \\
328 \\
268 \\
18\end{array}$ \\
\hline $\begin{array}{l}\text { TOTAL PIHAL } \\
\text { DEMA ED SBCTORS }\end{array}$ & $6,157,860$ & $3,273,556$ & $13.255,897$ & 8.551 .445 & - & $19,031,820$ & 4.676 .099 & $5,793,697$ & $60,740,374$ & $84 \pi$ \\
\hline $\begin{array}{l}\text { TRAHSPORAATIOH } \\
\text { BLECT RICITY GBH. } \\
\text { PETROLEOA PBODOCTS } \\
\text { BATORAL GAS } \\
\text { SYH-GAS }\end{array}$ & $\begin{array}{r}263,019 \\
-5,995,925 \\
-\end{array}$ & $\begin{array}{r}3.219 .627 \\
-2.229,350 \\
- \\
-\end{array}$ & $\begin{array}{r}-12,589,07 i \\
-\end{array}$ & $\begin{array}{r}78,774 \\
-6,531,169 \\
-1,339,919\end{array}$ & $\begin{array}{r}26,316,075 \\
-\end{array}$ & $\begin{array}{r}3.706,394 \\
1,199,634 \\
1,539,992 \\
-\end{array}$ & $\begin{array}{r}0,638,960 \\
= \\
=\end{array}$ & $\begin{array}{r}-4,994,555 \\
= \\
=\end{array}$ & $\begin{array}{r}10,912,219 \\
170,194 \\
200,073 \\
-\end{array}$ & $\begin{array}{r}15 \% \\
0 \% \\
0 \%\end{array}$ \\
\hline $\begin{array}{l}\text { MET POEL OSED } \\
\text { IN TBAHSPOBGA TICN }\end{array}$ & $-5,732,906$ & 990,277 & $-12,589,071$ & -7.792 .314 & 26.316 .075 & 6.446 .020 & $8,638,960$ & $-4,994,5 \leq 5$ & $11,282,486$ & $16 \%$ \\
\hline $\begin{array}{l}\text { TOTAL GROSS PLOHS } \\
\text { LOSSES } \varepsilon \text { OAISSI ORS }\end{array}$ & $\begin{array}{r}6.420,879 \\
390,068\end{array}$ & $\begin{array}{r}6,493,183 \\
-65,890\end{array}$ & $\begin{array}{r}13,255,897 \\
-419,670\end{array}$ & $\begin{array}{r}8,630,219 \\
-407,764\end{array}$ & $\begin{array}{r}26,316,075 \\
39,898\end{array}$ & $\begin{array}{r}25,477,840 \\
96, .475\end{array}$ & $\begin{array}{r}13,315,059 \\
221,390\end{array}$ & $\begin{array}{r}5,793,697 \\
463,354\end{array}$ & $\begin{array}{r}72.022 .860 \\
317,862\end{array}$ & $\begin{array}{r}100 \% \\
08\end{array}$ \\
\hline
\end{tabular}

\begin{tabular}{|c|c|c|c|c|c|c|c|c|c|c|}
\hline $\begin{array}{l}\text { SEPPLY OP EHERGY } \\
\text { POSSI L POEL } \\
\text { HYDRORLBCTRIC } \\
\text { HOCLBAR } \\
\text { GEO.E SOLAB }\end{array}$ & $\begin{array}{l}\overline{-} \\
\overline{-}\end{array}$ & $\overline{-}$ & - & $\begin{array}{l}\overline{-} \\
\overline{-}\end{array}$ & $\begin{array}{r}19.492 .839 \\
- \\
-\end{array}$ & $\begin{array}{r}24.481 .289 \\
- \\
-\end{array}$ & $\begin{array}{r}14.821 .811 \\
= \\
=\end{array}$ & $\begin{array}{r}927.948 \\
278.350 \\
7.471\end{array}$ & $\begin{array}{r}58,795.939 \\
927.948 \\
278.350 \\
7,471\end{array}$ & $\begin{array}{r}98 \% \\
2 \% \\
0 \% \\
0 \%\end{array}$ \\
\hline TCTAL SUPPLI & 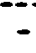 & 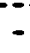 & - & - & 19.492 .839 & $24,481,289$ & $14,821,811$ & $1,213,769$ & $60,009,708$ & \\
\hline
\end{tabular}

MET IMPORTS

OF BEGIOY

$815,022 \quad 4,197,9+2$

247.155

351,366

$6,863,134$

$1,093,026-1,285,361$

$48,787 \quad 12,331,014$

MOTES:

1 TRALSFORAATION LOSS POB

2 TRALSFOBHATIOV LOSS FOR

TRABSPORAATIOH LOSS POR
BLECTRICITY GEH.

.TS $=68.60 \%$

CIS $=0.628$

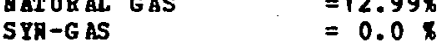


REGIDAAL ENBRGT BALAHCE STATEHENT

B? SECTOR AND POEL TYPE

\begin{tabular}{|c|c|c|c|c|c|c|c|c|c|}
\hline SECTOR & $\begin{array}{c}\text { DIS IILLATE } \\
\text { OIL }\end{array}$ & $\begin{array}{l}\text { RESI.DUAL } \\
\text { OIL }\end{array}$ & $\begin{array}{l}\text { GaSOLIN3 } \\
\text { (ALI D }\end{array}$ & $\begin{array}{l}\text { AYDEO- } \\
\text { CARBOHS } \\
\text { S IN } 10 * * 9\end{array}$ & $\begin{array}{c}\text { CRODE } \\
\text { OIL } \\
\text { ETU'S) }\end{array}$ & $\begin{array}{l}\text { NAT ORAI } \\
\text { GAS }\end{array}$ & $\operatorname{COAL}$ & ELECTEICITY & $\begin{array}{l}\text { SECTOR } \\
\text { TOTAL }\end{array}$ \\
\hline $\begin{array}{l}\text { FINAL DEAAND SECTORS } \\
\text { BBSIDB HTIAL, COBA. } \\
\text { IHDOSTRIAL } \\
\text { TRANSPORTATION } \\
\text { GISCELLAHEOUS OSES }\end{array}$ & $\begin{array}{r}613,000 \\
27,901 \\
60,101 \\
1,602\end{array}$ & $\begin{array}{r}243,166 \\
16.3,198 \\
53,999 \\
43,838\end{array}$ & 666.898 & $\begin{array}{r}92.899 \\
203.570 \\
82.421 \\
4.584\end{array}$ & $\begin{array}{l}- \\
-\end{array}$ & $\begin{array}{r}191.23 c \\
59,19 c \\
600 \\
7.16 c\end{array}$ & $\begin{array}{r}7.171 \\
46.765 \\
149 \\
-\end{array}$ & $\begin{array}{r}156.991 \\
74.771 \\
227 \\
1.056\end{array}$ & $\begin{array}{r}1.309 .466 \\
575.399 \\
864,395 \\
55.044\end{array}$ \\
\hline $\begin{array}{l}\text { TOTAL PINAL } \\
\text { DEEA AD SBCTORS }\end{array}$ & $7[2,1004$ & $50 \Xi, 201$ & 666,898 & 383.474 & - & $258,19=$ & 54,085 & 233.045 & $2,804,304$ \\
\hline $\begin{array}{l}\text { TRANSPORHATION } \\
\text { BLECTRICITY GRN. } \\
\text { PETBOLBDE PBODOCTS } \\
\text { MATURAL GAS } \\
\text { SFH-GAS }\end{array}$ & $\begin{array}{r}11.360 \\
- \\
=\end{array}$ & $\begin{array}{r}527.618 \\
- \\
=\end{array}$ & $\begin{array}{l}- \\
-\end{array}$ & $\begin{array}{r}1,295 \\
-15.167 \\
-\end{array}$ & 15. $16 \overline{-}$ & $\begin{array}{r}6.259 \\
691 \\
- \\
-\end{array}$ & $\begin{array}{r}30.261 \\
- \\
-\end{array}$ & $\begin{array}{r}-174.774 \\
- \\
-\end{array}$ & $\begin{array}{r}402,019 \\
685 \\
- \\
-\end{array}$ \\
\hline $\begin{array}{l}\text { NET POBL OSBD } \\
\text { IA TBANSPORGATION }\end{array}$ & 11,360 & 527,618 & - & $-13,872$ & 15,161 & 6.950 & 30,261 & $-174,774$ & 402,704 \\
\hline $\begin{array}{l}\text { TOTAL GROSS FLORS } \\
\text { LOSSES } 8 \text { OEISSIOHS }\end{array}$ & $\begin{array}{r}713,764 \\
t .3, \equiv 61\end{array}$ & $\begin{array}{r}1.033 .819 \\
-10.090\end{array}$ & $\begin{array}{l}666,898 \\
-21,113\end{array}$ & $\begin{array}{r}184,769 \\
-18,179\end{array}$ & $\begin{array}{r}15,161 \\
22\end{array}$ & $\begin{array}{r}265.147 \\
1.004\end{array}$ & $\begin{array}{r}84,366 \\
1,402\end{array}$ & $\begin{array}{r}233.045 \\
18.637\end{array}$ & $\begin{array}{r}3,207,008 \\
14,644\end{array}$ \\
\hline
\end{tabular}

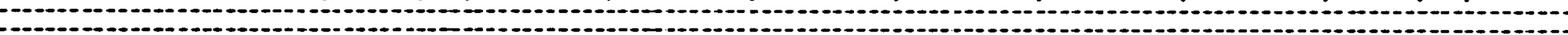

$\begin{aligned} & \text { SOPPLY OP ENERG } \\ & \text { FOSSIL POEL } \\ & \text { HYDROELRCTRIC } \\ & \text { NOCLEAR }\end{aligned}$
GEO.ESOLAR

\section{\$BT InPORTS}

OP REGIO

$757,725 \quad 1.023,328$

645,784

351,422

15,183

266,151

85,748

$9.975 \quad 3,154,719$

BOT ES:

1 TRARSFOREATION LOSS FOR

TRANS PORAATIOY LOSS POR

TRA usponatrion loss

BLECTRICITI EBE. $\quad=69.70 \mathrm{a}$

PETEOLEOH PRCDUCTS $=4.32 x$

SYN-GAS

$=0.0 \times$
$=0.0 \times$ 
REGIONAL BHERGY BALANCE STATEAENT

BY SBCTOR AND POBL TYPE

\begin{tabular}{|c|c|c|c|c|c|c|c|c|c|c|}
\hline SECTOR & $\begin{array}{l}\text { DISTILLATE } \\
\text { OII }\end{array}$ & $\begin{array}{l}\text { RES I DOAL } \\
\text { OIL }\end{array}$ & $\begin{array}{l}\text { GASOLIH } \\
\text { (ALL }\end{array}$ & $\begin{array}{c}\text { OTBER } \\
\text { GYDBO- } \\
\text { CARBOES } \\
\text { ITS I\& 10**9 }\end{array}$ & $\begin{array}{c}\text { CR ODE } \\
\text { OIL } \\
\text { BTO'SI }\end{array}$ & $\begin{array}{l}\text { MATUBAL } \\
\text { GAS }\end{array}$ & $\operatorname{COAL}$ & ELECTRICITY & $\begin{array}{l}\text { SECTOR } \\
\text { TOTAL }\end{array}$ & \\
\hline $\begin{array}{l}\text { FIHAL D BUAYD SECTORS } \\
\text { RESIDE BTIAL, COHE. } \\
\text { INDOSTRIAL } \\
\text { TRAHSP ORTATI ON } \\
\text { GISCBL LAGEOOS OSES }\end{array}$ & $\begin{array}{r}1,105,099 \\
83,105 \\
219,198 \\
6,701\end{array}$ & $\begin{array}{r}494.758 \\
276.706 \\
210.099 \\
75.043\end{array}$ & $1,804,900$ & $\begin{array}{r}239,202 \\
555,717 \\
352,980 \\
11,136\end{array}$ & - & $\begin{array}{r}1.078 .826 \\
605.312 \\
26.700 \\
36.678 \\
\end{array}$ & $\begin{array}{r}39.475 \\
1.100 .851 \\
595 \\
- \\
\end{array}$ & $\begin{array}{r}459,543 \\
321.681 \\
12.190 \\
8,968\end{array}$ & $\begin{array}{r}3,416,903 \\
2,943,372 \\
2,626,662 \\
138,526 \\
\end{array}$ & $\begin{array}{r}328 \\
27 \% \\
24 \% \\
1 \% \\
--\end{array}$ \\
\hline $\begin{array}{l}\text { FOTAL P IHAL } \\
\text { DEGA SBCTORS }\end{array}$ & $1,414,103$ & $1,056,606$ & $1,804,900$ & $1,159,035$ & - & $1,747,516$ & $1,140,921$ & 802,382 & $9,125,463$ & $85 \%$ \\
\hline $\begin{array}{l}\text { TRAYSPORUATIOH } \\
\text { BLECT RICITY GRH. } \\
\text { PETHOLBOA PBODOCTS } \\
\text { MATORAL GAS } \\
\text { SYH-GAS }\end{array}$ & $\begin{array}{r}129,409 \\
-749.754 \\
-\end{array}$ & $\begin{array}{r}884,545 \\
-287,674 \\
-\end{array}$ & $\begin{array}{r}-1.249 .176 \\
-\end{array}$ & $\begin{array}{r}-566,581 \\
-123 \\
-\end{array}$ & $2,740,454$ & $\begin{array}{r}96.498 \\
124,927 \\
141 \\
-\end{array}$ & $\begin{array}{r}1,135,891 \\
= \\
-\end{array}$ & $\begin{array}{r}-670.313 \\
- \\
-\end{array}$ & $\begin{array}{r}1,576,030 \\
12,196 \\
18 \\
-\end{array}$ & $\begin{array}{r}15 \% \\
0 \% \\
0 \%\end{array}$ \\
\hline $\begin{array}{l}\text { NBT POEL OSBD } \\
\text { IB TBAGSPOREATION }\end{array}$ & $-620,345$ & 596,871 & $-1,249,176$ & $-566,704$ & $2,740,454$ & 221,566 & $1,135,891$ & $-670,313$ & $1,588,244$ & $15 \%$ \\
\hline $\begin{array}{l}\text { TOTAL GROSS PLONS } \\
\text { LOSSES \& OHISSIOUS }\end{array}$ & $\begin{array}{r}1,543,512 \\
93,768\end{array}$ & $\begin{array}{r}1.941 .151 \\
-19.597\end{array}$ & $\begin{array}{r}1,804,900 \\
-57,141\end{array}$ & $\begin{array}{r}1,159,035 \\
-54,762\end{array}$ & $\begin{array}{r}2.740 .454 \\
4.154\end{array}$ & $\begin{array}{r}1,969,082 \\
7,456\end{array}$ & $\begin{array}{r}2,276.812 \\
37.856 \\
\end{array}$ & $\begin{array}{r}802,382 \\
64,171\end{array}$ & $\begin{array}{r}10,713,707 \\
75,805\end{array}$ & $\begin{array}{r}99 x \\
1 \% \\
\end{array}$ \\
\hline
\end{tabular}

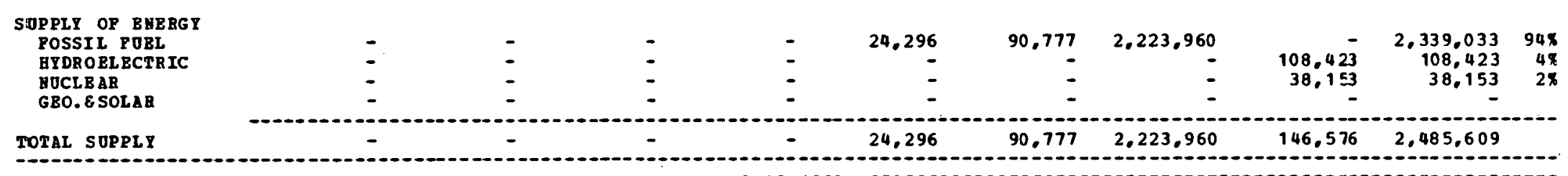

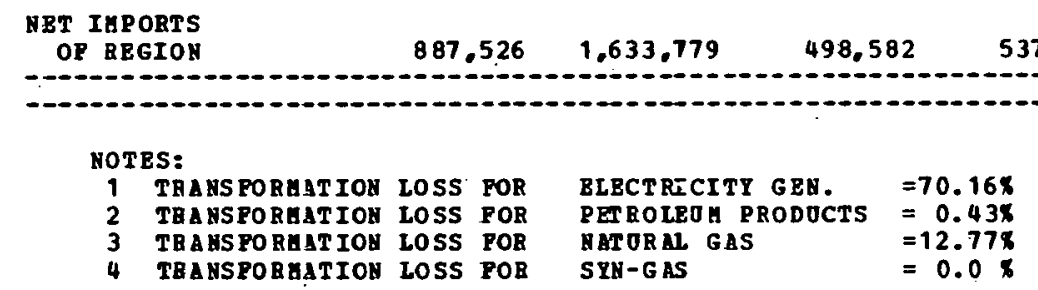


REGIOHAL 3NERGY BALANCE STATEIBNT

BY SECTOR AND POEL TYPE

\begin{tabular}{|c|c|c|c|c|c|c|c|c|c|c|}
\hline SECTOR & $\begin{array}{l}\text { DISTIELATE } \\
\text { OIL }\end{array}$ & $\begin{array}{l}\text { RES I DOAL } \\
\text { OII }\end{array}$ & $\begin{array}{l}\text { GASOL INZ } \\
\text { (ALL } 0\end{array}$ & $\begin{array}{c}\text { OTHER } \\
\text { AYDRO- } \\
\text { CARBOHS } \\
\text { NITS IN } 10 * * 9\end{array}$ & $\begin{array}{c}\text { CRODE } \\
\text { OIL } \\
\text { BTO'S) }\end{array}$ & $\begin{array}{l}\text { SAT ORAL } \\
\text { GAS }\end{array}$ & COAL & BLECTRICITY & $\begin{array}{r}\text { SECTOR } \\
\text { TOTAL }\end{array}$ & \\
\hline 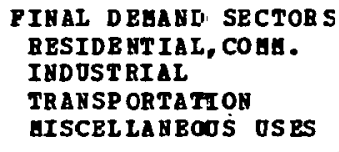 & $\begin{array}{r}601,802 \\
133,590 \\
375,800 \\
9.298\end{array}$ & $\begin{array}{r}86,568 \\
199,997 \\
6,197 \\
18,234\end{array}$ & $2,518,602$ & $\begin{array}{r}507.795 \\
823.971 \\
296.310 \\
22.922\end{array}$ & $\bar{z}$ & $\begin{array}{r}2,171,092 \\
1,710, .406 \\
65,701 \\
94.818\end{array}$ & $\begin{array}{r}103,338 \\
1,652,746 \\
548 \\
-\end{array}$ & $\begin{array}{r}571.921 \\
546.703 \\
1.865 \\
10.481\end{array}$ & $\begin{array}{r}4,042,516 \\
5,067,113 \\
3,265,023 \\
155,753\end{array}$ & $\begin{array}{r}27 \% \\
34 \% \\
22 \% \\
1 \%\end{array}$ \\
\hline $\begin{array}{l}\text { TOTAL FINAL } \\
\text { DBEA BD SETTOES }\end{array}$ & 1.120 .490 & $310,9.96$ & $2,518,602$ & $1,650,998$ & - & 4.042 .0 .17 & $1,756,332$ & $1,130,970$ & $12,530,405$ & $84 \%$ \\
\hline $\begin{array}{l}\text { TRANSPORBATDON } \\
\text { BLECTRICITY GEH. } \\
\text { PETROLEOH PRODDCTS } \\
\text { HATORAL GAS } \\
\text { SYH-GAS }\end{array}$ & $\begin{array}{r}44,051 \\
-1.049, .780 \\
-\end{array}$ & $\begin{array}{r}134,956 \\
-335,562 \\
-\end{array}$ & $\begin{array}{r}-2,386,506 \\
-\end{array}$ & $\begin{array}{r}9,116 \\
-1.024 .702 \\
-20.385\end{array}$ & $4.607 .57 \overline{-}$ & $\begin{array}{r}160,073 \\
215,472 \\
23,429\end{array}$ & $\begin{array}{r}2,962,582 \\
= \\
=\end{array}$ & $\begin{array}{r}-1.037 .964 \\
-\end{array}$ & $\begin{array}{r}2.272 .814 \\
26,401 \\
3.044\end{array}$ & $\begin{array}{r}158 \\
0 \% \\
0 \%\end{array}$ \\
\hline $\begin{array}{l}\text { NET FOEL OSID } \\
\text { IA TRANSPCRHATION }\end{array}$ & $-1,305,729$ & $r-200,606$ & $-2,386,506$ & $-1,035,971$ & 4.607 .579 & 398,974 & $2,962,582$ & $-1,037,964$ & $2,302,259$ & $15 \%$ \\
\hline $\begin{array}{l}\text { TOTAL GROSS PLOES } \\
\text { LOSSES } 8 \text { OELSSIOAS }\end{array}$ & $\begin{array}{r}1.164 .541 \\
76.745\end{array}$ & $\begin{array}{r}145,952 \\
-4,525\end{array}$ & $\begin{array}{r}2,518,502 \\
-79,736\end{array}$ & $\begin{array}{r}1,660,114 \\
-78,437\end{array}$ & $\begin{array}{r}4,607,579 \\
6,985\end{array}$ & $\begin{array}{r}4,440,5.91 \\
16,8.16\end{array}$ & $\begin{array}{r}4.718 .914 \\
78.461\end{array}$ & $\begin{array}{r}1,130,970 \\
90,4,50\end{array}$ & $\begin{array}{r}14,832,664 \\
100,759\end{array}$ & $\begin{array}{r}99 \% \\
1 \%\end{array}$ \\
\hline
\end{tabular}

\begin{tabular}{|c|c|c|c|c|c|c|c|c|c|c|}
\hline $\begin{array}{l}\text { SUPRLY OP EQBBG } \\
\text { FOSSIL FOEL } \\
\text { GYDROELECERIC } \\
\text { ROCLEAR } \\
\text { GBO. E SOLAR }\end{array}$ & $\bar{E}$ & ! & $\begin{array}{l}- \\
\overline{-}\end{array}$ & : & $\begin{array}{r}337,643 \\
= \\
-\end{array}$ & $\begin{array}{r}152,175 \\
= \\
=\end{array}$ & $\begin{array}{r}3,114,815 \\
= \\
=\end{array}$ & $\begin{array}{r}14,687 \\
96,719\end{array}$ & $\begin{array}{r}3,604,63 \equiv \\
14,68 \\
96,719\end{array}$ & $\begin{array}{r}97 \% \\
08 \\
3 \% \\
08\end{array}$ \\
\hline OPPLI & - & - & - & - & 337.643 & 152,175 & $3,114,815$ & 111.417 & $3,716,05$ & \\
\hline
\end{tabular}

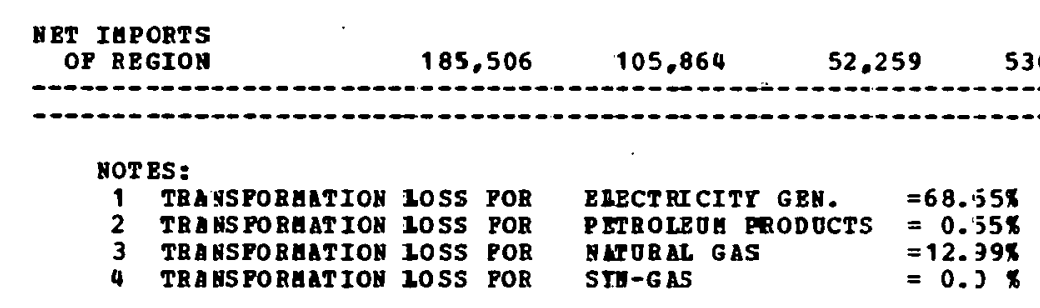


REG IONAL EHEGGT BALAACE STATEAENT

BI SECTOR AND FOEL TYPE

\begin{tabular}{|c|c|c|c|c|c|c|c|c|c|c|}
\hline SECTOR & $\begin{array}{l}\text { DISTILLATE } \\
\text { OIL }\end{array}$ & $\begin{array}{l}\text { RESI DOAL } \\
\text { O II }\end{array}$ & $\begin{array}{l}\text { GASOLIHE } \\
\text { (ALL OA] }\end{array}$ & $\begin{array}{c}\text { OTHER } \\
\text { GIDRO- } \\
\text { CARBONS } \\
\text { TS IN 10**9 }\end{array}$ & $\begin{array}{l}\text { CRODE } \\
\text { OIL } \\
\text { BTO'S) }\end{array}$ & $\begin{array}{l}\text { NAT ORAL } \\
\text { GAS }\end{array}$ & COAL & ELECTRICITY & $\begin{array}{r}\text { SECTOR } \\
\text { TOTAL }\end{array}$ & \\
\hline 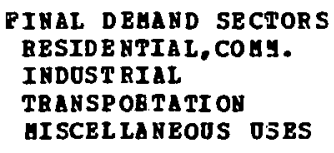 & $\begin{array}{r}213.900 \\
66,494 \\
210.902 \\
5,604\end{array}$ & $\begin{array}{r}15,256 \\
53,285 \\
4,301 \\
3,938\end{array}$ & $\begin{array}{r}- \\
1.249,999 \\
-\end{array}$ & $\begin{array}{r}327.599 \\
197.471 \\
121.266 \\
11.676\end{array}$ & $\begin{array}{l}- \\
-\end{array}$ & $\begin{array}{r}829.202 \\
683.899 \\
120.201 \\
49.893\end{array}$ & $\begin{array}{r}21.953 \\
165.263 \\
252 \\
-\end{array}$ & $\begin{array}{r}237,147 \\
130,908 \\
320 \\
2.950\end{array}$ & $\begin{array}{r}1.645 .057 \\
1.297 .320 \\
1.707 .241 \\
74,061\end{array}$ & $\begin{array}{r}308 \\
238 \\
31 \% \\
18\end{array}$ \\
\hline $\begin{array}{l}\text { TOTAL PINAL } \\
\text { DEEA ND SECTORS }\end{array}$ & 496,900 & 76.780 & $1,249,999$ & 658.012 & - & $1,683,195$ & 187,468 & 371.325 & $\begin{array}{r}4,723.679 \\
\end{array}$ & $85 \%$ \\
\hline $\begin{array}{l}\text { TRAYSPORHATIOK } \\
\text { BLECT RICITY GER. } \\
\text { PETBOLEOA PBODOCTS } \\
\text { NATORAL GAS }\end{array}$ & $\begin{array}{r}11.974 \\
-379.929 \\
-\end{array}$ & $\begin{array}{r}16,773 \\
-66,919 \\
-\end{array}$ & $-786,31 \overline{-}$ & $\begin{array}{r}283 \\
-281,036 \\
-70,306\end{array}$ & $1,452,582$ & $\begin{array}{r}413.850 \\
67.255 \\
80.803\end{array}$ & $\begin{array}{r}683.834 \\
- \\
-\end{array}$ & $\begin{array}{r}-326,247 \\
-\end{array}$ & $\begin{array}{r}800,467 \\
5,637 \\
10,497\end{array}$ & $\begin{array}{r}14 \% \\
0 \% \\
0 \%\end{array}$ \\
\hline $\begin{array}{l}\text { SYA-GAS } \\
\text { GET POEL OSED } \\
\text { IN TRANSPORHA TION }\end{array}$ & -367.955 & $-50,146$ & $-786,316$ & -351.059 & 1.452 .582 & 561,908 & 683,834 & -326.247 & 816,601 & $15 \%$ \\
\hline $\begin{array}{l}\text { TOTAL GROSS FLORS } \\
\text { LOSSES } \varepsilon \text { OUISSIOUS }\end{array}$ & $\begin{array}{r}508,874 \\
30,914\end{array}$ & $\begin{array}{r}93.553 \\
-949\end{array}$ & $\begin{array}{r}1.249,999 \\
-39,573\end{array}$ & $\begin{array}{l}658,295 \\
-31,103\end{array}$ & $\begin{array}{r}1.452,582 \\
2.202\end{array}$ & $\begin{array}{r}2.245,103 \\
8,501\end{array}$ & $\begin{array}{r}871.302 \\
14.487\end{array}$ & $\begin{array}{r}371.325 \\
29.696\end{array}$ & $\begin{array}{r}5,540,280 \\
14,175\end{array}$ & $\begin{array}{r}100 \% \\
0 \%\end{array}$ \\
\hline
\end{tabular}

(1)

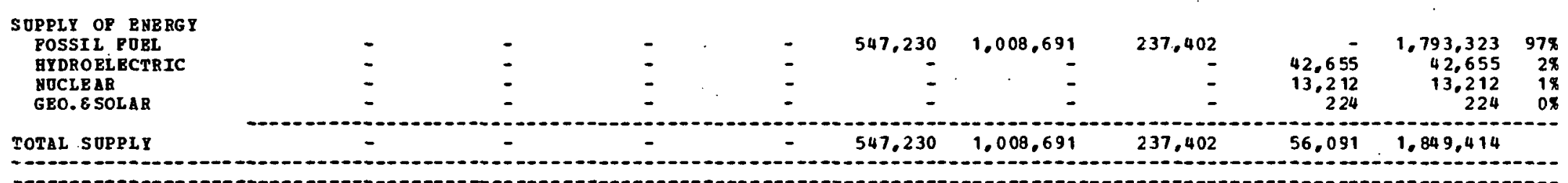

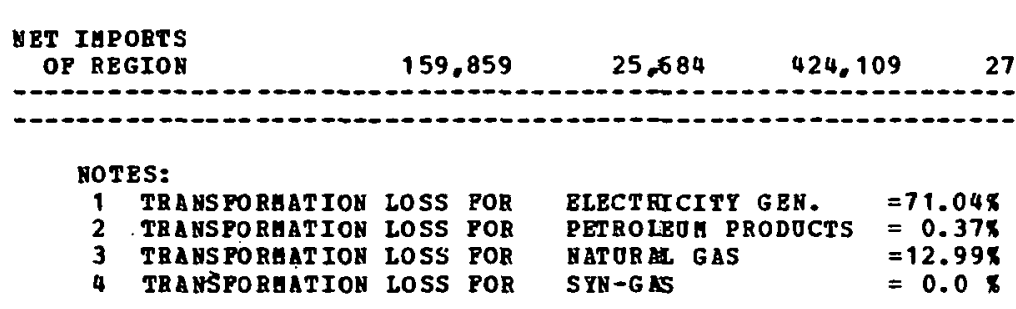


REGIONAL BNERGT BALANCE STATEYEHT

BY SECTOR AND POEL TYPE

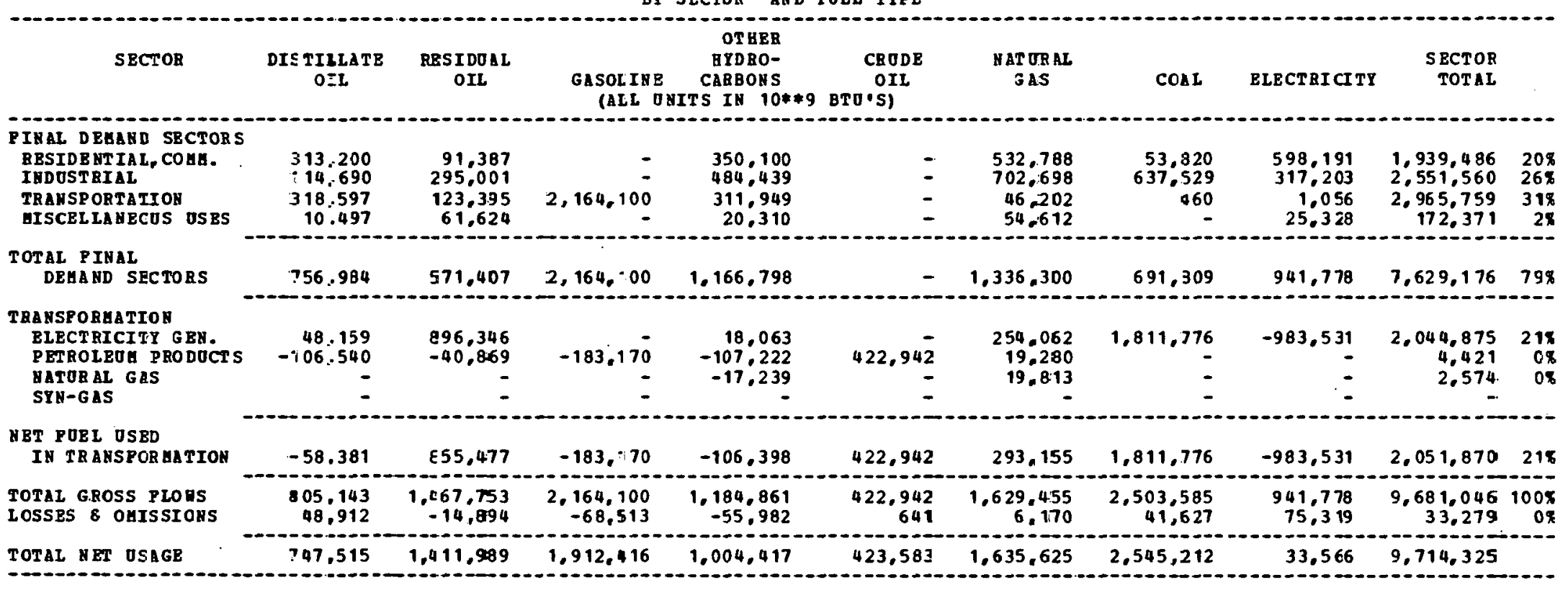

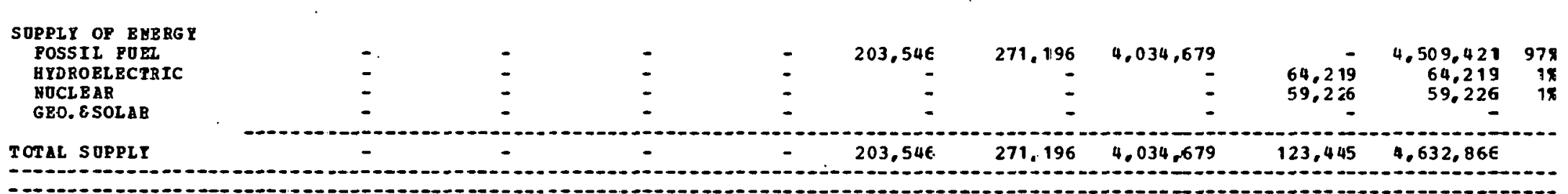

\begin{tabular}{l}
$\begin{array}{l}\text { MBT IUPORTS } \\
\text { OP REGIOU }\end{array}$ \\
\hdashline$\quad 147,515$
\end{tabular}


REGIONAL EMERGY BALANCE STA TELENT

BY SECTOR AND POEL TYPE

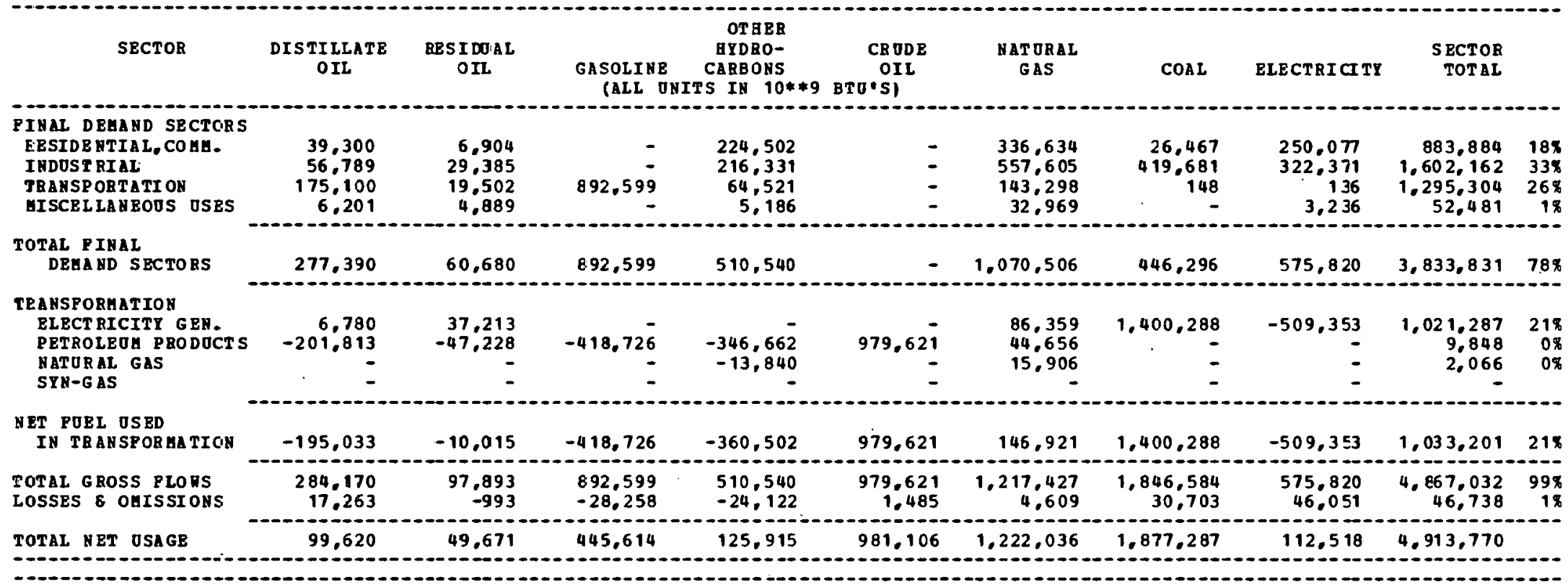

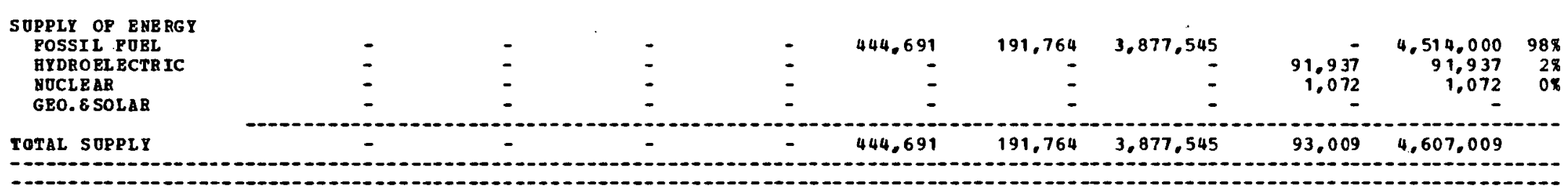

\section{NET IHPORTS}

99.620

49,671

445,614

125,915

$536,415 \quad 1,030,272 \quad-2,000,257$

19,509

306,761

NOTES:

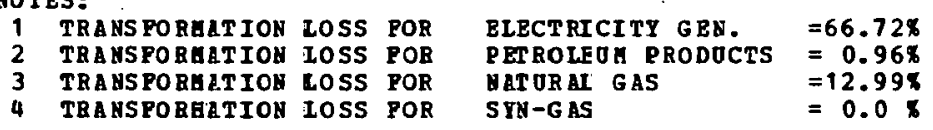


REGIONAL ENERGY BALAHCE STATEEEHT

BY SECTOB AND FOEL TYPE
BY TERG BALAHCE STAT

\begin{tabular}{|c|c|c|c|c|c|c|c|c|c|c|}
\hline SECTOR & $\begin{array}{c}\text { DIS TILLATE } \\
\text { OIL }\end{array}$ & $\begin{array}{c}\text { RE SIDVAL } \\
\text { OIL }\end{array}$ & $\begin{array}{l}\text { GASOLINE } \\
\text { (ALLL } 0\end{array}$ & $\begin{array}{c}\text { HYDBO- } \\
\text { CARBONS } \\
\text { IITS IH 10**9 }\end{array}$ & $\begin{array}{c}\text { CRODE } \\
\text { OIL } \\
\text { BTU-SI }\end{array}$ & $\begin{array}{l}\text { HAT OEAL } \\
\text { GAS }\end{array}$ & COAI & ELECTRICITY & $\begin{array}{r}\text { SECTOR } \\
\text { TOTAL }\end{array}$ & \\
\hline $\begin{array}{l}\text { PINAL DEHA D SECTORS } \\
\text { RESIDE HTIAL, COAA. } \\
\text { INDUSTRIAL } \\
\text { TRANSPORTATIOH } \\
\text { GISCELLA HEOUS OSBS }\end{array}$ & $\begin{array}{r}45,799 \\
129,583 \\
337.699 \\
15,900\end{array}$ & $\begin{array}{r}8,806 \\
70,795 \\
163.699 \\
13,690\end{array}$ & $.027,431$ & $\begin{array}{r}334,099 \\
1,119,875 \\
248,540 \\
10,508\end{array}$ & $\begin{array}{l}- \\
- \\
-\end{array}$ & $\begin{array}{r}706,950 \\
4.871,489 \\
231.083 \\
54,648\end{array}$ & $\begin{array}{r}10.721 \\
83.034 \\
302 \\
-\end{array}$ & $\begin{array}{r}373,583 \\
281,703 \\
276 \\
11,442\end{array}$ & $\begin{array}{r}1,479,958 \\
6,556,479 \\
2,508,920 \\
106,188\end{array}$ & $\begin{array}{r}128 \\
538 \\
20 \% \\
18\end{array}$ \\
\hline $\begin{array}{l}\text { TOTAL FINAL } \\
\text { DBVAND SECTORS }\end{array}$ & 528,981 & 256,990 & $1,527,401$ & $1,713,022$ & - & $5,864.050$ & 94.057 & 667.004 & $10,651,545$ & $86 \%$ \\
\hline $\begin{array}{l}\text { TRANSPORLATIOH } \\
\text { BLECTRICITY GER. } \\
\text { PETROLEOA ?BODOCTS } \\
\text { NATURAL GAS } \\
\text { SYN-GAS }\end{array}$ & $\begin{array}{r}1.271 \\
-2.545 .039 \\
-\end{array}$ & $\begin{array}{r}131.243 \\
-546.210 \\
-\end{array}$ & $\begin{array}{r}-5.318 .653 \\
- \\
-\end{array}$ & $\begin{array}{r}-2,846,191 \\
-1.065,057\end{array}$ & $\begin{array}{r}10,938,851 \\
-\end{array}$ & $\begin{array}{r}1,988,527 \\
498,65.5 \\
1,224,091 \\
-\end{array}$ & $\begin{array}{r}66,289 \\
\vdots \\
-\end{array}$ & $\begin{array}{r}-710,680 \\
= \\
=\end{array}$ & $\begin{array}{r}1,476,650 \\
81,413 \\
159,034 \\
-\end{array}$ & $\begin{array}{r}128 \\
18 \\
18\end{array}$ \\
\hline $\begin{array}{l}\text { NET POEL OSBD } \\
\text { IN TRAHSFOBAATIOH }\end{array}$ & $-2,543,768$ & $-414,967$ & $-5,318,653$ & $-3,911.248$ & $10,938,851$ & $3,711,273$ & 66.289 & $-710,680$ & $1.717,097$ & 14.8 \\
\hline $\begin{array}{l}\text { TOTAL GROSS PLORS } \\
\text { LOSSES } 8 \text { OUISSIOHS }\end{array}$ & $\begin{array}{r}5.30 .252 \\
32.212 \\
-\end{array}$ & $\begin{array}{r}388,233 \\
-3,939 \\
--9-0\end{array}$ & $\begin{array}{r}1,527,401 \\
-48,356\end{array}$ & $\begin{array}{r}1.713,022 \\
-80.937\end{array}$ & $\begin{array}{r}10,938,851 \\
16,584 \\
-\end{array}$ & $\begin{array}{r}9,575,363 \\
36,258 \\
\hdashline\end{array}$ & $\begin{array}{r}160, \equiv 46 \\
2, E 66\end{array}$ & $\begin{array}{r}667,004 \\
53,344 \\
\end{array}$ & $\begin{array}{r}12,368,642 \\
7,832 \\
-\end{array}$ & $\begin{array}{r}0.8 \\
0.8\end{array}$ \\
\hline
\end{tabular}

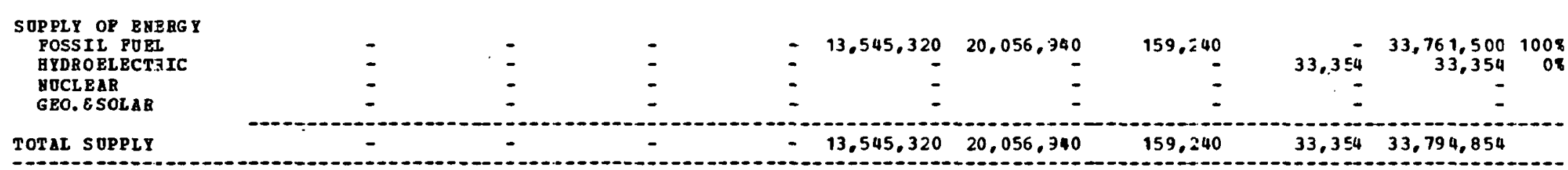

$\begin{array}{ll}-1 & \end{array}$

\section{NET IUPORTS}

OP REGION $\quad-2,382,574 \quad-161,916 \quad-3,839,6.08 \quad-2,279,163 \quad-2,589,884-10,445,318 \quad 3,772 \quad-23,685-21,418,379$

-

NOT BS:

TRANSPORAATION LUSS POR TRA FS FORATION LDSS POR TRANSPORUATION LUSS POR THA rS FORATION LOSS POB

BLECT RICITY $3 \mathrm{EN}$

PEROLEOA PR $=67.51 \mathrm{~s}$ MATOR AL GAS STW-G AS
$=67.518$
$=0.718$

$=12.998$ $=0.08$ 
REgIOHAL ENERGY BALANCE STATEHENT

BY SECTOR AND POEL TYPE

\begin{tabular}{|c|c|c|c|c|c|c|c|c|c|c|}
\hline SECTOR & $\begin{array}{l}\text { CIS TILLATE } \\
\text { OIL }\end{array}$ & $\begin{array}{l}\text { RES I DCAL } \\
\text { O Il }\end{array}$ & $\begin{array}{l}\text { GASOLINE } \\
\text { (ALL ONII }\end{array}$ & $\begin{array}{c}\text { OTHER } \\
\text { HYDRO- } \\
\text { CARBOHS } \\
\text { TS IN 10**9 }\end{array}$ & $\begin{array}{c}\text { CRODE } \\
\text { OIL } \\
\text { BTO'SI }\end{array}$ & $\begin{array}{l}\text { MAT ORAL } \\
\text { GAS }\end{array}$ & $\operatorname{COAL}$ & ELECTRICITY & $\begin{array}{l}\text { SECTOR } \\
\text { TOTAL }\end{array}$ & \\
\hline $\begin{array}{l}\text { FINAL DELAND SECTORS } \\
\text { RESIDE NTIAL, COAH. } \\
\text { INDOSTRIAL } \\
\text { TRANSPOBTATI OA } \\
\text { IISCELLANEOOS OSBS }\end{array}$ & $\begin{array}{r}63,602 \\
82,599 \\
164,004 \\
8,501\end{array}$ & $\begin{array}{r}9, E 20 \\
42, E 97 \\
2, \pm 00 \\
6, \pm 81\end{array}$ & $\begin{array}{r}- \\
704.997 \\
-\end{array}$ & $\begin{array}{r}156.303 \\
70.460 \\
113.632 \\
4.796\end{array}$ & $\begin{array}{l}- \\
\overline{-}\end{array}$ & $\begin{array}{r}421,990 \\
510,392 \\
73,099 \\
43,514\end{array}$ & $\begin{array}{r}18.297 \\
129.892 \\
131 \\
-\end{array}$ & $\begin{array}{r}166,108 \\
91,418 \\
302 \\
5.339\end{array}$ & $\begin{array}{r}835,920 \\
927,458 \\
1,058,365 \\
68,531\end{array}$ & $\begin{array}{r}248 \\
27 \% \\
31 \% \\
28\end{array}$ \\
\hline $\begin{array}{l}\text { TOTAL PINAL } \\
\text { DEHA ND SECTORS }\end{array}$ & 318,706 & $60, \varepsilon 98$ & 704.997 & 345,191 & - & $1.048,995$ & 148,320 & 263,167 & $2,890,274$ & $84 \pi$ \\
\hline $\begin{array}{l}\text { TRANSPORHATION } \\
\text { BLECTRICITY GEH. } \\
\text { PETROLEOH PRODOCTS } \\
\text { HATORAL GAS } \\
\text { SYH-GAS }\end{array}$ & $\begin{array}{r}4.049 \\
-265.430 \\
- \\
-\end{array}$ & $\begin{array}{r}57.734 \\
-69,346 \\
- \\
-\end{array}$ & $\begin{array}{r}-487.014 \\
-\end{array}$ & $\begin{array}{l}-201.228 \\
-125,793\end{array}$ & $\begin{array}{r}981,24 \overline{3} \\
-\end{array}$ & $\begin{array}{r}234.280 \\
44.731 \\
144.573 \\
-\end{array}$ & $\begin{array}{r}459,046 \\
= \\
=\end{array}$ & $\begin{array}{r}-231.243 \\
- \\
-\end{array}$ & $\begin{array}{r}523,866 \\
2,956 \\
18,780 \\
-\end{array}$ & $\begin{array}{r}15 \% \\
0 \% \\
18\end{array}$ \\
\hline $\begin{array}{l}\text { NET FOEL OSED } \\
\text { IN TRANSPOREATION. }\end{array}$ & $-261,381$ & $-11,612$ & $-487,014$ & $-327,021$ & 981,243 & 423,584 & 459,046 & $-231,243$ & 545,602 & $16 \%$ \\
\hline $\begin{array}{l}\text { TOTAL GROSS PLONS } \\
\text { LOSSES } \& \text { OUISSIONS }\end{array}$ & $\begin{array}{r}322.755 \\
19.607\end{array}$ & $\begin{array}{r}118,632 \\
-1,203\end{array}$ & $\begin{array}{l}704,997 \\
-22,319\end{array}$ & $\begin{array}{l}345,191 \\
-16,309\end{array}$ & $\begin{array}{r}981,243 \\
1,487\end{array}$ & $\begin{array}{r}1.472 .579 \\
5.576\end{array}$ & $\begin{array}{r}607,366 \\
10,098\end{array}$ & $\begin{array}{r}263,167 \\
21,046\end{array}$ & $\begin{array}{r}3,435,876 \\
17.983\end{array}$ & $\begin{array}{r}99 \% \\
1 \%\end{array}$ \\
\hline
\end{tabular}

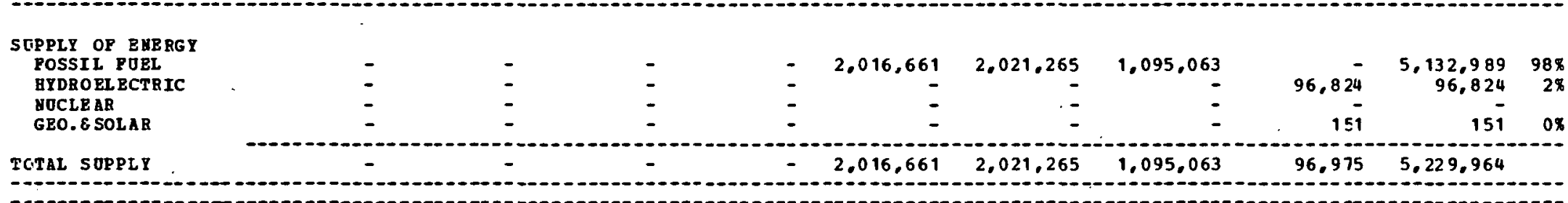

BRT IUPORTS
OP REGIOB


REGIONAL ELERST BALANCE STATEHENT

BY SECTOR AND POEL TYPE

\begin{tabular}{|c|c|c|c|c|c|c|c|c|c|c|}
\hline SECTOR & $\begin{array}{c}\text { DISTILLATE } \\
\text { OIL }\end{array}$ & $\begin{array}{l}\text { RES I DUAL } \\
\text { C IL }\end{array}$ & $\begin{array}{l}\text { GASOLIME } \\
\text { GALL O }\end{array}$ & $\begin{array}{c}\text { EYDRO- } \\
\text { CLRBONS } \\
\text { ITS IN 10**9 }\end{array}$ & $\begin{array}{c}\text { CRUDE } \\
\text { OIL } \\
\text { BTO'S) }\end{array}$ & $\begin{array}{l}\text { HAT ORAL } \\
\text { GAS }\end{array}$ & COAL & ELECTRI CITPY & $\begin{array}{r}\text { SECTOR } \\
\text { TOTAL }\end{array}$ & \\
\hline $\begin{array}{l}\text { PIHAL DEMAND SECTORS } \\
\text { RESIDENTIAL, COHA. } \\
\text { IHDOSTRIAL } \\
\text { TRANSPORTATIOH } \\
\text { GISCELLABEOUS OSES }\end{array}$ & $\begin{array}{r}1 \equiv 1,299 \\
1<7,704 \\
257,698 \\
5,201\end{array}$ & $\begin{array}{r}58.923 \\
14 L .494 \\
149.100 \\
20.481\end{array}$ & $1,726,401$ & $\begin{array}{r}171,501 \\
325,909 \\
452,781 \\
14,184\end{array}$ & $\begin{array}{l}- \\
-\end{array}$ & $\begin{array}{r}921,239 \\
953,304 \\
36,80: 3 \\
69,662\end{array}$ & $\begin{array}{r}15,273 \\
141,132 \\
406 \\
-\end{array}$ & $\begin{array}{r}487.577 \\
304.391 \\
643 \\
.15 .595\end{array}$ & $\begin{array}{r}1,785,811 \\
1,976,934 \\
2,663,829 \\
125,123\end{array}$ & $\begin{array}{r}24 \% \\
27 \% \\
36 \% \\
2 \%\end{array}$ \\
\hline $\begin{array}{l}\text { TOTAL P IYAL } \\
\text { DELA ND SECFORS }\end{array}$ & 541,902 & 372,998 & $1.726 .4 C 1$ & 364,375 & - & $1,981,0.04$ & 156.841 & 808,206 & 6.551 .697 & $89 \%$ \\
\hline $\begin{array}{l}\text { ZRANSEOREATIOI } \\
\text { ELECTRICITY GEN. } \\
\text { PETROLEO PBODOCT S } \\
\text { NATURAL GAS } \\
\text { SYN-GAS }\end{array}$ & $\begin{array}{r}5.966 \\
-5.97,640 \\
-\end{array}$ & $\begin{array}{r}533,199 \\
-835,542 \\
-\end{array}$ & $\begin{array}{r}-1.759,410 \\
- \\
-\end{array}$ & $\begin{array}{r}50,017 \\
-1,142,380 \\
-27,176 \\
\end{array}$ & $4,177,642$ & $\begin{array}{r}466,485 \\
183.967 \\
31.236 \\
-\end{array}$ & $\begin{array}{r}88,993 \\
- \\
-\end{array}$ & $\begin{array}{r}-350,450 \\
- \\
-\end{array}$ & $\begin{array}{r}794,211 \\
26,637 \\
4.060 \\
-\end{array}$ & $\begin{array}{r}118 \\
0 \% \\
0 \%\end{array}$ \\
\hline $\begin{array}{l}\text { NET POEI OSED } \\
\text { IA TR ANSPORGATION }\end{array}$ & $-591,674$ & $-302,343$ & $-1,759,410$ & $-1,115.539$ & 4.177 .642 & 681,689 & $88,9 \ni 3$ & $-350,450$ & 824,908 & $11 x$ \\
\hline $\begin{array}{l}\text { TOTAL GROSS FLOHS } \\
\text { LOSSES } 8 \text { OUISIIOHS }\end{array}$ & $\begin{array}{r}547.868 \\
33.282\end{array}$ & $\begin{array}{r}906,197 \\
-9,195\end{array}$ & $\begin{array}{r}1.726,401 \\
-54.656\end{array}$ & $\begin{array}{r}1,310,392 \\
-47,928\end{array}$ & $\begin{array}{r}4,177,642 \\
6,333\end{array}$ & $\begin{array}{r}2,662,693 \\
10,082\end{array}$ & $\begin{array}{r}245,804 \\
4,087\end{array}$ & $\begin{array}{r}808,206 \\
64,636\end{array}$ & $\begin{array}{r}7.376 .605 \\
6.642\end{array}$ & $\begin{array}{r}100 x \\
0 \times\end{array}$ \\
\hline $\begin{array}{l}\text { SOPPLY OP ENERGI } \\
\text { POSSIL PORI. } \\
\text { HYDROELECTEIC } \\
\text { NOCLEAR } \\
\text { GEO.ESOLAR }\end{array}$ & I & $\begin{array}{l}\overline{-} \\
\overline{-}\end{array}$ & $\overline{-}$ & - & $\begin{array}{r}2,373,452 \\
= \\
-\end{array}$ & $\begin{array}{r}688,481 \\
-\end{array}$ & $\begin{array}{r}79,107 \\
- \\
-\end{array}$ & $\begin{array}{r}456.584 \\
22.300 \\
7.085\end{array}$ & $\begin{array}{r}3,141,040 \\
456,584 \\
22,300 \\
7,085\end{array}$ & $\begin{array}{r}878 \\
13 \% \\
1 \% \\
08\end{array}$ \\
\hline
\end{tabular}

\section{YET IHPORTS}

OP Q EGION

$-16.489$

61.459

$-87.655$

$1,810,523$

$1,984,264$

170.784

$36.423 \quad 3,756,238$

NOT BS:

\begin{tabular}{|c|c|c|c|c|}
\hline $\begin{array}{l}\text { TRA NSPORAATION } \\
\text { TRABSPORATION } \\
\text { TRABSPOREATION }\end{array}$ & $\begin{array}{l}\text { LoS } \\
\text { LoS } \\
\text { LOS }\end{array}$ & $\begin{array}{l}\text { POB } \\
\text { POR } \\
\text { POB }\end{array}$ & $\begin{array}{l}\text { ELECT RICITY CEN. } \\
\text { PETROIRUA PRODOCTS } \\
\text { N ROURAL GAS }\end{array}$ & $\begin{array}{l}3.8 \\
1 \% \\
1 \%\end{array}$ \\
\hline & & & & \\
\hline
\end{tabular}




\section{INTERNAL DISTRIBUTION}

1. S. I. Auerbach

2. L. G. Berry

3. B. H. Bronfman

4. R. S. Carlsmith

5. W. S. Chern

6. F. L. Culler

7. R. M. Davis

8. R. C. DeVault

9. J. F. Dobson

10. W. Fulkerson

11. H. H. Herzog

12. E. A. Hirst

13. R. B. Honea

14. D. J. Jacobs

15. D. L. Kaserman

16. C. R. Kerley

17. A. S. Loebl

18. D. C. Parzyck

19. E. B. Peelle

20. H. Postma
21. B. J. Purdy

22. P. L. Rice.

23. C. R. Richmond

24. M. W. Rosenthal

25. R. H. Row

26. R. M. Rush

27. R. L. Spore

28. R. I. Van Hook

29. A. H. Voelker

30. D. P. Vogt

31- 32. Central Research Library

33. Document Reference Section

34- 35. Laboratory Records

36. Laboratory Records - RC

37. ORNL Patent Office

38-119. Regional Economic Analysis Group

Economic Analysis Section

Energy Division

\section{EXTERNAL DISTRIBUTION}

120. Director, Research and Technical Support Division, DOE-ORO. 121-147. Technical Information Center, DOE-ORO.

148-300. Economic Analysis Section Distribution, Energy Division. 